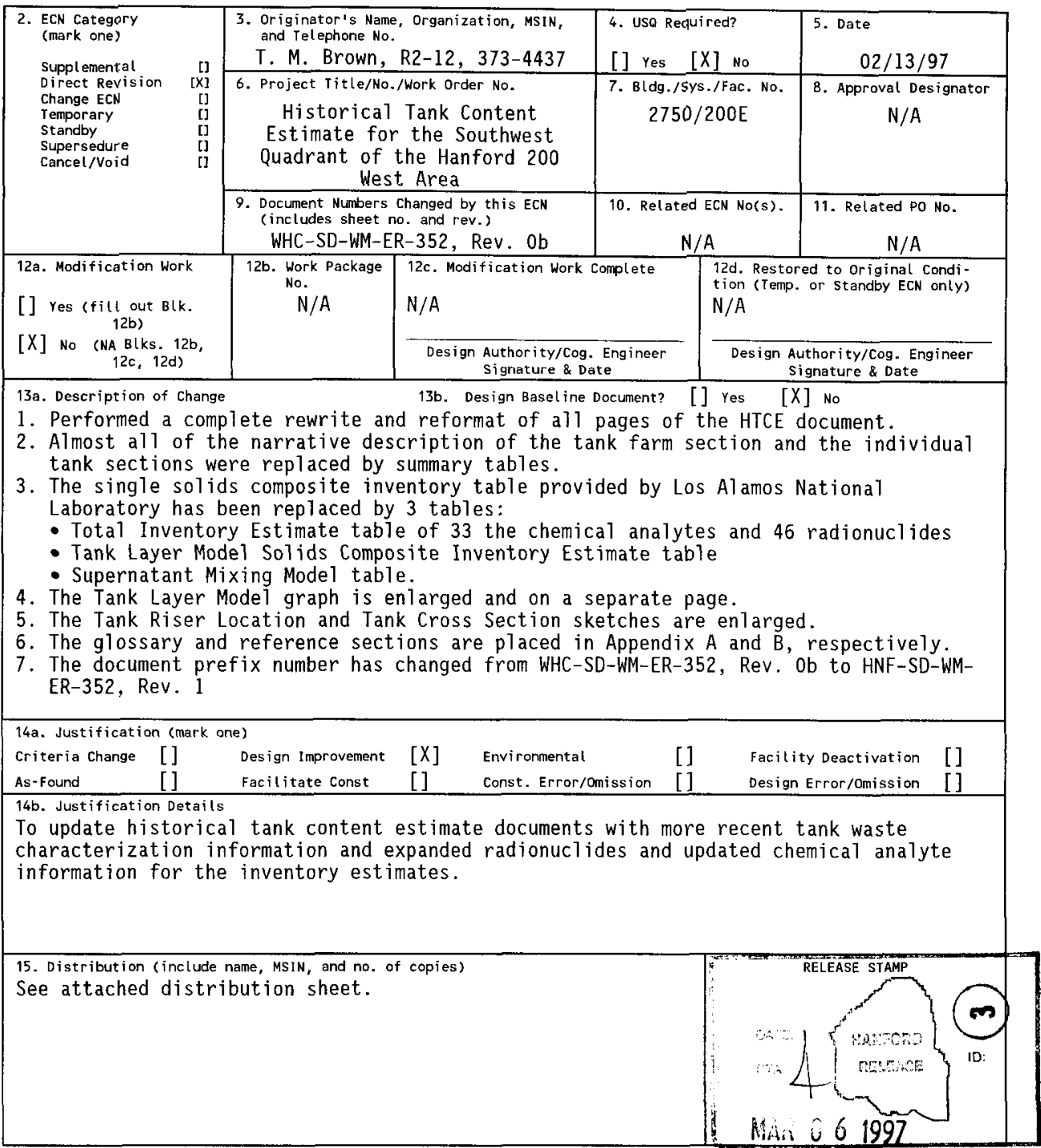

A-7900-013-2 (05/96) GEF095 


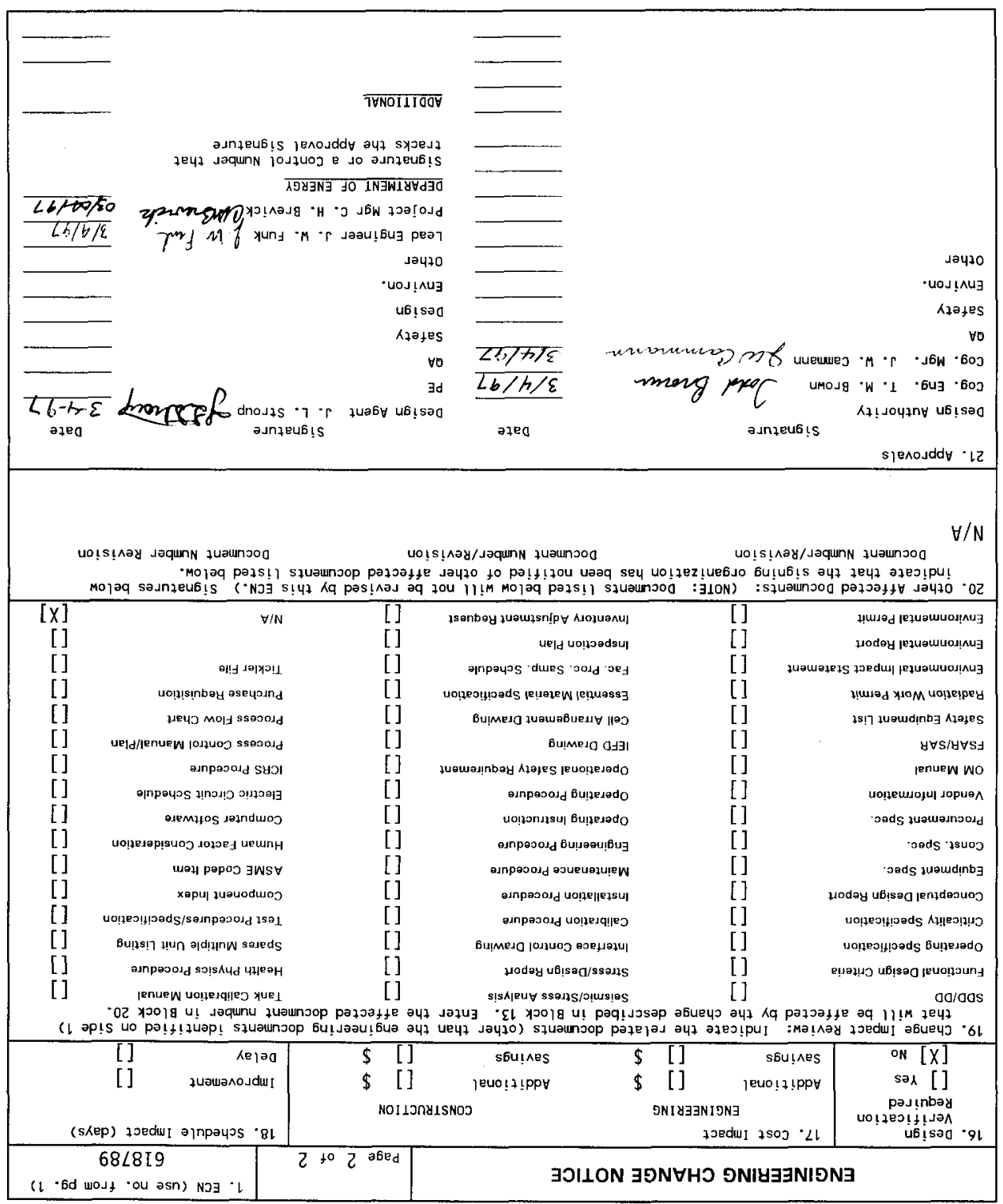




\title{
Historical Tank Content Estimate for the Southwest Quadrant of the Hanford 200 West Area
}

\author{
C. H. Brevick, J. L. Stroup, J. W. Funk \\ Fluor Daniel Northwest Inc., Richland, WA 99352 \\ U.S. Department of Energy Contract DE-AC06-96RL13200
}
EDT/ECN: 618789
UC: 2070
Org Code: 408
B\&R Code: EW3120074
Charge Code: El8675
Total Pages: $: 737527$

Key Words: Southwest quadrant, Historical Tank Content Estimate, tank farms, tank level, tank temperature, tank farm aerial photos, in-tank montages, TLM, SMM, inventory estimates, riser locations

Abstract: The Historical Tank Content Estimate for the Quadrant provides historical information on a tank-by- tank basis of the radioactive mixed wastes stored in the underground single-shell tanks for the Hanford 200 West Area. This report summarized historical information such as waste history, level history, temperature history, riser configuration, tank integrity, and inventory estimates on a tankby-tank basis. Tank farm aerial photographs and interior tank montages are also provided for each tank. A description of the development of data for the document of the inventory estimates provided by Los Alamos National Laboratory are also given in this report.

TRADEMARK DISCLAIMER. Reference herein to any specific comercial product, process, or service by trade name, trademark, manufacturer, or otherwise, does not necessarily constitute or imply its endorsement, recommendation, or favoring by the United States Government or any agency thereof or its contractors or subcontractors.

Printed in the United States of America. To obtain copies of this document, contact: Document Control Services, P.0. Box 950, Mailstop H6-08, Richland WA 99352, Phone (509) 372-2420;

Fax (509) 376-4989.
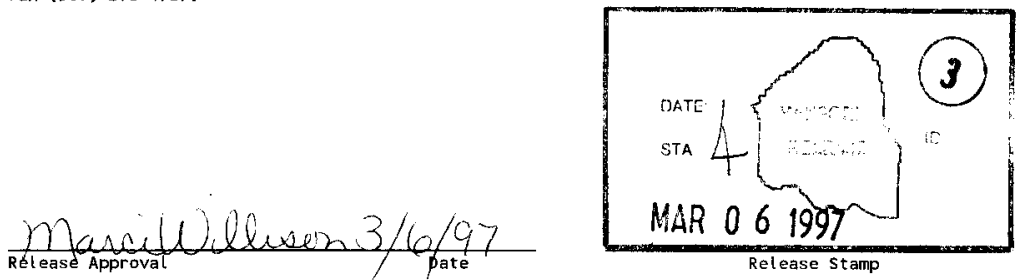

\section{Approved for Public Release}


(2) Title

Historical Tank Content Estimate for the Northwest quadrant of the Hanford 200 West Area

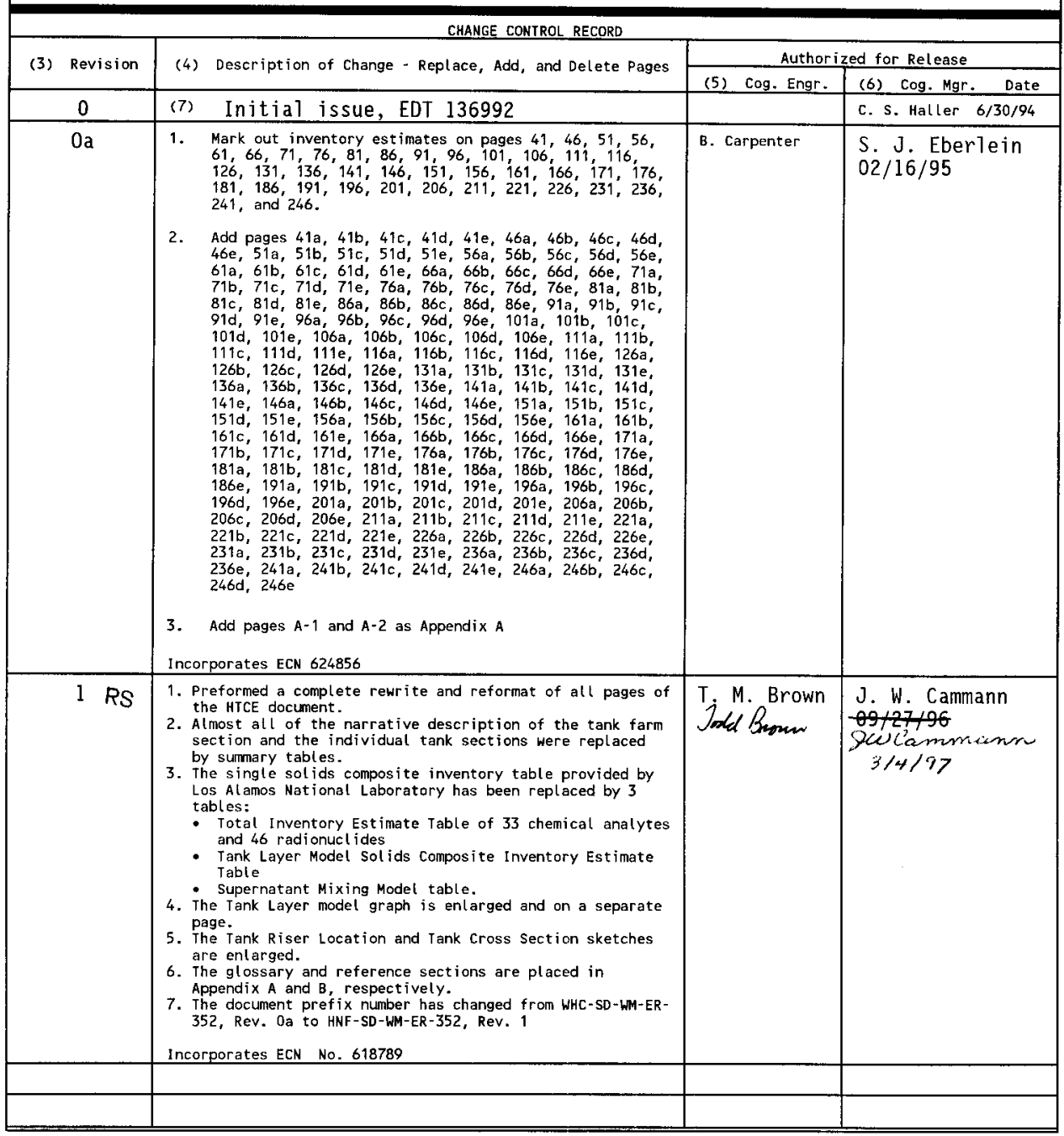




\title{
HISTORICAL TANK CONTENT ESTIMATE FOR THE SOUTHWEST QUADRANT OF THE HANFORD 200 WEST AREA
}

\author{
Prepared for \\ Lockheed Martin Hanford Corporation
}

January 1997

Prepared by

K. L. Ewer

J. W. Funk

R. G. Hale

G. A. Lisle

C. V. Salois

M. R. Umphrey

Fluor Daniel Northwest, Inc.

Richland, Washington 


\section{HISTORICAL TANK CONTENT ESTIMATE FOR THE SOUTHWEST QUADRANT OF THE HANFORD 200 WEST AREA}

WORK ORDER E18675

APPROVED:

Fluor Daniel Northwest, Inc.

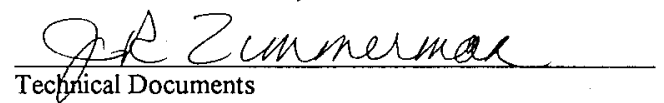

W. Foule
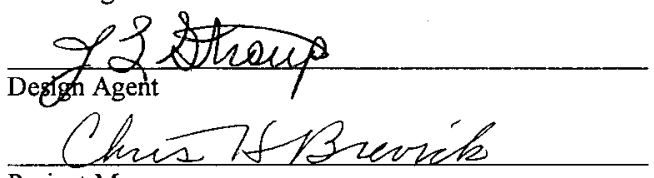

Project Manager
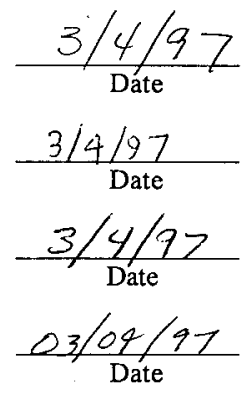

Lockheed Martin Hanford Corporation

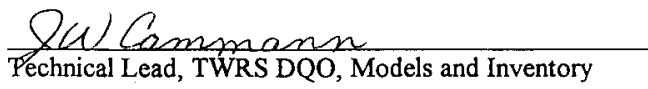

$\frac{3 / 4 / 97}{\text { Date }}$ 


\title{
ACKNOWLEDGMENTS
}

\begin{abstract}
A project of the this magnitude would not be possible without the help of a significant number of persons and organizations. Fluor Daniel Northwest, Inc., would like to acknowledge the contributions made by our Los Alamos National Laboratory counterparts: Stephen F. Agnew, Kenneth A. Jurgensen, Robert A. Corbin, Tomasita B. Duran, Bonnie L. Young, Theodore P. Ortiz, John FitzPatrick, and James Boyer. Also, Todd Brown, Brett Simpson and Jerry Cammann of Lockheed Martin Hanford Corporation are recognized for their contributions.
\end{abstract}




\section{INFORMATION FEEDBACK CARD}

\section{HISTORICAL TANK CONTENT ESTIMATE FOR THE SOUTHWEST QUADRANT OF THE HANFORD 200 WEST AREA}

\section{COMMENTS AND CONTRIBUTIONS}

The reader is requested to use this card to comment on this document, report any discrepancies, or contribute new information to improve the accuracy and content of the document. Please use the space provided, add additional pages if necessary, and return the comments to the person noted at the address listed below.

Send Comments to: Mr. Jerry W. Cammann

Lockheed Martin Hanford Corporation

TWRS DQO, Models and Inventory

P.O. Box 1970, MSIN R2-12

Richland, WA 99352 


\section{TRADEMARKS}

Microsoft Excel is a registered trademark of Microsoft Corporation.

ENRAF is a registered trademark of Delft Instruments.

AutoCAD is a registered trademark of Autodesk, Inc 


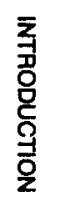




\subsection{Introduction}

\subsubsection{Purpose}

The purpose of this historical characterization document is to present the synthesized summaries of the historical records concerning the physical characteristics, and the radiological, and chemical composition of mixed wastes stored in underground single-shell tanks and the physical conditions of these tanks. The single-shell tanks are located on the United States Department of Energy's Hanford Site, approximately 25 miles northwest of Richland, Washington. The document will be used to assist in characterizing the waste in the tanks in conjunction with the current program of sampling and analyzing the tank wastes. Los Alamos National Laboratory (LANL) developed computer models that used the historical data to attempt to characterize the wastes and to generate estimates of each tank's inventory. A historical review of the tanks may reveal anomalies or unusual contents that could be critical to characterization and post characterization activities.

This document was developed by reviewing the operating plant process histories, waste transfer data, and available physical and chemical data from numerous resources. These resources were generated by numerous contractors from 1945 to the present.

Waste characterization, the process of describing the character or quality of a waste, is required by Federal law (Resource Conservation and Recovery Act [RCRA]) and state law (Washington Administrative Code [WAC] 173-303, Dangerous Waste Regulations). Characterizing the waste is necessary to determine methods to safely retrieve, transport, and/or treat the wastes.

This document is not intended for use as a total design basis document. Further investigations of the information may be required before using this data for design purposes or safety analysis.

\subsubsection{Scope}

The scope of this document is to provide a summary of the supporting documents (Brevick et al.,1997a, b, c) for the Southwest Quadrant (SW). The SW Quadrant covers three single-shell tank farms. These three tank farms, S, SX, and U, are located in the 200 West Area and are shown on the map in Figure 1. This summary includes waste transfer and level data, tank physical information, and surveillance data of the tanks and wastes for the SW Quadrant. The inventory estimates of waste types and volumes generated by the computer modeling programs developed by LANL are also included. A flow diagram showing the relationships between the sources of data, the HTCE, and the supporting documents is in Figure 2. The HTCE document also includes information on the safety issues affecting the tanks and the plants and processes that produced the waste in the underground waste storage tanks. For further explanation and development of the information, see the supporting documents.

\subsubsection{Approach}

This document was compiled from work performed by Fluor Daniel Northwest, Inc. (FDNW), LANL, and Lockheed Martin Hanford Corporation (LHMC), as well as work performed by 


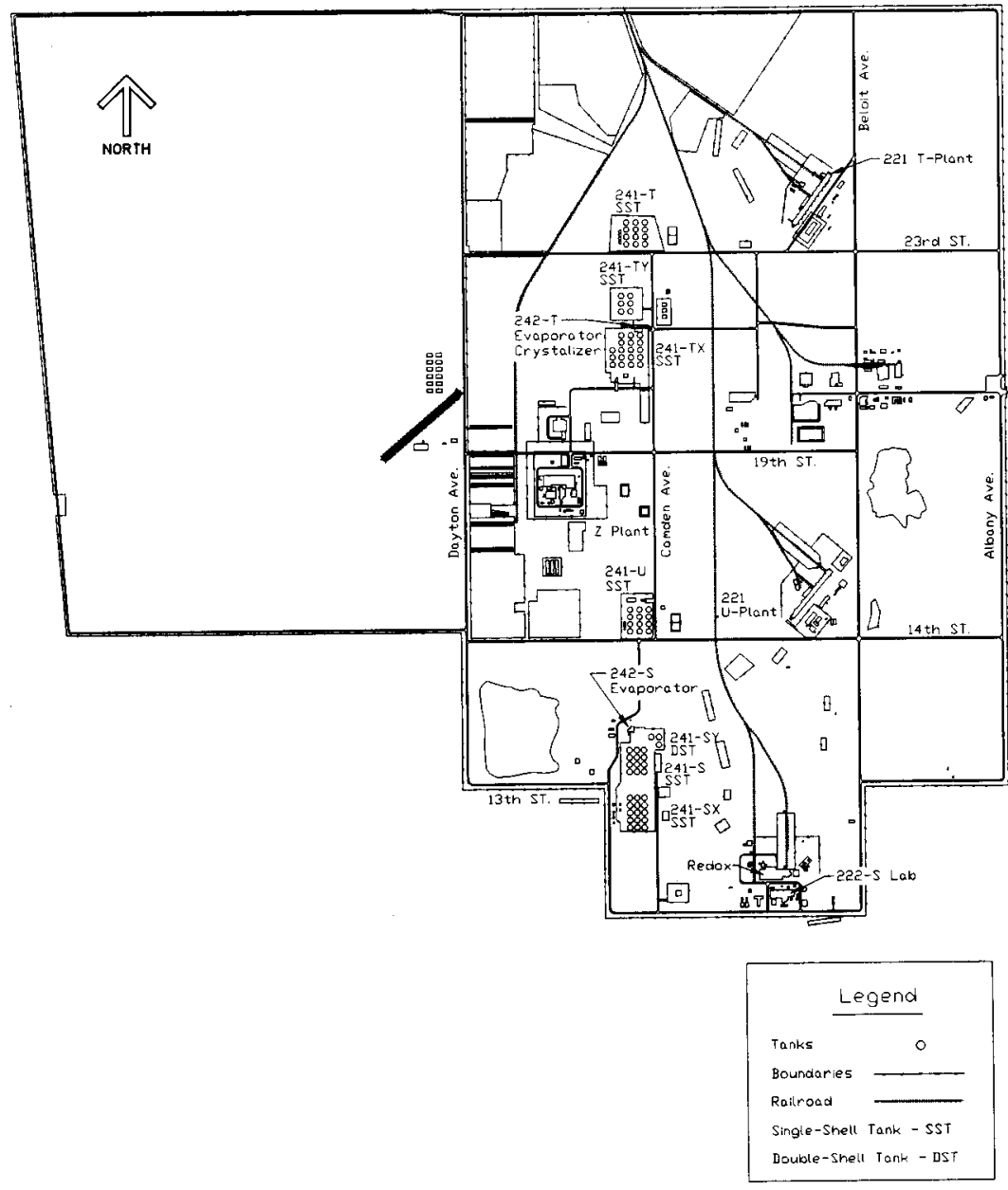

Figure 1. 200 West Area. 


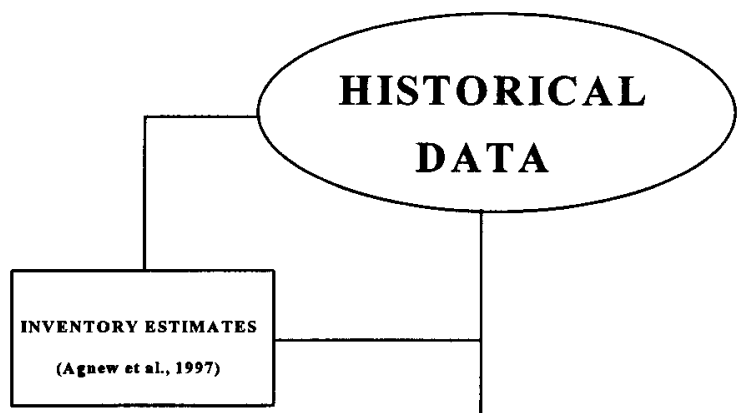

- historical tank Content estimate

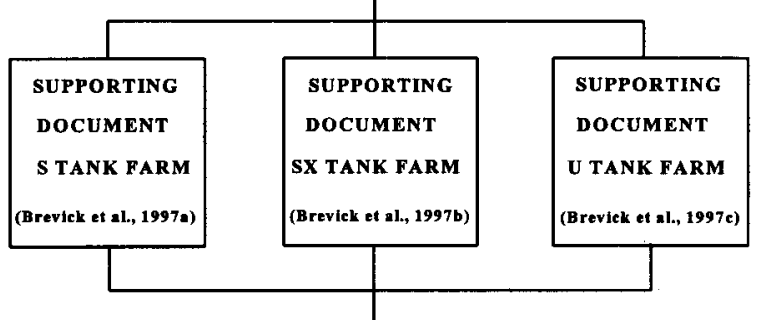

Figure 2. HTCE and Supporting Document Flow Diagram. 
Westinghouse Hanford Company. FDNW reviewed the historical records of the tanks and incorporated the inventory estimates and models of waste layers in the tanks being developed by LANL into this document.

\subsection{Safety Issues}

The safety issues that affect the tanks can be divided into two groups: watch list and non-watch list. The watch lists are listings of tanks believed to pose potential safety hazards to workers, the environment, and the public. Non-watch list issues are of concern because of their possible effect on workers and the environment. Occurrences are unusual events on the Hanford Site that sometimes are related to safety issues.

\subsubsection{Watch List Safety Issues}

Watch list safety issues for these tanks were identified as "issues/situations that contain most of the necessary conditions that could lead to worker (onsite) or offsite radiation exposure through an uncontrolled release of fission products" under Public Law 101-510, Section 3137, of the National Defense Authorization Act of Fiscal Year 1991 (i.e., the Wyden Amendment). As of June 30, 1996, 44 single-shell tanks and 6 double-shell tanks are on watch lists (Hanlon, 1996i). See the Approach for Tank Safety Characterization of Hanford Site Waste(Eberlein et al., 1995) for more information on the watch list issues.

\subsubsection{Non-Watch List Safety Issues}

Non-watch list issues include safety hazards such as leaking tanks. Tank leaks are a safety hazard because of their potential to release chemicals and radioactive liquids into the ground. Corrosion is the main cause of tank leaks. Three other safety issues that do not require a watch list and continual monitoring under the Wyden Amendment include criticality, tank bumps, and toxic vapor releases. The following sections provide a general description of the different non-watch list safety issues. See the Hanford Site Tank Farm Facilities Interim Safety Basis(Leach and Stahl, 1993) for more information.

\section{- Corrosion}

Corrosion is the most probable degradation mechanism of the steel tank liners resulting from contact with liquid, liquid-vapor, vapor, and solid phases of the wastes. The corrosion mechanisms that reduce the thickness of the carbon steel liners can be divided into two categories: localized and general or uniform. Localized corrosion occurs on a localized area of the liner surface. Some of the localized corrosion mechanisms include pitting corrosion, stress corrosion cracking, and crevice corrosion. General or uniform corrosion occurs over the entire liner surface. Corrosion of the steel tank liners may involve more than one of these mentioned mechanisms. Corrosion is a safety issue because it has the potential to degrade the tank liner to the point of causing a leak or, more seriously, structural failure of the tank. Either condition could release contamination to the environment.

\section{- Criticality}

Criticality is a self-sustained, nuclear chain reaction that can occur when a sufficient mass of fissile material is present in the proper configuration along with a neutron source to start the nuclear 
reaction. Criticality in the tank farms has been declared an unreviewed safety question, even though the Hanford Site Tank Farm Facilities Interim Safety Basis (Leach and Stahl, 1993) indicates that a "nuclear criticality accident in the tank farms is probably not an imminent risk." The unreviewed safety question on criticality in the tank farms remains because the inventory of fissile material and its distribution within the tanks cannot be confirmed as being within the approved safety envelope defined in the current safety analysis reports. Criticality is a safety issue because of the potential to release contamination to the environment.

\section{- Tank Bumps}

A tank bump is the sudden pressurization of the tank. This phenomenon occurs when solids overheat in the lower portion of the tank followed by uncontrolled mixing of these solids. The stirred hot solids rapidly transfer heat to the liquid in the tank, some of which quickly vaporizes. The rapid vapor generation causes a sudden internal tank pressurization that causes a bump. Uncontrolled mixing of heated solids can occur when an airlift circulator fails allowing the solids to heat up followed by rapid startup of the airlift circulator which causes rapid mixing. Uncontrolled mixing can also occur when a natural "rollover" of waste occurs in the tank. Tank bumps are a safety issue because of their potential to release contamination to the environment.

\section{Toxic Vapor Releases}

Toxic vapor releases are a recently analyzed safety concern at the Hanford Site. The entire issue of toxic gas releases at the tank farms is being investigated (Leach and Stahl, 1993).

\subsubsection{Occurrences}

Over the years, unusual events (occurrences) have occurred at several tanks in the SW Quadrant tank farms. An occurrence is an event that falls outside the normal operating, maintenance and/or construction procedures of the tank farm. Occurrences have been documented by various reporting methods including unusual occurrences reports, off-normal reports, event fact sheets, and occurrence reports. Before it was included in the supporting document, the occurrence documentation that could be located was evaluated for its significance in determining the waste content of the tanks. The types of significant occurrences included in the supporting documents are occurrences written about surface level changes, temperature changes, and radioactivity changes (activity in the drywells). This document does not contain information from the reports. For more information on occurrences, refer to the Occurrences Section for the specific tank in the supporting documents.

\subsection{Waste Generating Plants and Processes}

\subsubsection{Plants Processes}

Brief descriptions and histories of the plants and processes that generated waste now contained in the single-shell and double-shell tanks are presented in alphabetical order. Typically, the name of the plant and the process are synonymous. The dates and events described in the following brief histories are presented on time lines in Figures 3 and 4. Although not all of the processes listed below contributed waste directly to tanks in the Southwest Quadrant, the waste they generated could have been transferred indirectly from tank to tank. 


\section{- A Plant (PUREX)}

The Plutonium Uranium Extraction (PUREX) plant (i.e., A Plant) began operating in January 1956 (Gerber, 1993a). "The PUREX process is an advanced solvent extraction process that uses a tributyl phosphate in kerosene solvent for recovering uranium and plutonium from nitric acid solutions of irradiated uranium. Nitric acid is used instead of metallic nitrates to promote the extraction of uranium and plutonium from aqueous phase to an organic phase." (Wilson and Reep, 1991, p. B-4). Two campaigns of the Thorex process were conducted in 1966 and 1971 (Jungfleisch, 1984). The Thorex process recovered ${ }^{233} U$ from thorium irradiated in the Hanford Site reactors (Wilson and Reep, 1991). PUREX reprocessed aluminum-clad fuel elements and zirconium alloy-clad fuel elements, and provided plutonium for research reactor development, safety programs, and defense. Also, PUREX recovered slightly enriched uranium to be recycled as fuel in reactors generating electricity and plutonium (Rockwell, 1985). PUREX was put on standby in 1972 (Gerber, 1993a).

The PUREX plant was restarted in November 1983 but was shut down in December 1988 (see Figure 3). The plant was shut down due to the lack of steam pressure needed to operate the support backup safety equipment. There was a brief stabilization run in early 1990. In October 1990, PUREX was placed on standby by Secretary of Energy James Watkins. DOE issued the final closure order in December 1992 (Gerber, 1993b).

\section{- B Plant}

B Plant used the bismuth phosphate process at first, and later changed its processing capabilities to strontium and cesium fractionation. The bismuth phosphate process "separated plutonium from uranium and the bulk of fission products in irradiated fuel by co-precipitation with bismuth phosphate from a uranium nitrate solution. The plutonium was then separated from fission products by successive precipitation cycles using bismuth phosphate and lanthanum fluoride. The plutonium was isolated as a peroxide and, after dissolving in nitric acid, was concentrated as plutonium nitrate. The waste containing the uranium from which the plutonium had been separated, was made alkaline (neutralized) and stored in underground single-shell tanks. Other acid waste (which included most of the fission products) generated by this process was neutralized and stored in other single-shell tanks" (Wilson and Reep, 1991, p. B-3). "Some of the strontium and cesium fission products were removed (fractionated) from the waste and separately isolated to reduce the heat generation in the tanks. B Plant . . was modified in 1968 to permit removal of these fission products by a combination of precipitation, solvent extraction, and ion-exchange steps. The residual acid waste from the processing was neutralized and stored in single-shell tanks" (Wilson and Reep, 1991, pp. B-4 and B-5).

B Plant began its first batch run on April 13, 1945 (Anderson, 1990), and was shutdown in 1952 (Gerber, 1993b) (see Figure 3). Shortly after the renovations to B Plant were completed in December 1955, the 4X Program was abandoned. The 4X Program "planned to utilize the capabilities of all four Hanford processing plants (B, T, REDOX, and PUREX)" (Gerber, 1993b, p. 12); however, the large production and economic efficiency of the PUREX plant caused the $4 X$ Program to be abandoned (Gerber, 1993b). B Plant restarted in 1968 to recover cesium and strontium from stored liquid waste. Cesium and strontium recovery was completed in September 1983 and February 1985, respectively (Rockwell, 1985). 
HNF-SD-WM-ER-352, Rev. 1

\section{5-B (WESF)}

The Waste Encapsulation and Storage Facility (WESF) converted solutions of cesium and strontium nitrates recovered in B Plant to strontium fluoride and cesium chloride solids that are doubly encapsulated in metal (Ballinger and Hall, 1991). "Strontium and cesium capsules have been used in applications of fission byproducts for gamma and heat sources" (Wilson and Reep, 1991, p. B-5)

WESF was constructed in 1974 (see Figure 3). The process optimization for cesium and strontium was completed in 1978 and 1981, respectively (Rockwell, 1985). The cesium processing ended in 1983 and strontium encapsulation in 1985. The capsule return program started in 1988 and ended in 1995 (Gerber, 1996).

\section{- C Plant (Strontium Semiworks)}

The Strontium or Hot Semiworks Facility (i.e., C Plant) began operating in 1952 as a hot pilot plant for the REDOX process (see Figure 3). In 1954, the plant was converted to a pilot plant for the PUREX process and continued operating until 1956 (Ballinger and Hall, 1991). "The process building (201-C) contains three hot cells equipped only for contact maintenance and is supported by an aqueous makeup and control building (271-C) and a solvent handling building (276-C). The facility also includes a fiberglass exhaust filter and a 200-ft stack." (PNL, 1991, Vol. 1, p. 3.6). In 1960, the plant was reactivated as a pilot plant used to recover ${ }^{90} \mathrm{Sr},{ }^{147} \mathrm{Pm}$, and ${ }^{144} \mathrm{Cs}$ from PUREX waste. The plant was shut down in 1967 and the building and the site have been decontaminated and decommissioned (PNL, 1991).

\section{E S Plant (REDOX)}

The Reduction and Oxidation extraction (REDOX) plant (i.e., S Plant) began processing on January 9, 1952 (Anderson, 1990) (see Figure 3). "The REDOX extraction process was a secondgeneration recovery process and the first process to recover both plutonium and uranium. It used a continuous solvent extraction process to extract plutonium and uranium from dissolved fuel into a methyl isobutyl ketone (hexone) solvent. The slightly acidic wastestream contained the fission products and large quantities of aluminum nitrate that were used to promote the extraction of plutonium and uranium. This waste was neutralized and stored in single-shell tanks. The volume of high-level waste from this process was much smaller than that from the bismuth phosphate process, but larger than that from the PUREX process" (Wilson and Reep, 1991, pp. B-3 and B-4). REDOX operated until 1967 (Rockwell, 1985).

\section{T Plant}

T Plant was the first full-scale separations plant at the Hanford Site. T Plant used the bismuth phosphate process to separate plutonium from uranium and the bulk of fission products in irradiated fuel (B Plant used the same process). "The waste containing the uranium from which the plutonium had been separated was made alkaline (neutralized) and stored in underground single-shell tanks. Other acid waste (which included most of the fission products) generated by this process was neutralized and stored in other single-shell tanks" (Wilson and Reep, 1991, p. B-3).

T Plant began operating in 1944 (Rockwell, 1985) as a separations plant and continued until March 1956 (Gerber, 1994) (see Figure 4). T Plant's mission was changed in 1957 to the repair and high-level decontamination of equipment (Rockwell, 1985). T Plant was converted to a "central decontamination facility for the site. As such, failed and contaminated equipment was assessed and 
either repaired or discarded there for over three decades" (Gerber, 1994, p. 1). Early decontamination operations used steam, sand, chemicals, and detergents. "Smaller equipment pieces were immersed in decontamination solutions in 'thimble tanks,' and larger pieces were flushed with water, chemical solutions, sand-blasted, steam-blasted, high-pressure sprayed (using pressures up to 10,000 pounds per square inch), and/or scrubbed with detergents. During the initial years, a strong nitric acid flush (approximately 60\%) usually began the decontamination process, followed by a caustic wash with sodium hydroxide combined with sodium phosphate, boric acid, versene, sodium dichromate, sodium tartrate, or sodium citrate. However, it was learned that versene and tartrate, in particular, adversely affected the ability of soil cribs to absorb the rinsate materials. High-pressure sprays often used 1,1,1 trichloroethane or perchloroethylene, and detergents generally were chloride-based. By the mid-1960s, commercially prepared and trademarked chemical mixtures had replaced most of the simpler chemicals used in the early years. Many commercial products were based on oxalic acid, phosphates, nitric acidferrous ammonium sulfate combinations, potassium permanganate, and sodium bisulfate, with some unknown additives" (Gerber, 1994, pp. 40-42). The facility was modified in 1978 to store pressurized water reactor (PWR) core II fuel assemblies (Rockwell, 1985).

\section{U Plant}

U Plant (221-U) was built as one of three original bismuth phosphate process facilities, but it was not used for that purpose. U Plant was modified extensively and used for the uranium recovery process, operating from 1952 to 1958 (see Figure 4). Uranium in waste from the bismuth phosphate process initially was stored in the single-shell tanks. Later, the waste was sluiced, dissolved in nitric acid, and processed through a solvent extraction process using tributyl phosphate in kerosene to recover the uranium. The process was similar to that used later in the plutonium-uranium extraction (PUREX) process except that plutonium was not recovered. The acid waste from the uranium recovery process was made alkaline and retumed to single-shell tanks. The tributyl phosphate waste was treated with potassium ferrocyanide as a cesium and strontium scavenger. The recovery process resulted in an increase in nonradioactive salts and a small increase in waste volume (Wilson and Reep, 1991).

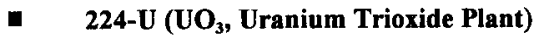

The 224-U Building was converted to a uranium trioxide $\left(\mathrm{UO}_{3}\right)$ plant that began operating in 1952 (see Figure 4). The $\mathrm{UO}_{3}$ plant was capable of handling the uranyl nitrate hexahydrate (UNH) stream from REDOX, U Plant, and PUREX. "The basic $\mathrm{UO}_{3}$ process, calcining, consisted of concentrating and then heating liquid $\mathrm{UNH}$ until it converted to a stable, orange-yellow powder. The nitric acid in the UNH solution could be recovered in the same process. The $\mathrm{UO}_{3}$ powder was the base material needed for the manufacture of uranium hexafluoride $\left(\mathrm{UF}_{6}\right)$, the primary feed material for the United States' gaseous diffusion plants. Because the largest of these plants was located in Ohio and Tennessee, it was considered safer to ship the material across the country in powder rather than in liquid form" (Gerber, 1993b, pp. 33-34). The UO $\mathrm{U}_{3}$ plant was shut down in 1972, but restarted in 1984. Since 1984, there have been 17 campaigns at the plant averaging 8 days each. Final deactivation of the plant was ordered in 1992. In April 1993, the $\mathrm{UO}_{3}$ plant resumed operations to convert 200,000 gallons of remaining $\mathrm{UNH}$ to $\mathrm{UO}_{3}$ powder. A final deactivation plan was written in the summer of 1993 (Gerber, 1993b).

\section{- Z Plant (PFP, Plutonium Finishing Plant)}

The Plutonium Finishing Plant (PFP) or Z Plant, previously called Plutonium Recovery and Finishing Operations, processed plutonium and prepared plutonium products. "Waste from this plant 
contained only minor amounts of fission products but did contain low concentration of plutonium and other transuranic elements and was high in metallic nitrates. Initially, this waste was discharged via cribs to soil columns, which absorbed the transuranic elements and retained them close to the point of discharge. Beginning in 1973, waste from PFP was stored with other waste in underground tanks" (Wilson and Reep, 1991, p. B-4). "Three types of feed materials are processed at the PFP to produce plutonium metal. Feed material types are handled differently in different process lines .... Historically, the main feed for the PFP was purified plutonium nitrate solution that was produced elsewhere in a fuel reprocessing plant. This feed was charged directly to one of the main process lines, which was initially a glovebox line. The glovebox line was replaced by remote mechanical lines, which were upgraded over the years. In time, processes were added to handle rework and scrap plutonium. These processes were used to convert the rework and scrap materials into a purified plutonium nitrate solution that could be handled by the main process" (Duncan and Mayancsik, 1993, pp. 2-1-2-2)

In July 1949, PFP began operations with a glovebox line (see Figure 4). The remote mechanical A line replaced the glovebox line in May 1953. Installment of the Recuplex Facility at PFP was completed in April 1955. The remote mechanical C line was installed in July 1960. In September 1961, the 232-Z Building had an incinerator and leaching equipment installed. In June 1964, the Plutonium Reclamation Facility (PRF) replaced the functions of the Recuplex Facility. Fabrication of plutonium metal nuclear weapon components ceased at the PFP in December 1965. In April 1973, the 232-Z Incinerator was shut down and the remote mechanical $\mathrm{C}$ line was placed on standby. The PRF was placed on standby in February 1979, and the remote mechanical A line was shutdown in December 1979. In January 1984, the PRF was restarted for a series of campaigns. The remote mechanical C line was restarted in June 1985 for a series of campaigns. In September 1986, operations at PFP were halted for nine months. This partial listing of the process history in the PFP is from Duncan et al. (1993)

\subsubsection{Waste Management Operations}

This section describes the different methods used to concentrate waste in the 200 Areas. Evaporating, and in-tank solidification are methods used to reduce the volumes of supernate. Brief descriptions and histories of the operations are presented in alphabetical order. The events and dates described in the brief histories are presented on a time line (Figure 5).

\section{2-A Evaporator-Crystallizer}

"The program objective was to reduce the volume of tanked waste liquors through the boiloff of water. This was accomplished by boiling the liquor in an enclosed vessel at reduced pressure. The evaporation was carried out until a slurry containing about $30 \mathrm{wt} \%$ solids was formed. The slurry was returned to underground waste tanks for cooling, crystallization, and settling. The principal products of waste solidification have been large volumes of sodium nitrate salt cakes and waste liquors that are rich in sodium hydroxide and sodium aluminate" (Wilson and Reep, 1991, p. B-5)

The 242-A Evaporator-Crystallizer began operating on March 18, 1977 (Anderson, 1990) (see Figure 5). In 1981, the evaporator was shut down for ten months to tie AW Tank Farm into the process (Rockwell, 1985). The evaporator was shut down in 1989 because of regulatory issues, but was restarted in 1994 after extensive modifications (Gerber, 1996). 
- 242-B Evaporator

"The first type of waste solidification facility, the 242-B and 242-T Concentrators, was originally used for concentration of bismuth phosphate process waste. In 1951, they began to concentrate cladding/first cycle waste. These concentrators were steam-heated pot evaporators operated outside the waste tanks and at atmospheric pressure. The liquors were partially boiled down and cycled to underground waste storage tanks" (Jungfleisch, 1984, p. 1-5). This evaporator ran for approximately four years (Anderson, 1990) (see Figure 5).

\section{2-S Evaporator-Crystallizer}

The 242-S Evaporator-Crystallizer was designed to boil off water from the waste in an enclosed vessel at reduced pressure, similar to the 242-A Evaporator-Crystallizer. "The evaporation was carried out until a slurry containing about $30 \mathrm{wt} \%$ solids was formed. The slurry was returned to underground waste tanks for cooling, crystallization, and settling. The principal products of waste solidification have been large volumes of sodium nitrate salt cakes and waste liquors that are rich in sodium hydroxide and sodium aluminate" (Wilson and Reep, 1991, p. B-5). The evaporator began operating on November 1, 1973 (Anderson, 1990) and was shut down in 1981 (Gerber, 1996) (see Figure 5).

\section{2-T Evaporator}

The 242-T Evaporator, like the 242-B Evaporator, began operating in 1951 (Gerber, 1992) to reclaim nonboiling waste storage capacity in existing tanks (see Figure 5). The evaporator was shut down in the summer of 1955 and modified for tributyl phosphate scavenging (Godfrey, 1965), although scavenging was never performed in this evaporator. The evaporator was restarted on December 3, 1965, and operated until April 15, 1976 (Anderson, 1990).

\section{In-Tank Solidification}

The in-tank solidification systems immobilized high level wastes, that were not self-boiling, by concentrating the waste directly inside the tanks to form radionuclide-bearing salt cakes (Shefcik, 1964). The first in-tank solidification unit (ITS-1) and the second in-tank solidification unit (ITS-2) operated in tanks in the BY Tank Farm (Caudill, 1965 and 1967). "...[O]ne used a hot air sparge (ITS-1) and the other used an immersed electrical heater (ITS-2). The ITS-1 operations were conducted in individual tanks. The ITS-2 concentrations were performed by heating the contents of one tank and moving the heated liquor through a series of other tanks" (Wilson and Reep, 1991, p. B-5).

ITS units 1 and 2 began operating on March 19, 1965, and February 17, 1968, respectively (see Figure 5). ITS-1 was converted to a cooler for ITS-2 on August 24, 1971. Both units were shut down on June 30, 1974 (Anderson, 1990).

\subsubsection{Miscellaneous Waste Sources and Equipment}

Wastes from various other sources on the Hanford Site have been added to the tanks. Some wastes are from the 300 Area, the 100 Area production reactors, various laboratories, and catch tanks.

\section{- Critical Mass Laboratories}

The critical mass laboratories were used to study the physics of plutonium solutions and solids to avoid accidently creating a criticality or self-sustained nuclear reaction. The first facility began operating in the 120 Building near $100 \mathrm{~F}$ in April 1950 and closed in December 1951. The second 
facility, the 209-E Building, was located next to the Strontium Semiworks and began operating in July 1961 (Ballinger and Hall, 1991). The plutonium used in the lab was reprocessed in PUREX.

\section{4-AR, -BXR, and -CR Process Vaults}

Three of the process vaults are the 244-AR Vault, the 244-BXR Vault, and the 244-CR Vault These vaults were composed of several process vessels or tanks used to prepare waste for treatment or storage. Specific wastes from tanks can be pumped temporarily to the vaults and later sent directly to desired tanks or processing facilities.

The AR Vault, located north and west of the A Tank Farm, was constructed in 1966. The vault facilities include a canyon building with process cells containing tanks. The AR Vault has been on standby since 1978 (Leach and Stahl, 1993).

The 244-BXR Vault, located south of the BX Tank Farm, began operating in 1952 (Rodenhizer, 1987) and became inactive in 1956. The waste in the vault was difficult to handle, so the vault was jetted with high-pressure steam in 1976. The 244-BXR Vault was used to process sludge in the recovery of uranium from bismuth phosphate metal waste in the tanks (Rodenhizer, 1987).

The 244-CR Vault, constructed in 1952, is located south of the C Tank Farm (Leach and Stahl, 1993). Salt-well waste from the C Tank Farm is interimly stored in the CR Vault. The 244-CR Vault was used to process sludge in the recovery of uranium from bismuth phosphate metal waste in the tanks (Rodenhizer, 1987).

\section{4-AR and 204-S Railroad Car Facilities}

The 204-AR rail car unloading facility built in 1981 (Leach and Stahl, 1993), replaced the 204-S rail car unloading facility. The facilities were built for pumping liquid radioactive waste from tank cars and sending the waste to 200 East Area tank farms (Leach and Stahl, 1993).

\subsubsection{Time Lines}

Time lines presented on the following pages represent many of the events that occurred during the history of the major plants and waste management operations on the Hanford Site. These are the same events as those described in the description of each facility. The plants, associated processes, and methods for managing waste are the main sources of the wastes stored in the tanks. Abbreviations are defined in the preceding text and in the glossary in Appendix A.

One time line represents the history of each of the tank farms in the Southwest Quadrant of the 200 West Area (Figure 6). The events represented include the dates of construction, the individual tank's entry into service and removal from service, and the deactivation of each tank farm. 


\section{PLANTS / PROCESS - TIME LINE}

A PLANT

B PLANT

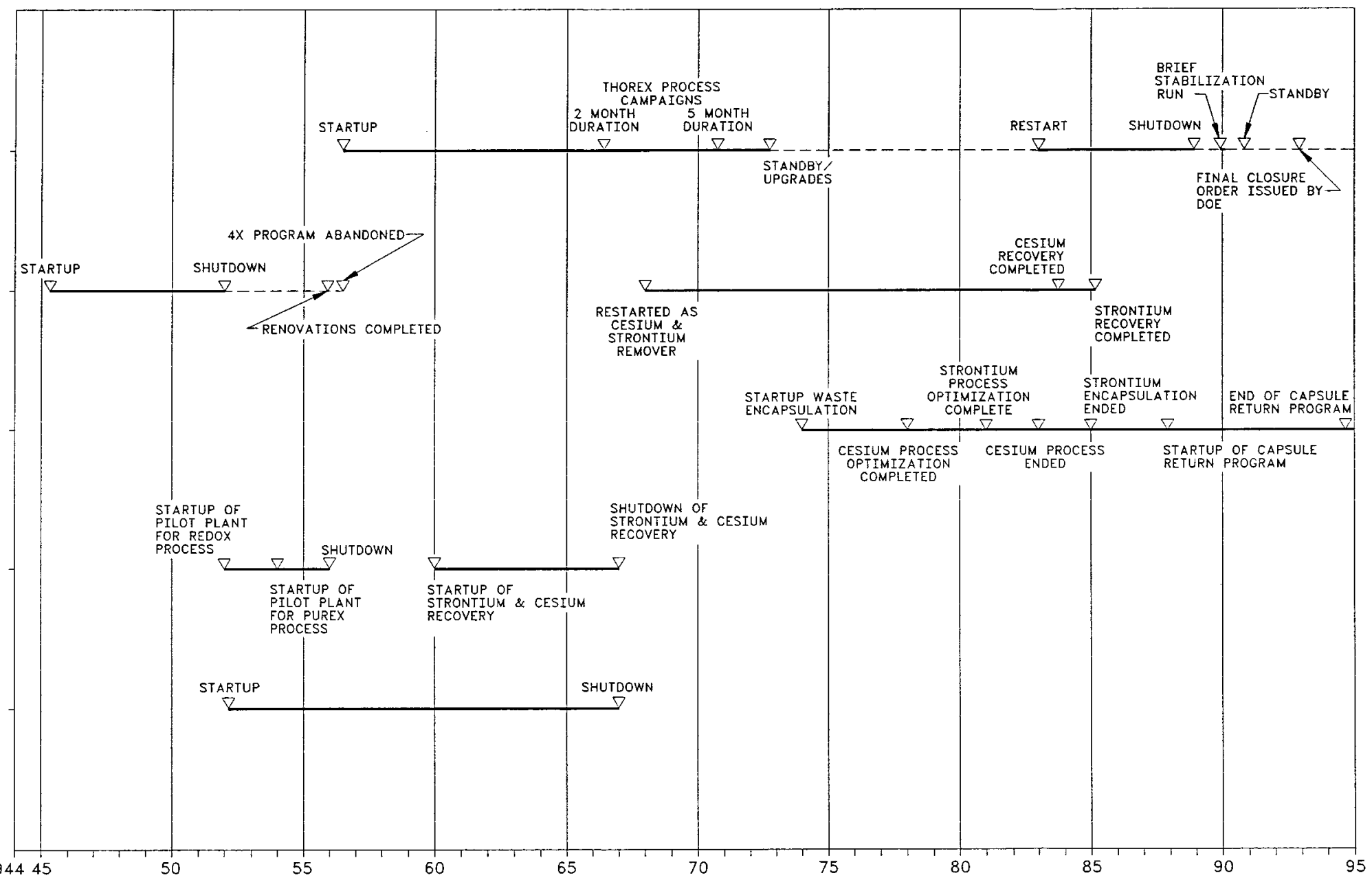

FIGURE 3 

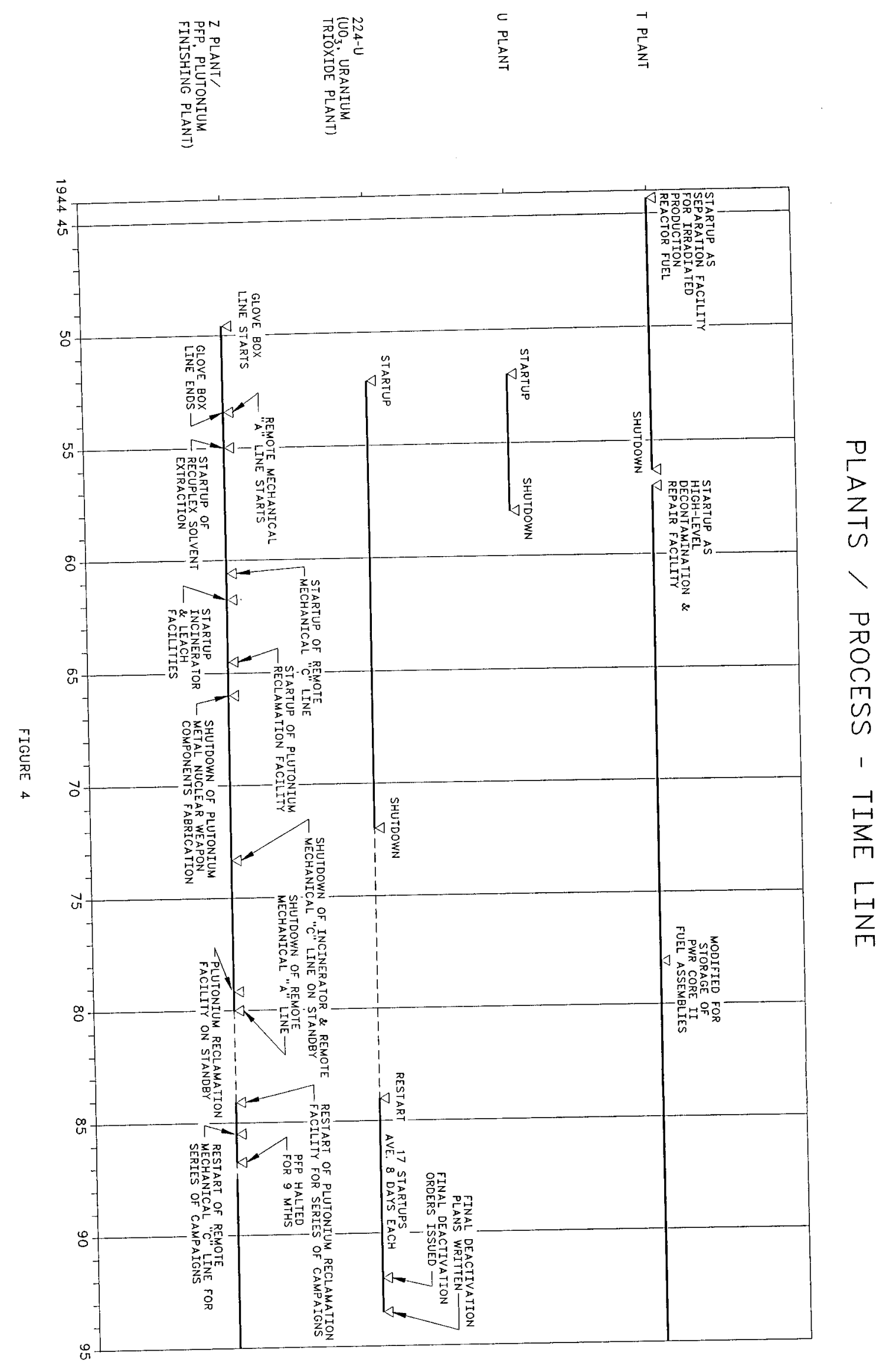

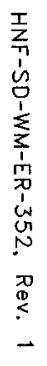


WASTE MANAGEMENT - TIME LINE

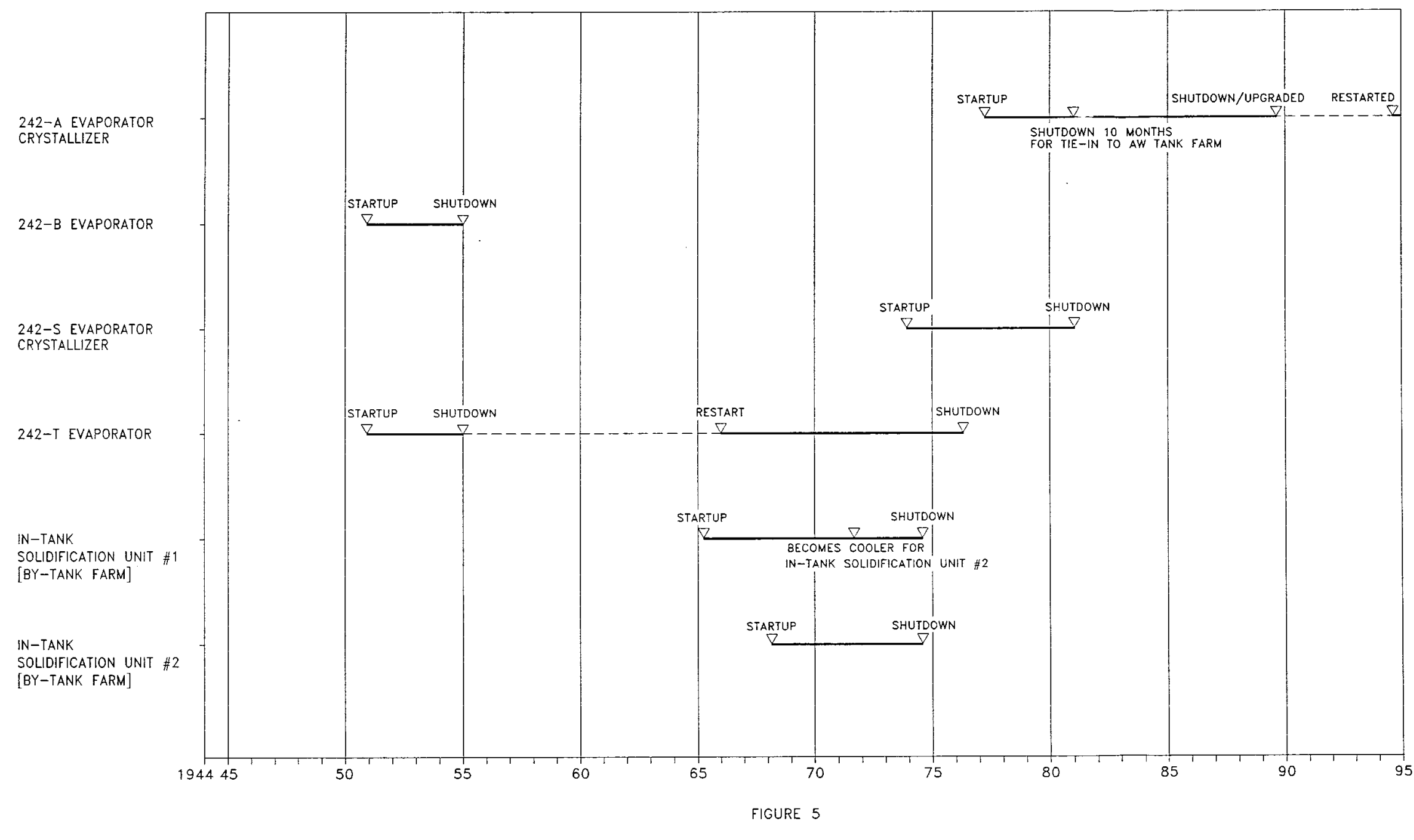




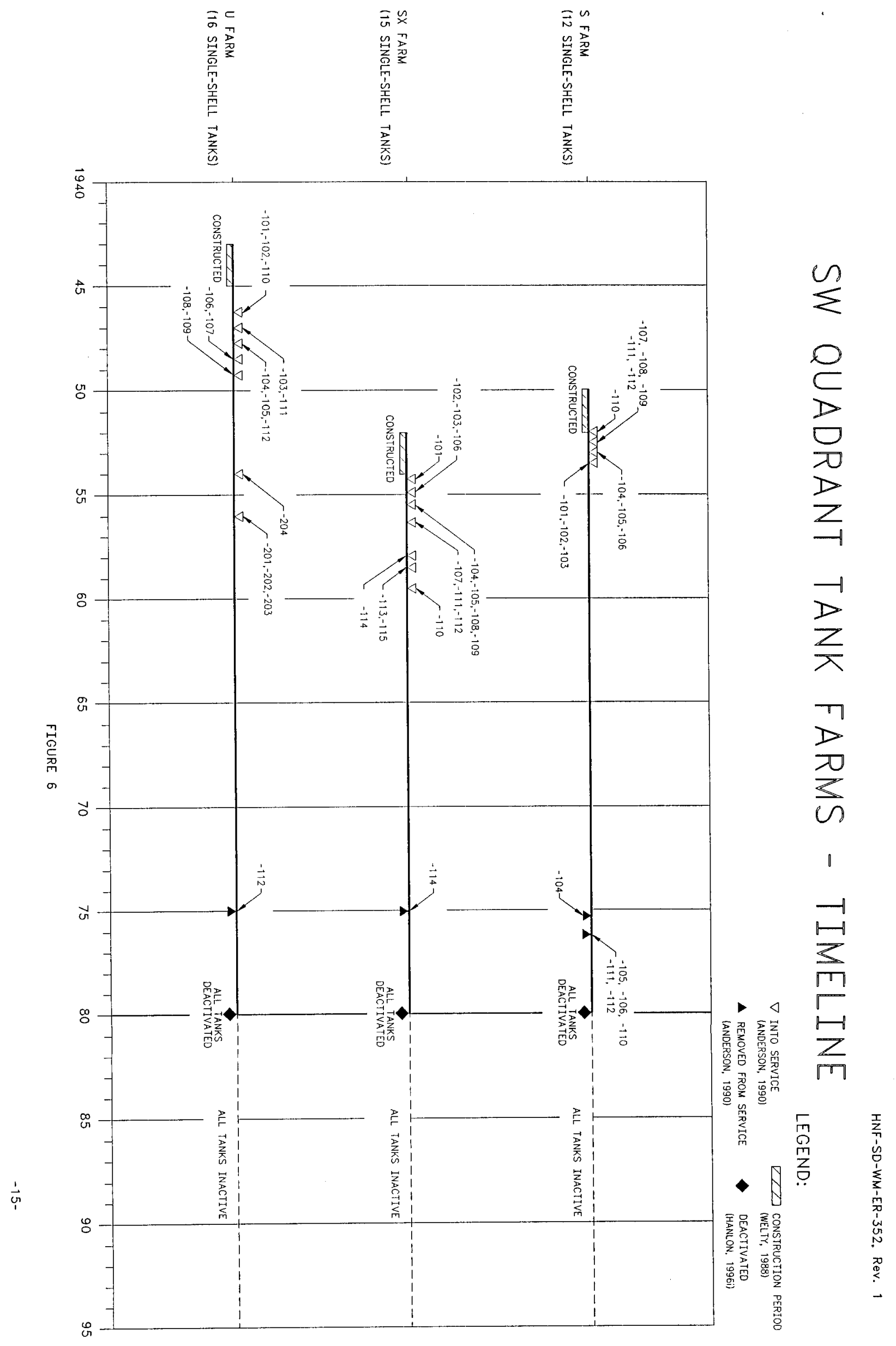


1.3 Tank and Tank Farm Summary Tables: Source and Development

The summary tables for each tank farm and each tank in the SW Quadrant comprise information from the supporting documents and the Waste Tank Summary Report for Month Ending June 30, 1996 (Hanlon, 1996i).

The information on the Tank Farm Summary tables includes the Tank Farm Description, Integrity, Tank Volumes, and Watch Lists sections selected from the supporting documents. The Waste Volume Section information was taken from the Waste Tank Summary Report for Month Ending June 30, 1996 (Hanlon, 1996i).

The information on the Tank Summary tables includes the Tank History, Tentatively Available Risers, Tank temperature, Tank Description, Interior Photographs and Waste Surface Level sections selected from the supporting documents. The Waste Volume section information was taken from the Waste Tank Summary Report for Month Ending June 30, 1996 (Hanlon, 1996i).

The "-" in the tank summary tables indicates the information is unknown, not applicable, and/or not available in the supporting documents.

The risers referred to in the Tentatively Available Riser section are risers that are available for interior tank waste sampling (Lipnicki, 1996). For more information, see the supporting documents.

The temperature and waste surface level data presented in the Tank Summary tables are from a continually updated database. Therefore, there are limits on the temperature and waste surface level data presented in the tables. All other information on the Tank Summary tables is from referenced documents.

The temperature data were queried from the Surveillance Analysis Computer System (SACS) database. The temperature data were queried for the period from January 1, 1950, through October 2, 1996. The end date for the queries varies for each tank farm (see supporting documents for further information). Temperature data prior to 1975 were not located. The temperature information presented is for data from 1975 through the date of the query for the specific tank. For a graphical representation of the data, further explanation about the development of the data, and the references, see the supporting documents.

The waste surface level data also were queried from the Surveillance Analysis Computer System (SACS) database. The waste surface level data were queried for the period from January 1, 1991, through October 14, 1996. The end date for the queries varies for each tank farm (see supporting documents for further information). The waste surface level information presented is for data from January 1, 1991 through the date of the query for the specific tank. The waste surface level device indicated on the Tank Summary tables is the most recent device being used based on the queried data. The maximum and minimum waste surface levels indicated on the Tank Summary tables do not always correlate to the most recent device indicated on the Tank Summary tables. For a graphical representation of the data, further explanation about the development of the data, and the references, see the supporting documents. 
For further information on the development of the data and more specific references on the Farm Summary tables and Tank Summary tables see the supporting documents.

\subsection{Waste and Level History: Source and Development}

The Waste and Level Histories combine the level history of the tank and the wastes added to the tank through out history. The Waste and Level Histories were incorporated from the Supporting Document for each tank. The supporting data were not included in this document. For the supporting data, further information on the development of the Waste and Level History sketches, and the references, see the supporting documents.

\subsection{Riser Configuration: Source and Development}

The riser configurations were incorporated directly from the supporting documents for each particular farm. The riser configurations were developed from numerous drawings and documents. For further information on the sizes of the risers, uses of the risers, references and the development of the riser configurations, see the supporting documents.

\subsection{Tank Layer Model: Source and Development}

The Tank Layer Model(TLM) graphs present the estimated waste types from the Hanford Tank Chemical and Radionuclide Inventories: HDW Model Rev. 4 (Agnew et al., 1997). The Tank Layer Model graphs were reformatted into individual tank graphs from Agnew's document to better illustrate what waste types Agnew has estimated. For further information on the TLM, see the supporting documents and Agnew's document.

\subsection{Inventory Estimates: Source and Development}

The Inventory Estimates present the estimated waste contents of the tanks from the Hanford Tank Chemical and Radiomuclide Inventories: HDWModel Rev. 4 (Agnew et al., 1997). The inventory estimates predict the physical characteristics and the chemical, and radiological constituents of the supernatant, the solids and the total volume for each tank. The inventory estimates for each tank include a Supernatant Mixing Model (SMM) Composite Inventory Estimate, a Tank Layer Model (TLM) Solids Composite Inventory Estimate and a Total Inventory Estimate. The SMM Composite Inventory Estimate is based on the output of the Supernatant Mixing Model and the TLM Solids Composite Inventory Estimate is based on the output of the Tank Layer Model. The Total Inventory Estimate is a combination of the SMM Composite Inventory Estimate and the TLM Solids Composite Inventory Estimate. The Inventory Estimates were incorporated directly from the supporting document which were incorporated directly from Agnew's document for each tank. For further information on the Inventory Estimates, see the supporting documents and Agnew's document.

\subsection{Tank Farm Photographs and Tank Montages: Source and Development}

The tank farm photographs and tank montages were incorporated directly from the supporting documents for each particular farm. The tank farm photographs and interior tank montages were 
reviewed in January 1996 to determine the clearest and most recent representation. For further information on the tank farm photograph and the interior tank montages, see the supporting documents. 
$\infty$
$\frac{1}{2}$
$\frac{2}{x}$
$\frac{1}{3}$
3 


\section{$2.0 \quad 241-S$ Tank Farm}

The S Tank Farm is located in the 200 West Area west of Beliot Avenue and north of 13th Street. The farm contains twelve 100 series, dish bottom design, single-shell tanks built in 1950 and 1951. The 100 series tanks are 75 feet in diameter with an operating capacity of 758,000 gallons. The tanks were designed to store non-boiling waste at a fluid temperature of $220^{\circ} \mathrm{F}$. The twelve tanks were constructed at different elevations with connecting overflow lines that allowed waste to cascade from tank to tank. The tank farm has four cascades of three tanks each. For more information on the design, construction, and waste contents of the tanks, refer to the S Tank Farm Supporting Document, HNF-SD-WM-ER-323, Rev. 1 (Brevick et al., 1997a).

The S Tank Farm tanks were built to store wastes from S Plant (the Reduction and Oxidation extraction plant [REDOX]). All the tanks were used to store the evaporator bottoms waste from the evaporator operations. Tank 241-S-102 was also used as a feed tank for the 242-S Evaporator (Agnew et al., 1997). The primary additions to the tanks were REDOX high-level waste (R1), REDOX cladding waste (CWR1, CWR2), water (WTR), REDOX saltcake (RsltCk) and partial nuetralization feed (NIT). (Agnew et al., 1995).

In 1953, surface condensers were installed to concentrate the waste in the first two cascades. The vapor condensate from the surface condensers was disposed of to the cribs (Leach and Stahl, 1993). Two of those cribs where the vapor condensate was disposed were 216-S-3 and 216-S-4 (Brevick et al., 1996). Tank 241-S-103 served as the emergency dump tank to the 242-S Evaporator and as a receiver for the 242-S cell sump drainage (Leach and Stahl, 1993).

The following table presents a summary of the 241-S Tank Farm. Refer to the glossary for waste type terminology.

\section{1-S TANK FARM SUMMARY}

\begin{tabular}{|c|c|c|c|}
\hline \multicolumn{2}{|c|}{ TANK FARM DESCRIPTION } & \multicolumn{2}{|c|}{ WASTE VOLUME (HANLON 1996i) } \\
\hline Shell Type & Single & Total Waste Volume & $5,510,000 \mathrm{gal}$ \\
\hline Number of Tanks & 12 & Waste Type & DSSF, NCPLX \\
\hline Construction Date & $1950-1951$ & Drainable Interstitial Liquids & $1,327,000 \mathrm{gal}$ \\
\hline \multicolumn{2}{|c|}{ INTEGRITY } & Pumpable Liquids & $1,323,000 \mathrm{gal}$ \\
\hline \multirow[t]{3}{*}{ Sound } & $101,102,103,105$ & Saltcake & $4,286,000 \mathrm{gal}$ \\
\hline & $106,107,108,109$ & Sludge & $1,166,000 \mathrm{gal}$ \\
\hline & $110,111,112$ & Supernatant & $58,000 \mathrm{gal}$ \\
\hline Assumed Leaker & 104 & WATCH & \\
\hline \multicolumn{2}{|c|}{ TANK VOLUMES } & Hydrogen & $102,111,112$ \\
\hline \multirow{3}{*}{\multicolumn{2}{|c|}{$12 @ 758,000 \mathrm{gal}$}} & Organic & 102,111 \\
\hline & & High Heat & none \\
\hline & & Ferrocyanide & none \\
\hline
\end{tabular}


The historical characterization and waste inventory information for each tank is assembled into a set of tables, sketches, graphs, and interior tank montage. The set consists of the following for each tank in S Tank Farm:

- Tank Summary table

- Waste and Level History sketch

- Riser Configuration sketch

- Tank Layer Model Estimate graph

- Total Inventory Estimate tables

- TLM Solids Composite Inventory Estimate tables

- SMM Composite Inventory Estimate tables

- Interior tank montage

The supporting document for $\mathrm{S}$ Tank Farm provides backup data and further explanation of the above tables, sketches, and graphs. 


\section{S TANK FARM}

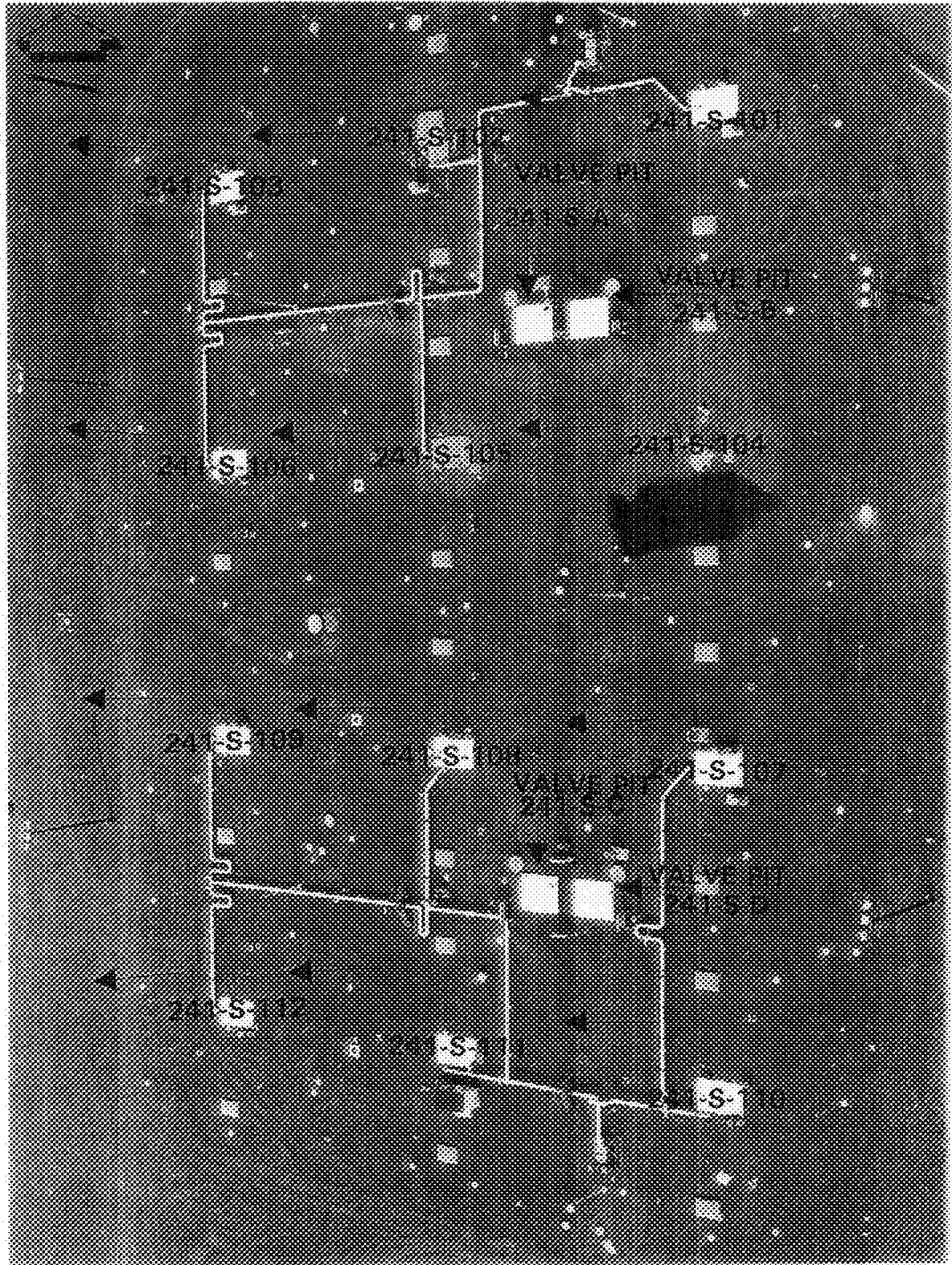


TANK 241-S-101 SUMMARY

\begin{tabular}{|c|c|c|c|}
\hline \multicolumn{2}{|c|}{ TANK HISTORY } & \multicolumn{2}{|c|}{ TANK DESCRIPTION } \\
\hline Entered Service & 3rd qtr 1953 & Diameter & $75 \mathrm{ft}$ \\
\hline Removed from Service & - & Bottom Shape & Dish \\
\hline Inactive & - & Nominal Capacity & $758,000 \mathrm{gal}$ \\
\hline Watch Lists & none & Cascade Tank & to $241-\mathrm{S}-102$ \\
\hline Integrity & Sound & Total Risers & 12 \\
\hline Assumed Leaker & - & \multicolumn{2}{|c|}{ WASTE VOLUME (HANLON 1996i) } \\
\hline Interim Stabilization (IS) & - & Total Waste Volume & $427,000 \mathrm{gal}$ \\
\hline Partial Interim Isolation (PI) & Dec 1982 & Waste Type & NCPLX \\
\hline Intrusion Prevention (IP) & - & Drainable Interstitial Liquids & $84,000 \mathrm{gal}$ \\
\hline \multicolumn{2}{|c|}{$\begin{array}{l}\text { TENTATIVELY AVAILABLE RISERS } \\
\end{array}$} & Pumpable Liquids & $127,000 \mathrm{gal}$ \\
\hline Riser Number(s) & Size & Saltcake & $171,000 \mathrm{gal}$ \\
\hline $4,11,16$ & 4 in & Sludge & $244,000 \mathrm{gal}$ \\
\hline 6 & 12 in & Supernatant & $\begin{array}{r}12,000 \mathrm{gal} \\
\end{array}$ \\
\hline \multicolumn{2}{|c|}{ TANK TEMPERATURE } & \multicolumn{2}{|c|}{ INTERIOR PHOTOGRAPHS } \\
\hline Average Tank Temperature & $110^{\circ} \mathrm{F}$ & Date & March 18, 1988 \\
\hline Maximum Temperature & $136.8^{\circ} \mathrm{F}$ & Montage Number & $94041033-40 \mathrm{CN}$ \\
\hline Date & Feb 6, 1989 & Photo Set Number & 8801412 \\
\hline Elevation from tank bottom & $0.48 \mathrm{ft}$ & \multicolumn{2}{|c|}{ WASTE SURFACE LEVEL } \\
\hline Riser Number & 14 & Devices & Manual ENRAF \\
\hline Minimum Temperature & $82.8^{\circ} \mathrm{F}$ & Max Level & 162.4 in \\
\hline Date & July 25, 1994 & Date & Aug 31, 1992 - Sept 23, 1992* \\
\hline Elevation from tank bottom & $24.44 \mathrm{ft}$ & Min Level & 160.82 in \\
\hline Riser Number & 14 & Date & March 27 and 28,1992 \\
\hline
\end{tabular}

\footnotetext{
- Numerous dates in this time span.
} 


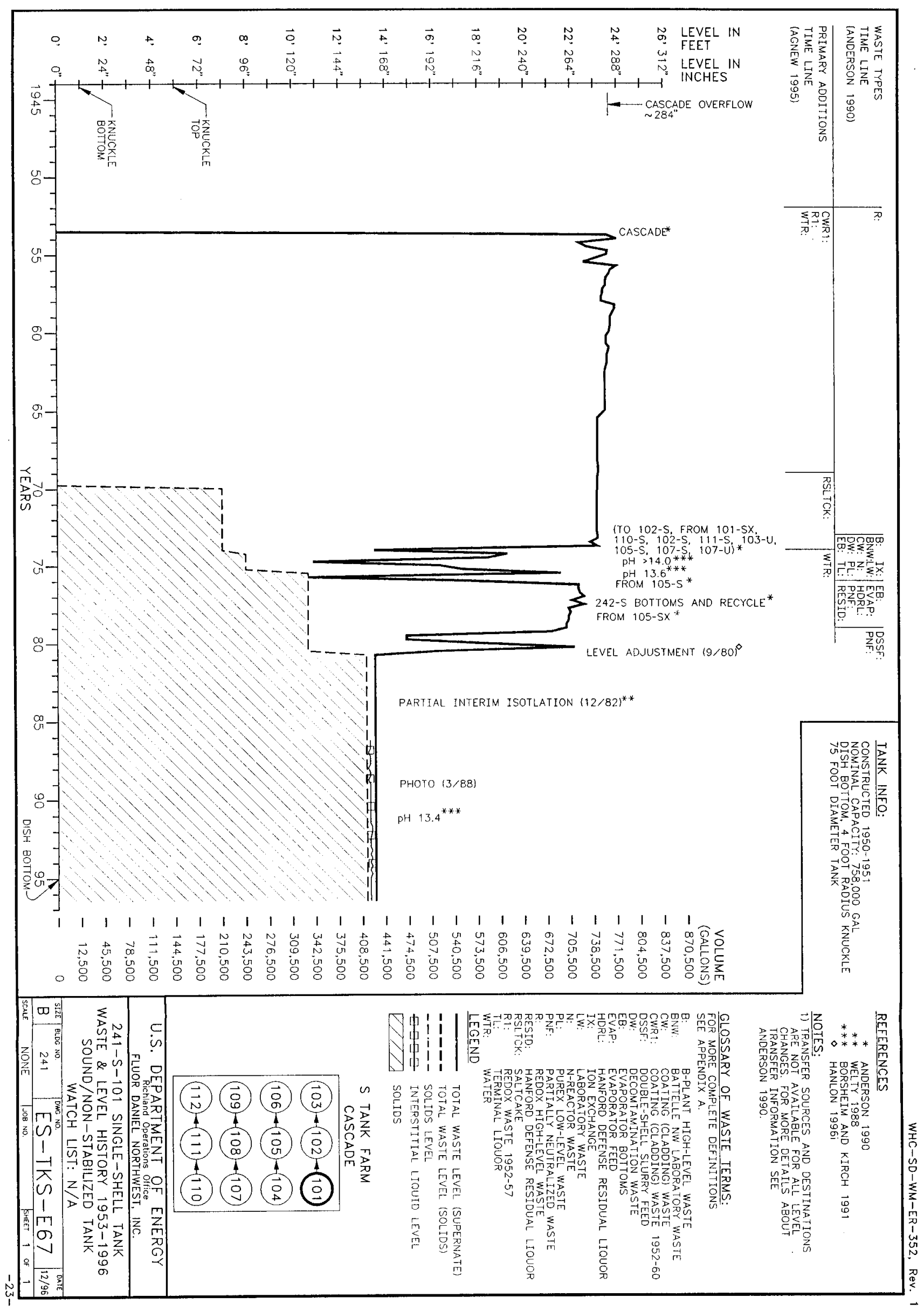




\section{$241-5-101$}

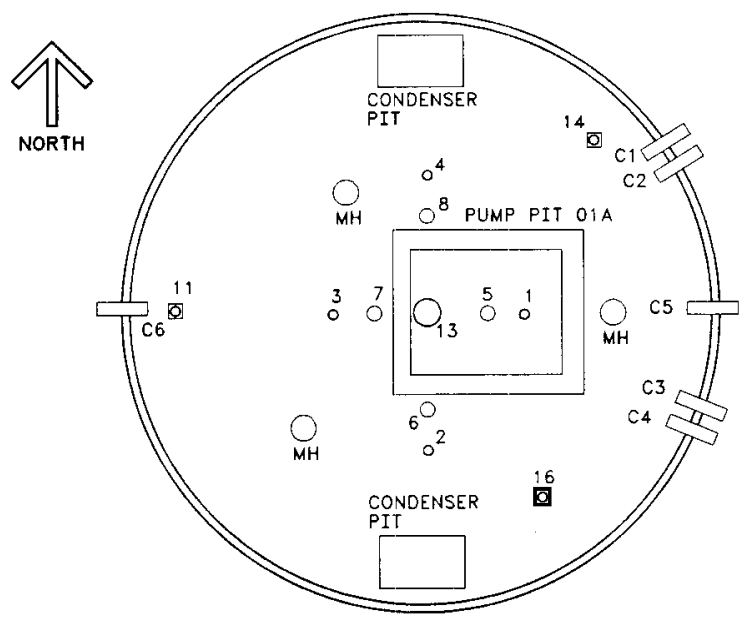

Ref: Alstad 1993

H-2-73181, Rev, 4

$\mathrm{H}-2-37524$, Rev. 1

\section{TANK RISER LOCATION}

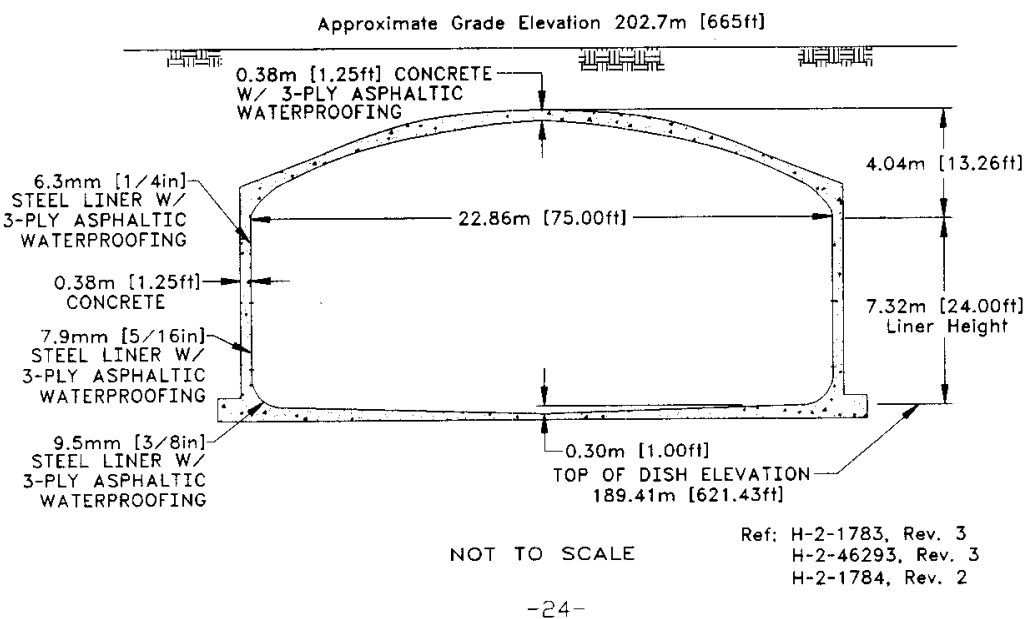


HNF-SD-WM-ER-352, Rev. 1

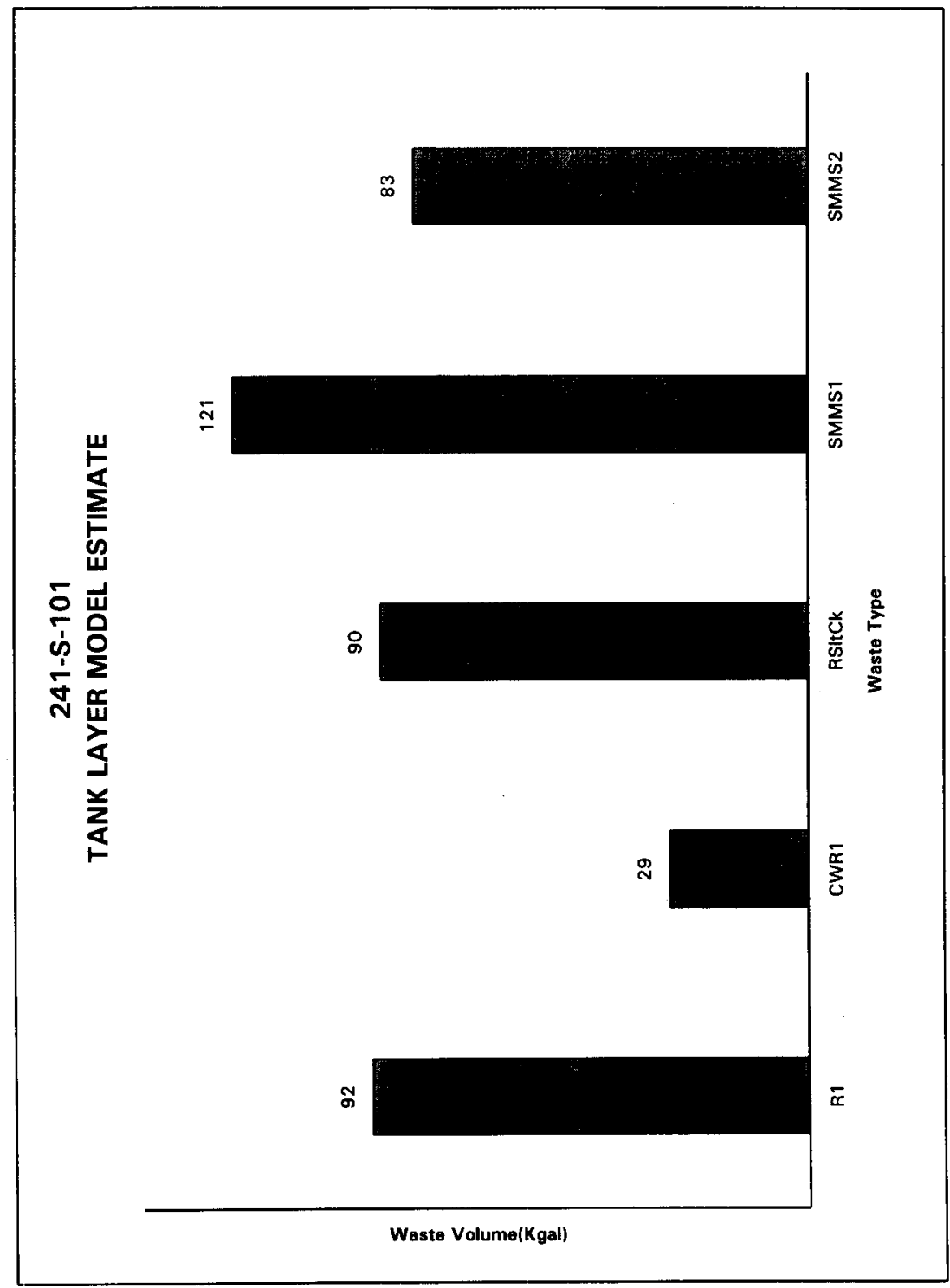


HNF-SD-WM-ER-352, Rev. 1

How Model Rev. 4

\begin{tabular}{|c|c|c|c|c|c|c|c|}
\hline \multicolumn{8}{|c|}{ Sinple-Shell Tank 241-S-101 } \\
\hline & \multicolumn{7}{|c|}{ TLM Solids Composite Inventory Estimate" } \\
\hline Physical & & & & & & & \\
\hline Properties : : & & $\cdots$ & & -95 CI & $67 \mathrm{CI}$ & $+67 \mathrm{CI}$ & $+95 \mathrm{CI}$ \\
\hline Total TIM Waste & $1.39 \mathrm{E}+06(\mathrm{~kg})$ & $(211 \mathrm{kga})$ & - & - & $\ldots$ & $\ldots$ & $\ldots$ \\
\hline Heat Load & $3.39(\mathrm{EW})$ & $(1.16 \mathrm{E}+04 \mathrm{BTU} / \mathrm{hr})$ & - & 2.77 & 3.13 & 3.56 & 3.64 \\
\hline Bulk Density & $1.74(g / \infty)$ & $\ldots$ & - & 1.62 & 1.64 & 1.89 & 1.95 \\
\hline Void Fraction & 0.658 & - & 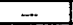 & 0.450 & 0.519 & 0.746 & 0.748 \\
\hline Water wt\% & 299 & - & $=$ & 17.4 & 21.4 & 36.4 & 38.2 \\
\hline TOC wt\% C (wet & 2.21E-03 & - & - & $1.97 E-03$ & 2.03E-03 & 2.38E-03 & $2.57 \mathrm{E}-03$ \\
\hline $\begin{array}{c}\text { Chenilow } \\
\text { Constituents }\end{array}$ & mold $\mathrm{L}$ & span & ke & $\begin{array}{l}95 \mathrm{cI} \\
\text { (nolit) }\end{array}$ & $\begin{array}{l}67 \mathrm{Cl} \\
(\mathrm{ma} / \mathrm{L})\end{array}$ & $\begin{array}{l}+67 \mathrm{CI} \\
(\text { molel })\end{array}$ & $\begin{array}{l}+95 \mathrm{CI} \\
\text { (mole } / \mathrm{L})\end{array}$ \\
\hline Nat & 10.4 & $1.37 \mathrm{E}+05$ & $1.90 \mathrm{E}+05$ & 8.15 & 8.37 & 13.2 & 14.8 \\
\hline All3+ & 6.03 & $9.36 \mathrm{E}+04$ & $1.30 \mathrm{E}+05$ & 5.20 & 5.44 & 6.67 & 7.32 \\
\hline Fe 3+ (total Fe) & 0.972 & $1.51 \mathrm{E}+04$ & $2.10 \mathrm{E}+04$ & 0.464 & 0.468 & 0.476 & 0.480 \\
\hline $\mathrm{Cr} 3+$ & 0.378 & $1.13 \mathrm{E}+0 \mathrm{~A}$ & $1.57 \mathrm{E}+04$ & 0.232 & 0.302 & 0.578 & 0.813 \\
\hline Bist & $2.93 \mathrm{E}-06$ & 0.352 & 0.499 & 2.49E-06 & $2.70 \mathrm{E}-06$ & 3.23E-06 & 355E-166 \\
\hline Let+ & $7.228-12$ & $5.76 \mathrm{E}-07$ & B.01E-07 & $6.33 \mathrm{E}-12$ & $6.83 \mathrm{E}-12$ & $7.98 \mathrm{E}-12$ & 8.T7E-12 \\
\hline $\mathrm{Hg} 2+$ & 5.61E-OA & 64.7 & 89.9 & $5.53 \mathrm{E}-04$ & $3.58 E-04$ & 5.64E-04 & $5.65 \mathrm{~S}-04$ \\
\hline $2 \mathrm{a}(\mathrm{arO} \mathrm{OH}) 2)$ & 2.93E-07: & I.53E-02 & $2.13 E-02$ & 2.69E- -07 & $2.80 \mathrm{E}-07$ & 3.07E-07 & 3.31E-07 \\
\hline $\mathrm{Pb2+}$ & $1.62 \mathrm{E}-02$ & $1.93 \mathrm{E}+03$ & $2.69 \mathrm{E}+03$ & 1.49E-02 & $1.57 E-02$ & $1.68 \mathrm{E}-02$ & $1.74 \mathrm{E}-02$ \\
\hline $\mathrm{Ni2t}$ & 287E-02 & 968 & $1.34 \mathrm{E}+03$ & $2.25 \mathrm{E}-02$ & $2.60 E-02$ & $3.07 \mathrm{E}-02$ & 3.03E-02 \\
\hline $\mathrm{Sr} 2+$ & 0 & 0 & 0 & 0 & 0 & $\mathbf{0}$ & 0 \\
\hline Mn4+ & 2.13E-05 & 0.674 & 0.936 & 1.52E-05 & $1.82 \mathrm{E}-05$ & 2.45E-05 & 2.76E-05 \\
\hline $\mathrm{Cot}$ & 0.145 & $3.35 E+03$ & $4.65 \mathrm{E}+03$ & 0.113 & 0.129 & 0.162 & 0.178 \\
\hline $\mathrm{K}+$ & $1.88 \mathrm{E}-02$ & 42 & 586 & I.44E-02 & $1.62 \mathrm{E}-02$ & 2.05E-02 & 2.13E-02 \\
\hline OH- & 24.9 & $2.43 \mathrm{E}+05$ & $3.38 \mathrm{E}+05$ & 21.0 & 22.3 & 27.6 & 30.3 \\
\hline NO3. & 4.62 & $1.65 \mathrm{E}+05$ & $2.29 E+05$ & 2.35 & 2.35 & 8.19 & 10.1 \\
\hline NO2. & 1.57 & $4.14 E+04$ & $5.75 \mathrm{E}+04$ & 0.987 & 1.19 & 1.87 & 1.87 \\
\hline Co32: & 0.147 & $5.08 E+03$ & $7,06 \mathrm{E}+03$ & 0.115 & 0.131 & 0.164 & 0.180 \\
\hline PO43- & $190 \mathrm{E}-04$ & 10.4 & 14.4 & 1.76E-04 & 1.84E-04 & 1.97E-04 & 2.15E-OA \\
\hline $5042-$ & $2.29 \mathrm{E}-02$ & $1.27 \mathrm{E}+03$ & $1.76 \mathrm{E}+03$ & 1.89E-02 & $2.05 \mathrm{E}-02$ & $2.45 \mathrm{E}-02$ & $2.61 \mathrm{E}-02$ \\
\hline $\mathrm{Si}(\mathrm{nsiO32-)}$ & $6.17 \mathrm{E}-02$ & 997 & $1.38 \mathrm{E}+03$ & 414E-02 & $5,40 \mathrm{E}-02$ & $7.02 \mathrm{E}-02$ & 8.31E-02 \\
\hline F. & $1.51 \mathrm{E}-04$ & 1,65 & 2.29 & 1.29E-04 & 1.42E-04 & $1.66 \mathrm{E}-04$ & I.79E-04 \\
\hline $\mathrm{Cl}$ & B.04E-02 & $1.64 E+03$ & $2.28 \mathrm{E}+03$ & 5.41E-02 & $6.70 E-02$ & 8.84E-02 & 0.126 \\
\hline C6H5OT- & $1.50 \mathrm{E}-04$ & 17.0 & 23.6 & 1.51E-04 & $1.54 E-04$ & $1.63 \mathrm{E}-04$ & $1.77 E-04$ \\
\hline EDTA4 & 6.08E-06 & 1.01 & 1.40 & 4.28E-06 & $5.26 \mathrm{E}-06$ & $7.16 \mathrm{E}-06$ & 8.24E-06 \\
\hline HEDTA3 & 5.05E-06 & $0.7 \%$ & 1.11 & $1.62 \mathrm{E}-06$ & $3.47 \mathrm{E}-06$ & 6.74E-06 & 8.39E-06 \\
\hline glycolente- & $2.20 \mathrm{E}-04$ & 9.51 & 13.2 & $1.20 \mathrm{E}-0 \mathrm{~A}$ & $1.74 E-04$ & $2.74 \mathrm{E}-04$ & $3.26 \mathrm{E}-04$ \\
\hline enetsits- & $2.29 \mathrm{E}-05$ & 0.776 & 1.08 & 2.22E-05 & $2.25 \mathrm{E}-05$ & $2.43 \mathrm{E}-05$ & 2.60E-05 \\
\hline oxalnte2- & $9.46 \mathrm{E}-12$ & 4.75E-67 & 6.65E-07 & 9.39E-12 & 8.90E-12 & 1.04E-11 & 1.14E-11 \\
\hline DEP & $13 \% E-04$ & 16.7 & 23.3 & $1.29 \mathrm{E}-04$ & $1.35 \mathrm{E}-04$ & 1.50E-04 & $1.62 \mathrm{E}-04$ \\
\hline Buthed & 1.39E-04 & 5.90 & 8.20 & $1.29 \mathrm{E}-0 \mathrm{~A}$ & 1.35E-04 & $1.50 \mathrm{E}-04$ & $1.62 \mathrm{E}-04$ \\
\hline $\mathrm{NH3}$ & 0.127 & $1.2+E+03$ & $1.72 \mathrm{E}+03$ & 4. $40 \mathrm{E}-02$ & 5.5TE-02 & 0.133 & 0.139 \\
\hline $\mathrm{Fe}(\mathrm{CN}) 64$ & 0 & 0 & 0 & 0 & 0 & 0 & 0 \\
\hline
\end{tabular}

"Unkeowns in tank solids invertory are evigred by Terk Leyoing Mode (TLM). 
NDW Model Rev. 4

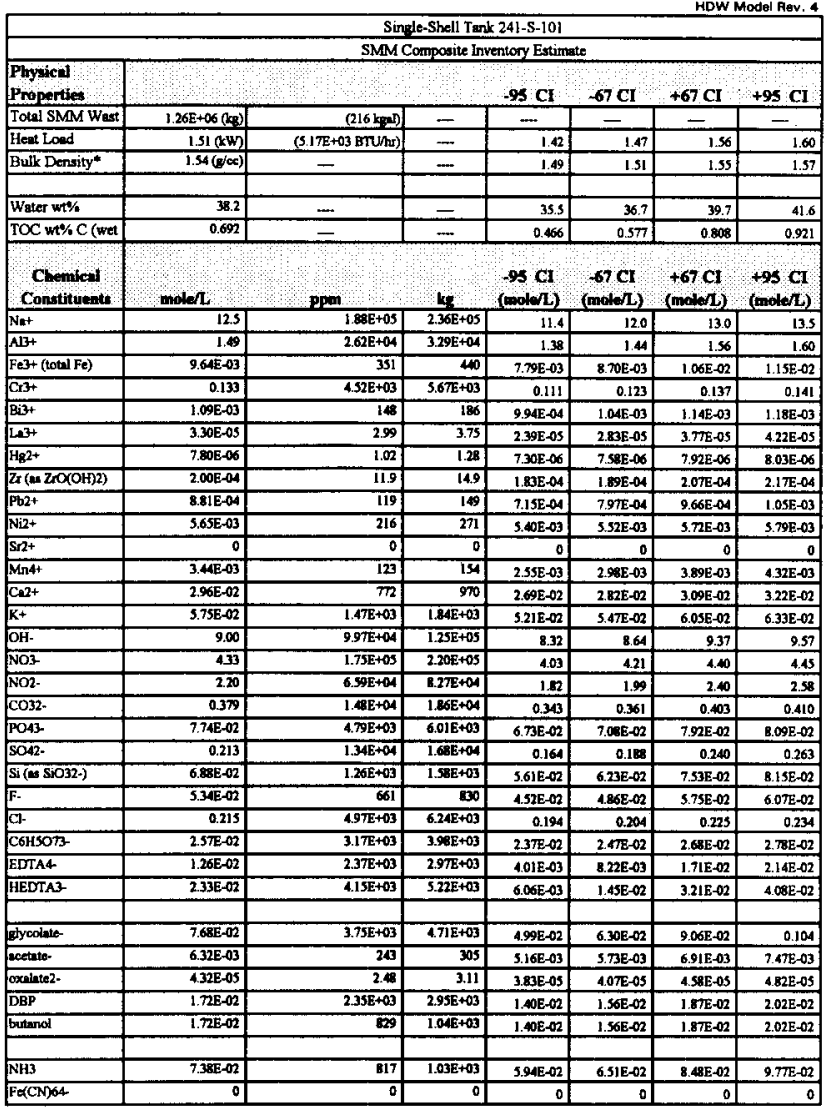

Density is celculated besed on $\mathrm{Na}$, OH-, and $\mathrm{AlO}$.

tWater $\mathrm{wt} \%$ derived from the difference of density and total dissolved apecies. 
HNF-SD-WM-ER-352, Rev. 1

\begin{tabular}{|c|c|c|c|c|c|c|c|}
\hline & \multicolumn{5}{|c|}{ Single-Shell Tark 241-S-101 } & \multicolumn{2}{|c|}{ HOW Model Rev, 4} \\
\hline & \multicolumn{7}{|c|}{ Total Inventory Estimate" } \\
\hline Payical & & & & & & & \\
\hline Properties & & & & $.95 \mathrm{CI}$ & $-67 \mathrm{CI}$ & $+67 \mathrm{Cl}$ & $+95 \mathrm{CI}$ \\
\hline Total Waste & $2.64 \mathrm{E}+06(\mathrm{~kg})$ & $(427 \mathrm{kgat})$ & $=$ & - & 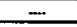 & - & - \\
\hline Heat Loed & $490(\mathrm{~km})$ & $(1.67 \mathrm{TE}+04 \mathrm{BTU} / \mathrm{hu})$ & - & 4.24 & 4.62 & 5.10 & 5.14 \\
\hline Bulk Density† & $1.64(0 / \infty)$ & -- & - & 1.57 & 1.58 & 1.72 & 1.74 \\
\hline Water wt\%t & 33.8 & - & $一$ & 27.0 & 28.8 & 37.6 & 38.4 \\
\hline TOC wt\% C (wet & 0.330 & - & - & 0.222 & 0.275 & 0.385 & 0.439 \\
\hline $\begin{array}{l}\text { Chezuic il } \\
\text { Contituents }\end{array}$ & molen $\mathrm{L}$ & pedi & Lo & $\left(m_{0, \mathrm{I}}\right)$ & $\begin{array}{l}67 \mathrm{CI} \\
\text { (nole/L) }\end{array}$ & $\begin{array}{l}+67 C \mathrm{CI} \\
\text { (male/L) }\end{array}$ & $\begin{array}{l}+9 \mathrm{CC} \\
\text { (mole L) }\end{array}$ \\
\hline $\mathrm{Na}+$ & 11.5 & $1.61 \mathrm{E}+05$ & $4.26 \mathrm{E}+0 \mathrm{~S}$ & 10.1 & 10.3 & 13.0 & 13.5 \\
\hline $\mathrm{Al3}+$ & 3.74 & $6.16 \mathrm{E}+04$ & $1.63 \mathrm{E}+05$ & 3.31 & 3.45 & 4.05 & 4.37 \\
\hline $\mathrm{Fe}^{3}+(\operatorname{cota} \mathrm{Fe})$ & 0.238 & $8.12 \mathrm{E}+03$ & $2.15 E+04$ & 0.234 & 0.236 & 0.240 & 0.242 \\
\hline $\mathrm{Cr} 3+$ & 0.254 & B.OBE+03 & $2.14 \mathrm{E}+0.4$ & 0.182 & 0.217 & 0.348 & 0.458 \\
\hline $\mathrm{Bi} 3+$ & \$.53E-04 & 70.6 & 187 & S.05E- -04 & $5.28 E-04$ & 5.77E-04 & $6.01 E-04$ \\
\hline Lat+ & 1.67E-05 & 1.42 & 3.75 & 1.21E-05 & $1.43 E-05$ & 1.91E-05 & 2.13E-05 \\
\hline $\mathrm{Hg2+}$ & 2.81E-04 & 34.5 & 912 & $2.77 \mathrm{E}-04$ & $2.80 \mathrm{E}-0.4$ & 2.82E- -04 & 2.83E-04 \\
\hline $\mathrm{Zr}(\mathrm{m} \mathrm{ZrO}(\mathrm{OH}) 2)$ & I.OIE-04 & 5.65 & 14.9 & 9.26E-05 & 9.57E-05 & 1.0SE-04 & $1.10 \mathrm{E}-04$ \\
\hline $\mathrm{Pb2+}$ & 8.4TE-03 & $1.07 \mathrm{E}+03$ & $2.84 E+03$ & $7,82 \mathrm{E}-03$ & $8.20 \mathrm{E}-03$ & B. $74 \mathrm{E}-03$ & 9.00E-03 \\
\hline Ni2t & $1.70 \mathrm{E}-02$ & 611 & $1.62 \mathrm{E}+03$ & 1. $\mathrm{OOE}-02$ & $1.57 \mathrm{E}-02$ & 1.80E- -02 & 1.73E-02 \\
\hline $8 x_{2+}$ & 0 & 0 & $\mathbf{0}$ & 0 & 0 & 0 & 0 \\
\hline Mn4t & $1.75 \mathrm{E}-03$ & 58.7 & 155 & $1.30 \mathrm{E}-03$ & 1.52E-03 & 1.98E.-03 & $2.20 E-03$ \\
\hline $\mathrm{Con}+$ & B.67E-02 & $2.12 \mathrm{E}+03$ & $5.62 \mathrm{E}+03$ & $7.15 \mathrm{E}-02$ & $7.90 \mathrm{E}-02$ & 9.45E-02 & 0.102 \\
\hline $\mathrm{K}+$ & $3.24 E-02$ & 917 & $2.43 \mathrm{E}+03$ & $3.56 E-02$ & $3.69 E-02$ & 3.991-02 & 4.13E-02 \\
\hline $\mathrm{OH}-$ & 16.8 & $1.75 \mathrm{E}+05$ & $4.63 \mathrm{E}+05$ & 14.8 & 15.5 & 18.2 & 19.6 \\
\hline $\mathrm{NO3}$ & 4.48 & $1.70 E+05$ & 4. $9 E+05$ & 3.30 & 3.34 & 6.22 & 7.11 \\
\hline NO2- & 1.89 & $530 \mathrm{E}+04$ & $1.40 \mathrm{E}+05$ & 1.56 & 1.71 & 2.05 & 2.08 \\
\hline Co32- & 0.265 & $9.71 E+03$ & $2.57 \mathrm{E}+04$ & 0.246 & 0.255 & 0.27 & 0.280 \\
\hline PO43 & $3.92 \mathrm{E}-12$ & $2.28 \mathrm{E}+03$ & $6.02 \mathrm{E}+03$ & $3.41 \mathrm{E}-02$ & $3.59 \mathrm{E}-02$ & 1.01E-02 & $4.10 E-02$ \\
\hline 5042. & 0.119 & $7.00 \mathrm{E}+03$ & $1.85 \mathrm{E}+04$ & $9.45 E-02$ & 0.106 & 0.133 & 0.144 \\
\hline Si (as SiO32.) & 6.53E-02 & $1.12 \mathrm{E}+03$ & $2.97 \mathrm{TE}+03$ & $5.66 \mathrm{E}-02$ & 6.15E-02 & 6.93E- -02 & $7.52 \mathrm{E}-02$ \\
\hline $\mathrm{F}-$ & $2.71 \mathrm{E}-02$ & 315 & B32 & $2.29 \mathrm{E}-02$ & 2.47E-02 & $2.92 \mathrm{E}-02$ & $3.08 \mathrm{E}-02$ \\
\hline C1 & 0.149 & $3.22 \mathrm{E}+03$ & $8.51 E+03$ & 0.129 & 0.139 & 0.154 & 0.170 \\
\hline C6HSOT3 & $1.31 \mathrm{E}-02$ & $1.51 \mathrm{E}+03$ & 4.00E+03 & $1.21 \mathrm{E}-02$ & 1.26E- -02 & $1.36 \mathrm{E}-02$ & $1.41 \mathrm{E}-02$ \\
\hline EDTA4- & $6.39 E-0.3$ & $1.12 \mathrm{E}+03$ & $2.97 E+03$ & $2.03 E-03$ & $4.16 \mathrm{E}-03$ & $8.63 \mathrm{E}-03$ & $1.08 \mathrm{E}-02$ \\
\hline HEDTA3. & 1.18E-02 & $197 \mathrm{E}+03$ & $3.22 \mathrm{E}+03$ & $3.07 \mathrm{E}-03$ & 7.32E-03 & 1.63E-02 & 2.06E-02 \\
\hline dycolnte & 3.90E-02 & $1.79 E+03$ & $4.72 E+03$ & 2.53E-02 & $3.20 \mathrm{E}-02$ & 4.60E-02 & $5.26 \mathrm{E}-02$ \\
\hline ecethts- & 3.21E-03 & 116 & 306 & $2.62 E-03$ & 2.91E-03 & 3.51E-03 & $3.79 \mathrm{E}-03$ \\
\hline oxsinte2. & 2.19E-05 & 1.18 & 3.11 & 1.94E-05 & 2.06E-0S & $2.31 \mathrm{E}-05$ & 2.44E-05 \\
\hline DBP & \$.75E-03 & $1.12 E+03$ & $2.97 \mathrm{E}+03$ & 7,17E-03 & $7.95 \mathrm{E}-03$ & 9.54E- 03 & 1.03E-02 \\
\hline butenol & $875 E-03$ & 397 & $1.05 E+03$ & $7.17 \mathrm{E}-03$ & $7.95 \mathrm{E}-03$ & 9.54E-03 & $1.03 \mathrm{E}-02$ \\
\hline NH3 & 1.00E-01 & $1.04 \mathrm{E}+03$ & $2.75 E+03$ & S.75E-02 & 6.41E-02 & 0.106 & 0.112 \\
\hline $\mathrm{Fe}(\mathrm{CN}) 64$ & 0 & 0 & 0 & 0 & 0 & 0 & 0 \\
\hline
\end{tabular}

Thkmowns in tark solids invertory are ancigned by Tenk Lyyering Model (TLM).

tWater wto derived from the differenoe of density and totul diveolved epecies. 
HDW Model Rev, 4

\begin{tabular}{|c|c|c|c|c|c|c|c|}
\hline & \multicolumn{7}{|c|}{ Single-Shell Tank 241-S-101 } \\
\hline & \multicolumn{7}{|c|}{ TLM Solids Composite Inventory Estimate* } \\
\hline Phyolical & & & & & & & \\
\hline Propertie: & & & & $95 \mathrm{CI}$ & $-67 \mathrm{CI}$ & $+67 \mathrm{Cl}$ & $+95 \mathrm{CI}$ \\
\hline Total TLM Weate & $1.39 \mathrm{E}+06(\mathrm{~kg})$ & (211 kaln) & - & $\ldots$ & - & $\ldots$ & - \\
\hline Heat Load & $3.39 \mathrm{(xw})$ & $(1.16 \mathrm{E}+04 \mathrm{BTU} / \mathrm{hs})$ & - & 2.77 & 3.13 & 3.56 & 3.64 \\
\hline Bulk Density & $1.74(g / \infty)$ & - & - & 1.62 & 1.64 & 1.89 & 1.95 \\
\hline Void Fraction & 0.658 & - & $\ldots$ & 0.450 & 0.519 & 0.746 & 0.748 \\
\hline Water wh & 29.9 & $\rightarrow$ & - & 17.4 & 21.4 & 36.4 & 38.2 \\
\hline TOC wt\% C (wet & $2.21 \mathrm{E}-03$ & $\ldots$ & - & 1.97E-03 & $2.03 \mathrm{E}-03$ & $2.38 \mathrm{E}-03$ & $2.57 \mathrm{E}-03$ \\
\hline Condionotest & $\mathrm{CM}$ & $\mathrm{MCV} / \mathrm{n}$ & CI & $\begin{array}{l}9 \mathrm{CI} \\
(\mathrm{CiN})\end{array}$ & $\begin{array}{l}67 \mathrm{Cl} \\
(\mathrm{CH})\end{array}$ & $\begin{array}{l}+67 \mathrm{Cl} \\
\text { (CDL) }\end{array}$ & $\begin{array}{l}+95 \mathrm{Cl} \\
\text { (CUL) }\end{array}$ \\
\hline $\mathrm{H}-3$ & $1.08 \mathrm{E}-04$ & $6.21 \mathrm{E}-02$ & 86.2 & $1,29 \mathrm{E}-0 \mathrm{~S}$ & 1.29E-05 & $1.13 E-04$ & $1.21 E-04$ \\
\hline $\mathrm{C}-14$ & $5.88 E-06$ & 3.38E-03 & 470 & $1.00 \mathrm{E}-06$ & $1.00 \mathrm{E}-\infty 6$ & 6.23E- -06 & $6.53 E-06$ \\
\hline Ni-59 & $9.39 \mathrm{E}-06$ & $5.40 E-03$ & 7.50 & 6.48E-06 & 6.48E- $-\infty 6$ & 9.99E-06 & 9.B8E-06 \\
\hline $\mathrm{Ni}-63$ & 8.88E-04 & 0.510 & 709 & 6.05E-04 & 6.05E-04 & 9.4AE-04 & $9.33 E-04$ \\
\hline$C_{0}-60$ & 1.79E-06 & $2.75 \mathrm{E}-03$ & 382 & $3.96 \mathrm{E}-07$ & $3.96 \mathrm{E}-07$ & 4.98E-06 & $5.38 \mathrm{E}-06$ \\
\hline $\operatorname{Se} 79$ & 3.18E-06 & $1.89 \mathrm{E}-03$ & 2.54 & $2.13 \mathrm{E}-07$ & 2.13E-07 & $3.25 \mathrm{E}-06$ & $6.09 \mathrm{E}-06$ \\
\hline Si-90 & 0.517 & 297 & $4.13 E+05$ & 0.401 & 0.467 & 0.556 & 0.563 \\
\hline$Y .90$ & 0.517 & 297 & $4.13 \mathrm{E}+05$ & 0.401 & 0.405 & 0.556 & 0.563 \\
\hline $2 r-93$ & $1,50 \mathrm{E}-05$ & 8.64E-03 & 12.0 & $1,00 E-06$ & 1.00E-06 & 1.54E-05 & $2.73 \mathrm{E}-05$ \\
\hline Nb-93m & $1.22 \mathrm{E}-05$ & $7.028-03$ & 9.75 & $8.21 \mathrm{E}-07$ & 8.21E-07 & $1.25 \mathrm{E}-05$ & 2.54E-05 \\
\hline To-99 & 4.49E-05 & $2.58 \mathrm{E}-02$ & 35.9 & $3.88 E-05$ & 4.13E-0S & 4.78E-05 & 5.11E-05 \\
\hline Ru.106 & $1.02 \mathrm{E}-49$ & 5.84E- -07 & E.11E-04 & 1.13E-12 & 1.13E-12 & $1.06 \mathrm{E}-09$ & 1.1SE-09 \\
\hline Cd-113m & $2.29 \mathrm{E}-05$ & 1.31E-02 & 18.3 & 3.10E-06 & 3.10E-06 & 4.70E-05 & 4. $55 \mathrm{E}-05$ \\
\hline Sb-125 & $1.63 E-05$ & 9.35E- 03 & 13.0 & 5.83E- 07 & $5.03 E-07$ & $1.70 \mathrm{E}-05$ & 1.84E-0.5 \\
\hline $5 n-126$ & 4.88E-06 & 2.80E-03 & 3.89 & 3.27E-07 & 3.27E-07 & 4.99E-06 & $9.55 \mathrm{E}-06$ \\
\hline $1-129$ & 8.54E-08 & 4.91E-05 & 6.22E-02 & $7.37 \mathrm{E}-\mathrm{Cs}$ & THA:-08 & $9.09 \mathrm{E}-0 \mathrm{~g}$ & $9.71 \mathrm{E}-08$ \\
\hline Cs-134 & 9.93E-07 & 5.71E-04 & 0.793 & 1.21E-08 & $1.21 \mathrm{E}-0 \mathrm{~B}$ & $1.03 \mathrm{E}-06$ & $1.11 \mathrm{E}-06$ \\
\hline Cs-137 & 0.163 & 93.6 & $1,30 E+05$ & 0.145 & 0.152 & 0.173 & 0.184 \\
\hline $\mathrm{Be}-137 \mathrm{~m}$ & 0.154 & 88.6 & $1.23 \mathrm{E}+05$ & 2.34E- 02 & 2.34E-02 & 0.161 & 0.169 \\
\hline Sm-151 & $1.13 E-02$ & 6.51 & $9.05 E+03$ & $7.59 \mathrm{E}-0 \mathrm{~A}$ & 7.59E-04 & $1.16 E-02$ & $2.21 \mathrm{E}-02$ \\
\hline Ev-152 & 6.86E-06 & 3.94E-03 & 5.4 & 3.08E- 06 & 3.08E- 06 & $6.89 \mathrm{E}-06$ & $6.93 \mathrm{E}-06$ \\
\hline Eu-154 & 1.15E-04 & 6.58E-02 & 91.5 & $9.57 \mathrm{E}-06$ & $9.5 \pi-06$ & $1,19 \mathrm{E}-04$ & 1.48E-04 \\
\hline Eu. 155 & 3.37E-04 & 0.194 & 269 & 1.45E-04 & 1. $45 E-04$ & $3.38 \mathrm{E}-04$ & 3.41E-Od \\
\hline Ra-226 & $6.72 E-10$ & 3.86E- -07 & $5.36 \mathrm{E}-04$ & $2.65 \mathrm{E}-10$ & 4.64E-10 & 8.79E-10 & $1008 \mathrm{E}-09$ \\
\hline $\mathrm{Ra}-228$ & 6.77E-49 & 3.86E- 06 & $5.37 \mathrm{E}-03$ & 4.08E-15 & 4.0.E-15 & $6.70 \mathrm{E}-10$ & 6.86E-09 \\
\hline Ac-227 & 3.25E- 09 & $1.87 \mathrm{E}-06$ & $2.60 \mathrm{E}-03$ & 1.31E-09 & 2.04E-09 & $4.46 \mathrm{E}-09$ & $5.61 \mathrm{E}-09$ \\
\hline $\mathrm{Pa}-231$ & 4.73E-09 & 272E- -6 & 3.76E-03 & 3.18E-10 & 3.18E-10 & $5.31 \mathrm{E}-09$ & 1.17508 \\
\hline Th-229 & $1.62 \mathrm{E}-10$ & $9,305-08$ & $1.29 \mathrm{E}-04$ & $7.76 \mathrm{E}-13$ & $7.76 \mathrm{E}-13$ & $1.63 \mathrm{E}-10$ & $1.65 \mathrm{E}-10$ \\
\hline Th-232 & $9.01 \mathrm{E}-11$ & 5.18E- 03 & $7.19 \mathrm{E}-05$ & $2.63 \mathrm{E}-16$ & 2.63E-16 & $1.14 \mathrm{E}-10$ & $1.37 \mathrm{E}-10$ \\
\hline $\mathrm{U}-232$ & $3,01 \mathrm{E}-09$ & 1.73E-05 & 2.40E-01 & 1.07E-08 & $1,9 \pi \mathrm{E}-08$ & 4.29E-08 & 5,7]E-08 \\
\hline $\mathrm{U}_{-233}$ & $1.15 \mathrm{SE}-07$ & 6.60E-05 & $9.18 E-02$ & 1.06E- 0 & $7.50 \mathrm{E}-08$ & 1.64E-0? & 2.1BE-07 \\
\hline $\mathrm{U}-234$ & $2.63 \mathrm{E}-06$ & 1.51E-03 & 2.10 & 2.32E- -06 & $2.4 \pi-06$ & $2.80 \mathrm{E}-06$ & 2.97E-06 \\
\hline U-235 & $1.11 \mathrm{E}-07$ & 6.36E-05 & B.85-02 & 9.02E-C8 & 1.04E-07 & 1.17E-07 & 1.24E-07 \\
\hline $\mathrm{U}-236$ & $6.81 \mathrm{E}-08$ & 3.91E-05 & 5.44E-02 & $5.59 E-08$ & 6.19E-08 & $7,45 E-08$ & 8.09E-08 \\
\hline$\underline{1238}$ & $2.51 \mathrm{E}-06$ & 1.4E- 03 & 2.00 & $2.24 E-06$ & $2.37 \mathrm{E}-06$ & $2.66 \mathrm{E}-06$ & $2.81 E-06$ \\
\hline Np-237 & 2.11E-07 & 1.21E-04 & 0.169 & 1.71E-07 & 1.8TE-07 & 2.27E-67 & 2.40E-07 \\
\hline Pu-238 & 7.34E-06 & 4.22E-03 & 5.86 & 6.53E- -06 & 6.92E-06 & $7.75 \mathrm{E}-06$ & 8.15E-06 \\
\hline PL-239 & 4.59E-04 & 0.264 & 367 & 4.01E-04 & 4.30E-04 & 4.89E-04 & S.17E-OA \\
\hline Pu-240 & $6.60 \mathrm{E}-05$ & 3.7. & 52.7 & 5.77E-05 & 6.18E-05 & $7,03 \mathrm{E}-05$ & 7.43E-05 \\
\hline Pu-24] & 4.15E-04 & 0.29 & 332 & $3.66 \mathrm{E}-04$ & $3.90 \mathrm{E}-04$ & 4.40E-04 & $4.64 E-04$ \\
\hline $\mathrm{Pu}-242$ & 1. $88 E-09$ & $1.08 \mathrm{E}-06$ & $1.50 \mathrm{E}-03$ & 1.65E-09 & $1.76 \mathrm{E}-09$ & $1.90 \mathrm{E}-09$ & $2.10 \mathrm{E}-09$ \\
\hline Am-241 & S.34E-05 & 3.0TE-02 & 426 & 3.18E-05 & 4.66E-05 & 6.06E-05 & 1.12E-04 \\
\hline Am-243 & $1.62 \mathrm{E}-09$ & 9.332 .07 & $1.30 \mathrm{E}-03$ & $7.645-10$ & $1.41 E-09$ & $1,7 \mathrm{AE}-00$ & $2.17 \mathrm{E}-09$ \\
\hline $\mathrm{Cm}-242$ & 7.14E-08 & 4.10E-05 & 5.70E-02 & $6.99 \mathrm{E}-08$ & 7.05E-08 & $7.20 \mathrm{E}-08$ & 7.20E-08 \\
\hline$C_{m}-243$ & 1.63E- $-\infty 9$ & 9.39E-07 & $1.30 \mathrm{E}-03$ & 1.605 .69 & $1.61 \mathrm{E}-09$ & $1.65 \mathrm{E}-09$ & $1.65 E-09$ \\
\hline Cm-244 & $120 \mathrm{E}-09$ & $7.34 \mathrm{E}-07$ & $1.02 \mathrm{E}-03$ & $2.33 \mathrm{E}-10$ & $6.51 \mathrm{E}-10$ & 1.70E- 09 & $170 \mathrm{E}-09$ \\
\hline Totels & $\mathbf{M}$ & $\mathrm{d} / \mathrm{s}$ & th: & $9 s \propto(\mathrm{M}$ & $\begin{array}{l}67 \mathrm{CO} \\
6, \mathrm{~A})\end{array}$ & $\begin{array}{l}+67 \mathrm{Cl} \mathrm{a} \\
\mathrm{c} \mathrm{Al}\end{array}$ & $\begin{array}{l}+95 \mathrm{Cl} \\
\text { Mor of })\end{array}$ \\
\hline Pu & $7.68 \mathrm{E}-03(\mathrm{n} / \mathrm{L})$ & - & 6.13 & $6.71 \mathrm{E}-03$ & 7.18E-03 & B.1n-03 & B.65E-03 \\
\hline $\mathrm{U}$ & 3.13E-02 & $431 E+03$ & $5.98 \mathrm{E}+03$ & $2.81 \mathrm{E}-02$ & 2.97E-02 & 3.33E-02 & $3.52 E-42$ \\
\hline
\end{tabular}

Unknowns in tenk solids inventory are asigned by Tent Loyering Model (ILM). 
HDW Model Rev. 4

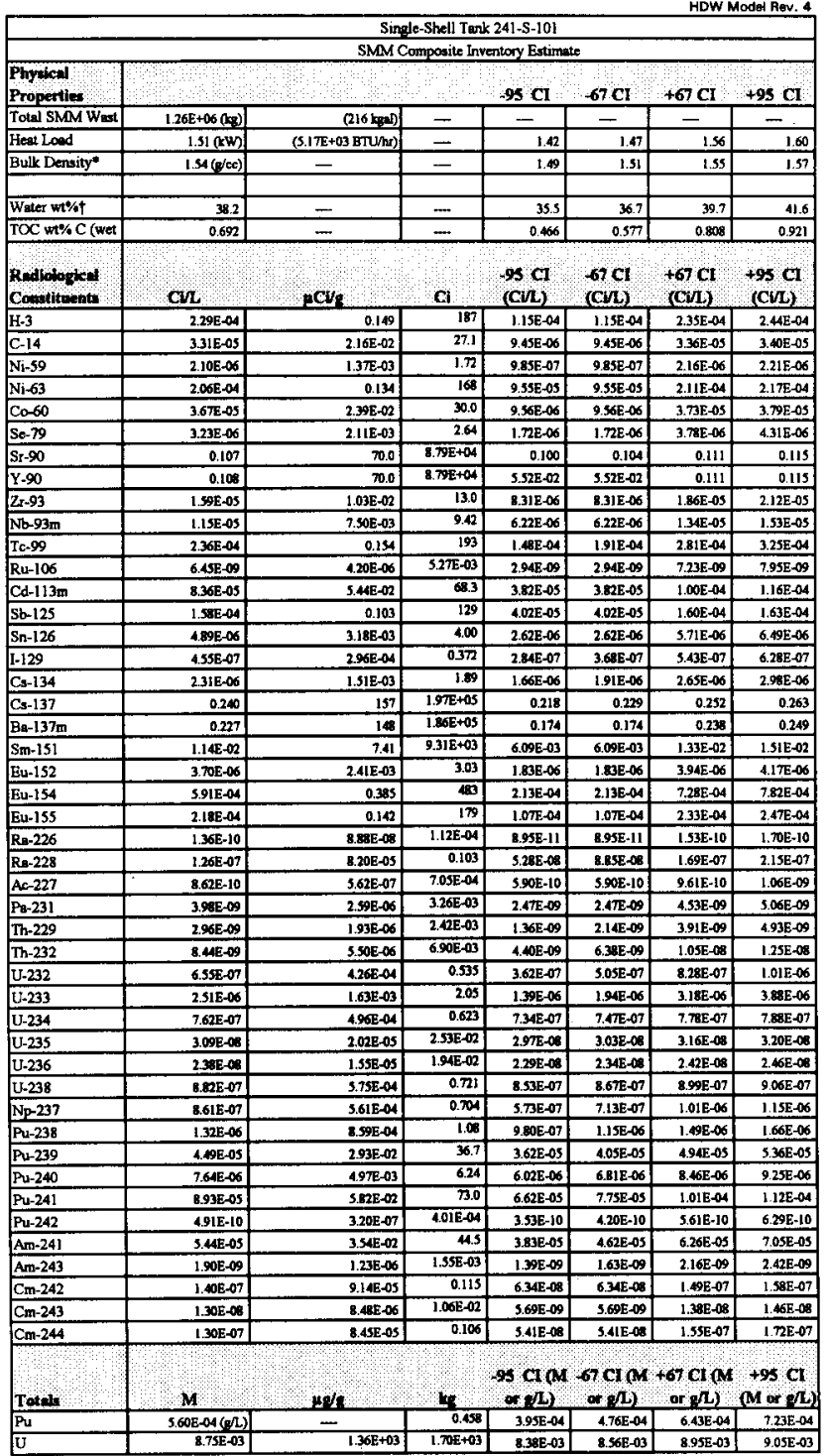

Density is colculated based on Nis, $\mathrm{OH}_{-}$and $\mathrm{AlO}_{2}$.

tWater wt\% detived foen the difference of dentity and total diusolved apecies. 


\begin{tabular}{|c|c|c|c|c|c|c|c|}
\hline \multirow[b]{3}{*}{$\begin{array}{l}\text { Phypical } \\
\text { Properties }\end{array}$} & \multicolumn{7}{|c|}{ Sinqle-Shell Tank 241-S-101 } \\
\hline & \multicolumn{7}{|c|}{ Total Inventory Estimale * } \\
\hline & & & & $95 \mathrm{CI}$ & $67 \mathrm{CI}$ & $+67 \mathrm{CI}$ & $+95 \mathrm{CI}$ \\
\hline Total Waste & $2.04 \mathrm{E}+06(\mathrm{gr})$ & (427 kgal) & $=$ & - & 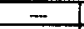 & - & - \\
\hline Heat Load & $490(\mathrm{~kW})$ & $(1.67 \mathrm{E}+04 \mathrm{BTU} / \mathrm{Mr})$ & $=$ & 4.24 & 4.62 & 5.10 & 5.14 \\
\hline Bulk Densityt & $1.64\left(y_{00}\right)$ & - & - & 1.57 & 1.58 & 1.72 & 1.74 \\
\hline & & & & & & & \\
\hline Water wt\% $\dagger$ & 33.8 & - & - & 27.0 & 28.8 & 37.6 & $\mathbf{3 8 . 4}$ \\
\hline TOC wt\% C (wet & 0.330 & - & - & 0.222 & 0.275 & 0.385 & 0.439 \\
\hline $\begin{array}{l}\text { Rediolodices } \\
\text { Conotitenents }\end{array}$ & $\mathrm{CM}$, & $\mu \mathrm{Q} / \mathrm{r}$ & $\mathrm{Cl}_{\mathrm{H}}$ & (c) Cl & $\begin{array}{l}6 \mathrm{Cl} \\
(\mathrm{CVL})\end{array}$ & $\begin{array}{c}+67 \mathrm{Cl} \\
(\mathrm{CH} / \mathrm{l})\end{array}$ & $\begin{array}{l}+9 \mathrm{ct} \\
(\mathrm{Cl} / \mathrm{L})\end{array}$ \\
\hline $\mathrm{H}-3$ & 1.69E-04 & 0.103 & 273 & $9.9 \pi-05$ & $9,97 \mathrm{E}-05$ & 1.72E-04 & $1.7 \pi-04$ \\
\hline $\mathrm{C}-14$ & 1.97E-05 & $1.20 \mathrm{E}-02$ & 31.8 & $7.69 \mathrm{E}-06$ & $7,69 \mathrm{E}-06$ & $1.99 \mathrm{E}-05$ & $2.01 \mathrm{E}-05$ \\
\hline Ni-59 & $5.70 \mathrm{E}-06$ & $3.48 E-03$ & 9.22 & $4.20 \mathrm{E}-06$ & $4.20 \mathrm{E}-06$ & $6.00 E-96$ & 593E-06 \\
\hline $\mathrm{Ni}-63$ & $5.03 \mathrm{E}-04$ & 0.332 & 37 & 3.97E-04 & $3.978-04$ & $5.70 E-04$ & $5.64 \mathrm{E}-04$ \\
\hline $\mathrm{Co}-60$ & 2.09E-05 & $1.28 \mathrm{E}-02$ & 33.8 & $7.20 \mathrm{E}-06$ & $7.20 \mathrm{E}-06$ & 2.12E-05 & $2.15 E-05$ \\
\hline Se. 79 & $3.21 E-06$ & $1.96 \mathrm{E}-03$ & 5.18 & $1.58 E-106$ & 1.58E-06 & $3.48 E-06$ & $4.57 E_{-06}$ \\
\hline Sr-90 & 0.310 & 189 & $5.01 E+03$ & 0.253 & 0.285 & 0.329 & 0.331 \\
\hline Y.90 & 0.310 & 109 & $5.01 \mathrm{E}+05$ & 0.252 & 0.252 & 0.329 & 0.331 \\
\hline $2 x-93$ & $1.55 \mathrm{E}-05$ & $9.45 E-03$ & 25.0 & 7.74E-06 & $7.74 \mathrm{E}-06$ & $1.68 \mathrm{E}-05$ & $2.12 \mathrm{E}-05$ \\
\hline $\mathrm{Nb}-93 \mathrm{~m}$ & $1.19 \mathrm{E}-05$ & 7.25E-03 & 19.2 & 5.68E-06 & 5.68E- -06 & $1.28 \mathrm{E}-05$ & $1.80 \mathrm{E}-05$ \\
\hline Tc-99 & 1.42E-04 & $8.65 \mathrm{E}-02$ & 229 & $9.69 \mathrm{E}-05$ & $1.19 E-04$ & $1.65 \mathrm{E}-04$ & 18TE-04 \\
\hline Ru-106 & 3.76E-09 & $2.30 \mathrm{E}-06$ & 6.09E-03 & $1.99 \mathrm{E}-09$ & $1.99 \mathrm{E}-09$ & 4.16E-09 & $4,52 \mathrm{E}-09$ \\
\hline $\mathrm{Cd}-113 \mathrm{~m}$ & 5.30E-05 & $3.27 \mathrm{E}-02$ & 8.6 & $3.06 \mathrm{E}-05$ & 306E-05 & $6.39 \mathrm{E}-05$ & $6.99 \mathrm{E}-05$ \\
\hline $\mathrm{Sb}-125$ & 8.78E- 05 & $5.37-02$ & 142 & 2.84E-05 & 2.24E-05 & 8.92E-05 & $9.06 \mathrm{E}-05$ \\
\hline $\mathrm{Sn}-126$ & 183E-06 & 2.98E-03 & 7.29 & $2.40 E-06$ & $2.40 E-06$ & $530 \mathrm{E}-06$ & 7.07E-06 \\
\hline 1-129 & $2.72 \mathrm{E}-07$ & 1.66E-04 & 0.440 & $1.068-07$ & 2.28E-07 & $3.1 \pi-07$ & $3.00 \mathrm{E}-07$ \\
\hline Cs-134 & $1.66 \mathrm{E}-06$ & $1.02 E-03$ & 26 & $9.74 E-07$ & $9.74 E-97$ & $1.83 \mathrm{E}-06$ & $2.00 \mathrm{E}-06$ \\
\hline Cs.137 & 0.202 & 124 & $3.27 \mathrm{E}+05$ & 0.191 & 0.190 & 0.210 & 0.219 \\
\hline $\mathrm{Ba}-137 \mathrm{~m}$ & 0.191 & 117 & $3.09 E+05$ & $9.96 \mathrm{E}-02$ & $9.96 \mathrm{E}-02$ & 0.197 & 0.202 \\
\hline Sm-15i & 1.14E-02 & 6.94 & $1.8 \mathrm{EE}+04$ & 5.58E-03 & 5.5SE-03 & $1.23 \mathrm{E}-02$ & $1.64 E-02$ \\
\hline Eu-152 & $5.26 \mathrm{E}-06$ & 3.22E-03 & 8.50 & $3.30 E-06$ & 3.30E-06 & $3.38 E-06$ & $5.50 \mathrm{E}-06$ \\
\hline Eut-154 & 3.56E-04 & 0.217 & 575 & $1.64 \mathrm{E}-04$ & $1.64 \mathrm{E}-04$ & $4.25 E-04$ & 4.52E-04 \\
\hline Eu-155 & $2.77 \mathrm{E}-04$ & 0.169 & 40 & $1,70 \mathrm{E}-04$ & $1.7 \mathrm{aE}-04$ & 2.85E-04 & $2.92 \mathrm{E}-04$ \\
\hline Ra-226 & 4.01E-10 & $2.45 \mathrm{E}-07$ & 6.4BE-04 & 2.10E-10 & $2.97 \mathrm{E}-10$ & $4.98 E-10$ & $5.91 \mathrm{E}-10$ \\
\hline$R_{m-228}$ & $6.70 \mathrm{E}-08$ & 409E-05 & 0.109 & $3.00 \mathrm{E}-0 \mathrm{e}$ & 4:EIE-08 & B.89E- -68 & 1.12E-07 \\
\hline Ac-227 & $2.04 E-09$ & $1.25 \mathrm{E}-06$ & $3.30 \mathrm{E}-03$ & 1.13E-09 & 1.43E-09 & 2.61E-09 & 3.15E-09 \\
\hline Pa-23| & $435 E-09$ & $2.66 \mathrm{E}-06$ & $7.035-03$ & 2.01E-09 & $2.01 E-09$ & $4.63 \mathrm{E}-09$ & $1.63 \mathrm{E}-09$ \\
\hline Th-229 & 1.58E-09 & $9.64 \mathrm{E}-07$ & 2.5SE-03 & $7.66 \mathrm{E}-10$ & $1.16 E-09$ & $2.06 \mathrm{E}-09$ & 2.57E-09 \\
\hline Th-232 & 1.31E-08 & $2.648-06$ & $6.97 \mathrm{E}-03$ & $2.27 \mathrm{E}-09$ & $3.2 \pi=00$ & $5,36 \mathrm{E}-09$ & $6.36 \mathrm{E}-09$ \\
\hline $\mathbf{U}-232$ & 3.46E-07) & 2.11E-04 & 0.559 & 1.98E-07 & $2.70 \mathrm{E}-07$ & 4.30E-07) & 5.28E-07 \\
\hline U.233 & $1.33 E-06$ & B.11E.04 & 2.14 & $7.595-07$ & $1.04 E-06$ & $1.66 \mathrm{E}-06$ & $2.02 E-06$ \\
\hline $\mathrm{U}-\mathbf{2 3 4}$ & $1.68 E-06$ & $1.03 E-03$ & 2.72 & $1.53 \mathrm{E}-06$ & $1.61 \mathrm{E}-26$ & $1.77 \mathrm{E}-06$ & $1.85 E-06$ \\
\hline U-235 & $7.03 E-08$ & 4.30E-03 & 0.114 & $6.42-08$ & $6.71 E-6$ & $7.37 \mathrm{E}-08$ & $7.71 E-28$ \\
\hline U.236 & 1.5TEA8 & 2.79E-05 & $7.36 \mathrm{E}-02$ & $3.97 \mathrm{E}-\infty 8$ & $4.27 \mathrm{E}-98$ & $4.88 \mathrm{E}-08$ & $5.20 E-08$ \\
\hline U-238 & $1.69 E-06$ & 1.03E-03 & 27 & $1.55 \mathrm{E}-\infty 6$ & $1.62 E-06$ & $1.76 \mathrm{E}-06$ & $1.84 E-06$ \\
\hline $\mathrm{Np}-237$ & 5.40E-07 & 3.30E- -4 & 0.873 & $3.94 \mathrm{E}-07$ & 4.65E- 07 & 6.15E-07 & $6.88 \mathrm{E}-07$ \\
\hline Pu-228 & 4.29E-06 & $2.62 E-03$ & 6.94 & $3.91 E-06$ & 4.10E-06 & $4.49 \mathrm{E}-166$ & 4.67E-06 \\
\hline Pu-239 & $2.50 \mathrm{E}-04$ & 0.153 & 404 & $2.22 \mathrm{E}-\mathrm{AM}$ & $2.365-04$ & $2.64 \mathrm{E}-\mathrm{OA}$ & $2 . \pi \mathrm{E}-04$ \\
\hline Pu-240 & $3.65 \mathrm{E}-05$ & $2.23 E-02$ & 59.0 & $3.26 \mathrm{E}-0.05$ & 3.45E-05 & 3.8SE-05 & 4.04E-05 \\
\hline$P u-241$ & $2.505-04$ & 0.153 & 405 & $2.27 \mathrm{E}-04$ & 2.30E-04 & $2.62 \mathrm{E}-0.04$ & $2.73 E-04$ \\
\hline Pu-242 & $1.18 E-09$ & 7.18E-07 & 1.90E.03 & $1.07 E-09$ & $1.12 E-09$ & $1.23 \mathrm{E}-09$ & 1.28E-09 \\
\hline Am-241 & 5.99E-05 & $3.29 \mathrm{E}-02$ & $\$ 7.1$ & 4.16E-05 & $497 \mathrm{k}-05$ & $5.81 \mathrm{E}-05$ & 8.15E-05 \\
\hline $\mathrm{Am}-243$ & $1.76 \mathrm{E}-09$ & $1.08 E-06$ & 2.8SE-03 & $1.28 \mathrm{E}-09$ & $1.62 E-09$ & 1.90E-09 & 2.03E-09 \\
\hline $\mathrm{Cm}-242$ & 1.06E-07 & 6.49E-05 & 0.172 & $6.73 \mathrm{E}-0 \mathrm{~B}$ & $6.73 E-18$ & 1.11E-07 & 1.15E 07 \\
\hline $\mathrm{Cm}-243$ & 7.40E-09 & $4.52 E-06$ & $1.20 \mathrm{E}-02$ & $3.69 E-109$ & $3.69 \mathrm{E}-09$ & $7.81 \mathrm{E}-09$ & 8.21E-09 \\
\hline $\mathrm{Cm}-244$ & $6.63 E-08$ & 1.05E-05 & 0.107 & 2.00E-08 & 2.00E-08 & $7,92 E-08$ & 8.75E- 08 \\
\hline Totik & $\mathrm{M}$ & ets & $\mathrm{L}, \mathrm{H}$ & $\begin{array}{l}90 \mathrm{M} \\
0, \mathrm{~L})\end{array}$ & $\begin{array}{l}6 \mathrm{cl}(\mathrm{M} \\
\mathrm{r}, \mathrm{L})\end{array}$ & $\begin{array}{l}+67 \mathrm{~d} \mathrm{M} \\
\text { or } \mathrm{LL})\end{array}$ & $\begin{array}{c}+9 s \mathrm{Q} \\
\mathrm{M} \in \mathrm{M}\end{array}$ \\
\hline $\mathrm{Pu}$ & $4.08 \mathrm{E}-03(\mathrm{~g} / \mathrm{L})$ & - & 6.50 & $3.62 \mathrm{E}-03$ & $384 \mathrm{E}-03$ & $4.31 \mathrm{E}-03$ & 1.53E-03 \\
\hline $\mathrm{U}$ & $2.00 \mathrm{E}-02$ & $2.91 \mathrm{E}+03$ & $7.68 \mathrm{E}+03$ & $1.83 \mathrm{E}-02$ & $1.91 E-02$ & $2.09 \mathrm{E}-02$ & 2.18E-02 \\
\hline
\end{tabular}

"Unknowns in turk solids inventory ane turiened by Tank Loyering Model (TLM).

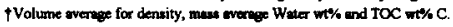




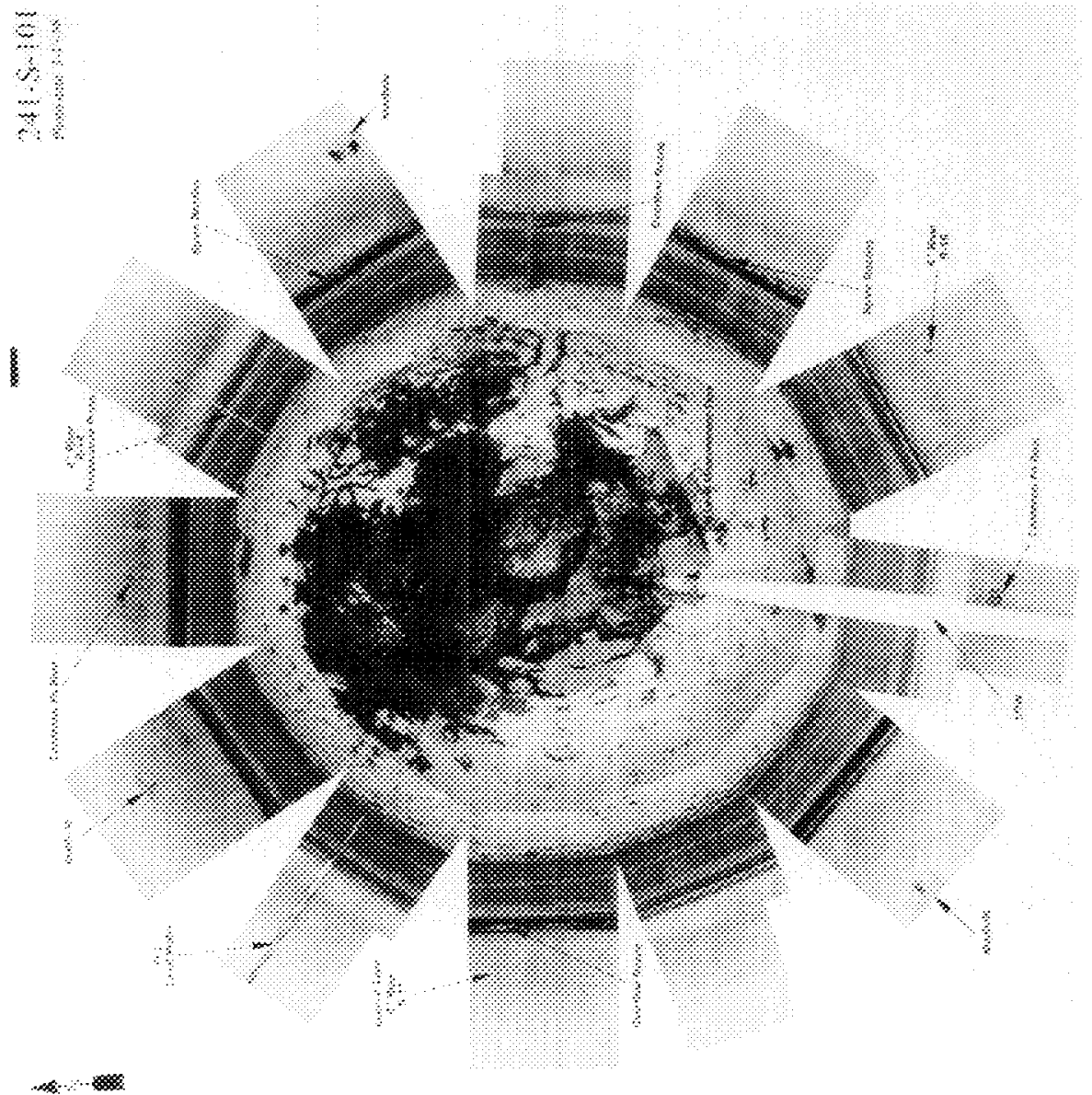


TANK 241-S-102 SUMMARY

\begin{tabular}{|c|c|c|c|}
\hline \multicolumn{2}{|c|}{ TANK HISTORY } & \multicolumn{2}{|c|}{ TANK DESCRIPTION } \\
\hline Entered Service & 3rd qtr 1953 & Diameter & $75 \mathrm{ft}$ \\
\hline Removed from Service & - & Bottom Shape & Dish \\
\hline Inactive & 1980 & Nominal Capacity & $758,000 \mathrm{gal}$ \\
\hline Watch Lists & Hydrogen \& Organics & Cascade Tank & to $241-\mathrm{S}-103$ \\
\hline Integrity & Sound & Total Risers & 12 \\
\hline Assumed Leaker & - & $\begin{array}{r}\text { WASTE VOLUN } \\
\end{array}$ & LON 1996i) \\
\hline Interim Stabilization (IS) & - & Total Waste Volume & $549,000 \mathrm{gal}$ \\
\hline Partial Interim Isolation (PI) & Dec 1982 & Waste Type & DSSF \\
\hline Intrusion Prevention (IP) & - & Drainable Interstitial Liquids & $230,000 \mathrm{gal}$ \\
\hline \multicolumn{2}{|c|}{ TENTATIVELY AVAILABLE RISERS } & Pumpable Liquids & $239,000 \mathrm{gal}$ \\
\hline Riser Number(s) & Size & Saltcake & $545,000 \mathrm{gal}$ \\
\hline $1,11,14,16$ & 4 in & Sludge & $4,000 \mathrm{gal}$ \\
\hline 7 & 12 in & Supernatant & $0 \mathrm{gal}$ \\
\hline \multicolumn{2}{|c|}{ TANK TEMPERATURE } & \multicolumn{2}{|c|}{ INTERIOR PHOTOGRAPHS } \\
\hline Average Tank Temperature & $102^{\circ} \mathrm{F}$ & Date & March 18,1988 \\
\hline Maximum Temperature & $122^{\circ} \mathrm{F}$ & Montage Number & $94041033-39 \mathrm{CN}$ \\
\hline Date & May 2, and June 6, 1988 & Photo Set Number & 8801411 \\
\hline Elevation from tank bottom & $2.44 \mathrm{ft}, 4.44 \mathrm{ft}, 6.44 \mathrm{ft}$ & \multicolumn{2}{|c|}{\begin{tabular}{|c|} 
WASTE SURFACE LEVEL \\
\end{tabular}} \\
\hline Riser Number & 3 & Devices & Manual ENRAF \\
\hline Minimum Temperature & $71.2^{\circ} \mathrm{F}$ & Max Level & 206.1 in \\
\hline Date & April 18, 1992 & Date & June 13,1994 \\
\hline Elevation from tank bottom & $24.44 \mathrm{ft}$ & Min Level & 202 in \\
\hline Riser Number & 3 & Date & Jan 21, 1991 \\
\hline
\end{tabular}




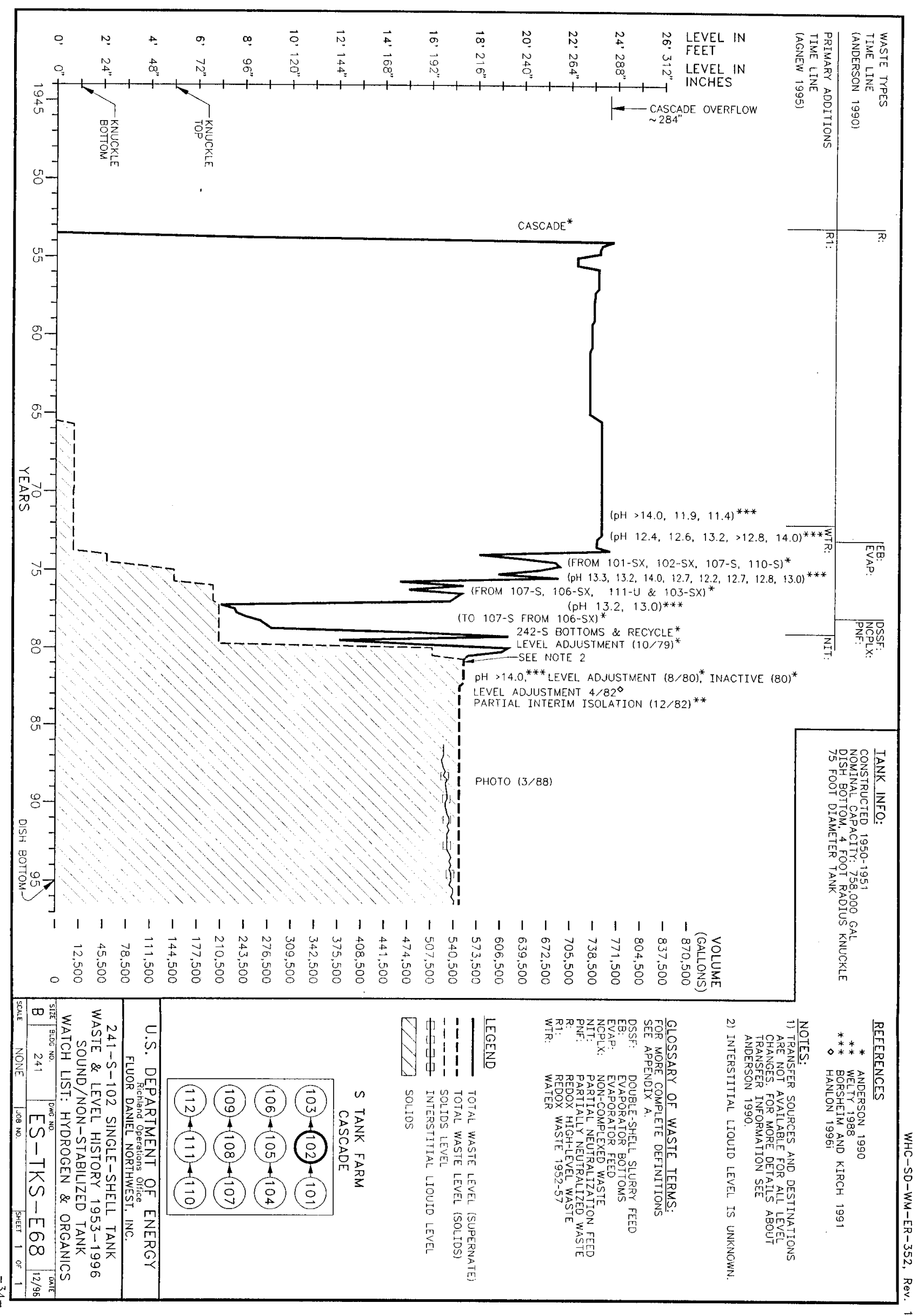




\section{$241-5-102$}

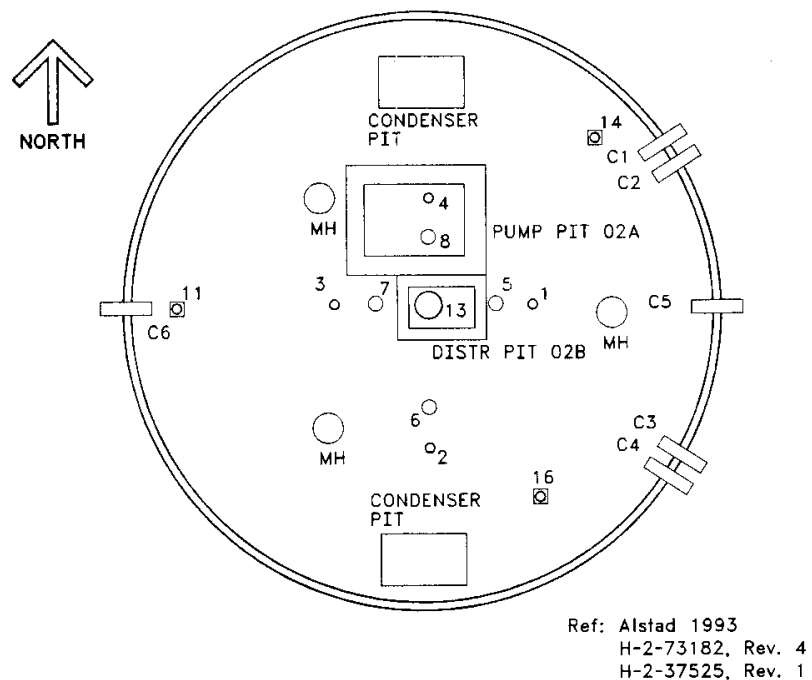

\section{TANK RISER LOCATION}

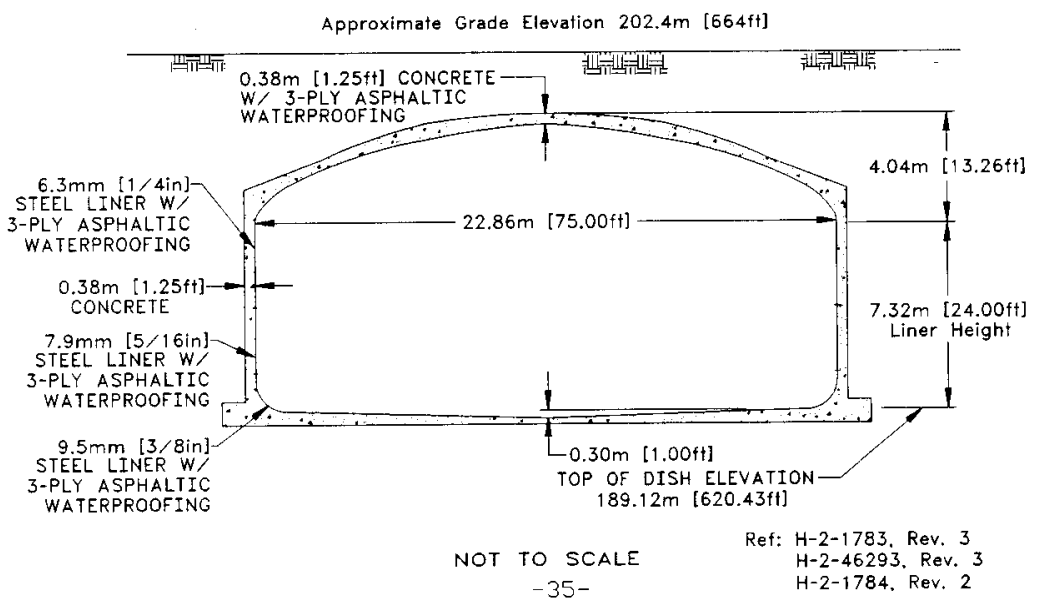


HNF-SD-WM-ER-352, Rev. 1

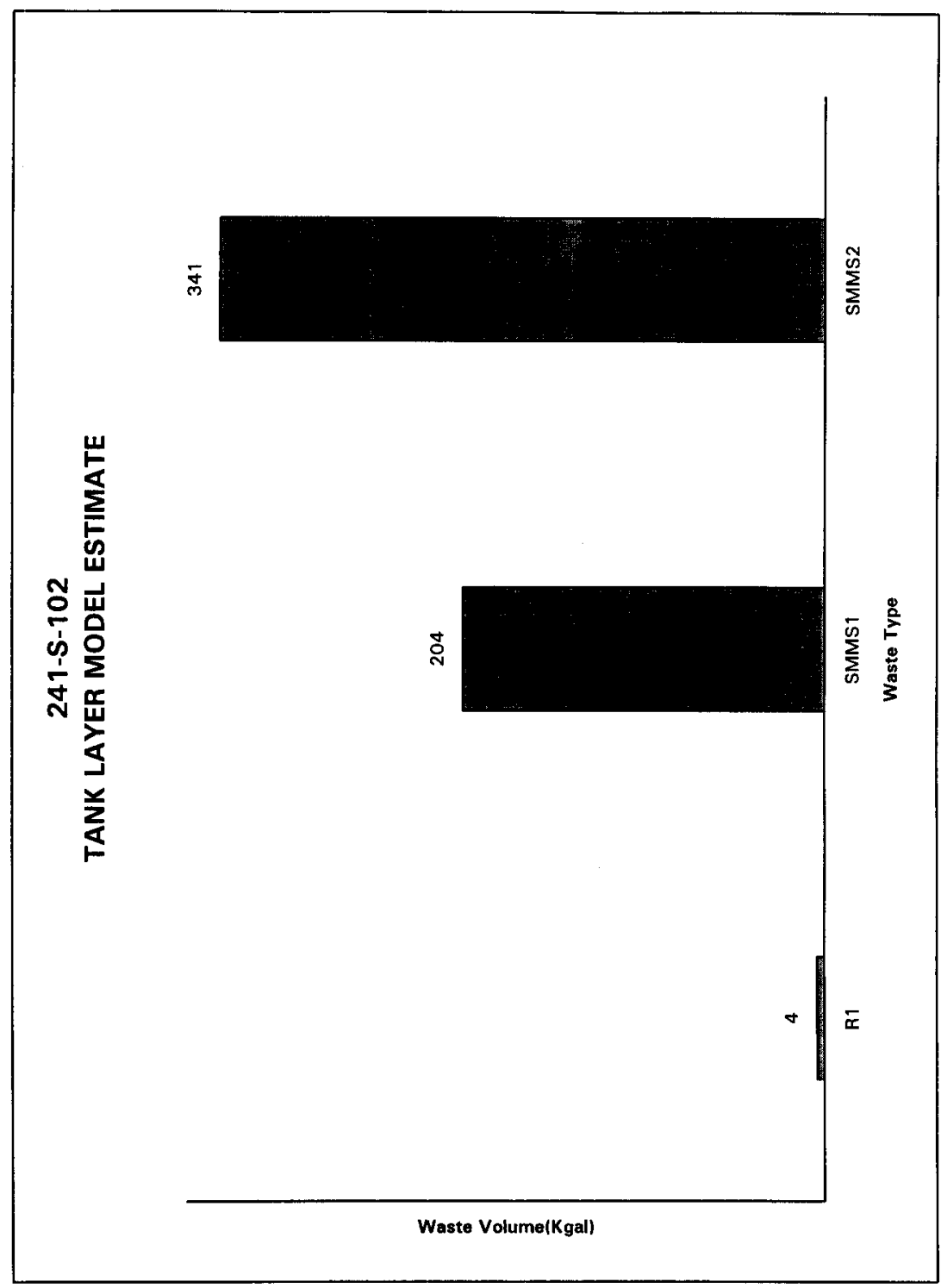

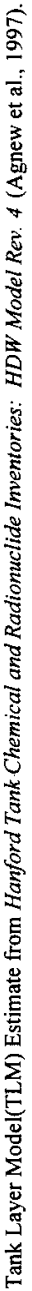


HNF-SD-WM-ER-352, Rev, 1

HDW Model Rev. 4

\begin{tabular}{|c|c|c|c|c|c|c|c|}
\hline \multicolumn{8}{|c|}{ Sirngle-Shell Tank 241-S-102 } \\
\hline & \multicolumn{7}{|c|}{ ThM Solids Composite Inventory Estimate* } \\
\hline Physici I & & & & & & & \\
\hline Propertiea & & & & $-9 \mathrm{SCI}$ & $-67 \mathrm{CI}$ & $+67 \mathrm{CI}$ & $+95 \mathrm{CI}$ \\
\hline Total TLM Waste & $2.66 \mathrm{E}+04(\mathrm{~kg})$ & $(4.00 \mathrm{~kg})$ & - & - & $\cdots$ & - & - \\
\hline Heat Load & $9.86 E-02(\mathrm{KW})$ & (337 BTUhr) & $=$ & $7.15 E-02$ & B. $74 \mathrm{E}-02$ & 0.106 & 0.109 \\
\hline Bullk Density & $1.76(\mathrm{~g} / \mathrm{cc})$ & - & $\ldots$ & 1.51 & 1.53 & 2.11 & 2.24 \\
\hline Void Fraction & 0.516 & - & $\cdots$ & $6.00 \mathrm{E}-02$ & 0.217 & 0.737 & 0.743 \\
\hline Water wt\% & 27.0 & $\ldots$ & - & 2.64 & 10.0 & 42.6 & 442 \\
\hline TOC wt\% C (wet & 0 & $\ldots$ & - & 0 & 0 & 0 & 0 \\
\hline $\begin{array}{l}\text { Clentled } \\
\text { Constítuents }\end{array}$ & moled & Dod & 14 & $\begin{array}{l}95 \mathrm{Cl} \\
(\mathrm{mol} / \mathrm{L})\end{array}$ & $\begin{array}{l}67 \mathrm{CI} \\
(\mathrm{mole} / \mathrm{L})\end{array}$ & $\begin{array}{r}+69 \mathrm{CI} \\
(\mathrm{molel} \text { ) }\end{array}$ & $\begin{array}{l}+95 \mathrm{Cl} \\
(\mathrm{mold} / \mathrm{L})\end{array}$ \\
\hline Net & 8.13 & $1.06 \mathrm{E}+05$ & $2.83 \mathrm{E}+03$ & 3.05 & 3.56 & 14.7 & 18.2 \\
\hline $\mathrm{Al}^{3+}$ & 6.99 & $1.07 \mathrm{E}+05$ & 2.85E+03 & 6.58 & 6.74 & 7.15 & 7.15 \\
\hline $\mathrm{Fe}+$ (totil Fe) & 1.01 & $3.22 E+04$ & 856 & 0.994 & 1.00 & 1.02 & 1.03 \\
\hline $\mathrm{Cr}^{3+}$ & $6.19 E-02$ & $1.83 \mathrm{E}+03$ & 48.7 & $7,00 \mathrm{E}-03$ & $339 \mathrm{E}-02$ & 0.522 & 1.06 \\
\hline Bi3 & 0 & 0 & 0 & 0 & 0 & 0 & 0 \\
\hline Lest & 0 & (1) & 0 & 0 & 0 & 0 & 0 \\
\hline $\mathrm{Hg} 2+$ & 0 & 0 & 0 & 0 & 0 & 0 & 0 \\
\hline $\mathrm{Zr}(\mathrm{as} 2 \mathrm{rO}(\mathrm{OH}) 2)$ & 0 & 0 & 0 & 0 & 0 & 0 & 0 \\
\hline $\mathrm{Pb} 2+$ & 0 & 0 & 0 & 0 & 0 & 0 & 0 \\
\hline $\mathrm{Ni2}+$ & S.07E-02 & 1.69E+03 & $\mathbf{4 5 0}$ & $3.66 \mathrm{E}-02$ & 4.45E-02 & $5.54 \mathrm{E}-\mathrm{A} 2$ & 5 45E-02 \\
\hline$s+2+$ & 0 & 0 & 0 & 0 & 0 & 0 & 0 \\
\hline Mn4t & 0 & 0 & 0 & 0 & 0 & 0 & 0 \\
\hline $\mathrm{Con}+$ & 0.220 & $5.02 E+03$ & 133 & 0.146 & 0.182 & 0.258 & 0.294 \\
\hline $\mathrm{K}+$ & $1.12 \mathrm{E}-02$ & 250 & 6.64 & $1.28 \mathrm{E}-03$ & S.2SE- 03 & $1.52 \mathrm{E}-02$ & $1.52 \mathrm{E}-02$ \\
\hline OH- & 25.6 & $2.48 E+05$ & $6.59 \mathrm{E}+03$ & 23.1 & 24,3 & 26.9 & 28.4 \\
\hline $\mathrm{NO3}$ & 529 & $1.87 \mathrm{E}+05$ & $496 \mathrm{E}+03$ & 6.03E-02 & $6.002-02$ & 13.5 & 17.9 \\
\hline NO2- & 1.40 & $3.82 E+04$ & $3.01 \mathrm{E}+03$ & 0.132 & 0.995 & 2.15 & 2.15 \\
\hline Co32- & 0.270 & $7.51 E+03$ & 200 & 0.146 & 0.182 & 0.258 & 0.294 \\
\hline $\mathrm{PO} 43$ & 0 & 0 & 0 & 0 & 0 & 0 & 0 \\
\hline SO42- & $1.04 E-02$ & 369 & 15.1 & $1.19 \mathrm{E}-\mathbf{0 3}$ & $4.87 E-03$ & $1.41 E-02$ & $1.41 E-02$ \\
\hline $\mathrm{Si}(\mathrm{suS} \mathrm{Si}$ 32-) & 8.06E-03 & 129 & 3.42 & 9.21E-04 & $3.7 / \mathrm{E}-03$ & $1.09 \mathrm{E}-02$ & 1.09E-O2 \\
\hline F- & 0 & 0 & 0 & 0 & 0 & 0 & 0 \\
\hline Cl. & $5.16 \mathrm{E}-02$ & $1.04 \mathrm{E}+03$ & 27.7 & S.91E-03 & $2.42 \mathrm{E}-02$ & $700 \mathrm{E}-02$ & 0.157 \\
\hline C6HSOT3 & 0 & 0 & 0 & 0 & 0 & 0 & 0 \\
\hline EDTA4 & 0 & 0 & 0 & 0 & 0 & 0 & 0 \\
\hline HEDTA3 & 0 & 0 & 0 & 0 & 0 & 0 & 0 \\
\hline ghycolate- & 0 & 0 & 0 & 0 & 0 & 0 & 0 \\
\hline acetate. & 0 & 0 & 0 & 0 & 0 & 0 & 0 \\
\hline oxulate2- & 0 & 0 & 0 & 0 & 0 & 0 & 0 \\
\hline DBP & 0 & 0 & 0 & 0 & 0 & 0 & 0 \\
\hline butanol & 0 & 0 & 0 & 0 & 0 & 0 & 0 \\
\hline $\mathrm{NH3}$ & 0.256 & $2.47 \mathrm{E}+03$ & 65.7 & $6.55 \mathrm{E}-02$ & $9.25 E-02$ & 0.270 & 0.284 \\
\hline $\mathrm{Fe}(\mathrm{CN}) 64$ & 0 & 0 & 0 & 0 & 0 & 0 & 0 \\
\hline
\end{tabular}

"Unknowns in tonk solids inventory are maignod by Tunk Layering Moded (TLM). 
HDW Model Rav. 4

\begin{tabular}{|c|c|c|c|c|c|c|c|}
\hline \multirow{4}{*}{$\begin{array}{l}\text { Physical } \\
\text { Properties }\end{array}$} & \multicolumn{7}{|c|}{ Single-Shell Tank 241-S-102 } \\
\hline & \multicolumn{7}{|c|}{ SMM Composite Inventory Estingte } \\
\hline & & & & & & & \\
\hline & & & & $98 \mathrm{CI}$ & $-67 \mathrm{Cl}$ & $+67 \mathrm{CI}$ & $+95 \mathrm{Cl}$ \\
\hline Total SMM Wast & $2.64 \mathrm{E}+06(\mathrm{~kg})$ & (545 kyst) & - & - & - & سم & $\ldots$ \\
\hline Heat Loed & $1.99(\mathrm{~kW})$ & (6BOE+03 BTU/4s) & $=$ & 1.88 & 1.93 & 2.05 & 2.11 \\
\hline Bullk Density" & $1.28(\mathrm{~g} / \mathrm{cc})$ & - & - & 1.26 & 1.27 & 1.29 & 1.30 \\
\hline & & & & & & & \\
\hline Water wot & 61.4 & - & - & 59.5 & 60.4 & 62.5 & 63.7 \\
\hline TOC wh\% C (wet & 0.413 & - & - & 0.274 & 0.342 & 0.484 & 0.553 \\
\hline $\begin{array}{l}\text { Chembil } \\
\text { Conistitueati }\end{array}$ & moled & $\mathrm{pom}$ & 10 & $\begin{array}{l}-93 \mathrm{CI} \\
(\mathrm{noder})\end{array}$ & $\begin{array}{l}67 \mathrm{Cl} \\
\text { (noled) }\end{array}$ & $\begin{array}{l}+67 \mathrm{CI} \\
(\text { moter })\end{array}$ & $\begin{array}{c}+98 \mathrm{C} \\
(\mathrm{mal} / \mathrm{L})\end{array}$ \\
\hline $\mathrm{Na}+$ & 6.50 & $1.17 \mathrm{E}+05$ & $3.008+05$ & 5,95 & 6.22 & 6.73 & 6.95 \\
\hline Al3+ & 0.779 & $1.64 E+04$ & $4.33 \mathrm{E}+04$ & 0.719 & 0.747 & 0.813 & 0.830 \\
\hline Fe3+ (total Fe) & $4.88 \mathrm{E}-03$ & 213 & 562 & $3,98 E-03$ & 4.42E- 03 & $\$ 34 E-03$ & $5.78 \mathrm{E}-03$ \\
\hline $\mathrm{Cr} 3+$ & $7.01 \mathrm{E}-02$ & $2.85 \mathrm{E}+03$ & $7.52 \mathrm{E}+03$ & $579 \mathrm{E}-02$ & $6.45 E-02$ & $7.23 \mathrm{E}-02$ & $7.44 \mathrm{E}-02$ \\
\hline Bist & $5.71 \mathrm{E}-04$ & 93.3 & 246 & $5.19 \mathrm{E}-04$ & $5.44 E-04$ & $5.97 \mathrm{E}-04$ & $0.23 E-04$ \\
\hline Lat & $1.54 E-05$ & 1.67 & 4.41 & $1.11 \mathrm{E}-05$ & 1.32E-05 & $1.76 E-05$ & $1.96 \mathrm{E}-05$ \\
\hline $\mathrm{H} \notin 2+$ & $3.99 \mathrm{E}-06$ & 0.626 & 1.65 & 3.72E-D6 & $3.87 \mathrm{E}-06$ & 4.05E -06 & 4.10E-06 \\
\hline $\mathrm{Zr}(\mathrm{ar} \mathrm{ZO}(\mathrm{OH}) 2)$ & $1.07 \mathrm{E}-04$ & 7.66 & 20.2 & $9.79 \mathrm{E}-05$ & $1.01 \mathrm{E}-04$ & $1.1 \mathrm{E}-0.04$ & $1.17 \mathrm{E}-04$ \\
\hline $\mathrm{Pb2}+$ & $4.43 E-04$ & 71.8 & 189 & $3.61 \mathrm{E}-04$ & $4.01 \mathrm{E}-04$ & $4.84 \mathrm{E}-04$ & $5.24 \mathrm{E}-04$ \\
\hline Nizt & 2.91E-03 & 133 & 352 & 278E-03 & 2.84E-03 & $2.94 \mathrm{E}-03$ & $2.97 \mathrm{~T}-03$ \\
\hline $82+$ & 0 & 0 & 0 & 0 & 0 & 0 & 0 \\
\hline Mnd+ & $1.738-03$ & 75.2 & 198 & 1.32E-03 & $1.53 E-03$ & $1.97 \mathrm{E}-03$ & $2.1 \mathrm{BE}-03$ \\
\hline $\mathrm{Con}+$ & 1.52E-02 & 47 & $1.26 \mathrm{E}+03$ & $1.39 \mathrm{E}-02$ & 1.45E-02 & $1.99 \mathrm{E}-02$ & $1.65 E-02$ \\
\hline$k+$ & $2.97 \mathrm{E}-02$ & 908 & $2.39 \mathrm{E}+03$ & $270 \mathrm{E}-02$ & $2.63 E-02$ & $3.11 \mathrm{e}-02$ & 3.25E-02 \\
\hline OH- & 4.67 & $6.21 \mathrm{E}+04$ & $1.64 E+05$ & 4.30 & 4.47 & 4.88 & 4.95 \\
\hline $\mathrm{NO} 3$ & 2.29 & $1.11 \mathrm{E}+0 \mathrm{~S}$ & $2.93 \mathrm{E}+05$ & 2.12 & 2.22 & 2.33 & 2.34 \\
\hline NO2- & 1.14 & 409E+01 & $1,06 \mathrm{E}+05$ & 0.952 & 1.04 & 1.23 & 1.32 \\
\hline Co32. & 0.191 & E.95E+03 & $2.36 \mathrm{E}+04$ & 0.17 & 0.1100 & 0.204 & 0.206 \\
\hline $\mathrm{PO43}$ & $3.91 \mathrm{E}-02$ & $2.91 E+03$ & $7.66 \mathrm{E}+03$ & $3.37 \mathrm{E}-02$ & $3.56 \mathrm{E}-02$ & $4.01 E-02$ & 4.10E-02 \\
\hline SO42- & 0.108 & $8.13 E+03$ & $2.14 \mathrm{E}+04$ & $8.4 \mathrm{E}-02$ & $9.57 \mathrm{E}-02$ & 0.121 & 0.132 \\
\hline Si (as SiO32-) & $3.52 \mathrm{E}-02$ & 74 & $2.04 \mathrm{E}+03$ & $2.90 \mathrm{E}-02$ & $3.20 \mathrm{E}-02$ & 3. $84 \mathrm{E}-02$ & $4.14 \mathrm{E}-02$ \\
\hline F. & $2.80 E-02$ & 416 & $1.10 \mathrm{E}+03$ & $2.34 E-02$ & $2.53 \mathrm{E}-02$ & $3.02 \mathrm{E}-02$ & $3.19 E-02$ \\
\hline $\mathrm{Cl}-$ & 0.112 & $310 \mathrm{E}+03$ & $8.16 \mathrm{E}+03$ & 0.101 & 0.106 & 0.117 & 0.121 \\
\hline CofSOT3 & $1.25 E-02$ & $1.85 E+03$ & $4.89 \mathrm{E}+03$ & 1.15E-02 & $1.20 \mathrm{E}-02$ & $1.30 \mathrm{E} .02$ & $1.35 \mathrm{E}-02$ \\
\hline EOTA4 & $6.31 \mathrm{E}-03$ & $1.42 E+03$ & $3.75 \mathrm{E}+03$ & 1.95E-03 & $4.08 E-03$ & $8.56 \mathrm{E}-03$ & $1.08 \mathrm{E}-02$ \\
\hline HEDTA2 & 1.18E-02 & $2.53 \mathrm{E}+03$ & $6.6 \mathrm{TE}+03$ & $3.06 \mathrm{E}-03$ & $7.32 E-03$ & $1.63 \mathrm{E}-02$ & $2.07 \mathrm{E}-02$ \\
\hline Glycolate- & $3.53 \mathrm{E}-02$ & $2.25 \mathrm{E}+03$ & $5.93 E+0.03$ & $249 \mathrm{E}-02$ & 3.15E-02 & 4.51E-02 & $5.17 \mathrm{E}-02$ \\
\hline acetute- & 2.67E-03 & 123 & 325 & 2.19E-03 & $2.43 E-03$ & 2.92E-03 & 3.16E-03 \\
\hline axalexe2- & 2.01E-05 & 1.39 & 3.65 & $1.7 \mathrm{EE}-05$ & $1.90 E-05$ & $2,13 \mathrm{E}-05$ & $2.24 E-05$ \\
\hline DBP & 8.44E-03 & $1.39 E+03$ & $3.66 \mathrm{E}+03$ & $6.92 \mathrm{E}-03$ & $7.6 \mathrm{TE}-03$ & $9.20 \mathrm{E}-03$ & 9.93E-03 \\
\hline butaniol & 8.44E-03 & 40 & $129 \mathrm{E}+03$ & $6.92 E-03$ & $7.67 \mathrm{E}-03$ & $9.20 \mathrm{E}-03$ & $9.93 E-03$ \\
\hline NH3 & 3.94E-02 & 511 & $1.35 \mathrm{E}+03$ & $3.14 E-02$ & $3.42 \mathrm{E}-02$ & $4.38 \mathrm{E}-02$ & S.01E-02 \\
\hline $\mathrm{Fe}(\mathrm{CN}) 64$ & 0 & 0 & 0 & 0 & 0 & 0 & 0 \\
\hline
\end{tabular}

-Density is calculoted besed on Na, OH-, and $\mathrm{AlO}_{2}$ -

tWater wh\% derived from the difference of density and totai dissolved epecies. 


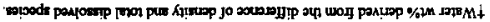

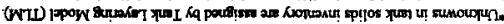

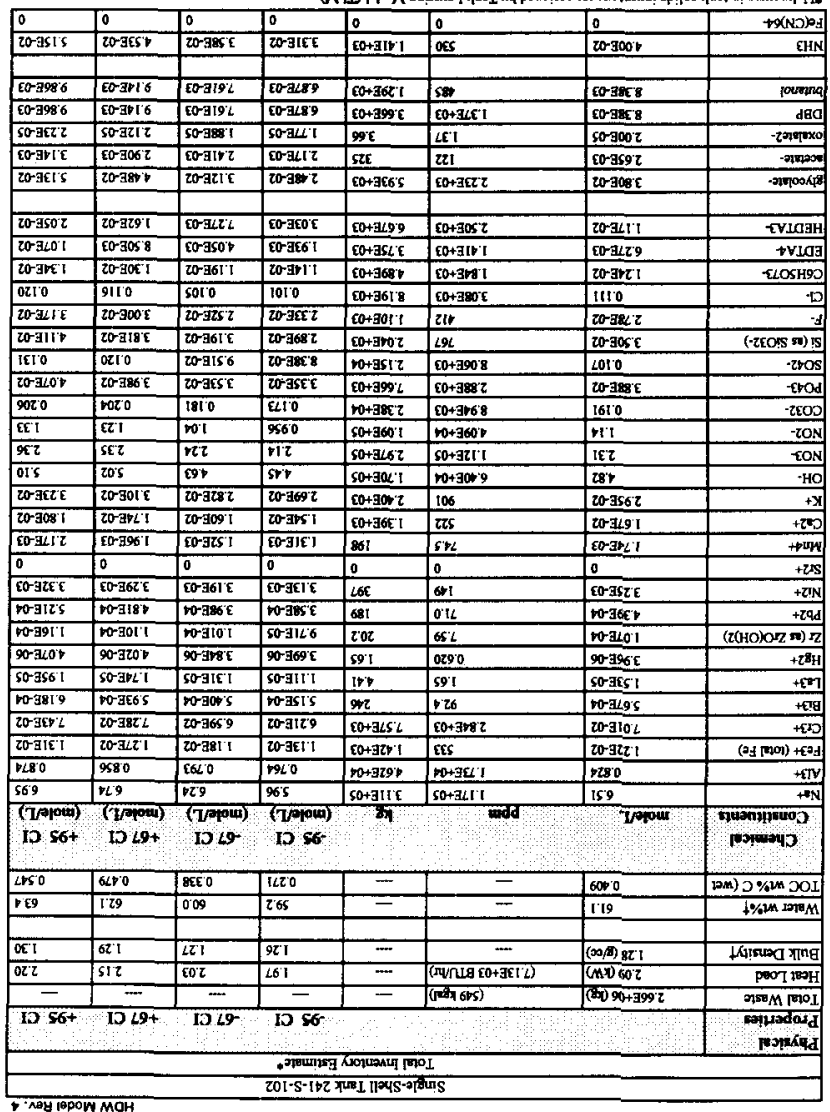


HNF-SD-WM-ER-352, Rev. I

HOW Model Rav. 4

\begin{tabular}{|c|c|c|c|c|c|c|c|}
\hline \multirow{3}{*}{$\begin{array}{l}\text { Phyilet } \\
\text { Propertices }\end{array}$} & \multicolumn{7}{|c|}{ Single-Shell Tank 241-S-102 } \\
\hline & \multicolumn{7}{|c|}{ TLM Solids Composite Inventory Estimale * } \\
\hline & & & & $95 \mathrm{CI}$ & $67 \mathrm{Cl}$ & $+67 \mathrm{CI}$ & $+95 \mathrm{CI}$ \\
\hline Total TLM Weste & $2.66 \mathrm{E}+04(\mathrm{~kg})$ & $(4.00 \mathrm{kgand})$ & - & $\ldots$ & $\ldots$ & - & - \\
\hline Heat Load & $9.86 \mathrm{E}-02(\mathrm{CW})$ & (337 BTU/4s) & $=$ & $7.15 E-02$ & $8.74 E-02$ & 0.106 & 0.109 \\
\hline Bulk Density & $1.76(g / \infty)$ & $\ldots$ & $\ldots$ & 1.51 & 1.53 & 2.11 & 2.24 \\
\hline Void Fraction & 0.536 & $\overline{-}$ & - & $6.00 \mathrm{E}-02$ & 0.217 & 0.737 & 0.743 \\
\hline Water why & 27.0 & - & - & 2.64 & 10.0 & 42.6 & 44.2 \\
\hline TOC wt\% C (wet & 0 & $=$ & $=$ & o] & 0 & 0 & 0 \\
\hline $\begin{array}{l}\text { R } \\
\text { Contioloted }\end{array}$ & CUL & $\mathrm{HCV}$ & Ci & $\begin{array}{l}95 \mathrm{Cl} \\
\text { (CU) }\end{array}$ & $\begin{array}{l}67 \mathrm{Cl} \\
\text { (C)L })\end{array}$ & $\begin{array}{l}+6 \mathrm{CI} \\
(\mathrm{C} / \mathrm{L})\end{array}$ & $\begin{array}{l}+95 \mathrm{Cl} \\
\text { (CUL) }\end{array}$ \\
\hline $\mathrm{H}-3$ & 2.92E-05 & $1.67 \mathrm{E}-02$ & 0.443 & $3.05 E-06$ & $1.47 \mathrm{E}-05$ & 4.10E-05 & 4.10E-05 \\
\hline $\mathrm{C}-14$ & $2.26 \mathrm{E}-06$ & $1.29 \mathrm{E}-03$ & 3.43E-42 & 2.59E-07 & $1.06 \mathrm{E}-06$ & $3.07 E-06$ & $3.07 E-06$ \\
\hline $\mathrm{Ni}-59$ & $1.49 E-05$ & 8.46E-03 & 0.225 & $1.0 \pi-05$ & $1.31 E-05$ & 1.62E-0s & $1.60 \mathrm{E}-0 \mathrm{~s}$ \\
\hline Ni-63 & 1.39E-03 & 0.790 & 21.0 & $1.00 \mathrm{E}-03$ & $1.22 \mathrm{E}-03$ & $1.52 \mathrm{E}-03$ & 1. $4 \mathrm{E}-03$ \\
\hline Co-60 & 8.94E-07 & $5.09 \mathrm{E}-04$ & 1.35E-02 & $1.02 \mathrm{E}-07$ & $4.18 E-07$ & $1.21 \mathrm{E}-06$ & $1.21 \mathrm{E}-06$ \\
\hline Se-79 & 4.81E- -07 & 2.74E-04 & $7.27-03$ & $5.50 \mathrm{E}-08$ & $2.25 \mathrm{E}-07$ & 6.51E-07 & 7.15E-06 \\
\hline Sr-90 & 0.928 & 529 & $1.4 \mathrm{E}+04$ & 0.662 & 0.812 & 1.02 & 1.03 \\
\hline$Y-90$ & 0.929 & 529 & $1.41 \mathrm{E}+04$ & 0.662 & 0.812 & 1.02 & 1.03 \\
\hline $2 x-93$ & $2.2 \pi-06$ & $1.291-03$ & $3.43 E-02$ & 2.59E-07 & $1.06 \mathrm{E}-06$ & $3.08 \mathrm{E}-06$ & 3.03E-05 \\
\hline $\mathrm{Nb}-93 \mathrm{~m}$ & $1.85 \mathrm{E}-06$ & $1.06 \mathrm{E}-03$ & 2.81E-02 & $2.12 \mathrm{E}-07$ & 8.68E-07 & $2.51 E-06$ & 3.21E-05 \\
\hline Tc-99 & 1.S8RE-05 & $9.02 \mathrm{E}-03$ & 0.200 & $1,81 \mathrm{E}-06$ & 7.42E-06 & 2.15E-05 & 2.1SE-05 \\
\hline Ru-106 & 2 SOE- 12 & $1.42 \mathrm{E}-09$ & 3. $\pi \mathrm{EE}-08$ & $2.86 \mathrm{E}-13$ & $1.17 \mathrm{E}-12$ & $3.38 \mathrm{E}-12$ & 3.38E.12 \\
\hline Cd-113m & $6.99 \mathrm{E}-06$ & $3.98 \mathrm{E}-03$ & 0.106 & $8.00 \mathrm{E}-07$ & $3.27 \mathrm{E}-06$ & 9.4BE-06 & 9.4BE-06 \\
\hline $\mathrm{Sb}-125$ & $1.31 E-06$ & $7.47 \mathrm{E}-04$ & $1.99 \mathrm{E}-02$ & 1 SOE- -07 & 6.14E-07 & $1.7 \mathrm{BE}-26$ & $178 E-06$ \\
\hline Sn-126 & $7.39 \mathrm{E}-07$ & $4.21 \mathrm{E}-04$ & $1.12 \mathrm{E}-02$ & 8.4SE-08 & 3.46E-07 & $1,00 E-06$ & $1.14 E-05$ \\
\hline $1-129$ & 3.0NEAB & 1.7BE-0S & 4.61E-04 & 3.48E-09 & $1.42 E-08$ & 4.13E-08 & 4. $13 \mathrm{EE}-08$ \\
\hline Cs-134 & $272 \mathrm{E}-08$ & 1.5SE-05 & 4.1IE-04 & $7.19 \mathrm{E}-09$ & $1.52 \mathrm{E}-08$ & $3.52 \mathrm{E}-08$ & $3.52 \mathrm{E}-08$ \\
\hline Cs-137 & $5.60 \mathrm{E}-12$ & 31.9 & 80 & 1. $\triangle 4 E-02$ & $3.13 E-02$ & $7.26 \mathrm{E}-122$ & $7.26 \mathrm{E}-02$ \\
\hline $\mathrm{B}-137 \mathrm{~m}$ & $5.30 E-02$ & 30.2 & 802 & 1.40E-02 & $2.96 \mathrm{E}-02$ & $6.87 \mathrm{E}-02$ & $6.87 \mathrm{E}-02$ \\
\hline $5 m-151$ & 1.7E-03 & 0.976 & 26.0 & $1.96 \mathrm{E}-\mathrm{MA}$ & B.03EA4 & $2.33 E-03$ & $2.64 \mathrm{E}-02$ \\
\hline $\mathrm{Eu}-152$ & $7.00 E-06$ & $3.98 \mathrm{E}-03$ & 0.106 & $6.85 E-06$ & 6.91E-06 & 7.065-06 & $7.06 \mathrm{E}-06$ \\
\hline Eu-154 & $2.16 E-05$ & $1.23 \mathrm{E}-02$ & 0.327 & $2.47 \mathrm{E}-106$ & 1.018-0s & $2.93 E-05$ & $2.93 E-05$ \\
\hline Eu-155 & 3.30E-04 & 0.188 & 5.00 & 3.23E-04 & $3.26 \mathrm{E}-04$ & 3.33E-04 & 3.33E-04 \\
\hline $\operatorname{Ra}-226$ & $1.08 \mathrm{E}-09$ & $6.156-07$ & $1.63 \mathrm{E}-0 \mathrm{~s}$ & 1. AEE-10 & 6.CAE-10 & $1.56 \mathrm{E}-09$ & $2.01 E-09$ \\
\hline Ra-22B & $9.26 \mathrm{E}-15$ & $5.28 \mathrm{E}-12$ & $1.40 \mathrm{E}-10$ & $9.07 \mathrm{E}-15$ & $9.15 E-15$ & $9.34 \mathrm{E}-15$ & 9.34E-15 \\
\hline Ac-227 & 4.76E-09) & 2.71E-06 & 7.20E-65 & $3.06 \mathrm{E} \cdot 10$ & $2.00 E-09$ & $7.52 \mathrm{E}-09$ & $1.02 E-08$ \\
\hline Pa-23! & $7.18 \mathrm{EE}-10$ & 4.09E-07 & $1.09 \mathrm{E}-\mathrm{SB}$ & B.21E-11 & 3.36E-10 & 2.04E-09 & $1.68 \mathrm{E}-08$ \\
\hline Th-229 & $1.7 \pi-12$ & 1.01E-09 & $2.6 \mathrm{TE}-08$ & 1.73E-12 & $1.75 E-12$ & $1.78 \mathrm{BE}-12$ & 1.7.te-12 \\
\hline Th-232 & 5.91E-16 & 3.36E-13 & 8.4E-12 & $6.76 \mathrm{E}-17$ & $2.77 \mathrm{E}-16$ & $8.01 E-16$ & 8.01E-16 \\
\hline $\mathrm{U}-232$ & 5.24E-12 & 2.9ek-09 & $7.92 \mathrm{E}-08$ & 3,90E-13 & 2.4SE-12 & $7.10 \mathrm{E}-12$ & 7.10E-12 \\
\hline U-233 & 2.00E-13 & $1.14 E-10$ & $3.02 \mathrm{E}-09$ & $2.29 \mathrm{E}-14$ & 9.34E-14 & 2.71E-13 & 2.7LE-13 \\
\hline U-234 & 1.19E-07) & $6.78 E-05$ & 3.00E-03 & $1.36 \mathrm{E}-00 \mathrm{~B}$ & $5.5 \times 2-06$ & $1.62 \mathrm{E}-\mathrm{-07}$ & $1.62 \mathrm{E}-07$ \\
\hline U-235 & 5.13E-09 & 2.92E-06 & 7.76E-0s & $5.86 E-10$ & $2.40 \mathrm{E}-09$ & $6.95 E-09$ & $6.95 \mathrm{E}-09$ \\
\hline U-236 & 1.89E-09 & $1.072-06$ & 2.85E-05 & 2.16E-10 & $8.828-10$ & $2.56 \mathrm{E}-09$ & $2.860-09$ \\
\hline U-238 & 1.22E-07 & 6.92E-05 & $1.8 \mathrm{EE}-03$ & $1.39 \mathrm{E}-08$ & $5.69 \mathrm{E}-08$ & $1.65 \mathrm{E}-07$ & $1.65 E-07$ \\
\hline Np-237 & $1.03 E-07$ & S. BSE-05 & $1.56 \mathrm{E}-03$ & 1.18E- -18 & 4.81EA8 & 1.39E-07 & $1.395-07$ \\
\hline Pu-238 & $6.61 \mathrm{E}-06$ & $3.7 \pi-63$ & 0.100 & 4.75E-06 & $5.66 \mathrm{E}-96$ & 7.57E-06 & 8.48E-06 \\
\hline Pu-239 & $4.72 \mathrm{E}-04$ & 0.269 & 7.14 & $3.39 \mathrm{E}-04$ & 4.04E-04 & 3.40E-04 & $6.05 E-04$ \\
\hline $\mathrm{Pu}-240$ & $6.76 \mathrm{E}-05$ & 3.85E-02 & 1.02 & 4.8SE-05 & $5.7 \mathrm{RE}-05$ & 7.73E-05 & $8.66 \mathrm{E}-05$ \\
\hline $\mathrm{Pu}-241$ & $3.97 \mathrm{E}-04$ & 0.226 & 6.01 & 2.85E-04 & $3.40 \mathrm{E}-\mathrm{04}$ & 4.55E-04 & $5.09 \mathrm{E}-04$ \\
\hline $\mathrm{Pu}-242$ & 1.82E-09 & 1.04E-06 & $2.76 \mathrm{E}-05$ & 1.31E-09 & $1.56 \mathrm{E}-09$ & $2.08 E-09$ & 2.34E-09 \\
\hline Am-24l & 8.09E- 06 & 4.61E-03 & 0.122 & $9.20 \mathrm{E}-07$ & $3.79 \varepsilon-06$ & $1.10 \mathrm{E}-0 \mathrm{~s}$ & $1.43 \mathrm{E}-04$ \\
\hline Aml-243 & $7.57 \mathrm{E}-11$ & 4.31E-A8 & $1.15 \mathrm{E}-06$ & 8.66E-12 & 3.5AE-11 & $1.03 \mathrm{E}-10$ & 1.34E-09 \\
\hline $\mathrm{C}_{\mathrm{m}}-242$ & 1.61E-07) & $9.16 \mathrm{E}-05$ & $2.44 E-03$ & 1.58E-07 & 1.59E- -07 & $1.62 E-07$ & $1.62 \mathrm{E}-07$ \\
\hline $\mathrm{C}_{\mathrm{m}-243}$ & $3.69 \mathrm{E}-09$ & 2.10E- $-\infty$ & $5.58 E-05$ & $3.61 \mathrm{E}-09$ & 3.64E-09 & $3.72 \mathrm{E}-09$ & $3.72 \mathrm{E}-09$ \\
\hline $\mathrm{C}_{\mathrm{m}-244}$ & $2.70 \mathrm{E}-09$ & $1.54 \mathrm{E}-06$ & 4.09E-05 & $3.09 E-10$ & $1.2 \pi-09$ & $3.67 \mathrm{E}-09$ & 3.67E-09 \\
\hline Totial & $\mathbf{M}$ & es/s. & 18 & $\begin{array}{c}95 \mathrm{cloM} \\
\text { of }\end{array}$ & $67 \mathrm{cr}(\mathrm{M}$ & $\begin{array}{l}6, \mathrm{C}(\mathrm{M} \\
\text { or } / \mathrm{L})\end{array}$ & $\begin{array}{c}+95 \mathrm{O} \\
\mathrm{Mor} / \mathrm{L})\end{array}$ \\
\hline Pu & $7.89 \mathrm{E}-03(\mathrm{~g} / \mathrm{L})$ & - & 0.119 & $3.67 \mathrm{E}-03$ & 6.76E-03 & $9.03 \mathrm{E}-03$ & $1.01 \mathrm{E}-02$ \\
\hline $\mathrm{U}$ & $1.53 \mathrm{E}-03$ & 207 & 5.51 & 1.75E-04 & $7.16 E-04$ & $2.07 E-03$ & $2.07 \mathrm{E}-03$ \\
\hline
\end{tabular}

*Unkowns in tank solids inventory ere maigned by Tank Leyering Model (TLM) 
HNF-SD-WM-ER-352, Rev. 1

HDW Model Rev, 4

\begin{tabular}{|c|c|c|c|c|c|c|c|}
\hline \multirow[b]{3}{*}{$\begin{array}{l}\text { Phystcal } \\
\text { Proportios }\end{array}$} & \multicolumn{7}{|c|}{ Sinde-Shell Tank $241-S-102$} \\
\hline & \multicolumn{7}{|c|}{ SMOM Corrposite Inventory Extimate } \\
\hline & & & & $-95 \mathrm{CI}$ & $-67 \mathrm{CI}$ & $+67 \mathrm{CI}$ & $+95 \mathrm{Cl}$ \\
\hline Total SMM Wast & $2.64 E+06(\mathrm{~kg})$ & (545 kgal) & - & - & - & - & $-\infty$ \\
\hline Heat Loed & $1.99(\mathrm{~kW})$ & $(6.80 \mathrm{E}+03 \mathrm{BTU} / \mathrm{hs})$ & - & 1.88 & 1.93 & 2.05 & 2.11 \\
\hline Bulk Density* & $1.28(\mathrm{r} / \mathrm{cos})$ & - & 一 & 1.26 & 1.27 & 1.29 & 1.30 \\
\hline Water wt\%t & 61.4 & - & . & 99.5 & 60.4 & 62.5 & 63.7 \\
\hline TOC wt\% C (wet & 0.413 & 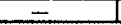 & $\div$ & 0.274 & 0.342 & 0.484 & 0.553 \\
\hline 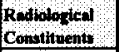 & $\mathrm{cin}$ & $\Delta C v_{2}$ & $\mathbf{C I}$ & $\begin{array}{l}95 \mathrm{CI} \\
(\mathrm{C} / \mathrm{L})\end{array}$ & $\begin{array}{l}67 \mathrm{Cl} \\
(\mathrm{C} N \mathrm{~L})\end{array}$ & $\begin{array}{c}+67 \mathrm{Cl} \\
(\mathrm{CU} / \mathrm{L})\end{array}$ & $\begin{array}{l}+8 \mathrm{C} \\
\text { (Ci) }\end{array}$ \\
\hline $\mathrm{H}-3$ & $1.1 \pi-04$ & 9.14E-02 & 241 & $6.17 \mathrm{E}-05$ & $6.1 \pi-05$ & $1.20 \mathrm{E}-04$ & $1.24 E-04$ \\
\hline $\mathrm{C}-14$ & $1.66 \mathrm{E}-05$ & 1.30E-02 & 34.2 & 5.05E-06 & 5.05E-06 & $1.68 E-05$ & $1.70 \mathrm{E}-0.5$ \\
\hline Ni-59 & 1.07E-06 & 8.36E-04 & 2.20 & S.26E-07 & $5.26 \mathrm{E}-07$ & $1.10 E-06$ & 1.12E- -6 \\
\hline $\mathrm{Ni}-63$ & $1.05 \mathrm{E}-04$ & 8. $18 E-02$ & 216 & 5.09E-05 & S.09E-05 & $1.07 \varepsilon-04$ & $1.10 \mathrm{E}-04$ \\
\hline Co-60 & 1.83E-05 & 1. $13 \mathrm{E}-02$ & 37.6 & 5.04E-06 & S.04E-06 & 1.86E.05 & $1.89 \mathrm{E}-05$ \\
\hline Se-79 & 1.65E-06 & 1.29E- -03 & 3.40 & $9.12 \mathrm{E}-07$ & Q.12E-07 & 191E.06 & 2.17E-06 \\
\hline St -90 & $5.46 \mathrm{E}-02$ & 42.7 & $1.13 \mathrm{E}+05$ & $5,09 \mathrm{E}-02$ & $5,27 \mathrm{E}-02$ & $5.64 E-02$ & $5.82 \mathrm{E}-02$ \\
\hline$Y-90$ & S.46E-02 & 42.7 & $1.13 \mathrm{E}+05$ & 2.91E-02 & 2.91E-02 & $5.64 \mathrm{E}-02$ & 5.82E-02 \\
\hline$Z r-93$ & 8.08E-06 & $6.33 \mathrm{E}-03$ & 16.7 & 4.41E- -06 & 4.41E-06 & 9,4]E-06 & 1.07E-05 \\
\hline $\mathrm{Nb}-93 \mathrm{~m}$ & $5.87 \mathrm{E}-06$ & $460 \mathrm{E}-03$ & 12.1 & $3.30 \mathrm{E}-06$ & $3.30 E-06$ & 6.81E.06 & $7.70 \mathrm{E}-06$ \\
\hline Tc-99 & 1.18E-04 & $9.24 \mathrm{E}-02$ & 24 & 7.51E-05 & 9.61E-05 & 1.40E-04 & $1.62 \mathrm{E}-04$ \\
\hline Ru-106 & $3.22 \mathrm{E}-09$ & 2.52E- 06 & 6.64E-03 & $1.51 \mathrm{E}-09$ & 1.51E-09 & $3.60 \mathrm{E}-09$ & $3.95 \mathrm{E}-09$ \\
\hline Cd.113m & $423 \mathrm{E}-05$ & 3.31E-02 & 87.3 & 2.03E-0.5 & $2.03 E-05$ & $5.03 \mathrm{E}-05$ & $5.00 \mathrm{E}-05$ \\
\hline Sb-125 & $7.81 \mathrm{E}-05$ & 6.11E-012 & 161 & $2.10 \mathrm{E}-05$ & $2.10 \mathrm{E}-05$ & 7.96E-05 & $8.09 E-0.5$ \\
\hline Sn-126 & 2.49E-06 & $1.95 \mathrm{E}-03$ & 5.14 & 1.39E-06 & $1.39 \mathrm{E}-06$ & $2.89 \mathrm{E}-06$ & $3.27 \mathrm{E}-06$ \\
\hline I-129 & $2.28 \mathrm{E}-07$ & 1.78E-04 & 0.470 & 1.44E-07 & 1.85E-07 & $2.70 \mathrm{E}-07$ & 3.12E-07 \\
\hline $\mathrm{Cs}-134$ & $1.20 \mathrm{E}-06$ & 9.41E- 04 & 2.8 & 8.72E-07 & 9.86E-07 & $1.37 \mathrm{E}-06$ & 1.54E-06 \\
\hline Ce-137 & 0.128 & 99.8 & $263 \mathrm{E}+05$ & 0.116 & 0.121 & 0.134 & 0.139 \\
\hline Ba- $137 \mathrm{~m}$ & 0.121 & 94.4 & 2. $6 E+05$ & 9.19E-02 & 9.69E-02 & 0.126 & 0.132 \\
\hline Sm-151 & $5.80 \mathrm{E}-0 \mathrm{3}$ & 4.54 & $1.20 \mathrm{E}+04$ & 3.22E- -03 & 3.22E- -03 & $6.7 \mathrm{SE}-03$ & $7.63 \mathrm{E}-03$ \\
\hline Eu-152 & $1.86 \mathrm{E}-\infty 6$ & 1.46E- 03 & $3 B A$ & 9.51E-07 & 9.51E-07 & 1.98E-06 & $2.10 \mathrm{E}-06$ \\
\hline Eu-154 & 2.95E-04 & 0.232 & 611 & $1.12 E-04$ & 1.12E-04 & $3.63 \mathrm{E}-04$ & $3.89 \mathrm{E}-04$ \\
\hline Eu-155 & $1.10 \mathrm{E}-04$ & 8.60E- -22 & 227 & 5.56E-05 & 5.56E-05 & 1.1TE-04 & 1.25E-04 \\
\hline Ra-226 & $7.03 \mathrm{E}-11$ & $5.50 E-08$ & 1.45E-01 & 4.75E-11 & 4.75E-11 & $7.858-11$ & 8.65E-11 \\
\hline Ra-228 & 6.33E-08 & 495E- 05 & 0.131 & $2.63 \mathrm{E}-08$ & 4.4E- 01 & B.52E- 08 & 1.09E-07 \\
\hline$A c-227$ & 4.42E-10 & $3.46 E-07$ & 9.13E-04 & $3.10 \mathrm{E}-10$ & 3.10E-10 & $4.90 \mathrm{E}-10$ & $5.36 \mathrm{E}-10$ \\
\hline$P(-23)$ & $203 E-09$ & $1.59 \mathrm{E}-06$ & 4.19E-03 & 1.30E-09 & $1.30 \mathrm{E}-09$ & $2.30 E-09$ & 2.55E-09 \\
\hline$T h-229$ & $1,400-09$ & 1.16E- -06 & 3.07E-03 & 6.74E-10 & $1.075-09$ & $1.9 \pi-09$ & $2.48 \mathrm{E}-09$ \\
\hline Th.232 & 4.21E-09 & $3.30 \mathrm{E}-06$ & 865-03 & $2.16 \mathrm{E}-99$ & 3.17209 & $5.26 \mathrm{E}-109$ & $6.26 \mathrm{E}-09$ \\
\hline$U-232$ & 3.30E-07 & $2.58 \mathrm{E}-04$ & 0.60 & 1.81E-07 & 2.54E-07 & 4.17E-07 & 3.11E-07 \\
\hline $\mathrm{U}-233$ & $1.26 E-06$ & 9.89E-04 & 2.61 & $6.96 \mathrm{E}-07$ & 9.73E-07 & $1.605-06$ & $1.96 \mathrm{E}-06$ \\
\hline U.234 & 3.91E- 07 & $3.00 \mathrm{E}-04$ & 0807 & $3.76 \mathrm{E}-07$ & $3,83 \mathrm{E}-07$ & 100E-07 & $4.04 \mathrm{E}-07$ \\
\hline U-235 & $1.59 \mathrm{E}-0 \mathrm{~B}$ & $1.24 \mathrm{E}-0 \mathrm{~s}$ & $3.205-02$ & $1.52 \mathrm{E}-08$ & 1.55E-08 & $163 E-68$ & $1.64 E-08$ \\
\hline $\mathrm{U}-236$ & $1.22 E-0$ & $9.53 \mathrm{E}-06$ & 2.51E-02 & 1.J8E-08 & $1.20 \mathrm{E}-08$ & $124 E-08$ & $1.26 \mathrm{E}-08$ \\
\hline $\mathrm{U}-238$ & 4.48E-07 & 3.SOE- 04 & 0.924 & 4.32E-07 & 439E-07 & A57E-07 & 4.60E- 07 \\
\hline $\mathrm{Np}-237$ & 4.34E-07 & 3.00E-04 & 0.895 & $2.94 E-07$ & $3.62 \mathrm{E}-07$ & S.06E-07 & $5.76 \mathrm{E}-07$ \\
\hline Pu-238 & 6.63E-07 & S. $19 \mathrm{E}-\mathrm{OA}$ & 1.37 & 498E-07 & 5.79E- -07 & $7.47 \mathrm{E}-07$ & 8.28E-07 \\
\hline Pu-239 & 2.28E-05 & $1.78 \mathrm{E}-02$ & 47.0 & $1.86 E-05$ & $2.06 E-05$ & 2.49E-05 & $2.70 \mathrm{E}-0 \mathrm{~S}$ \\
\hline Pu-240 & 3.86E-06 & $3.02 \mathrm{E}-03$ & 7.96 & 3.0TE-06 & 3.46E-06: & $4.26 \mathrm{E}-06$ & $4.64 E-06$ \\
\hline Pu-241 & 4.4BE-05 & $3.50 \mathrm{E}-02$ & $\mathbf{9 . 3}$ & 3.35E-05 & 3.90E-05 & 5.05E-05 & $5.60 \mathrm{E}-05$ \\
\hline Pu-242 & 2.46E-10 & 1.92E-07 & $5.07 E-04$ & $1.79 \mathrm{E}-10$ & 2.11E-10 & 280E-10 & $3.13 \mathrm{E}-10$ \\
\hline Am-241 & 2.7BE-0S & $2.17 \mathrm{E}-02$ & 573 & $2.00 \mathrm{E}-05$ & 2.38E-05 & $3.18 \mathrm{E}-05$ & $3.56 \mathrm{E}-05$ \\
\hline Am-243 & $9.55 \mathrm{E}-10$ & $7.48 \mathrm{E}-07$ & $1.9 \pi .03$ & $7.10 \mathrm{E} \cdot 10$ & B.27E-10 & 1.092 .00 & $121 \mathrm{E}-09$ \\
\hline $\mathrm{Cm}-242$ & 7.01E-08 & $5.49 \mathrm{E}-05$ & 0.145 & $3.27 \mathrm{E}-08$ & $3.27 E-06$ & $7,47 \mathrm{E}-08$ & $7.92 \mathrm{E}-08$ \\
\hline $\mathrm{Cm}-243$ & $6.49 E-09$ & $5.08 \mathrm{E}-06$ & $1.34 \mathrm{E}-02$ & $297 \mathrm{E}-09$ & 2.52E-D9 & 690E- 09 & $7.30 \mathrm{E}-09$ \\
\hline $\mathrm{Cm}-244$ & 6. $46 \mathrm{E}-08$ & $5.06 \mathrm{E}-05$ & 0.133 & $2.7 \mathrm{EE}-08$ & $2.78 E-08$ & $7.71 \mathrm{E}-08$ & 8.50E- 08 \\
\hline Totats & $\mathbf{M}, \quad$ & pty & $t \leq$ & $98 \mathrm{MO}$ & $\begin{array}{l}67 \mathrm{ClO} \\
\text { or } / \mathrm{L})\end{array}$ & $\begin{array}{r}\$ 67 \mathrm{Cl}(\mathrm{M} \\
0, \mathrm{~L})\end{array}$ & oM or $1 \mathrm{~L}$ \\
\hline Pu & 2.BSE-04 (g/L) & - & 0.587 & 2.05E-O4 & $2.44 E-04$ & $3.25 \mathrm{E}-04$ & $364 E-A 4$ \\
\hline $\mathrm{U}$ & 4.6E-03 & 836 & $2.20 \mathrm{E}+03$ & $4.29 \mathrm{E}-03$ & $4.38 \mathrm{E}-03$ & 4.61E- -03 & 4.64E-03 \\
\hline
\end{tabular}

tWater wt\% derived from the difference of denvity and total dissolvad species. 
HDW Model Pev. 4

\begin{tabular}{|c|c|c|c|c|c|c|c|}
\hline \multirow[b]{3}{*}{$\begin{array}{l}\text { Phyaled } \\
\text { Propentiles }\end{array}$} & \multicolumn{7}{|c|}{ Single-Shell Tank 241-S-102 } \\
\hline & \multicolumn{7}{|c|}{ Total Inventory Estimate* } \\
\hline & & & & $9 \mathrm{CI}$ & $-67 \mathrm{CI}$ & $+67 \mathrm{CI}$ & $+95 \mathrm{CI}$ \\
\hline Total Waste & $2.66 \mathrm{E}+06(\mathrm{~kg})$ & (5.49 kgat) & - & - & $\ldots$ & $\ldots$ & - \\
\hline Heat Load & $2.09(\mathrm{~kW})$ & (7.13E+03 BTU/hr) & - & 1.92 & 2.03 & 2.15 & 2.20 \\
\hline Bulk Density† & $1.28(\mathrm{~g} / \infty)$ & $\ldots$ & - & 1.26 & 1.27 & 1.29 & 1.30 \\
\hline Wrter wt\%t & & 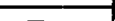 & 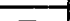 & $\infty 9$ & & & \\
\hline $\mathrm{TOC}$ wh\% C (wet & $\frac{61.1}{0.409}$ & - & $=$ & 0.271 & $\frac{60.0}{0.338}$ & $\frac{62.1}{0.479}$ & $\begin{array}{r}63.4 \\
0.547\end{array}$ \\
\hline 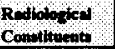 & $\mathrm{Cl} / \mathrm{L}$ & $\mathrm{nC} / \mathrm{l}$ & a & $\begin{array}{l}\text { os CI } \\
\text { (CVin) }\end{array}$ & $\begin{array}{l}-67 \mathrm{C} \\
(\mathrm{CH})\end{array}$ & $\begin{array}{l}+6 \mathrm{ct} \\
(\mathrm{cl}) \mathrm{L}\end{array}$ & $\begin{array}{l}+95 \mathrm{CI} \\
(\mathrm{Cu})\end{array}$ \\
\hline $\mathrm{H}-\mathrm{3}$ & $1.16 \mathrm{E}-04$ & 9.07E-02 & 241 & 6.15E-05 & $6.15 \mathrm{E}-05$ & $3.20 \mathrm{E}-04$ & $1.24 \mathrm{E}-04$ \\
\hline $\mathrm{C}-14$ & $1.65 E-05$ & $1.29 \mathrm{E}-02$ & 34.2 & $5.03 \pm-06$ & 5.03E-06 & $1.6 \pi-05$ & $1.69 E-05$ \\
\hline $\mathrm{Ni}-59$ & $1.1 \pi-06$ & $9.12 \mathrm{E}-04$ & 2.43 & $6.30 \mathrm{E}-07$ & $6.30 \mathrm{E}-07$ & $1.20 E-06$ & $1.22 \mathrm{E}-06$ \\
\hline $\mathrm{Ni}-63$ & 1.14E-04 & 8.99E-02 & 237 & $6.07 \mathrm{E}-05$ & $6.07 \mathrm{E}-05$ & 1. $1 \pi-04$ & 1.19E-04 \\
\hline $\operatorname{Co}-60$ & 1.81E-05 & 1.41E-02 & 37.6 & $5.015-06$ & 5.01E-06 & 1.84E-05 & $1.87 \mathrm{E}-05$ \\
\hline Se-79 & 1.64E-06 & 1.25E-03 & 3.41 & 9.68E-07 & 9.03E-07 & $190 E-06$ & $2.16 \mathrm{E}-06$ \\
\hline Sr-90 & 6.00E-02 & 47.3 & $1.2 \pi+05$ & $3.73 \mathrm{E}-02$ & S.91E-02 & $6.27 \mathrm{E}-02$ & 6.45E-02 \\
\hline$Y-90$ & 6.09E-02 & 47.5 & $1.27 \mathrm{E}+05$ & $3,57 \mathrm{E}-02$ & $3.5 \pi \mathrm{E}-02$ & $6.28 \mathrm{E}-02$ & $6.45 \mathrm{E}-02$ \\
\hline$Z r-93$ & 8.04E-06 & $6.286-03$ & 16.7 & $4.39 \mathrm{E}-06$ & $4.39 \mathrm{E}-06$ & $9.36 E-06$ & $1.06 E-05$ \\
\hline $\mathrm{Nb}-93 \mathrm{~m}$ & S.85E-06 & 4.56E-03 & 12.1 & $3.29 E-06$ & $3.29 E-06$ & 6.7TE-0S & $7.66 \mathrm{E}-06$ \\
\hline Tc-99 & $1.17 \mathrm{E}-04$ & $9.16 \mathrm{E}-02$ & 241 & $7.47 \mathrm{E}-05$ & 9.55E-05 & 1.39E-04 & 1.61E-04 \\
\hline Ru-106 & $3.20 \mathrm{E}-09$ & 2.49E- 06 & 6.6AE-03 & $1.50 \mathrm{E}-09$ & 1.50E-09 & $3.57 \mathrm{E}-09$ & $3.92 \mathrm{E}-09$ \\
\hline Cd.113m & 4.21E-05 & 3.28E-02 & 87.4 & $2.02 E-05$ & $2.02 E-05$ & $5.00 E-03$ & 5.76E-05 \\
\hline Sb-125 & $7.76 \mathrm{E}-05$ & $6.05 \mathrm{E}-02$ & 161 & $2.09 E-05$ & 2.09E-05 & 7,90E-05 & 8.04E-05 \\
\hline $\mathrm{Sn}-126$ & $2.49 \mathrm{E}-06$ & $1.93 \mathrm{E}-03$ & 5.15 & 1.38E- -06 & $1.395-06$ & $2.87 \mathrm{E}-06$ & $3.25 \mathrm{SE}-06$ \\
\hline I-129 & $226 \mathrm{E}-07$ & 1. T7E-OA & 0.40 & 1.44E-07 & 134E-A7 & $2.69 \mathrm{E}-07$ & $3.10 \mathrm{E}-07$ \\
\hline Cs-134 & 1.19E-06 & $9.32 E-04$ & 2.43 & 8.66E-07 & 9.79E-07 & $1.36 \mathrm{E}-0.05$ & 1.53E- 06 \\
\hline Cs-137 & 0.127 & 99.1 & $2.64 E+05$ & 0.115 & 0.121 & 0.133 & 0.139 \\
\hline Ba-137m & 0.120 & 93.8 & $2.50 \mathrm{E}+05$ & $9.16 \mathrm{E}-02$ & 9.16E-02 & 0.126 & $0.13 !$ \\
\hline $\mathrm{Sm}=151$ & $5.77 \mathrm{E}-03$ & 4.50 & $1.20 \mathrm{E}+0 \mathrm{~A}$ & $3.21 \mathrm{E}-03$ & 3.21E-03 & 6.70E-03 & $7.58 \mathrm{E}-03$ \\
\hline $\mathrm{Eu}-152$ & $1,900-06$ & 1.4BE-03 & 3.95 & 9.95E-07 & 9.95E-07 & $2.02 \mathrm{E}-06$ & $2.14 E-06$ \\
\hline Eu-154 & 2.94E-04 & 0.229 & 611 & $1.1 \mathrm{E}-\mathrm{A}$ & $1.116-04$ & $3.60 \mathrm{E}-04$ & $3.86 \mathrm{E}-04$ \\
\hline Eu-155 & 1.21E-04 & 8.70E-02 & 232 & 3.76t-0s & 5.70E-0s & 1.19E-04 & $1.26 \mathrm{E}-04$ \\
\hline $\operatorname{Re}-226$ & $7.76 \mathrm{E}-11$ & $6.06 \mathrm{E}-08$ & $1.61 \mathrm{E}-04$ & $5.50 \mathrm{E}-11$ & 5.50E-11 & 8.59E-11 & 9.37E-11 \\
\hline Re-228 & $6.2815-08$ & 4.90E-05 & 0.131 & $2.61 E-08$ & 4.41E-08) & 7. $46 \mathrm{E}-08$ & $1.08 \mathrm{E}-07$ \\
\hline Ac-227 & A.74E-10 & 3.70E-07 & 9.BSE-04 & $3.42 \mathrm{E}-10$ & 3.A2E-10 & $5.21 \mathrm{E}-10$ & $5.67 \mathrm{E}-10$ \\
\hline $\mathrm{PB}-231$ & 2.02E-09 & $1,98 \mathrm{E}-06$ & $4.20 \mathrm{E}-03$ & 1.29E-09 & $1.29 E-09$ & $2.29 E-09$ & 2.54E-09 \\
\hline Th-229 & 1.4PE-AS & 1.15E-06 & $3.07 \mathrm{E}-03$ & $6.69 \mathrm{E}-10$ & $1.06 \mathrm{E}-09$ & $1.95 E-69$ & $2.46 \mathrm{E}-09$ \\
\hline Th-232 & $418 \mathrm{E}-09$ & $3.26 \mathrm{E}-06$ & 8.69E-03 & $2.15 E-09$ & 3.14E-09 & $5.225-09$ & $6.21 E-09$ \\
\hline $\mathrm{U}-232$ & $3.2 \pi-07$ & 2.55E-A4 & 0.60 & 1.BOE-07 & 2.52E-07 & 414E-07 & $5.07 \mathrm{E}-07$ \\
\hline $\mathrm{U}-233$ & $1.25 E-05$ & $9.79 \mathrm{E}-\mathrm{OH}$ & 261 & $6.91 \mathrm{E}+07$ & 9.66507 & $1.59 \mathrm{E}-06$ & $1.94 E-06$ \\
\hline U. 234 & 3.00E-07 & 3.04E-04 & 0809 & $3.74 E-07$ & 3.81E-0? & 390E-07 & 4.01E-07 \\
\hline U-235 & $1.58 \mathrm{E}-0.0$ & $1.23 E-05$ & 329E-0 & $1.52 E-08$ & 1.55E-CO & $1.62 \mathrm{E}-08$ & $1,63 E-08$ \\
\hline U-236 & $1.21 \mathrm{E}-08$ & 9.45E-06 & $2525-00$ & $1.17 \mathrm{E}-08$ & $1.19 \mathrm{E}-0$ & $1.23 E-08$ & $1.25 \mathrm{E}-48$ \\
\hline U-238 & 4.45E-O7 & 3.4.0E-04 & 0.925 & $430 \mathrm{E}-07$ & 4.37E-07 & 4.5AE-07 & 4.5TE-0? \\
\hline $\mathrm{Np}-237$ & 431E-07 & $3.3 \mathrm{TE}-04$ & 0.67 & 2.92E-07 & 3.60E-07 & S.03E-07 & 5.73E-0? \\
\hline Pu-238 & 7,06E-07 & $5.51 \mathrm{E}-04$ & 1.9 & $5,42 E-07$ & 6.23E-07. & 7.905-07 & 8.70E-07 \\
\hline Pu-239 & 2.61E-05 & $2.03 \mathrm{E}-02$ & 54.2 & 2.19E-05 & 2.39E-05 & $2.82 E-05$ & 3.02E-05 \\
\hline $\mathrm{Pu}-240$ & 4.32E- -06 & 3.37E-03 & 8.98 & 3.54E-06 & $3.93 \mathrm{E}-06$ & 172E-06 & S.10E-06 \\
\hline Pu-241 & 4.73E-05 & $3.69 \mathrm{E}-02$ & 98,4 & $3.62 E-05$ & 4.16E-05 & $530 \mathrm{E}-05$ & $5.85 E-05$ \\
\hline Pu-242 & $2.57 \mathrm{E}-10$ & $2.01 E-07$ & 53:E-OA & $1.91 \mathrm{E}-10$ & 2.23E-10 & 2.91E-10 & $3.24 E-10$ \\
\hline Am-24I & $2.76 \mathrm{E}-05$ & $2.16 \mathrm{E}-02$ & 57,4 & $1.99 E-05$ & 2375-05 & 3.16E-0S & 3. SAE-05 \\
\hline$A m-243$ & 9.49E-10 & $7,41 E-07$ & $1.9 \mathrm{TE}-03$ & $7.05 \mathrm{E}-10$ & B.22E-10 & $1.08 E-09$ & $1.20 \mathrm{E}-09$ \\
\hline $\mathrm{Cm}-242$ & 7.03E- & $5.52 \mathrm{E}-0 \mathrm{~S}$ & 0.147 & $3.3 \pi \times$ & 337E-06 & $7.5 \mathrm{E}-68$ & 7.98E-08 \\
\hline $\mathrm{Cm}-243$ & 6.47E-09 & 5.0SE-06 & $1.34 E-02$ & $292 E-09$ & $2.92 \mathrm{E}-09$ & 6.88E-09 & $7.28 \mathrm{E}-09$ \\
\hline $\mathrm{Cm}-244$ & 6.42E-06 & S.01E-05 & 0.133 & 2.76E-08 & $2.76 \mathrm{E}-08$ & 7.65E-08 & 8.4AE-OB \\
\hline Tots & $\mathrm{M}$ ? & 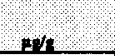 & $1+$ & os ct $(\mathrm{M}$ & $\begin{array}{l}67 \mathrm{Cl}(\mathrm{l}) \\
\mathrm{c}, \mathrm{P}, \mathrm{L})\end{array}$ & $\begin{array}{l}+67 \mathrm{Cl}(\mathrm{M} \\
6 \mathrm{r} / \mathrm{L})\end{array}$ & $\begin{array}{l}+9 s \mathrm{Cl} \\
\text { Mor ph }\end{array}$ \\
\hline $\mathrm{Pu}$ & 3.40E-OA $(\Omega)$ & - & 0.07 & 2.61E-04 & $3.00 \mathrm{E}-04$ & 3.80E-04 & 4.19E-04 \\
\hline $\mathrm{U}$ & 4.47E-03 & 800 & $2.21 \mathrm{E}+03$ & $4.2 \pi-03$ & $4.37 E-03$ & 4.58E-03 & 4.61E-03 \\
\hline
\end{tabular}

"Unknown in tenk solids inventory me marigned by Tenk Layering Model (TLM)

tVolume everuge for density, mase averge Woter we\% and TOC w\% C. 
MN.

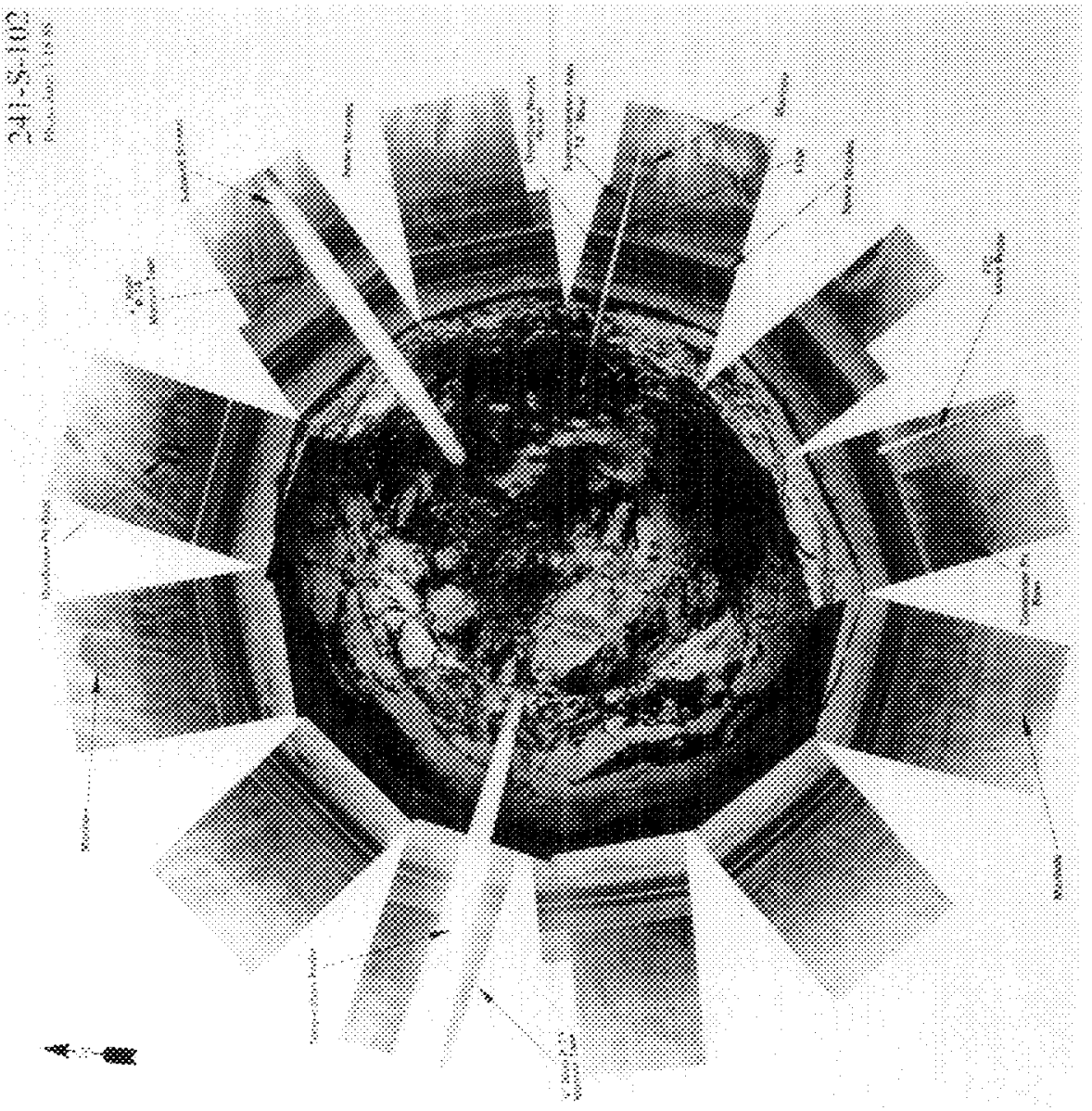




\begin{tabular}{|c|c|c|c|}
\hline 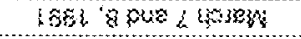 & 5100 & $\xi$ & sogunto sosib \\
\hline$\quad 218783$ & $\left.3 \times 33^{2}\right)$ & 2088 & 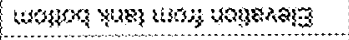 \\
\hline 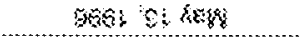 & $23 \times 3$ & $368=z \sin$ & 3320 \\
\hline 4 30 & $\operatorname{sen} x \operatorname{sen}$ & $\triangle \times 13$ & 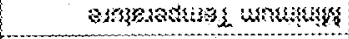 \\
\hline SVYNA lenugsi nise onv & 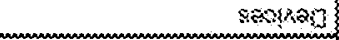 & $\xi$ & coquma sasis \\
\hline \multicolumn{2}{|c|}{ 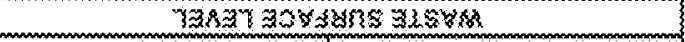 } & 450 & 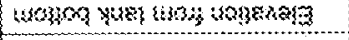 \\
\hline \$t: $0805 \tilde{3}$ & 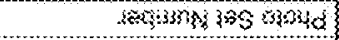 & $805: 2 x<1$ & 3186 \\
\hline 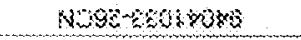 & 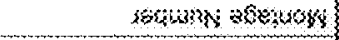 & $x_{0} 66$ & 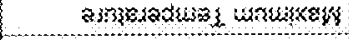 \\
\hline $8805^{\prime} ; 20 \%$ & axa & 3,89 & 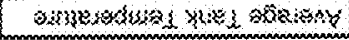 \\
\hline \multicolumn{2}{|c|}{ 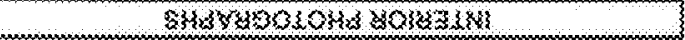 } & \multicolumn{2}{|c|}{ 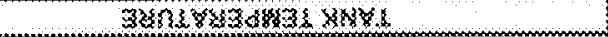 } \\
\hline $86000<$ & 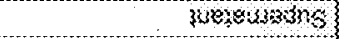 & 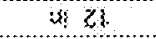 & 88 \\
\hline $8 \% 0000:$ & astrsts & i3) 1 & $3+18$ is \\
\hline $19 \$ 000^{\prime}, Z 2$ & 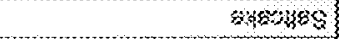 & 238 & (O) Bopurps 39,4 \\
\hline $10600 \%$ & 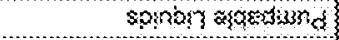 & \multicolumn{2}{|c|}{ 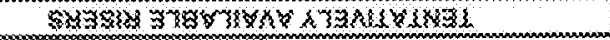 } \\
\hline 6000098 & 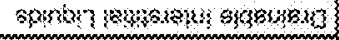 & $\ldots$ & 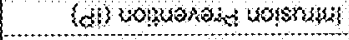 \\
\hline stso & $\left.\approx 3 \pi_{j} \approx \xi \leqslant \&\right\}$ & $7861 \cdot 330$ & 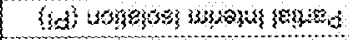 \\
\hline $306000 \times 3$ & aumpon alsen bsal & $\cdot+\cdots$ & 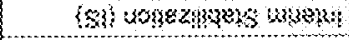 \\
\hline \multicolumn{2}{|c|}{ 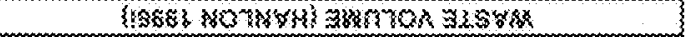 } & $\therefore$ & Japen pouksst \\
\hline$z^{3}$ & 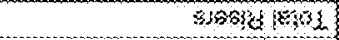 & puns & 5) \\
\hline sutas & 4hes apsosens & suks & ang genk \\
\hline 30900060 & 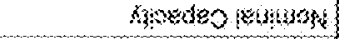 & $986 \%$ & sngongs \\
\hline $4 \$$ & 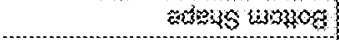 & $\cdot \cdot$ & 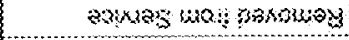 \\
\hline if $9 l$ & 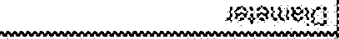 & 8564 do 143 & 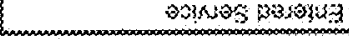 \\
\hline \multicolumn{2}{|c|}{ 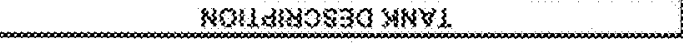 } & \multicolumn{2}{|c|}{ MHOLSIH HNYL } \\
\hline
\end{tabular}

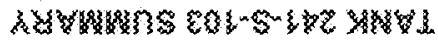




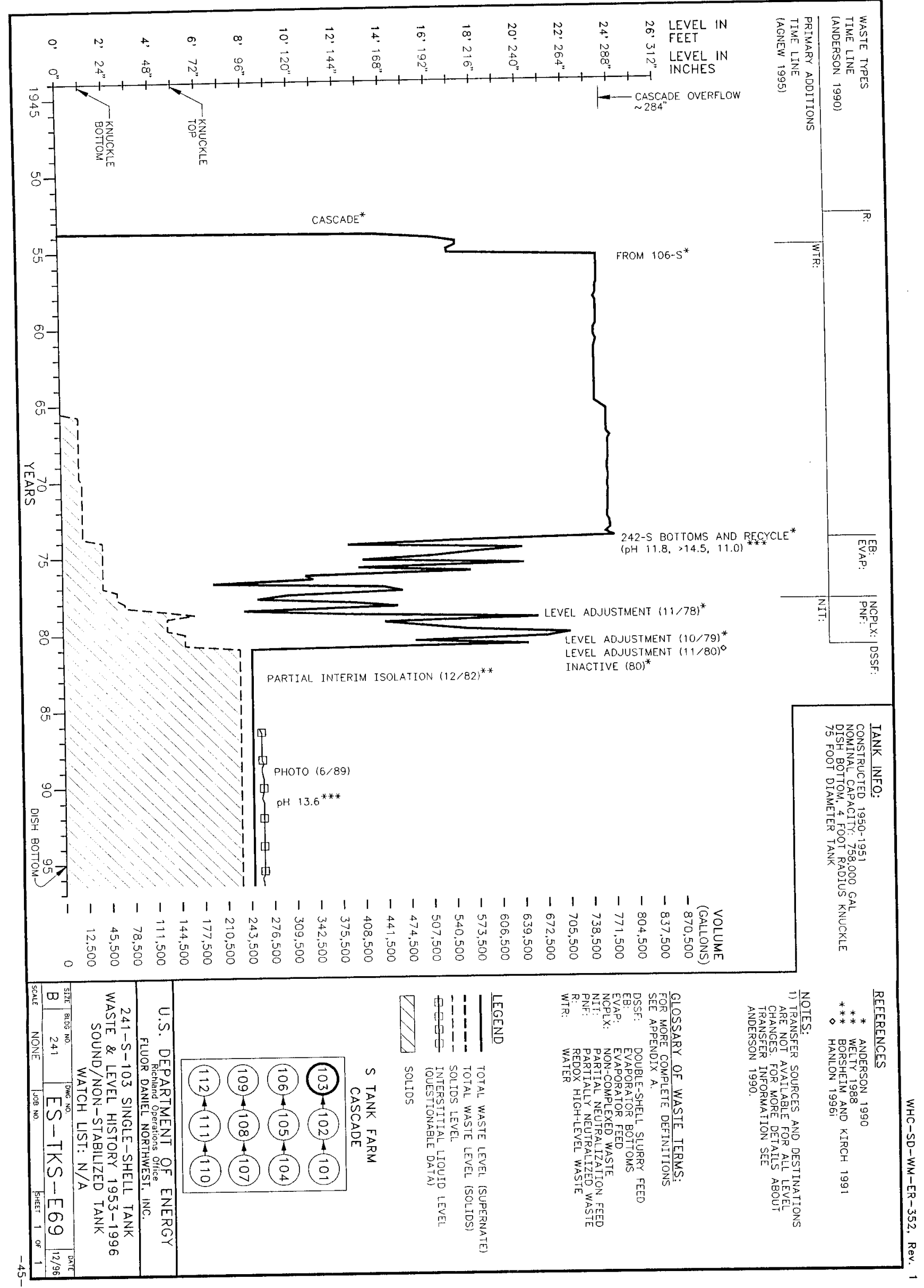




$$
241-5-103
$$

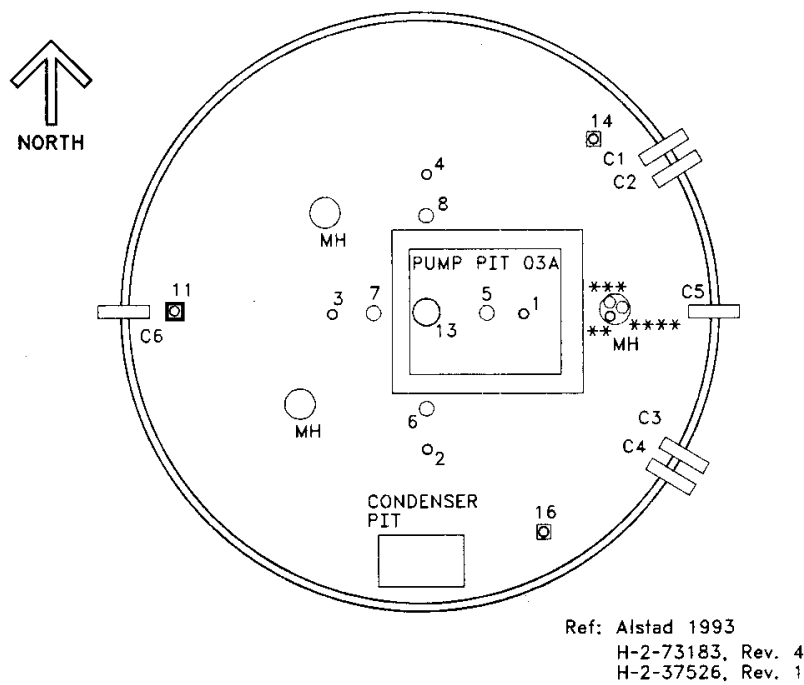

\section{TANK RISER LOCATION}

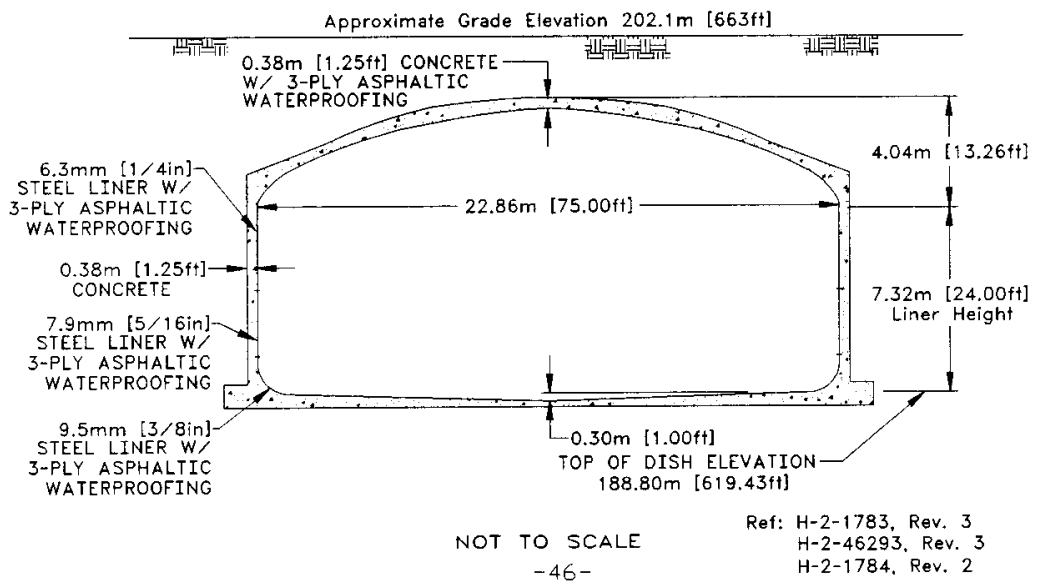




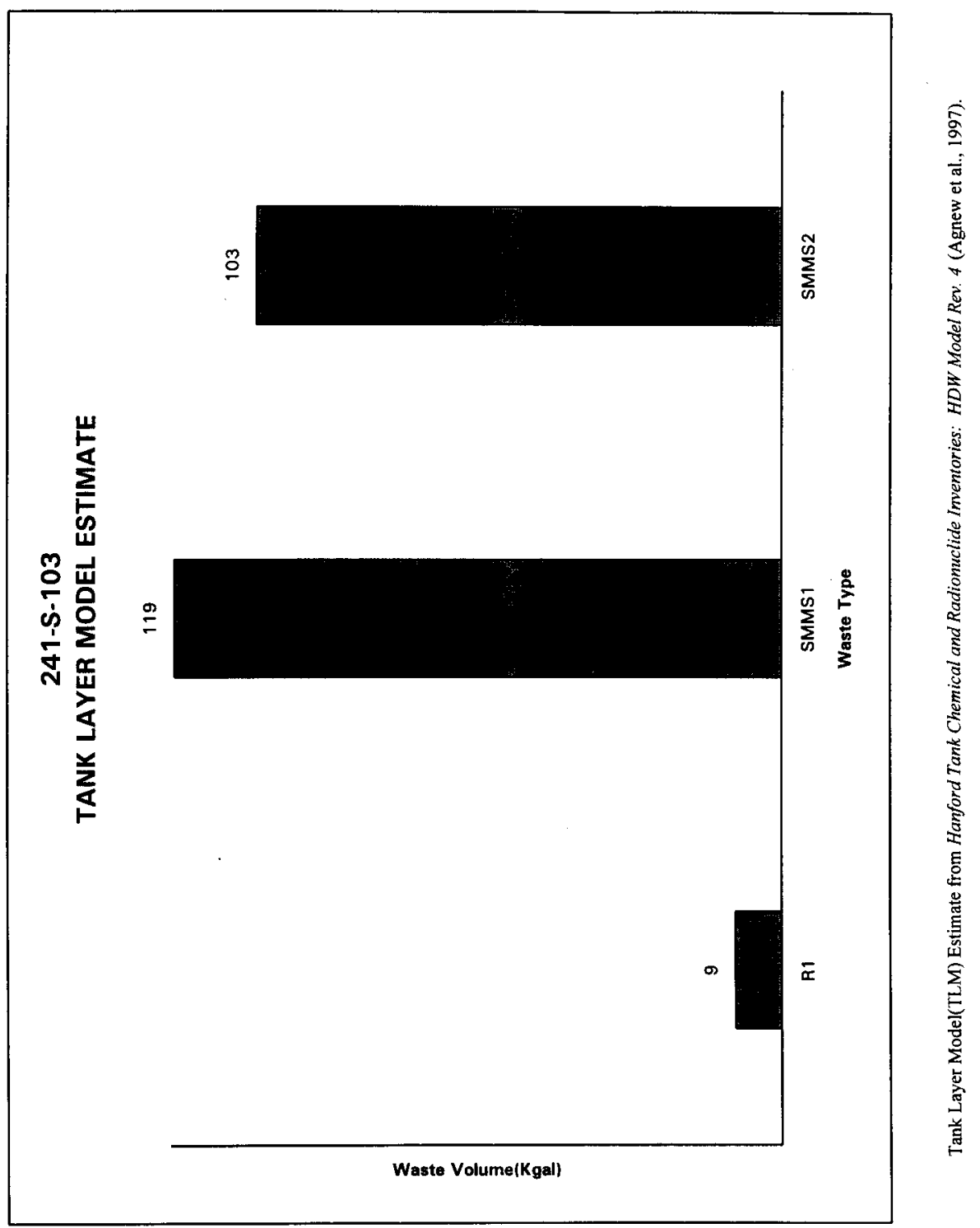


HNF-SD-WM-ER-352, Rev. 1

HDW Model Aev. 4

\begin{tabular}{|c|c|c|c|c|c|c|c|}
\hline & \multicolumn{7}{|c|}{ Single-Shell Tank 241-S-103 } \\
\hline & \multicolumn{7}{|c|}{ TLM Solids Composite Inventory Estimate* } \\
\hline Physical & & & & & & & \\
\hline Froperties & & & & $95 \mathrm{CI}$ & $-67 \mathrm{CI}$ & $+67 C I$ & $+95 \mathrm{CI}$ \\
\hline Total TLM Weste & $5.98 \mathrm{E}+04(\mathrm{~kg})$ & $(9.00 \mathrm{kgl})$ & - & - & - & - & - \\
\hline Heat Loed & $0.222(\mathrm{kw})$ & (7se BTUhr) & $\ldots$ & 0.161 & 0.197 & 0.238 & 0.246 \\
\hline Bulk Density & $1.76(1 / \infty)$ & - & - & 1.51 & 1.53 & 2.11 & 2.24 \\
\hline Void Fraction & 0.536 & - & ז. & $6.00 \mathrm{E}-02$ & 0.217 & 0.737 & 0.743 \\
\hline Water wt\% & 27.0 & - & - & 2.64 & 10.0 & 42.6 & 44.2 \\
\hline TOC wt\% C (wet & 0 & $\ldots$ & - & 0 & 0 & 0 & 0 \\
\hline $\begin{array}{l}\text { Chentent } \\
\text { Constituents }\end{array}$ & mole/L & $\mathrm{POW}$ a & Mr & $\begin{array}{l}98 \mathrm{CI} \\
\text { (mole L) }\end{array}$ & $\begin{array}{l}67 \mathrm{CI} \\
(\mathrm{mole} / \mathrm{L})\end{array}$ & $\begin{array}{l}+67 \mathrm{Cl} \\
(\mathrm{molall})\end{array}$ & $\begin{array}{l}+95 \mathrm{CL} \\
\text { (boleli) }\end{array}$ \\
\hline $\mathrm{Na}+$ & 8.13 & $1.06 \mathrm{E}+05$ & $6.36 E+03$ & 3.05 & 3.56 & 147 & 18.2 \\
\hline $\mathrm{Al}^{3}+$ & 6.99 & $10 \pi+05$ & $6.42 \mathrm{E}+03$ & 6.58 & 6.74 & 7.15 & 7.15 \\
\hline $\mathrm{Fe} 3+($ total $\mathrm{Fe})$ & 1.01 & $3.22 \mathrm{E}+04$ & $1,93 \mathrm{E}+03$ & 0.994 & 1.00 & 1.02 & 1.03 \\
\hline $\mathrm{Cr}^{3+}$ & 6.19E-02 & $1.83 \mathrm{E}+03$ & 110 & $7.08 E-03$ & 3.39E- -02 & 0.522 & 1.06 \\
\hline Biz+ & 0 & 0 & 0 & 0 & 0 & 0 & 0 \\
\hline $1+3+$ & 0 & 0 & 0 & 0 & 0 & 0 & 0 \\
\hline fig2t & 0 & 0 & 0 & 0 & 0 & 0 & 0 \\
\hline $\mathrm{Zr}(\mathrm{a} \mathrm{ZOO}(\mathrm{OH}) 2)$ & 0 & 0 & 0 & 0 & 0 & 0 & 0 \\
\hline $\mathrm{Pb}_{2}+$ & 0 & 0 & 0 & 0 & 0 & 0 & 0 \\
\hline Ni2+ & $5.07 E-02$ & $1.69 \mathrm{E}+0.3$ & 101 & $3.66 \mathrm{E}-02$ & 4.4SE-O2 & $5.54 \mathrm{E}-02$ & 5.45E-02 \\
\hline $\mathbf{S}-2+$ & 0 & 0 & 0 & 0 & 0 & 0 & 0 \\
\hline Mndt & 0 & 0 & 0 & 0 & 0 & 0 & 0 \\
\hline $\mathrm{Ca2t}$ & 0.220 & $5,02 \mathrm{E}+03$ & 300 & 0.146 & 0.182 & 0.258 & 0.294 \\
\hline $\mathbf{K}+$ & 1.12E-02 & 250 & 149 & 1.28E-03 & $5.25 \mathrm{E}-03$ & $1.52 E-02$ & $1.52 \mathrm{E}-02$ \\
\hline OH- & 25.6 & $2.48 \mathrm{E}+05$ & $1.48 \mathrm{E}+04$ & 23.1 & 243 & 26.9 & 28.4 \\
\hline NO3. & 5.29 & $187 \mathrm{E}+05$ & $1.12 \mathrm{E}+04$ & $6.03 \mathrm{E}-02$ & $6.05 E-02$ & 23.5 & 17.9 \\
\hline NO2- & 1.46 & $3.82 E+04$ & $2.28 \mathrm{E}+03$ & 0.132 & 0.595 & 2.15 & 2.15 \\
\hline $\cos 2$. & 0.220 & $7,51 \mathrm{E}+03$ & 49 & 0.146 & 0.182 & 0.258 & 0.294 \\
\hline 1043 & 0 & 0 & 0 & 0 & 0 & 0 & 0 \\
\hline SO42. & $1.04 \mathrm{E}-02$ & 569 & 34.0 & $1.19 \mathrm{E}-03$ & 4.87E-03 & I.4IE-02 & $1.41 E-02$ \\
\hline Si (as SiO32-) & 8.06E-03 & 129 & 7.71 & $9.2] E-04$ & 3. $77 \mathrm{E}-03$ & $109 E-02$ & $1.09 \mathrm{E}-02$ \\
\hline F- & 0 & 0 & 0 & 0 & 0 & 0 & 0 \\
\hline Cl. & $5.16 \mathrm{E}-02$ & $1.04 E+03$ & 62.3 & $5.91 \mathrm{E}-03$ & 2.42E-02 & $700 \mathrm{E}-02$ & 0.157 \\
\hline C6HSOT & 0 & 0 & 0 & 0 & 0 & 0 & 0 \\
\hline EDTA4 & 0 & 0 & 0 & 0 & 0 & 0 & 0 \\
\hline HEDTA3 & 0 & 0 & 0 & 0 & 0 & 0 & 0 \\
\hline Sycolate- & 0 & 0 & 0 & 0 & 0 & 0 & 0 \\
\hline acetnte. & 0 & 0 & 0 & 0 & 0 & 0 & 0 \\
\hline axalute2- & 0 & O & 0 & 0 & 0 & 0 & 0 \\
\hline DBP & 0 & 0 & 0 & 0 & 0 & 0 & 0 \\
\hline butenol & 0 & 0 & 0 & 0 & 0 & 0 & 0 \\
\hline $\mathrm{NH3}$ & & & & $145 \mathrm{~m}$ & & & \\
\hline Fe(CN)64 & $\frac{0.256}{0}$ & $\frac{2.47 \mathrm{t}+03}{0}$ & $-\frac{148}{0}$ & $\frac{6.35 \mathrm{E}-02}{0}$ & $\begin{array}{r}9.25 \mathrm{E}-02 \\
0\end{array}$ & 0.270 & $\frac{0.284}{0}$ \\
\hline
\end{tabular}

*Unkrowns in tank woids iviventory are ascigned by Tonk Loyeing Model (TLM). 
HNF-SD-WM-ER-352, Rev. 1

HOW Model Rev. 4

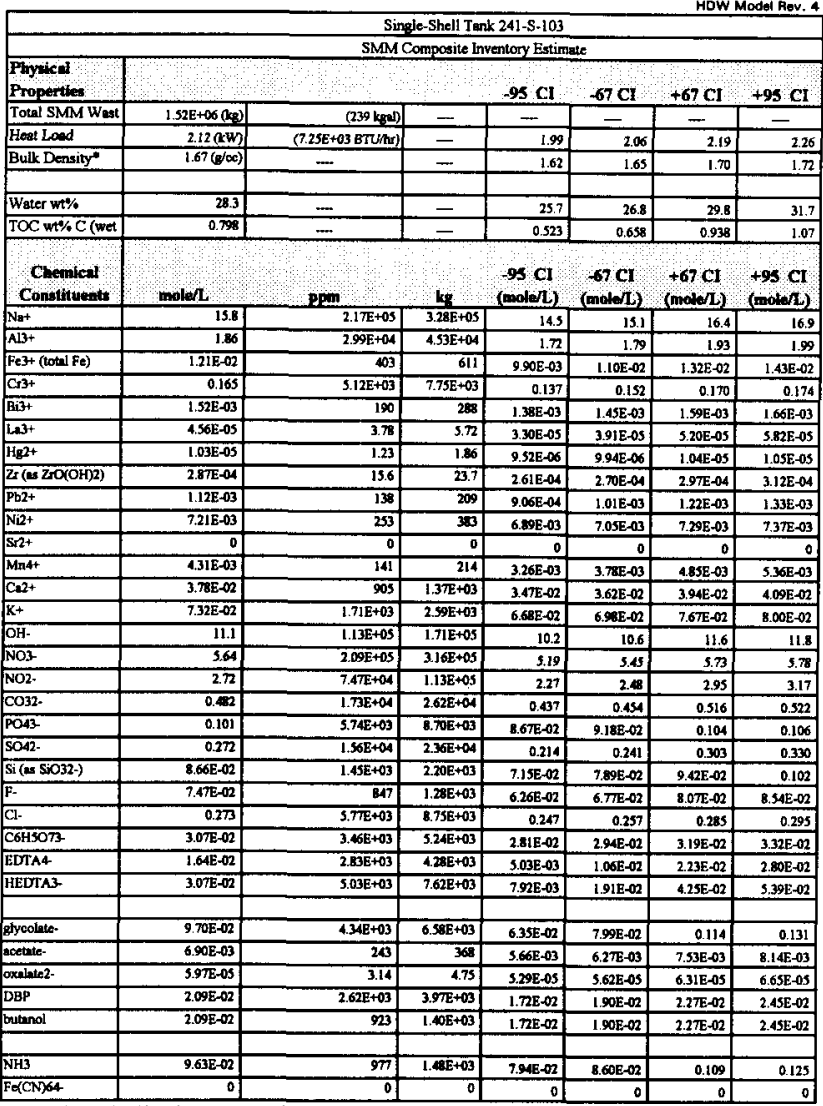

Density is calculeted besed on $\mathrm{Nh}, \mathrm{OH}$-, and $\mathrm{AlC2}$.

tWater wt\% derived from the difference of dersity end woul diasolved epecies. 
HNF-SD-WM-ER-352, Rev. I

\begin{tabular}{|c|c|c|c|c|c|c|c|}
\hline \multirow{4}{*}{$\begin{array}{l}\text { Phyicied } \\
\text { Properties } \\
\end{array}$} & \multicolumn{7}{|c|}{ Single-Shell Terk 241-S-103 } \\
\hline & \multicolumn{7}{|c|}{ Total Inventory Estimate* } \\
\hline & & & & & & & \\
\hline & & & & $-95 \mathrm{CI}$ & $-67 \mathrm{CI}$ & $+67 \mathrm{CI}$ & $+95 \mathrm{Cl}$ \\
\hline Total Waste & $1.58 \mathrm{SE}+06(\mathrm{kQ})$ & $(248 \mathrm{~kg} d)$ & $=$ & - & $\cdots$ & $\ldots$ & - \\
\hline Heat Loed & $2.35(\mathrm{~kW})$ & $(8.01 E+03 \mathrm{BTU} / \mathrm{hu})$ & - & 221 & $2.2 B$ & 2.41 & 2.48 \\
\hline Bulk Densityt & $1.68(\mathrm{~g} / 0 \mathrm{c})$ & $\ldots$ & - & 1.63 & 1.65 & 1.70 & 1.72 \\
\hline & & & & & & & \\
\hline Water wt\% ${ }^{\dagger}$ & 28.3 & $=$ & $\ldots$ & 25.7 & 26.8 & 29.7 & 31.5 \\
\hline TOC wt\% C (wet & 0.768 & - & $\ldots$ & 0.503. & 0.633 & 0.903 & 1.03 \\
\hline $\begin{array}{l}\text { Chomict } \\
\text { Constituents }\end{array}$ & mole/L & phe & $\mathrm{kg}$ & (nolel) & $\begin{array}{l}67 \mathrm{CL} \\
\text { (moled })\end{array}$ & $\begin{array}{l}+67 \mathrm{Cl} \\
\text { (mold } 1 \text { ) }\end{array}$ & $\begin{array}{l}+95 \mathrm{cl} \\
\text { (moleh) }\end{array}$ \\
\hline $\mathrm{Nat}^{+}$ & 15.5 & $2.12 E+05$ & $3.35 E+05$ & 14.2 & 14.9 & 16.1 & 16.6 \\
\hline $\mathrm{Al}^{3+}$ & 2.04 & $3.29 E+04$ & $5.17 \mathrm{E}+04$ & 1.91 & 1.98 & 2.11 & 2.17 \\
\hline $\mathrm{Fe}+$ (total Fe) & $4.84 E-02$ & $1.61 \mathrm{E}+03$ & $2.5 \mathrm{AE}+03$ & $4.63 \mathrm{E}-02$ & 4.75E-02 & $4.95 \mathrm{E}-02$ & $5.05 E-02$ \\
\hline $\mathrm{Cr}^{3+}$ & 0.161 & $4.99 E+03$ & $7.86 \mathrm{E}+03$ & 0.143 & 0.152 & 0.172 & 0.171 \\
\hline $\mathrm{Bi3+}$ & $1.47 \mathrm{E}-03$ & 183 & 28 & $1.33 E-03$ & $1.40 €-03$ & $1.54 E_{-03}$ & $1.60 \mathrm{E}-03$ \\
\hline Le3+ & 4.39E-05 & 3.63 & 5.72 & 3.18E-05 & 3.77E-05 & 5.01E-05 & $5.61 \mathrm{E}-0.5$ \\
\hline $\mathrm{Hg}_{2+}$ & $9.88 \mathrm{E}-06$ & 0.18 & 186 & $9.17 \mathrm{E}-06$ & 9.5TE.06 & $1.00 \mathrm{E}-05$ & $1.02 E-05$ \\
\hline Zr(as $\left.\mathrm{ZrO}(\mathrm{OH})_{2}^{2}\right)$ & $2.76 \mathrm{E}-04$ & 15.0 & 237 & $2.52 \mathrm{E}-04$ & $2.60 \mathrm{E}-04$ & $2.66 \mathrm{E}-04$ & $3.00 \mathrm{E}-04$ \\
\hline $\mathrm{PO} 2+$ & $1.08 \mathrm{E}-03$ & 133 & 209 & $8.73 E-04$ & 9.73E-04 & $1.18 E-03$ & $1.28 \mathrm{E}-03$ \\
\hline Ni2+ & $8.79 \mathrm{E}-03$ & 307 & 84 & 8.28E-03 & 8.56E-03 & 8.96E-03 & $8.94 E-03$ \\
\hline $\mathrm{S} 2+$ & 0 & 0 & 0 & 0 & 0 & 0 & 0 \\
\hline Mn4t+ & $4.15 E-03$ & 136 & 214 & $3.14 E-03$ & $3.64 E-03$ & 4.6TE-03 & $5.17 \mathrm{E}-03$ \\
\hline $\mathrm{Cu} 2+$ & $4,44 E-02$ & $1.00 \mathrm{E}+03$ & $1.6 \pi+03$ & 4.14E-02 & 4.29E-02 & $4.60 E-02$ & $4.74 \mathrm{E}-02$ \\
\hline $\mathrm{K}+$ & 7.09E-02 & $1.65 \mathrm{E}+03$ & $2600+03$ & 6.48E- -22 & 6.77E-0. & $7,43 \mathrm{E}-02$ & $7.75 \mathrm{E}-02$ \\
\hline $\mathrm{OH}-$ & 11.6 & $3.18 \mathrm{E}+05$ & $1.85 E+05$ & 10.8 & 11.2 & 120 & 12.3 \\
\hline $\mathrm{NO}$ & 5.62 & $2.68 E+05$ & $3.27+03$ & 5.19 & 5.40 & 58 & 5.87 \\
\hline NO2- & 2.67 & $7.33 E+04$ & $1.15 E+05$ & 2.24 & 2.44 & 2.90 & 3.11 \\
\hline Co32. & 0.472 & $1.69 \mathrm{E}+04$ & $2666+04$ & 0.429 & 0.446 & 0.506 & 0.511 \\
\hline PO43- & $9.76 \mathrm{E}-02$ & $5.53 E+03$ & $8.70 \mathrm{E}+03$ & $8.36 E-02$ & 8.85E-02 & 0.100 & 0.103 \\
\hline SO42. & 0.262 & $1.50 \mathrm{E}+0 \mathrm{~A}$ & $2.36 \mathrm{E}+04$ & 0.207 & 0.233 & 0.292 & 0.319 \\
\hline Si (u SiO32-) & $8.37 E-02$ & $1.40 \mathrm{E}+03$ & $2.21 E+03$ & $6.92 \mathrm{E}-02$ & $7.63 \mathrm{E}-02$ & 9.11E-02 & $9.82 \mathrm{E}-02$ \\
\hline F. & $7.20 \mathrm{E}-02$ & 815 & $1.28 \mathrm{E}+03$ & $6.03 \mathrm{E}-12$ & $6.52 E-02$ & $7.77 \mathrm{E}-02$ & $8.23 \mathrm{E}-02$ \\
\hline Cl- & 0.265 & $5.59 \mathrm{E}+03$ & $8.81 E+03$ & 0.240 & 0.249 & 0.276 & 0.286 \\
\hline C6H5O73- & $2,95 \mathrm{E}-02$ & $3.33 \mathrm{E}+03$ & $5.24 \mathrm{E}+03$ & 2.71E- -2 & $2.83 E-02$ & 3.08E-02 & 3.20 $\mathrm{E}-02$ \\
\hline EDTA4- & 1.SSE-02 & $2.72 E+03$ & $4.28 \mathrm{E}+03$ & 4.85E-03: & $102 E-02$ & 2.15E-02 & $2.70 \mathrm{E}-02$ \\
\hline HEDTA3 & $2.96 \mathrm{E}-02$ & $4.84 E+03$ & $7.62 \mathrm{E}+03$ & 7.63E-03 & 1. BAE-02 & $4.09 \mathrm{E}-02$ & $5.20 \mathrm{E}-02$ \\
\hline glyeolinte- & $9.35 E-02$ & $4.18 E+03$ & $6.56 \mathrm{E}+03$ & $6.12 \mathrm{E}-12$ & $7.70 E-02$ & 0.110 & 0.126 \\
\hline acetate- & 6.6SE- 03 & 234 & 368 & 5.46E-03 & $6.04 E-03$ & $7.26 \mathrm{E}-03$ & $7.85 \mathrm{E}-03$ \\
\hline oxalate2-2. & $5,75 E-05$ & 3.02 & 4.75 & 5.09E-05 & 5.42E-05 & $6.09 \mathrm{E}-05$ & $6.41 \mathrm{E}-05$ \\
\hline DBP & 2.01E-02 & $2.52 E+03$ & $397 \mathrm{E}+03$ & $1.65 E-02$ & $1.83 E-02$ & 2.19E-02 & $2.36 \mathrm{E}-02$ \\
\hline butanol & 2.01E-02 & 838 & 1.40E+03 & $1.65 \mathrm{E}-02$ & 1.83E-02 & 2.19E-02 & $2.36 \mathrm{E}-02$ \\
\hline $\mathrm{NH3}$ & 0.102 & $1.03 E+03$ & $1.63 \mathrm{E}+03$ & 8.58E-02 & $9.22 \mathrm{E}-02$ & 0.115 & 0.129 \\
\hline $\mathrm{Fe}(\mathrm{CN}) 64$ & 0 & -6 & 0 & 0 & 0 & 0 & 0 \\
\hline
\end{tabular}

- inknowns in tenk solids inventory we masigned by Temk Leyering Model (TLM)

+Water w\% derived from the difference of density and totel dimolved species. 
HDW Model Rev. 4

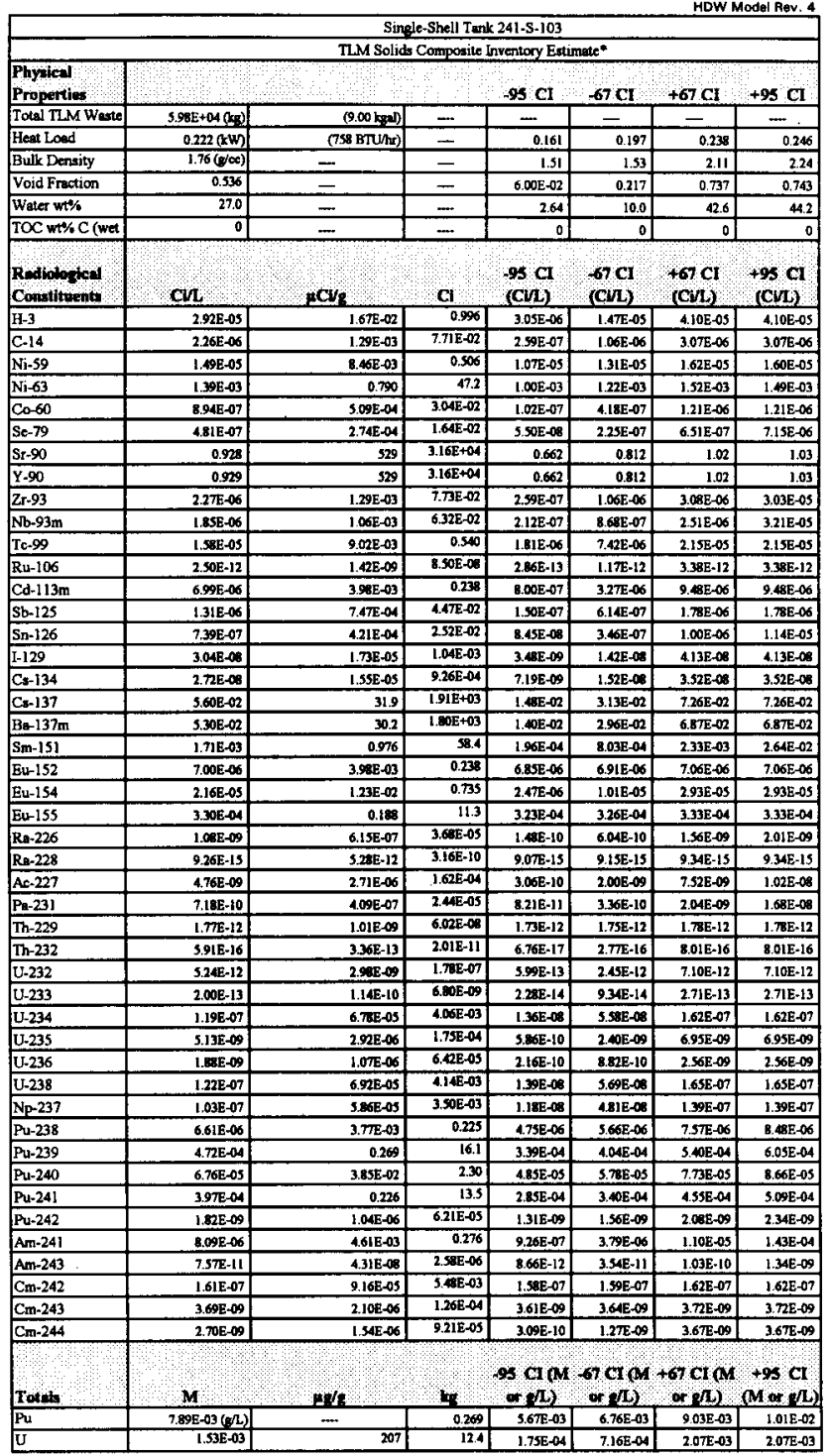

Urkenows in tenk solids inventory are saigned by Tenk Lyywing Model (TLM). 
HNF-SD-WM-ER-352, Rev. 1

HDW Model Rev, 4

\begin{tabular}{|c|c|c|c|c|c|c|c|}
\hline \multirow[b]{3}{*}{$\begin{array}{l}\text { Phypical } \\
\text { Properties }\end{array}$} & \multicolumn{7}{|c|}{ Single-Shell Tank 241-S-103 } \\
\hline & \multicolumn{7}{|c|}{ SMM Composite Inventory Estimete } \\
\hline & & 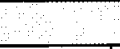 & & $-95 \mathrm{CI}$ & $67 \mathrm{CI}$ & $+67 \mathrm{CI}$ & $+95 \mathrm{CI}$ \\
\hline Total SMM Wart & $152 \mathrm{E}+06(\mathrm{~kg})$ & (239 kpal $)$ & - & $\ldots$ & $\longrightarrow$ & - & - \\
\hline \begin{tabular}{|l|} 
Heat Lasd \\
\end{tabular} & $2.12(\mathrm{cw})$ & $(7.25 \mathrm{E}+03 \mathrm{BTU} / \mathrm{hu}$ & - & 1.99 & 2.06 & 2.19 & 2.26 \\
\hline Bulk Density* & $1.67(\mathrm{~g} / \propto)$ & - & $\ldots$ & 1.62 & 1.65 & 1.70 & 1.72 \\
\hline Waler wt\% $\dagger$ & 28.3 & 一 & - & 25.7 & 26.8 & 29.8 & 31.7 \\
\hline TOC w\% C (wet & 0.79 & $\ldots$ & $=$ & 0.523 & 0.659 & 0.938 & 1.07 \\
\hline $\begin{array}{l}\text { Roundoted } \\
\text { Contituents }\end{array}$ & CVL & $\mathrm{PC} / \mathrm{s}$ & Cl & $\begin{array}{l}95 \mathrm{CI} \\
\text { (GV) }\end{array}$ & $\begin{array}{l}-67 \mathrm{CI} \\
\text { (CUL) }\end{array}$ & $\begin{array}{r}+67 \mathrm{CI} \\
(\mathrm{CV}) \\
\end{array}$ & $\begin{array}{l}+95 \mathrm{CI} \\
\text { (CVI) }\end{array}$ \\
\hline $\mathrm{H}-3$ & $2.80 \mathrm{E}-04$ & 0.167 & 253 & $1.46 \mathrm{E}-04$ & $1.46 \mathrm{E}-04$ & $2.89 \mathrm{E} \cdot 04$ & $3.00 \mathrm{E}-04$ \\
\hline$C-14$ & 4.03E-05 & 2.41E-02 & 36.4 & $1.23 \mathrm{E}-05$ & 1.23E-05 & $4.09 \mathrm{E}-0 \mathrm{~S}$ & 4.15E-05 \\
\hline $\mathrm{Ni}-59$ & $2.61 E-\infty$ & $1.56 \mathrm{E}-03$ & 2.36 & 1.29E-06 & $1.29 E-06$ & $2.67 \mathrm{E}-06$ & 2.74E-06 \\
\hline $\mathrm{Ni}-63$ & $2.558-04$ & 0.153 & 231 & $1.25 \mathrm{E}-0 \mathrm{~A}$ & $1.25 E-04$ & $2.62 \mathrm{E}-04$ & 2.08E-04 \\
\hline $\mathrm{Co}-60$ & 4.46E-05 & $2.66 \mathrm{E}-02$ & 0.3 & $1.26 \mathrm{E}-05$ & $1.26 \mathrm{E}-05$ & 4.S4E-05 & 4.62E-05 \\
\hline Se-79 & $4,00 \mathrm{E}-06$ & $2.39 \mathrm{E}-03$ & 3.62 & $2.21 \mathrm{E}-06$ & 2.21E-06 & $4.65 \mathrm{E}-06$ & $5.27 \mathrm{E}-06$ \\
\hline Sr-90 & 0.133 & 79.6 & $1.21 \mathrm{E}+0 \mathrm{~S}$ & 0.124 & 0.129 & 0.138 & 0.142 \\
\hline$Y-90$ & 0.133 & 79.6 & $1.21 E+05$ & $7.16 \mathrm{E}-02$ & $7.16 \mathrm{E}-02$ & 0.138 & 0.142 \\
\hline $2 \mathrm{~s}-93$ & 1.96E-OS & 1.1TE-02 & 17.8 & $1.07 \mathrm{E}-05$ & $1.07 \mathrm{E}-05$ & $2.29 \mathrm{E}-05$ & $2.60 \mathrm{E}-05$ \\
\hline $\mathrm{Nb}-93 \mathrm{~m}$ & 1.43E-05 & 8.S1E-03 & 12.9 & B.01E-06 & B.01E-60 & $1.65 \mathrm{E}-05$ & $1.87 \mathrm{E}-0 \mathrm{~s}$ \\
\hline Tc-99 & 2.87E-04 & 0.171 & 260 & $1.83 E-04$ & $2.34 \mathrm{E}-04$ & $341 \mathrm{E}-04$ & $3.92 E-04$ \\
\hline Ru-106 & $7.93 \mathrm{E}-09$ & $4.73 E-06$ & $7.17 \mathrm{E}-03$ & $3.79 E-09$ & $3.79 \mathrm{E}-09$ & 8.85E-09 & $9.69 \mathrm{E}-09$ \\
\hline $\mathrm{Cd}-113 \mathrm{~m}$ & $1.03 \mathrm{E}-04$ & 6.15E-02 & 93.2 & 494E-05 & 4.94E-05 & $1.22 \mathrm{E}-04$ & 1.41E-04 \\
\hline $5 b-125$ & $1.92 E-04$ & 0.114 & 17 & $5.30 \mathrm{E}-05$ & 5.30E-05 & $1.95 E-04$ & $1.99 \mathrm{E}-04$ \\
\hline $5 n-126$ & $6.05 \mathrm{E}-06$ & $3.61 \mathrm{E}-03$ & 5.47 & $3.37 \mathrm{E}-06$ & $3.37 \mathrm{E}-06$ & $7.02 E-06$ & $7.94 \mathrm{E}-06$ \\
\hline $1-129$ & S.53E-07 & $3.30 \mathrm{E}-04$ & 0.500 & $3.51 E-07$ & $450 \mathrm{E}-07$ & $6.57 \mathrm{E}-07$ & $7.57 \mathrm{E}-07$ \\
\hline Ca-134 & $3.03 E-06$ & 1.81E-03 & 2.74 & $2.16 \mathrm{E}-06$ & 2.55E-06 & $3.47 \mathrm{E}-06$ & $3.90 E-06$ \\
\hline Cs- 137 & 0.310 & 185 & $2.80 \mathrm{E}+05$ & 0.279 & 0.294 & 0.325 & 0.341 \\
\hline $\mathrm{Bo}-137 \mathrm{~m}$ & 0.293 & 175 & $2.65 E+05$ & 0.230 & 0.230 & 0.308 & 0.322 \\
\hline Sm-151 & $1.41 \mathrm{E}-02$ & 8.41 & $1.27 \mathrm{E}+04$ & TEAE-03 & $7.84 E-03$ & 1.63E-02 & $1.85 \mathrm{E}-02$ \\
\hline Eu-152 & $4.61 \mathrm{E}-0 \mathrm{~S}$ & $2.75 \mathrm{E}-03$ & 4.17 & $2.40 \mathrm{E}-00$ & 2.40E-06 & 4.93E-06 & $5.24 E-06$ \\
\hline Eu-154 & $723 E-04$ & 0.432 & 654 & $2.77 \mathrm{E} 04$ & 2.TTE-A4 & $8.85 \mathrm{E}-04$ & $9.49 \mathrm{E}-04$ \\
\hline Eu-155 & $2.73 \mathrm{E}_{04}$ & 0.163 & 247 & 1.41E-04 & $1.41 \mathrm{E}-04$ & $2.92 \mathrm{E}-04$ & $3.11 \mathrm{E}-04$ \\
\hline Rs-226 & $1.71 \mathrm{E}-10$ & $1.02 \mathrm{E}-07$ & $1.55 \mathrm{E}-04$ & $1.16 \mathrm{E}-10$ & $1.16 \mathrm{E}-10$ & $1.91 \mathrm{E}-10$ & $2.10 \mathrm{E}-10$ \\
\hline Ra-228 & $1.67 \mathrm{E}-07$ & 9.95E-05 & 0.151 & $6.98 E-68$ & $1.17 \mathrm{E}-07$ & $2.24 \mathrm{E}-07$ & 2.85E-07 \\
\hline$A c-227$ & $1.08 \mathrm{E}-09$ & 6. $46 \mathrm{E}-07$ & $9.79 \mathrm{E}-04$ & $7.6 \mathrm{E}-10$ & $7,615-10$ & $1.20 \mathrm{E}-09$ & 1.31E-09 \\
\hline $\mathrm{Pz}-231$ & 4.94E-09 & 2.96- 6 & $4.49 \mathrm{E}-03$ & 3.18E-09 & $3.18 E-49$ & $5.61 E-09$ & $6.23 E-09$ \\
\hline Th-229 & $3.91 \mathrm{E}-09$ & $2.33 \mathrm{E}-06$ & $3.54 E-03$ & 1.77E-09 & $2.82 \mathrm{E}-09$ & $5.17 E-09$ & $6.52 E-09$ \\
\hline Th-232 & 1.11E-08 & $6.63 E-06$ & $1,00 \mathrm{E}-02$ & 5.74E- -9 & 8.37E-69 & $1.38 \mathrm{E}-0 \mathrm{~s}$ & 1.65E-08 \\
\hline $\mathrm{U}-232$ & 8.58E-07 & $5.12 \mathrm{E}-04$ & 0.776 & $4.69 \mathrm{E}-07$ & $6.99 \mathrm{E}-07$ & $1.09 \mathrm{E}-06$ & $1.33 \mathrm{E}-\infty 6$ \\
\hline U-233 & $3.29 \mathrm{E}-06$ & $1.96 \mathrm{E}-03$ & 297 & $1.00 \mathrm{E}-06$ & $2.53 \mathrm{E}-06$ & 4.1TE-06 & $5.11 \mathrm{E}-06$ \\
\hline $\mathrm{U}-234$ & $9.64 \mathrm{E}-07$ & $3.76 \mathrm{E}-04$ & 0.87 & 9.28E-07 & 9.46E-07 & 9.845-07 & $9.94 \mathrm{E}-07$ \\
\hline U.235 & $3.91 \mathrm{E}-08$ & 2.34E-05 & 3.SE-D2 & $3.76 \mathrm{E}-0 \mathrm{~s}$ & $3.83 \mathrm{E}-03$ & $4.00 \mathrm{E}-08$ & 4.04E- 08 \\
\hline $\mathrm{U}-236$ & $3.02 \mathrm{E}-0 \mathrm{~B}$ & $1.80 \mathrm{E}-05$ & $2.75-02$ & $2.91 \mathrm{E}-08$ & $2.97 \mathrm{E}-08$ & $3.06 \mathrm{E}-08$ & $3.11 \mathrm{E}-08$ \\
\hline U.238 & $1.12 \mathrm{E}-06$ & $6.67 \mathrm{E}-04$ & 1.01 & $1.08 \mathrm{E}-06$ & $1.10 \mathrm{E}-06$ & $1.14 \mathrm{E}-06$ & 1.15E-06 \\
\hline $\mathrm{Np}-237$ & $1.05 \mathrm{E}-06$ & $6.26 \mathrm{E}-04$ & 0.948 & $7.07 \mathrm{E}-07$ & 8.74E-07 & $1.22 \mathrm{E}-06$ & $1.39 \mathrm{E}-06$ \\
\hline Pu-238 & $1.63 \mathrm{E}-06$ & $9.75 E-04$ & I.48 & $1.23 \mathrm{E}-06$ & $1.43 \mathrm{E}-06$ & 1.84E- 06 & 2.03E-106 \\
\hline Pu-239 & $3.615-05$ & $3.35 \mathrm{E}-02$ & 50.8 & $4.58 \mathrm{E}-0 \mathrm{~S}$ & $5.09 \mathrm{E}-05$ & 6.13E-05 & $6.63 E-05$ \\
\hline $\mathrm{Pu}-240$ & $9,51 E-06$ & $5.6 \mathrm{E}-03$ & 8.60 & $7.60 \mathrm{E}-06$ & $8.54 E-06$ & $1.05 E-05$ & $1.14 E-05$ \\
\hline$P(4-241$ & $1.10 \mathrm{E}-04$ & $6.59 \mathrm{E}-02$ & 99.8 & $83 \mathrm{IE}-05$ & $9.64 E-05$ & 1.24E-04 & $1.38 \mathrm{E}-04$ \\
\hline Pu-242 & $6.06 \mathrm{E}-10$ & $3.62 \mathrm{E}-07$ & $5.48 E-0.4$ & 4.43E-10 & $5.23 \mathrm{E}-10$ & $6.89 \mathrm{E} \cdot 10$ & $7.69 \mathrm{E}-10$ \\
\hline Am-241 & $6.81 \mathrm{E}-05$ & $406 \mathrm{E}-02$ & 61.6 & 4.91E-0S & $5.84 \mathrm{E}-0 \mathrm{~S}$ & 7.78E-05 & $8.71 \mathrm{E}-05$ \\
\hline$A m=243$ & $2,36 \mathrm{E}-09$ & 1.4IE-06 & 2.13E-03 & $1.76 \mathrm{E}-09$ & $2.05 \mathrm{E}-09$ & $2.68 \mathrm{E}-09$ & 2.98E- 09 \\
\hline $\mathrm{Cm}-242$ & $1.752-07$ & 1.04E-04 & 0.15 & $8.40 \mathrm{E}-08$ & $8.40 \mathrm{E}-0 \mathrm{~B}$ & $1.87 \mathrm{E}-07$ & $1.99 \mathrm{E}-07$ \\
\hline $\mathrm{Cm}-243$ & $1.62 \mathrm{E}-08$ & $9.60 \mathrm{E}-06$ & $1.46 \mathrm{E}-02$ & 7.52E-09 & $7.32 \mathrm{E}-09$ & $1.73 \mathrm{E}-08$ & $183 E-08$ \\
\hline $\mathrm{Cm}-244$ & $1.60 \mathrm{E}-07$ & $9.55 \mathrm{E}-0 \mathrm{~S}$ & 0.145 & $7.07 \mathrm{E}-68$ & 7.0TE-08 & $1.90 \mathrm{E}-07$ & 2.10E-07 \\
\hline Totill: & $\mathbf{M}$ & $y=8$ & lot & $\begin{array}{l}6 \mathrm{cL}(\mathrm{M} \\
\text { ot }\end{array}$ & $\begin{array}{l}67 \mathrm{Cl}(\mathrm{M} \\
\text { or }+\mathrm{L})\end{array}$ & $\begin{array}{l}+6 \mathrm{Cta} \\
\text { or git) }\end{array}$ & $\begin{array}{r}+95 \mathrm{CI} \\
(\mathrm{M} \mathrm{or} \mathrm{pL})\end{array}$ \\
\hline $\mathrm{Pu}$ & $7.03 \mathrm{E}-04(\mathrm{r} / \mathrm{L})$ & - & 0.636 & $5.09 \mathrm{E}-04$ & $6.04 E-04$ & $8.02 \mathrm{E}-04$ & 8.96-04 \\
\hline $\mathrm{U}$ & $1.10 \mathrm{E}-02$ & $1.57 \mathrm{E}+03$ & $2.38 \mathrm{E}+03$ & $1.06 \mathrm{E}-02$ & $1.08 \mathrm{E}-02$ & 1.13E-02 & $1.14 \mathrm{E}-02$ \\
\hline
\end{tabular}

-Dersity is calculated based on $\mathrm{Na}, \mathrm{OH}$, and $\mathrm{AOO} 2$

tWater wt\% derived from the difference of dersity and total dizsolved apecies. 
HNF-SD-WM-ER-352, Rev. 1

HoW Model Rev. 4

\begin{tabular}{|c|c|c|c|c|c|c|c|}
\hline \multicolumn{8}{|c|}{ Single-Shell Tank 24l-S-103 } \\
\hline & \multicolumn{7}{|c|}{ Total Inventory Estimate* } \\
\hline $\begin{array}{l}\text { Phyincal } \\
\text { Propertios }\end{array}$ & & & & $-95 \mathrm{CI}$ & $67 \mathrm{CI}$ & $+67 \mathrm{CL}$ & $+95 \mathrm{CI}$ \\
\hline Total Waste & $1.58 E+\infty 6(k s)$ & (248 kgat) & - & $\ldots$ & -- & $\ldots$ & + \\
\hline Heat Load & $2.35(\mathrm{~kW})$ & (8.01E+03 BTU/ur) & $\ldots$ & 2.21 & 2.28 & 2.41 & 2.48 \\
\hline Bulk Densityt & $1.68(\mathrm{p} / \mathrm{cc})$ & - & $\ldots$ & 1.63 & 1.65 & 1.70 & 1.72 \\
\hline Water wt\% $\uparrow$ & 28.3 & $\ldots$ & - & 75 & & & 315 \\
\hline TOC wt\% C (wet & 0.768 & $m$ & - & 0503 & 0.633 & 0.903 & 1.03 \\
\hline $\begin{array}{l}\text { Roupopicel } \\
\text { Conatitienda }\end{array}$ & $\mathbf{C V}, \mathrm{L}$ & $\mu C w_{\Omega}$ & $\mathbf{C i}$ & $\begin{array}{l}-95 \text { C1 } \\
\text { (CV) }\end{array}$ & $\begin{array}{l}-67 \mathrm{C} \\
(\mathrm{CVI})\end{array}$ & $\begin{array}{l}+67 \mathrm{Cl} \\
(\mathrm{CV} \mathrm{L})\end{array}$ & $\begin{array}{r}+93 \mathrm{CI} \\
(\mathrm{Cu} L)\end{array}$ \\
\hline $\mathrm{H}-3$ & 2.7IE-04 & 0.161 & 254 & 1.42E-04 & $1.42 \mathrm{E}-04$ & 2.79E-04 & $2.90 \mathrm{E}-04$ \\
\hline $\mathrm{C}-14$ & $3.89 \mathrm{E}-05$ & $2.32 E-02$ & 36.5 & $1.19 \mathrm{E}-05$ & $1.19 E-05$ & $3.95 \mathrm{E}-05$ & 4.01E-05 \\
\hline $\mathrm{Ni}-59$ & $3.05 \mathrm{E}-06$ & $1.82 \mathrm{E}-03$ & 2.86 & 1.78E-06 & $1.78 E-06$ & $3.12 \mathrm{E}-06$ & 3.1BE- -06 \\
\hline $\mathrm{N}-63$ & $2.97 \mathrm{E}-04$ & 0.17 & 278 & 1.71E-04 & 1.71E-04 & $3.03 \mathrm{E}-04$ & 3.09E-04 \\
\hline $\mathrm{C}-60$ & 4.30E-05 & $2.56 \mathrm{E}-02$ & 40.4 & $1.21 \mathrm{E}-05$ & 1.21E-05 & 4.38E-05 & 4.46E-05 \\
\hline Se-79 & $3.87 \mathrm{E}-06$ & $2.31 E-03$ & 3.64 & 2.15E-00 & 2.15E-06 & 4.50E-06 & S.09E-06 \\
\hline Sr-90 & 0.162 & 96.6 & $1.52 E+05$ & 0.152 & 0.158 & 0.166 & 0.171 \\
\hline Y.90 & 0.162 & 96.6 & $1.52 \mathrm{E}+05$ & 0.103 & 0.103 & 0.166 & 0.171 \\
\hline $2 x-93$ & 1.90E-05 & $1.13 E-02$ & 17.8 & 1.04E-05 & $1.042-05$ & 2.21E-05 & 2.51E-05 \\
\hline $\mathrm{Nb}-93 \mathrm{~m}$ & $1.38 E-05$ & B.23E-03 & 13.0 & $7.78 E-05$ & $7.78 \mathrm{E}-06$ & 1.60E-05 & 1.B1E-0S \\
\hline$T_{c-99}$ & 2.77E-04 & 0.165 & 260 & 1.77E-04 & $2.26 \mathrm{E}-04$ & 3.29E-04 & 3.79E-04 \\
\hline Ru-106 & $7.64 E-09$ & $4.55 E-06$ & $7.17 \mathrm{E}-03$ & $3,65 E-09$ & $3.65 \mathrm{E}-09$ & B. 53E- 09 & 9.3AE-09 \\
\hline $\mathrm{Cd}-113 \mathrm{~m}$ & 9.95E-05 & $3.93 \mathrm{E}-02$ & 93.4 & $4.79 \mathrm{E}-05$ & 4.79E-05 & $1.18 E-04$ & $1.36 \mathrm{E}-04$ \\
\hline $\mathrm{Sb}-125$ & $1.85 E-0.4$ & 0.110 & 173 & $5.11 \mathrm{E}-05$ & 5.11E-05 & $1.88 \mathrm{E}-04$ & $1.92 \mathrm{E}-04$ \\
\hline Sn-126 & $5.85 \mathrm{E}-166$ & $3.49 \mathrm{E}-03$ & 5.49 & $32 \pi-06$ & $3.27 \mathrm{E}-06$ & $6.79 E-06$ & $7.68 \mathrm{E}-06$ \\
\hline $1-129$ & 5.34E-07) & $3.18 E-04$ & 0.501 & $3,40 E-07$ & 435E-07 & 6.34E- -07 & 7.31E-67 \\
\hline Cs-134 & $2.92 \mathrm{E}-06$ & $1.74 \mathrm{E}-03$ & 2.74 & $2.09 \mathrm{E}-06$ & 246E-06 & 3.35E-06 & $3.76 \mathrm{E}-06$ \\
\hline Cs-137 & 0.300 & 179 & $2.52 E+05$ & 0.271 & 0.285 & 0.316 & 0.331 \\
\hline $\mathrm{Ba} \cdot 137 \mathrm{~m}$ & 0.284 & 169 & $2.67 \mathrm{E}+05$ & 0.223 & 0.223 & $\begin{array}{r}0.299 \\
\end{array}$ & 0.313 \\
\hline $5 m-151$ & $1.36 \mathrm{E}-02$ & 8.13 & $1.23 E+04$ & $7,63 \mathrm{E}-03$ & 7.6 $\mathrm{JE}-03$ & $1.59 \mathrm{E}-02$ & $1.79 \mathrm{E}-02$ \\
\hline Eu-152 & 4.70E- 06 & $2.00 \mathrm{E}-03$ & 4.41 & $2.57 \mathrm{E}-06$ & 257E-06 & 5.00E-06 & $5.30 \mathrm{E}-06$ \\
\hline Eu-154 & $6.9 \mathrm{gE}-04$ & 0.416 & 655 & $2.68 \mathrm{E}-04$ & 2.68E-04 & B. $53 \mathrm{E}-0 \mathrm{~A}$ & $9.16 \mathrm{E}-04$ \\
\hline Eu-155 & 2.75E-04 & 0.164 & 258 & 1.48E-04 & 1. 40 E-04 & 2.93E-04 & $3.12 E-04$ \\
\hline Re-226 & $2.04 \mathrm{E}-10$ & $1.22 \mathrm{E}-07$ & 1.92E-04 & 1.51E-10 & 1.51E-10 & $2.23 E-10$ & $2.42 \mathrm{E} \cdot 10$ \\
\hline $\mathrm{R}=-228$ & 1.61E-07 & 9.58E-05 & 0.151 & $6.72 \mathrm{E}-08$ & 1.13E-07 & 2.16E-07 & 2.75E-07 \\
\hline$A C-227$ & 1.22E-09 & $7.25 \mathrm{E}-07$ & 1.14E-03 & $9.06 \mathrm{E}-10$ & $9.06 \mathrm{E}-10$ & $133 \mathrm{E}-09$ & $1.44 E-09$ \\
\hline Pa-231 & 4.81E- -9 & 2.87E-06 & 4.52E-03 & $3.09 \mathrm{E}-09$ & $3.09 E-09$ & 5.43E-09 & $6.03 \mathrm{E}-09$ \\
\hline Th-229 & 3.7TE-09 & 2.25E- -06 & 3.S4E-03 & 1.72E- -09 & $27 \mathrm{E}-09$ & 4.98E- 09 & $6.28 \mathrm{E}-09$ \\
\hline Th-232 & $1.0 \pi-08$ & $6.38 E-06$ & $1.00 \mathrm{E}-02$ & $5,53 E-09$ & $8.07 \mathrm{TE}-09$ & $1.33 E-08$ & 1.59E-08 \\
\hline $\mathrm{U}-232$ & 8.2TE-07 & 4.93E-04 & 0.776 & 4.52E-07 & $6.35 \mathrm{E}-07$ & I.05E-AS & $1.29 \mathrm{E}-06$ \\
\hline $\mathrm{U}-233$ & $3.1 \pi 006$ & L.OE-03 & 2.97 & 1.74E-06 & 244E-06 & $402 \mathrm{E}-00$ & 4.92E-06 \\
\hline $\mathrm{U}-234$ & $9302-07$ & $5.56 \mathrm{E}-0 \mathrm{~A}$ & 0.876 & 899E-07 & $9.16 \mathrm{E}-07$ & $9.51 E-07$ & $9.62 \mathrm{E}-07$ \\
\hline $\mathrm{U}-235$ & 3.79E-00 & 2.26E-05 & 3.50E-O2 & 3.64E-08 & $3.7 \mathrm{E}-08$ & $3.86 \mathrm{E}-08$ & 391E-08 \\
\hline $\mathrm{U}-236$ & 2.91E-0 & 1.74E-05 & $2.74 E-02$ & 2.81E- -08 & 287E-08 & $2.96 \mathrm{E}-08$ & $3.00 \mathrm{E}-08$ \\
\hline $\mathrm{U}-238$ & $1.008-06$ & 6.4E-04 & 101 & 1.05E-06 & 1.06E-06 & 1.10E-0.5 & 1.1]E-06 \\
\hline $\mathrm{Np}-237$ & $1.01 E-06$ & 6.04E-04 & 0.952 & 6.B6E-07 & 8. $46 E-07$ & $1.18 \mathrm{E}-06$ & 1.35E-06 \\
\hline Pu-238 & 1.81E-06 & $1.08 E-03$ & $1 . \pi$ & $1.43 E-06$ & $1.62 \mathrm{E}-06$ & 2.01E-06 & $2.20 \mathrm{E}-06$ \\
\hline Pu-239 & 7.12E-05 & 4.24E-02 & 6.8 & 6.13E-05 & 6.62E-05 & 7.62E-05 & 8.10E-0S \\
\hline Pu-240 & 1.16E-05 & $6.92 E-03$ & 10.9 & 9.78E-06 & 1.07E-05 & 1.26E-05 & 1.35E-05 \\
\hline Pu-24l & $1.21 \mathrm{E}-04$ & $7.20 \mathrm{E}-02$ & 113 & 9.45E-0S & 1.07E-04 & $1.34 \mathrm{E}-\mathrm{OA}$ & $1.47 \mathrm{E}-04$ \\
\hline Pu-242 & 6.5OE-10 & $3.80 \mathrm{E}-07$ & 6.10E-04 & $4.93 E-10$ & $5,70 \mathrm{E}-10$ & $7.30 \mathrm{E}-10$ & 8.08E-10 \\
\hline Ann-241 & 6.59E-05 & 3.93E-02 & 61.8 & 4.76E-05 & $5.66 \mathrm{E}-05$ & $7.52 \mathrm{E}-05$ & 8.42E-05 \\
\hline $\mathrm{Am}-243$ & $2.28 \mathrm{E}-09$ & $1.36 \mathrm{E}-06$ & 2. LAE-03 & $1.70 \mathrm{E}-09$ & $1.98 E-09$ & 2.58E-09 & 2.88E-09 \\
\hline $\mathrm{Cm}-242$ & 1.74E-07 & $1.04 \mathrm{E}-04$ & 0.164 & 8.6BE-08 & 9.6BE-08 & 1.86E-07 & 1.97E-07 \\
\hline $\mathrm{Cm}-243$ & 1.5TE-08 & $9.37 \mathrm{E}-06$ & 1. $4 \mathrm{E}-02$ & $7.39 \mathrm{E}-09$ & $7,38 \mathrm{E}-09$ & 1.68E-08 & $1.78 \mathrm{E}-0 \mathrm{~s}$ \\
\hline $\mathrm{Cm}+244$ & 1.54E-07 & $9.20 \mathrm{E}-05$ & 0.145 & $6.83 E-08$ & 6.83E-08 & $1.83 E-07$ & $2.02 \mathrm{E}-07$ \\
\hline Totds & $\mathbf{M} \quad \mathbf{}$ & $t h$ & ta & $\begin{array}{l}9 \mathrm{CIOA} \\
0+1 \mathrm{~L}\end{array}$ & $\begin{array}{c}67 \mathrm{Ct}(\mathrm{M} \\
0 \mathrm{OH} / \mathrm{L}\end{array}$ & $\begin{array}{l}+67 \mathrm{CI}(\mathrm{u} \\
\mathrm{\alpha} \mathrm{L})\end{array}$ & $\begin{array}{l}+95 \mathrm{Cl} \\
\mathrm{M} \mathrm{ar} / \mathrm{L})\end{array}$ \\
\hline $\mathrm{Pu}$ & $9.64 \mathrm{E}-04(\Omega / \mathrm{L})$ & - & 0.005 & $7 . \pi \mathrm{E}-04$ & $8.69 \mathrm{E}-04$ & $1.06 \mathrm{E}-03$ & $1.15 \mathrm{E}-03$ \\
\hline $\mathrm{U}$ & $1.072-02$ & $1.52 E+03$ & $2.39 \mathrm{E}+03$ & $1.03 E-02$ & $1.05 \mathrm{E}-02$ & $1.09 \mathrm{E}-02$ & $1,10 \mathrm{E}-02$ \\
\hline
\end{tabular}

- Unkonowns in tank solids inventory are assigned by Tenk Lyyeing Model (TLM).

† Volume average for density, mass averse Water wt\% and TOC wt\% C. 


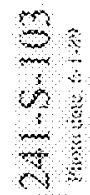


TANK 241-S-104 SUMMARY

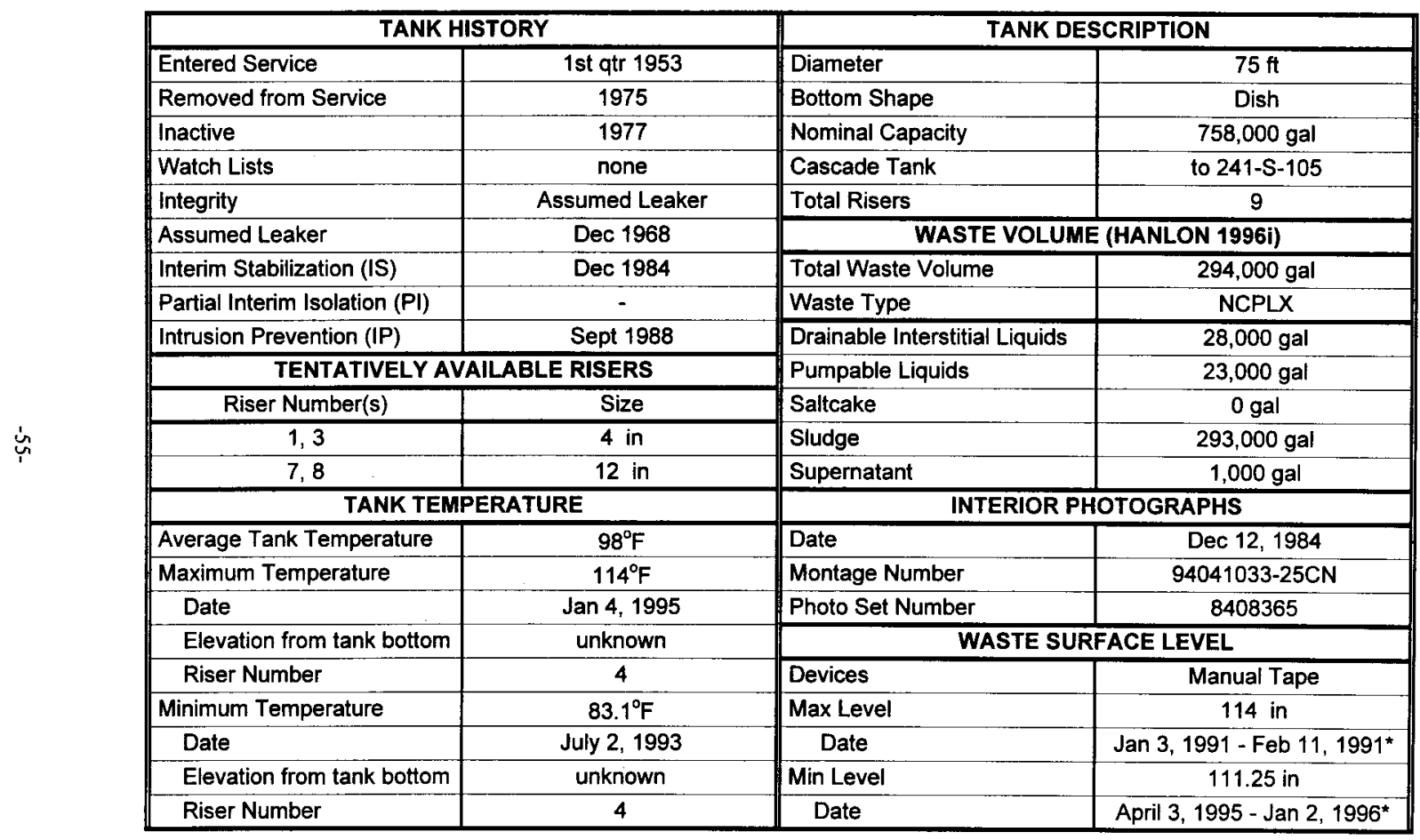

- Numerous dates in this time span. 


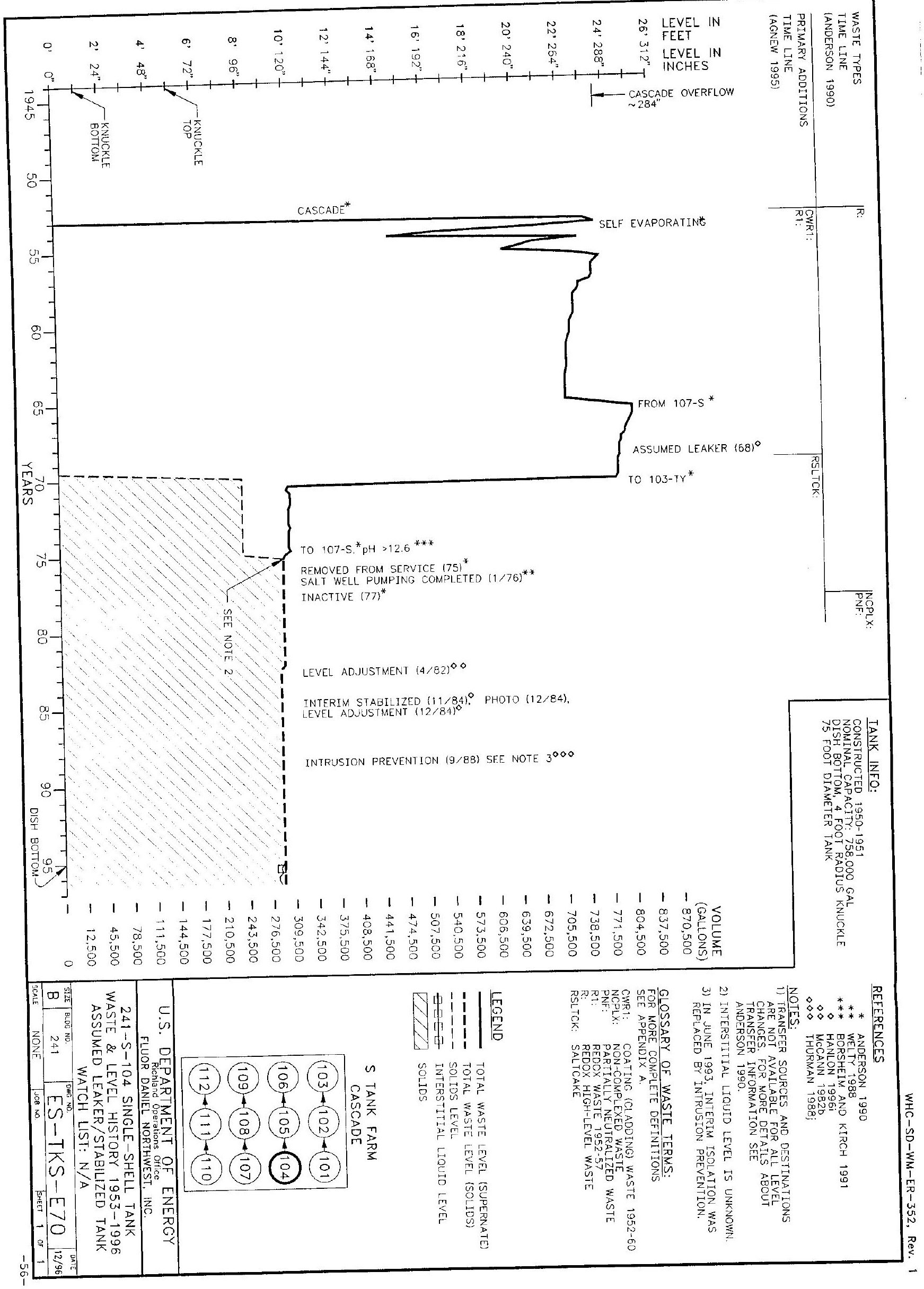




$$
241-5-104
$$

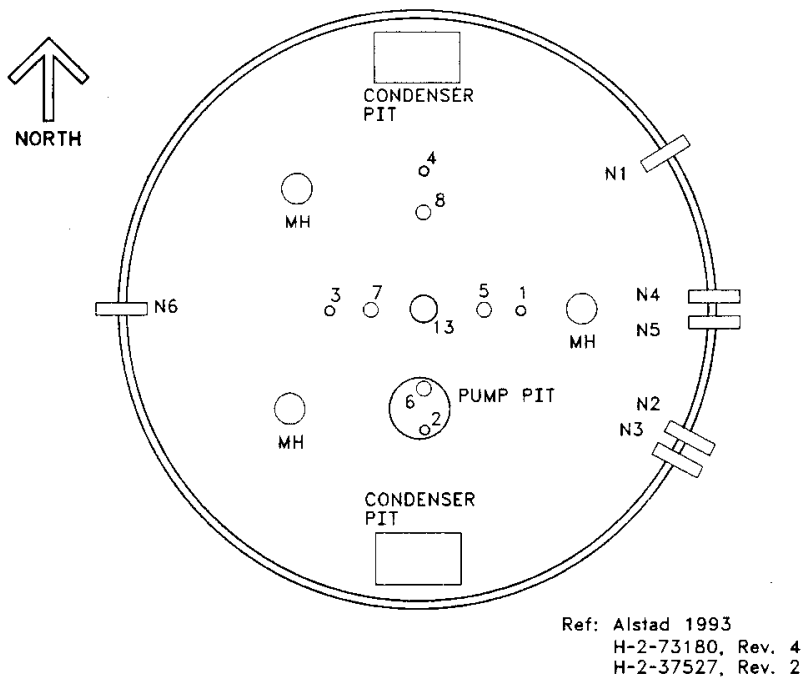

\section{TANK RISER LOCATION}

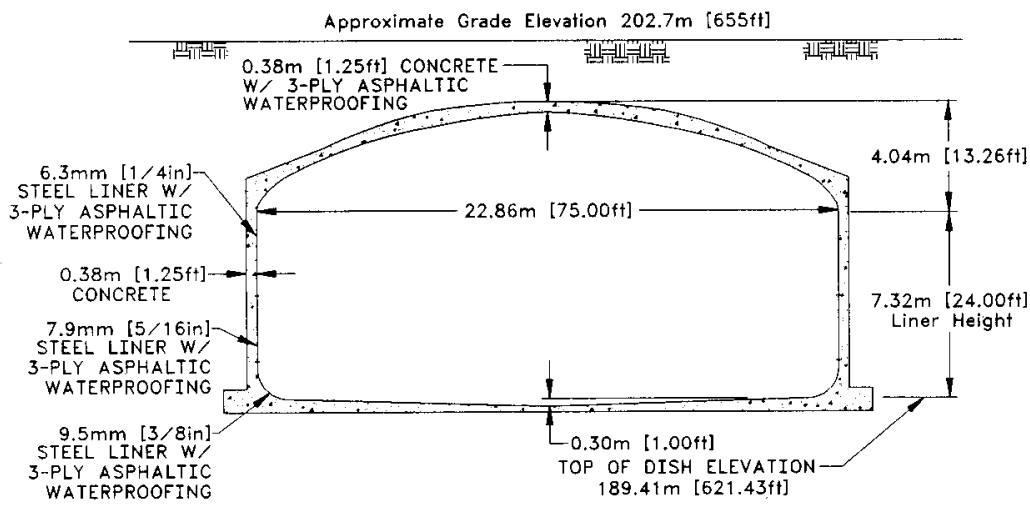

$$
\begin{gathered}
\text { NOT TO SCALE } \\
-57-
\end{gathered}
$$

Ref: H-2-1783, Rev. 3 H-2-46293, Rev, 3 H-2-1784, Rev. 2 
HNF-SD-WM-ER-352, Rev. 1

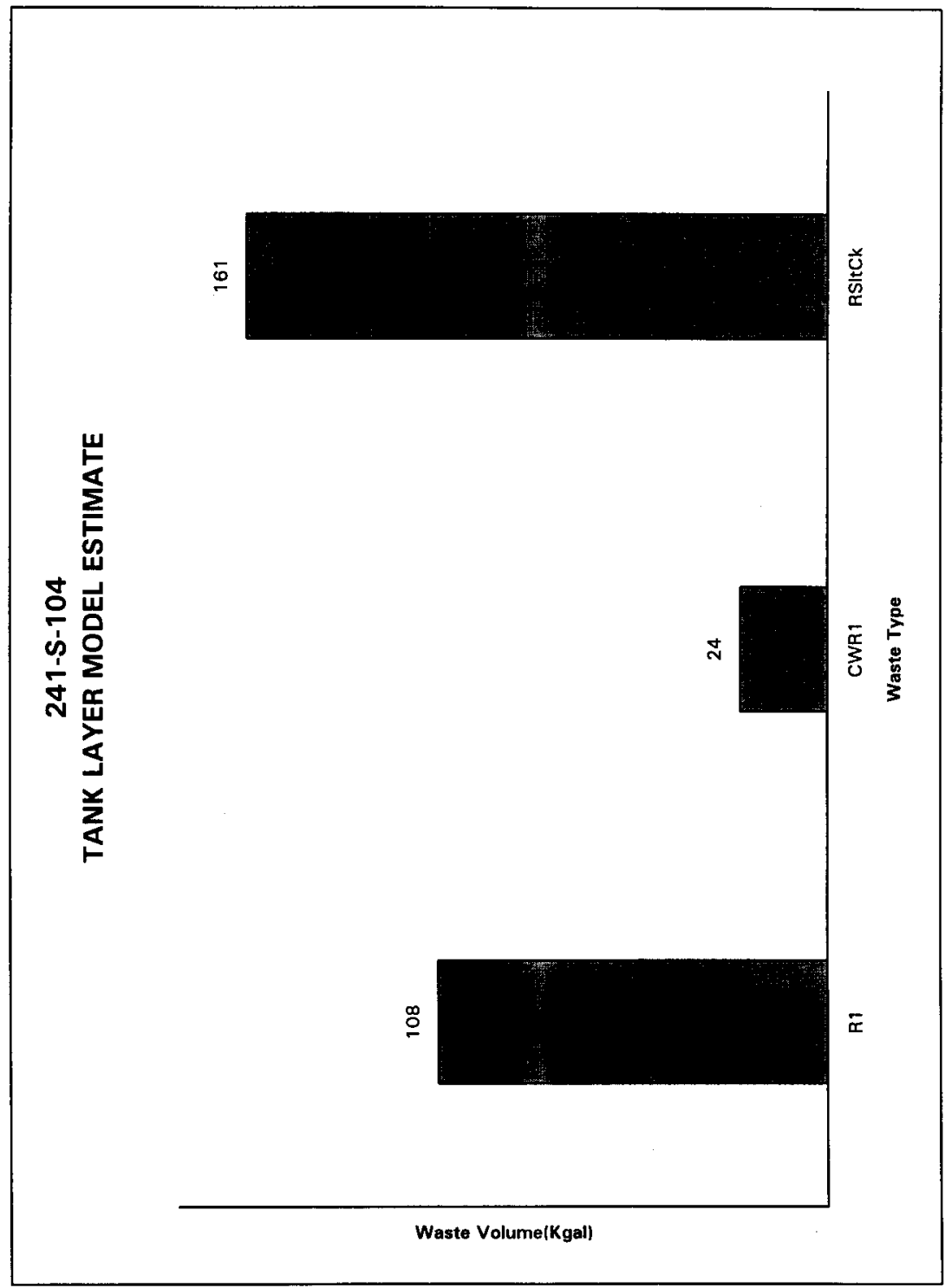


HDW Model Rev. 4

\begin{tabular}{|c|c|c|c|c|c|c|c|}
\hline & \multicolumn{7}{|c|}{ Single-Shell Tank 241-S-104 } \\
\hline & \multicolumn{7}{|c|}{ TLM Solids Composite Inventory Estimate" } \\
\hline $\begin{array}{l}\text { Physical } \\
\text { Proponties }\end{array}$ & & & & $-95 \mathrm{CI}$ & $-67 \mathrm{CI}$ & $+67 \mathrm{CI}$ & $+95 \mathrm{Cl}$ \\
\hline Total TLM Waste & $1.92 \mathrm{E}+06(\mathrm{kP})$ & $(293 \mathrm{kgal})$ & $\ldots$ & $\ldots$ & - & - & - \\
\hline Heat Load & $4.67(\mathrm{~kW})$ & $(1.59 \mathrm{E}+04 \mathrm{BTU} / \mathrm{hr})$ & - & 3.94 & 437 & 4.87 & 4.96 \\
\hline Bulk Density & $1.73(0 / \infty)$ & + & $\overline{-}$ & 1.58 & 1.69 & 1.86 & 1.91 \\
\hline Void Fraction & 0.682 & 一 & - & 0.507 & 0.565 & 0.756 & 0.788 \\
\hline Water wt\% & 310 & - & - & 20.1 & 23.6 & 36.5 & 42.0 \\
\hline TOC wt\% C (wet & $2.85 E-03$ & $\rightarrow$ & $\ldots$ & $2.58 \mathrm{E}-03$ & $2.65 E-03$ & $3.06 \mathrm{E}-03$ & $3.33 E-03$ \\
\hline $\begin{array}{l}\text { Chented } \\
\text { Conntituents }\end{array}$ & moled L & $\operatorname{pom~} \quad \therefore$ & tre & (ns Cl & $\begin{array}{l}67 \mathrm{CX} \\
(\mathrm{mol} / \mathrm{A})\end{array}$ & $\begin{array}{l}+67 \mathrm{Cl} \\
(\mathrm{mate} / \mathrm{L})\end{array}$ & $\begin{array}{l}+95 \mathrm{CI} \\
(\mathrm{molah})\end{array}$ \\
\hline $\mathrm{Nat+}$ & 11.0 & $1.46 \mathrm{E}+05$ & $2.81 E+05$ & 8.34 & 9.35 & 13.5 & 14.7 \\
\hline $\mathrm{Al}^{3}+$ & 5.36 & $8.34 \mathrm{E}+04$ & $160 \mathrm{E}+05$ & 4.28 & 460 & 6.18 & 7.01 \\
\hline $\mathrm{Fe} 3+($ total $\mathrm{Fe})$ & 0.397 & $1.28 \mathrm{E}+04$ & $2.46 \mathrm{E}+04$ & 0.390 & 0.393 & 0.400 & 0.404 \\
\hline $\mathrm{Cr} 3 \mathrm{H}$ & 0.475 & $1.42 \mathrm{E}+04$ & $2.74 E+04$ & 0.287 & 0.376 & 0.644 & 0.842 \\
\hline Biz+ & 37RE- 0 & 0.455 & 0.875 & $3.19 \mathrm{E}-06$ & 3.48E-06 & 4.16E-06 & $4.57 \mathrm{E}-06$ \\
\hline $\mathrm{Ln}^{3+}$ & $9.30 E-12$ & $7.45 \mathrm{E}-07$ & $1.43 E-06$ & 8.15E-12 & 8. BOE-12 & $1.03 \mathrm{E}-11$ & 1.13E-11 \\
\hline $\mathrm{Fg}+$ & $3352-04$ & 38.7 & 74.5 & $3.30 \mathrm{E}-04$ & 333E-04 & $3.36 \mathrm{E}-04$ & $3.37 \mathrm{E}-04$ \\
\hline $\mathbf{Z r}(\operatorname{es} \mathbf{Z r O} \times \mathrm{OH}) 2)$ & 3.T7E-07 & $1.9 \mathrm{E}-02$ & 3.BIE-02 & $3.46 \mathrm{E}-07$ & $3.6 \mathrm{HE}-07$ & $3.95 \mathrm{E}-07$ & 4.26E-07 \\
\hline $\mathrm{Pb} 2+$ & 9.73E-03 & $1.16 \mathrm{E}+03$ & $2.24 \mathrm{E}+03$ & 8.95E-03 & $9.38 E-03$ & $1.01 \mathrm{E}-02$ & $1.04 \mathrm{E}-02$ \\
\hline $\mathrm{Ni2+}$ & $2.71 \mathrm{E}-02$ & 917 & $1.76 \mathrm{E}+03$ & $2.19 \mathrm{E}-02$ & $2.48 \mathrm{E}-02$ & $2.80 E-02$ & 2.85E-02 \\
\hline $\mathrm{Sr} 2+$ & 0 & 0 & 0 & 0 & 0 & 0 & 0 \\
\hline Minst & 2.75E-0s & 0.871 & 1.67 & $1.96 \mathrm{E}-05$ & 2.35E-05 & 3.15E-05 & 355E-05 \\
\hline $\mathrm{Co2+}$ & 0.133 & $3.08 \mathrm{E}+03$ & $5.92 E+03$ & 0.106 & 0.119 & 0.147 & 0.161 \\
\hline $\mathbf{K}+$ & $2.1 \mathrm{BE}-0.2$ & 493 & 947 & I.82E-02 & $196 \mathrm{E}-02$ & $2.34 \mathrm{E}-02$ & 2.51E-02 \\
\hline OH- & 23.1 & $2.27 \mathrm{E}+0 \mathrm{~s}$ & $436 \mathrm{E}+05$ & 18.2 & 19.8 & 26.7 & 30.2 \\
\hline $\mathrm{NO3}$ & 4.88 & $1.75 \mathrm{E}+03$ & $3.36 \mathrm{E}+05$ & 296 & 2.96 & 7,89 & 9.53 \\
\hline $\mathrm{NO2-}$ & 1.64 & $4.36 \mathrm{E}+04$ & $8.39 E+04$ & 1.15 & 133 & 1.90 & 1.90 \\
\hline $\cos 2-$ & 0.136 & $4.70 \mathrm{E}+03$ & $9.04 E+03$ & 0.108 & 0.122 & 0.150 & 0.163 \\
\hline $\mathrm{PO43-}$ & $2.4 \mathrm{E}-04$ & 13.4 & 257 & $2.2 \pi \mathrm{E}-04$ & $2.37 \mathrm{E}-04$ & 2.54E-04 & 2.77E-04 \\
\hline SO42. & $2.6 \pi-02$ & 1.48E+03] & 2.8SE+03 & $233 \mathrm{E}-02$ & $2+\pi E-02$ & $2.87 \mathrm{E}-02$ & $3.08 \mathrm{E}-02$ \\
\hline Si(ns sio32-) & $761 \mathrm{E}-02$ & $1.23 \mathrm{E}+03$ & $2.37 \mathrm{E}+03$ & $4.98 \mathrm{E}-02$ & $6.61 \mathrm{E}-02$ & 8.60E-02 & 9.5TE-02 \\
\hline$F$ & $1,95 E-04$ & 2.13 & 4.10 & $1.66 \mathrm{E}-04$ & 1.83E-04 & 2. IAE-OA & $2.31 E-04$ \\
\hline Cl- & $9.29 \mathrm{E}-42$ & $1.90 \mathrm{E}+03$ & $3.65 \mathrm{E}+03$ & S.90E-02 & 7.56E-02 & 0.100 & 0.132 \\
\hline C6HSOT- & 2.01E-04 & 21.9 & 42.2 & $1.95 \mathrm{E}-\mathrm{AS}$ & $1.98 \mathrm{E}-04$ & $2.10 \mathrm{E}-04$ & 2.28E-04 \\
\hline EDTA4 & 7.BAE-06 & 1.30 & 250 & S.51E-OS & $6.78 \mathrm{E}-06$ & $9.23 \mathrm{E}-06$ & 1.06E-05 \\
\hline HEDTA3 & $6.51 E-05$ & 1.03 & 1.98 & $2.09 E-06$ & $4.47 \mathrm{E}-06$ & $8.69 \mathrm{E}-06$ & 1.08E-05 \\
\hline glycoluts- & $2.84 E-04$ & 12.3 & 23.6 & 1.5AE-OA & $2.25 \mathrm{E}-\mathrm{OA}$ & $3.53 \mathrm{E}-04$ & $4.19 \mathrm{E}-04$ \\
\hline ocetate- & 295E-05 & 1.00 & 1.93 & 2.85E-05 & 2.90E-05 & 3.13E-05 & $3.35 E-05$ \\
\hline Oxalute2- & $1.22 \mathrm{E}-11$ & 6.) $9 E-07$ & 1.19E-06 & $1.08 \mathrm{E}-11$ & $1.15 \mathrm{E}-11$ & 1.34E-11 & 1.4TE-11 \\
\hline DBP & $1.78 \mathrm{E}-\mathrm{OA}$ & 21.6 & 41.6 & $1.67 E-04$ & $1.74 \mathrm{E}-04$ & $1.93 \mathrm{E}-0.4$ & $2.08 \mathrm{E}-04$ \\
\hline butanal & 1.78E-04 & 7.63 & 14.7 & $167 E-04$ & $1.74 E-04$ & $1.938-04$ & $2.08 E-04$ \\
\hline $\mathrm{NH3}$ & 0.114 & $1.12 \mathrm{E}+03$ & $2.15 E+03$ & 4.40E-02 & $5.39 \mathrm{E}-02$ & 0.119 & 0.125 \\
\hline $\mathrm{Fo}(\mathrm{CN}) 64$ & 0 & 0 & 0 & 0 & 0 & 0 & 0 \\
\hline
\end{tabular}

"Unlonowns in tank solids inventory we woigned by Tank Loyajing Model (TLM) 
HDW Model Rev. 4

\begin{tabular}{|c|c|c|c|c|c|c|c|}
\hline & \multicolumn{7}{|c|}{ Single-Sheil Tank 241-S-104 } \\
\hline & \multicolumn{7}{|c|}{ SMM Cormposite Inventory Estimate } \\
\hline Phylicel : & & & & & & & \\
\hline Propertie & & & & $95 \mathrm{CI}$ & $-67 \mathrm{CI}$ & $+67 \mathrm{CI}$ & $+95 \mathrm{CI}$ \\
\hline Total SMM Weat & $4.89 \mathrm{E}+03(\mathrm{~kg})$ & $(1.01 \mathrm{kgal})$ & 一 & - & $\ldots$ & $\ldots$ & - \\
\hline Heat Load & $4.58 E-03(\mathrm{~kW})$ & (15.6 BTU/h) & 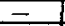 & $3.75 E-03$ & d.15E-03 & S.OTE-03 & 5.45E-03 \\
\hline Bulk Density & $1.28(9 / \infty)$ & - & - & 1.24 & 1.26 & 1.29 & 1.30 \\
\hline & & & & & & & \\
\hline Water wt\% & 63.4 & - & $\ldots$ & 61.0 & 02.1 & 64.9 & 67.2 \\
\hline TOC wt\% C (wet & $557 \mathrm{E}-03$ & 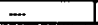 & - & 4.15E-03 & 4.83E-03 & $6.2 \pi \cdot 03$ & $6.92 E-03$ \\
\hline $\begin{array}{l}\text { Chernicil } \\
\text { Conithituents. }\end{array}$ & inoled & $\mathrm{ppm}$ - $\quad$ & Wa: & $\begin{array}{l}98 \mathrm{CI} \\
(\mathrm{mol} / \mathrm{L})\end{array}$ & $\begin{array}{l}67 \text { (1) } \\
\text { (molal) }\end{array}$ & $\begin{array}{c}67 \mathrm{Ci} \\
\text { (nowlt) }\end{array}$ & $\begin{array}{l}+98 \mathrm{CI} \\
(\mathrm{mol} / \mathrm{L})\end{array}$ \\
\hline $\mathrm{Nat}$ & 6.39 & $1.15 \mathrm{E}+05$ & 561 & 5.36 & 3.92 & 6.79 & 7.14 \\
\hline All+ & 0.994 & $2.10 \mathrm{E}+04$ & 102 & 0.915 & 0.957 & 1.03 & 1.07 \\
\hline $\mathrm{Fe} 3+($ total $\mathrm{Fe})$ & $1.24 E-03$ & 34.3 & 0.265 & $6.87 \mathrm{E}-04$ & $9.59 \mathrm{E}-04$ & $1.53 \mathrm{E}-03$ & $1.80 \mathrm{E}-03$ \\
\hline $\mathrm{Cr}_{3+}+$ & $7.46 \mathrm{E}-02$ & $3.03 \mathrm{E}+03$ & 14.8 & 4,25E-02 & $5.82 \mathrm{E}-02$ & 9.09E-02 & 0.107 \\
\hline Bi3+ & 5.44E-06 & 0.869 & $4.34 E-03$ & $4.59 \mathrm{E}-06$ & 5.01E-06 & $5.8 T E-06$ & $6.28 \mathrm{E}-06$ \\
\hline $2+3+$ & 1.34E-II & 1.46E- -06 & $7.11 \mathrm{E}-09$ & $9.43 \mathrm{E}-12$ & 1.14E-11 & 1.54E-11 & $1.72 \mathrm{E}-11$ \\
\hline $\mathrm{Hg} 2+$ & 8.5.4E-07 & 0.134 & $6.55 E-04$ & $7.70 \mathrm{E}-07$ & $8.12 E-07$ & $8.975-07$ & $9.38 \mathrm{E}-07$ \\
\hline $\mathrm{Zr}$ (as $\mathrm{ZrO}(\mathrm{OH}) 2)$ & $5,43 E-07$ & 3.87E-02 & 1.69E 04 & 4.29E-07 & 474E-07 & 5.90E-07 & $6.50 \mathrm{E}-07$ \\
\hline $\mathrm{Pb} 2+$ & $1.35 \mathrm{E}-04$ & 21.9 & 0.107 & 7.39E-AS & $1.04 E-04$ & $1.67 \mathrm{t}-04$ & $1.97 \mathrm{E}-04$ \\
\hline $\mathrm{Ni2+}$ & $1.12 \mathrm{E}-03$ & 51.4 & 0.251 & $1.01 \mathrm{E}-03$ & $1.06 \mathrm{E}-03$ & $1.18 \mathrm{E}-03$ & 1.23E-03 \\
\hline $\mathrm{Sr} 2+$ & 0 & 0 & 0 & 0 & 0 & 0 & 0 \\
\hline Mn4t & 396E-05 & 1.70 & B.31E-03 & 2.88E-05 & 3.41E-05 & $4.5 ! \mathrm{E}-05$ & 5.03E-05 \\
\hline $\mathrm{Con}+$ & $5.59 \mathrm{E}-03$ & 175 & O.BS6 & $3.41 E-03$ & $4.49 E-03$ & $6.71 \mathrm{E}-03$ & $7,78 E-03$ \\
\hline $\mathbf{K}+$ & $2.53 \mathrm{E}-02$ & 73 & 3.78 & $1.92 \mathrm{E}-02$ & $2.21 E-02$ & 2.86E-02 & $3.19 \mathrm{E}-02$ \\
\hline OH- & 6.20 & B.24E+04 & 402 & 5.42 & 596 & 6.44 & 6.69 \\
\hline NO3. & 1.74 & B.44E+04 & 412 & 1.41 & 1.57 & 1.91 & 2.07 \\
\hline NO2- & 1.48 & $5.33 E+04$ & 260 & 1.03 & 1.26 & 1.63 & 1.73 \\
\hline CO32- & 9.52E-03 & 47 & 2.18 & 733E-03 & $8.40 E-03$ & $1,00 \mathrm{E}-02$ & $1.17 E-02$ \\
\hline PO4,3- & $3.52 \mathrm{E}-04$ & 26.1 & 0.128 & $2.73 E-04$ & $320 E-04$ & $3.75 \mathrm{E}-04$ & $3.92 \mathrm{E}-04$ \\
\hline SO42. & $3.20 \mathrm{E}-02$ & $240 E+03$ & 11.7 & 2.43E-02 & $280 \mathrm{E}-02$ & $3.61 \mathrm{E}-02$ & 4.01E-O2 \\
\hline $\mathrm{Si}(\mathrm{es} \mathrm{SiO32-)}$ & $2.11 \mathrm{E}-02$ & 469 & 2.27 & $1.07 E-02$ & $1,58 E-02$ & $2.65 \mathrm{E}-02$ & $3.16 \mathrm{E}-02$ \\
\hline F. & $2.81 \mathrm{E} 04$ & $\begin{array}{r}4.17 \\
\end{array}$ & $2.04 \mathrm{E}-02$ & $2.01 \mathrm{E}-04$ & $237 \mathrm{E}-04$ & $3.20 \mathrm{E}-04$ & $3.52 \mathrm{E}-04$ \\
\hline $\mathrm{Cl} \cdot$ & 0.106 & $2.93 E+03$ & 14.3 & 5.68E-02 & $8.07 \mathrm{E}-02$ & 0.116 & 0.116 \\
\hline C6HSO73- & $2.90 \mathrm{E}-04$ & 2.9 & 0.209 & $2.65 E-04$ & $2.67 \mathrm{E}-0 \mathrm{~A}$ & $3.11 \mathrm{E}-04$ & $3.30 \mathrm{E}-04$ \\
\hline EDTA4 & $1.13 \mathrm{E}-0 \mathrm{~S}$ & 2.54 & $1.24 \mathrm{E}-02$ & $6.38 E-06$ & $8.76 \mathrm{E}-0 \mathrm{~s}$ & $1.38 \mathrm{E}-05$ & $1.62 \mathrm{E}-05$ \\
\hline HEDTA3- & $9.38 \mathrm{E}-06$ & 2.01 & $9.82 \mathrm{E}-03$ & 2.42E-06 & 5.77E-06 & 1.30E-0S & 1.65E-05 \\
\hline glycolate & $4.09 \mathrm{E}-04$ & 24.0 & 0.117 & 1. $\mathrm{REE}-0 \mathrm{~A}$ & $2.90 \mathrm{E}-04$ & $5.27 \mathrm{E}-04$ & $6.40 \mathrm{E}-04$ \\
\hline acctater & 4.25E-05 & 1.86 & $9.57 \mathrm{E}-13$ & 3.35E-05 & 3.79E-05 & 4.68E-05 & $5.09 \mathrm{E}-0 \mathrm{~S}$ \\
\hline oxolate2- & $1.76 \mathrm{E}-11$ & 1.21E-65 & 5.90E-09 & 1.55E-11 & 1.65E-11 & $1.86 \mathrm{E}-11$ & $1.96 \mathrm{E}-11$ \\
\hline DBP & $2.57 \mathrm{E}-0 \mathrm{~A}$ & 42.3 & 0.206 & $1.93 \mathrm{E}-04$ & 2.24E-04 & 2.88E-04 & 3.13E-04 \\
\hline butanol & $2.57 \mathrm{E}-04$ & 14.9 & $7.238-02$ & 1.93E-04 & $2.24 \mathrm{E}-04$ & $2.88 \mathrm{E}-04$ & 3.JBE-04 \\
\hline NH3 & 2.85E-02 & $3 \pi$ & 1.85 & $1.772-02$ & $2.28 \mathrm{E}-02$ & $3.37 \mathrm{E}-02$ & $3.84 \mathrm{E}-02$ \\
\hline $\mathrm{Fo}(\mathrm{CN}) 64$ & 0 & 0 & 0 & 0 & 0 & 0 & 0 \\
\hline
\end{tabular}

"Density is calculated besed on Ne, OH-, and $\mathrm{AlO} 2$.

tWater wh\% derived from the difference of density and tosal disalved species. 
HNF-SD-WM-ER-352, Rev. 1

\begin{tabular}{|c|c|c|c|c|c|c|c|}
\hline \multicolumn{8}{|c|}{ Single-Shell Tank 241-S-104 } \\
\hline & \multicolumn{7}{|c|}{ Total Inventory Fstimate* } \\
\hline $\begin{array}{l}\text { Phytical } \\
\text { Properties }\end{array}$ & & & & $95 \mathrm{CI}$ & $-67 \mathrm{CI}$ & $+67 \mathrm{CI}$ & $+95 \mathrm{CI}$ \\
\hline Total Waste & $1.93 \mathrm{E}+06(\mathrm{~kg})$ & (294 kgal) & $\ldots$ & - & $\ldots$ & - & - \\
\hline Heat Load & $4.67(\mathrm{~kW})$ & (1.60E+04 BTU/hs) & - & 3.94 & 4.37 & 4.87 & 4.96 \\
\hline Bulk Density† & $1.73(g / \infty)$ & - & $\ldots$ & 1.58 & 1,65 & 1.86 & 1.91 \\
\hline & & & & & & & \\
\hline Water wt\%! & 31.1 & $\cdots$ & - & 20.2 & 23.7 & 36.6 & 42.1 \\
\hline TOC wi\% C (wet & $2.86 E-03$ & $=$ & - & $2.59 \mathrm{E}-03$ & $2.66 \mathrm{E}-03$ & $307 E-03$ & $3.33 \mathrm{E}-03$ \\
\hline $\begin{array}{l}\text { Chemical } \\
\text { Constituents }\end{array}$ & $\operatorname{mote} / \mathrm{L}$ & Ppm X & log: & $\begin{array}{l}95 \mathrm{Ct} \\
\text { (nolen) }\end{array}$ & $\begin{array}{c}67 \mathrm{Cl} \\
\text { (molel }\end{array}$ & $\begin{array}{l}+67 \mathrm{Cl} \\
\text { (mole/ } / \text { ) }\end{array}$ & $\begin{array}{r}+95 \mathrm{Cl} \\
\text { (moleh) }\end{array}$ \\
\hline $\mathrm{Na+}$ & 11.0 & $1.46 \mathrm{E}+05$ & $2.82 \mathrm{E}+03$ & 8.33 & 9.34 & 13.4 & 14.7 \\
\hline $\mathrm{Al} 13+$ & 5.34 & $8.32 \mathrm{E}+04$ & $1.60 \mathrm{E}+05$ & 4.27 & 4.59 & 6.16 & 6.99 \\
\hline Fe3t (total Fe) & 0.395 & $1.27 E+04$ & $2.46 E+04$ & 0.388 & 0.392 & 0.399 & 0.402 \\
\hline $\mathrm{Cr} 3+$ & 0.473 & $1.42 E+04$ & $2.74 E+04$ & 0.286 & 0.375 & 0.642 & 0.840 \\
\hline $\mathrm{Bi3+}$ & $3.78 E-06$ & 0.436 & 0.879 & $3.19 \mathrm{E}-06$ & $3.48 \mathrm{~B}-06$ & 4.16E-06 & $4.58 \mathrm{E}-06$ \\
\hline Le?t & 9.3!E-12 & $7.47 \mathrm{E}-07$ & $144 \mathrm{E}-6$ & B.16E-12 & $8.81 E-12$ & $1.03 \mathrm{E}-11$ & $1.13 \mathrm{E}-11$ \\
\hline $\mathrm{Hg}^{2+}$ & $334 \mathrm{E}-04$ & 38.6 & 74.5 & $3.29 \mathrm{E}-04$ & 3.32E-04 & 3.3SE-04 & $3.36 \mathrm{E}-04$ \\
\hline $\mathrm{Zr}(\Delta \mathrm{ZrO}(\mathrm{OH}) 2)$ & $3.7 \pi-07$ & $1.99 \mathrm{E}-02$ & 3.83E-02 & $3,47 E-07$ & 3.62E-07 & 3.96E-07 & 4.2TE-07 \\
\hline $\mathrm{Pb} 2+$ & $9.70 \mathrm{E}-03$ & $1.16 \mathrm{E}+03$ & $2.24 \mathrm{E}+03$ & $8.92 E-03$ & 9.35E-03 & $1.00 E-02$ & $1.04 E-02$ \\
\hline Ni2 + & $2.70 \mathrm{E}-02$ & 914 & $1.76 \mathrm{E}+03$ & $2.18 E-02$ & $2.47 E-02$ & $2.87 \mathrm{k}-02$ & $2.84 \mathrm{E}-02$ \\
\hline $\mathrm{S} \sqrt{2+}$ & 0 & 0 & 0 & 0 & 0 & 0 & 0 \\
\hline Mn4t & 2.75E-05 & 0.573 & 1.68 & J.97E-05 & 2.35E-05 & $3.16 E-05$ & $3.56 \mathrm{E}-0.5$ \\
\hline $\mathrm{Co}_{2+}+$ & 0.133 & $3.07 z+03$ & $5.92 \mathrm{E}+03$ & 0.105 & 0.119 & 0.147 & 0.160 \\
\hline $\mathbf{K +}$ & $2.19 \mathrm{E}-02$ & 494 & 951 & $1.82 \mathrm{E}-02$ & 1.97E-02 & $2.34 \mathrm{E}-02$ & $2.52 \mathrm{E}-02$ \\
\hline $\mathrm{OH}-$ & 23.1 & $2.26 E+05$ & $4.36 \mathrm{E}+05$ & 18.1 & 19.7 & 26.6 & 30.1 \\
\hline NO3- & 4.87 & $1.74 E+05$ & $3.36 E+05$ & 2.95 & 2.95 & 7.87 & 9.50 \\
\hline NO2- & 1.64 & $4.36 \mathrm{E}+04$ & $8.41 \mathrm{E}+04$ & 1.16 & 1.33 & 1.90 & 1.90 \\
\hline Co32- & 0.135 & $4.69 \mathrm{E}+03$ & 9.04E+03 & 0.108 & 0.121 & 0.149 & 0.163 \\
\hline PO4,3- & 2.45E-04 & 13.4 & 25.9 & $2.27 \mathrm{~T}-04$ & $2.37 \mathrm{E}-04$ & $2.55 \mathrm{E}-04$ & $2.77 \mathrm{E}-04$ \\
\hline 5042. & $2.68 \mathrm{E}-02$ & $1.48 E+03$ & $2.86 \mathrm{E}+03$ & $2.34 \mathrm{E}-02$ & 2.47E-02 & $2.87 E-02$ & $3.08 \mathrm{E}-02$ \\
\hline $\mathrm{Si}(\mathrm{As} \mathrm{SiO} 2 \mathrm{O})$ & $7.59 \mathrm{E}-02$ & $1.23 E+03$ & $2.3 \pi+03$ & 4.97E-02 & 6. SOE- -02 & 8.5BE-02 & 9.54E-02 \\
\hline F. & $1.95 \mathrm{E}-04$ & 2.14 & 412 & $1.66 \mathrm{E}-04$ & $1.84 E-04$ & $2.14 \mathrm{E}-04$ & $2.31 \mathrm{E}-04$ \\
\hline $\mathrm{Cl}$ & $9.30 \mathrm{E}-02$ & $1.90 \mathrm{E}+03$ & $3.67 \mathrm{E}+03$ & $5.90 \mathrm{E}-02$ & 7.56E-02 & 0.100 & 0.132 \\
\hline C6: 35073 & $2.02 \mathrm{E}-04$ & 22.0 & 42.4 & 1.95E-04 & $1.98 \mathrm{E}-04$ & $2.10 \mathrm{E}-04$ & $2.29 \mathrm{E}-04$ \\
\hline EDTA4 & $7.85 E-06$ & 1.31 & 2.52 & $5.52 E-06$ & $6.79 E-06$ & $9.24 E-06$ & $1.06 \mathrm{E}-05$ \\
\hline HEDTA3- & $6.52 \mathrm{E}-06$ & 1.03 & 1.99 & 2.09E-06 & $4.48 E-06$ & $8.70 E-06$ & 1.08E- 05 \\
\hline & & & & & & & \\
\hline glycolate- & 284E-04 & 12.3 & 23.7 & 1.54E-04 & $2.25 E-04$ & $354 E-04$ & $4.20 \mathrm{E}-04$ \\
\hline wectate- & 2.95E-05 & 1.01 & 1.94 & $2.86 \mathrm{E}-05$ & $2.905-05$ & $3.14 E-05$ & 3.35E-05 \\
\hline axalnte2- & $1.22 \mathrm{E}-11$ & $6.20 \mathrm{E}-07$ & $1.19 \mathrm{E}-06$ & $1.08 \mathrm{E}-11$ & 1.15E-11 & $1.34 \mathrm{E}-11$ & $1.4 \mathrm{TE}-11$ \\
\hline DBP & 1.79E-04 & 21.7 & 41.8 & $1.67 \mathrm{TE}-04$ & $1.74 E-04$ & $1.93 \mathrm{E}-04$ & $2.09 \mathrm{E}-04$ \\
\hline butunol & 1.79E-04 & 7.65 & 14.7 & $1.67 \mathrm{E}-04$ & 1.74E-A4 & $193 \mathrm{E}-04$ & $209 \mathrm{E}-04$ \\
\hline $\mathrm{NH3}$ & 0.114 & $1.12 \mathrm{E}+03$ & $2.15 E+03$ & 4.39E-02 & $538 E-02$ & 0.119 & 0.124 \\
\hline $\mathrm{Fe}(\mathrm{CN}) 64$ & 0 & 0 & 0 & 0 & 0 & 0 & 0 \\
\hline
\end{tabular}

-Unknowns in tank solids inventory arc assigned by Tenk Laycring Model (TLM).

†Water wt\% derived from the difference of density and tokal dissolved species. 
HDW Model Rev. 4

\begin{tabular}{|c|c|c|c|c|c|c|c|}
\hline & & $\sin$ & le-Shell Tan & nk 241-S-104 & & & \\
\hline & & TLM Solid & Composile & Inventory Est & imate* & & \\
\hline Physical & & & & & & & \\
\hline Propertien & & & & $95 \mathrm{CI}$ & $-67 \mathrm{CI}$ & $+67 \mathrm{CI}$ & $+95 \mathrm{CI}$ \\
\hline Total TLM Waste & $1.92 \mathrm{E}+06(\mathrm{~kg})$ & $(293$ leacl) & - & - & - & - & - \\
\hline Heat Loed & $4.67(\mathrm{~kW})$ & $(1.59 \mathrm{E}+04 \mathrm{BTT} / \mathrm{hu})$ & - & 3.94 & 4.37 & 4.87 & 4.96 \\
\hline Bulk Density & $1.73(g / c)$ & $\ldots$ & $=$ & 1.58 & 1.65 & 1.86 & 1.91 \\
\hline Void Fraction & 0.682 & $\ldots$ & - & 0.507 & 0.565 & 0.756 & 0.788 \\
\hline Water wt\% & 31.0 & - & - & 20.1 & 23.6 & 36.5 & 42.0 \\
\hline TOC wt\% C (wet & $2.85 \mathrm{SE}-03$ & - & - & $2.58 \mathrm{E}-03$ & $2.65 \mathrm{E}-03$ & $3.06 \mathrm{E}-03$ & $3.33 E-03$ \\
\hline $\begin{array}{l}\text { Rudiolootyd } \\
\text { Constituent: }\end{array}$ & $C / L$ & $\mathrm{ect} / \mathrm{L}$ & $\mathrm{Cl}$ & $\begin{array}{l}95 \mathrm{CI} \\
\text { (CN) }\end{array}$ & $\begin{array}{l}67 \mathrm{cl} \\
(\mathrm{CVL})\end{array}$ & $\begin{array}{r}+67 \text { CI } \\
\text { (CUL) }\end{array}$ & $\begin{array}{r}+9 \mathrm{cl} \\
\mathrm{cnL})\end{array}$ \\
\hline $\mathrm{H}-3$ & $1.33 \mathrm{E}-04$ & $7.69 \mathrm{E}-02$ & 148 & $1.09 \mathrm{E}-05$ & 1.09E-05 & 1.39E-04 & $1.51 E-04$ \\
\hline $\mathrm{C}-14$ & $7.13 E-06$ & 4.1JE-03 & 790 & 8.43E-07 & $8.43 E-07$ & $7.42 \mathrm{E}-06$ & $7.96 \mathrm{E}-06$ \\
\hline Ni-59 & $9.22 \mathrm{E}-06$ & $5.32 \mathrm{E}-03$ & 10.2 & $5.48 E-06$ & $5.4 \mathrm{AE}-06$ & 9.73E- -6 & $9.64 \mathrm{E}-06$ \\
\hline $\mathrm{Ni}-63$ & B.7SE-04 & 0.505 & 971 & $5.12 \mathrm{E}-04$ & 5. $12 E-04$ & $9.23 \mathrm{E}-04$ & $9.14 E-04$ \\
\hline$C 0-60$ & 5.99E-06 & 3.46E-03 & 6.64 & $3.33 \mathrm{E}-07$ & $3.33 \mathrm{E}-07$ & $6.23 \mathrm{E}-06$ & $6.75 \mathrm{E}-06$ \\
\hline Se-79 & $4.00 E-06$ & 2.31E-03 & 4.43 & $1.79 \mathrm{E}-07$ & 1.7DE-07 & $4.06 \mathrm{E}-06$ & $6.46 \mathrm{E}-06$ \\
\hline Sr-90 & 0.487 & 281 & $5.40 \mathrm{E}+05$ & 0.399 & 0.44 & 0.520 & 0.525 \\
\hline Y-90 & 0.487 & 281 & $5.40 E+05$ & 0.342 & 0.342 & 0.520 & 0.525 \\
\hline Zr-93 & $1.89 E-05$ & $109 \mathrm{E}-02$ & 21.0 & Q.45E-07 & 8.45E-07 & $1.92 \mathrm{E}-05$ & $2.93 \mathrm{E}-05$ \\
\hline $\mathrm{Nb}-93 \mathrm{~m}$ & $1.54 \mathrm{E}-05$ & 8.86E-03 & 17.0 & 6.91E-017 & 6.91E-07 & $1.56 \mathrm{E}-05$ & $2.65 E-05$ \\
\hline Tc-99 & 5.48E-05 & 3.16E-02 & 60.7 & 4.96E-05 & $5.16 \mathrm{E}-05$ & 5.84E-05 & $6.26 \mathrm{E}-\Delta 5$ \\
\hline Ru-106 & 1.31E-09 & $7.55 \mathrm{E}-07$ & $1.45 E-03$ & 9.43E-13 & $9,43 E-13$ & $1.37 \mathrm{E}-09$ & $1.49 \mathrm{E}-09$ \\
\hline $\mathrm{Cd}-113 \mathrm{~m}$ & 2.8IE-05 & $1.62 \mathrm{E}-02$ & 31.1 & $2.61 E-06$ & $2.61 \mathrm{E}-06$ & 5.91E-05 & $5.72 \mathrm{E}-05$ \\
\hline $\mathrm{Sb}-125$ & 2.07E-05 & $1.19 E-02$ & 23.0 & 4.90E-07 & $4.90 \mathrm{E}-07$ & $2.16 E-05$ & $2.34 E-05$ \\
\hline Sn-126 & $6.14 E-06$ & 3.54E-03 & 6.80 & $2.75 E-07$ & $2.75 \mathrm{E}-07$ & $6.23 \mathrm{E}-06$ & $1.01 E-05$ \\
\hline $\mathrm{I}-129$ & $1.04 E-0 ?$ & $6.00 \mathrm{E}-05$ & 0.115 & 9.4IE-08 & $9.81 E-08$ & L.11E-07 & 1.19E-07 \\
\hline Cs.134 & 1.27E-06 & $7,35 E-04$ & 1.41 & $1.02 E-08$ & $1.02 \mathrm{E}-08$ & 1.32E-06 & $1.42 E-06$ \\
\hline $\mathrm{C}_{3}-137$ & 0.199 & 115 & $2.20 \mathrm{E}+05$ & 0.184 & 0.190 & 0.212 & 0.226 \\
\hline $\mathrm{Ba}-137 \mathrm{~m}$ & 0.188 & 108 & $2.09 \mathrm{E}+05$ & L.97E-02 & $1.97 \mathrm{E}-02$ & 0.194 & 0.208 \\
\hline $\mathrm{Sm}-151$ & 1.43E-02 & 8.22 & $1.58 E+04$ & 6395-04 & $6.39 \mathrm{E}-04$ & 1.45E-02 & $2.33 \mathrm{E}-02$ \\
\hline Eu-152 & $7.47 \mathrm{E}-06$ & $4.31 E-03$ & B.28 & $2.59 \mathrm{E}-96$ & $2.59 E-06$ & $7.50 E-06$ & $7.56 \mathrm{E}-06$ \\
\hline Eu-154 & $1.43 \mathrm{E}-04$ & $8.2 \pi-02$ & 199 & C.05E-06 & 8.05E-06 & 1.49E-04 & $1.85 E-04$ \\
\hline Eu-155 & 3.70E-04 & 0.213 & 410 & $1.22 E-04$ & $1.22 \mathrm{E}-04$ & $371 \mathrm{E}-04$ & $3.74 E-04$ \\
\hline $\operatorname{Ra}-226$ & $6.56 \mathrm{E}-10$ & $3.79 E-0 ?$ & $7.28 \mathrm{E}-04$ & $3.13 E-10$ & 3.98E-10 & $8.32 E-10$ & $1.00 \mathrm{E}-09$ \\
\hline $\mathrm{Ra}-228$ & $8.66 \mathrm{E}-09$ & $4.99 \mathrm{E}-06$ & $9.60 \mathrm{E} 03$ & $3.44 E-15$ & $3.4 \mathrm{E}-15$ & 8.74E-09 & $8.84 E-69$ \\
\hline Ac-227 & 327e-09 & $1.85 \mathrm{E}-06$ & $3.62 E-03$ & $1.62 E-09$ & $1.76 \mathrm{E}-09$ & 4.28E-09 & $5.26 \mathrm{E}-09$ \\
\hline $\mathrm{Pa}-231$ & 5.95E-09 & $3.43 \mathrm{E}-06$ & $6.60 \mathrm{E}-03$ & $2.67 \mathrm{E}-10$ & $2.67 \mathrm{~T}-10$ & $6.4 \mathrm{E}-19$ & $1.19 \mathrm{E}-08$ \\
\hline Th-229 & $208 \mathrm{E}-10$ & $1.20 \mathrm{E}-07$ & 2.31E-04 & $6.55 E-13$ & 6.55E-13 & 2.10E-10 & $2.12 E-10$ \\
\hline Th-232 & 1.16E-10 & $6.69 \mathrm{E}-08$ & $1.29 \mathrm{E}-04$ & $2.21 \mathrm{E}-16$ & $2.21 E-16$ & $1.46 \mathrm{E}-10$ & $1.76 \mathrm{E}+10$ \\
\hline U.232 & $3975-08$ & $2.23 E-05$ & 1.29E-02 & $13 T E-08$ & $2.53 E-08$ & S.S2E-08 & $7.34 E-08$ \\
\hline U-233 & $1.43 E-07$ & $8.548-05$ & 0.164 & $5.23 \mathrm{E}-08$ & $9.66 \mathrm{E}-08$ & 2.11E-07 & $2.81 E-07$ \\
\hline U.234 & $1.96 \mathrm{E}-06$ & $1.13 E-03$ & 2.17 & 1.56E-06 & 1.75E-06 & $2.17 \mathrm{E}-06$ & $2.39 \mathrm{E}-06$ \\
\hline U-235 & 8. 16E-08 & 4.71E-05 & 9.05e-13 & $6.57 \mathrm{E}-08$ & $7.3 \mathrm{AE}-0$ & $9.05 E-08$ & $9.94 \mathrm{E}-08$ \\
\hline U-236 & $5.63 E-08$ & $3.25 \mathrm{E}-05$ & $6.25 \mathrm{E}-02$ & $4.06 \mathrm{E}-08$ & 4.84E-08 & $6.46 E-08$ & $7.28 \mathrm{E}-08$ \\
\hline U-238 & 1.84E-06 & $1.06 \mathrm{E}-03$ & 2.04 & 1.49E-06 & $1.66 \mathrm{E}-06$ & $2.03 E-06$ & $2.23 \mathrm{E}-06$ \\
\hline $\mathrm{Np}-237$ & 7.51E- 07 & 1.45E-04 & 0.279 & 2.18E-07 & 2.31E-07 & $2.69 \mathrm{E}-07$ & $2.89 \mathrm{E}-07$ \\
\hline Pu-238 & 5.79E- 06 & $3.34 E-03$ & 6.4 & $4.88 E-06$ & 5.32E-06 & 6.25E-06 & $6.70 \mathrm{E}-06$ \\
\hline Pu-239 & $3.49 \mathrm{E}-04$ & 0.200 & 385 & $2.98 \mathrm{E}-04$ & $3.23 \mathrm{E}-04$ & 3.73E-04 & $397 \mathrm{E}-04$ \\
\hline Pu-240 & 5.04E-05 & 2.91E-02 & 35.9 & 4.34E-DS & $4.68 \mathrm{E}-05$ & 5,40E-05 & $5.74 \mathrm{E}-0.5$ \\
\hline Pu-24l & $3.26 \mathrm{E}-04$ & 0.188 & 362 & $2.80 \mathrm{E}-04$ & $3.03 \mathrm{E}-04$ & 3.50E-04 & $373 \mathrm{E}-04$ \\
\hline Pu-242 & $1.51 E-09$ & $8.69 E-07$ & $1.6 \pi-03$ & $1.26 \mathrm{E}-09$ & $1.38 \mathrm{~B}-09$ & $1.63 \mathrm{E}-09$ & $1.75 \mathrm{E}-09$ \\
\hline Amn-24! & $6.71 E-05$ & $3.87 \mathrm{E}-02$ & 74.5 & 3.94E-05 & $5.85 E-05$ & 7.65E-05 & $1.17 \mathrm{E}-04$ \\
\hline Am-243 & $2.07 \mathrm{E}-09$ & $1.20 \mathrm{E}-06$ & $2,30 E-03$ & $9.69 \mathrm{E}=10$ & $1.20 \mathrm{E}-09$ & $2.28 E-09$ & $2.54 E-09$ \\
\hline $\mathrm{Cm}-242$ & $6.05 \mathrm{E}-08$ & $3.49 \mathrm{E}-05$ & $6.71 E-02$ & $5.92 E-08$ & $5.96 E-08$ & 6.10E- 08 & $6.10 \mathrm{E}-08$ \\
\hline Cn-243 & 1.38E-09 & $7.99 \mathrm{E}-07$ & $1.54 E-03$ & $1.36 E-09$ & $1,37 \mathrm{TE}-09$ & $1.40 E-09$ & $1.40 \mathrm{E}-09$ \\
\hline Cm-244 & $1.11 \mathrm{E}-09$ & 6.41E-07 & $1.23 \mathrm{E}-03$ & $2.29 \mathrm{E}-10$ & $5.81 E-10$ & $1.47 E-09$ & $1.47 \mathrm{E}-09$ \\
\hline Tatals & $\mathbf{M} \quad \mathbf{L}$ & ends & 10 & $\begin{array}{l}95 \boldsymbol{Q}(\mathrm{M} \\
\text { or } \mathrm{L})\end{array}$ & $\begin{array}{l}67 \mathrm{ClOM} \\
\text { or } \mathrm{L})\end{array}$ & $+67 \mathrm{Cl} \mathrm{M}$ & $\begin{array}{l}+9 s \mathrm{CI} \\
\mathrm{M} \text { or } \mathrm{\alpha} / \mathrm{L})\end{array}$ \\
\hline Pu & $5.81 E-03(\mathrm{~g} / \mathrm{L})$ & - & 6.44 & 4.99E-03 & $5.39 \mathrm{E}-03$ & $6.23 \mathrm{E}-03$ & $6.63 \mathrm{E}-03$ \\
\hline $\mathrm{u}$ & $2.30 \mathrm{E}-02$ & $3.16 \mathrm{E}+03$ & $6.07 \mathrm{E}+03$ & $1.87 \mathrm{E}-02$ & $2,08 \mathrm{E}-92$ & $2.54 \mathrm{E}-02$ & $2.78 E-02$ \\
\hline
\end{tabular}

"Triknowns in tank solids inventory are assigned by Tenk Leyering Model (TLM) 
HNF-SD-WM-ER-352, Rev. 1

HDW Modal Rev. 4

\begin{tabular}{|c|c|c|c|c|c|c|c|}
\hline \multirow{3}{*}{\begin{tabular}{|l|} 
\\
Phyolicd \\
Properties \\
\end{tabular}} & \multicolumn{7}{|c|}{ Single-Shall Tank 241-S-104 } \\
\hline & \multicolumn{7}{|c|}{ SMM Composite Inventory Estimate } \\
\hline & & & & $-98 \mathrm{CI}$ & $67 \mathrm{Cl}$ & $+67 \mathrm{CI}$ & $+95 \mathrm{Cl}$ \\
\hline Total SMM Wast & $4.69 \mathrm{E}+03(\mathrm{~kg})$ & $(1.01 \mathrm{kgat})$ & - & $=$ & $=$ & $\ldots$ & $\cdots$ \\
\hline Heat Load & 4.58E-03 (kW) & (15.6 BTU/me) & - & $3.75 E-03$ & 4. $1.5 \mathrm{E}-03$ & $5.01 E-03$ & $5,45 \mathrm{E}-03$ \\
\hline Bulk Density" & $1.28(\mathrm{~g} / \mathrm{ec})$ & $m$ & - & 1.24 & 1.26 & 1.29 & 1.30 \\
\hline Water wt\%t & 63.4 & - & - & 61.0 & 62.1 & 64.9 & 67.2 \\
\hline TOC wt\% C (wet) & 5.5TE-03 & - & 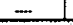 & 4. $15 E-03$ & $4.83 \mathrm{E}-03$ & $6.27 \mathrm{E}-03$ & $6.92 \mathrm{E}-03$ \\
\hline Rouliosownt & $\mathrm{CM}, \mathrm{L}$ & $\mathrm{ACv}_{\mathrm{s}}$ & Ci & $\begin{array}{l}98 \mathrm{CI} \\
\text { (ciL) }\end{array}$ & $\begin{array}{l}67 \mathrm{Cl} \\
(\mathrm{Cl} / \mathrm{L})\end{array}$ & $\begin{array}{l}+67 \mathrm{C} \\
(\mathrm{CVL})\end{array}$ & $\begin{array}{l}+95 \mathrm{Cl} \\
\text { (CVL) }\end{array}$ \\
\hline $\mathrm{H}-3$ & $1.76 E-04$ & 0.138 & 0.674 & $9.18 \mathrm{E}-10$ & 9.1BE-10 & $1.7 \pi-04$ & $1.77 \mathrm{E}-04$ \\
\hline C-14 & $9.06 \mathrm{E}-06$ & $7.08 E-03$ & $3.46 \mathrm{E}-02$ & 7.13E-11 & 7.13E-11 & 9.14E-06 & $9.22 \mathrm{E}-06$ \\
\hline Ni-59 & 5.05E-07 & 3.95E-04 & $1.935-03$ & $9.02 E-12$ & $9.02 \mathrm{E}-12$ & $5.32 E-07$ & $5.58 E-07$ \\
\hline $\mathrm{Ni}-63$ & $4.90 \mathrm{E}-05$ & $3.83 \mathrm{E}-02$ & 0.187 & $8.43 \mathrm{E}-10$ & 8.43E-10 & $5.17 \mathrm{E}-05$ & $5,42 \mathrm{E}-05$ \\
\hline $\mathrm{Co}-60$ & $8.15 \mathrm{E}-06$ & $6.37 \mathrm{E}-03$ & 3.11E-02 & $2.84 \mathrm{E}-11$ & 2.84E-11 & $822 \mathrm{E}-06$ & 8.29E-06 \\
\hline Se-79 & L.24E-06 & $9.72 E-04$ & 4.75E-03 & $151 E-11$ & $1.51 \mathrm{E}-11$ & $169 \mathrm{E}-06$ & 209E-06 \\
\hline Sr-90 & 2.11E-02 & 16.5 & 80.7 & $1.81 \mathrm{E}-02$ & $1.96 \mathrm{E}-02$ & $2.2 \pi-02$ & 2.42E-02 \\
\hline$Y-90$ & 2.11E-02 & 16.5 & $\begin{array}{r}0.7 \\
\end{array}$ & 5.85E-07 & 5.85E-07 & $2.2 \pi \mathrm{E}-02$ & 2.42E-02 \\
\hline ZI.93 & 6.22E-06 & 4.B6E-03 & $23 \pi-02$ & $7.15 \mathrm{~S}-11$ & $7.15 \mathrm{E}-11$ & $8.46 \mathrm{E}-06$ & $9.97 \mathrm{E}-0 \mathrm{~s}$ \\
\hline $\mathrm{Nb}-93 \mathrm{~m}$ & $435 E-\infty 6$ & $3.40 E-03$ & $1.66 E-02$ & SBAE-11 & 5.84E-11 & $5.92 E+06$ & $7.43 E-06$ \\
\hline Tc-99 & 7.04E-05 & $5.50 \mathrm{E}-02$ & 0.269 & $5.49 \mathrm{E}-05$ & $0.24 \mathrm{E}-0.05$ & 7.84E-05 & 8.65E-05 \\
\hline Ru-106 & $1.805-09$ & $1.47 \mathrm{E}-06$ & 7.19E-06 & $1.78 E \cdot 16$ & $1.7 \mathrm{BE}-16$ & $190 \mathrm{E}-09$ & $192 \mathrm{E}-49$ \\
\hline $\mathrm{Cd}-113 \mathrm{~m}$ & $3.67 \mathrm{E}-05$ & 2.87t-02 & 0.10 & $2.21 \mathrm{E}-10$ & $2.21 \mathrm{E}-10$ & $3,70 \mathrm{E}-0 \mathrm{~S}$ & $3.735-05$ \\
\hline Sb-125 & $2.91 \mathrm{E}-0.5$ & $2.29 E-02$ & 0.111 & $4.27 \mathrm{E}-11$ & $4.27 \mathrm{E}-11$ & 2.93E-05 & $2.96 \mathrm{E}-05$ \\
\hline Sn-126 & $1.86 E-06$ & $1.46 E-03$ & $7.125-03$ & $2.33 E-11$ & 2.33E-11 & 2.54E-06 & $300 \mathrm{E}-0.6$ \\
\hline $\mathrm{I}-129$ & 1.34E-07 & $1.04 E-04$ & $5.10 \mathrm{E}-04$ & $1.04 E-07$ & 1.18E-07 & 1.49E-07 & $1,64 \mathrm{E}-07$ \\
\hline$C_{5}-134$ & $1.60 \mathrm{E}-06$ & $1.25 E-03$ & $6.12 E-03$ & $7.87 \mathrm{E}-13$ & $7.87 \mathrm{E}-13$ & $1.62 E-06$ & $1.63 \mathrm{E}-06$ \\
\hline Cs-137 & 0.226 & 176 & $\$ 2$ & 0.179 & 0.201 & 0.250 & 0.274 \\
\hline $\mathrm{Ba}-137 \mathrm{~m}$ & 0.213 & 167 & $\$ 15$ & $139 E-06$ & $1.39 E-06$ & 0.215 & 0.217 \\
\hline Sm-151 & 4.35E-03 & 3.40 & 16.6 & $5.41 E-08$ & $5.41 \mathrm{E}-08$ & $5.92 \mathrm{E}-03$ & $7.385-09$ \\
\hline Eu-152 & $7.06 \mathrm{E}-07$ & $5.52 E-04$ & 2.70E-D3 & 5.34E-12 & $5.34 \mathrm{E}-12$ & $7.12 \mathrm{E}-07$ & $7.19 E-07$ \\
\hline Eu-154 & 1.95E-04 & 0.152 & 0.744 & $6.85 \mathrm{E}-10$ & 6.BSE-10 & $1.96 \mathrm{E}-0 \mathrm{~d}$ & $1.98 \mathrm{E}-04$ \\
\hline Eu-155 & $3.58 \mathrm{E}-05$ & 2.80E-A2 & 0.137 & $2.53 E-10$ & $2.53 \mathrm{E}-10$ & $3.61 \mathrm{E}-0 \mathrm{~S}$ & 3.65E-05 \\
\hline Ra-226 & $3.85 \mathrm{E}-11$ & $3.01 E-08$ & 1.47E-07 & $1.05 \mathrm{E}-15$ & $1.05 E-15$ & 5.25E-11 & $6.58 \mathrm{E}-11$ \\
\hline Re-228 & 1.25E-09 & $9.80 \mathrm{E}-07$ & 4.795-06 & $7.12 \mathrm{E}-21$ & 7.12E-2! & $1.27 \mathrm{E}-09$ & $1.28 \mathrm{E}-09$ \\
\hline$A c-227$ & 2.24E-10 & $1.75 E-07$ & B.55E-07 & 6.10E-15 & $6.10 \mathrm{E}-15$ & $3.05 \mathrm{E}-10$ & $3.82 \mathrm{E}+10$ \\
\hline $\mathrm{Pe}-231$ & $1.24 \mathrm{E}-49$ & 9.72E-07 & 4.75E-06 & $2.26 \mathrm{E}-14$ & $2.26 \mathrm{E}-14$ & $1.69 \mathrm{E}-09$ & $2.12 \mathrm{E}-09$ \\
\hline Th-229 & $3.01 \mathrm{E}-11$ & $2.35 \mathrm{E}-08$ & 1.15E-07) & $1.345-18$ & $1.348-18$ & 3.04E-11 & $306 \mathrm{E}-11$ \\
\hline Th-232 & 1.6TE-10 & $1.31 \mathrm{E}-07$ & $6.39 \mathrm{E}-07$ & I. $29 \mathrm{E}-20$ & $1.89 \mathrm{E}-20$ & $2.09 \mathrm{E}-10$ & 2. $49 \mathrm{E}-10$ \\
\hline $\mathrm{U}_{-232}$ & $1.27 \mathrm{E}-0 \mathrm{~s}$ & 994E-06 & 4.85E-05 & $1.04 \mathrm{E}-08$ & $1.17 \mathrm{E}-08$ & $1.37 \mathrm{E}-0 \mathrm{~s}$ & $1.4 \pi \mathrm{E}-0 \mathrm{~s}$ \\
\hline $\mathrm{U}-233$ & $4.80 \mathrm{E}-08$ & 3.0en-0s & 1.86E-04 & 3.97E-08 & 4.49E-08 & $5.25 E-08$ & $5.61 \mathrm{E}-08$ \\
\hline U-234 & $2.305-07$ & 1.0E-04 & B.T7E-OA & 2.07E-07 & $2.18 E-07$ & $2,41 E-07$ & 2.52E-07 \\
\hline $\mathrm{U}-235$ & $9.28 E-09$ & $7.25 E-06$ & 3.54E-05 & 8.35E- -9 & B SOE-09 & $9.74 \mathrm{E}-09$ & $1.02 \mathrm{E}-08$ \\
\hline $\mathrm{U}-236$ & 9.51E-09 & 7.44E-06 & 3.63E-05 & $8.54 \mathrm{E}-09$ & $9.01 E-09$ & $1.00 \mathrm{E}-08$ & $1.05 E-08$ \\
\hline U-238 & 2.01E-07 & $1.5 \pi-04$ & $7.69 \mathrm{E}-04$ & $1.81 \mathrm{E}-07$ & 1.91E-07 & $2.11 \mathrm{E}-07$ & $2.21 \mathrm{E}-07$ \\
\hline $\mathrm{Np}-237$ & $307 \mathrm{E}-07$ & 2. $40 E-04$ & $1.17 \mathrm{E}-03$ & $236 \mathrm{E}-07$ & 2,70E-07 & $3.45 \mathrm{E}-07$ & $3.82 \mathrm{E}-07$ \\
\hline Pu-238 & $1.71 E-07$ & $1.34 E-04$ & $6.53 \pm-04$ & $5.09 \mathrm{E}-08$ & $1.10 \mathrm{E}-07$ & $2.32 E-07$ & 2.91E-07 \\
\hline Pu.239 & $5.52 \mathrm{E}-06$ & 4.32E-03 & $2.11 \mathrm{E}-02$ & $1.60 \mathrm{E}-06$ & $355 \mathrm{E}-06$ & $7.50 \mathrm{E}-05$ & $9.39 \mathrm{E}-06$ \\
\hline Pu-240 & 9.16E-07 & $7,16 E-04$ & $3.50 \mathrm{E}-03$ & $2.74 E-07$ & $5.85 \mathrm{E}-07$ & 124E-06 & $156 \mathrm{E}-06$ \\
\hline Pu-241 & $8.77 \mathrm{E}-06$ & $6.86 \mathrm{E}-03$ & $335 \mathrm{E}-02$ & $2.63 \mathrm{E}-06$ & 5.64E-06 & 1.19E-05 & 1. $49 \mathrm{E}-05$ \\
\hline$P u-242$ & $4.65 \mathrm{E}-11$ & $3.64 E-08$ & $1.7 B E-07$ & $1,39 \mathrm{E}-11$ & $2.99 \mathrm{E}-11$ & 6.32E.11 & $7.91 \mathrm{E}-11$ \\
\hline Am-241 & $1.86 \mathrm{E}-05$ & 1.46E-02 & $7.12 \mathrm{E}-02$ & $5.42 \mathrm{E}-06$ & 1.19E-05 & 2.54E-05 & $3.19 \mathrm{E}-0 \mathrm{~s}$ \\
\hline Am-243 & $5.95 \mathrm{E}-10$ & 4.65E-07 & $2.27 \mathrm{E}-06$ & $1.40 \mathrm{E} \cdot 10$ & $3.33 \mathrm{E}-10$ & 8.72E-10 & $1.15 E-09$ \\
\hline $\mathrm{Cm}-242$ & $1.26 \mathrm{E}-10$ & 9.86E-03 & 481E-07 & $1.22 \mathrm{E}-13$ & $1.22 \mathrm{E}-13$ & $1.20 \mathrm{E}-10$ & $1.29 \mathrm{E}-10$ \\
\hline $\mathrm{Cm}-243$ & $2.58 \mathrm{E}-12$ & $2.0 ! E-09$ & 9.84E-69 & 2.BOE-15 & $2.80 \mathrm{E}-15$ & 2.61E-12 & $2.63 \mathrm{E}-12$ \\
\hline $\mathrm{Cm}-244$ & $1.50 \mathrm{~B}-10$ & $1.17 \mathrm{E}-07$ & 5.73E-07 & $8.52 \mathrm{E}-14$ & 3.52E-14 & $1.52 \mathrm{E}-10$ & $1.53 \mathrm{E}-10$ \\
\hline Totals & $M$ & thls & wa & $\begin{array}{r}95 \mathrm{Ml} \\
+\mathrm{L})\end{array}$ & $67 \mathrm{Cl}(\mathrm{M}$ & $\begin{array}{r}+67 \mathrm{Cl} \mathrm{(M} \\
0+\mathrm{pL}\end{array}$ & $\begin{array}{l}+95 \mathrm{CI} \\
\mathrm{M} \text { or } \mathrm{H} / \mathrm{L}\end{array}$ \\
\hline $\mathrm{Pu}$ & $9.20 \mathrm{E}-05(z / \mathrm{L})$ & $=$ & 3.51E-04 & $2.68 \mathrm{E}-05$ & 5.BTE-0S & $1.25 \mathrm{E}-04$ & $1.57 \mathrm{E}-0 \mathrm{4}$ \\
\hline $\mathrm{U}$ & 2.49E-03 & 463 & 2.26 & 2.24E-03 & $2.36 \mathrm{E}-03$ & $2.61 E-03$ & $2, T 3 E-03$ \\
\hline
\end{tabular}

"Density is calculated beed on Na, OH-, and $\mathrm{AO}_{2}$ -

tWeter wr\% derived from the differerce of density and toeal disenolved species. 
HDW Model Rov. 4

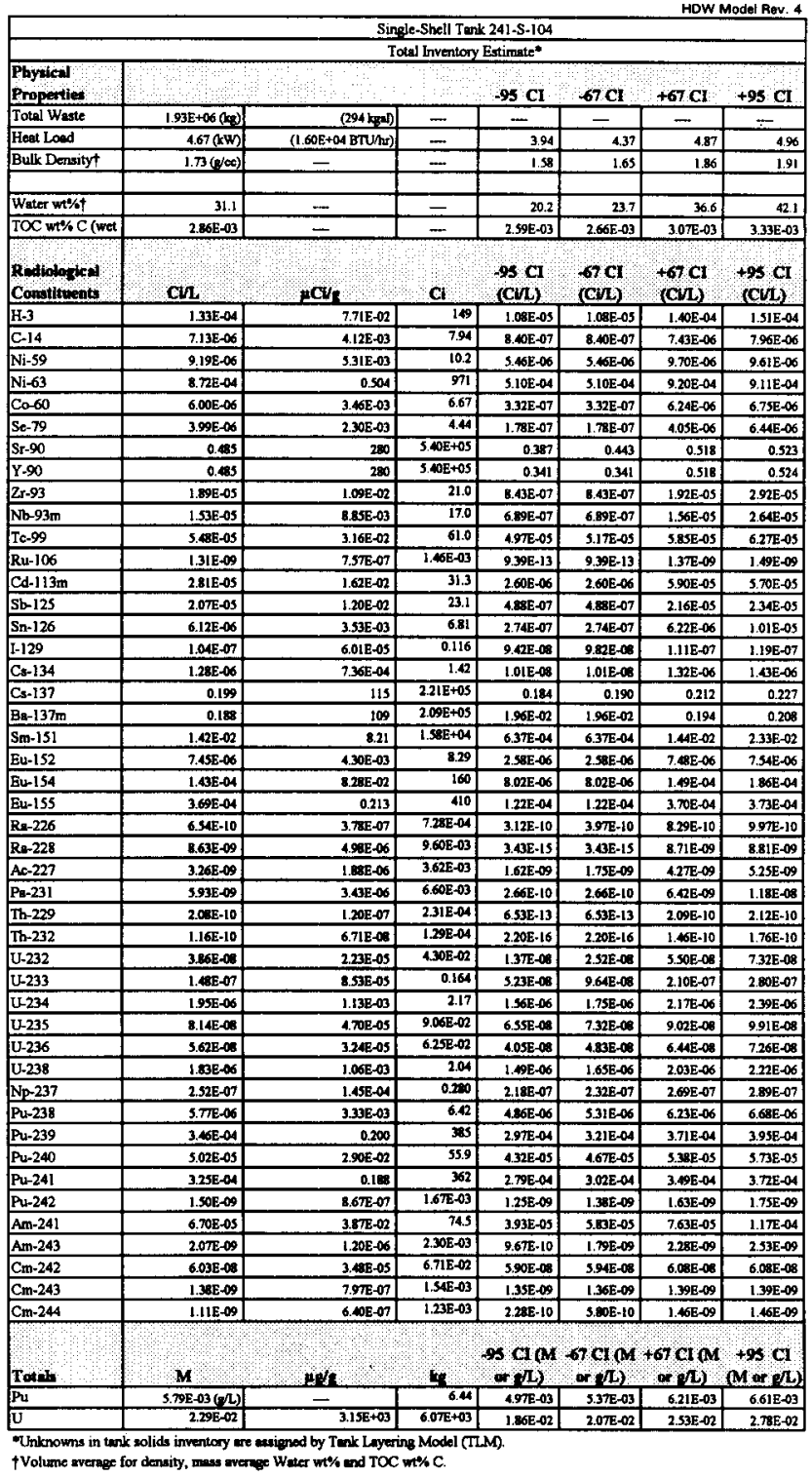




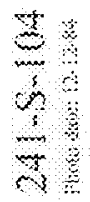

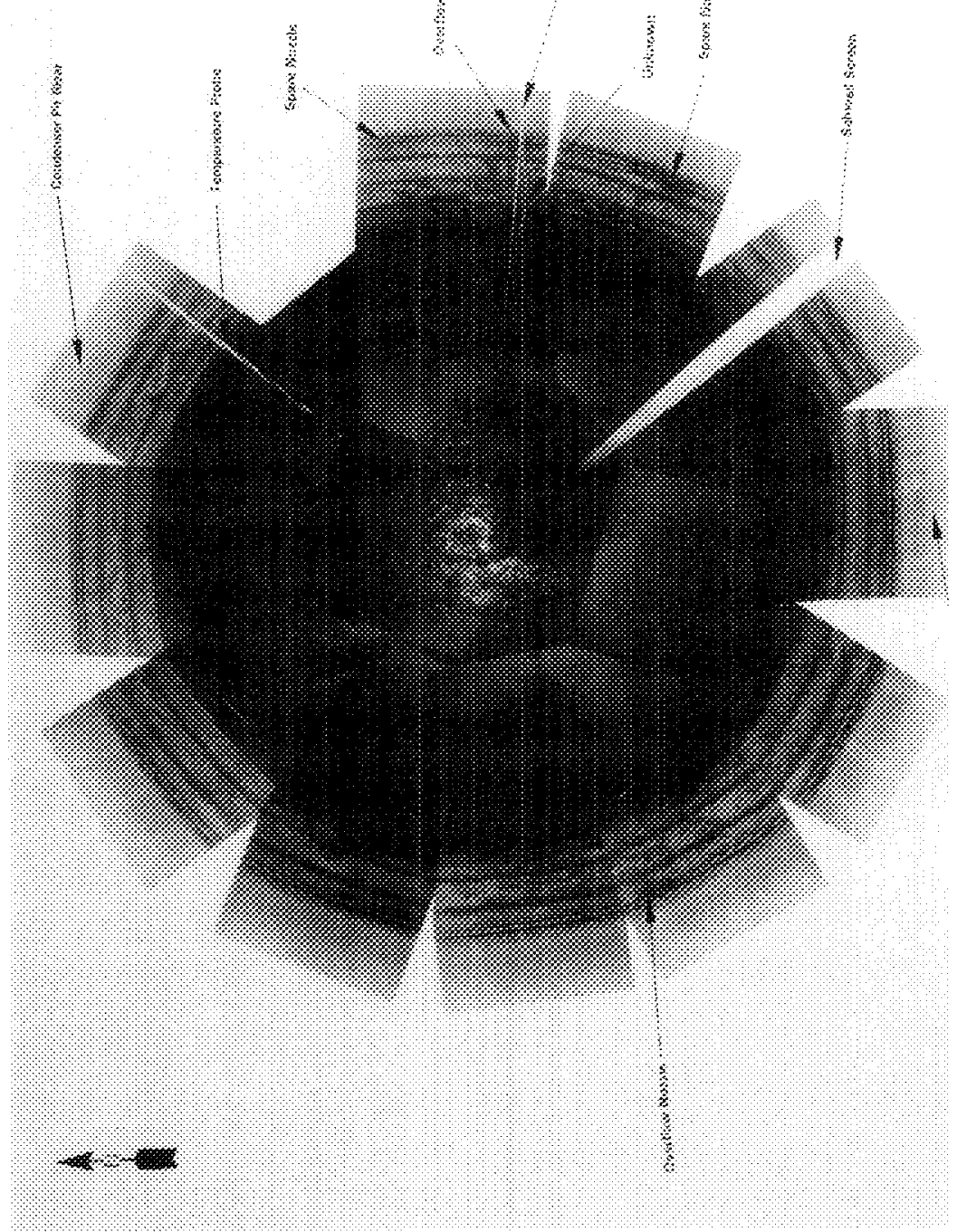


TANK 241-S-105 SUMMARY

\begin{tabular}{|c|c|c|c|}
\hline \multicolumn{2}{|c|}{ TANK HISTORY } & \multicolumn{2}{|c|}{ TANK DESCRIPTION } \\
\hline Entered Service & 2nd qtr 1953 & Diameter & $75 \mathrm{ft}$ \\
\hline Removed from Service & 1975 & Bottom Shape & Dish \\
\hline Inactive & 1978 & Nominal Capacity & $758,000 \mathrm{gal}$ \\
\hline Watch Lists & none & Cascade Tank & to $241-S-106$ \\
\hline Integrity & Sound & Total Risers & 12 \\
\hline Assumed Leaker & - & \multicolumn{2}{|c|}{ WASTE VOLUME (HANLON 1996i) } \\
\hline Interim Stabilization (IS) & Sept 1988 & Total Waste Volume & $456,000 \mathrm{gal}$ \\
\hline Partial Interim Isolation (PI) & Dec 1982 & Waste Type & NCPLX \\
\hline Intrusion Prevention (IP) & Sept 1990 & Drainable Interstitial Liquids & $35,000 \mathrm{gal}$ \\
\hline \multicolumn{2}{|c|}{$\begin{array}{l}\text { TENTATIVELY AVAILABLE RISERS } \\
\end{array}$} & Pumpable Liquids & 13,000 gal \\
\hline Riser Number(s) & Size & Saltcake & $454,000 \mathrm{gal}$ \\
\hline $2,11,16$ & 4 in & Sludge & $2,000 \mathrm{gal}$ \\
\hline 6,8 & 12 in & Supernatant & 0 gal \\
\hline \multicolumn{2}{|c|}{ TANK TEMPERATURE } & \multicolumn{2}{|c|}{ INTERIOR PHOTOGRAPHS } \\
\hline Average Tank Temperature & $73^{\circ} \mathrm{F}$ & Date & April 12, 1989 \\
\hline Maximum Temperature & $82.58^{\circ} \mathrm{F}$ & Montage Number & $94041033-22 \mathrm{CN}$ \\
\hline Date & $\operatorname{Jan} 30,1996$ & Photo Set Number & 89041307 \\
\hline Elevation from tank bottom & $0.56 \mathrm{ft}$ & \multicolumn{2}{|c|}{ WASTE SURFACE LEVEL } \\
\hline Riser Number & 4 & Devices & Manual ENRAF \\
\hline Minimum Temperature & $58.64^{\circ} \mathrm{F}$ & Max Level & 158 in \\
\hline Date & May $20-22,1996$ & Date & April 11, 1995 \\
\hline Elevation from tank bottom & $10.56 \mathrm{ft}$ & Min Level & 155.1 in \\
\hline Riser Number & 4 & Date & July 8 and 29,1991 \\
\hline
\end{tabular}




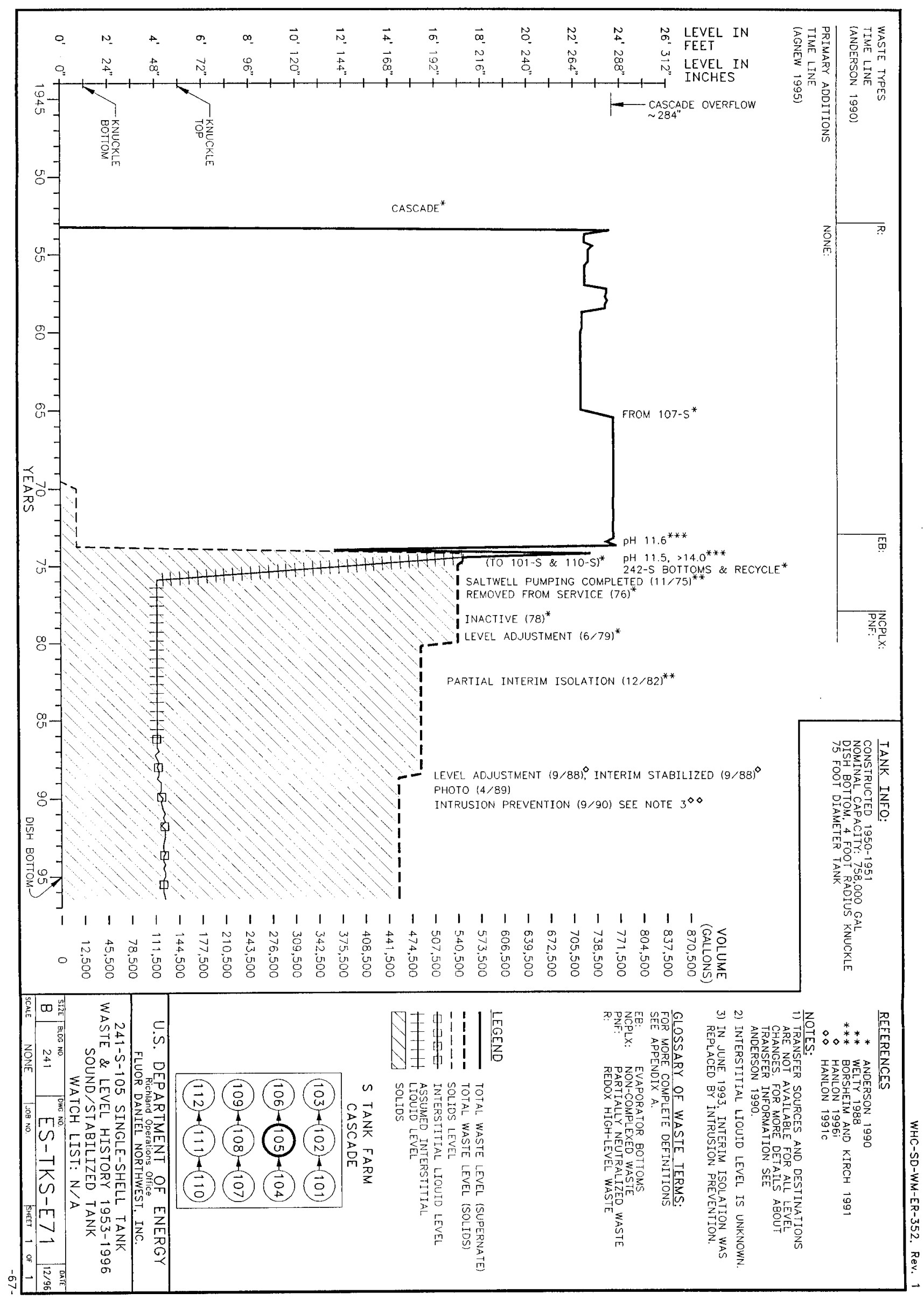




$$
241-5-105
$$

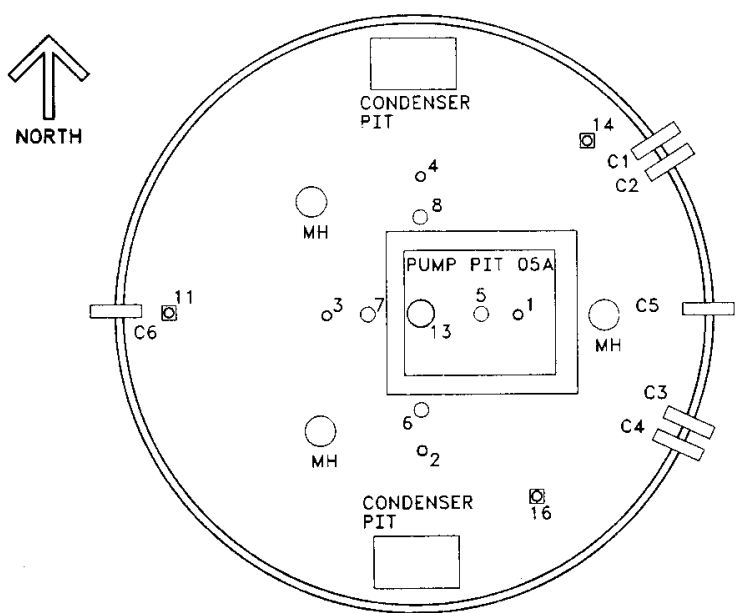

Ref: Alstad 1993

$\mathrm{H}-2-73184$, Rev. 4

$\mathrm{H}-2-37528$, Rev. 2

\section{TANK RISER LOCATION}

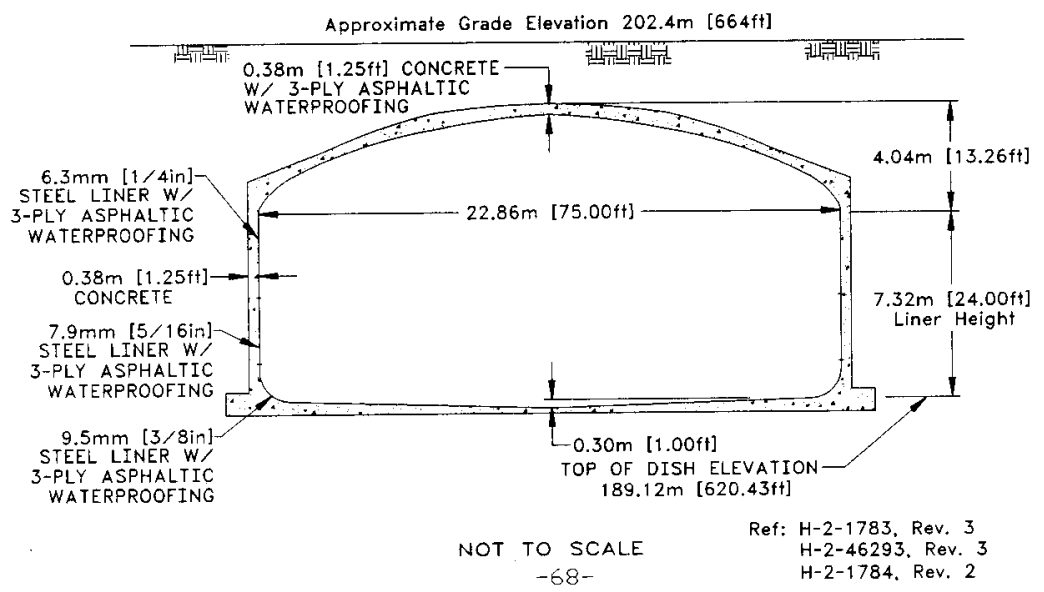


HNF-SD-WM-ER-352, Rev. 1

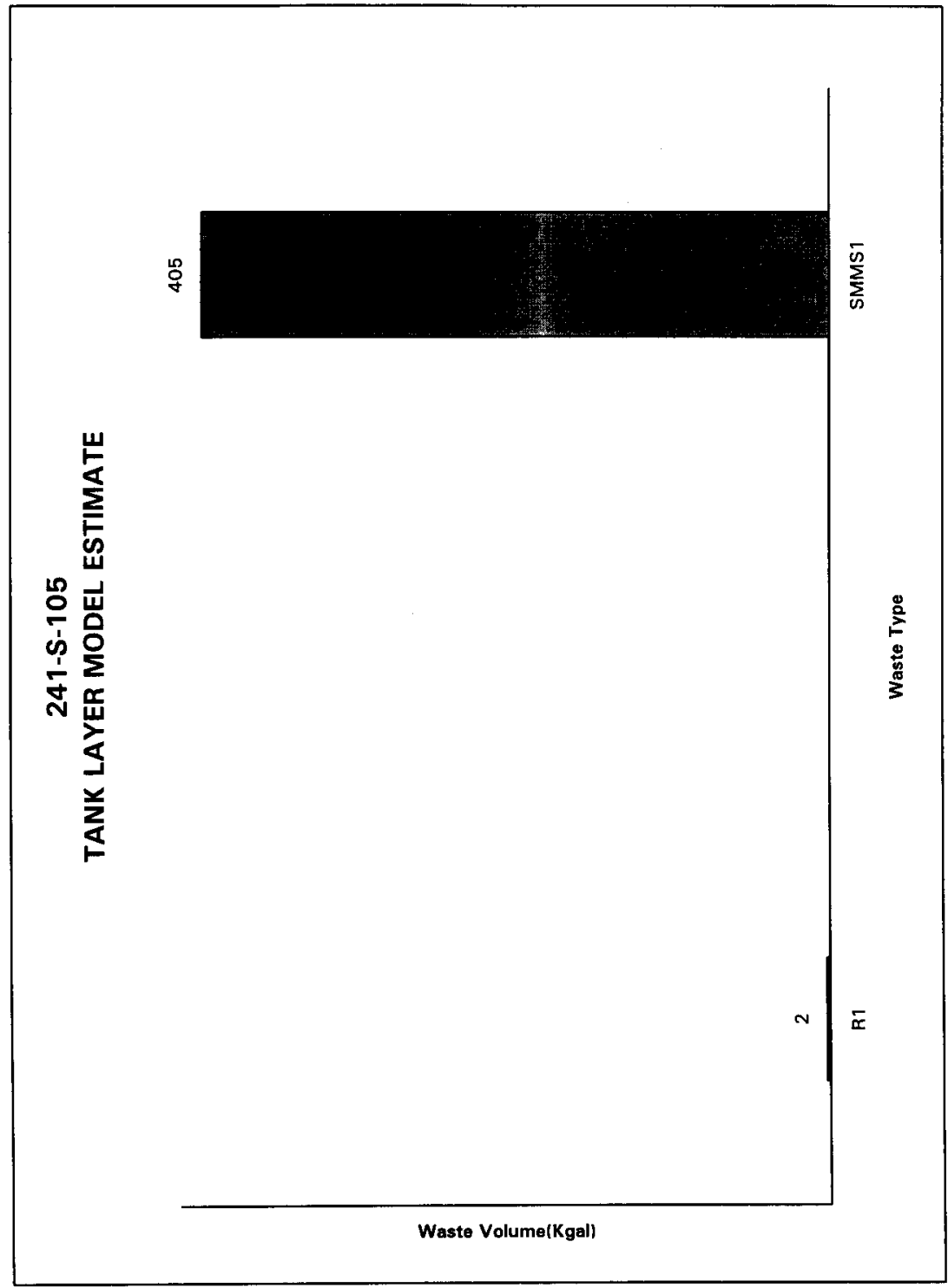

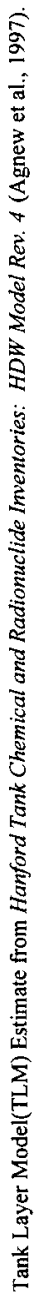


HDW Model Rev. 4

\begin{tabular}{|c|c|c|c|c|c|c|c|}
\hline & \multicolumn{7}{|c|}{ Single-Shell Tank 241-S-105 } \\
\hline & \multicolumn{7}{|c|}{ TLM Solids Composite Inventory Estimete" } \\
\hline Phyiced & & & & & & & \\
\hline Propentien & & & $\therefore \because$ & $95 \mathrm{CI}$ & $-67 \mathrm{CI}$ & $+67 \mathrm{CI}$ & $+95 \mathrm{CI}$ \\
\hline Total TLM Warte & $1.33 \mathrm{E}+04(\mathrm{Cg})$ & $(2.00 \mathrm{kgl})$ & - & - & - & - & - \\
\hline Heat Load & 493E-02 (kW) & (168 BTU/hr) & $\ldots$ & $35 \mathrm{FT}-02$ & $4.37 \mathrm{E}-02$ & $5.29 \mathrm{E}-02$ & $5.46 \mathrm{E}-02$ \\
\hline Bulk Density & $1.76(0 / \infty)$ & - & 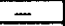 & 1.51 & 1.53 & 2.11 & 2.24 \\
\hline Void Fraction & 0.536 & $=$ & - & $6.00 \mathrm{E}-02$ & 0.217 & 0.737 & 0.743 \\
\hline Weter wt\% & 27.0 & - & - & 2.64 & 10.0 & 42.6 & 44.2 \\
\hline $\mathrm{TOC}$ wt\% C (wet & 0 & $=$ & - & 0 & 0 & 0 & 0 \\
\hline 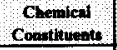 & nollo $/ \mathrm{L} \quad$ & Ppen $\quad \cdots$ & ks & $\begin{array}{l}98 \mathrm{Cl} \\
\text { (andl } \mathrm{L} \text { ) }\end{array}$ & $\begin{array}{l}67 \mathrm{Cl} \\
(\text { Wholol } \\
\end{array}$ & $\begin{array}{l}+67 \mathrm{Cl} \\
(\mathrm{mot}=\mathrm{L})\end{array}$ & $\begin{array}{l}+9 \mathrm{Cl} \\
(\operatorname{mol} \mathrm{L})\end{array}$ \\
\hline $\mathrm{Nat}$ & 8.13 & $1.06 \mathrm{E}+05$ & $1.41 E+03$ & 1.05 & 3.56 & 14.7 & 18.2 \\
\hline $\mathrm{Al3+}$ & 6.99 & $1.07 E+05$ & $1,43 \mathrm{E}+03$ & 6.58 & 6.74 & 7.15 & 7.15 \\
\hline $\mathrm{Fe} 3+$ (total Fe) & 101 & $3.22 \mathrm{E}+04$ & 428 & 0.994 & 1.00 & 1.02 & 1.03 \\
\hline $\mathrm{Cr} 3+$ & $6.19 \mathrm{E}-02$ & $1.83 \mathrm{E}+03$ & 24.3 & $7.09 \mathrm{E}-03$ & $3.39 \mathrm{E}-02$ & 0.522 & 1.06 \\
\hline Bist & 0 & 0 & 0 & 0 & 0 & 0 & 0 \\
\hline Let & 0 & 0 & 0 & 0 & 0 & 0 & 0 \\
\hline $\mathrm{Hg} 2+$ & 0 & 0 & 0 & 0 & 0 & 0 & 0 \\
\hline $2 x(a \mathrm{2x}(\mathrm{OOH}) 2)$ & 0 & 0 & 0 & 0 & 0 & 0 & 0 \\
\hline $\mathrm{Pb} 2+$ & 0 & 0 & 0 & 0 & 0 & 0 & 0 \\
\hline $\mathrm{Ni2}+$ & 5.07E-02 & $1.69 \mathrm{E}+03$ & 22.5 & $3.66 \mathrm{E}-02$ & $4.45 E-02$ & $5.54 \mathrm{E}-02$ & S.45E-02 \\
\hline $3+2+$ & 0 & 0 & 0 & 0 & 0 & 0 & 0 \\
\hline Mndt & 0 & 0 & 0 & 0 & 0 & 0 & 0 \\
\hline $\mathrm{Can}+$ & 0.220 & $5.02 \mathrm{E}+03$ & 66.6 & 0.146 & 0.182 & 0.258 & 0.294 \\
\hline $\mathrm{K}+$ & $1.12 E-02$ & 250 & 3.32 & 1.28E-03 & $5.25 \mathrm{E}-0.03$ & $1.52 \mathrm{E}-02$ & $1.52 E-02$ \\
\hline OH- & 25.6 & 2.48E+05 & $3.29 \mathrm{E}+03$ & 23.1 & 243 & 26.9 & 28.4 \\
\hline NO3- & 5.29 & $1.87 \mathrm{E}+05$ & $2.45+03$ & $6.03 \mathrm{E}-02$ & $6.03 \mathrm{E}-02$ & 13.5 & 17.9 \\
\hline NO2- & 1.46 & $3.22 \mathrm{E}+04$ & 507 & 0.132 & 0.595 & 2.15 & 2.15 \\
\hline Cos2- & 0.220 & $7.51 \mathrm{E}+03$ & 99.8 & 0.146 & $0.1 \mathrm{L2}$ & 0.258 & 0.294 \\
\hline $\mathrm{PO43}$ & 0 & 0 & 0 & 0 & 0 & 0 & 0 \\
\hline SO42- & 1.04E-02 & 36 & 7.56 & 1.19E-03 & $4.87 \mathrm{E}-93$ & $1.41 \mathrm{E}-02$ & 1.4JE-02 \\
\hline $\mathrm{Si}(\mathrm{ar} \mathrm{SiO} 2-\mathrm{)}$ & B.06E-03 & 129 & 1.71 & 9.21E-04 & 3.77E-03 & $1.09 \mathrm{E}-02$ & $1.09 E-02$ \\
\hline F. & 0 & 0 & 0 & $a$ & 0 & 0 & 0 \\
\hline c1- & $5.16 \mathrm{E}-02$ & $1.04 \mathrm{E}+03$ & 13.8 & S.91E-03 & 2.42E-02 & $7,00 \mathrm{E}-02$ & 0.157 \\
\hline C6HSO73- & 0 & 0 & 0 & 0 & 0 & 0 & 0 \\
\hline EDTAL & 0 & 0 & 0 & 0 & 0 & 0 & 0 \\
\hline HEDTA3 & 0 & 0 & 0 & 0 & 0 & 0 & 0 \\
\hline glyeohte- & 0 & 0 & 0 & 0 & 0 & 0 & 0 \\
\hline anetate- & 0 & 0 & 0 & 0 & 0 & 0 & 0 \\
\hline oxeninte?-2 & 0 & 0 & 0 & $\theta$ & 0 & a & 0 \\
\hline$\overline{D B P}$ & 0 & 0 & 0 & 0 & 0 & 0 & 0 \\
\hline buttenol & 0 & 0 & 0 & 0 & 0 & 0 & 0 \\
\hline $\mathrm{NH13}$ & 0.256 & $2.47 \mathrm{E}+03$ & 32.9 & $6.55 \mathrm{E}-02$ & $9.25 \mathrm{E}-02$ & $0.270^{\circ}$ & 0.284 \\
\hline $\mathrm{Fe}(\mathrm{CN}) 64$ & 0 & 0 & 0 & 0 & 0 & 0 & 0 \\
\hline
\end{tabular}

*Unknowns in tank solids inventory ere escigred by Tank Leyering Model (TLM). 


\begin{tabular}{|c|c|c|c|c|c|c|c|}
\hline \multirow[b]{3}{*}{$\begin{array}{l}\text { Poypich } \\
\text { Properties }\end{array}$} & \multicolumn{7}{|c|}{ Single-Shell Tunk 241-S-105 } \\
\hline & \multicolumn{7}{|c|}{ SMM Composite Inventory Estimste } \\
\hline & & & & $95 \mathrm{CI}$ & $67 \mathrm{Cl}$ & $+67 \mathrm{CI}$ & $+95 \mathrm{CL}$ \\
\hline Total SMM Wast & $2.46 \mathrm{E}+06(\mathrm{~kg})$ & (405 kgal) & 一 & $\ldots$ & $\ldots$ & - & - \\
\hline Heat Load & $2.59(\mathrm{kw})$ & (9.89E+03 BTU/mr) & 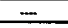 & 2.69 & 2.79 & 3.01 & 3.01 \\
\hline Bulk Density* & $1.61(g / \infty)$ & $\ldots$ & م- & 1.54 & 1.58 & 1.63 & 1.63 \\
\hline & & & & & & & \\
\hline Water wi\% & $\mathbf{3 4 . 2}$ & - & - & 32.4 & 33.1 & 35.9 & 38.5 \\
\hline TOC wt $\% \mathrm{C}$ (wet & 0.264 & - & - & 0.235 & 0.249 & 0.27 & 0.292 \\
\hline $\begin{array}{l}\text { Chenificl } \\
\text { Conntutueato }\end{array}$ & mole/L & pem & $x=$ & $\begin{array}{l}95 \mathrm{Cl} \\
\text { (nolwh }\end{array}$ & $\begin{array}{l}67 \mathrm{CI} \\
(\mathrm{mole} / \mathrm{L})\end{array}$ & $\begin{array}{c}+67 \mathrm{CI} \\
(\mathrm{mbl} / \mathrm{L})\end{array}$ & (mostal \\
\hline $\mathrm{Net+}$ & $\begin{array}{r}13.9 \\
\end{array}$ & $1992+05$ & 4. $50 \mathrm{E}+0.5$ & 12.4 & 13.2 & 14.4 & 14.6 \\
\hline Al34 & 1.97 & $3.32 E+04$ & $8.17 E+04$ & 1.70 & 1.83 & 2.13 & 2.03 \\
\hline Fe3+ (total Fe) & 8.26E-03 & 287 & 707 & $6.92 \mathrm{E}-03$ & $7,58 \mathrm{E}-03$ & $8,93 E-03$ & $9.59 \mathrm{E}-03$ \\
\hline $\mathrm{Crr}^{3+}$ & 0.204 & $6.59 \mathrm{E}+03$ & $1.62 \mathrm{E}+04$ & 0.147 & 0.178 & 0.209 & 0.213 \\
\hline Bi3t & $7.36 \mathrm{E}-04$ & 95.7 & 236 & $6.61 \mathrm{E}-04$ & $6.97 \mathrm{E}-04$ & 2.74E-04 & 8.11E-04 \\
\hline Lent & 9.36E-10 & $8.09 \mathrm{E}-05$ & 1.99E-04 & $7.17 \mathrm{E}-10$ & 8.25E-10 & 1.05E-09 & $1.16 \mathrm{E}-09$ \\
\hline $\mathrm{Hg} 2+$ & 6. I $14-06$ & 0.767 & 1.09 & $3.75 \mathrm{E}-06$ & $5.9 \pi-06$ & $6.23 \mathrm{E}-06$ & 6.31E-06 \\
\hline $\mathrm{Z}(\mathrm{a} \mathrm{ZrO}(\mathrm{OH}) 2)$ & 1.5AE-04 & 8.72 & 21.5 & 1.40E-04 & 1.45E-04 & $1.59 \mathrm{E}-04$ & $1.6 \mathrm{TE}-0.4$ \\
\hline $\mathrm{Pb} 2+$ & $7.23 \mathrm{E}-04$ & 93.2 & 230 & $5.900-04$ & $6.60 \mathrm{E}-04$ & $7, \mathrm{~B} 6 \mathrm{E}-04$ & 8.47E-04 \\
\hline $\mathrm{Ni} 2+$ & $5.575-03$ & 203 & 501 & $5.35 \mathrm{E}-03$ & S.45E-03 & $5.68 E-03$ & $5.78 E-03$ \\
\hline $\mathrm{Si} 2+$ & 0 & 0 & 0 & 0 & 0 & 0 & 0 \\
\hline $\mathrm{Mn4t}$ & 2.30E-03 & 81.6 & 201 & 1.75E-03 & $2.06 \mathrm{E}-03$ & 2.71E-03 & $3.03 \mathrm{E}-03$ \\
\hline Con+ & 2.81E-02 & 709 & $1.75 E+03$ & 242E-02 & $2.63 \mathrm{E}-02$ & $3,06 \mathrm{E}-02$ & $3.26 \mathrm{E}-02$ \\
\hline $\mathrm{K}^{+}$ & $5,80 \mathrm{E}-02$ & $1.41 E+03$ & 3. $4 \mathrm{GE}+03$ & $5.13 \mathrm{E}-02$ & $5.45 E-02$ & $6.20 \mathrm{E}-12$ & $6.22 \mathrm{E}-02$ \\
\hline OH- & 11.9 & $1.26 \mathrm{E}+05$ & 3.JOE+0S & 10.2 & 11.0 & 12.9 & 12.3 \\
\hline NO3- & 470 & $1.81 \mathrm{E}+05$ & 4.47E+05 & 4.26 & 4.48 & 4.88 & 4.88 \\
\hline NO2- & 2.62 & $7.50 \mathrm{E}+04$ & $1.85 \mathrm{E}+05$ & 2.25 & 2.46 & 2.76 & 2.90 \\
\hline CO32- & 0.234 & $8.74 E+03$ & 2.15E+od & 0.212 & 0.219 & 0.253 & 0.235 \\
\hline POA3- & $5.2 \pi-02$ & 3.11E+03 & $7.67 \mathrm{E}+03$ & $4.028-02$ & 4.85E- 02 & $5,41 \mathrm{E}-02$ & $5.54 \mathrm{E}-02$ \\
\hline SO42: & 0.161 & $9.60 \mathrm{E}+03$ & $2.36 \mathrm{E}+04$ & 0.125 & 0.142 & 0.180 & 0.196 \\
\hline Si (s Sio32-) & $6.27 \mathrm{E}-02$ & $1.10 \mathrm{E}+03$ & $2.70 \mathrm{E}+03$ & $5.36 E-02$ & $5,80 \mathrm{E}-02$ & $6.74 E-02$ & 7.19E-02 \\
\hline F- & $3.52 \mathrm{E}-02$ & 417 & $1.03 E+03$ & 2.87E-02 & $3.14 \mathrm{E}-02$ & $3.85 E-02$ & $4.10 E-02$ \\
\hline Cl- & 0.236 & $5.19 \mathrm{E}+03$ & $1.28 E+04$ & 0.204 & 0.219 & 0.253 & 0.249 \\
\hline C6HSO73. & I.71E-02 & $2.01 E+03$ & $4,96 E+03$ & 1.58E-02 & $1.64 \mathrm{E}-02$ & $1.782-02$ & 1.84E-02 \\
\hline EDTA4- & 1.92E-03 & 34 & $\begin{array}{r}846 \\
\end{array}$ & 9.55E-04 & 1.42E-03 & 2.41E-03 & 2.89E-03 \\
\hline HEDTA2 2 & $3.07 \mathrm{E}-03$ & 523 & $1.29 \mathrm{E}+03$ & $1.15 E-03$ & $2.06 \mathrm{E}-03$ & 4.06E-03 & $5,02 E-03$ \\
\hline glyediate- & 2.65E-02 & $1.24 E+03$ & $3.04 E+03$ & $1.53 \mathrm{E}-02$ & 2.08E-02 & $3.22 \mathrm{E}-02$ & $3.76 \mathrm{E}-02$ \\
\hline coctate- & $2.46 \mathrm{E}-03$ & $\$ 0.4$ & $\mathbf{2 3}$ & $1.99 \mathrm{E}-03$ & $2.22 \mathrm{E}-03$ & 2.70E-03 & $293 \mathrm{E}-03$ \\
\hline oxalinte2. & $1.23 \mathrm{E}-09$ & $6.72 \mathrm{E}-05$ & $1.65 \mathrm{E}-04$ & 1.11E-09 & $1.17 \mathrm{E}-09$ & $1.29 \mathrm{E}-09$ & $1.34 \mathrm{E}-69$ \\
\hline DBP & 1.19E-02 & $1.55 E+03$ & 3.83E+03 & 9.63E-03 & $1.07 \mathrm{E}-02$ & 1.30E-02 & $1.41 \mathrm{E}-02$ \\
\hline butenol & 1.19E-02 & 54: & $1.35 \mathrm{E}+03$ & 9.63E-03 & 1.07E-02 & $1.30 E-02$ & I.41E-02 \\
\hline $\mathrm{NH3}$ & $0.80 E-02$ & 728 & $1.79 \mathrm{E}+03$ & S.85E-02 & $6.26 E-02$ & $7.68 \mathrm{E}-02$ & B.6] $\mathrm{E}-02$ \\
\hline $\mathrm{Fe}(\mathrm{CN}) 64$ & 0 & 0 & 0 & 0 & 0 & 0 & 0 \\
\hline
\end{tabular}

-Density is calculated based on Na, OH-, and AO2.

$\uparrow$ Water wt\% derived from the difference of denaity and total diswolved species. 
HNF-SD-WM-ER-352, Rev. 1

\begin{tabular}{|c|c|c|c|c|c|c|c|}
\hline \multirow{4}{*}{$\begin{array}{l}\text { Poynical } \\
\text { Properties }\end{array}$} & \multicolumn{7}{|c|}{ Single-Shell Tank 241-\$-105 } \\
\hline & \multicolumn{7}{|c|}{ Total Inventory Estimate } \\
\hline & & & & & & & \\
\hline & & & & $-95 \mathrm{CI}$ & $-67 \mathrm{CI}$ & $+67 \mathrm{CI}$ & $+98 \mathrm{CI}$ \\
\hline Total Waste & $2.48 \mathrm{E}+06(\mathrm{~kg})$ & $(407 \mathrm{kgah})$ & $\ldots$ & - & - & $=$ & $\rightarrow$ \\
\hline Heat Load & $2.94(\mathrm{kw})$ & (1.00E+04 BrU/hr) & - & 2.73 & 2.83 & 3.06 & 3.06 \\
\hline Bulk Dersity & $1.61( \pm / \infty)$ & - & - & 1.54 & 1.58 & 1.63 & 1.63 \\
\hline Water wt\%t & $\mathbf{3 4 2}$ & $\ldots$ & & & & & \\
\hline & 0262 & $=$ & $\cdots$ & 32.3 & 33.0 & 359 & 38.6 \\
\hline 100 w\% c (wet) & 0.202 & - & - & 0.233 & 0.247 & $0.2 \pi$ & 0.290 \\
\hline $\begin{array}{l}\text { Choputical } \\
\text { Contutituethts }\end{array}$ & noterL & ppen & Le : & $\begin{array}{c}95 \mathrm{Cl} \\
(\text { moleh })\end{array}$ & $\begin{array}{c}67 \mathrm{CI} \\
(\text { wolla) }\end{array}$ & $\begin{array}{l}+67 \mathrm{CI} \\
(\mathrm{mole} / \mathrm{L})\end{array}$ & $\begin{array}{l}+95 \mathrm{Cl} \\
\text { (noled } 1)\end{array}$ \\
\hline $\mathrm{Nat+2}$ & $\begin{array}{r}13.9 \\
\end{array}$ & $1.99 \mathrm{E}+05$ & $4.92 \mathrm{E}+05$ & 12.3 & 13.2 & 14.4 & 14.5 \\
\hline $\mathrm{Al} 3+$ & 2.00 & $3.36 \mathrm{E}+04$ & $8.31 \mathrm{E}+0 \mathrm{~d}$ & 1.72 & 185 & 2.16 & 2.05 \\
\hline $\mathrm{Fe}^{3}+($ total Fe) & $1.32 E-02$ & 459 & $1.13 \mathrm{E}+03$ & $1.19 E-02$ & $1.25 E-02$ & 1.39E- -02 & 1.45E-02 \\
\hline $\mathrm{Cr} 3+$ & 0.203 & $6.56 \mathrm{E}+03$ & $1.63 \mathrm{E}+0 \mathrm{~d}$ & 0.152 & 0.179 & 0.209 & 0.212 \\
\hline Bis+ & $7.32 E-0-4$ & 95.2 & 236 & $6.57 \mathrm{E}-04$ & $6.94 \mathrm{E}-04$ & $7.70 \mathrm{E}-04$ & 8.07E-04 \\
\hline Les+ & $9.32 \mathrm{E}-10$ & B.0SE-05 & $1990-4$ & $7.14 \mathrm{E}-10$ & $8.20 \mathrm{E}-10$ & $1.042-09$ & 1.15E-09 \\
\hline $\mathrm{Hg} 2+$ & 6.11E-06 & 0.763 & 1.89 & $5.72 E-06$ & $5.95 \mathrm{E}-06$ & $6.20 \pm-06$ & $6.28 \mathrm{E}-06$ \\
\hline $2 \pi(\omega \mathrm{ZO}(\mathrm{OH}) 2)$ & $1.53 \mathrm{E}-04$ & 8.67 & 21.5 & 1.39E-04 & 1.44E-04 & $1.588-04$ & $1.66 \mathrm{E}-04$ \\
\hline $\mathrm{Pb} 2+$ & $7.19 \mathrm{E}-0 \mathrm{M}$ & 92.7 & 230 & S.96E-04 & $6.56 \mathrm{E}-04$ & $7,82 \mathrm{E}-04$ & 8.43E-04 \\
\hline $\mathrm{Ni2+}$ & 5.79E-03 & 211 & 523 & $5.598-03$ & $5.69 \mathrm{E}-03$ & $5.89 E-03$ & 5.98E- -03 \\
\hline $\mathrm{s} 2+$ & 0 & 0 & 0 & 0 & 0 & 0 & 0 \\
\hline Mn4t & 2.37E- -93 & 81.2 & 201 & $1.74 E-03$ & 2.05E- 03 & 2.70E- -03 & $3.01 \mathrm{E}-03$ \\
\hline $\mathrm{Ce} 2+$ & 2.94E-02 & 733 & $1.81 \mathrm{E}+03$ & $2.55 E-02$ & $2.74 \mathrm{E}-02$ & $3.13 \mathrm{E}-02$ & $3.32 \mathrm{E}-02$ \\
\hline $\mathrm{K}+$ & $5.78 \mathrm{E}-02$ & $1.41 E+03$ & $3.48 \mathrm{E}+03$ & $5.11 \mathrm{E}-02$ & 5.43E-02 & $6.17-02$ & 6.19E-02 \\
\hline $\mathrm{OH}-$ & 12.0 & $1.27 E+05$ & $3.13 E+05$ & $\begin{array}{r}10.2 \\
-\quad 10 \\
\end{array}$ & 11.1 & 12.9 & 12.4 \\
\hline NO3. & 4.71 & J.82E+05 & 4.95E+05 & 4.33 & 491 & $\$ .85$ & 4.66 \\
\hline $\mathrm{NO}_{2-}$ & 2.61 & $7.4 \mathrm{E}+04$ & $1.85 E+05$ & 2.25 & 2.46 & 2.75 & 2.89 \\
\hline CO32- & 0.234 & 6.73E+03 & 2.16E+04 & 0.212 & 0.219 & 0.252 & 0.255 \\
\hline PO43. & $5.24 E-02$ & $3.10 \mathrm{E}+03$ & $7.6 \pi+03$ & 4.60E-02 & ABRE- O2 & $538 \mathrm{E}-02$ & $5.52 \mathrm{E}-02$ \\
\hline SO42. & 0.160 & $9.55 \mathrm{E}+03$ & $2.30 E+04$ & 0.125 & 0.141 & 0.179 & 0.195 \\
\hline $\mathrm{Si}$ (as $\mathrm{SiO} 32$ ) & $6.246-02$ & $1.09 \mathrm{E}+07$ & $270 E+03$ & $5.33 E-02$ & $578 \mathrm{E}-02$ & $6.71 \mathrm{E}-02$ & $7.16 \mathrm{E}-02$ \\
\hline F- & 3.51E-02 & 415 & $1.03 E+03$ & 2.86E-02 & $3.13 E-02$ & $3.83 E-02$ & $4.08 \mathrm{E}-02$ \\
\hline $\mathrm{Cl}$ & 0.235 & $5.17 E+03$ & $1.28 \mathrm{E}+04$ & 0.204 & 0.218 & 0.252 & 0.248 \\
\hline $\mathrm{C} 6 \mathrm{HSO} 73$ & $1.70 \mathrm{E}-02$ & 2.DOE +03 & 4.96E+03 & $1.57 \mathrm{E}-02$ & $1.64 \mathrm{E}-02$ & 1.T7E-02 & $1.83 \mathrm{E}-02$ \\
\hline EDTA4 & 1.91E-03 & 30 & 845 & 9.51E-A4 & 1.42E-03 & $2.40 \mathrm{E}-03$ & $2.88 \mathrm{E}-03$ \\
\hline HEDTA & $3.05 \mathrm{E}-03$ & 520 & $1.29 \mathrm{E}+03$ & $1.14 E-03$ & $2.07 E-03$ & 4.04E-03 & 5.00E-03 \\
\hline Rifycolinto- & $2.63 \mathrm{E}-02$ & $1.23 \mathrm{E}+03$ & $304 \mathrm{E}+03$ & 1.53E- -02 & 2.07E-02 & $3.20 \mathrm{E}-02$ & 3.74E-02 \\
\hline acetalte- & $2.45 E-03$ & 89,9 & 223 & 1.99E-03 & $2.21 \mathrm{E}-03$ & $2.69 \mathrm{E}-03$ & $2.91 \mathrm{E}-03$ \\
\hline oxalate?- & $1.22 E-09$ & 6.68E-05 & 1.65ed & $1.10 \mathrm{E}-09$ & $1.16 \mathrm{E}-09$ & $1.28 E-99$ & $134 \mathrm{E}-09$ \\
\hline DBP & $1.18 E-02$ & $1.55 \mathrm{E}+03$ & $3.03 E+03$ & $9 . \sec -03$ & $1.0 \mathrm{TE}-02$ & $1.29 \mathrm{E}-02$ & $1,40 \mathrm{E}-02$ \\
\hline butanol & $1.18 E-02$ & 345 & $1.35 \mathrm{E}+03$ & 9.5OE-03 & $1.07 \mathrm{E}-02$ & $1.29 \mathrm{E}-02$ & $1.40 \mathrm{E}-02$ \\
\hline $\mathrm{NH3}$ & $6.97 \mathrm{E}-12$ & 737 & $183 \mathrm{E}+03$ & $5.95 \mathrm{E}-02$ & $6.35 \mathrm{E}-\mathrm{OH}$ & $776 \mathrm{E}-02$ & $869 \mathrm{E}-0$ \\
\hline $\mathrm{Fe}(\mathrm{CN}) 64$ & 0 & 0 & 0 & 0 & 0 & 0 & 0 \\
\hline
\end{tabular}

"Unlonowns in tank solida inventory ere maigrod by Tonk Leyering Model (TLM).

fWater wh\% derived from the difference of denvity and totel dienolved spocies. 


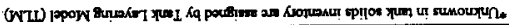

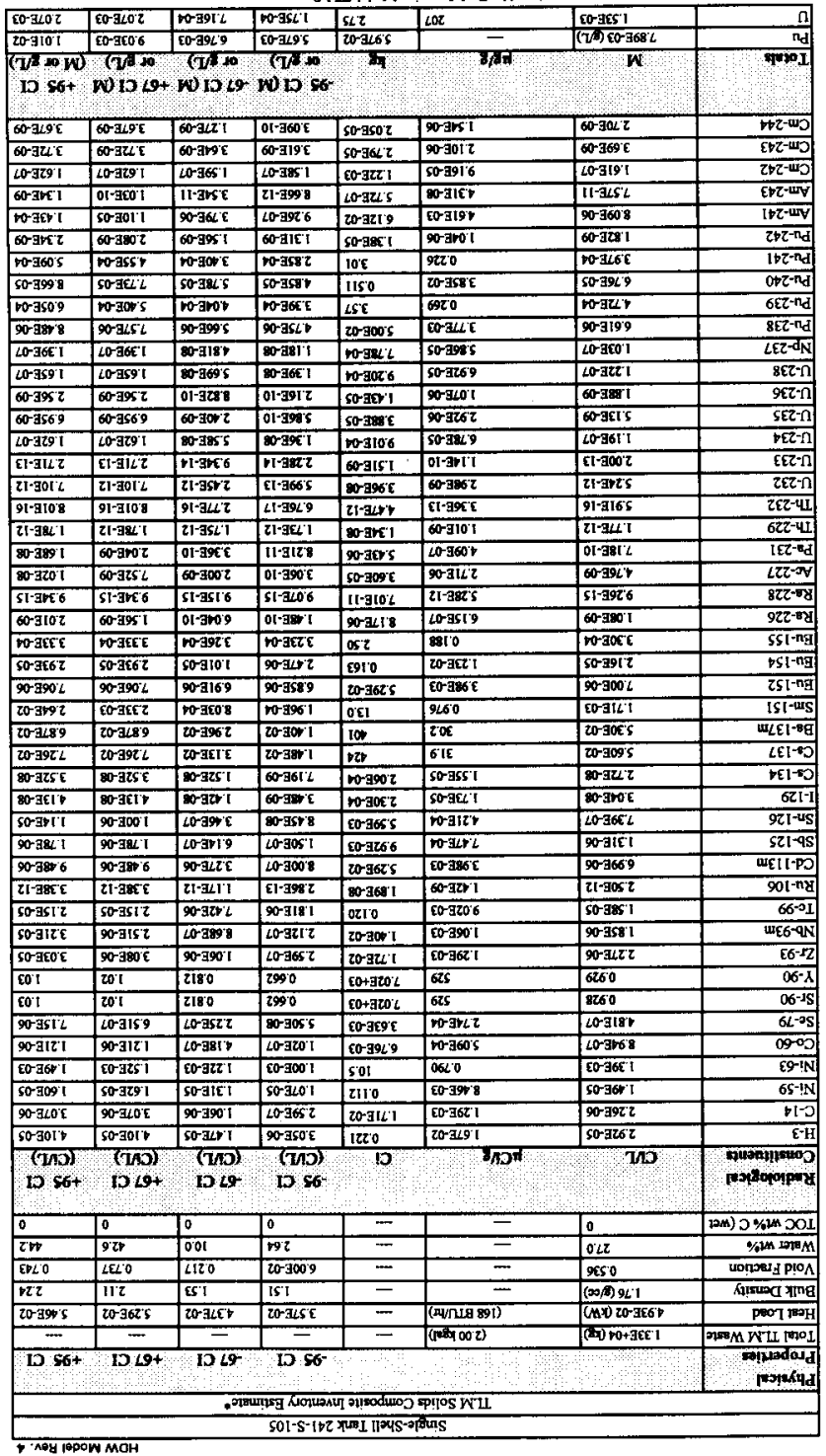


HNF-SD-WM-ER-352, Rev. 1

HDW Model Rev. 4

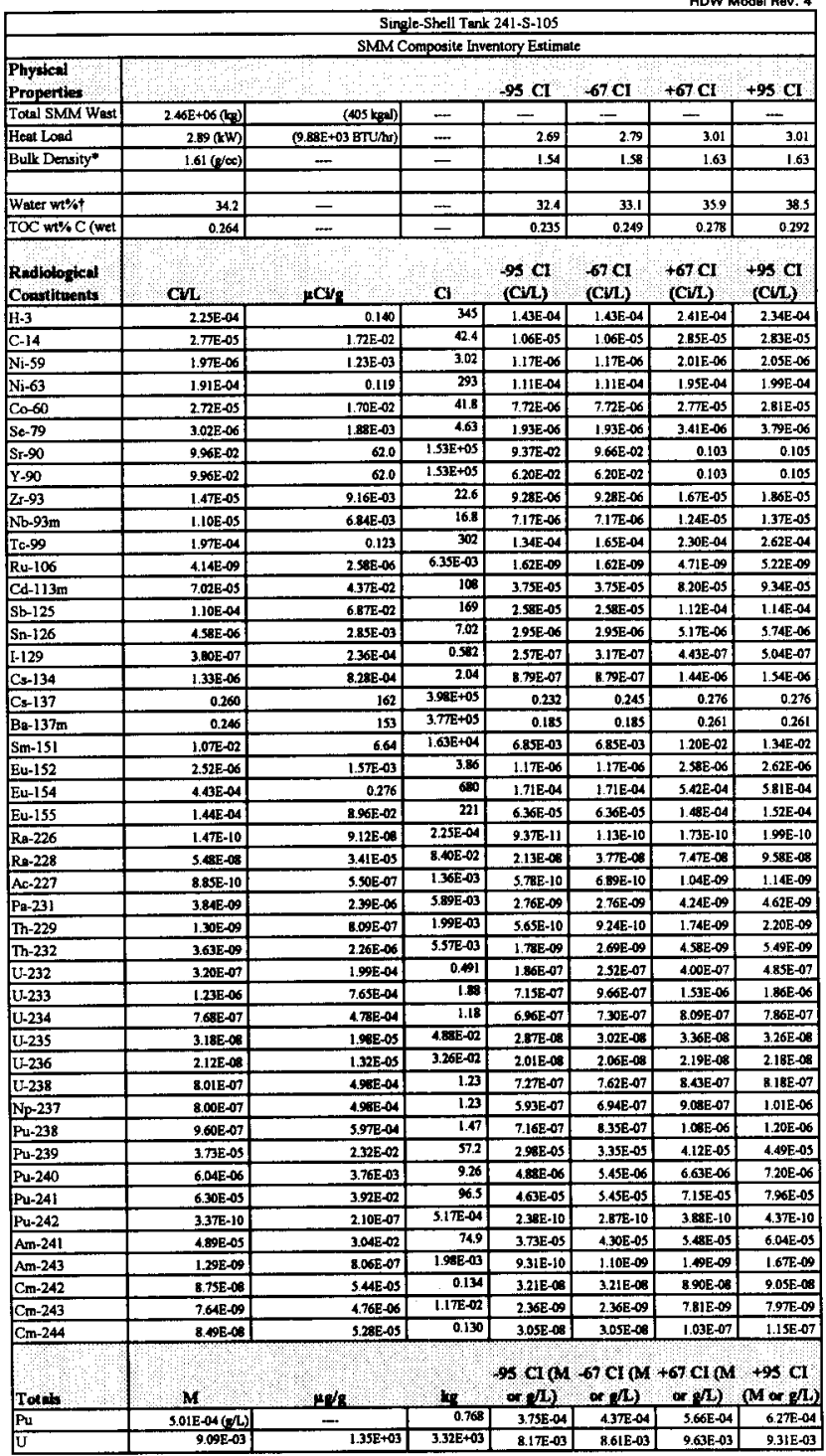

Density is calculated besed on Na, OH-, and AlO2-

tWoter wt\% derived from the difference of dersity and total diwolved species 
HNF-SD-WM-ER-352, Rev. 1

\begin{tabular}{|c|c|c|c|c|c|c|c|}
\hline \multicolumn{8}{|c|}{ Single-Shell Tank 241-S-105 } \\
\hline & \multicolumn{7}{|c|}{ Tots! Inventory Estimate" } \\
\hline Propertiles & & & & $95 \mathrm{CI}$ & -6701 & $+67 \mathrm{CI}$ & $+95 \mathrm{CI}$ \\
\hline Total Waste & $2.48 \mathrm{E}+0.6(\mathrm{~kg})$ & $(407 \mathrm{k} 1)$ & - & $\ldots$ & $\ldots$ & - & - \\
\hline Heas Lond & $2.94(\mathrm{~kW})$ & $(1.00 \mathrm{E}+04 \mathrm{BT} / \mathrm{hr})$ & - & 2.73 & 2.83 & 3.06 & 3.06 \\
\hline Bulk Density ${ }^{\prime}$ & $1.61(g / \infty)$ & $\ldots$ & - & 1.54 & 1.58 & 1.63 & 1.63 \\
\hline Water wh\% & 34.2 & $\ldots$ & - & 32.3 & 33.0 & 35.9 & 38.6 \\
\hline TOC wt\% C (wet & 0.262 & 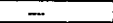 & - & 0.233 & 0.247 & 0.277 & 0.290 \\
\hline $\begin{array}{l}\text { Rodiolod dea } \\
\text { Contituents }\end{array}$ & cul & \#CUs: & $\mathrm{cl}$ & (c) & $\begin{array}{l}67 \mathrm{ct} \\
\text { (CnL) }\end{array}$ & $\begin{array}{l}+67 \mathrm{Cl} \\
\text { (CVL) }\end{array}$ & $\begin{array}{l}+95 \mathrm{cl} \\
\text { (CU) }\end{array}$ \\
\hline $\mathrm{H}-\mathbf{3}$ & 2.24E-04 & 0.139 & 345 & $1.43 \mathrm{E}-04$ & $1.43 \mathrm{E}-04$ & $2.40 \mathrm{E}-04$ & $2.33 E-04$ \\
\hline C-14 & $2.75 E-05$ & $1.71 \mathrm{E}-02$ & 4.4 & $1.06 \mathrm{E}-05$ & $1.06 \mathrm{E}-0 \mathrm{~S}$ & $2.83 E-05$ & $2.82 E-05$ \\
\hline Ni-59 & $2.03 E-06$ & $1.2 T E-03$ & 3.13 & $1.24 \mathrm{E}-06$ & $1.24 \mathrm{E}-06$ & $2.0 \pi-06$ & $2.11 \mathrm{E}-06$ \\
\hline Ni-63 & $1.9 \pi \mathrm{E}-04$ & 0.122 & 303 & $1.18 \mathrm{E}-04$ & 1.18E-OS & $2.01 E-04$ & $205 E-04$ \\
\hline $\cos 60$ & 2.7IE-05 & $1.69 \mathrm{E}-02$ & 41.8 & $7.69 E-06$ & $7.69 \mathrm{E}-66$ & $2.76 \mathrm{E}-05$ & $2.80 \mathrm{E}-05$ \\
\hline Se-79 & $3.01 E-06$ & $1.87 \mathrm{E}-03$ & 4.63 & $1.92 E-06$ & $1.92 \mathrm{E}-\infty 6$ & $3.40 E-06$ & $37 \mathrm{TE}-06$ \\
\hline Sr-90 & 0.104 & 64.5 & $1.60 \mathrm{E}+05$ & $9.83 E-02$ & 0.101 & 0.106 & 0.109 \\
\hline$Y-90$ & 0.104 & 04.5 & $1.60 \mathrm{E}+05$ & $6.62 \mathrm{E}-02$ & $6.62 \mathrm{E}-02$ & 0.106 & 0.109 \\
\hline Zr-93 & $1.4 \pi-05$ & $9.12 E-03$ & 22.6 & $9.24 E-06$ & $9.24 E-06$ & $1.66 \mathrm{E}-05$ & $1.85 \mathrm{E}-0.5$ \\
\hline $\mathrm{Nb}-93 \mathrm{~m}$ & $1.09 \mathrm{E}-05$ & $6.81 E-03$ & 16.9 & $7.15 E-06$ & $7.15 E-06$ & $1.23 E-05$ & 1.36E-05 \\
\hline Tc-99 & $196 \mathrm{E}-\mathrm{OA}$ & 0.122 & 303 & 1.33E-04 & $1.64 E-04$ & $2.29 \mathrm{E}-04$ & $2.60 \mathrm{E}-04$ \\
\hline $\mathrm{Ru}-106$ & 4. $12 \mathrm{E}-09$ & $2.56 E-06$ & $6.355-03$ & $1.61 \mathrm{E}-09$ & $1.61 E-09$ & 4.68E-09 & $5.19 E-09$ \\
\hline Cd-113m & $6.99 \mathrm{E}-05$ & 4.35E-02 & 108 & $3.74 E-05$ & $3.74 E-05$ & $8.1 \pi-05$ & $9.29 \mathrm{E}-05$ \\
\hline$S b-125$ & $1.10 \mathrm{E}-04$ & $6.83 \mathrm{E}-02$ & 16 & $2.57 \mathrm{E}-\mathrm{OS}$ & $257 \mathrm{E}-05$ & $1.12 \mathrm{E}-04$ & $1.14 \mathrm{E}-0 \mathrm{al}$ \\
\hline $\mathrm{s}_{\mathrm{n}-126}$ & 4.56E- -06 & 2.0ME-03 & 7.02 & $2.93 \mathrm{E}-06$ & $2.93 E-06$ & $5.15 E-06$ & $5.71 \mathrm{E}-06$ \\
\hline I-129 & 3.78E-07 & $2.35 E-64$ & 0.562 & $2.56 E-07$ & $3.15 E-07$ & 4.4IE-07 & $5.02 \mathrm{E}-07$ \\
\hline Cs. 134 & $1.32 \mathrm{E}-06$ & $8.24 E-04$ & 2.04 & 8.75E-07 & 8.75E-07 & $1,43 E-06$ & $1.53 \mathrm{E}-06$ \\
\hline Cs-137 & 0.259 & 161 & 3.96E+05 & 0.231 & 0.24 & 0.275 & 0.273 \\
\hline $\mathrm{Ba}-137 \mathrm{~m}$ & 0.245 & 152 & 3.77t+05 & 0.185 & 0.185 & 0.260 & 0.260 \\
\hline $\mathrm{Sm}-151$ & $1.06 \mathrm{E}-02$ & 6.60 & $1.64 \mathrm{E}+0 \mathrm{~A}$ & $6.83 \mathrm{E}-03$ & $6.83 \mathrm{E}-03$ & $1.20 \mathrm{E}-02$ & $1.33 \mathrm{E}-02$ \\
\hline Eu-152 & 2.5AE- 06 & $1.56 \mathrm{E}-03$ & 3.91 & $1.20 E-06$ & $1.20 \mathrm{E} .06$ & $2.60 \mathrm{E}-006$ & $2.64 \mathrm{E}-06$ \\
\hline Eu-154 & A.AIE-AN & 0.275 & 600 & 1.70E-AS & $1.70 \mathrm{E}-9$ & $5.39 \mathrm{E}-04$ & $57 \pi \mathrm{E}-04$ \\
\hline Eu-155 & 1.45E-04 & $9.01 \mathrm{E}-02$ & 223 & $6.49 \mathrm{E}-05$ & $6.49-05$ & $1,49 \mathrm{E}-04$ & $1.52 \mathrm{E}-04$ \\
\hline$R=-226$ & 1.51E-10 & $9.40 \mathrm{E}-08$ & $2.33 \mathrm{E}-04$ & $1.03 \mathrm{E}-10$ & $1.18 E \cdot 10$ & $1.76 \mathrm{E}-10$ & $1.99 \mathrm{E}-10$ \\
\hline $\operatorname{Ra-228}$ & $5.45 E-08$ & $3.39 \mathrm{E}-05$ & $8.00 E-02$ & $2.12 \mathrm{E}-08$ & $3.75 E-08$ & $7.43 E-08$ & $9.54 \mathrm{E}-08$ \\
\hline Ac-227 & 9.04E-10 & $3,62 E-07$ & $1.39 \mathrm{E}-03$ & $0.25 E-10$ & $7.09 \mathrm{~B}-10$ & 1.05E-09 & $1.13 E-09$ \\
\hline $\mathrm{Pa}-231$ & $3.83 E-09$ & $2.36 E-06$ & $5.90 \mathrm{E}-03$ & $2.75 E-09$ & $2.73 E-09$ & $4.22 E-09$ & $4.60 E-09$ \\
\hline Th-229 & $1.29 \mathrm{E}-109$ & $8.05 E-07$ & $1.99 \mathrm{E}-03$ & $5.62 \mathrm{E}-10$ & $9.20 \mathrm{E}-10$ & 1.73E-09 & $2.19 \mathrm{E}-09$ \\
\hline$T h-232$ & $3.61 E-09$ & 2.25E-06 & 5.57E-03 & $1.77 \mathrm{E}-09$ & $2.67 \mathrm{E}-09$ & 4.56E-09 & $5.46 \mathrm{E}-09$ \\
\hline U.232 & 3.19E-01 & 1.9E-OA & 0.49 & 1.8SE-07 & 2.51E-07. & $3.96 E-07$ & $4.83 E-07$ \\
\hline U-233 & $1.22 \mathrm{E}-06$ & 7.61E-04 & 1.8 & 7.11E-07 & $9.61 \mathrm{E}-07$ & $1.53 \mathrm{E}-06$ & $1.85 E-06$ \\
\hline U-234 & $7.64 \mathrm{E}-07$ & 4.76E-04 & 1.18 & $6.93 \mathrm{E}-07$ & $7.27 \mathrm{E}-07$ & $8.05 E-07$ & $7.83 E-07$ \\
\hline U-235 & $3.1 \pi-08$ & $1.97 \mathrm{E}-65$ & 4.6NE-02 & $2.86 \mathrm{E}-08$ & 3.01E- -8 & 3.34E-08 & $3.24 E-08$ \\
\hline U-236 & $2.11 E-\infty 6$ & $1.328-05$ & $3.26 \mathrm{E}-02$ & $2.00 E-08$ & $206 \mathrm{E}-08$ & $2.18 E-08$ & $2.17 \mathrm{E}-08$ \\
\hline U-238 & $7.97 \mathrm{E}-07$ & 4.96E- -94 & 1.23 & $7.24 E-07$ & $7.59 \mathrm{E}-07$ & 8.39E-07 & B.15E-O7 \\
\hline Np-237 & $797:-07$ & $4.968-04$ & 1.23 & $5.90 \mathrm{DE}-07$ & 6.91E-07 & $9.04 E-07$ & $1.01 E-06$ \\
\hline$P u-238$ & $9.895-07$ & $6.14 E-04$ & 1.52 & $7.45 E-07$ & 8.64E-97 & L.11E- -06 & $1.23 \mathrm{E}-06$ \\
\hline Pu-239 & $3.95 E-05$ & $2.46 \mathrm{E}-02$ & 60.8 & $3.26 E-05$ & 3.60E-05 & 4.30E-05 & $4.63 \mathrm{E}-05$ \\
\hline $\mathrm{PL}-240$ & 6.31E-06 & $3.94 E-03$ & 9.7 & $5.18 E-06$ & $5.75 E-26$ & $6.93 \mathrm{E}-06$ & $730 \mathrm{E}-06$ \\
\hline Pu-241 & 6.46E-05 & $4.02 \mathrm{E}-02$ & 99.5 & 4.80E-05 & $5.61 E-05$ & $7.31 E-05$ & B.12E-05 \\
\hline$P L-242$ & $3.45 E-10$ & $2.14 \mathrm{E}-07$ & $3.31 E-04$ & $2.46 \mathrm{E}-10$ & $2.94 \mathrm{E}=10$ & 3.95E-10 & 4.44E-10 \\
\hline$A m-241$ & $4.8 \pi-03$ & $3.03 \mathrm{E}-02$ & 75.0 & $3.718-05$ & 1.28E-05 & $5.46 E-05$ & $6.02 \mathrm{E}-05$ \\
\hline Am-243 & $1.29 \mathrm{E}-09$ & $8.02 E-07$ & 1.99E-03 & $9.2 \mathrm{TE}-10$ & $1.10 E-09$ & $1.48 \mathrm{E}-09$ & $1.67 \pi-09$ \\
\hline$C_{m-242}$ & $8.7 E-\infty 8$ & 5.46E-03 & 0.135 & 3.2TE-08 & $3.27-08$ & 8.94E-06 & $9.08 \mathrm{E}-08$ \\
\hline $\mathrm{C}_{\mathrm{Cm}}-243$ & $7.63 \mathrm{E}-09$ & $4.74 E-06$ & $1.17 \overline{1702}$ & $2.37 E-09$ & $2.37 E-09$ & $7,79 E-09$ & $795 \mathrm{E}-09$ \\
\hline$C_{m}-244$ & 8.45E-08 & $5.26 \mathrm{E}-03$ & 0.130 & $3.03 E-00$ & $3.03 E-08$ & $1.03 E-07$ & I.15E-07 \\
\hline Tokds & $\mathbf{M}$ & $\mathrm{epd}$ & tre & $95 \mathrm{clM}$ & $67 \mathrm{ct}(\mathrm{M}$ & $+6 \mathrm{Cr}(\mathrm{M}$ & $\begin{array}{c}95 \mathrm{Cl} \\
\mathrm{M} \text { or } \mathrm{gl}\end{array}$ \\
\hline Pu & $3.38 \mathrm{E}-0 \mathrm{04}(\mathrm{g} / \mathrm{L})$ & - & 0.828 & $4.20 \mathrm{E}-04$ & 4.78E-0A & $5.97 \mathrm{E}-04$ & $6.55 E-04$ \\
\hline U & $9.06 \mathrm{E}-03$ & $1.34 E+03$ & $3.32 \mathrm{E}+03$ & $8.14 E-03$ & $8.57 \mathrm{E}-03$ & $9.58 E-03$ & $9.27 \mathrm{E}-03$ \\
\hline
\end{tabular}

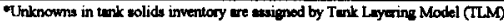

† Volume average for density, mase average Water wt\% and TOC wo\% C. 


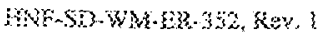

3
2
1
1

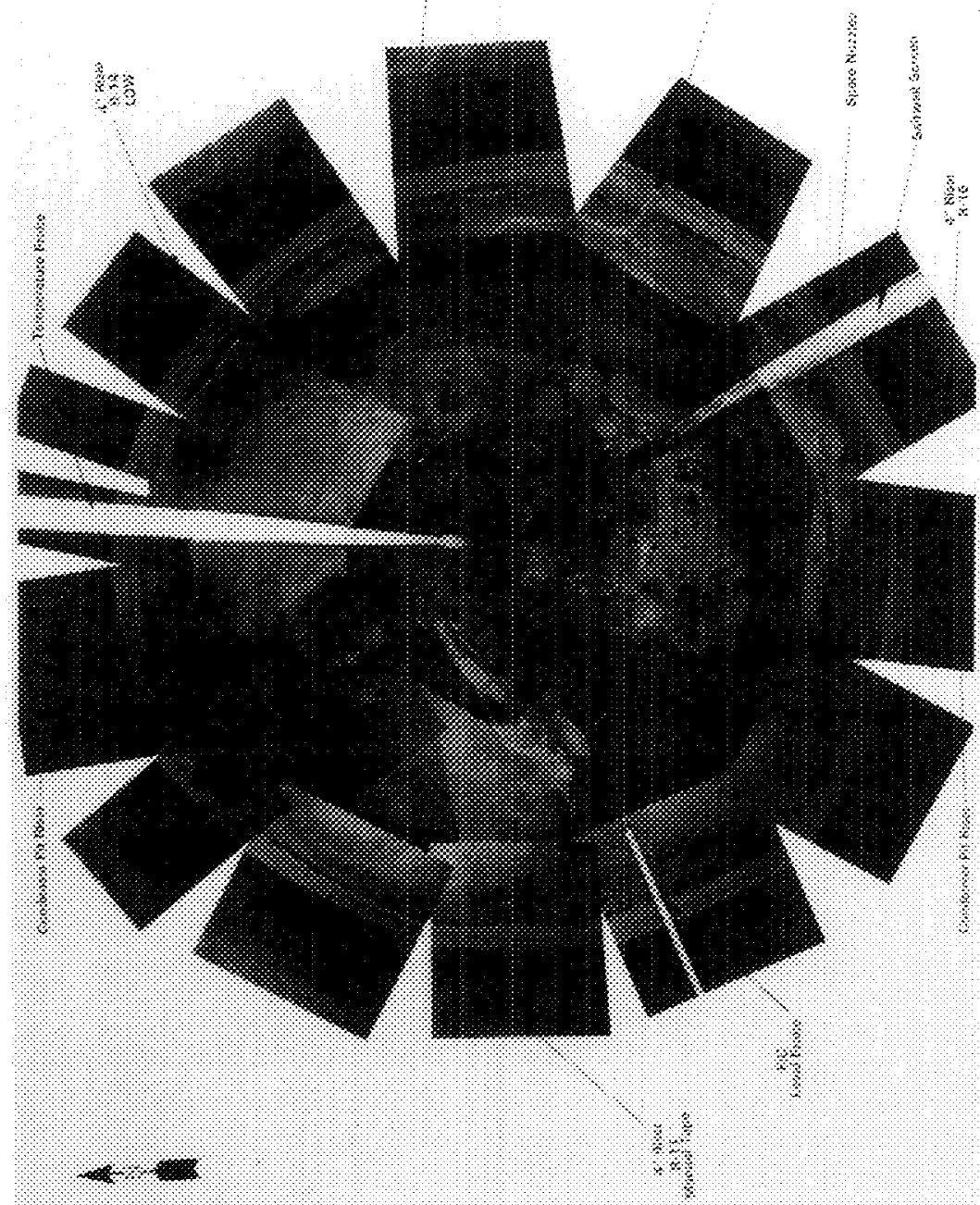


TANK 241-S-106 SUMMARY

\begin{tabular}{|c|c|c|c|}
\hline \multicolumn{2}{|c|}{ TANK HISTORY } & \multicolumn{2}{|c|}{ TANK DESCRIPTION } \\
\hline Entered Service & 2nd qtr 1953 & Diameter & $75 \mathrm{ft}$ \\
\hline Removed from Service & 1976 & Bottom Shape & Dish \\
\hline Inactive & - & Nominal Capacity & $758,000 \mathrm{gal}$ \\
\hline Watch Lists & none & Cascade Tank & none \\
\hline Integrity & Sound & Total Risers & 12 \\
\hline Assumed Leaker & - & \multicolumn{2}{|c|}{ WASTE VOLUME (HANLON 1996i) } \\
\hline Interim Stabilization (IS) & - & Total Waste Volume & $479,000 \mathrm{gal}$ \\
\hline Partial Interim Isolation (PI) & Dec 1982 & Waste Type & NCPLX \\
\hline Intrusion Prevention (IP) & - & Drainable Interstitial Liquids & $186,000 \mathrm{gal}$ \\
\hline \multicolumn{2}{|c|}{ TENTATIVELY AVAILABLE RISERS } & Pumpable Liquids & $168,000 \mathrm{gal}$ \\
\hline Riser Number(s) & Size & Saltcake & $447,000 \mathrm{gal}$ \\
\hline $11,14,16$ & 4 in & Sludge & $28,000 \mathrm{gal}$ \\
\hline 6,8 & 12 in & Supernatant & $4,000 \mathrm{gal}$ \\
\hline \multicolumn{2}{|c|}{ TANK TEMPERATURE } & \multicolumn{2}{|c|}{ INTERIOR PHOTOGRAPHS } \\
\hline Average Tank Temperature & $76^{\circ} \mathrm{F}$ & Date & March 17, 1989 \\
\hline Maximum Temperature & $87^{\circ} \mathrm{F}$ & Montage Number & $94041033-23 \mathrm{CN}$ \\
\hline Date & $\operatorname{Jan} 1,1992$ & Photo Set Number & 89032049 \\
\hline Elevation from tank bottom & $4.46 \mathrm{ft}$ & \multicolumn{2}{|c|}{ WASTE SURFACE LEVEL } \\
\hline Riser Number & 4 & Devices & Auto and Manual ENRAF \\
\hline Minimum Temperature & $62.06^{\circ} \mathrm{F}$ & Max Level & 181.06 in \\
\hline Date & March 19, $21 \& 24,1996$ & Date & Dec 13, 1995 \\
\hline Elevation from tank bottom & $16.46 \mathrm{ft}, 18.46 \mathrm{ft}$ & Min Level & 174.5 in \\
\hline Riser Number & 4 & Date & April 8 and 22,1991 \\
\hline
\end{tabular}




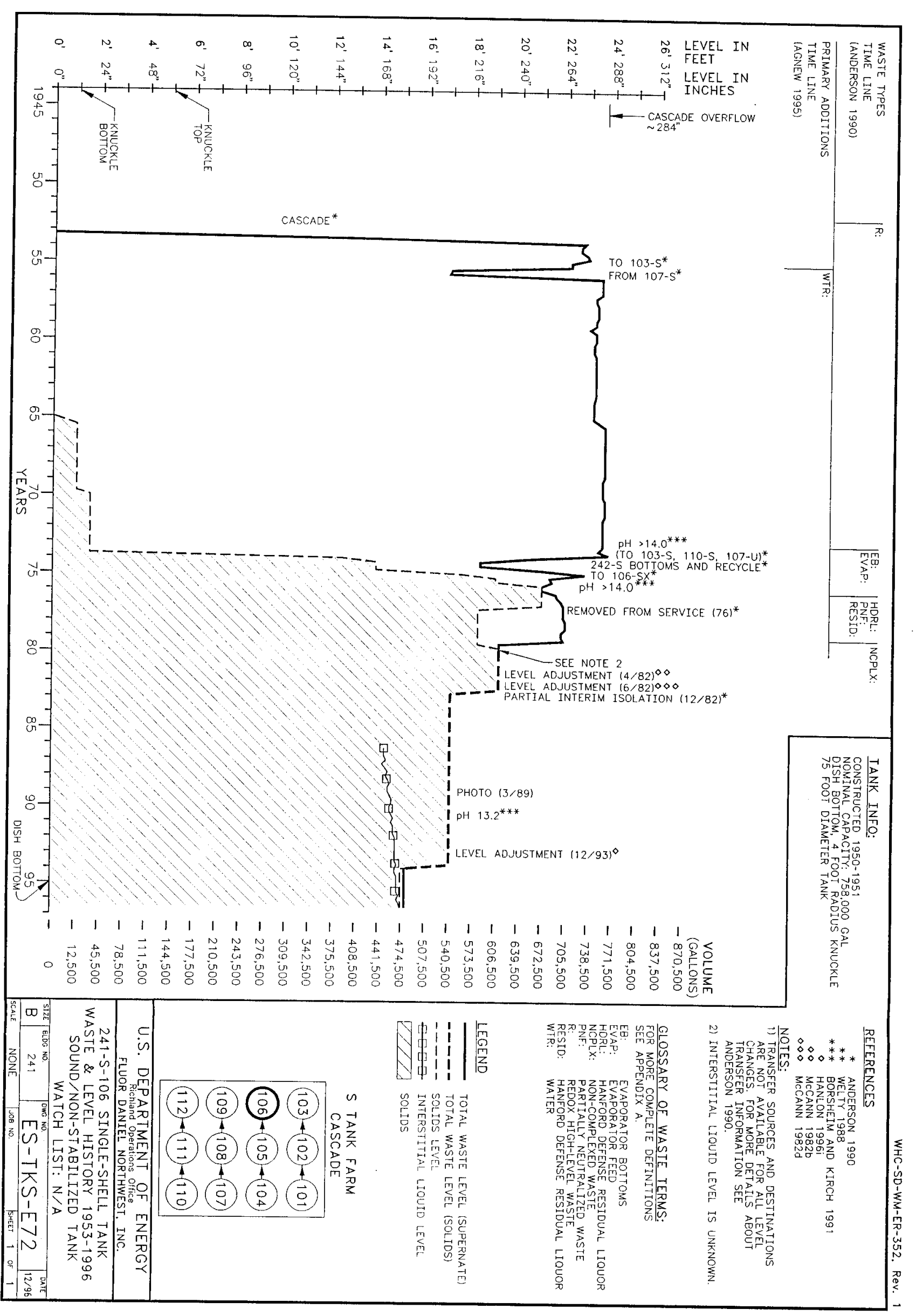




\section{$241-5-106$}

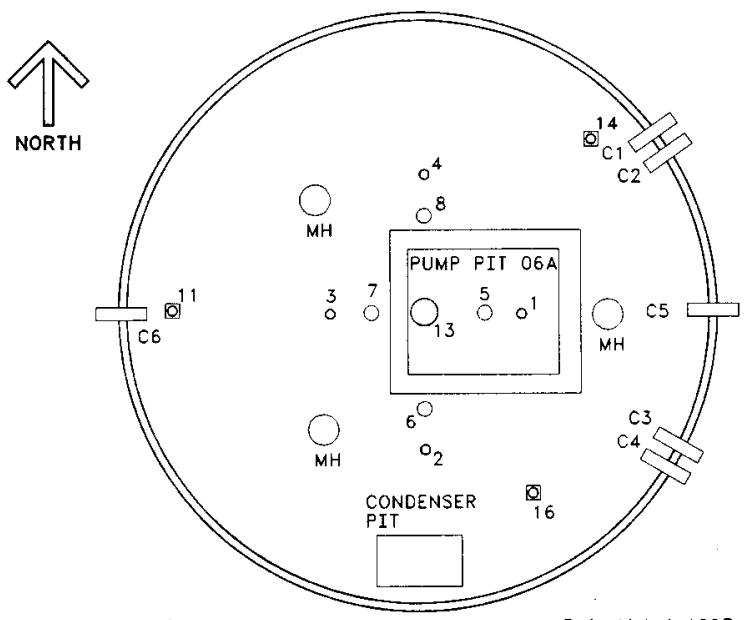

Ref: Alstad 1993

$\mathrm{H}-2-73185$. Rev. 4

$\mathrm{H}-2-37829$. Rev. 2

\section{TANK RISER LOCATION}

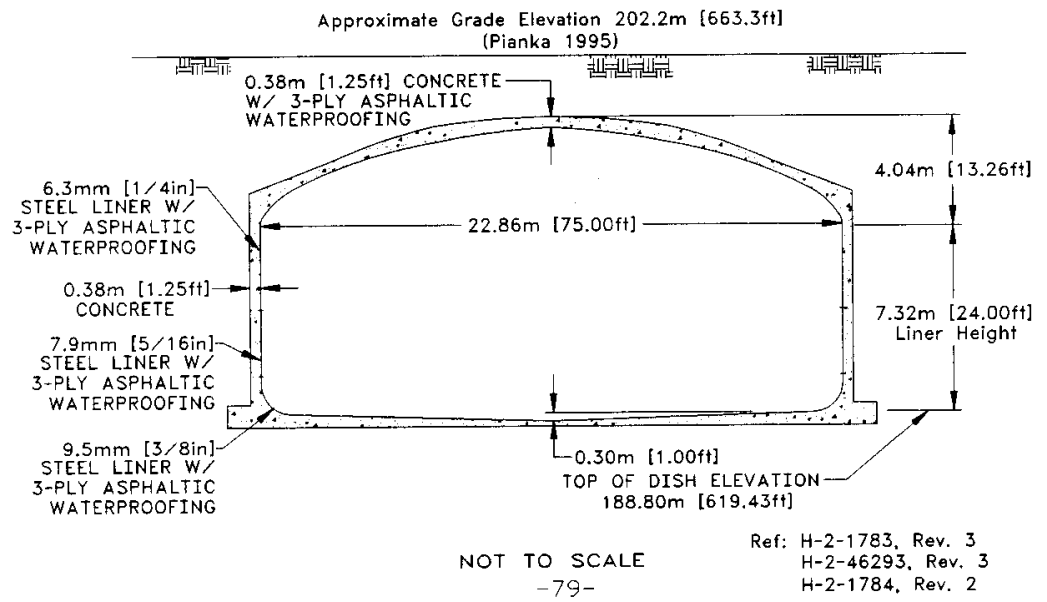




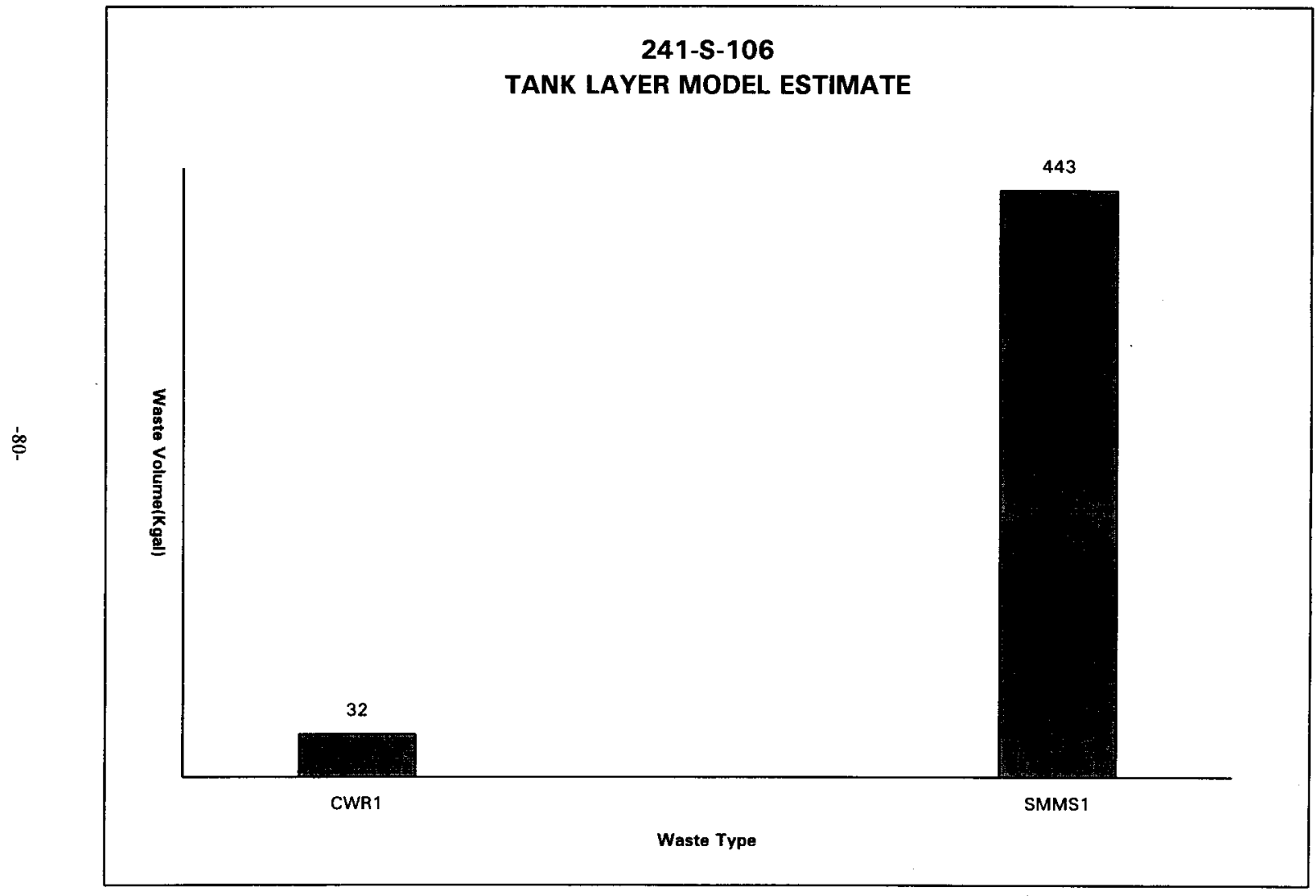


HDW Madgl Pav, 4

\begin{tabular}{|c|c|c|c|c|c|c|c|}
\hline & \multicolumn{7}{|c|}{ Single-Shell Tank 241-S-106 } \\
\hline & \multicolumn{7}{|c|}{ TLM Solids Composice Inventory Estimate* } \\
\hline Phycical & & & & & & & $\therefore$ \\
\hline Properties & & & & $95 \mathrm{CI}$ & $67 \mathrm{CI}$ & $+67 \mathrm{CI}$ & $+95 \mathrm{CI}$ \\
\hline Total TLM Waste & $2.15 \mathrm{E}+05(\mathrm{~kg})$ & $(32.0 \mathrm{kgal})$ & - & - & - & - & - \\
\hline Heat Load & $3.00 E-03(\mathrm{~kW})$ & $(10.3$ BTU/hr) & $=$ & $2.52 E-03$ & 2.90E-03 & $3.1 ! E-03$ & $3.20 E-03$ \\
\hline Bulk Density & $1.77(1 / \infty)$ & $=$ & - & 1.47 & 1.57 & 1.92 & 2.02 \\
\hline Void Fraction & 0.651 & $\ldots$ & - & 0.488 & 0.557 & 0.780 & 0.825 \\
\hline Water wt\% & 24.5 & - & $=$ & 4.37 & 12.2 & 45.5 & 55.1 \\
\hline TOC wt\%C (wet & 0 & + & - & 0 & 0 & 0 & 0 \\
\hline $\begin{array}{l}\text { Clented } \\
\text { Condtituents }\end{array}$ & moleh & pep $\quad \square$ & 40 & $\begin{array}{c}95 \mathrm{Cl} \\
(\mathrm{bolel})\end{array}$ & $\begin{array}{l}67 \mathrm{CI} \\
\text { (mald } \mathrm{L})\end{array}$ & $\begin{array}{l}+67 \mathrm{CI} \\
\text { (noterL) }\end{array}$ & $\begin{array}{l}+95 \mathrm{Cl} \\
\text { (molent) }\end{array}$ \\
\hline Not & 7.86 & $1.02 \mathrm{E}+03$ & $2.19 \mathrm{E}+04$ & 2.23 & 3.91 & 10.7 & 129 \\
\hline $\mathrm{All+}$ & 11.2 & $1.71 \mathrm{E}+05$ & $3.66 \mathrm{E}+04$ & 5.84 & 7.28 & 14.0 & 16.1 \\
\hline $\mathrm{Fe} 3+(\operatorname{total} \mathrm{Fe})$ & 0.165 & $5.20 E+03$ & $1.12 \mathrm{E}+03$ & 0.153 & 0.160 & 0.170 & 0.175 \\
\hline Cr3t & 2.04E-03 & 59.8 & 12.8 & 1.71E-03 & 1.97E-03 & $2.10 \mathrm{E}-03$ & 2.17E-03 \\
\hline Biz+ & 0 & 0 & 0 & 0 & 0 & 0 & 0 \\
\hline Let+ & $\begin{array}{r}0 \\
\end{array}$ & 0 & 0 & 0 & 0 & 0 & 0 \\
\hline $\mathrm{Hg} 2+$ & $4.08 \mathrm{E}-03$ & 462 & 99.1 & 4.02E- -03 & $4.06 \mathrm{E}-03$ & 4.10E-03 & 4.11E-03 \\
\hline $\mathrm{Z}_{\mathrm{S}(\mathrm{ns} \mathrm{ZO} \mathrm{OOH}) \mathrm{n})}$ & 0 & 0 & 0 & 0 & 0 & 0 & 0 \\
\hline $\mathrm{Pb} 2+$ & 0.118 & $1.38 E+04$ & 2.95E+03 & 0.108 & 0.113 & 0.122 & 0.126 \\
\hline $\mathrm{Ni2+}$ & $1.02 \mathrm{E}-03$ & 33.7 & 7.24 & $8.54 \mathrm{E}-04$ & 9.84E-04 & 1.5]E-03 & $3.79 \mathrm{E}-03$ \\
\hline $8 n+$ & 0 & 0 & 0 & 0 & 0 & 0 & 0 \\
\hline Mndt & 0 & 0 & 0 & 0 & 0 & 0 & 0 \\
\hline $\mathrm{Ca} 2+$ & 0.121 & $2.73 E+03$ & 586 & $6.72 \mathrm{E}-02$ & $9.87 \mathrm{E}-02$ & 0.141 & 0.161 \\
\hline $\mathrm{k+}$ & 1.54E-03 & 339 & 7,27 & 1.29E-03 & 1. $4 \mathrm{EE}-03$ & $1.59 E-03$ & $1.64 \mathrm{E}-03$ \\
\hline OH- & 41.7 & $4.00 \mathrm{E}+05$ & $8.59 \mathrm{E}+04$ & 20.0 & $\begin{array}{r}25.9 \\
\end{array}$ & 53.0 & 61.2 \\
\hline $\mathrm{NO}$ & 0.57 & $2.00 \mathrm{E}+04$ & $4.30 \mathrm{E}+03$ & 0.85 & 0.554 & 0.591 & 0.610 \\
\hline NO2- & 0.958 & 2. $69 \mathrm{E}+04$ & $5.345+03$ & 0.79 & 0.925 & 0.990 & 1.02 \\
\hline CO32- & 0.121 & $4.09 \mathrm{E}+03$ & 87 & $6.72 \mathrm{E}-02$ & 9.87E-02 & 0.141 & 0.151 \\
\hline PO43. & 0 & 0 & 0 & 0 & 0 & 0 & 0 \\
\hline$\$ 042$ & 8.39E-03 & 455 & 97.6 & 7.04E-03 & B. $10 \mathrm{E}-03$ & $8.67 \mathrm{E}-03$ & 8.94E-03 \\
\hline $\mathrm{Si}(\mathrm{as} \mathrm{SiO32-)}$ & $2.01 \mathrm{E}-02$ & 319 & 684 & $1.69 E-02$ & $1.94 \mathrm{E}-02$ & $8.18 \mathrm{E}-02$ & 0.175 \\
\hline F. & 0 & 0 & 0 & 0 & 0 & 0 & 0 \\
\hline c1. & $7.07 \mathrm{E}-03$ & 141 & 30.3 & 5,93E-03 & 6., $3 \mathrm{BE}-03$ & $7.30 \mathrm{E}-03$ & 7.53E-03 \\
\hline C6HSOT3 & 0 & 0 & 0 & 0 & 0 & 0 & 0 \\
\hline EDTA4 & 0 & 0 & 0 & 0 & 0 & 0 & 0 \\
\hline HEDTA3- & 0 & 0 & 0 & 0 & 0 & 0 & 0 \\
\hline Elycolnte- & 0 & 0 & 0 & 0 & 0 & 0 & 0 \\
\hline ecente- & 0 & 0 & 0 & 0 & 0 & 0 & 0 \\
\hline Gxalute2- & 0 & 0 & 0 & 0 & 0 & 0 & 0 \\
\hline $\mathrm{DBP}$ & 0 & 0 & 0 & 0 & 0 & 0 & 0 \\
\hline tuttanol & 0 & 0 & 0 & 0 & 0 & 0 & 0 \\
\hline $\mathrm{NH3}$ & $2.05 \mathrm{E}-04$ & 1.97 & 0.422 & $1.61 \mathrm{E}-04$ & $1.80 E-04$ & $2.38 \mathrm{E}-04$ & $2.49 E-04$ \\
\hline $\left.\mathrm{F}_{\mathrm{E}} \mathrm{CN}\right) 64$ & 0 & 0 & 0 & 0 & 0 & $\mathbf{0}$ & 0 \\
\hline
\end{tabular}

"Unkowns in tark colids inventory ue ssigred by Tark Layeting Model (TLM). 
HNF-SD-WM-ER-352, Rev. 1

HOW Model Rev. 4

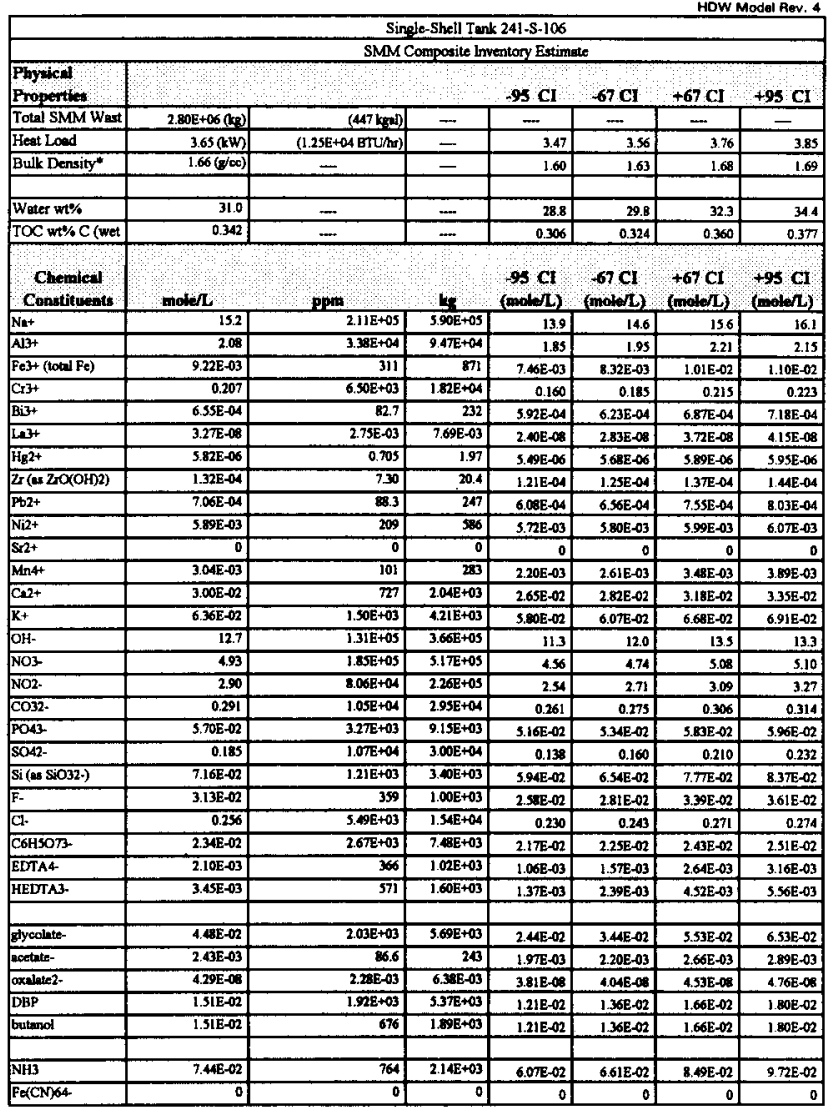

Density is calculated besed on $\mathrm{Na}$, OH-, and AJO2.

tWater wh\% derived from the difference of deraty and total dineolved apecies. 
HNF-SD-WM-ER-352, Rev. 1

HDW Model Rev. 4

\begin{tabular}{|c|c|c|c|c|c|c|c|}
\hline \multirow[b]{3}{*}{$\begin{array}{l}\text { Physical } \\
\text { Properties }\end{array}$} & \multicolumn{7}{|c|}{ Single-Shell Tank 241-S-106 } \\
\hline & \multicolumn{7}{|c|}{ Total Inventory Estimate* } \\
\hline & & $\therefore \therefore$ & & $95 \mathrm{CI}$ & $-67 C I$ & $+67 \mathrm{Cl}$ & $\begin{array}{l}+95 \mathrm{CI} \\
+\end{array}$ \\
\hline Total Waste & $3.02 \mathrm{E}+06(\mathrm{R})$ & $(479 \mathrm{kgal})$ & $\ldots$ & - & - & - & - \\
\hline Heat Loed & $3.66(\mathrm{~kW})$ & $(1.25 \mathrm{EE}+04 \mathrm{BTU} / \mathrm{hr})$ & . & 3.47 & 3.56 & 3.76 & 3.85 \\
\hline Bulk Densityt & $1.66(g / \infty)$ & $\ldots$ & $=$ & 1.61 & 1.64 & 1.68 & 1.70 \\
\hline Water wt\% & 30.6 & - & - & 28.5 & 29.3 & 32.0 & 33.6 \\
\hline TOC wt\% C (wet & 0.317 & - & $\rightarrow$ & 0.284 & 0.300 & 0.334 & 0.350 \\
\hline $\begin{array}{l}\text { Chomped } \\
\text { Conitituents }\end{array}$ & motal $\mathrm{L}$ & Dep $=$ & $k g$ & $\begin{array}{l}\text { ss Cl } \\
\text { (molef }\end{array}$ & $\begin{array}{l}67 \mathrm{CI} \\
(\mathrm{mol} / \mathrm{h})\end{array}$ & $\begin{array}{l}+67 \mathrm{CI} \\
(\mathrm{molel})\end{array}$ & $\begin{array}{l}+95 \mathrm{CI} \\
\text { (molets) }\end{array}$ \\
\hline Nat & 14.7 & $2.03 \mathrm{E}+0 \mathrm{~S}$ & $6.12 \mathrm{E}+05$ & 13.5 & 14.2 & 15.1 & 15.5 \\
\hline Allt & 2.69 & $4.36 \mathrm{E}+04$ & $1.31 \mathrm{E}+05$ & 2.31 & 2.43 & 2.86 & 2.99 \\
\hline $\mathrm{Fe} 3+(\operatorname{toth} \mathrm{Fe})$ & $1.95 E-02$ & 659 & $1.99 \mathrm{E}+03$ & $1.80 E-02$ & $1.88 E-02$ & $2.05 E-02$ & $2.13 E-02$ \\
\hline $\mathrm{Cr} 3+$ & 0.193 & $6.04 E+03$ & $1.82 \mathrm{E}+04$ & 0.150 & 0.173 & 0.201 & 0.208 \\
\hline $\mathrm{Bi}+$ & $6.11 \mathrm{E}-04$ & 76.8 & 232 & S.52E-04 & $5.81 \mathrm{E}-04$ & 6.41E-04 & $6,70 \mathrm{E}-04$ \\
\hline Le3+ & 3.05E-18 & 2.5SE-03 & $7.60 \mathrm{E}-03$ & $2.24 E-08$ & $2.64 \mathrm{E}-08$ & $3.47 \mathrm{E}-08$ & $3.87 \mathrm{E}-08$ \\
\hline $\mathrm{Hg} 2+$ & $2.78 \mathrm{E}-04$ & 33.5 & 101 & $2.74 \mathrm{E}-04$ & $2.76 \mathrm{E}-04$ & $279 \mathrm{E}-04$ & $2.80 \mathrm{E}-04$ \\
\hline $2 x(\div 25$ (OH)2 $)$ & $1.24 \mathrm{E}-04$ & 6.78 & 20.4 & $1.13 \mathrm{E}-04$ & $1.17 \mathrm{E}-04$ & 1.28E-04 & 1.34E-04 \\
\hline $\mathrm{Pb2+}$ & 8.52E-03 & $1.06 E+03$ & $3.20 \mathrm{E}+03$ & 7.68E-03 & $8.26 \mathrm{E}-49$ & $8.73 \mathrm{E}-03$ & 8.98E-03 \\
\hline $\mathrm{Ni2+}$ & $5.57 \mathrm{D}-03$ & 197 & 393 & $5,40 \mathrm{E}-03$ & $5.48 \mathrm{E}-03$ & $5.65 \mathrm{E}-03$ & 5.78E-03 \\
\hline $8 \times 2+$ & 0 & 0 & 0 & 0 & 0 & 0 & 0 \\
\hline Mn4t+ & 2.84E-03 & 93.8 & 283 & 2.05E-03 & $2.44 E-03$ & $3.24 \mathrm{E}-03$ & $3.63 E-03$ \\
\hline Ca2t & $3.61 \mathrm{E}-02$ & 869 & $2.62 \mathrm{E}+03$ & $3.25 \mathrm{E}-02$ & $3.44 \mathrm{E}-02$ & $3.7 \mathrm{E}-02$ & 3.93E-02 \\
\hline $\mathrm{K}+$ & 5.95E-02 & $1.40 \mathrm{E}+03$ & $4.22 \mathrm{E}+03$ & $5.42 \mathrm{E}-02$ & $5.67 \mathrm{E}-02$ & $6.25 \mathrm{E}-02$ & 6.46E-02 \\
\hline$\overline{\mathrm{OH}}-$ & 14.7 & I.5OE+05 & $4.52 \mathrm{E}+05$ & 13.2 & $13 \mathrm{~T}$ & 15.4 & 15.9 \\
\hline NO3- & 4.64 & $1.7 \mathrm{E}+0 \mathrm{~S}$ & $5.22 \mathrm{E}+0 \mathrm{~s}$ & 4.30 & 4.46 & $4 \pi$ & 480 \\
\hline NO2- & 27 & $7.67 \mathrm{E}+04$ & $2.31 E+05$ & 2.43 & 2.59 & 2.95 & 3.11 \\
\hline $\cos 2-$ & 0.279 & $1.01 \mathrm{E}+04$ & $3.04 \mathrm{E}+04$ & 0.252 & 0.265 & 0.294 & 0.301 \\
\hline $\mathrm{PO43}$ & $5.32 \mathrm{E}-02$ & 3.04E+03 & $9.15 E+03$ & $4.8] E-02$ & $4.99 \mathrm{E}-02$ & $5,44 E-02$ & $5.56 \mathrm{E}-02$ \\
\hline SO42- & 0.173 & $9.98 \mathrm{E}+03$ & $3.01 \mathrm{E}+04$ & 0.129 & 0.150 & 0.19 & 0.217 \\
\hline Si (as SiO32-) & $6.81 \mathrm{E}-02$ & $1.15 E+03$ & $3.47 \mathrm{E}+03$ & $5.68 E .02$ & $6.24 \mathrm{E}-02$ & $7.39 \mathrm{E}-02$ & $7,94 \mathrm{E}-02$ \\
\hline F. & $292 \mathrm{E}-02$ & 333 & $1.00 \mathrm{E}+03$ & $240 \mathrm{E}-02$ & $2.62 \mathrm{E}-02$ & 3. 17E-02 & $3.37 \mathrm{E}-02$ \\
\hline Cl. & 0.240 & $3.11 \mathrm{E}+03$ & $1.54 E+04$ & 0.216 & 0.227 & 0.254 & 0.256 \\
\hline C6HSO73 & $2.18 \mathrm{E}-02$ & $2.48 \mathrm{E}+03$ & $7,48 E+03$ & $2.02 \mathrm{E}-02$ & $2.10 \mathrm{E}-02$ & $2.26 \mathrm{E}-02$ & $2,34 \mathrm{E}-02$ \\
\hline EDTA4 & $1.96 \mathrm{E}-05$ & 340 & $1.02 \mathrm{E}+03$ & $9.93 \mathrm{E}-04$ & $1.47 \mathrm{E}-03$ & $2.46 \mathrm{E}-03$ & 2.95E-03 \\
\hline HEDTA3- & 3.22E-03 & 531 & $1.60 \mathrm{E}+03$ & $1.28 \mathrm{E}-03$ & $2.22 \mathrm{E}-03$ & $4.22 \mathrm{E}-03$ & $5.19 \mathrm{E}-03$ \\
\hline glycolate- & $4.18 \mathrm{E}-02$ & $1.89 \mathrm{E}+03$ & $5.69 \mathrm{E}+03$ & $2.28 E-02$ & 3.21E-02 & 5.16E-02 & $6.09 \mathrm{E}-02$ \\
\hline cectute- & $2.27 \mathrm{E}-03$ & 80.5 & 243 & 1BAE-03 & $2.05 E-03$ & $2.49 \mathrm{E}-03$ & $2.70 \mathrm{E}-03$ \\
\hline oxalute2- & $400 \mathrm{E}-0$ & $2.12 \mathrm{E}-03$ & 6.3EE-03 & $3.56 \mathrm{E}-08$ & $3.77 \mathrm{E}-\mathrm{OB}$ & 4.23E- $A B$ & 4.44E-08 \\
\hline DBP & $1.41 \mathrm{E}-02$ & $1.7 \mathrm{RE}+03$ & $5.37 \mathrm{E}+03$ & 1.13E-02 & $1.27 \mathrm{E}-02$ & $1.55 \mathrm{E}-02$ & $1.68 \mathrm{E}-02$ \\
\hline butanol & 1.41E-02 & 623 & $1.89 \mathrm{E}+03$ & $1.13 E-02$ & $1.27 \mathrm{E}-02$ & $1.55 \mathrm{E}-02$ & $1.68 \mathrm{E}-02$ \\
\hline $\mathrm{NH3}$ & $6.94 \mathrm{E}-02$ & 710 & $2.14 \mathrm{E}+03$ & $5.6 \pi \mathrm{E}-02$ & 6.1TE-D2 & $7.92 \mathrm{E}-02$ & $9.0 \pi \mathrm{e}-02$ \\
\hline $\mathrm{Fe}(\mathrm{CN}) 64$ & 0 & 0 & 0 & 0 & 0 & 0 & 0 \\
\hline
\end{tabular}


HNF-SD-WM-ER-352, Rev. 1

HDW Model Rev, 4

\begin{tabular}{|c|c|c|c|c|c|c|c|}
\hline \multirow[b]{3}{*}{$\begin{array}{l}\text { Physical } \\
\text { Properties }\end{array}$} & \multicolumn{7}{|c|}{ Single-Shell Tank 241-S-106 } \\
\hline & \multicolumn{7}{|c|}{ TLM Solids Componite Inventory Estimate* } \\
\hline & $\because \quad \therefore$ & & & $49 \mathrm{CI}$ & $-67 \mathrm{CI}$ & $+67 \mathrm{CI}$ & $+95 \mathrm{Cl}$ \\
\hline Total TLM Waste & $2.15 E+05(\mathrm{~kg})$ & (32.0 kgal) & $=$ & - & $=$ & - & - \\
\hline Heet Load & $3.00 \mathrm{E}-03(\mathrm{~kW})$ & $(10.3 \mathrm{BTU} / \mathrm{hr})$ & - & 2.52E-03 & $2.90 \mathrm{E}-03$ & $3.11 \mathrm{E}-03$ & $3.20 \mathrm{E}-03$ \\
\hline Bulk Density & $1.7(g / \infty)$ & - & - & t.47 & 1.57 & 1,92 & 2.02 \\
\hline Void Fraction & 0.651 & - & - & 0.488 & 0.557 & 0.780 & 0.825 \\
\hline Water wt\% & 245 & 一 & - & 4.37 & 12.2 & 45.5 & 55.1 \\
\hline $\mathrm{TOC}$ wt\% C (wet & 0 & - & - & 0 & 0 & 0 & 0 \\
\hline $\begin{array}{l}\text { Rodbalotes } \\
\text { Constltutents }\end{array}$ & $c t h$ & $\mathrm{nc} / \mathrm{s}$ & $\mathbf{C}$ & $\begin{array}{l}95 \mathrm{Cl} \\
\text { (Cllt) }\end{array}$ & $\begin{array}{l}67 \mathrm{cl} \\
(\mathrm{C}) \mathrm{L})\end{array}$ & $\begin{array}{c}+67 \mathrm{Cl} \\
(\mathrm{CU}) \mathrm{L})\end{array}$ & $\begin{array}{l}+95 \mathrm{Cl} \\
\text { (CYL) }\end{array}$ \\
\hline $\mathrm{H}-3$ & 1.01E-06 & S.7LE-04 & 0.123 & $6.33 \mathrm{E}-07$ & $8.90 E-07$ & $1.13 \mathrm{E}-06$ & 1.24E-06 \\
\hline C-14 & 1.09E-07 & 6.16E-05 & $1.32 \mathrm{E}-02$ & $9.15 \mathrm{E}-08$ & 1.0SE-07 & 1.13E-07 & 1.16E-07 \\
\hline $\mathrm{Ni}-59$ & $300 \mathrm{E}-08$ & $1.75 \mathrm{E}-05$ & 3.75E-03 & 2.S9E-08 & $2.99 \mathrm{E}-0 \mathrm{8}$ & $4.60 \mathrm{E}-08$ & 1.15E-07 \\
\hline $\mathrm{Ni}-63$ & 290E-06 & 1.64E-03 & 0.351 & 2.43E-06 & $2.00 \mathrm{E}-06$ & 4.31E-06 & 1.00E-05 \\
\hline $\mathrm{Co}-60$ & $4.71 \mathrm{E}-0 \mathrm{~s}$ & $2.66 \mathrm{E}-0 \mathrm{~S}$ & $5.71 \mathrm{E}-03$ & $3.96 \mathrm{E}-08$ & 4.56E-08 & 4.97E-08 & $5.02 E-08$ \\
\hline Se-79 & 2.37E-08 & $1.34 E-05$ & 2.8TE-03 & $1.990-08$ & $2.29 \mathrm{E}-0 \mathrm{~g}$ & 2.45E-08 & 2.53E-08 \\
\hline Sr-90 & 2.05E-03 & 1.16 & 248 & 1.72E-03 & 1.99E-03 & 2.12E-03 & 2.18E-03 \\
\hline Y-90 & 2.05E-03 & 1.16 & 248 & $1.72 E-03$ & 1.9EE-03 & 2.12E.03 & 2.18E-03 \\
\hline 2r.93 & 1.12E-07 & $6.34 E-05$ & 1.36E- 02 & $9,42 E-68$ & 1.09E-07 & 1.16E-0? & 1.20E-07 \\
\hline Nb-93m & 9.13E-08 & 5.15E-05 & 1.1JE-02 & $7.66 \mathrm{E}-08$ & B.82E-08 & 9,43E-08 & 9.73E.08 \\
\hline Tc-99 & 7.84E-07 & 4.4.2E-04 & 9. $4 \mathrm{E}-02$ & $6.5 \pi \mathrm{E}-07$ & $7.57 \mathrm{E}-07$ & $8.10 \mathrm{E}-07$ & $8.35 \mathrm{E}-07$ \\
\hline Ru-106 & 2.76E-13 & $1.56 \mathrm{E}-10$ & 3.34E-00 & 2.31E-13 & $2.66 \mathrm{E}-13$ & $2.85 \mathrm{E}-13$ & $2.94 \mathrm{E}-13$ \\
\hline Cd-113m & 3.51E-07 & $1.90 \mathrm{E}-04$ & 4.25E-02 & $2.94 \mathrm{E}-07$ & 3.39E-07 & 3.62E-07 & $3.74 E-07$ \\
\hline $5 b-125$ & $7.89 \mathrm{E}-48$ & 4.46E-05 & 9.56E-03 & $6.62 \mathrm{E}-0 \mathrm{G}$ & $7.63 \mathrm{E}-08$ & B.16E-08 & 8.41E-08 \\
\hline Sn-126 & $3.61 \mathrm{E}-08$ & 2.04E-05 & 430-03 & $3.03 \mathrm{E}-03$ & 3.49E-08 & 1.7. -08 & $3.85 \mathrm{E}-0 \mathrm{~s}$ \\
\hline $\mathrm{I}-129$ & $1.50 \mathrm{E}-\infty$ & $8.47 \mathrm{E}-07$ & I.,R2E-04 & $126 \mathrm{E}-09$ & $1.45 E-09$ & $1.55 \mathrm{E}-09$ & $1.60 \mathrm{E}-09$ \\
\hline Cs-134 & 1.65E-09 & 9.34E-07 & $200 \mathrm{E}-04$ & $1.39 \mathrm{E}-09$ & $1.60 \mathrm{E}-09$ & 1.71E-09 & $1.76 \mathrm{E}-09$ \\
\hline Cs-137 & 2.35E-03 & 1.33 & 255 & $1.97 \mathrm{E}-03$ & $2.27 E-03$ & 2.43E-03 & $2.50 E-03$ \\
\hline $\mathrm{Be}-137 \mathrm{~m}$ & 2.22E-03 & 1.26 & 269 & $1.87 \mathrm{E}-03$ & 2.15E-03 & $2.30 E-03$ & $2.3 \%-03$ \\
\hline Sm-151 & 4.47E-05 & 4.7BE- -02 & 10.3 & $7.10 E-05$ & B.18E-OS & .75E-05 & $9.02 \mathrm{E}-05$ \\
\hline $\mathrm{Eu}-152$ & $1.75 \mathrm{E}-07$ & 9.89E-05 & $2.12 \mathrm{E}-02$ & 1.74E-07 & $1.75 E-07$ & $1.76 \mathrm{E}-07$ & $1.76 \mathrm{E}-07$ \\
\hline Eu-154 & $1.13 E-06$ & 6.48E-04 & 0.139 & $9.63 \mathrm{E}-07$ & $1.11 E-06$ & 1.19E- -06 & $1.22 \mathrm{E}-06$ \\
\hline Eu-155 & B.33E- -06 & 4.7JE-03 & 1.01 & $8.27 \mathrm{E}-06$ & $8.32 \mathrm{E}-\infty 6$ & 8.15E-06 & 8.36E-06 \\
\hline Ro-226 & 2.83E-12 & 1.00E-09 & 3.42E-07 & $2.37 \mathrm{E}-12$ & 2.73E-12 & $2.92 E-12$ & $3.01 \mathrm{E}-12$ \\
\hline Ra-228 & 2.52E-16 & 1.65E-13 & 3.5.E-H & $2.90 \mathrm{E}-16$ & 2.91E-16 & $2.92 \mathrm{E} \cdot 16$ & $2.93 \mathrm{E}-16$ \\
\hline$A c-227$ & $1.45 \mathrm{E}-11$ & 8.19E-09 & $1.76 \mathrm{E}-06$ & 1.22E-11 & 1. $40 \mathrm{E}-11$ & 1.50E-11 & 1.55E-11 \\
\hline $\mathrm{Pa}-231$ & $3.44 E+11$ & $1.94 \mathrm{E}-08$ & $4.175-06$ & 2.89E-11 & 3.32E-11 & $3.55 \mathrm{E} \cdot 11$ & $3.67 \mathrm{E}-11$ \\
\hline Th-229 & 4.13E-14 & 2.33E-11 & 5.00E-09 & $4.09 \mathrm{E}-14$ & 4.12E-14 & $4.13 \mathrm{E}-14$ & 4.14E-14 \\
\hline Th-232 & 404E-17 & 2.28E- 14 & 40E-12 & 3.39E- 17 & 3.90E-17 & $41 \pi-17$ & $4.30 \mathrm{E}-17$ \\
\hline U-232 & $6.4 \pi-10$ & 3.65E-07 & 7,BE-05 & $5.62 \mathrm{E}-10$ & $6.12 \mathrm{E}-10$ & $6.728-10$ & $690 \mathrm{E}-10$ \\
\hline U-233 & 2.39E-11 & $1.35 \mathrm{E}-0$. & 2,90506 & 2.0BE-11 & 2.26E-11 & $2.49 \mathrm{E}-11$ & $2.55 \mathrm{E}-11$ \\
\hline U.234 & $1.48 \mathrm{E}-03$ & 8.36E-03 & 179 & 1.29E-0S & 1. $10 \mathrm{E}-05$ & 1.S4E-0S & $1.58 E-05$ \\
\hline U.235 & 6.29E-07 & 3.5SE-04 & $7,625-02$ & $5.46 \mathrm{E}-07$ & $5.95 \mathrm{E}-07$ & $6.53 \mathrm{E}-07$ & $6.71 \mathrm{E}-07$ \\
\hline $\mathrm{U}-236$ & $3.26 \varepsilon-07$ & 1.84E-04 & 3.95E-O2 & 2.83E-07 & 3.08E-07 & $3.39 \mathrm{E}-07$ & 3.48E-07 \\
\hline U-238 & 1.44E-05 & 8.14E-03 & 1.73 & 1.25E-0S & 1.36E-05 & 1.508-05 & 1.54E-05 \\
\hline $\mathrm{Np}-237$ & $5.48 E-09$ & $3.09 E-06$ & 6.64E-04 & 4.60E-09 & $5.30 \mathrm{E}-09$ & $5.6 \%-09$ & S.84E-09 \\
\hline Pu-238 & 2.51E-05 & 1. $22 \mathrm{E}-02$ & 3.04 & 2.40E-0S & 2.45E-05 & $2.57 \mathrm{E}-03$ & $2.62 \mathrm{E}-05$ \\
\hline Pu-239 & 1.61E-03 & 0.908 & 193 & 1.S4E-03 & $1.57 \mathrm{E}-03$ & $1.64 E-03$ & $1.68 \mathrm{E}-03$ \\
\hline Pu.240 & $2.27=-04$ & 0.128 & 27.5 & $2.17 E-04$ & 2.22E-04 & $2.325-04$ & $2.37 \mathrm{E}-04$ \\
\hline Pu-241 & $1.39 \mathrm{E}-03$ & 0.782 & 168 & 1.32E-03 & 1.35E-03 & $1.42 E-03$ & $1.45 \mathrm{E}-03$ \\
\hline Pu-242 & 5.BPE-09 & $3.32 \mathrm{E}-06$ & $7,122-04$ & $3.62 \mathrm{E}-09$ & $5.75 \mathrm{E}-09$ & $6.012-09$ & $6.14 E-09$ \\
\hline Am-241 & 4.09E-07 & 2.31E-04 & 4.95E-02 & 3.43E-07 & 3.95E-07 & $4.22 E-07$ & $436 \mathrm{E}-07$ \\
\hline Am-243 & 3.7nE-12 & $2.10 \mathrm{E}-09$ & 4.SIE-07 & 3.13E-12 & $3.60 \mathrm{E}-12$ & $3.85 E-12$ & $3.97 \mathrm{E}-12$ \\
\hline $\mathrm{Cm}-242$ & $3.65 E-09$ & $2.06 \mathrm{E}-06$ & 4.42E-04 & $3.62 \mathrm{E}-09$ & $3.65 \mathrm{E}-09$ & $3.60 \mathrm{E}-09$ & $3.67 \mathrm{E}-09$ \\
\hline $\mathrm{Cm}-243$ & B.34E-11 & 4.71E- 08 & b.01E-05 & $8.2 \pi-11$ & $8.32 \mathrm{E}-11$ & $8.35 \mathrm{E}-11$ & $8.36 \mathrm{E} \cdot 11$ \\
\hline Cm-244 & $1.30 \mathrm{E} \cdot 10$ & $7.33 \mathrm{E}-0 \mathrm{~s}$ & $1.57 \mathrm{E}-05$ & $1.09 \mathrm{E}-10$ & $1.25 \mathrm{E}-10$ & $1,3 \mathrm{AE}-10$ & $1.38 \mathrm{E}-10$ \\
\hline Totels: & $\mathbf{M}$ & $1 / \mathrm{d}$ & 4 & $\begin{array}{l}9 \mathrm{Ol} \\
\mathrm{N} / \mathrm{L})\end{array}$ & $\begin{array}{l}67 \mathrm{CO} \\
\text { or } \mathrm{L} \text {, }\end{array}$ & $\begin{array}{l}67 C 1(4 \\
6, \Lambda)\end{array}$ & $\begin{array}{l}+901 \\
(M+1 L)\end{array}$ \\
\hline Pu & $2.69 \mathrm{E}-02(\mathrm{~g} / \mathrm{L})$ & - & 3.26 & $2.57 \mathrm{E}-02$ & 2.63E-02 & $2.75 E-02$ & $2.81 \mathrm{E}-02$ \\
\hline $\mathbf{U}$ & 0.18I & $244 E+04$ & $5.23 E+03$ & 0.158 & 0.17 & 0.188 & 0.194 \\
\hline
\end{tabular}

"Unknowns in tink solids inventory are assignad by Tenk Layering Model (TLM). 


\begin{tabular}{|c|c|c|c|c|c|c|c|}
\hline \multirow[b]{3}{*}{$\begin{array}{l}\text { Physical } \\
\text { Properties }\end{array}$} & \multicolumn{7}{|c|}{ Single-Sheil Tank 241-S-106 } \\
\hline & \multicolumn{7}{|c|}{ SMM Composite Inventory Estimate } \\
\hline & & & & $.95 \mathrm{CI}$ & $-67 \mathrm{Cl}$ & $+67 \mathrm{CI}$ & $+95 \mathrm{CI}$ \\
\hline Totel SMM Wast & $2.80 \mathrm{E}+06(\mathrm{~kg})$ & (447 kged $)$ & $=$ & - & $\cdots$ & - & - \\
\hline Heat Load & $3.65(\mathrm{~kW})$ & $(1.25 \mathrm{E}+04 \mathrm{BTU} / \mathrm{hr})$ & - & 3.47 & 3.56 & 3.76 & 3.85 \\
\hline Bulk Density* & $1.66(\mathrm{~g} / \infty)$ & - & $\ldots$ & 1.60 & 1.63 & 1.68 & 1.69 \\
\hline Water wt $\% \dagger$ & 310 & $\ldots$ & - & 28.8 & 29.8 & 32.3 & 34.4 \\
\hline TOC w1\% C (wet & 0.342 & - & $\ldots$ & 0.306 & 0.324 & 0.360 & 0.377 \\
\hline $\begin{array}{l}\text { Roidjological } \\
\text { Comstituents }\end{array}$ & CVL & $\mu \mathrm{C} / \mathrm{R}$ & $\mathbf{C A}$ & $\begin{array}{l}95 \mathrm{C} \\
(\mathrm{C} / \mathrm{L})\end{array}$ & $\begin{array}{l}67 \mathrm{Cl} \\
\text { (C/L) }\end{array}$ & $\begin{array}{l}+6 \mathrm{Cl} \\
(\mathrm{C} / \mathrm{L})\end{array}$ & $\begin{array}{l}+95 \mathrm{Cl} \\
\text { (CVL) }\end{array}$ \\
\hline $\mathrm{H}-3$ & $2.82 \mathrm{E}-04$ & 0.171 & 478 & $1.74 E-04$ & $1.74 \mathrm{E}-04$ & 2.95E-04 & $2.90 \mathrm{E}-04$ \\
\hline $\mathrm{C}-14$ & 3.45E-05 & $2.09 E=02$ & S8.4 & $1.19 \mathrm{E}-05$ & $1.19 \mathrm{E}-05$ & $3.52 E-05$ & $3.51 E-05$ \\
\hline Ni-59 & $2.26 \mathrm{E}-06$ & $1.36 \mathrm{E}-03$ & 3.82 & $1.20 E-06$ & $1.20 \mathrm{E}-06$ & $2.31 E-06$ & $2.36 \mathrm{E}-06$ \\
\hline Ni-63 & $2.20 \mathrm{E}-04$ & 0.133 & 372 & $1.15 E-04$ & $1.15 E-04$ & $2.75 E-04$ & $2.30 E-04$ \\
\hline Co-60 & 3.59E-05 & $2.14 E-02$ & 59.9 & $9.52 E-06$ & $9.52 \mathrm{E}-06$ & $3.58 E-05$ & $3.61 \mathrm{E}-05$ \\
\hline Se-79 & $3.57 \mathrm{E}-06$ & $2.16 \mathrm{E}-03$ & 6.04 & $2.13 E-06$ & $2.13 E-06$ & $4.09 E-06$ & $4.998-06$ \\
\hline St -90 & 0.112 & 67.6 & 1 1.99E+05 & 0.105 & 0.108 & 0.116 & 0.119 \\
\hline$Y-90$ & 0.112 & 67.6 & $1.89 \mathrm{E}+05$ & $6.21 \mathrm{E}-02$ & 6.21E-02 & 0.116 & 0.119 \\
\hline Zr-93 & $1.75 E-05$ & $1.06 E-02$ & 29.6 & $1.03 E-05$ & $1.03 E-05$ & 2.01E-05 & $2.26 \mathrm{E}-05$ \\
\hline $\mathrm{Nb}-93 \mathrm{~m}$ & $1.29 E-05$ & 7, 7TE-03 & 21.8 & $7.81 \mathrm{E}-06$ & $7.81 E-06$ & $1.47 \mathrm{E}-0.5$ & 1.64E-05 \\
\hline Te-99 & $2.47 E-04$ & 0.149 & 48 & $1.63 \mathrm{E}-04$ & $2.04 E-04$ & $2.90 \mathrm{E}-04$ & 3.32E-04 \\
\hline Ru-106 & $5.80 \mathrm{E}-69$ & $3.50 E-06$ & 9.81E-03 & 2.46E-09 & 2.46E-09 & $6.55 \mathrm{E}-09$ & 7.23E- 09 \\
\hline $\mathrm{Cd}-113 \mathrm{~m}$ & $8.82 E-05$ & $5.33 \mathrm{E}-02$ & 10 & 4.49E-05 & 4.49E-05 & $1.04 E-04$ & 1.19E-04 \\
\hline St-125 & 1.40E-04 & B.80E-02 & 246 & 3.37E-05 & 3.37E-05 & $1.47 \pi-04$ & 1.49E-04 \\
\hline Sn-126 & 5.41E-06 & $3.27 E-03$ & 9.15 & $3.25 E-06$ & 3.25E-06 & $6.19 \mathrm{E}-06$ & $6.94 \mathrm{E}-06$ \\
\hline I-129 & 4.76E-07 & 2.87E--04 & 0805 & 3.13E- -07 & 3.92E-07 & 5.60 - -07 & 6.41E-07 \\
\hline Cs-134 & $1.72 \mathrm{E}-06$ & 1.04E-03 & 2.92 & $9.10 \mathrm{E}-07$ & 9.10E-07 & $1.82 E-06$ & 1.91E-06 \\
\hline Cs-137 & 0.300 & 181 & $5.07 \mathrm{E}+05$ & 0.276 & 0.288 & 0.313 & 0.325 \\
\hline Be-1 $137 \mathrm{~m}$ & 0.284 & 177 & $4.80 \mathrm{E}+05$ & 0.175 & 0.175 & 0.296 & 0.298 \\
\hline $5 \mathrm{~m}-15]$ & $1.26 \mathrm{E}-02$ & 7.61 & $2.13 \mathrm{E}+0.4$ & 7,55E-03 & 7.55E -03 & $1.44 E-02$ & $1.62 E-02$ \\
\hline Eu-152 & $3.14 \mathrm{E}-66$ & $1.90 \mathrm{E}-03$ & 3.32 & $1.36 \mathrm{E}-06$ & $1.36 E-06$ & $320 \mathrm{E}-06$ & 325E-06 \\
\hline Eu-154 & 5.7RE-04 & 0.349 & 978 & $2.18 E-04$ & $2.18 E-04$ & $7.08 \mathrm{E}-04$ & $7.60 \mathrm{E}-0 \mathrm{de}$ \\
\hline Eu-155 & $1.80 \mathrm{E}-\mathrm{MA}$ & 0.109 & 305 & $7,368-05$ & 7.39E-05 & $1.84 \mathrm{E}-04$ & $1.87 \mathrm{E}-0 \mathrm{~A}$ \\
\hline Ra-226 & $1.57 \mathrm{E}-10$ & $9.51 \mathrm{E}-08$ & $2.665-001$ & 1.13E-10 & $1.13 E-10$ & $1.80 E-10$ & 2.01E-10 \\
\hline $\mathrm{Ra}-228$ & $5.17 \mathrm{E}-08$ & 3.12E-05 & 8.74E-02 & $2.06 E-08$ & 3.58E- -08 & $7.01 E-0$ & 8.97E- -88 \\
\hline Ac-227 & 9.48E-10 & $5.73 \mathrm{E}-07$ & $1.60 \mathrm{E}-03$ & 6.B9E-10 & $6.89 \mathrm{E}-10$ & $1.08 E-09$ & $1.16 \mathrm{E}-09$ \\
\hline $\mathrm{Pa}-231$ & 4.28E-09 & $2.58 E-06$ & $7.24 E-03$ & 2.BAE-09 & 2.845-09 & $4.80 E-09$ & $5.30 \mathrm{E}-09$ \\
\hline Th-229 & $1.24 \mathrm{E}-09$ & 7.4TE-07 & 2.00E-03 & $5.55 \mathrm{E}-10$ & B.BEE-10 & $1.64 E-09$ & 2.07E-09 \\
\hline $7 \mathrm{~h}-232$ & $3.56 \mathrm{E}-09$ & 2.15E-06 & $6.03 \mathrm{E}-03$ & $1.85 E-00$ & 2.69E- -09 & 4.44E- -09 & $5.28 \mathrm{E}-09$ \\
\hline U-232 & $3.23 \mathrm{E}-07$ & $1.95 E-04$ & 0.547 & $1.99 E-07$ & $2.59 \mathrm{E}-07$ & $3.97 \mathrm{E}-07$ & 4.75E- -07 \\
\hline U-233 & $1.24 \mathrm{E}-06$ & 1.49E-04 & 2.10 & $7.63 \mathrm{E}-07$ & 9.96E-07. & $1.52 \mathrm{E}-06$ & 1.82E-06 \\
\hline U-234 & $8.07 \mathrm{E}-07$ & $4.83 \mathrm{E}-0 \mathrm{a}$ & 1.37 & 7.4TE-07 & $776 E-07$ & 8.41E-07) & 8.31E-07 \\
\hline U-235 & $3.33 E-08$ & $2.01 \mathrm{E}-05$ & $5.63 E-02$ & $3.0 \pi \mathrm{E}-08$ & $3.19 E-08$ & $3.48 E-08$ & $3.43 E-08$ \\
\hline U-236 & $2.39 \mathrm{E}-18$ & $1.44 E-05$ & 4.04E- -22 & 2.29E-08 & $2.34 E-08$ & $2.4 \mathrm{E}-\mathrm{A} B$ & $2.46 \mathrm{E}-88$ \\
\hline U-238 & $3.30 \mathrm{E}-07$ & 5.01E-04 & 1.40 & 7.69E-07 & $7.98 E-07$ & 8.6SE-07 & 8.53E-07 \\
\hline $\mathrm{Np}-237$ & $9.66 \mathrm{E}-07$ & 5.8AE-OA & 1.64 & $6.91 E-07$ & 8.26E-07 & $1.11 \mathrm{E}-06$ & 1.25E-06 \\
\hline Pu-238 & $1.18 E-\infty 6$ & $7.138-04$ & 200 & 8.SBE- -7 & $1.02 E-06$ & $1.35 \mathrm{EE}-06$ & 1.50E-06 \\
\hline Pu-239 & 4.26E-05 & 2.5TE-02 & 7.0 & $3.43 E-05$ & 384E-05 & 4.68E-05 & $5.08 E-05$ \\
\hline$P u-240$ & $7.06 E-06$ & $4.27 E-03]$ & 12.0 & $5.52 E-06$ & $6.28 \mathrm{E}-06$ & $7.85 \mathrm{SE}-06$ & $8.60 \mathrm{E}-06$ \\
\hline Pu-241 & 7.81E-05 & $4.72 \mathrm{E}-02$ & 132 & $3.61 \mathrm{E}-05$ & 6.69E-05 & 8.94E-05 & 1.00E-04 \\
\hline $\mathrm{Pu}-242$ & $4.26 \mathrm{E}-10$ & 2.5TE-07 & $7.2010-04$ & 2.94E-10 & 3.59E-10 & $4.93 \mathrm{E}-10$ & $5.57 \mathrm{E}-10$ \\
\hline Am-24] & $3.79 \mathrm{E}-05$ & $3.50 \mathrm{E}-02$ & 97.9 & 4.25E-05 & 5.01E-05 & 6.57E-05 & $7.32 \mathrm{E}-05$ \\
\hline $\mathrm{Am}-243$ & $1.75 E-09$ & $1.06 \mathrm{E}-06$ & 2.96E-03 & $1.27 E-09$ & 1.S0E-09 & 2.01E-09 & 2.25E-09 \\
\hline $\mathrm{Cm}-242$ & $1.09 \mathrm{E}-07$ & $6.57 \mathrm{E}-03$ & 0.184 & 3.55E-08 & 3.55E-08 & $1.12 E-07$ & 1.14E-07 \\
\hline $\mathrm{Cm}-243$ & $9.83 E-09$ & 5.94E-06 & $1.66 \mathrm{E}-02$ & 2.8AE-09 & 2.8AE-09 & $1.01 E-08$ & $1.04 E-08$ \\
\hline $\mathrm{Cm}-244$ & $1.10 \mathrm{E}-07$ & $6.65 E-05$ & 0.186 & 3.BOE- 08 & $3.80 E-08$ & $1.35 \mathrm{E}-07$ & $1.50 \mathrm{E}-07$ \\
\hline Tots & $\mathbf{M}$ & $\mu / s$ & is: & $\begin{array}{l}95 \mathrm{CM} \\
\text { ors L) }\end{array}$ & $\begin{array}{r}67 \mathrm{Cl} M \\
\text { or } \mathrm{L}) \\
\end{array}$ & $\begin{array}{c}+67 \mathrm{cl} \mathrm{M} \\
\quad+1 \mathrm{~L})\end{array}$ & $\begin{array}{c}+95 \mathrm{C} \\
\mathrm{M} \text { or } \mathrm{L} \text { ) }\end{array}$ \\
\hline Pu & $5.4 \pi-04(\mathrm{~g} / \mathrm{L})$ & - & 0.926 & $391 \mathrm{E}-04$ & $4.68 \mathrm{E}-04$ & $6.27 \mathrm{E}-04$ & $7.03 \mathrm{E}-04$ \\
\hline $\mathrm{U}$ & $9.47 \pi-03$ & $1.36 \mathrm{E}+03$ & $3.81 E+03$ & 8.70E-03 & $9.06 \mathrm{E}-03$ & 9.91E-03 & $9.75 E-03$ \\
\hline
\end{tabular}

"Density is calculated based on $\mathrm{Na} \mathrm{OH}$-, and $\mathrm{AO} 2$ -

tWater wt\% derived from the difference of density and total diveolved specice. 
HNF-SD-WM-ER-352, Rev. 1

HDW Model Rev. 4

\begin{tabular}{|c|c|c|c|c|c|c|c|}
\hline \multicolumn{8}{|c|}{ Single-Sheli Tonk 241-S-106 } \\
\hline & \multicolumn{7}{|c|}{ Total Inventory Estimate" } \\
\hline Phybical & & & & & & & \\
\hline Propentios & & & & $95 \mathrm{CI}$ & $67 \mathrm{CI}$ & $+67 \mathrm{CI}$ & $+95 \mathrm{Cl}$ \\
\hline Total Waste & $3.02 \mathrm{E}+06(\mathrm{~kg})$ & (479 kFal) & - & - & - & - & - \\
\hline Heat Load & $3.66(\mathrm{cW})$ & $(1.25 \mathrm{E}+04 \mathrm{BTU} / \mathrm{hr})$ & - & 3.47 & 3.56 & 3.76 & 3.85 \\
\hline Bulk Densityt & $1.66\left(y^{\prime} / c\right)$ & - & - & 1.61 & 1.64 & 1.68 & 1.70 \\
\hline & & & & & & & \\
\hline Waler wat $\dagger$ & 30.6 & $\ldots$ & $\ldots$ & 28.5 & 29.3 & 32.0 & 33.6 \\
\hline TOC wt\% C (wet & 0.317 & $\ldots$ & - & 0.284 & 0.300 & 0.334 & 0.350 \\
\hline 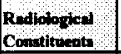 & ch & pC/s & $\mathbf{C i}$ & $\begin{array}{l}9 \mathrm{Cl} \\
\text { (CiL) }\end{array}$ & $\begin{array}{l}67 \mathrm{cl} \\
(\mathrm{Cub})\end{array}$ & $\begin{array}{r}+67 \mathrm{Cl} \\
\text { (CUL) }\end{array}$ & $\begin{array}{c}+95 \mathrm{Cl} \\
\text { (ch) }\end{array}$ \\
\hline $\mathrm{H}-3$ & $2.63 \mathrm{E}-04$ & 0.158 & 478 & $1.63 E-04$ & 1.63E-04 & $2.76 \mathrm{E}-04$ & $2.71 \mathrm{E}-04$ \\
\hline $\mathrm{C}-14$ & $322 \mathrm{E}-05$ & $1.94 \mathrm{E}-02$ & 58.4 & 1.11E-05 & 1.11E-05 & 3.28E-05 & $3.2 T E-05$ \\
\hline $\mathrm{Ni-59}$ & $2.11 \mathrm{E}-06$ & $1.2 \mathrm{E}-03$ & 3.82 & $1.12 \mathrm{E}-06$ & $1.12 E-06$ & $2.16 \mathrm{E}-06$ & $2.21 E-06$ \\
\hline Ni-63 & 2.05E-04 & 0.123 & 372 & $1.07 E-04$ & $1.07 \mathrm{E}-04$ & $2.10 \mathrm{E}-04$ & $2.15 E-04$ \\
\hline$C_{0}-60$ & $3.30 \mathrm{E}-05$ & $1.99 \mathrm{E}-02$ & 59.9 & 8.89E-06 & 8.89E-C6 & 3.34E-05 & $3.37 z-05$ \\
\hline Se-79 & 3.34E-06 & $2.01 E-03$ & 6.05 & $1.99 \mathrm{E}-06$ & $1.99 \mathrm{E}-96$ & $3.82 E-06$ & $4.29 \mathrm{E}-06$ \\
\hline Sr-90 & 0.105 & 62.9 & $1.90 \mathrm{E}+05$ & $9.80 \mathrm{E}-02$ & 0.101 & 0.108 & 0.111 \\
\hline$Y-90$ & 0.105 & 62.9 & $1.90 \mathrm{E}+05$ & $5.81 \mathrm{E}-02$ & $5.81 E-02$ & 0.108 & 0.111 \\
\hline Zr-93 & 1.63E-0s & $9.82 \mathrm{E}-03$ & 29.6 & 9.60E-06 & $9.60 E-\infty$ & 1.88E-05 & $2.11 \mathrm{E}-05$ \\
\hline $\mathrm{Nb}-93 \mathrm{~m}$ & $1.20 \mathrm{E}-05$ & $7.22 \mathrm{E}-03$ & 21.8 & $7.30 \mathrm{E}-06$ & $7.30 \mathrm{E}-96$ & $1.37 \mathrm{E}-05$ & $1.53 E-05$ \\
\hline Tc-99 & $2.31 \mathrm{E}-04$ & 0.139 & 418 & $1.52 \mathrm{E}-0 \mathrm{As}$ & $1.90 \mathrm{E}-04$ & $2.71 \mathrm{E}-04$ & $3.10 \mathrm{E}-04$ \\
\hline $\mathrm{Ru}-106$ & $5.41 \mathrm{E}-09$ & 3.25E-06 & 9.81E-03 & 2.29E-09 & $229 \mathrm{E}-99$ & 6.11E-09 & $6.74 E-09$ \\
\hline $\mathrm{Cd}-113 \mathrm{~m}$ & $8.23 \mathrm{E}-0 \mathrm{~s}$ & 4.95E-02 & 149 & 4.19E-05 & 4.19E-05 & $9.69 \mathrm{E}-05$ & $1.11 E-04$ \\
\hline Sb-125 & $1.36 \mathrm{E}-04$ & 8.17E-02 & 240 & $3.14 E-05$ & 3.14E-05 & $1.38 \mathrm{E}-04$ & $1.39 E-04$ \\
\hline Sn-126 & $5.05 E-06$ & $3.04 \mathrm{E}-03$ & 9.15 & $3.03 \mathrm{E}-06$ & $3.03 E-60$ & $5.78 E-06$ & $6.48 E-06$ \\
\hline I-129 & 4.44E-07 & $2.672-04$ & 0.805 & 2.92E-07 & 3.66E-07 & $5.22 \mathrm{E}-07$ & $5.98 E-07$ \\
\hline Cs-134 & $161 E-06$ & $9.67 \mathrm{E}-04$ & 2.92 & 8.49E-07 & 8.49E-07 & $1.70 E-06$ & $1,76 \mathrm{E}-06$ \\
\hline Cs-137 & 0.280 & 16 & 5.068405 & 0.258 & 0.269 & 0.293 & 0.303 \\
\hline Ba-137m & 0.265 & 199 & $4.00 \mathrm{E}+05$ & 0.164 & 0.164 & $0.2 \pi$ & $\begin{array}{l}0.278 \\
\end{array}$ \\
\hline Sm-151 & 1.18E-02 & 7.07 & $2.13 E+04$ & $7.05 E-03$ & 7.05E-03 & 1.35E-02 & $1.31 E-02$ \\
\hline Eu-152 & 2.95E-06 & $\begin{array}{ll}.77 \mathrm{E}-03 \\
\end{array}$ & 5.34 & $1.28 E-06$ & $1.28 \mathrm{E}-06$ & $3.00 \mathrm{E}-06$ & $3.04 \mathrm{E}-06$ \\
\hline Eu-154 & 5.39E-04 & 0.324 & 97 & $2.03 \mathrm{E}-04$ & $2.03 \mathrm{E}-04$ & $6.61 \mathrm{E}-04$ & $7.10 \mathrm{E}-04$ \\
\hline Eu-155 & $1.69 \mathrm{E}-04$ & 0.101 & 306 & 6.94E-05 & $6.94 \mathrm{E}-05$ & 1.72E-04 & $1,75 E-04$ \\
\hline $\mathrm{Ra}-226$ & $1.47 \mathrm{E}-10$ & 8.85E-08 & $2.67 \mathrm{E}-04$ & $1.05 \mathrm{E}-10$ & $1.05 E-10$ & $1.68 \mathrm{E} \cdot 10$ & $1.88 \mathrm{E}-10$ \\
\hline Ra-228 & 4.82E-08 & $2.90 \mathrm{E}-05$ & 774E-02 & $1.93 \mathrm{E}-08$ & $3.34 E-08$ & $6.54 \mathrm{E}-08$ & $8.37 E-08$ \\
\hline Ac-227 & $8.65 \mathrm{E}-10$ & $5.33 \mathrm{E}-07$ & T.61E-03 & $6.44 \mathrm{E}-10$ & $6.44 \mathrm{E}-10$ & $1.01 E-09$ & $1.08 E-09$ \\
\hline $\mathrm{Pa}-231$ & $400 \mathrm{E}-09$ & 2.40E-06 & $7.24 \mathrm{E}-03$ & $2.65 E-09$ & $2.65 \mathrm{E}-09$ & 4. $19 \mathrm{E}-09$ & $4.95 \mathrm{E}-09$ \\
\hline Th-229 & $1.15 \mathrm{E}-09$ & $6.93 \mathrm{E}-07$ & 2.09E-03 & $5.18 \mathrm{E}-10$ & 1.28E-10 & $1.53 E-09$ & $1.93 \mathrm{E}-09$ \\
\hline Th-232 & $3.33 \mathrm{E}-49$ & $2.00 \mathrm{E}-06$ & 6.03E-03 & $1.72 E-09$ & 2.51E-09 & 4.14E-09 & $493 \mathrm{E}-09$ \\
\hline $\begin{array}{l}\mathrm{U}-232 \\
\end{array}$ & $3.028-07$ & 1.81E-04 & 0.57 & $1.85 \mathrm{E}-07$ & 2.42E- -07 & 3.70E-07 & 4.44E-07 \\
\hline U-233 & $1.16 \mathrm{E}-06$ & 696E-04 & 2.10 & $7.12 \mathrm{E}-07$ & 9.30E-07 & 1. $2 \mathrm{E}-06$ & $1.70 \mathrm{E}-06$ \\
\hline U.234 & $1,74 \mathrm{E}-06$ & $1.056-03$ & 3.16 & $1.61 \mathrm{E}-06$ & $1.69 \mathrm{E}-06$ & $1.78 E-\infty 6$ & $1.81 \mathrm{E}-06$ \\
\hline U.235 & $7.31 \mathrm{E}-08$ & 439E-05 & 0.132 & $6.76 \mathrm{E}-08$ & $7.08 E-08$ & $7,47 \mathrm{E}-08$ & 7.59E-08 \\
\hline U-236 & 4.41]E-08 & 2.65E-05 & $7.99 \mathrm{E}-02$ & $4.12 E-00$ & $4.29 \mathrm{E}-08$ & 4. $49 \mathrm{E}-0 \mathrm{~B}$ & 4.5SE-0.3 \\
\hline U-238 & $1.74 E-66$ & $1.04 E-03$ & \begin{tabular}{r|}
3.15 \\
\end{tabular} & $1.61 \mathrm{E}-06$ & $1.68 E-06$ & 1.75E-06 & $1.80 \mathrm{E}-06$ \\
\hline $\mathrm{Np}-237$ & $9.02 \mathrm{E}-07$ & $5.42 \mathrm{E}-04$ & 1.64 & $6.46 \mathrm{E}-07$ & $9.71 \mathrm{E}-07$ & $1.03 \mathrm{E}-\infty 6$ & $1.16 \mathrm{E}-06$ \\
\hline Pu-238 & 2.7BE-06 & $1.67 \mathrm{E}-03$ & 5.04 & $2.48 E-06$ & $2.63 \mathrm{E}-06$ & $2.93 \mathrm{E}-06$ & $3.08 E-06$ \\
\hline Pu-239 & 1. $47 \mathrm{TE}-04$ & $8.85 E-02$ & 267 & $1.39 \mathrm{E}-0 \mathrm{~A}$ & $1.43 E-04$ & 1.51E-04 & 1.55E-04 \\
\hline $\mathrm{Pu}-240$ & $2.17-05$ & $131 \mathrm{E}-02$ & 39.4 & 2.03E-05 & 2.10E-05 & 2.25E-05 & 2.32E-05 \\
\hline$P u-241$ & $1.65 \mathrm{E}-04$ & 9.95E-02 & 300 & 1.45E-04 & 1.55E-04 & $1.76 \mathrm{E}-04$ & $1.86 E-04$ \\
\hline Pu-242 & $7.00 E-10$ & $4.75 E-07$ & 1.8BE-03 & $6.67 \mathrm{E}-10$ & $7.27 \mathrm{E}-10$ & $8.53 \mathrm{E}-10$ & $9.13 E-10$ \\
\hline Am-241 & 5.40E-05 & $3.25 E-02$ & 93.0 & $3.9 \pi-05$ & 4.6TE-0S & 6.13E-05 & 6.53E-05 \\
\hline Am-243 & $1.63 \mathrm{E}-09$ & 9.87E- -07 & $2.66-03$ & $1.18 \mathrm{BE}-09$ & $1.40 \mathrm{E}-09$ & $1.87 E-09$ & 2.10E-09 \\
\hline $\mathrm{Cm}-242$ & $1.02 \mathrm{E}-07$ & 6.12E-05 & 0.18 & 3.34E-08 & $3.34 E-08$ & $1.040-07$ & $1.07 \mathrm{E}-07$ \\
\hline$C_{m-243}$ & $9.18 E-109$ & $5.52 \mathrm{E}-06$ & 1.66E-02 & $2.66 \mathrm{E}-09$ & $2.66 \mathrm{E}-09$ & $9.46 E-09$ & 9.73E- 09 \\
\hline$C_{m-244}$ & $1.03 E-07$ & 6.18E-05 & 0.186 & 335E-08 & 3.55E-108 & $1.26 \mathrm{E}-07$ & 1.40E.07 \\
\hline Tofles & $\mathbf{M}$ & Eds & $6, \quad$ & $\begin{array}{l}95 \mathrm{ClO} \\
0 \mathrm{~L})\end{array}$ & $\begin{array}{l}67 \mathrm{clom} \\
\text { or r L) }\end{array}$ & $\begin{array}{c}+67 \mathrm{Cl}(\mathrm{M} \\
\text { or } \mathrm{kL})\end{array}$ & $\begin{array}{c}+95 \mathrm{Cl} \\
\mathrm{a} / \mathrm{L})\end{array}$ \\
\hline PL & $2.31 \mathrm{E}-03(\mathrm{~g} / \mathrm{L})$ & - & 4.18 & $2.16 \mathrm{E}-03$ & $2.23 \mathrm{E}-03$ & $2.38 \mathrm{E}-03$ & $2.45 E-03$ \\
\hline $\mathrm{U}$ & $2.10 \mathrm{E}-02$ & $3.00 \mathrm{E}+03$ & $9.04 E+03$ & 1.94E-02 & $2.03 E-02$ & $2.14 E-02$ & 2.1BE-02 \\
\hline
\end{tabular}


TANK 241-S-107 SUMMARY

\begin{tabular}{|c|c|c|c|}
\hline \multicolumn{2}{|c|}{ TANK HISTORY } & \multicolumn{2}{|c|}{ TANK DESCRIPTION } \\
\hline Entered Service & 4th qtr 1952 & Diameter & $75 \mathrm{ft}$ \\
\hline Removed from Service & - & Bottom Shape & Dish \\
\hline Inactive & 1980 & Nominal Capacity & $758,000 \mathrm{gal}$ \\
\hline Watch Lists & none & Cascade Tank & to $241-S-108$ \\
\hline integrity & Sound & Total Risers & 12 \\
\hline Assumed Leaker & - & \multicolumn{2}{|c|}{$\begin{array}{l}\text { WASTE VOLUME (HANLON 1996i) } \\
\end{array}$} \\
\hline Interim Stabilization (IS) & - & Total Waste Volume & $376,000 \mathrm{gal}$ \\
\hline Partial Interim Isolation (PI) & Dec 1982 & Waste Type & NCPLX \\
\hline Intrusion Prevention (IP) & - & Drainable Interstitial Liquids & $45,000 \mathrm{gal}$ \\
\hline \multicolumn{2}{|c|}{$\begin{array}{l}\text { TENTATIVELY AVAILABLE RISERS } \\
\end{array}$} & Pumpable Liquids & $88,000 \mathrm{gal}$ \\
\hline Riser Number(s) & Size & Saltcake & 69,000 gal \\
\hline $2,11,14,16$ & 4 in & Sludge & $293,000 \mathrm{gal}$ \\
\hline 6 & 12 in & Supernatant & $14,000 \mathrm{gal}$ \\
\hline \multicolumn{2}{|c|}{ TANK TEMPERATURE } & \multicolumn{2}{|c|}{ INTERIOR PHOTOGRAPHS } \\
\hline Average Tank Temperature & $101^{\circ} \mathrm{F}$ & Date & March 12, 1987 \\
\hline Maximum Temperature & $123^{\circ} \mathrm{F}$ & Montage Number & $94041033-24 \mathrm{CN}$ \\
\hline Date & May 2, 1988 & Photo Set Number & 8701464 \\
\hline Elevation from tank bottom & $0.48 \mathrm{ft}$ & \multicolumn{2}{|c|}{\begin{tabular}{|c|} 
WASTE SURFACE LEVEL \\
\end{tabular}} \\
\hline Riser Number & 4 & Devices & Auto and Manual ENRAF \\
\hline Minimum Temperature & $78.3^{\circ} \mathrm{F}$ & Max Level & 146.49 in \\
\hline Date & Feb 13, 1993 & Date & Sept 15,1996 \\
\hline Elevation from tank bottom & $24.44 \mathrm{ft}$ & Min Level & 142.1 in \\
\hline Riser Number & 4 & Date & $\operatorname{Jan} 1,1991$ - May $16,1991^{*}$ \\
\hline
\end{tabular}

- Numerous dates in this time span. 


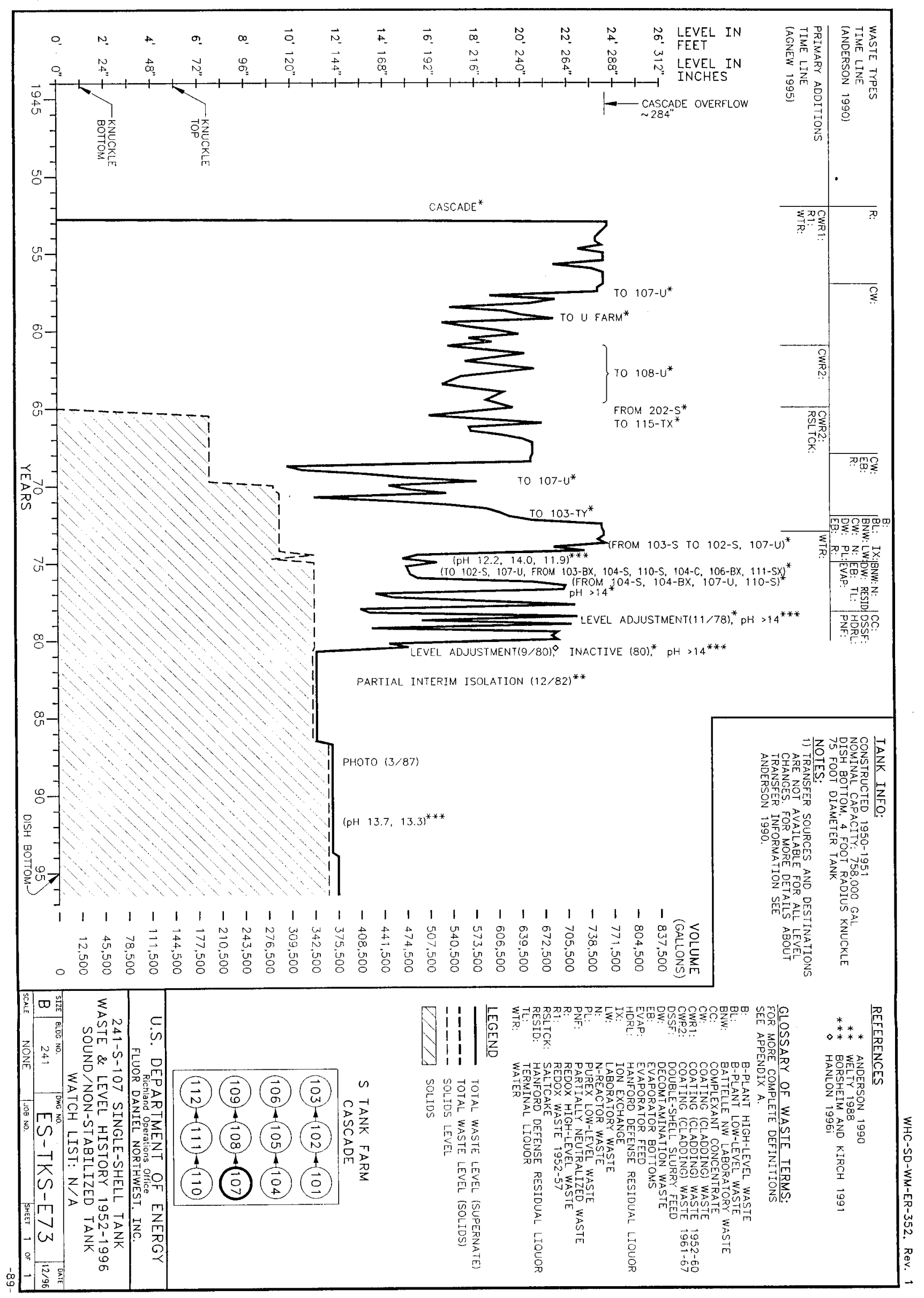




$$
241-5-107
$$

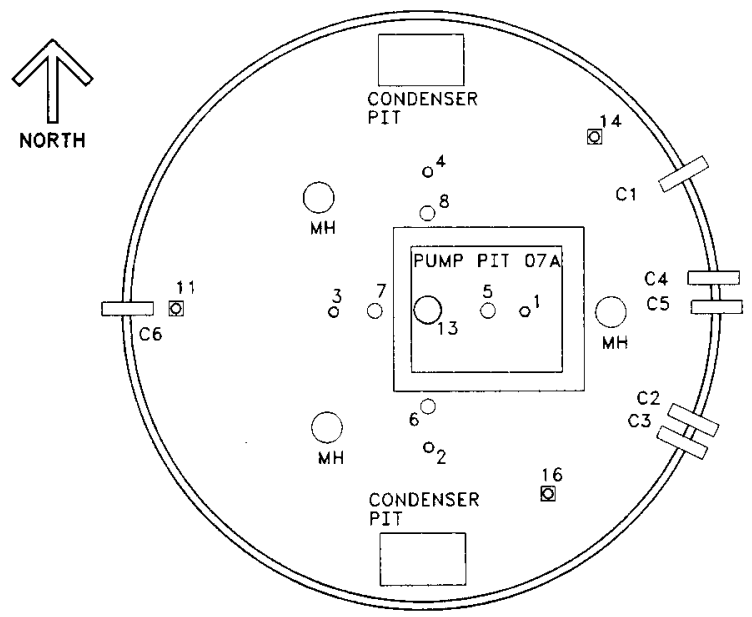

Ref: Alstad 1993

$\mathrm{H}-2-73186$. Rev. 4

$\mathrm{H}-2-37530$. Rev. 2

\section{TANK RISER LOCATION}

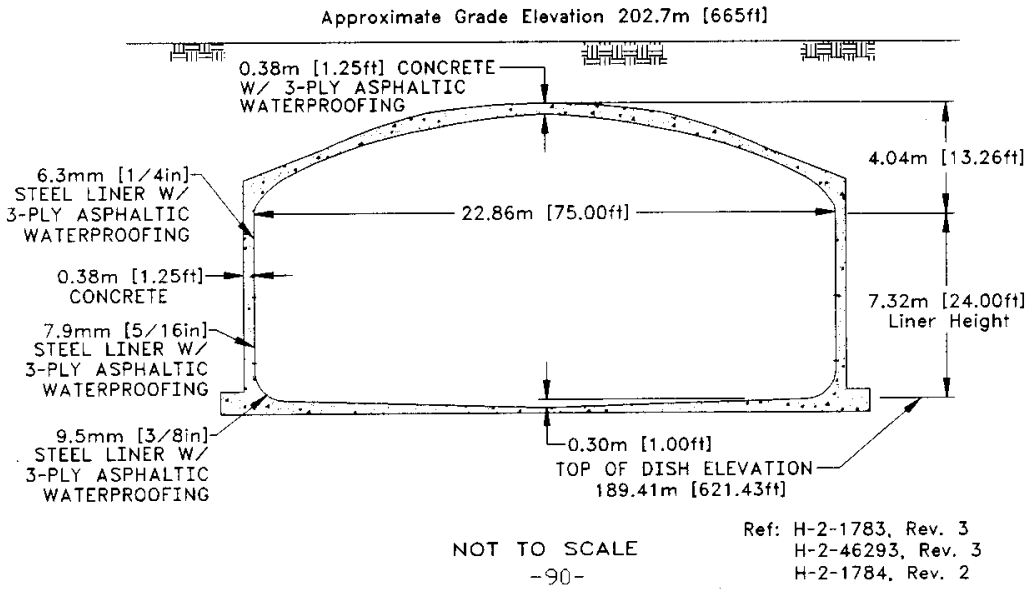




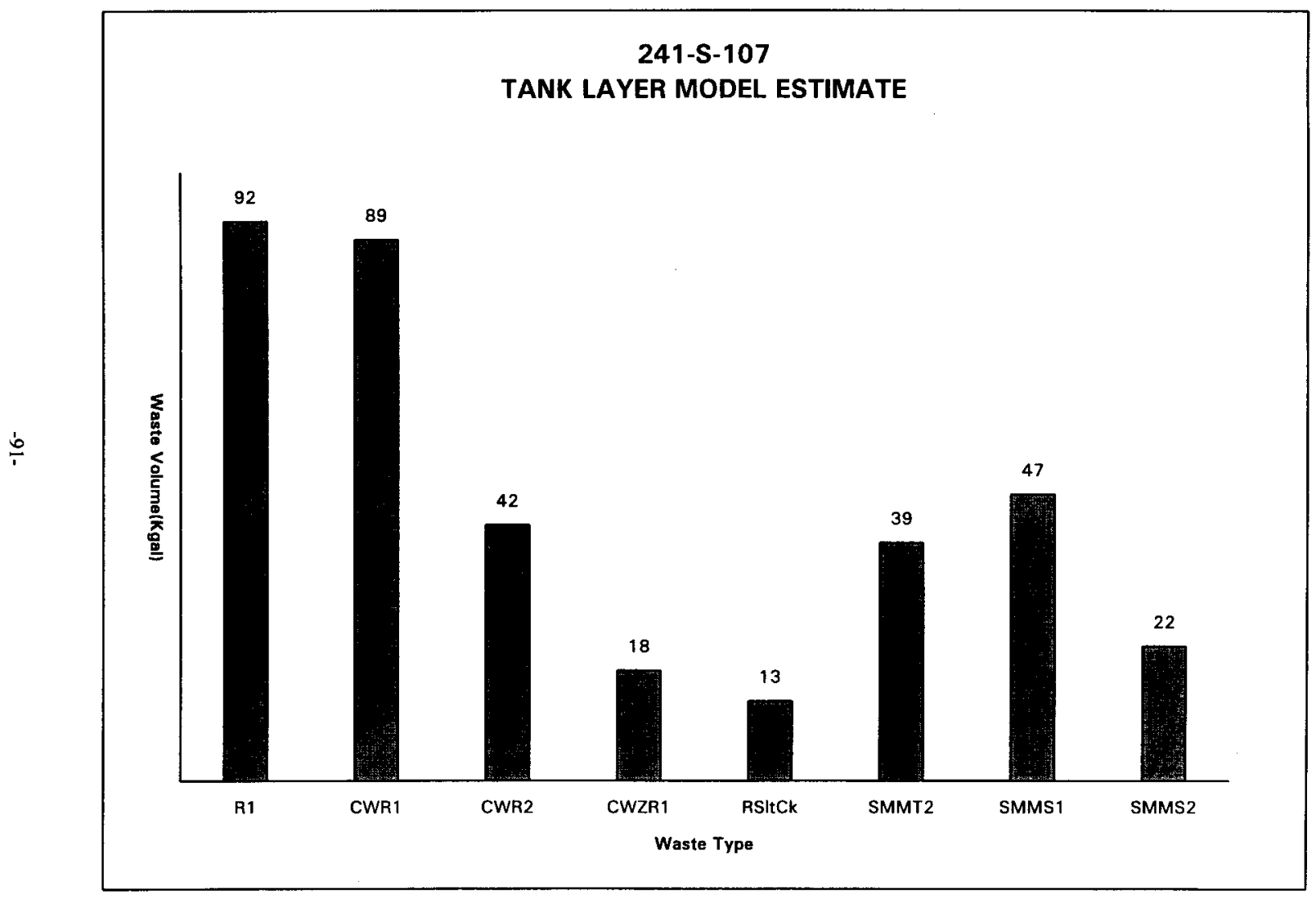


HNF-SD-WM-ER-352, Rev. 1

HDW Madol Rev. 4

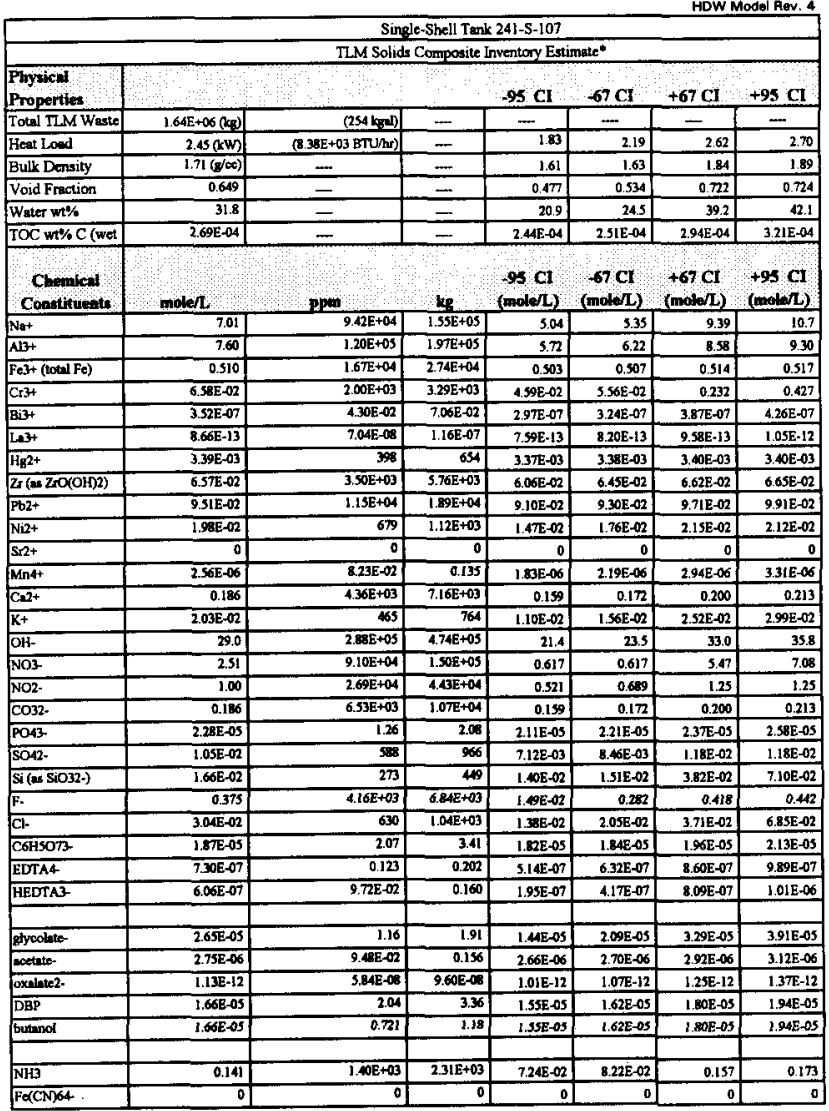

Unlowowns in tank wolids inventory are ascigned by Tenk Layering Moded (TLM) 
HNF-SD-WM-ER-352, Rev. 1

HDW Model Rev. 4

\begin{tabular}{|c|c|c|c|c|c|c|c|}
\hline \multicolumn{8}{|c|}{ Single-Shell Tank 241-\$-107 } \\
\hline & \multicolumn{7}{|c|}{ SMM Connposite Inventory Estimnte } \\
\hline $\begin{array}{l}\text { Phyuctal } \\
\text { Properties }\end{array}$ & a $\quad \cdots$ & & & $25 \mathrm{CI}$ & $-67 \mathrm{CI}$ & $+67 \mathrm{CI}$ & $+95 \mathrm{CI}$ \\
\hline Total SMM Wast & $6.46 \mathrm{E}+05(\mathrm{gs})$ & (122 lagal) & $\ldots$ & $\ldots$ & $\ldots$ & - & - \\
\hline Hest Loed & $0.732(\mathrm{~kW})$ & $(2.50 \mathrm{E}+03 \mathrm{ETU} / \mathrm{hr})$ & - & 0.670 & 0.710 & 0.755 & 0.77 \\
\hline Bulk Dersity* & $1.40\left(g^{\prime} \infty\right)$ & - & - & 1.37 & 1.38 & 1.41 & 1.42 \\
\hline Water wt\% & 49.0 & - & - & 46.8 & 47.8 & so.4 & 51.9 \\
\hline TOC wo $\mathrm{C}$ (wet & 0.898 & - & - & 0.468 & 0.679 & 1.12 & 1.33 \\
\hline Conemifel & $\mathrm{nold}$ & on & Le & $9 \mathrm{CI}$ & $\begin{array}{c}67 \mathrm{CL} \\
\text { (noleL) }\end{array}$ & $\begin{array}{l}+67 \mathrm{Cl} \\
\text { (molel } \mathrm{L})\end{array}$ & $\begin{array}{l}+93 \mathrm{CI} \\
(\text { (welan) }\end{array}$ \\
\hline Nat & 9.39 & $1.54 \mathrm{E}+05$ & $9.97 \mathrm{E}+04$ & 8.60 & 9.00 & 9.7 & 10.0 \\
\hline Alb & 1.05 & $2.03 \mathrm{E}+04$ & $1.31 E+04$ & 0.975 & 1.01 & 1.09 & 1.13 \\
\hline $\mathrm{Fe} 3+($ total Fe) & $7.79 E-03$ & 311 & 201 & $6.49 \mathrm{E}-03$ & $7.13 \mathrm{E}-03$ & $8.46 \mathrm{E}-03$ & $9.095-03$ \\
\hline $\mathrm{Cr}^{3+}$ & $9.20 \mathrm{E}-02$ & $3.45 E+03$ & $2.23 E+03$ & $7.83 \mathrm{E}-02$ & 8.61E-02 & 9.5SE-02 & $9.81 \mathrm{E}-02$ \\
\hline $\mathrm{Bi3+}$ & B.42E-04 & 126 & 81.2 & $7,67 \mathrm{E}-04$ & s.04E-04 & 179E-04 & $9.16 \mathrm{E}-04$ \\
\hline $\mathrm{L} 23+$ & $2.73 E-05$ & 2.72 & 1.75 & $1.98 \mathrm{E}-05$ & $2.35 E-05$ & 3.12E-05 & $3.49 \mathrm{E}-05$ \\
\hline $\mathrm{Hg} 2+$ & SEOE-06 & 0.832 & 0.537 & $5.41 \mathrm{E}-06$ & $5.63 \mathrm{E}-06$ & 5.89E-06 & $3.97 \mathrm{E}-06$ \\
\hline $\mathrm{Zr}(\mathrm{a} \mathrm{2r} \times(\mathrm{OH}) 2)$ & 1.5SE-04 & 10.1 & 6.52 & 1.41E-04 & $1.46 \mathrm{E}-04$ & $160 \mathrm{E}-04$ & $1.68 \mathrm{E}-04$ \\
\hline Pb2+ & 6.4AE-01 & 95.4 & 61.6 & $3.20 \mathrm{E}-0.4$ & 5.81E-04 & $7.07 \mathrm{E}-04$ & $7.68 \mathrm{E}-0.4$ \\
\hline $\mathrm{Ni2+}$ & $4.33 \mathrm{E}-03$ & 182 & 117 & 4.14E-03 & 424E-03 & $4.38 E-09$ & 4.43E-03 \\
\hline $8 x^{2}+$ & 0 & 0 & 0 & 0 & 0 & 0 & 0 \\
\hline $\mathrm{Mns+}$ & $2.64 k-03$ & 104 & 67.0 & $2.02 \mathrm{E}-03$ & $232 \mathrm{E}-13$ & $296 \mathrm{E}-03$ & $3.27 E-03$ \\
\hline $\mathrm{C.2+}$ & $2.33 E-02$ & 668 & 41 & 2.14E-02 & $2.23 \mathrm{E}-02$ & $2.43 \mathrm{E}-02$ & $2.52 \mathrm{E}-02$ \\
\hline $\mathbf{K}+$ & 4.35E-02 & $1.22 E+03$ & 706 & $3.97 \mathrm{E}-02$ & 4.15E-02 & 4.56E-02 & 4.76E-02 \\
\hline OH- & 6.38 & $7.76 \mathrm{E}+04$ & $5.01 E+04$ & 5.93 & 6.14 & 6.63 & 6.78 \\
\hline NO3- & 3.21 & $1.02 \mathrm{E}+05$ & $9.19 E+04$ & 2.97 & 3.11 & 3.26 & $3.3]$ \\
\hline NO2- & 1.38 & $5.21 E+04$ & $337 \mathrm{E}+04$ & 1.32 & 1.4 & 1.72 & 1.86 \\
\hline Co32- & 0.319 & $1.37 \mathrm{E}+04$ & $8,86 \mathrm{E}+03$ & 0.286 & 0.303 & 0.398 & 0.348 \\
\hline PO43- & $6.02 E-02$ & $4.09 \mathrm{E}+03$ & $2.64 \mathrm{E}+03$ & $5.23 \mathrm{E}-02$ & S.51E-02 & $6.16 \mathrm{E}-02$ & $6.30 \mathrm{E}-02$ \\
\hline 5042. & 0.171 & $1.17 \mathrm{E}+04$ & $7.59 E+03$ & 0.137 & 0.153 & 0.190 & 0.206 \\
\hline Si (as SiO32-) & $5.5 \%-02$ & $1.12 \mathrm{E}+03$ & 723 & $4.68 \mathrm{E}-02$ & $5.12 \mathrm{E}-02$ & 6.03E-02 & $6.47 \mathrm{E}-02$ \\
\hline F. & 4.13E-M & 562 & 363 & $3.49 \mathrm{E}-02$ & $376 E-02$ & $4,45 E-02$ & $4,70 \mathrm{E}-02$ \\
\hline Cl- & 0.162 & $4.12 E+03$ & $2.66 \mathrm{E}+03$ & 0.147 & 0.154 & 0.170 & 0.175 \\
\hline C6HSOT3 & $1.90 E-02$ & $2.57 \mathrm{E}+03$ & $1.66 \mathrm{E}+03$ & $1.72 \mathrm{E}-02$ & 1.81E-02 & $2.00 \mathrm{E}-02$ & $2.09 \mathrm{E}-02$ \\
\hline EDTA4 & 2.04E-02 & $4.20 \mathrm{E}+03$ & $2.71 E+03$ & $5.54 \mathrm{E}-03$ & $1298=02$ & 2.80E-02 & 3.5SE-02 \\
\hline HEDTA 3 & 3.94E-02 & $7.72 \mathrm{E}+03$ & $4.99 \mathrm{E}+03$ & $9,66 \mathrm{E}-03$ & $242 \mathrm{E}-02$ & $5.47 \mathrm{E}-02$ & $6.96 \mathrm{E}-02$ \\
\hline glyoalnte- & 8.TYE- 02 & $472 \mathrm{E}+03$ & $3.05 E+03$ & $5,02 E-02$ & $7.2 \pi-02$ & 0.103 & 0.118 \\
\hline sestete- & $4.52 E-03$ & 191 & 121 & 3.71E-03 & 4. 10E-03 & 493E-03 & $5.33 \mathrm{E}-03$ \\
\hline oxnhinte2. & $3.5 \mathrm{EE}-0 \mathrm{~s}$ & 2.25 & 1.46 & $3.17 \mathrm{E}-0 \mathrm{~S}$ & $3.37-05$ & $3,79 \mathrm{E}-05$ & $3.99 \mathrm{E}-05$ \\
\hline DBP & $1.245-02$ & $1.97 E+03$ & $1.21 \mathrm{E}+0$. & 1.02E-02 & $1.13 E-02$ & I.35E-02 & $1.46 E-02$ \\
\hline buttunol & $1.24 E-02$ & 660 & 426 & $1.02 E-02$ & $1.13 E-02$ & 1.35E-02 & $1.46 \mathrm{E}-02$ \\
\hline NH3 & $5.525-02$ & 671 & 433 & 4.51E-02 & 491E-02 & 6.30E-02 & $7.20 E-02$ \\
\hline Fe(CN) & 0 & 0 & 0 & 0 & o & 0 & 0 \\
\hline
\end{tabular}

"Density is calculated besed on $\mathrm{Ne}, \mathrm{OH}$, ind $\mathrm{AlO} 2$ -

+Water wt\% derived from the difference of dersity and total dinsolved apecies. 
HNF-SD-WM-ER-352, Rev. 1

HDW Madel Rev. 4

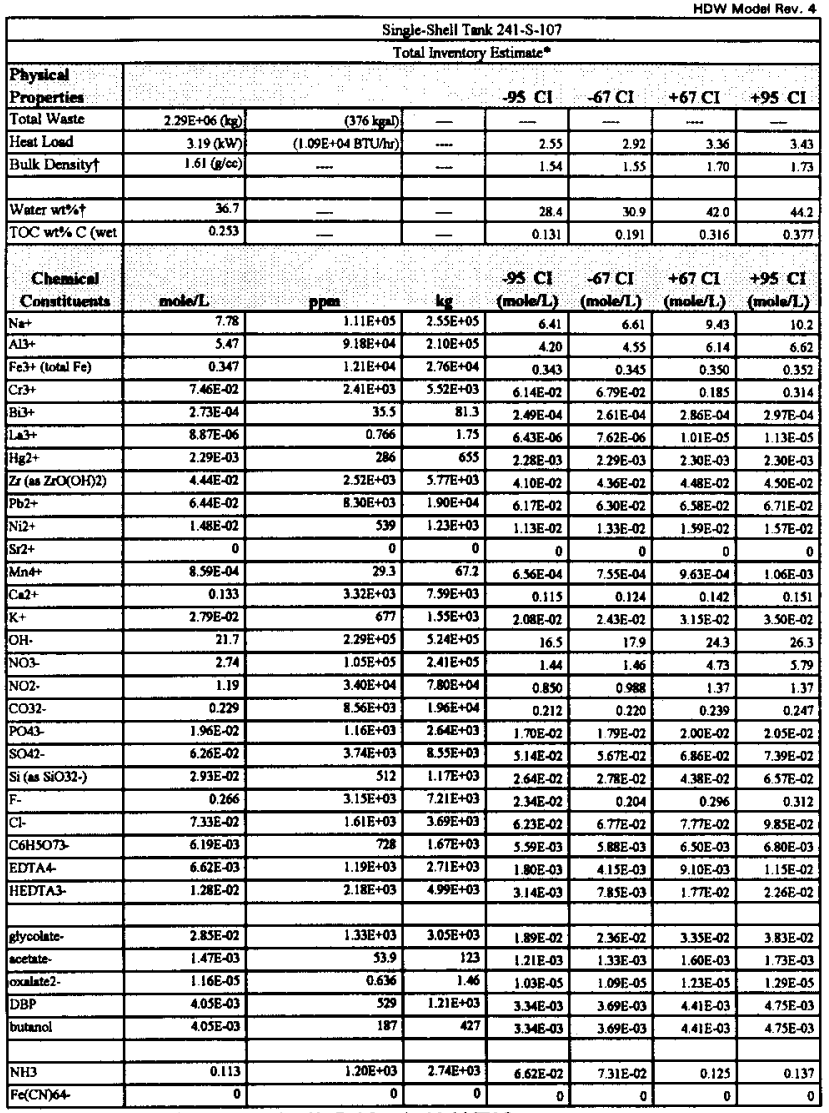

Unknowns in tunk solids inventory wre saigned by Tenk Loyering Model (TLM).

tWater wt\% derived from the difference of dencity and total dissolved epecies. 
HNF-SD-WM-ER-352, Rev. 1

HOW Model Rov. 4

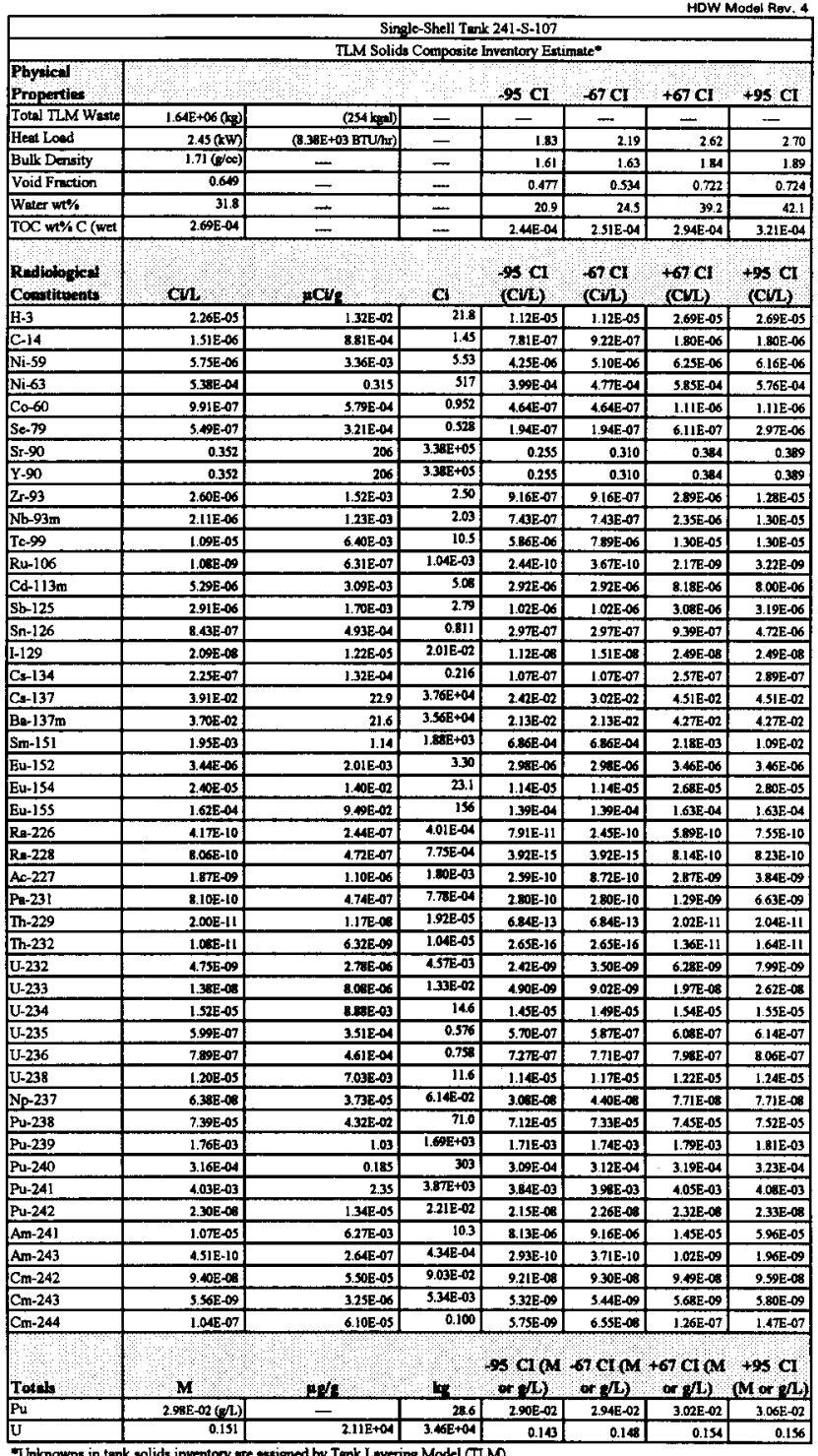

-Unionowns in tenk solids inventory tre sestigned by Tenk Lyering Model (TLM) 
HNF-SD-WM-ER-352, Rev. 1

HDW Model Rev. 4

\begin{tabular}{|c|c|c|c|c|c|c|c|}
\hline \multicolumn{8}{|c|}{ Single-Shell Tank 241-S-107 } \\
\hline \multicolumn{8}{|c|}{ SMaM Connposite Inventory Estimate } \\
\hline $\begin{array}{l}\text { Phyled } \\
\text { Properties }\end{array}$ & & & & $95 \mathrm{CI}$ & $-67 \mathrm{Cl}$ & $+67 \mathrm{CI}$ & $+95 \mathrm{CI}$ \\
\hline Total SMM Wast & $6.46 \mathrm{E}+05(\mathrm{~kg})$ & $(122 \mathrm{kgal})$ & - & - & $\rightarrow$ & $\ldots$ & - \\
\hline Heat Load & $0.732(\mathrm{~kW})$ & (2.50E+03 ETU/hs) & $=$ & 0.670 & 0.710 & 0.755 & $0.7 m$ \\
\hline Bulk Density* & $1.40(0 / \infty)$ & $\ldots$ & - & 1.37 & 1.38 & 1.41 & 1.42 \\
\hline Water wt\% $\%$ & 49.0 & - & - & 46.8 & 47.8 & 50.4 & 51.9 \\
\hline TOC w1\% C (wet & 0.898 & $\cdots$ & - & 0.468 & 0.679 & 1.12 & 133 \\
\hline $\begin{array}{l}\text { Rediofowici! } \\
\text { Constituents }\end{array}$ & $\mathrm{Cu}$ & $\mu C U_{R}$ & Cl & $\begin{array}{l}\$ \mathrm{Cl} \\
(\mathrm{C} / \mathrm{L})\end{array}$ & $\begin{array}{l}67 \mathrm{CI} \\
(\mathrm{CL} / \mathrm{L})\end{array}$ & $\begin{array}{c}+67 \mathrm{CI} \\
\text { (CVL) }\end{array}$ & $\begin{array}{l}+95 \mathrm{CI} \\
(\mathrm{CH} / \mathrm{L})\end{array}$ \\
\hline $\mathrm{H}-3$ & $1.69 \mathrm{E}-04$ & 0.121 & 78.2 & 8.95E-05 & 8.95E-05 & 1.81E-04 & 1.95E-04 \\
\hline $\mathrm{C}-14$ & 2.51E-0S & $1.80 \mathrm{E}-02$ & II.6 & 8.45E-06 & 8.45E-06 & 2.55E.05 & 2.58E-05 \\
\hline Ni-59 & $1,62 \mathrm{E}-06$ & $1.16 \mathrm{E}-03$ & 0.751 & 8.41E-07 & 8.4IE-07 & $1.66 \mathrm{E}-06$ & $1.70 \mathrm{E}-06$ \\
\hline $\mathrm{Ni}-63$ & $1.59 \mathrm{E}-04$ & 0.114 & 73.6 & $8.19 \mathrm{E}-05$ & 8.19E-05 & $1.63 \mathrm{E}-04$ & $1.67 \mathrm{E}-04$ \\
\hline Co-60 & 2.86E-05 & 2.04E-02 & 13.2 & 9.49E-06 & 9. $49 E-06$ & $2.94 \mathrm{E}-05$ & $3.03 \mathrm{E}-05$ \\
\hline Se-79 & 2.69E-06 & $1.88 \mathrm{E}-03$ & 1.22 & $1.57 \mathrm{E}-06$ & $1.57 \mathrm{E}-06$ & $3.02 \mathrm{E}-\infty 6$ & $3.39 \mathrm{E}-06$ \\
\hline St-90 & $9.03 E-02$ & 64.6 & $4 . \overline{E+04}$ & 8.4. & 8.76E-02 & $9.30 \mathrm{E}-02$ & 9.55E-02 \\
\hline Y.90 & $9.03 E-02$ & 64.6 & $4.1 \mathrm{E}+04$ & $5.36 \mathrm{E}-02$ & $5.36 \mathrm{E}-02$ & $9.30 \mathrm{E}-02$ & $9.56 \mathrm{E}-02$ \\
\hline$z-93$ & 1.29E-05 & $9.25 \mathrm{E}-03$ & 5.97 & $7.61 \mathrm{E}-06$ & 7.61E-06 & $1.49 \mathrm{E}-05$ & 1.67E-05 \\
\hline $\mathrm{Nh}-93 \mathrm{~m}$ & 9.36E-06 & 6.70E-03 & 4.32 & 5.64E-06 & S.64E-06 & $1.07 \mathrm{e}-05$ & $1.20 \mathrm{E}-0 \mathrm{~S}$ \\
\hline Tc-99 & 1.79E-04 & 0.128 & 825 & $1.16 \mathrm{E}-04$ & $1.47 \mathrm{E}-04$ & $2.11 \mathrm{E}-04$ & 2.41E-04 \\
\hline Ru-106 & $5.56 \mathrm{E}-09$ & $3.97 \mathrm{E}-06$ & $2.57 \mathrm{E}-03$ & $3.09 \mathrm{E}-09$ & 3.00E- 00 & $6.11 E-\infty 9$ & $6.61 \mathrm{E}-09$ \\
\hline Cd-113m & 6.83E-05 & 492E-02 & 31.8 & $3.69 \mathrm{E}-05$ & $3.69 \mathrm{E}-05$ & 8.03E-05 & $9.14 E-05$ \\
\hline$\$ b-125$ & $1.25 \mathrm{E}-0 \mathrm{~A}$ & 8.05E-02 & 57.8 & $4.2 \mathrm{SE}-05$ & $425 \mathrm{E}-05$ & $130 \mathrm{E}-04$ & $1.35 E-04$ \\
\hline $\mathrm{Sn}-126$ & 3.99E-06 & 2.85E-03 & 184 & $2.38 \mathrm{E}-06$ & 2.38E-06 & $4.56 \mathrm{E}-06$ & $5.11 \mathrm{E}-06$ \\
\hline $1-129$ & 3.44E-07 & $2.46 \mathrm{E}-04$ & 0.159 & $2.24 \mathrm{E}-07$ & 2.83E-07 & $4.06 \mathrm{E}-07$ & $4.66 \mathrm{E}-07$ \\
\hline Cs-134 & 2.70E-06 & 1.93E-03 & 1.25 & $1.58 E-06$ & 2.12E-OS & $3.28 \mathrm{E}-06$ & 3.BAE-OG \\
\hline$C s-137$ & 0.200 & 19 & $9.63 \mathrm{E}+04$ & 0.188 & 0.198 & 0.219 & 0.229 \\
\hline Ba-137m & 0.197 & $14]$ & $9.11 \mathrm{E}+04$ & 0.163 & 0.163 & 0.207 & 0.217 \\
\hline$S m-151$ & $9.2 \pi-03$ & 6.63 & $4.28 E+03$ & $5.55 E-03$ & 5.55E-03 & $1.06 \mathrm{E}-02$ & $1.19 E-02$ \\
\hline Eu-152 & 3.48E-06 & 2. $\triangle 9 E-03$ & 1.61 & $2.16 E-06$ & $2.16 \mathrm{E}-06$ & $3.89 \mathrm{E}-06$ & 4.29E- -06 \\
\hline Eu-154 & 4.8AE-04 & 0.346 & 224 & 2.1BE-04 & $2.18 E-04$ & $5.80 E-04$ & $6.19 \mathrm{E}-04$ \\
\hline Eu-155 & 2.09E-04 & 0.14 & 96.0 & $1.29 \mathrm{E}-0 \mathrm{~d}$ & 1.20E-04 & $2.33 \mathrm{E}-04$ & $2.58 \mathrm{E}-04$ \\
\hline $\operatorname{Ra}-226$ & $1.09 \mathrm{E}-10$ & $7.78 \mathrm{E}-08$ & $5.02 \mathrm{E}-05$ & 7.58E-11 & 7. S0E-11 & $1.21 \mathrm{E}-10$ & $1.32 \mathrm{E}-10$ \\
\hline Ra-228 & $9.63 \mathrm{E} \cdot 0 \mathrm{~B}$ & $6.89 \mathrm{E}-05$ & 4.45E-02 & 1.09E-08 & $6.80 \mathrm{E}-08$ & 1.29E-07 & 1.64E-07 \\
\hline$A c-227$ & 6.84E-10 & 4.89E-07 & $3.16 \mathrm{E}-04$ & 4.92E-10 & $4,92 \mathrm{E}-10$ & 7.53E-10 & 8.20E-10 \\
\hline$P_{0.231}$ & $3.17 \mathrm{E}-09$ & 2.27E-06 & $1.4 \pi-03$ & $2.11 \mathrm{E}-09$ & $2.11 \mathrm{E}-09$ & $3.56 \mathrm{E}-09$ & $3.93 \mathrm{E}-09$ \\
\hline Th-229 & $2.26 \mathrm{E}-09$ & $1.62 \mathrm{E}-06$ & 1.05E-A3 & 1.05E-69 & 1.64E-09 & 2.98E-09 & $3.75 \mathrm{E}-09$ \\
\hline Th-232 & $6.50 \mathrm{E}-09$ & 465E-06 & $3.00 \mathrm{E}-03$ & 3.43E-09 & 493E-09 & $8.06 \mathrm{E}-09$ & $9.57 \mathrm{~T}-09$ \\
\hline U-232 & 49:9E-07 & $3,56 \mathrm{E}-04$ & 0.230 & $2.76 \mathrm{E}-07$ & 3.84E-07 & $6.30 \mathrm{E}-07$ & $7.70 \mathrm{E}-07$ \\
\hline $\mathrm{U} \cdot \mathbf{2 3 3}$ & 1.91E-06 & $1.37 \mathrm{E}-03$ & 0.62 & $1.06 \mathrm{E}-06$ & 1.4TE- 06 & $2.41 \mathrm{E}-06$ & 2.95E-06 \\
\hline U-234 & $5.63 \mathrm{E}-07$ & 1.03E-04 & 0.260 & $5.428-07$ & $5.53 \mathrm{E}-07$ & $5.74 E-07$ & $5.81 \mathrm{E}-07$ \\
\hline U-235 & $2.28 \mathrm{E}-08$ & 1.63E-05 & 1.05E-02 & $2.20 \mathrm{E}-08$ & $2.24 E-48$ & $2.33 \mathrm{E}-08$ & $2.36 \mathrm{E}-08$ \\
\hline $\mathrm{U}-236$ & 1.T2E-08 & $1.27 \mathrm{E}-05$ & 8.19E-03 & 1.71E-0s & 1.75E-08 & 1.60E-08 & 1.83E- 08 \\
\hline U-238 & 6.53E-07 & 4.67E-0A & 0.302 & 6.33E-07 & 6.49E-07 & 6.64E-07 & $6.70 \mathrm{E}-07$ \\
\hline $\mathrm{Np}-237$ & $6.43 \mathrm{E}-07$ & 4.60E-04 & 0.297 & 4.40E-07 & 5.39E-07 & 7.47E-07 & 8.48E-07 \\
\hline Pu-238 & $1.18 E-06$ & 8. $46 \mathrm{E}-0 \mathrm{~A}$ & 0.546 & $9.44 E-07$ & 1.06E-06 & $1.30 \mathrm{E}-06$ & $1.42 \mathrm{E}-06$ \\
\hline $\mathrm{Pu}-239$ & $4.00 \mathrm{E}-05$ & 2.86E-02 & 18.5 & $3.39 \mathrm{E}-0 \mathrm{~S}$ & 3.60E-05 & $4.31 \mathrm{E}-05$ & 4.61E-05 \\
\hline $\mathrm{Pu}-240$ & 6.85E- 06 & $4.90 E-63$ & 3.26 & $3.71 \mathrm{E}-06$ & $6.2 \pi-06$ & $7.42 \mathrm{E}-\mathrm{-06}$ & $7.985-06$ \\
\hline $\mathrm{Pu}-241$ & B. IOE-05 & $5.600-02$ & 37.4 & 6.48E-05 & $7.27 \mathrm{E}-0 \mathrm{~s}$ & 8.93E-05 & 9.73E-05 \\
\hline Pu-242 & 4.45E.10 & $3.19 \mathrm{E}-07$ & 2.06E-04 & 3.48E-10 & $3.96 \mathrm{E}-10$ & 4.95E-10 & $5.43 E-10$ \\
\hline Am-241 & 4 46E-0S & $3.19 \mathrm{E}-02$ & 20.6 & $3.33 \mathrm{E}-0 \mathrm{~S}$ & 3.89E-05 & $5.04 \mathrm{E}-0 \mathrm{~S}$ & $5.60 \mathrm{E}-05$ \\
\hline Am-243 & 1.64E-49 & $1.18 \mathrm{E}-06$ & $7.59 \mathrm{E}-0.9$ & 1.26E-09 & 1.4AE-09 & $188 \mathrm{E}-09$ & 2.09E-09 \\
\hline $\mathrm{Cm}-242$ & 1.32E-07 & 9.46E-05 & $6.11 \mathrm{E}-02$ & $7.8 \mathrm{JE}-08$ & $7.81 \mathrm{E}-08$ & 1.48E-07 & 1.63E-07 \\
\hline $\mathrm{Cm}+243$ & $1.22 \mathrm{E}-08$ & 8.71E-06 & $5.62 \mathrm{E}-03$ & $7015-09$ & 7.01E-99 & $1.36 \mathrm{E}-08$ & 1.50E-08 \\
\hline $\mathrm{C} m-244$ & $1.12 E-07$ & $8.02 \mathrm{E}-05$ & 5.18E-02 & 5.D9E-08 & 5. $995-0 \mathrm{~B}$ & $1.30 \mathrm{E}-07$ & $1.42 \mathrm{E}-07$ \\
\hline Totels & $\mathbf{M}$ & $i d s$ & w & $\begin{array}{l}95 \mathrm{al} \\
-\mathrm{m})\end{array}$ & $\begin{array}{l}67 \mathrm{CH} \\
\text { of } \mathrm{Cl})\end{array}$ & $\begin{array}{c}+67 C R Q \\
\alpha+L)\end{array}$ & $\begin{array}{l}+95 \mathrm{Cl} \\
\mathrm{M} \text { or } \mathrm{AJ})\end{array}$ \\
\hline Pu & $4.60 E-04(\% / L)$ & - & 0.212 & $3.44 E-04$ & 4,01E-04 & S.18E-04 & 5.75E-04 \\
\hline $\mathrm{U}$ & 6.45E- 03 & $1.10 \mathrm{E}+03$ & 70 & $6.20 \mathrm{E}-03$ & $6.32 \mathrm{E}-03$ & $6.59 \mathrm{E}-03$ & $6.66 \mathrm{E}-03$ \\
\hline
\end{tabular}

-Density is calculated based on $\mathrm{Na}$ OH, and $\mathrm{AlO}_{2}$.

tWater wt\% derived from the diffarence of density and total diswolved apecies 
HNF-SD-WM-ER-352, Rev. 1

MDW Model Rev. 4

\begin{tabular}{|c|c|c|c|c|c|c|c|}
\hline \multirow[b]{3}{*}{$\begin{array}{l}\text { Phyrieal } \\
\text { Properties }\end{array}$} & \multicolumn{7}{|c|}{ Single-Shell Tank 241-S-107 } \\
\hline & \multicolumn{7}{|c|}{ Total Inventory Estimate* } \\
\hline & \&: & & & $-95 \mathrm{CI}$ & $67 \mathrm{CI}$ & $+67 \mathrm{Cl}$ & $+95 \mathrm{Cl}$ \\
\hline Total Waste & $229 \mathrm{E}+06(\mathrm{~kg})$ & (376 k데) & - & - & - & - & - \\
\hline Heat Loed & $3.19(\mathrm{~kW})$ & $(1.09 \mathrm{E}+04 \mathrm{BTU} / \mathrm{hr})$ & - & 2.55 & 2.92 & 3.36 & 3.43 \\
\hline Bulk Density† & $1.61(g / c)$ & \begin{tabular}{l|}
- \\
-
\end{tabular} & - & 1.54 & 1.55 & 1.70 & 1.73 \\
\hline & & & & & & & \\
\hline Water wt\% $\phi$ & 36.7 & - & - & 28.4 & 30.9 & 42.0 & 44.2 \\
\hline TOC wt $\%$ C (wet & 0.253 & - & - & 0.131 & 0.191 & 0.316 & 0.37 \\
\hline $\begin{array}{l}\text { Rollototed } \\
\text { Conntituenth }\end{array}$ & $\mathrm{CH}$ & $\mathrm{pC}_{\mathbf{l}}$, & c) & $\begin{array}{l}\text { os CI } \\
\text { (cn) }\end{array}$ & (Cis) & $\begin{array}{l}+6 \mathrm{Cl} \\
\text { (CUL) }\end{array}$ & $\begin{array}{l}+95 \mathrm{CI} \\
\text { (CUL) }\end{array}$ \\
\hline $\mathrm{H} \cdot \mathbf{3}$ & T.02E-05 & $4.36 \mathrm{E}-02$ & 99.9 & 4,43E-05 & 4.43E-05 & $7.40 \mathrm{E}-05$ & $7.87 \mathrm{E}-0 \mathrm{~S}$ \\
\hline C-14 & $9.1 \pi-06$ & $5.70 \mathrm{E}-03$ & 13.1 & $3.76 \mathrm{E}-06$ & $3.76 \mathrm{E}-06$ & $9.37 \mathrm{~T}-06$ & $9.40 \mathrm{E}-06$ \\
\hline Ni-59 & 4.41E-06 & $2.74 \mathrm{E}-03$ & 6.28 & $3.40 \mathrm{E}-06$ & $3.97 E_{-06}$ & 4. $75 \mathrm{EE}-06$ & $4.68 \mathrm{E}-06$ \\
\hline $\mathrm{Ni}-63$ & 4.15E-04 & 0.258 & 591 & $3.21 \mathrm{E}-04$ & $3.74 \mathrm{E}-\mathrm{as}$ & 4.4TE-04 & 4.40E-04 \\
\hline $\mathrm{Co}-60$ & 9.94E-OS & 6.18E-03 & 14.2 & $3.75 E-06$ & $3.75 E-06$ & $1.02 E-05$ & 1.0SE-05 \\
\hline Se-79 & $1.23 \mathrm{E}-06$ & $7.62 \mathrm{E}-04$ & 1.74 & B.BOE-07 & 8.BOE-07 & $1.35 E-06$ & 2.83E-06 \\
\hline $\mathrm{Sr}-90$ & 0.267 & 166 & 3.80E+05 & 0.202 & 0.238 & 0.289 & 0.292 \\
\hline Y.SO & 0.267 & 166 & $3.00 \mathrm{E}+05$ & 0.202 & 0.239 & 0.289 & 0.292 \\
\hline $\mathrm{Zx}-93$ & 5.95E-06 & $3.20 \mathrm{E}-03$ & 8.47 & $4.23 \mathrm{E}-06$ & 4.23E-96 & $6.58 \mathrm{E}-96$ & $1.2 T \mathrm{E}-0 \mathrm{~S}$ \\
\hline $\mathrm{Nb}-93 \mathrm{~m}$ & 4.45E-06 & $2.77 \mathrm{E}-03$ & 6.35 & $3.25 \mathrm{E}-06$ & $325 \mathrm{E}-\infty$ & $490 \mathrm{E}-06$ & 1.17E-0S \\
\hline Tc-99 & $6.53 \mathrm{E}-05$ & 4.06E-02 & 93.0 & $4.52 \mathrm{E}-05$ & 5.50E-05 & $7.57 \mathrm{E}-05$ & 8.58E-05 \\
\hline Ru-106 & $2.53 \mathrm{E}-09$ & $1.57 \mathrm{E}-06$ & $3.60 \mathrm{E}-03$ & $1.73 E-199$ & $1,73 \mathrm{E}-09$ & $3.26 \mathrm{E}-09$ & $3.96 \mathrm{E}-09$ \\
\hline $\mathrm{Cd}-113 \mathrm{~m}$ & $2.59 \mathrm{E}-03$ & $1.61 \mathrm{E}-02$ & 36.9 & L.SSE-05 & 1.55E-05 & 2.96E-05 & $3.32 E-05$ \\
\hline$S b-125$ & 4.26E-05 & 2.65E- -22 & 60.6 & $1.57 E-05$ & $1.572-05$ & 4.4LE-0s & 4.56E-05 \\
\hline $5 n-126$ & $1.86 \mathrm{E}-06$ & $1,16 E-03$ & 2.65 & L.34E-A6 & $1.34 E-96$ & 2.05E- 06 & $4.43 E-06$ \\
\hline 1.129 & $1.26 E-07$ & 7.83E-05 & 0.179 & B.GE- $\infty$ & $1.06 E-07$ & $1.46 \mathrm{E}-07$ & 1,65E -07 \\
\hline Cs-134 & $1.03 \mathrm{E}-06$ & $6.39 \mathrm{E}-04$ & 1.46 & 6.63E-07 & 8.42E-07 & $1.22 E-06$ & 1.40E-06 \\
\hline Cs-137 & 9.41E-02 & 38.5 & $1.3 \mathrm{EE}+05$ & 8.0E-02 & 8.91E-02 & $9.81 E-02$ & 0.101 \\
\hline Ba-137m & $8.90 \mathrm{E}-02$ & 55.3 & $1.2 \pi+05$ & $6.72 \mathrm{E}-02$ & $6.72 \pm-02$ & $9.28 \mathrm{E}-02$ & $9.54 \mathrm{E}-02$ \\
\hline Sm-151 & 433E-03 & 2.69 & $6.16 \mathrm{E}+03$ & $3.12 E-03$ & $3.12 E-03$ & 4.7TE-03 & $1.02 \mathrm{E}-02$ \\
\hline $\mathrm{Eu}_{\mathrm{U}} 152$ & 3.45E-06 & $2.15 E-03$ & 491 & $3.02 \mathrm{E}-06$ & $3.02 E-06$ & $3,58 E-06$ & 3.71E- -6 \\
\hline Eu-154 & $1.73 E-0.9$ & 0.108 & 247 & 2.70E-05 & 8.70E-05 & $2.05 E-04$ & $2.1 T E-04$ \\
\hline Eu-155 & 1.T7E-04 & 0.110 & 252 & $1.52 \mathrm{E}-04$ & .52E-04 & 1.85E-04 & 1.93E-04 \\
\hline Ra-226 & $3.172-10$ & $1.97-07$ & 4.51E-04 & 9.31E-11 & 2.03E-10 & $4.31 \mathrm{E}-10$ & 9.41E-10 \\
\hline$R_{g}-228$ & $3.18 \mathrm{E}-08$ & 1.98E-05 & $4.53 \mathrm{E}-02$ & 1.38E-08 & $2.26 \mathrm{E}-08$ & 4.25E-AB & $5.38 E-68$ \\
\hline Ac-227 & $1.49 \mathrm{E}-09$ & $9.25 \mathrm{E}-07$ & 2.12E-03 & 4.1BE-10 & 8.24E-10 & 2.15E-09 & 2.79E-09 \\
\hline $\mathrm{Pa}-231$ & $1.58 E-09$ & 9.00E-07 & $2.24 E-03$ & 1.15E-09 & $1.15 \mathrm{E}-09$ & $1.89 \mathrm{E}-09$ & 5.43E-09 \\
\hline Th-229 & 7.48E-10 & 4.65E-07 & $1.06 \mathrm{E}-03$ & $3.53 E-10$ & $3.46 \mathrm{E} \cdot 10$ & $9.82 E-10$ & 1.23E-09 \\
\hline Th-232 & $2.12 \mathrm{E}-09$ & 1.32E-06 & $3.01 \mathrm{E}-03$ & $1.12 \mathrm{E}-9$ & $1.61 E-09$ & 2.62E-09 & 3.11E-09 \\
\hline U-232 & 1.65E-07 & $1.02 E-04$ & 0.235 & $9.2 \pi-A B$ & 1.28E-07 & $2.08 E-07$ & 2.53E-07 \\
\hline $\mathrm{U}-233$ & $6.29 \mathrm{E}-07$ & 391E-04 & 0.695 & 3.53E-07 & 48E-07 & $7.93 E-07$ & $9.67 \mathrm{~T}-07$ \\
\hline $\mathrm{U}-234$ & $1.04 E-05$ & 6.0E-03 & 14.9 & 9.98E-06 & $1.02 \mathrm{E}-05$ & $1.065-05$ & 1.0TE-05 \\
\hline $\mathrm{U}-235$ & $4.12 \mathrm{E}-07$ & $2.56 \mathrm{E}-04$ & 0.587 & 3.93E-97 & 4.04E-97 & $4.18 E-07$ & 4.22E-07 \\
\hline U-236 & $5,39 E-07$ & 3.35E-04 & 0.767 & 4.97E-07 & $5.2 \pi-07$ & $5.44 E-07$ & 5.50E-07 \\
\hline U-238 & 8.33E-06 & 5.18E-03 & 11.9 & $7.8 E-\infty$ & B.14E-06 & 8.46E-06 & 8.56E-06 \\
\hline $\mathrm{Np}-237$ & $2.52 E-07$ & 1.56E- -94 & 0.350 & 1.06E-07 & $2.18 E-07$ & $2.86 \mathrm{E}-07$ & $3.19 \mathrm{E}-07$ \\
\hline Pu-238 & $5.03 E-05$ & 3.13E-02 & 71.6 & 4.85E-05 & 4.99E-05 & 5.07E-05 & S.11E-05 \\
\hline Pu-239 & $1.20 \mathrm{E}-03$ & 0.748 & $1.7 \mathrm{IE}+03$ & $1.17 E-03$ & 1.19E-03 & 1.22E-03 & $1.24 E-03$ \\
\hline Pu-240 & $2.15 E-04$ & 0.134 & 307 & 2.11E-A4 & $2.13 \mathrm{E}-0 \mathrm{~A}$ & $2.18 \mathrm{E}-0.4$ & $220 \mathrm{E}-04$ \\
\hline Pu-24! & $2.75 E-03$ & 1.71 & $3.91 E+03$ & $2.62 E-03$ & $2.72 E-03$ & $2.76 \mathrm{E}-03$ & 2.78E-03 \\
\hline Pu-242 & $1.57 \mathrm{E}-08$ & 9.75E- -66 & $2.23 E-02$ & 1.47E-08 & 1.54E-08 & $1.58 E-08$ & $1.59 \mathrm{E}-08$ \\
\hline Am-24! & $2.17 \pi-05$ & $1.35 E-02$ & 30.9 & 1.81E-05 & $1.99 \mathrm{E}-05$ & $2.43 \mathrm{E}-0 \mathrm{~S}$ & $5.41 E-05$ \\
\hline Am-243 & $8.38 \mathrm{E}-10$ & $3.21 \mathrm{E}-07$ & $1.19 \mathrm{E}-03$ & $7.14 \mathrm{E}-10$ & $7.71 \mathrm{E}-10$ & $1.22 \mathrm{E}-09$ & $1.94 \mathrm{E}-09$ \\
\hline $\mathrm{Cm}-242$ & $1,06 \mathrm{E}-07$ & 6.61E-05 & 0.151 & $8.88 E-08$ & $8.88 E-08$ & 1.11E-07 & 1.16E-07 \\
\hline $\mathrm{Cm}-243$ & $7.70 \mathrm{E}-09$ & 4.79E- -6 & $1.10 \mathrm{E}-02$ & $6.03 \mathrm{E}-09$ & $6.03 \mathrm{E}-09$ & $8.16 \mathrm{E}-09$ & $8.61 \mathrm{E}-09$ \\
\hline $\mathrm{Cm}-244$ & 1.07E-07 & 6.64E-05 & 0.152 & 4.01E-0 & 2.06E-08 & 1.21E-07 & 1.35E-07 \\
\hline Totels & $\mathbf{M}$ & $\Delta \mathrm{s} / \mathrm{s}$ & $\mathbf{H}^{\prime}$ & $\begin{array}{l}95 \mathrm{M} \\
\text { on } \mathrm{L})\end{array}$ & $\begin{array}{l}6 \%(\mathrm{M} \\
\text { or L) }\end{array}$ & $\begin{array}{l}+67 \mathrm{ct}(\mathrm{M} \\
0, \mathrm{p} / \mathrm{L})\end{array}$ & $\begin{array}{c}+54 \mathrm{Cl} \\
(\mathrm{M} \alpha \mathrm{L})\end{array}$ \\
\hline$P$ & $2.03 E-02(\alpha)$ & - & 288 & $1.97 \mathrm{E}-02$ & $2.00 E-02$ & 2.05E-02 & $2.08 \mathrm{E}-02$ \\
\hline $\mathrm{U}$ & 0.104 & $1.54 E+24$ & $3.54 \mathrm{EE}+04$ & $9.37 t-02$ & 0.102 & 0.106 & 0.107 \\
\hline
\end{tabular}




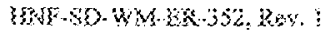
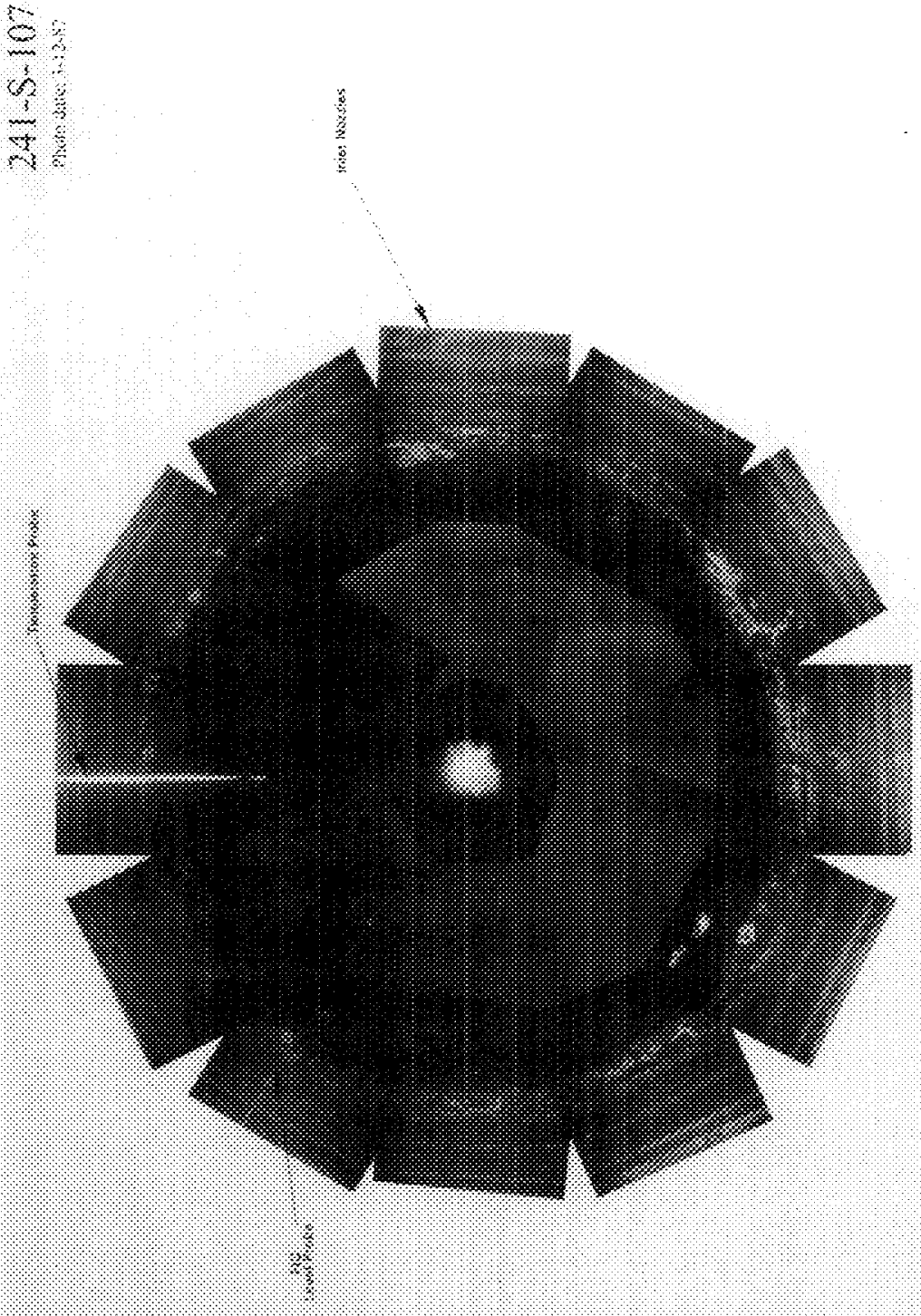

1,

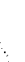


TANK 241-S-108 SUMMARY

\begin{tabular}{|c|c|c|c|}
\hline \multicolumn{2}{|c|}{ TANK HISTORY } & \multicolumn{2}{|c|}{ TANK DESCRIPTION } \\
\hline Entered Service & 4th qtr 1952 & Diameter & $75 \mathrm{ft}$ \\
\hline Removed from Service & - & Bottom Shape & Dish \\
\hline Inactive & 1979 & Nominal Capacity & $758,000 \mathrm{gal}$ \\
\hline Watch Lists & none & Cascade Tank & to $241-S-109$ \\
\hline Integrity & Sound & Total Risers & 12 \\
\hline Assumed Leaker & - & \multicolumn{2}{|c|}{\begin{tabular}{|c|} 
WASTE VOLUME (HANLON 1996i) \\
\end{tabular}} \\
\hline Interim Stabilization (IS) & - & Total Waste Volume & $604,000 \mathrm{gal}$ \\
\hline Partial Interim Isolation (PI) & Dec 1982 & Waste Type & NCPLX \\
\hline Intrusion Prevention (IP) & - & Drainable Interstitial Liquids & $92,000 \mathrm{gal}$ \\
\hline \multicolumn{2}{|c|}{ TENTATIVELY AVAILABLE RISERS } & Pumpable Liquids & $105,000 \mathrm{gal}$ \\
\hline Riser Number(s) & Size & Saltcake & $600,000 \mathrm{gal}$ \\
\hline 11,14 & 4 in & Sludge & $4,000 \mathrm{gal}$ \\
\hline 7,8 & 12 in & Supernatant & $0 \mathrm{gal}$ \\
\hline \multicolumn{2}{|c|}{\begin{tabular}{|c|} 
TANK TEMPERATURE \\
\end{tabular}} & \multicolumn{2}{|c|}{ INTERIOR PHOTOGRAPHS } \\
\hline Average Tank Temperature & $81^{\circ} \mathrm{F}$ & Date & March 12, 1987 \\
\hline Maximum Temperature & $94.64^{\circ} \mathrm{F}$ & Montage Number & $94041033-32 \mathrm{CN}$ \\
\hline Date & Dec 15, 1995 & Photo Set Number & 8701462 \\
\hline Elevation from tank bottom & $10.45 \mathrm{ft}$ & \multicolumn{2}{|c|}{\begin{tabular}{|c|} 
WASTE SURFACE LEVEL \\
\end{tabular}} \\
\hline Riser Number & 4 & Devices & Manual ENRAF \\
\hline Minimum Temperature & $57^{\circ} \mathrm{F}$ & Max Level & 191 in \\
\hline Date & July 2, 1994 & Date & Feb 4, 1991 \\
\hline Elevation from tank bottom & $30.45 \mathrm{ft}$ & Min Level & 158.8 in \\
\hline Riser Number & 4 & Date & April 3, 1995 \\
\hline
\end{tabular}




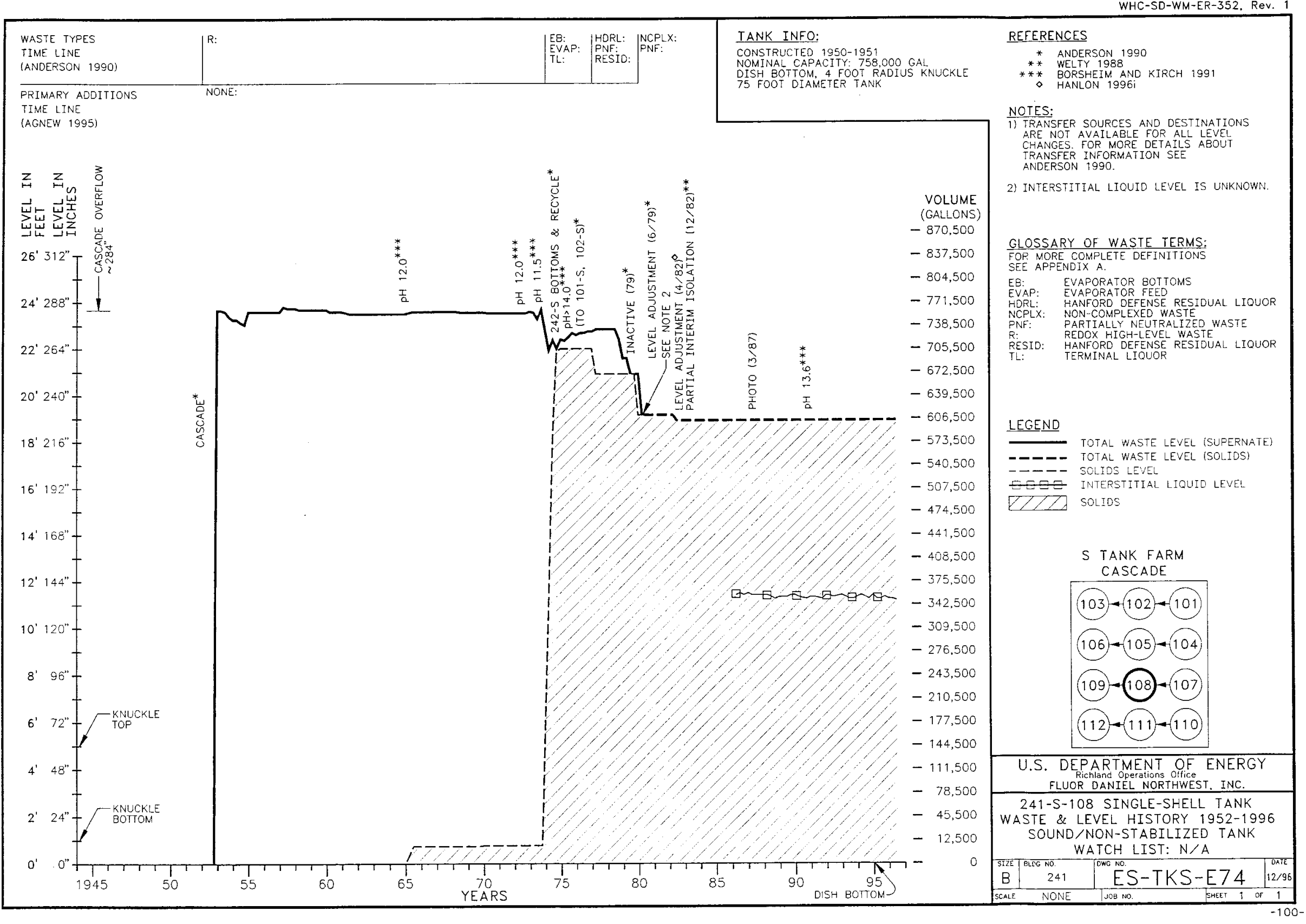




\section{$241-5-108$}

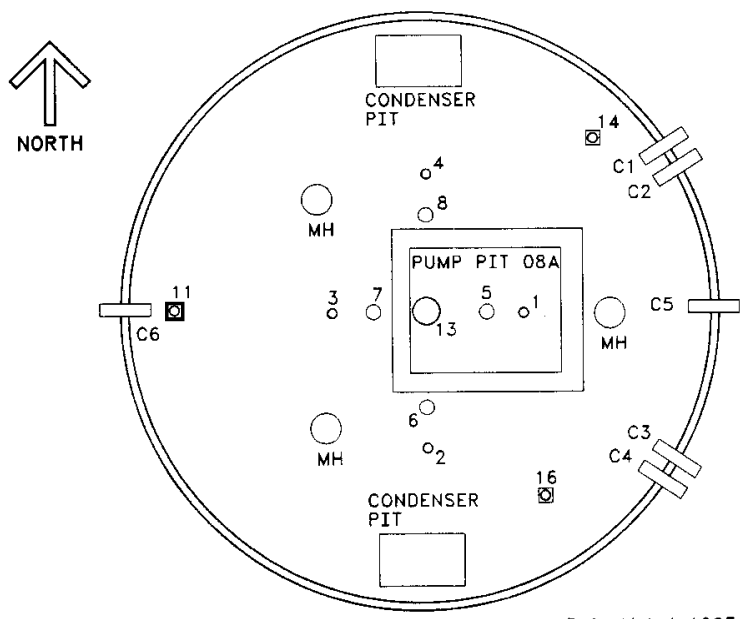

Ref: Alstad 1993

$\mathrm{H}-2-73187$. Rev. 4

H-2-37531, Rev. 2

\section{TANK RISER LOCATION}

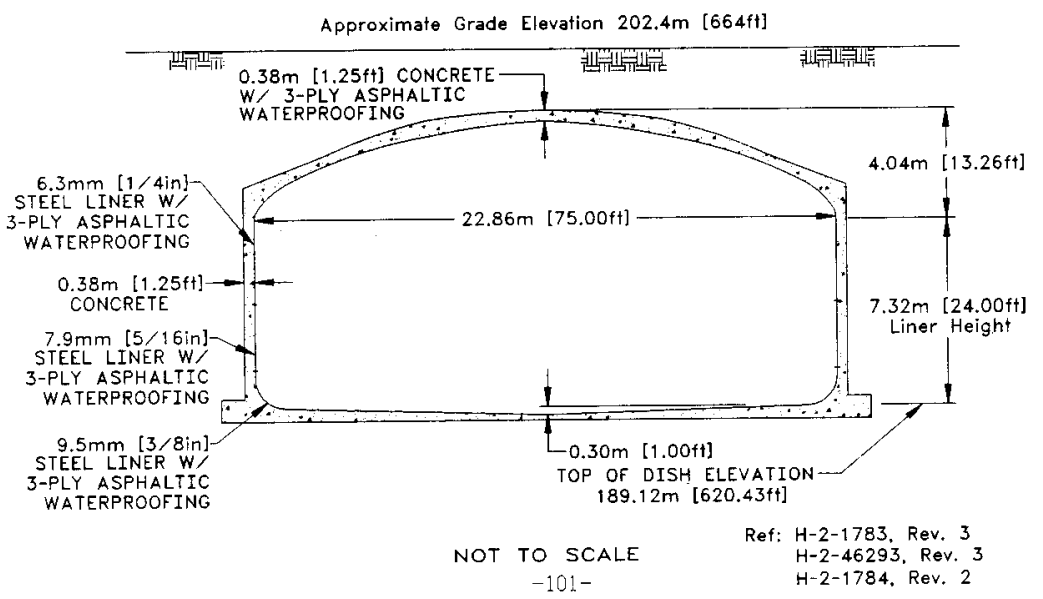


HNF-SD-WM-ER-352, Rev. 1

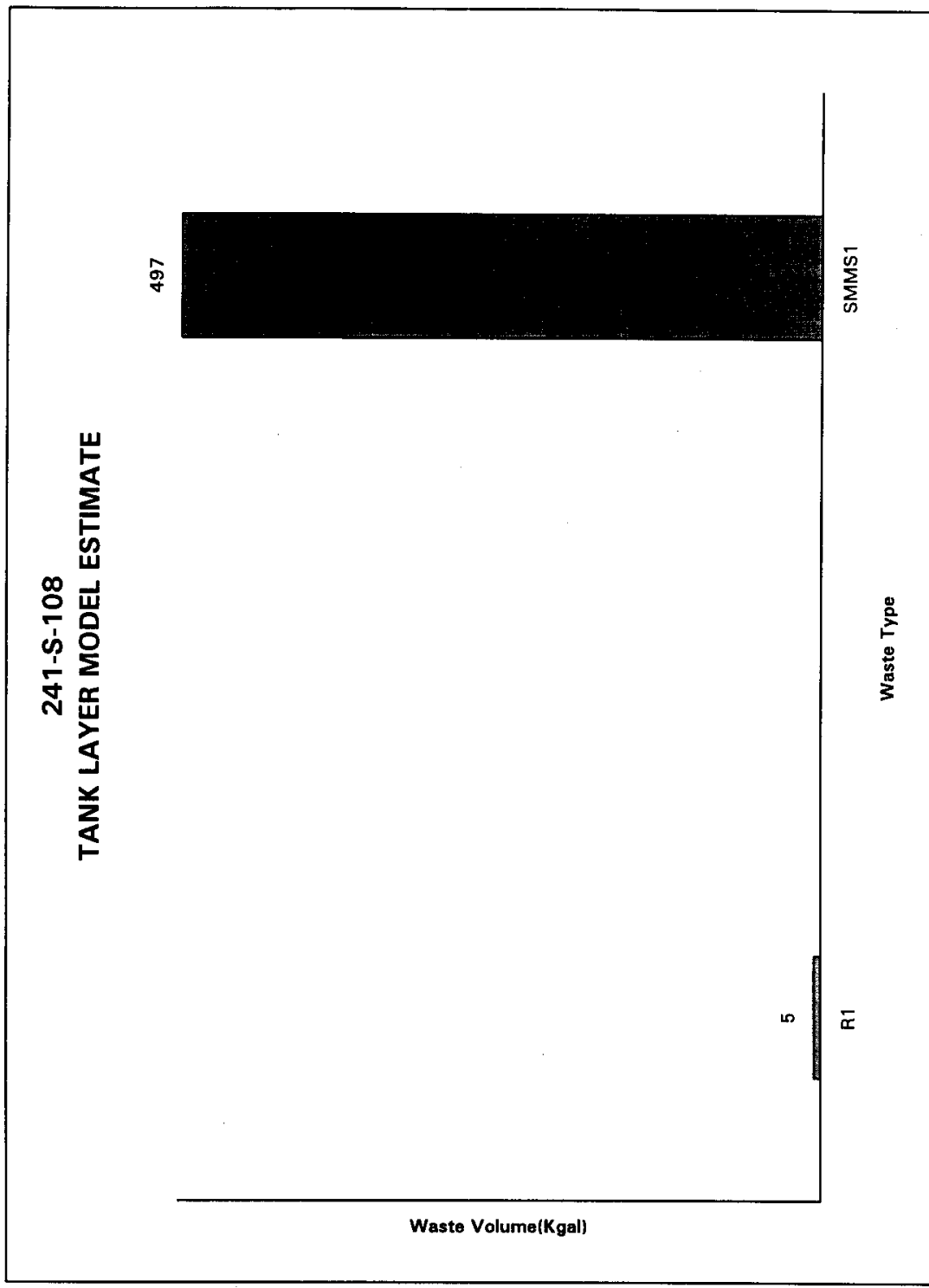

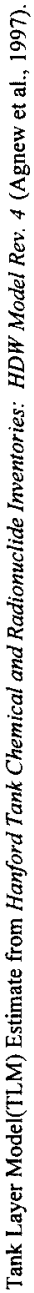


HDW Model Aev. 4

\begin{tabular}{|c|c|c|c|c|c|c|c|}
\hline \multirow[b]{3}{*}{$\begin{array}{l}\text { Phypicat } \\
\text { Propertias }\end{array}$} & \multicolumn{7}{|c|}{ Sirgle-Shell Tank 241-S-108 } \\
\hline & \multicolumn{7}{|c|}{ TLM Solids Composite Inventory Estimate* } \\
\hline & & & & -9s CI & $-67 \mathrm{CI}$ & $+67 \mathrm{CI}$ & $+95 \mathrm{Cl}$ \\
\hline Totel TLM Waste & $3.32 \mathrm{E}+04(\mathrm{~kg})$ & (5.00 kgtil) & - & $\ldots$ & - & - & $\ldots$ \\
\hline Fieat Lond & $0.123(\mathrm{kw})$ & (42) BTU/hr) & $\ldots$ & 8.94E-02 & 0.109 & 0.132 & 0.136 \\
\hline Bulk Density & $1.76(\mathrm{~g} / \mathrm{cx})$ & - & $\ldots$ & 1.51 & 1.53 & 2.11 & 2.24 \\
\hline Void Fraction & 0.536 & - & $\ldots$ & $600 \mathrm{E}-02$ & 0.217 & 0.737 & 0.743 \\
\hline Water wi\% & 27.0 & - & - & 2.64 & 10.0 & 42.6 & 44.2 \\
\hline TOC wt\% C (wet & 0 & - & - & 0 & 0 & 0 & 0 \\
\hline $\begin{array}{l}\text { Chemiled } \\
\text { Conthitumats }\end{array}$ & molert & pon & $\mathrm{kg}: \mathrm{:}$ & $\begin{array}{l}95 \mathrm{CT} \\
(\mathrm{n}=\mathrm{W} / \mathrm{A})\end{array}$ & $\begin{array}{l}-67 \mathrm{CI} \\
\text { (1nolih) }\end{array}$ & $\begin{array}{l}+67 \mathrm{CI} \\
(\mathrm{mol} / \mathrm{L})\end{array}$ & $\begin{array}{l}+95 \mathrm{CI} \\
\text { (anoleh } \mathrm{L})\end{array}$ \\
\hline $\mathrm{Na}+$ & 8.13 & $1.06 \mathrm{E}+05$ & $3.53 \mathrm{E}+03$ & 3.05 & 3.56 & 14.7 & 18.2 \\
\hline $\mathrm{Al3}^{+}$ & 6.99 & $1.07 \mathrm{E}+05$ & $3.56 \mathrm{E}+03$ & 6.58 & 6.74 & 7.15 & 7.15 \\
\hline Fey+(totel Fe) & 1.01 & $3.22 \mathrm{E}+0.24$ & $1.07 E+03$ & 0.994 & 1.00 & 1.02 & 1.03 \\
\hline $\mathrm{Cr} 3+$ & $6.19 \mathrm{E}-02$ & $1.83 \mathrm{E}+03$ & 60.8 & 7.09E-03 & $3.39 \mathrm{E}-02$ & 0.522 & 1.06 \\
\hline Bi3+ & 0 & 0 & 0 & 0 & 0 & 0 & 0 \\
\hline La3+ & 0 & 0 & 0 & 0 & 0 & 0 & 0 \\
\hline $\mathrm{Hg} 2+$ & 0 & 0 & 0 & 0 & 0 & 0 & 0 \\
\hline $\mathrm{Z}(\mathrm{w} \mathrm{ZO}(\mathrm{OH}) 2)$ & 0 & 0 & 0 & 0 & 0 & 0 & 0 \\
\hline $\mathrm{Pb} 2+$ & 0 & 0 & 0 & 0 & 0 & 0 & 0 \\
\hline $\mathrm{Ni2+}$ & $5.07 \mathrm{E}-02$ & $1.69 \mathrm{E}+03$ & 36.3 & 3.66E-02 & 4.45E-02 & 5.54E-02 & 5.45E-02 \\
\hline $5 \times 2+$ & 0 & 0 & 0 & 0 & 0 & 0 & 0 \\
\hline Mn4t & 0 & 0 & 0 & 0 & 0 & 0 & 0 \\
\hline $\mathrm{Co} 2+$ & 0.220 & $5.02 E+03$ & 167 & 0.146 & 0.182 & 0.258 & 0.294 \\
\hline $\mathrm{K}+$ & $1.12 E-02$ & 250 & 8.30 & 1.28E-03 & 5.25E-03 & $1.52 \mathrm{E}-02$ & $1.52 \mathrm{E}-02$ \\
\hline OH- & 25.6 & $2.48 E+05$ & $8.23 \mathrm{E}+03$ & 23.1 & 24.3 & 26.9 & 28.4 \\
\hline $\mathrm{NO3}$ & 5.29 & 1.87E+05 & $6.20 \mathrm{E}+03$ & 6.03E-02 & 6.03E-02 & 13.5 & 17.9 \\
\hline NO2- & 1.46 & $3.82 E+04$ & $1.27 \mathrm{E}+03$ & 0.132 & 0.595 & 2.15 & 2.19 \\
\hline $\cos 2$ & 0.220 & $7.51 E+63$ & 249 & 0.146 & 0.182 & 0.258 & 0.294 \\
\hline PO43- & 0 & 0 & 0 & 0 & 0 & 0 & 0 \\
\hline SO42- & $1.04 E-02$ & 569 & 18.9 & 1.19E-03 & 4,8TE-03 & $1.41 E-02$ & $1.41 \mathrm{E}-02$ \\
\hline $\mathrm{Si}(\mathrm{cosiO} 32-)$ & $8.06 k-03$ & 129 & 4.28 & 9.21E-04 & $3.77 \mathrm{E}-03$ & $1.09 \mathrm{E}-02$ & $1.09 \mathrm{E}-02$ \\
\hline F. & 0 & 0 & 0 & 0 & 0 & 0 & 0 \\
\hline cl- & $5.16 \mathrm{E}-02$ & $1.04 \mathrm{E}+03$ & 346 & S.91E-03 & 2.42E-02 & $7.00 \mathrm{E}-02$ & 0.157 \\
\hline C6HSO73 & 0 & 0 & 0 & 0 & 0 & 0 & 0 \\
\hline EDTA4 & 0 & 0 & 0 & 0 & 0 & 0 & 0 \\
\hline HEDTA3 & 0 & 0 & 0 & 0 & 0 & 0 & 0 \\
\hline glycolnte- & 0 & 0 & 0 & 0 & 0 & 0 & 0 \\
\hline scetates- & 0 & 0 & 0 & 0 & 0 & 0 & 0 \\
\hline oxulate2. & 0 & 0 & 0 & 0 & 0 & 0 & 0 \\
\hline DEP & 0 & 0 & 0 & 0 & 0 & 0 & 0 \\
\hline butunol & 0 & 0 & 0 & 0 & 0 & 0 & 0 \\
\hline $\mathrm{NH3}$ & 0.256 & $2.47 \mathrm{E}+03$ & 82.1 & $6.55 \mathrm{E}-02$ & $9.25 \mathrm{E}-02$ & 0.270 & 0.284 \\
\hline $\mathrm{Fo}(\mathrm{CN}) 64$ & 0 & 0 & 0 & 0 & 0 & 0 & 0 \\
\hline
\end{tabular}

-Unlknowns in tunk solids inventery are ascigned by Tenk Leyeting Model (TLM). 
HOW Model Aev. 4

\begin{tabular}{|c|c|c|c|c|c|c|c|}
\hline \multirow[b]{3}{*}{$\begin{array}{l}\text { Plypical } \\
\text { Proponties }\end{array}$} & \multicolumn{7}{|c|}{ Single-Shell Tonk 241-S-108 } \\
\hline & \multicolumn{7}{|c|}{ SMM Cornposite Inventory Estimete } \\
\hline & & & & $95 \mathrm{CI}$ & $-67 \mathrm{CI}$ & $+67 \mathrm{CI}$ & $+95 \mathrm{CI}$ \\
\hline Total SMM Wat & $3.14 \mathrm{E}+06(\mathrm{mg})$ & $(497 \mathrm{kpa})$ & $\cdots$ & $\ldots$ & - & $\cdots$ & - \\
\hline Heat Load & $3.98(\mathrm{~kW})$ & $(1.36 \mathrm{E}+04 \mathrm{BTU} / \mathrm{hr})$ & $=$ & 3.73 & 3.85 & 4.12 & 4.14 \\
\hline Bulk Density" & $1.67(g / \infty)$ & - & - & 1.60 & 1.64 & 1.70 & $1 . \%$ \\
\hline Waiter $w t / \%$ & 30.0 & E & $\ldots$ & 27.8 & 28.7 & 31.5 & 34.0 \\
\hline TOC w\% C (wet & 0.321 & - & $=$ & 0.286 & 0.303 & 0.338 & 0.355 \\
\hline 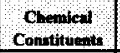 & $=1$ olde $\mathrm{L}$. & Ppm $n$ - & lys & ( $98 \mathrm{~d}$ & 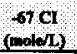 & $\begin{array}{l}+67 \mathrm{CI} \\
(\mathrm{mols} \mathrm{L})\end{array}$ & $\begin{array}{l}49 \mathrm{CI} \\
\text { (moleA) }\end{array}$ \\
\hline $\mathrm{Na}+$ & 15.4 & 2.13E+0s & $6.65 \mathrm{E}+05$ & 13.9 & 14.8 & 15.9 & 16.3 \\
\hline Al3t & 2.15 & 3.47E+04 & $1.09 \mathrm{E}+05$ & 1.86 & 2.00 & 2.31 & 2.22 \\
\hline $\mathrm{Fe}^{3}+($ totel Fe) & $9.59 \mathrm{E}-03$ & 320 & $1.01 \mathrm{E}+03$ & $7.68 \mathrm{E}-03$ & 8.72E-03 & $1.055-02$ & $1.13 E-02$ \\
\hline Cr3+ & 0.221 & $6.875+03$ & $2.16 \mathrm{E}+04$ & 0.163 & 0.194 & 0.226 & 0.231 \\
\hline $\mathrm{Bi3+}$ & 8.02E-04 & 100 & 315 & $T .21 E-04$ & $7.61 E-04$ & 8.43E-04 & $8.82 E-04$ \\
\hline Le3+ & $1.17 \mathrm{E}-09$ & 9.74E-OS & $3.06 E-04$ & 8.91E-10 & $103 E-09$ & $1.32 \mathrm{E}-09$ & 1.45E- 09 \\
\hline He2+ & $6.71 \mathrm{E}-06$ & 0.005 & 2.53 & $6.295-00$ & $6.53 E-06$ & $6.80 \mathrm{E}-05$ & $6.89 \mathrm{E}-00$ \\
\hline $\mathrm{Z}(\mathrm{a} \mathrm{ZTO}(\mathrm{OH}) 2)$ & $1.66 \mathrm{E}-04$ & 9.05 & 28.5 & $1.5 t E-09$ & $136 E-04$ & $1.72 \mathrm{E}-04$ & 1.60E-04 \\
\hline $\mathrm{Pb} 2+$ & $7,92 \mathrm{E}-04$ & 9.3 & 309 & 6.64E-04 & $7.2 \pi-04$ & 8.S8E-04 & $9.21 \mathrm{E}-04$ \\
\hline $\mathrm{Ni2+}$ & $6.26 \mathrm{E}-03$ & 220 & 61 & 6.04E-03 & 6.15E-03 & $6.37 \mathrm{E}-03$ & 6. $48 E-03$ \\
\hline $8 \sqrt{2+}$ & 0 & 0 & 0 & 0 & 0 & 0 & 0 \\
\hline Mnt+ & 3.01E-03 & 9.8 & 311 & 2.18E-03 & $2.59 \mathrm{E}-03$ & 3.42E-03 & $3.83 E-03$ \\
\hline $\mathrm{Ca} 2+$ & $3.20 \mathrm{E}-02$ & 767 & $2.41 E+03$ & $2.77 \mathrm{E}-02$ & $2,98 \mathrm{E}-02$ & 3.41E-02 & $3.62 \mathrm{E}-02$ \\
\hline $\mathrm{K}+$ & 6.48E-02 & $1.52 \mathrm{E}+03$ & $4.77 \mathrm{E}+03$ & $5.79 E-02$ & $6.11 \mathrm{E}-02$ & $6.89 \mathrm{E}-02$ & $7.01 E-02$ \\
\hline OH- & 13.0 & 1.32E+05 & $4.16 \mathrm{E}+05$ & 11.2 & 12.1 & 14.0 & 13.5 \\
\hline NO3- & 5.17 & $1.92 E+05$ & $6,03 E+05$ & 4.7 & 4.94 & 3.35 & 5.35 \\
\hline NO2- & 292 & S.03E+04 & $2.53 \mathrm{E}+05$ & 2.55 & 2.73 & 3.10 & 3.27 \\
\hline $\cos 2-$ & 0.284 & $1.02 E+04$ & $3.21 E+04$ & 0.256 & 0.268 & 0.304 & 0.307 \\
\hline PO43- & $6.335-02$ & $3.60 \mathrm{E}+03$ & $1.13 \mathrm{E}+0 \mathrm{~A}$ & $5.64 t-02$ & 5.BBE-02 & $6.48 \mathrm{E}-02$ & $6.62 \mathrm{E}-02$ \\
\hline SO42- & 0.188 & $1.08 \mathrm{E}+04$ & $3.40 \mathrm{E}+04$ & 0.143 & 0.165 & 0.213 & 0.234 \\
\hline Si ( S So32-) & 7.18E-02 & $1.21 \mathrm{E}+03$ & $3.79 \mathrm{E}+03$ & $6.00 \mathrm{E}-02$ & 6.58E-02 & $7.78 \mathrm{E}-02$ & 8.35E-02 \\
\hline F. & $3.83 E-02$ & 436 & $1.37 \mathrm{E}+03$ & 3.13E-02 & $3.43 E-02$ & 4.18E-02 & $4,45 \mathrm{E}-02$ \\
\hline $\mathrm{Cl} \cdot$ & 0.261 & $53 \times E+03$ & $1.74 E+04$ & 0.229 & 0.244 & 0.279 & 0.278 \\
\hline C6HSOT3- & $2.20 E-02$ & $2.48 E+03$ & $7.81 E+03$ & $2.03 E-02$ & $211 \mathrm{E}-02$ & $220 \mathrm{E}-02$ & $2.36 \mathrm{E}-02$ \\
\hline EDTA4 & $2.372-03$ & $\$ 9$ & $1.29 \mathrm{E}+03$ & 1.15E-03 & $1.75 \mathrm{E}-03$ & $3.00 E-03$ & $3.61 \mathrm{E}-03$ \\
\hline HEDTA 3 . & $3.90 \mathrm{E}-03$ & $6+10$ & $2.01 E+03$ & $1.46 \mathrm{E}-03$ & 2.65E-03 & S.15E-03 & $6.38 E-03$ \\
\hline glyoolatio- & $3.44 \mathrm{E}-02$ & $1.55 E+03$ & $486 E+43$ & $1.99 \mathrm{E}-02$ & 2.70E- -02 & 4.19E-02 & $4.90 \mathrm{E}-02$ \\
\hline metate. & $2.73 E-03$ & 96.3 & 303 & $2.21 E-03$ & $2.46 \mathrm{E}-03$ & $2.90 \mathrm{E}-03$ & $3.24 E-03$ \\
\hline oxalute?- & 1.54E-09 & 8.0\%E-45 & 2.5AE-A4 & $1.38 \mathrm{E}-09$ & 1.46E-0s & $1.61 E-09$ & $1.69 \mathrm{E}-09$ \\
\hline DBP & 1.48E-02 & $1.8 \pi+03$ & $5.87 E+03$ & 1.19E-M & $1,34 \mathrm{E}-02$ & $1.63 E-02$ & $1, \pi \mathrm{E}-02$ \\
\hline butanal & $1.8 \mathrm{E}-02$ & 658 & $2.07 \varepsilon+03$ & $1.19 E-02$ & $1.34 \mathrm{E}-02$ & $1.63 \mathrm{E}-02$ & 1.T/E-02 \\
\hline NH3 & $7.75 E-02$ & $7 m$ & $2.48 E+03$ & $6.4 B E-02$ & $6.95 E-02$ & $878 E-02$ & 9,9TE-02 \\
\hline $\mathrm{Fe}(\mathrm{CN}) 64$ & 0 & 0 & 0 & 0 & 0 & 0 & 0 \\
\hline
\end{tabular}

-Density is celeuluted besed on Na $\mathrm{OH}$, and $\mathrm{AlO}_{2}$.

†Water wh\% derived from the difference of density and cotel diewolved epecies. 
HDW Model Rev. 4

\begin{tabular}{|c|c|c|c|c|c|c|c|}
\hline \multicolumn{8}{|c|}{ Single-Shell Tank 241-S-108 } \\
\hline & \multicolumn{7}{|c|}{ Total Inventory Estimete* } \\
\hline $\begin{array}{l}\text { Phyiled } \\
\text { Properties }\end{array}$ & & & $\because \because \quad \vdots$ & $-95 \mathrm{CI}$ & $-67 \mathrm{CI}$ & $+67 \mathrm{CI}$ & $+9 \mathrm{~S} \mathrm{CI}$ \\
\hline Total Waste & $3.18 \mathrm{E}+06(\mathrm{gg})$ & $(502 \mathrm{kgal})$ & - & $=$ & - & - & - \\
\hline Heat Load & $4.10(\mathrm{~kW})$ & (1.40E+04 BTU/hu) & - & 3.82 & 3.95 & 4.26 & 4.26 \\
\hline Bulk Densityt & $1.67(\mathrm{~g} / \mathrm{cc})$ & - & - & 1.60 & 1.64 & 1.70 & 1.71 \\
\hline Water wtyt & 29.9 & $\cdots$ & $\ldots$ & 27.8 & 28.7 & 31.6 & 34.1 \\
\hline TOC wt\% C (wet & 0.318 & - & $\ldots$ & 0.283 & 0.300 & 0.335 & 0.351 \\
\hline Chonewl & not $w U$ & pon & $\mathbf{k g}$ & $\begin{array}{l}95 \mathrm{CI} \\
(\mathrm{mol} / \mathrm{L})\end{array}$ & $\begin{array}{l}67 \mathrm{Cr} \\
(\mathrm{nold} / \mathrm{L})\end{array}$ & $\begin{array}{l}+67 \mathrm{CI} \\
\text { (mold } / \mathrm{L})\end{array}$ & $\begin{array}{c}\text { ss Ct } \\
\text { (mole/L) }\end{array}$ \\
\hline $\mathrm{Na}+$ & 15.4 & $2.11 \mathrm{E}+05$ & $6.72 \mathrm{E}+05$ & 13.8 & 14.7 & 15,9 & 16.2 \\
\hline $\mathrm{Al3+}$ & 2.19 & $3.54 \mathrm{E}+04$ & $1.13 E+05$ & 1.92 & 2.05 & 2.35 & 2.26 \\
\hline $\mathrm{Fez}+(100+1 \mathrm{Fe})$ & $1.96 \mathrm{E}-02$ & 64 & $2.09 \mathrm{E}+03$ & $1.79 E-02$ & $1.87 \mathrm{E}-02$ & 2.04E- -02 & 2.13E-02 \\
\hline $\mathrm{Cr}^{3+}$ & 0.219 & $6.81 E+03$ & $2.16 \mathrm{E}+04$ & 0.172 & 0.197 & 0.227 & 0.229 \\
\hline $\mathrm{Bi3+}$ & $7.94 E-04$ & 99.2 & 315 & $7.14 \mathrm{E}-04$ & $7.53 \mathrm{E}-64$ & 8.34E-04 & 8.73E-04 \\
\hline Lot & $1.16 \mathrm{E}-09$ & $9.64 E-05$ & $3.06 \mathrm{E}-04$ & $8.82 \mathrm{E}-10$ & $1.02 E-09$ & $1.30 E-09$ & 1.44E-09 \\
\hline $\mathrm{H} \mathrm{g}^{2+}$ & 6.64E-06 & 0.797 & 2.53 & $6.23 \mathrm{E}-06$ & 6. $46 E-06$ & $6.73 E-06$ & $6.82 E-06$ \\
\hline $2 \mathrm{r}(\mathrm{ar} \mathrm{ZRO}(\mathrm{OH}) 2)$ & 1.6AEA & 8.96 & 28.5 & $1.50 \mathrm{E}-04$ & 1.55E-04 & $1.70 \mathrm{E}-04$ & 1.79E-04 \\
\hline Po2t+ & 7.85E-04 & 97.2 & 309 & 6.57E-04 & 7.19E-04 & 8. SOE-04 & $9.12 E-04$ \\
\hline $\mathrm{Ni2}+$ & 6.70E-03 & 235 & 748 & $6.52 E-03$ & $6.61 E-03$ & $6.79 \mathrm{E}-03$ & $6.885-03$ \\
\hline $5 \sqrt{2+}$ & 0 & 0 & 0 & 0 & 0 & 0 & 0 \\
\hline $\mathrm{MnAt}$ & $2908-03$ & 978 & 311 & $2.16 \mathrm{E}-03$ & 2568.03 & 3.39E-03 & $3.79 \mathrm{E}-03$ \\
\hline $\mathrm{Ca} 2+$ & $3.38 \mathrm{E}-02$ & 8II & $2.58 E+03$ & $3.03 \mathrm{E}-02$ & $3.20 \mathrm{E}-02$ & $3.56 \mathrm{E}-12$ & $3,73 \mathrm{E}-02$ \\
\hline $\mathrm{K}+$ & 6.43E-02 & $1.50 \mathrm{E}+03$ & $47 \mathrm{E}+03$ & $5.74 \mathrm{E}-02$ & $6.07 E-02$ & $6.81 \mathrm{IE}-02$ & $6.95 \mathrm{E}-02$ \\
\hline $\mathrm{OH} \cdot$ & 13.1 & $1.34 \mathrm{E}+05$ & $4.24 \mathrm{E}+05$ & 11.4 & 12.2 & 14.1 & 13.6 \\
\hline NO3. & 5.17 & $1.92 E+05$ & $6.09 E+05$ & 478 & 5.00 & 5.30 & 5.30 \\
\hline NO2. & 290 & $7.99 \mathrm{E}+04$ & 2.54E+05 & 2.54 & 2.72 & 3.08 & 3.26 \\
\hline Co32- & 0.224 & $102 \mathrm{E}+\mathrm{C}+\mathrm{A}$ & $3.23 \mathrm{E}+0 \mathrm{~A}$ & 0.256 & 0.268 & 0.303 & 0.306 \\
\hline PO43 & $6.27 E-02$ & $3.56 \mathrm{E}+03$ & $1.13 E+04$ & $5.58 \mathrm{E}-02$ & $582 \mathrm{E}-12$ & 6.41E-02 & 6.56E-02 \\
\hline SO42- & 0.187 & $1.07 \mathrm{E}+04$ & $3,41 E+04$ & 0.142 & 0.163 & 0.211 & 0.232 \\
\hline $\mathrm{Si}(\mathrm{as} \mathrm{SiO32-)}$ & $7.11 \mathrm{E}-02$ & $1.20 \mathrm{E}+03$ & $3.80 \mathrm{E}+03$ & 5.95E-02 & 6.52E- 02 & $7.71 \mathrm{E}-02$ & 8.28E-02 \\
\hline F- & $37 \mathrm{NE}-02$ & 431 & $1.372+03$ & $3.10 \mathrm{E}-02$ & 3,39E-02 & 4.13E-02 & 4.40E-02 \\
\hline Ct & 0.259 & $5.49 \mathrm{E}+03$ & $1.74 E+04$ & 0.228 & 0.243 & 0.277 & 0.276 \\
\hline C6HSO73- & $2.1 \pi-02$ & $2.46 \mathrm{E}+03$ & $7,81 \mathrm{E}+03$ & $2.01 \mathrm{E}-02$ & 2.09E- 02 & $2.26 \mathrm{E}-02$ & $2.34 \mathrm{E}-02$ \\
\hline EDTA4 & $2.35 \mathrm{E}-03$ & 405 & $1.29 \mathrm{E}+03$ & 1.14E-03 & 1.73E-03 & $2.97 \mathrm{E}-03$ & $3.58 \mathrm{BE}-03$ \\
\hline HEDTA3 & $3.86 \mathrm{E}-03$ & 633 & $2.01 E+03$ & $1.45 \mathrm{E}-03$ & 2.63E-03 & $5.10 \mathrm{E}-03$ & $6.3] E-03$ \\
\hline grycolate- & 3.41E-02 & $1.53 \mathrm{E}+03$ & $4.86 \mathrm{E}+03$ & $1.97 \mathrm{E}-02$ & $2.67 \mathrm{E}-02$ & 4.15E-02 & $4.86 \mathrm{E}-02$ \\
\hline mectute. & $2706-03$ & 95.3 & 303 & $2.19 \mathrm{E}-03$ & 2.44E-03 & 2.96E-03 & $3.21 \mathrm{E}-03$ \\
\hline oxalite2- & $1.52 \mathrm{E}-09$ & 8.00E-05 & 2.54E-04 & $1.37 \mathrm{E}-09$ & 1.4E-09 & 1.60E-09 & $1.67 \mathrm{E}-09$ \\
\hline DBP & $1.4 \pi-02$ & 1.85E+03 & $5.87 \mathrm{E}+03$ & $1,18 \mathrm{BE}-02$ & $1.32 E-02$ & $1.61 \mathrm{E}-02$ & $1.75 \mathrm{E}-02$ \\
\hline butanol & 1.47E-02 & 651 & $2.07 \mathrm{E}+03$ & 1.18E-02 & $1.32 \mathrm{E}-02$ & 1.61E-02 & 1.75E-02 \\
\hline NH3 & $7,93 \mathrm{E}-02$ & 807 & $2.56 \mathrm{E}+03$ & $6.62 \mathrm{E}-02$ & $7.14 \mathrm{E}-122$ & 8.94E-02 & 0.101 \\
\hline Fe(CN) 64 & 0 & 0 & 0 & 0 & 0 & 0 & 0 \\
\hline
\end{tabular}

*Unknowns in tenk solike inventory ere asigred by Tenk Isyering Model (TLM).

† Water wt\% derived from the difference of density and totel diveoived species. 
HNF-SD-WM-ER-352, Rev. 1

HDW Model Rov. 4

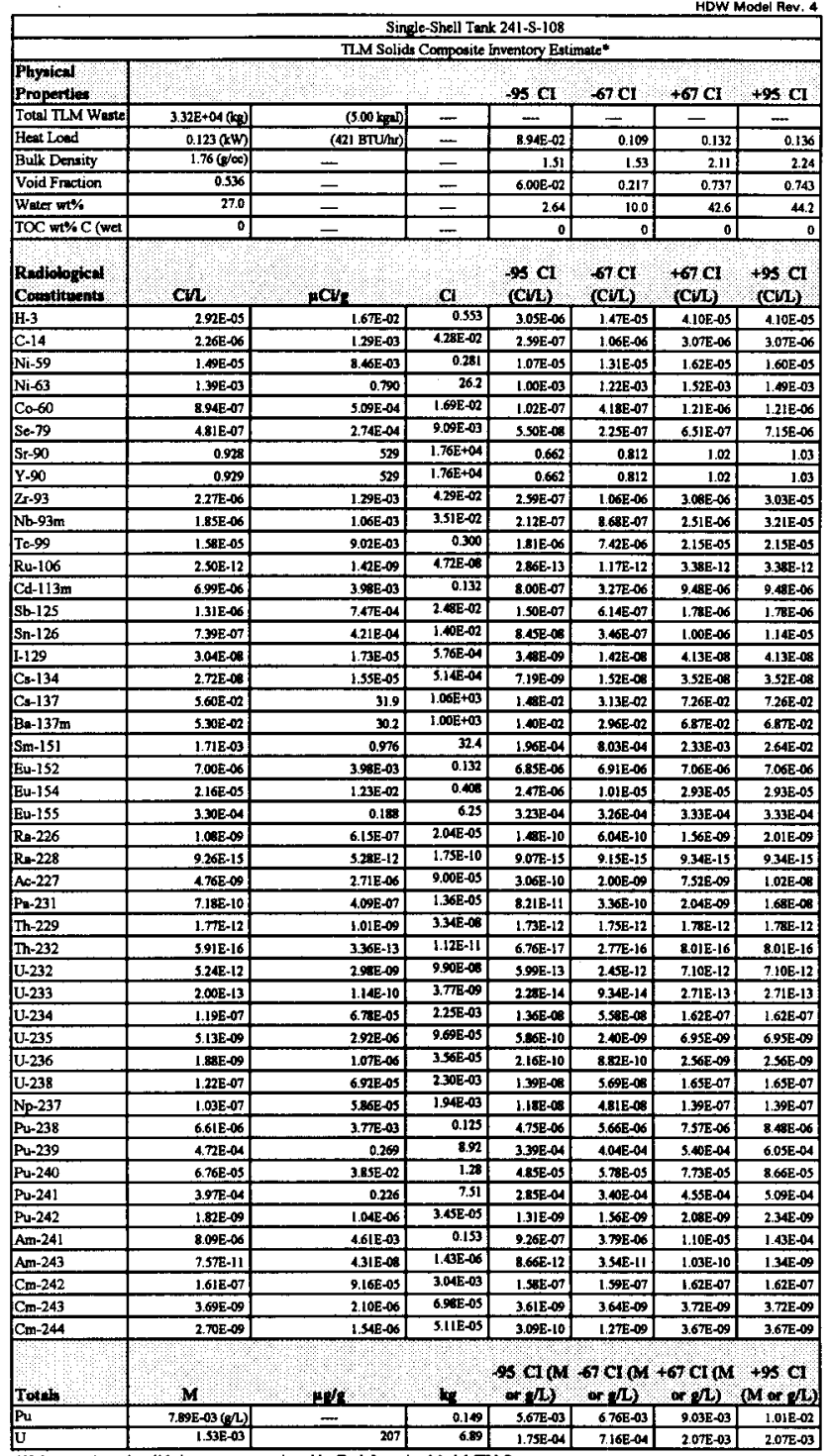

Hinknowns in tenk solids inventory ere watored by Tenk Loyerine Model (TLM). 
HNF-SD-WM-ER-352, Rev. 1

HDW Model Rev. 4

\begin{tabular}{|c|c|c|c|c|c|c|c|}
\hline \multirow{4}{*}{$\begin{array}{l}\text { Phyoled } \\
\text { Propenties }\end{array}$} & \multicolumn{7}{|c|}{ Single-Shell Tenk 241-S-108 } \\
\hline & \multicolumn{7}{|c|}{ SMM Composite Inventory Estimate } \\
\hline & & & & & & & \\
\hline & & 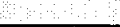 & & $95 \mathrm{CI}$ & $-67 \mathrm{Cl}$ & $+67 \mathrm{CI}$ & $+95 \mathrm{CI}$ \\
\hline Total SMM Wnat & $3.14 \mathrm{E}+06(\mathrm{~kg})$ & $(497 \mathrm{kpa} /)$ & $\cdots$ & - & $\ldots$ & - & - \\
\hline Heat Load & $3.98(\mathrm{tW})$ & $(1.36 \mathrm{E}+04 \mathrm{BTU} / \mathrm{hr})$ & $=$ & 3.73 & 3.85 & 4.12 & 4.14 \\
\hline Bulk Density* & $1.67(g / c)$ & - & - & 1.60 & 1.64 & 1.70 & 1.70 \\
\hline & & & & & & & \\
\hline Water wt\%† & 30.0 & - & - & 27.8 & 28.7 & 31.5 & 34.0 \\
\hline TOC wt\% C (wet & 0.321 & $=$ & $\bar{E}$ & 0.286 & 0.303 & 0.338 & 0.355 \\
\hline $\begin{array}{l}\text { Roulotofical } \\
\text { Constituent }\end{array}$ & CUL & $\mathrm{ACV}_{2}$ & c) & $\begin{array}{l}95 \mathrm{CI} \\
\text { (CV) }\end{array}$ & $\begin{array}{l}67 \mathrm{Cl} \\
(\mathrm{CVL})\end{array}$ & $\begin{array}{r}+67 \mathrm{Cl} \\
(\mathrm{CUL})\end{array}$ & $\begin{array}{l}95 \mathrm{Cl} \\
\text { (cil) }\end{array}$ \\
\hline $\mathrm{H} \cdot \mathbf{3}$ & $2.62 \mathrm{E}-04$ & 0.157 & 494 & $1.58 \mathrm{E}-04$ & $1.58 E-04$ & $2.78 E-04$ & $2.72 \mathrm{E}-04$ \\
\hline $\mathrm{C}-14$ & $3.34 E-05$ & $2.00 \mathrm{E}-02$ & 62.9 & $1.15 \mathrm{E}-0 \mathrm{~S}$ & 1.15E-05 & $3.42 E-05$ & 3.4 IE-05 \\
\hline Ni-59 & $2.29 E-06$ & $1.37 \mathrm{TE}-03$ & 4.30 & $1.26 \mathrm{E}-06$ & $1.26 \mathrm{E}-06$ & $2.34 E-\infty 6$ & $2.39 \mathrm{E}-06$ \\
\hline $\mathrm{Ni}-63$ & 2.22E-04 & 0.133 & 418 & $1.20 \mathrm{E}-04$ & $1.20 \mathrm{E}-04$ & $2.2 \pi \mathrm{E}-04$ & $2.32 \mathrm{E}-04$ \\
\hline $\cos 60$ & $3372-05$ & $2.02 E-02$ & 63.5 & $8.66 \mathrm{E}-06$ & $8.66 \mathrm{E}-06$ & 3.42E-05 & $3.4 \pi-05$ \\
\hline Se-79 & $3.49 E-16$ & $2.09 \mathrm{E}-03$ & 6.57 & $2.09 \mathrm{E}-06$ & 2.09E- -06 & $4.00 \mathrm{E}-66$ & 4.48EE-06 \\
\hline St-90 & 0.114 & 68.5 & 2.ISE+05 & 0.108 & 0.111 & 0.118 & 0.121 \\
\hline $\mathrm{Y} .90$ & 0.114 & 68.5 & $2.15 E+03$ & $6.62 \mathrm{E}-02$ & $6.62 \mathrm{E}-02$ & 0.118 & 0.121 \\
\hline Zx-93 & $1.71 E-05$ & $1.02 E-02$ & 32.1 & $1.01 \mathrm{E}-05$ & $1.01 \mathrm{E}-05$ & $1.96 \mathrm{E}-05$ & $2.20 \mathrm{E}-05$ \\
\hline $\mathrm{Nb}-93 \mathrm{~m}$ & $1.27 \mathrm{E}-05$ & $7.57 \mathrm{E}-03$ & 23.8 & $7.76 \mathrm{E}-06$ & $7.76 \mathrm{E}-6$ & $1.44 E-05$ & $1.61 \mathrm{E}-0 \mathrm{~s}$ \\
\hline Tc-99 & $2.385-04$ & 0.143 & 48 & $1.572-04$ & 1.968-04 & $2.80 \mathrm{E}-04$ & $3.218-04$ \\
\hline Ru-106 & $5.19 E-08$ & $3.111 E-06$ & $9.76 \mathrm{E}-03$ & $1958-09$ & 1.95E-09 & $5.91 \mathrm{E}-09$ & $6.57 \mathrm{E}-09$ \\
\hline Cd-113m & $8.335-05$ & 4.98E-02 & 157 & $4.135-05$ & 4.13E-05 & 9.85E-05 & 1.13E-04 \\
\hline Sb-125 & 1.38E-04 & $8.28 E-02$ & 260 & $297 \mathrm{E}-05$ & $297 \mathrm{E}-25$ & 1.40E-04 & $1.43 E-04$ \\
\hline Sn-126 & $5.29 \mathrm{E}-06$ & $3.17 \pi-03$ & 9.96 & $3.198-06$ & 3.19E- -6 & $6.05 E-06$ & $6.78 E-06$ \\
\hline I-129 & 4SAE-M & 2.75E- -4 & 0.863 & $3.01 E-07$ & $378 E-07$ & $5.40 \mathrm{E}-07$ & 6.19E-07 \\
\hline Cs-134 & $1.56 \mathrm{E}-06$ & $9.31 \mathrm{E}-04$ & 2.93 & $1.04 E-06$ & 1.04E-06 & $1.67 \mathrm{E}-06$ & $1.79 E-06$ \\
\hline Cs-137 & 0.287 & 172 & 5.40E+05 & 0.258 & 0.272 & 0.303 & 0.305 \\
\hline $\mathrm{Ba}-137 \mathrm{~m}$ & 0.271 & 162 & 5.10E+05 & 0.203 & 0.203 & 0.287 & 0.289 \\
\hline Sm-151 & 1.23E- -12 & 7.38 & $2.32 \mathrm{E}+04$ & $7.43 \mathrm{E}-03$ & $7.43 E-03$ & I.4IE-02 & $1.58 E-02$ \\
\hline Eu-152 & 3.05E- -16 & $1.83 \mathrm{E}-03$ & 5.74 & $1.32 E-06$ & 1.32E-06 & $3.11 E-06$ & $3.1 \pi E-06$ \\
\hline Eu-154 & $5.42 \mathrm{E}-0 \mathrm{~A}$ & 0.325 & $1.02 \mathrm{E}+03$ & $1.93 \mathrm{E}-04$ & $1.93 \mathrm{E}-04$ & $6.69 \mathrm{E}-04$ & $7.19 \mathrm{E}-\mathrm{As}$ \\
\hline Eu-155 & $1.75 E-04$ & 0.105 & 330 & $7.20 \mathrm{E}-05$ & $7.20 \mathrm{E}-05$ & $1.80 \mathrm{E}-04$ & $1.84 E-04$ \\
\hline Ra-226 & $1.63 \mathrm{E}-10$ & $9.78 E-08$ & 3.075 .04 & $1.10 E-10$ & $1.20 E-10$ & 1.91E-10 & $2.17 \mathrm{E}-10$ \\
\hline $\mathrm{Re}=228$ & S.2AE-08 & $3.50 \mathrm{E}-05$ & 0.110 & $2.28 E-08$ & 4.02E-08 & $7.96 \mathrm{E}-0.98$ & $1.02 \mathrm{E}-07$ \\
\hline Ac-227 & $9.86 E-10$ & $9.90 E-07$ & 1.85E-03 & $6.74 \mathrm{E}-10$ & $7.34 \mathrm{E}-10$ & $1.15 \mathrm{E}-00$ & $1.24 \mathrm{E}-09$ \\
\hline $\mathrm{Pa}-231$ & $4.35 E-09$ & $2.60 \mathrm{E}-06$ & 8.18E-03 & 2.95E-09 & 2.95E-09 & 4.86E-09 & 5.34E-09 \\
\hline Th-229 & 1.39E- -09 & $8.33 \mathrm{E}-07$ & 2.62E-03 & $6.10 \mathrm{E}-10$ & $9.92 \mathrm{E}-10$ & $1.86 \mathrm{E}-09$ & $2.35 E-09$ \\
\hline Th-232 & 3.93E-09 & 2.35E-06 & $7.40 E-03$ & $1.96 E-09$ & 293E-09 & 4.94E-09 & $5.91 \mathrm{E}-09$ \\
\hline U-232 & 3.53E-07 & 2.11E-04 & 0.664 & $2.10 E-97$ & 2.00E-07 & $4,39 E-07$ & 3.28E-07 \\
\hline U-233 & 1.33E- -6 & 8.10E-0. & 2.53 & 8.0TE-07 & 1.0TE-06 & $1.69 \mathrm{E}-06$ & $2.02 E-06$ \\
\hline U-234 & 8.55E-07 & $5.125-04$ & 1.61 & $7.82 E-07$ & $8.1 \pi E-07$ & $8.98 E-07$ & 8.79E-0? \\
\hline U-235 & $3.54 E-08$ & $2.12 E-05$ & $6.66 \mathrm{E}-02$ & $3.22 \mathrm{E}-18$ & $3.3 T \mathrm{E}-08$ & $3.72 \mathrm{E}-18$ & $3.64 E-08$ \\
\hline U-236 & $2.42 E-08$ & $1.458-05$ & 4.56-02 & $2.31 E-08$ & $2.36 \mathrm{E}-08$ & 2.49E-08 & $2.49 \mathrm{E}-08$ \\
\hline U.238 & 8.89E-07 & $5.32 \mathrm{E}-04$ & 1.67 & 8.14E-07 & 8.50E-07 & $9.33 \mathrm{E}-07$ & $9.12 \mathrm{E}-07$ \\
\hline Np-237 & 9.44E-07 & $5.65 E-04$ & 1.78 & $6.77 \mathrm{E}-07$ & $8.08 E-07$ & $1.09 \mathrm{E}-06$ & $1.21 \mathrm{E}-06$ \\
\hline $\mathrm{Pu}-\mathbf{2 3 8}$ & $1.16 \mathrm{E}-06$ & $6.94 \mathrm{E}-04$ & 2.18 & 8.47E-07 & $1.00 \mathrm{E}-06$ & 1.32E-06 & $1.47 \mathrm{E}-06$ \\
\hline Pu-239 & 4.36E-05 & $2.61 \mathrm{E}-02$ & B.1. & 3.66E-05 & 3.95E-05 & 4. $7 \mathrm{k}-05$ & $5.16 \mathrm{E}-05$ \\
\hline Pu-240 & $7.14 \mathrm{E}-06$ & $4.27 \mathrm{t}-03$ & 13.4 & 5.64E-06 & $6.37 \mathrm{E}-16$ & $7.90 \mathrm{E}-\infty 6$ & $8.63 E-06$ \\
\hline Pu-241 & 7.65E-05 & 4.S8E-02 & 144 & $5.52 E-05$ & $6.56 \mathrm{E}-05$ & . $.75 E .05$ & 9.80E-05 \\
\hline Pu-242 & $4.13 \mathrm{E}-10$ & $2.47 \mathrm{E}-07$ & $7.77 \mathrm{E}-04$ & 2.86E-10 & $3.4 \mathrm{EE}-10$ & $4.7 \mathrm{EE}-10$ & $5.41 \mathrm{E}-10$ \\
\hline Am-241 & $5.64 \mathrm{E}-05$ & $3.38 E-02$ & 106 & $4.16 E-05$ & 4.88E-05 & 6.40E-05 & $7.13 E-05$ \\
\hline Am-243 & $1.57 \mathrm{E}-09$ & $9.41 \mathrm{E}-07$ & $2.66 \mathrm{E}-03$ & 1.11E-09 & 1.33E-09 & $1.82 \mathrm{E}-09$ & $2.06 \mathrm{E}-199$ \\
\hline $\mathrm{Cm}-242$ & 1.08E-07 & $6.495-05$ & 0.204 & $3.73 E-08$ & $3.73 \mathrm{E}-08$ & L.10E-07 & $1.12 E-07$ \\
\hline $\mathrm{Cm}-243$ & $9.64 E-09$ & $5.77 E-06$ & $1.81 E_{-02}$ & $2.86 \mathrm{E}-109$ & 2.86E-09 & $9.86 \mathrm{E}-09$ & J.01E-08 \\
\hline $\mathrm{Cm}_{\mathrm{m}-244}$ & $1.06 \mathrm{E}-07$ & $6.35 \mathrm{SE}-0 \mathrm{~S}$ & 0.200 & $3.63 \mathrm{E}-108$ & $3.63 E-08$ & $1.30 \mathrm{E}-07$ & $1.45 E-07$ \\
\hline Tokts & $\mathrm{M}$ & $n \pi / \pi$ & $\mathrm{H}, \mathrm{k}$ & $\begin{array}{l}95 \mathrm{cl}(\mathrm{M} \\
\text { (en })\end{array}$ & $\begin{array}{l}6 \mathrm{Cl}(\mathrm{A} \\
\text { or } / \mathrm{L})\end{array}$ & $\begin{array}{r}67 \mathrm{ct} \mathrm{M} \\
\text { or } / \mathrm{L})\end{array}$ & $\begin{array}{l}+9 s \text { cl } \\
(M \text { os } / 2)\end{array}$ \\
\hline $\mathrm{Pu}$ & $5.71 E-04(\mathrm{~K} / \mathrm{L})$ & - & 1.07 & 1.19E-0A & 4.94E-04 & $6.48 \mathrm{E}-04$ & $7.22 \mathrm{E}-04$ \\
\hline $\mathrm{U}$ & $1.01 \mathrm{E}-02$ & $1.4 \bar{E}+03$ & $4.52 \mathrm{E}+03$ & $9.16 \mathrm{E}-03$ & $9.60 \mathrm{E}-03$ & $1.06 \mathrm{E}-02$ & $1.04 \mathrm{E}-02$ \\
\hline
\end{tabular}

tWater w\% derived from the differenoe of density and total divoolved species. 


\begin{tabular}{|c|c|c|c|c|c|c|c|}
\hline \multirow{4}{*}{$\begin{array}{l}\text { Phylail } \\
\text { Propertios }\end{array}$} & \multicolumn{7}{|c|}{ Singie-Shell Tank 241-S-108 } \\
\hline & \multicolumn{7}{|c|}{ Totel Inventory Estimete* } \\
\hline & & & & & & & \\
\hline & & & & $95 \mathrm{CI}$ & $-67 \mathrm{CI}$ & $+67 \mathrm{CI}$ & $+9 \mathrm{~s} \mathrm{CI}$ \\
\hline Total Waste & $3.18 E+06(\mathrm{~kg})$ & $(502$ logal) & 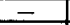 & - & $\cdots$ & - & - \\
\hline Heat Lood & $4.10(\mathrm{~kW})$ & $(1,40 \mathrm{E}+0 \mathrm{~A}$ BTU/4r) & $\ldots$ & 3,82 & 3.95 & 4.26 & 4.26 \\
\hline Bulk Density & $1.67(\mathrm{~g} / \infty)$ & $\ldots$ & $=$ & 1.60 & 1.64 & 1.70 & $1.7 !$ \\
\hline Weter wt\%t & 29.9 & - & & & & & \\
\hline TOC wt\% C (wet & 0.318 & & $=$ & 27.8 & 28.7 & 31.6 & 34.1 \\
\hline 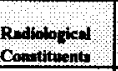 & $\mathrm{C} / \mathrm{L} / \mathrm{L}$ & torl & $\mathrm{Cl}$ & $\begin{array}{l}28 \mathrm{Cl} \\
(\mathrm{O} / \mathrm{t})\end{array}$ & $\begin{array}{l}-67 \mathrm{CI} \\
(\mathrm{CH})\end{array}$ & $\begin{array}{r}+67 \mathrm{Ct} \\
(\mathrm{CH})\end{array}$ & $\begin{array}{l}+95 \mathrm{Cl} \\
(\mathrm{Cl} / \mathrm{L})\end{array}$ \\
\hline $\mathrm{H}-3$ & $2.60 E-04$ & 0.156 & 494 & $1.56 \mathrm{E}-04$ & $1.56 \mathrm{E}-04$ & $2.76 \mathrm{E}-04$ & 2.70E-04 \\
\hline $\mathrm{C}-14$ & 3.31E-05 & $1.9 \mathrm{gE}-02$ & 62.9 & $1.14 \mathrm{E}-05$ & 1.14E-05 & $3.39 \mathrm{E}-05$ & 3.33E-05 \\
\hline $\mathrm{Ni}-59$ & 2.41E-06 & $1.44 \mathrm{E}-0 \mathrm{3}$ & 4.58 & $1.39 \mathrm{E}-06$ & $1.39 \mathrm{E}-05$ & 2.46E-A6 & $2.51 \mathrm{E}-06$ \\
\hline $\mathrm{Ni}-63$ & $2.34 \mathrm{E}-04$ & 0.140 & 444 & $1.33 \mathrm{E}-\mathrm{OA}$ & $1.33 \mathrm{E}-04$ & $2.39 \mathrm{E}-04$ & 2.44E-04 \\
\hline $\mathrm{Ca}-60$ & $3.34 E-05$ & $2.00 E-02$ & 63.5 & B.58E-06 & B.58E-06 & $3.39 \mathrm{E}-05$ & 3.44E-05 \\
\hline Se-79 & 3.46E-06 & 2.07E-03 & 6.58 & $2.06 \mathrm{E}-06$ & $2.08 E-06$ & $3.96 \mathrm{E}-06$ & 444E-06 \\
\hline Sr-90 & 0.123 & 7.3 & $233 \mathrm{E}+05$ & 0.116 & 0.119 & 0.126 & 0.129 \\
\hline Y.90 & 0.123 & 73.3 & $2.33 \mathrm{E}+05$ & $7.47 \mathrm{E}-02$ & $7.47 \mathrm{E}-02$ & 0.126 & 0.129 \\
\hline$Z r-93$ & 1.69E-0s & $1.01 E-02$ & 32.1 & $9.99 \mathrm{E}-0 \mathrm{~S}$ & $9.99 \mathrm{E}-06$ & $1.94 \mathrm{E}-05$ & 2.18E-05 \\
\hline $\mathrm{Nb}-93 \mathrm{~m}$ & $1.25 \mathrm{E}-45$ & $7.51 \mathrm{E}-03$ & 23.8 & $7.70 \mathrm{E}-06$ & $7.70 \mathrm{E}-06$ & $1.43 \mathrm{E}-0.5$ & $1,60 \mathrm{E}-05$ \\
\hline Te-99 & $2.36 E-04$ & 0.141 & 48 & $1.55 E-04$ & 1.95E-04 & $2.78 E-04$ & $3.18 \mathrm{E}-04$ \\
\hline Ru-106 & $5.14 \mathrm{E}-09$ & $3.07 \mathrm{E}-06$ & $9.76 \mathrm{E}-03$ & $1,93 E-09$ & $1.93 \mathrm{E}-09$ & $5.86 \mathrm{E}-09$ & $6.51 \mathrm{E}-09$ \\
\hline Cd-113m & 8.25E-05 & $494 \mathrm{E}-02$ & 157 & 4.10E-05 & $4.10 E-05$ & $9.76 \mathrm{E}-05$ & $1.12 \mathrm{~F}-04$ \\
\hline$S b-125$ & $1.37 \mathrm{E}-04$ & 8.19E-02 & 260 & 2,94E-05 & $2.94 \mathrm{E}-05$ & $1.39 E-04$ & $1.41 \mathrm{E}-04$ \\
\hline Sn-126 & $5.25 \mathrm{E}-06$ & $3.14 \mathrm{E}-03$ & 9.97 & $3.17 \mathrm{E}-06$ & $3.17 \mathrm{E}-06$ & 6.00E-06 & 6.72E-06 \\
\hline $\mathrm{I} 129$ & $4.55 E-07$ & $2.72 \mathrm{E}-04$ & 0.864 & 2.99E-07 & $3.74 E-07$ & 5.35E-07 & 6.13E-07 \\
\hline $\mathrm{Cs}-134$ & 1.5AE-D6 & $9.21 \mathrm{E}-04$ & 2.93 & $103 \mathrm{E} 0 \mathrm{OS}$ & $1.03 \mathrm{E}-06$ & $1.66 \mathrm{E}-06$ & $1.77 \mathrm{E}-06$ \\
\hline Cs-137 & 0.285 & 170 & $5.41 E+05$ & 0.256 & 0.270 & 0.301 & 0.303 \\
\hline $\mathrm{Ba}-137 \mathrm{~m}$ & 0.269 & 161 & $5.118+05$ & 0.201 & 0.201 & 0.284 & 0.286 \\
\hline$S m-151$ & $1.22 \mathrm{E}-02$ & 7.31 & $2.32 \mathrm{~g}+0 \mathrm{4}$ & $7.37 \mathrm{E}-03$ & $7.37 \mathrm{E}-03$ & 1. $40 \mathrm{E}-02$ & $1.5 T E-02$ \\
\hline Eu-152 & 3.09E-06 & 1.BSE-03 & 5.87 & $1.37 \mathrm{E}-06$ & $1372-06$ & $3.15 \mathrm{E}-06$ & $3.20 E-06$ \\
\hline Eu-154 & $5.37 \mathrm{E}-04$ & 0.321 & $1.02 E+13$ & $191 \mathrm{E}-04$ & $1.91 \mathrm{E}-0 \mathrm{~d}$ & $6.62 \mathrm{E}-04$ & $7.12 \mathrm{E}-04$ \\
\hline Eu-155 & $1.7 \mathrm{BE}-\mathrm{Ad}$ & 0.106 & 336 & 7,46E-05 & 7. 6 EE-05 & $1.81 E-0.4$ & 1.85E-04 \\
\hline Rn-226 & $1.73 \mathrm{E}-10$ & $1.03 \mathrm{E}-07$ & $3.28 \mathrm{E}-04$ & $1.29 \mathrm{E}-10$ & $1.30 \mathrm{E}-10$ & $1.95 \mathrm{E}-10$ & 2.17E-10 \\
\hline$R a-228$ & $5.79 \mathrm{E}-08$ & $3.46 \mathrm{E}-05$ & 0.110 & $2.26 \mathrm{E}-08$ & 3.99E-09 & $700-0$ & $1.01 \mathrm{E}-07$ \\
\hline Ac-227 & $1.02 E-09$ & $6.12 \mathrm{E}-07$ & $1.94 \mathrm{E}-03$ & $7.68 \mathrm{E}-10$ & $7.74 \mathrm{E}-10$ & 1. $15 E-09$ & $1.23 \mathrm{E}-09$ \\
\hline $\mathrm{P}_{\mathrm{a}-231}$ & $4.31 \mathrm{E}-09$ & $2.58 E-06$ & 8.19E-03 & 2.93E-00 & $2,93 E-09$ & $4.81 \mathrm{E}-09$ & $5.30 \mathrm{E}-09$ \\
\hline Th-229 & $1,38 E-\infty$ & 8.24E-07 & $2.62 \mathrm{E}-03$ & $6.04 E-10$ & 9.83E-10 & $184 E-09$ & $2.33 \mathrm{E}-09$ \\
\hline Th-232 & 3.90E- -9 & $2338-06$ & $7.406-03$ & $1.945-09$ & $2,90 E-09$ & $489 \mathrm{E}-09$ & $5.85 E-09$ \\
\hline $\mathrm{U}-232$ & 3.49E-07 & 2.095-04 & 0.664 & $2.08 E-07$ & $2.7 \mathrm{E}-07$ & $433 \mathrm{E}-07$ & $5.22 E-07$ \\
\hline U.233 & $1.34 E-6$ & $8.02 \mathrm{E}-04$ & 2.55 & $7.995-07$ & $1.06 E-06$ & $1.66 \mathrm{E}-06$ & $2.00 E-06$ \\
\hline $\mathrm{U}-234$ & 8.48E-07 & $5.07 \mathrm{E}-04$ & $\begin{array}{r}1.61 \\
\end{array}$ & 7.75E-07 & $8.10 \mathrm{E}-07$ & $8.89 \mathrm{E}-07$ & 8.71E-07 \\
\hline U-235 & $3.51 E-08$ & $2.10 \mathrm{E}-0 \mathrm{~s}$ & $6.67:-02$ & $3.20 \mathrm{E}-0 \mathrm{~s}$ & 3.34E-08 & $3.69 \mathrm{E}-08$ & $3.60 \mathrm{E}-08$ \\
\hline $\mathrm{U}-236$ & 2.40E-08 & 1.44E-AS & 4SGE-02 & $2.29 \mathrm{E}-0 \mathrm{~s}$ & 2.34E-08 & $2.47 \mathrm{E}-08$ & $2.47 \mathrm{E}-0 \mathrm{~B}$ \\
\hline U-238 & 8.82E-07 & $5.2 \pi-04$ & 1.68 & 8.08E-07 & $84 \mathrm{BE}-07$ & $9.24 E-07$ & 9.04E-07 \\
\hline $\mathrm{Np}-237$ & 9.36E .07 & $5.600-04$ & 1.7 & $6.72 \mathrm{E}-07$ & 8.01E-07 & $1.07 \mathrm{E}-06$ & $1.20 \mathrm{E}-06$ \\
\hline Pu-238 & $1.21 E-06$ & $7.26 \mathrm{E}-04$ & 2.31 & $9.04 \mathrm{E}-07$ & $1.005-06$ & $1.3 \%=0$ & $1.53 \mathrm{E}-06$ \\
\hline $\mathrm{Pu}-239$ & 4.79E-05 & 2.86E-02 & 91.0 & $3.99 \mathrm{E}-0 \mathrm{~S}$ & 438E-05 & 5.19E-0S & $5.58 E-05$ \\
\hline $\mathrm{Pu}-240$ & $7.74 \mathrm{E}-06$ & $4,63 \mathrm{E}-03$ & 14.7 & $6.26 \mathrm{E}-06$ & $6.98 \mathrm{E}-06$ & B. $49 \mathrm{E}-06$ & $9.21 \mathrm{E}-06$ \\
\hline Pu-241 & $7.97 \mathrm{E}-05$ & $4.77 \mathrm{E}-02$ & 151 & $5.86 \mathrm{E}-05$ & 6.01:-05 & 9.05E-05 & $1.01 \mathrm{E}-04$ \\
\hline $\mathrm{Pu}-242$ & 4.27E-10 & $2,56 \mathrm{E}-07$ & 8.12E-04 & $3.01 \mathrm{E}-10$ & $3.635-10$ & $492 \mathrm{E}-10$ & $5.54 \mathrm{E}-10$ \\
\hline Am-241 & $5.60 \mathrm{E}-05$ & $3.35 \mathrm{E}-02$ & 106 & $4.12 \mathrm{E}-05$ & $484 \mathrm{E}-05$ & $6.35 \mathrm{E}-05$ & $7.072-05$ \\
\hline$A_{m-243}$ & $1.56 \mathrm{E}-09$ & $9,32 \mathrm{E}-07$ & $2.96 \mathrm{E}-03$ & $1.10 E-09$ & $1.32 \mathrm{E}-09$ & $1,00 E-99$ & $2.04 \mathrm{E}-09$ \\
\hline $\mathrm{Cm}-242$ & $1.09 \mathrm{E}-07$ & 6.51E-05 & 0.207 & $3.85 E-08$ & 3.85E- 08 & $1.11 \mathrm{E}-07$ & $1.13 \mathrm{E}-07$ \\
\hline $\mathrm{Cm}-243$ & 9.58E-09 & 5.73E-06 & $182 \mathrm{E}-02$ & $2.87 \mathrm{E}-09$ & $28 \pi \mathrm{E}-09$ & $900 \mathrm{E}-09$ & $1.00 \mathrm{E}-08$ \\
\hline $\mathrm{Cm}-244$ & $1.05 E-07$ & $6.29 \mathrm{E}-05$ & 0.200 & $3.59 \mathrm{E}-0 \mathrm{~s}$ & $350 \mathrm{E}-08$ & $1.29 \mathrm{E}-07$ & 1.4AE-07 \\
\hline Totelia & $\mathbf{M} \gg$ & ests & 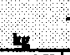 & $95 \mathrm{dr}$ & $67 \mathrm{CO}$ & $\begin{array}{l}+6 \mathrm{Cl} \text { (M } \\
+4 \mathrm{~L} \text { L) }\end{array}$ & $\begin{array}{l}+\$ \mathrm{Cl} \\
(\mathrm{N}, \mathrm{ar} / \mathrm{L})\end{array}$ \\
\hline $\mathrm{Fu}$ & $6.44 \mathrm{E}-04(\Omega / \mathrm{L})$ & - & 1.22 & 4.94E-04 & $5.63 \mathrm{E}-04$ & $7.21 \mathrm{E}-04$ & $7.94 \mathrm{E}-\mathrm{OA}$ \\
\hline $\mathrm{U}$ & $1.00 E-02$ & 1.43E+03 & $4.53 E+03$ & $9.08 E-03$ & $9.53 \mathrm{E}-03$ & $1.05 \mathrm{E}-02$ & $1.03 \mathrm{E}-02$ \\
\hline
\end{tabular}

Lriknowns in terk solids imentory we astignad by Tenk Lyering Model (TLM).

†Volume average for density, meas averinge Water wt\% and $\mathrm{TOC}$ wt\% C. 


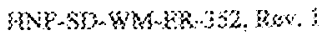

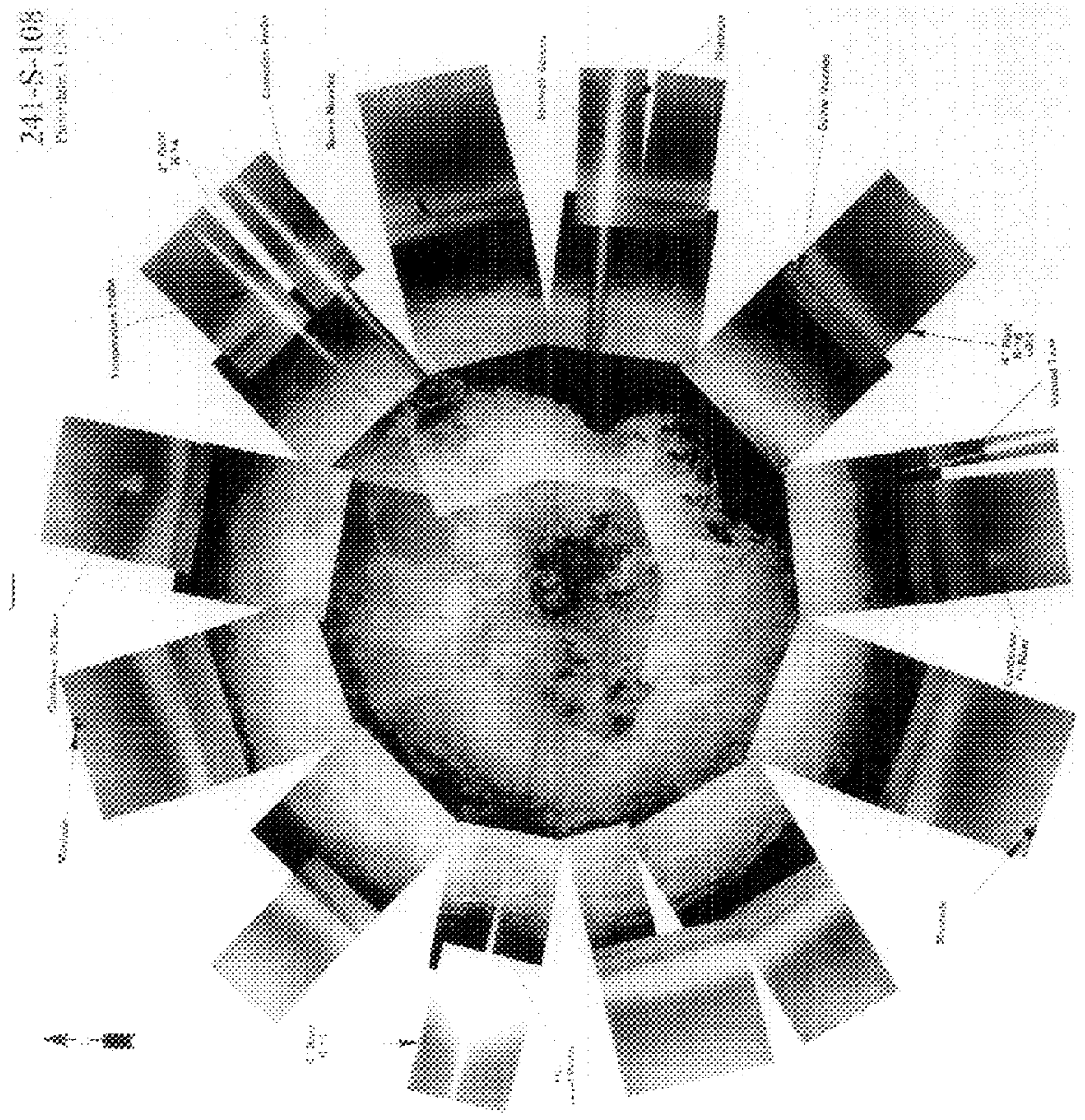


TANK 241-S-109 SUMMARY

\begin{tabular}{|c|c|c|c|}
\hline \multicolumn{2}{|c|}{ TANK HISTORY } & \multicolumn{2}{|c|}{ TANK DESCRIPTION } \\
\hline Entered Service & 4th qtr 1952 & Diameter & $75 \mathrm{ft}$ \\
\hline Removed from Service & - & Bottom Shape & Dish \\
\hline Inactive & - & Nominal Capacity & $758,000 \mathrm{gal}$ \\
\hline Watch Lists & none & Cascade Tank & none \\
\hline Integrity & Sound & Total Risers & 12 \\
\hline Assumed Leaker & - & WASTE VOLUN & LON 1996i) \\
\hline Interim Stabilization (IS) & - & Total Waste Volume & $568,000 \mathrm{gal}$ \\
\hline Partial Interim Isolation (PI) & Dec 1982 & Waste Type & NCPLX \\
\hline Intrusion Prevention (IP) & - & Drainable Interstitial Liquids & $141,000 \mathrm{gal}$ \\
\hline \multicolumn{2}{|c|}{$\begin{array}{l}\text { TENTATIVELY AVAILABLE RISERS } \\
\end{array}$} & Pumpable Liquids & $119,000 \mathrm{gal}$ \\
\hline Riser Number(s) & Size & Saltcake & $555,000 \mathrm{gal}$ \\
\hline $2,11,14,16$ & 4 in & Sludge & 13,000 gal \\
\hline 6 & 12 in & Supernatant & $0 \mathrm{gal}$ \\
\hline \multicolumn{2}{|c|}{ TANK TEMPERATURE } & \multicolumn{2}{|c|}{ INTERIOR PHOTOGRAPHS } \\
\hline Average Tank Temperature & $79^{\circ} \mathrm{F}$ & Date & Aug 24, 1984 \\
\hline Maximum Temperature & $89.42^{\circ} \mathrm{F}$ & Montage Number & $94041033-18 \mathrm{CN}$ \\
\hline Date & Jan 30, 1996, Feb $1 \&$ 2, 1996 & Photo Set Number & 8905333 \\
\hline Elevation from tank bottom & $4.49 \mathrm{ft}$ & \multicolumn{2}{|c|}{\begin{tabular}{|c} 
WASTE SURFACE LEVEL \\
\end{tabular}} \\
\hline Riser Number & 4 & Devices & Manual ENRAF \\
\hline Minimum Temperature & $55.3^{\circ} \mathrm{F}$ & Max Level & 172.8 in \\
\hline Date & Feb 13, 1993 & Date & Sept 6, 1993 \\
\hline Elevation from tank bottom & unknown & Min Level & 171.1 in \\
\hline Riser Number & 4 & Date & July 1,1995 \\
\hline
\end{tabular}




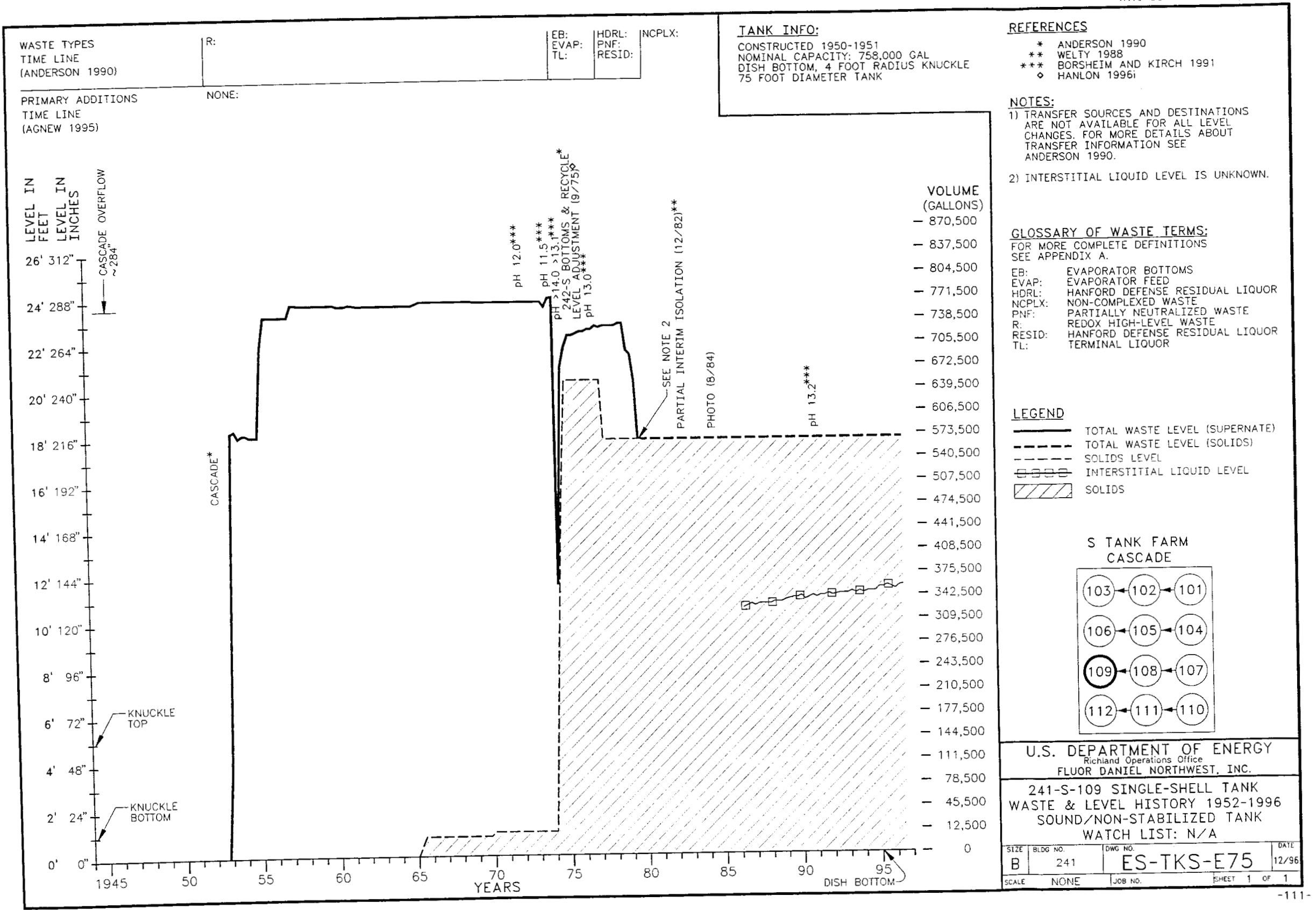




\section{$241-5-109$}

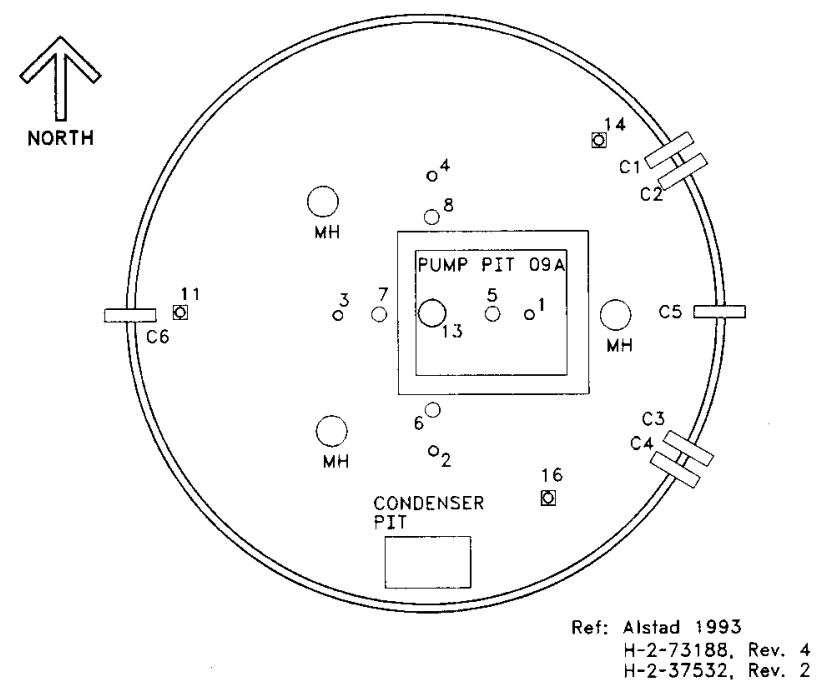

TANK RISER LOCATION

Approximate Grade Elevation 202.2m [663.3ft]

(Pianka 1995)

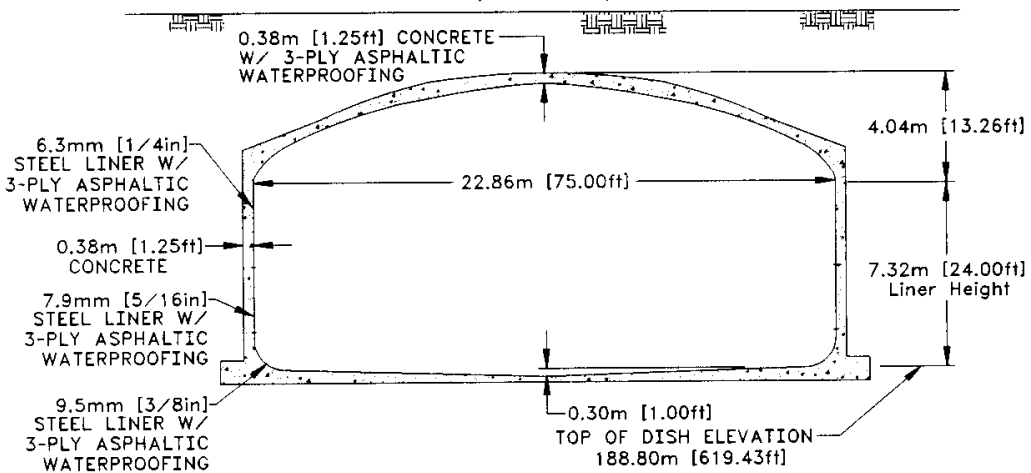

$$
\begin{gathered}
\text { NOT TO SCALE } \\
\text {-112- }
\end{gathered}
$$

Ref: $\mathrm{H}-2-1783$, Rev. 3 $\mathrm{H}-2-46293$, Rev. 3 H-2-1784, Rev, 2 


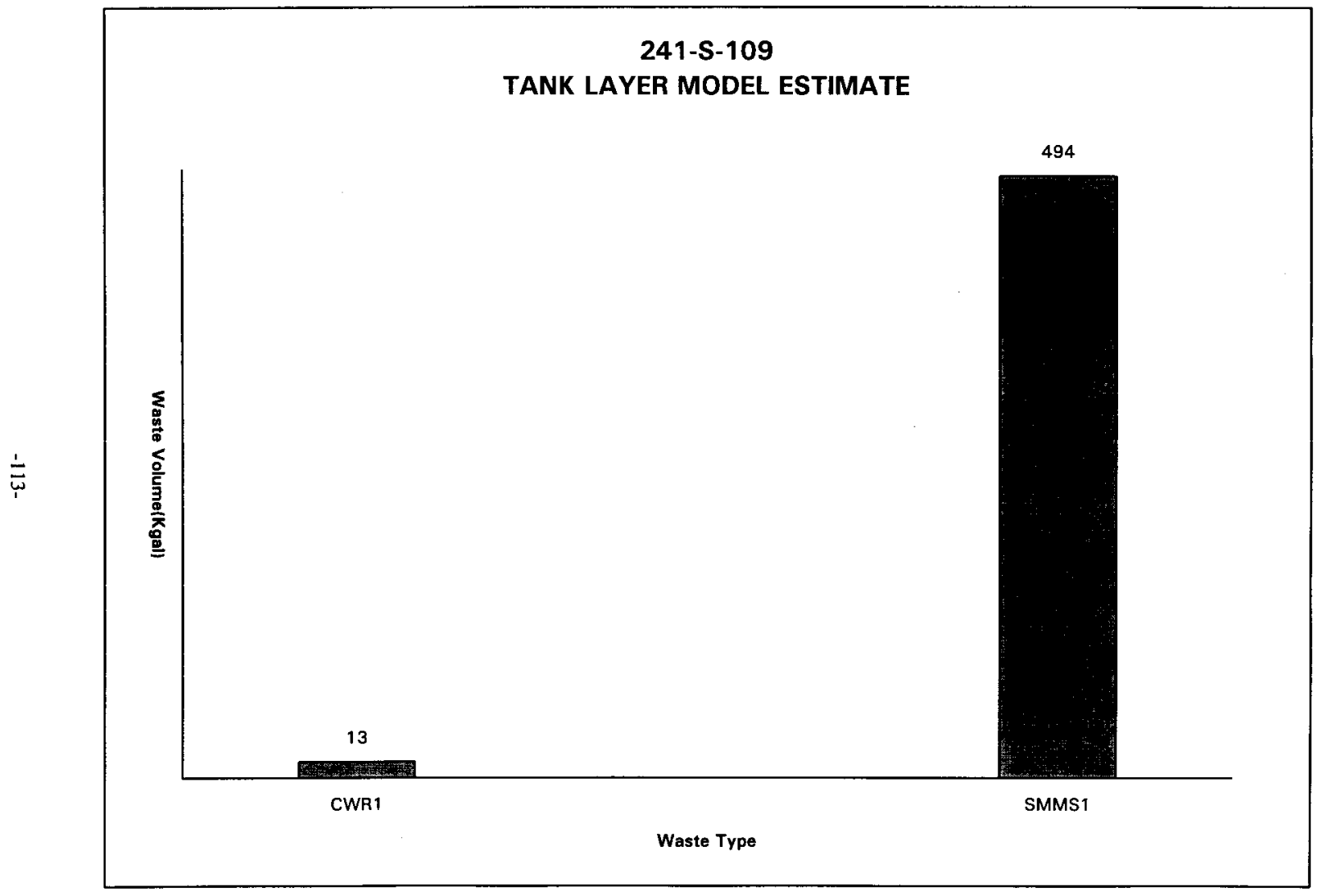

Tank Layer Model(TLM) Estimate from Hanford Tank Chemical and Radionuclide Inventories: HDW Model Rev. 4 (Agnew et al., 1997). 
HNF-SD-WM-ER-352, Rev. 1

HDW Madel Rov. 4

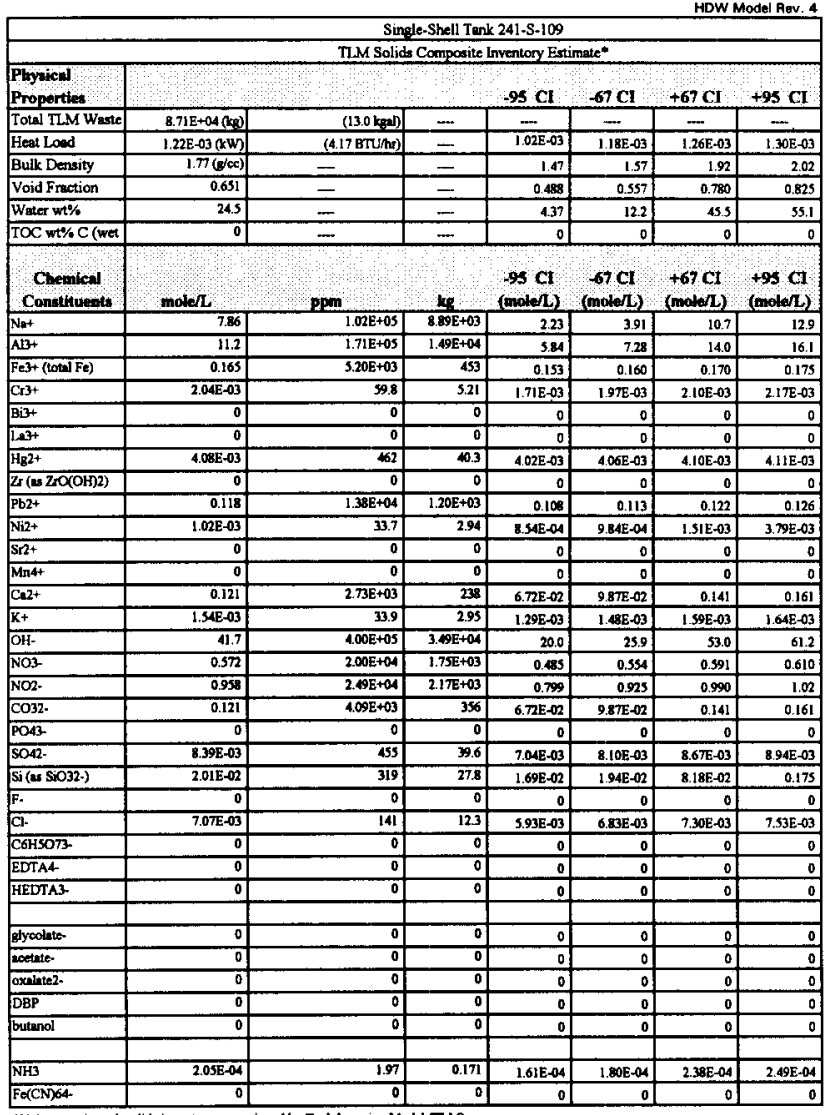

"Unkowowns in tunk solids inventory we wedigned by Tenk Lyyering Model (TLM). 
HNF-SD-WM-ER-352, Rev. 1

HDW Modet Aev. 4

\begin{tabular}{|c|c|c|c|c|c|c|c|}
\hline \multicolumn{8}{|c|}{ Single-Shell Tank 241-S-109 } \\
\hline & \multicolumn{7}{|c|}{ SMM Composite Inventory Estimate } \\
\hline $\begin{array}{l}\text { Physicid } \\
\text { Properties }\end{array}$ & & & & $-95 \mathrm{CI}$ & $-67 \mathrm{CI}$ & $+67 \mathrm{CI}$ & $+95 \mathrm{CI}$ \\
\hline Total SMM West & $3.10 E+06(\mathrm{~kg})$ & (494 kgal) & $\cdots$ & $=$ & - & 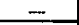 & 二 \\
\hline Heat Load & $3.87(\mathrm{~kW})$ & (1.32E+04 BTU/hr) & - & 3.64 & 3.75 & 4.00 & 4.02 \\
\hline Bulk Density* & $1.66(\mathrm{~g} / \mathrm{ec})$ & - & - & 1.59 & 1.63 & 1.68 & 1.69 \\
\hline Water $w \%$ & 30.9 & $\ldots$ & - & 28.6 & 29.6 & 32.3 & 347 \\
\hline TOC wt\% C (wet & 0.340 & 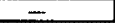 & $\rightarrow$ & 0.304 & 0.321 & 0.358 & 0.375 \\
\hline $\begin{array}{l}\text { Chephical } \\
\text { Conutituients }\end{array}$ & mole/L & pra $\quad$ - & bis & $\begin{array}{l}9 \mathrm{CI} \\
(\mathrm{mol} / \mathrm{L})\end{array}$ & $\begin{array}{l}67 \mathrm{Cl} \\
\text { (molel })\end{array}$ & $\begin{array}{l}+67 \mathrm{CI} \\
\text { (moled })\end{array}$ & $895 \mathrm{Cl}$ \\
\hline Nat & 15.1 & $2.10 E+05$ & 6.5IE+05 & 13.7 & 14.5 & 15.6 & 16.0 \\
\hline All + & 2.08 & $3.38 E+04$ & $1.05 E+05$ & 1.81 & 1.94 & 2.23 & 2.15 \\
\hline $\mathrm{Fe}+($ total $\mathrm{Fe}$ ) & $9.65 E-03$ & 325 & $1.01 E+03$ & 7.85E-03 & 8.73E-03 & $1.06 \mathrm{E}-02$ & $1.14 E-02$ \\
\hline $\mathrm{Cr}^{3+}$ & 0.213 & $6.68 E+03$ & $207 E+04$ & 0.159 & 0.188 & 0.218 & 0.223 \\
\hline Bij+ & $7 . \pi 5-04$ & 98.2 & 304 & $7.01 \mathrm{E}-04$ & $7,39 \mathrm{E}-04$ & B.17E-04 & 8.55E-04 \\
\hline Lent & $175 E-68$ & $1.47 \mathrm{E}-03$ & 4.54E-43 & $1.29 \mathrm{E}-0 \mathrm{~B}$ & $1.52 E-08$ & 1.98E-08 & $220 \mathrm{E}-08$ \\
\hline $\mathrm{Hg} 2+$ & $6.59 E-00$ & 0.797 & 2.47 & $6.17 \mathrm{E}-06$ & 6.40E-06 & $6.66 \mathrm{E}-06$ & $6.74 \mathrm{E}-66$ \\
\hline $\mathrm{Zr}(\mathrm{ar} \mathrm{ZrO}(\mathrm{OH}) 2)$ & $1,60 \mathrm{E}-04$ & 8.82 & 27.3 & $1.46 \mathrm{E}-04$ & I.51E-04 & $1.66 \mathrm{E}-04$ & $1.74 \mathrm{E}-04$ \\
\hline Pb2+ & $7.81 E-04$ & 97.7 & 303 & $6.60 \mathrm{E}-04$ & $7.19 \mathrm{E}-04$ & 8.43E-04 & $9.02 \mathrm{E}-04$ \\
\hline $\mathrm{Ni2}^{+}$ & $6.20 E-03$ & 220 & 681 & $6.00 \mathrm{E}-03$ & $6.10 E-03$ & $6.31 E-03$ & $6.41 E-03$ \\
\hline $\mathrm{sn} 2+$ & 0 & 0 & 0 & 0 & 0 & 0 & 0 \\
\hline Mndt & 3.14E-03 & 104 & 322 & $2.27 \mathrm{E}-03$ & $2.70 E-03$ & $3.58 E-03$ & $400 \mathrm{E}-03$ \\
\hline $\mathrm{Ce} 2+$ & $3.17 \mathrm{E}-02$ & 767 & $2.37 \mathrm{E}+03$ & $2.77 \mathrm{E}-02$ & $2.96 \mathrm{E}-02$ & $3.37 \mathrm{E}-02$ & $3.57 E-02$ \\
\hline $\mathbf{K}+$ & $6.372-02$ & $1.50 E+03$ & $4.652+03$ & $5.72 \mathrm{E}-02$ & $6.03 E-02$ & $6.74 \mathrm{E}-02$ & $6.93 E-02$ \\
\hline $\mathrm{OH}-$ & 12.6 & $1.30 \mathrm{E}+05$ & 1.01E+05 & 11.0 & 11.7 & 13.5 & 13.2 \\
\hline $\mathrm{NO3}$ & 5.04 & $1.89 E+05$ & $5.85 E+05$ & 4.62 & 483 & 5.21 & 5.21 \\
\hline NO2- & 2.85 & $7.93 E+04$ & 2.45E+0S & 2.48 & 2.65 & 3.05 & 3.23 \\
\hline $\cos 2-$ & 0.295 & $1.07 \mathrm{E}+04$ & $3.32 E+04$ & 0.266 & 0.280 & 0.315 & 0.317 \\
\hline POA3- & $6.496-02$ & $3.72 \mathrm{E}+03$ & I.15E+04 & $5.93 E-02$ & $6.06 \mathrm{E}-02$ & $6.64 \mathrm{E}-02$ & 6.79E-02 \\
\hline SO42- & 0.190 & $1.10 \mathrm{E}+04$ & $3.42 \mathrm{E}+04$ & 0.143 & 0.165 & 0.216 & 0.239 \\
\hline Si (es SiO32-) & 7.16E-02 & $1.21 \mathrm{E}+03$ & $3.76 E+03$ & $5.92 \mathrm{E}-02$ & $6.53 E-02$ & $7.79 \mathrm{E}-02$ & 8. $40 \mathrm{E}-02$ \\
\hline F. & $3.72 E-02$ & 27 & $1,32 E+03$ & $3.05 E-02$ & $3.33 \mathrm{E}-02$ & 4.05E-02 & $431 \mathrm{E}-02$ \\
\hline $\mathrm{Cl}-$ & 0.256 & $5.47 \mathrm{E}+03$ & $1.6 \mathrm{EE}+04$ & 0.226 & 0.240 & 0.273 & 0.274 \\
\hline C6HSO73- & $2.33 \mathrm{E}-02$ & $2.66 \mathrm{E}+03$ & $8.23 \mathrm{E}+03$ & 2.15E-02 & $2.24 \mathrm{E}-02$ & $2.42 \mathrm{E}-02$ & $2.50 \mathrm{E}-02$ \\
\hline EDTA4- & $2.32 E-03$ & 403 & $1.25 \mathrm{E}+03$ & 1.15E-03 & 1.72E-03 & $2.92 \mathrm{E}-03$ & 3.50E-03 \\
\hline HEUTA & 3.00E-03 & 629 & $1.95 \mathrm{E}+03$ & $1.47 \mathrm{E}-03$ & $260 E-03$ & $5.00 E-03$ & 6. $1 \pi-03$ \\
\hline Elycolnte- & 3.85E-02 & $1.74 E+03$ & $5.40 E+03$ & $2.1 \pi-02$ & $2.99 \mathrm{E}-02$ & $4.71 E-02$ & S.52E-02 \\
\hline neetate- & $2.70 \mathrm{E}-03$ & 96.3 & 20 & 2.19E-03 & $2.44 E-43$ & 2.96E-03 & 3.21E.09 \\
\hline oxalate2. & $2.29 E-08$ & $1.22 \mathrm{E}-0 \mathrm{3}$ & $3.7 T \mathrm{E}-\mathrm{A3}$ & $2.04 \mathrm{E}-08$ & $2,16 \mathrm{E}-08$ & 2.4IE-08 & $2.53 \mathrm{E}-08$ \\
\hline DBP & 1.35E-02 & $1.97 \mathrm{TE}+03$ & $6.06 E+03$ & $1.24 E-02$ & $1.39 \mathrm{E}-\mathrm{O} 2$ & 1.70E-02 & 1.B5E-02 \\
\hline buttenal & $1.55 E-02$ & 693 & 2.15E+03 & 1.24E-02 & $1,39 \mathrm{E}-02$ & L.70E-02 & 1.85E-02 \\
\hline $\mathrm{NH3}$ & $7.63 \mathrm{E}-02$ & 78 & $2.42 E+03$ & $6.235-02$ & $6.78 E-02$ & 8.70E-02 & 9.95E-02 \\
\hline Fe(CN)S4 & 0 & 0 & 0 & 0 & 0 & 0 & 0 \\
\hline
\end{tabular}

Density is colculated besed on Ne, OH-, und $\mathrm{AO}_{2}$.

+Water wt\% derived from the difference of density and lotal dissolvod species. 
HDW Model Rov. 4

\begin{tabular}{|c|c|c|c|c|c|c|c|}
\hline \multicolumn{8}{|c|}{ Single-Shell Tank 241-S-109 } \\
\hline & \multicolumn{7}{|c|}{ Total Inventory Estimate" } \\
\hline Pthyiled & & & & & & & \\
\hline Propenties & & & & $-9 \mathrm{CI}$ & $-67 \mathrm{CI}$ & $+67 \mathrm{CI}$ & $+95 \mathrm{CI}$ \\
\hline Total Waste & $3.18 \mathrm{E}+06(\mathrm{~kg})$ & $(507 \mathrm{kgal})$ & - & - & 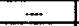 & - & - \\
\hline Heat Load & $3.87(\mathrm{~kW})$ & (1.32E+04 BTU/hr) & $\ldots$ & 3.64 & 3.75 & 4.01 & 4.03 \\
\hline Bulk Density & $1.60(\delta / \infty)$ & - & - & 1.60 & 1.63 & 1.68 & 1.69 \\
\hline Water $w t^{*} \% \dagger$ & 30.7 & ימה & - & 28.4 & 29.4 & 32.1 & 34.4 \\
\hline TOC wt\% C (wet & 0.331 & - & - & 0.295 & 0.313 & 0,348 & 0.365 \\
\hline $\begin{array}{l}\text { Chenent } \\
\text { Conslituents }\end{array}$ & molol,$\quad$ & Ppen & ke & $\begin{array}{l}95 \mathrm{CI} \\
\text { (nimled })\end{array}$ & $\begin{array}{l}67 \mathrm{Cl} \\
(\mathrm{wot} / \mathrm{L})\end{array}$ & $\begin{array}{l}+67 \text { C1 } \\
\text { (nole/4) }\end{array}$ & $\begin{array}{l}+95 \mathrm{CI} \\
\text { (molen) }\end{array}$ \\
\hline Nat & 14.9 & $2.07 \mathrm{E}+05$ & $6.60 \mathrm{E}+05$ & 13.5 & 14.3 & 15.4 & 15.8 \\
\hline Albt & 2.31 & $3.76 \mathrm{E}+04$ & $1.20 \mathrm{E}+05$ & 205 & 2.18 & 2.46 & 2.41 \\
\hline $\mathrm{Fe}=3+(\operatorname{total} \mathrm{Fe})$ & $1.36 \mathrm{E}-02$ & 459 & $1.46 \mathrm{E}+03$ & 1.19E-02 & $1.278-02$ & 1.45E-02 & 1.54E-02 \\
\hline$\overline{\mathrm{Cr} 3+}$ & 0.207 & $6.99 \mathrm{E}+03$ & $2.0 \pi \mathrm{E}+04$ & 0.155 & 0.183 & 0.212 & 0.217 \\
\hline $\mathrm{Biz}+$ & $7.53 E-04$ & 95.5 & 304 & $6.63 \mathrm{E}-04$ & $7.20 \mathrm{E}-04$ & $7.90 \mathrm{E}-04$ & $8.33 E-04$ \\
\hline Layt & $1.70 \mathrm{E}-08$ & 1.43E-03 & 4,54E-03 & $1.26 \mathrm{E}-08$ & $1.48 \mathrm{E}-08$ & 1.93E- -88 & 2.15E-08 \\
\hline $\mathrm{Hg} 2+$ & 1.11E-04 & 13.4 & 42.7 & 1.09E-04 & 1.10E-04 & $1.11 \mathrm{E}-04$ & 1.12E-04 \\
\hline $\mathrm{Zr}(\mathrm{As} \mathrm{ZO}(\mathrm{OH}) 2)$ & $1.56 \mathrm{E}-04$ & 8.57 & 27.3 & 1.42E-04 & $1.47 \mathrm{E}-04$ & $1.62 \mathrm{E}-04$ & 1.69E-04 \\
\hline PO2+ & 3.78E-03 & 47 & $1.50 \mathrm{E}+03$ & $3.53 \mathrm{E}-03$ & 3.68E-03 & 3.85E -03 & 3.90E-03 \\
\hline $\mathrm{Ni2+}$ & 6.07E-03 & 215 & 684 & S.87E-03 & $5.97 \mathrm{E}-03$ & $6.17 E-03$ & $6.27 \mathrm{E}-03$ \\
\hline $5 n+$ & 0 & 0 & 0 & 0 & 0 & 0 & 0 \\
\hline Mndt & $3.06 \mathrm{E}-03$ & 101 & 322 & 2.21E-03 & $2.63 E-03$ & 3.49E-03 & $390 \mathrm{E}-03$ \\
\hline Cent & $3.40 \mathrm{E}-02$ & 820 & $2.61 \mathrm{E}+03$ & $3.00 \mathrm{E}-02$ & $3.20 \mathrm{E}-02$ & 3.59E-02 & 3. $\mathrm{nEE}-02$ \\
\hline$k+$ & $6.21 \mathrm{E}-02$ & $1.46 \mathrm{E}+03$ & $4.66 \mathrm{E}+03$ & $5.58 \mathrm{E}-02$ & 5.68E-02 & 6.57E-012 & $675 \mathrm{E}-02$ \\
\hline OH- & 13.4 & $1.37 \mathrm{E}+05$ & $436 \mathrm{E}+05$ & 11.8 & 12.5 & 14.2 & 13.9 \\
\hline $\mathrm{NO}$ & 4.93 & 1.84E+05 & $5.86 \mathrm{E}+0.5$ & 4.52 & 4.72 & 5.10 & 510 \\
\hline NO2. & 2.80 & $7.78 E+04$ & $2.48 \mathrm{E}+05$ & 2.44 & $2.6 !$ & 299 & 3.17 \\
\hline Co32- & 0.291 & $1.05 E+04$ & $3.35 \mathrm{E}+0.4$ & 0.262 & 0.276 & 0.310 & 0.312 \\
\hline PO43. & $6.325-02$ & $3.62 \mathrm{E}+03$ & $1.15 E+a$ & $5.69 \mathrm{E}-02$ & $5.90 \mathrm{E}-02$ & 6.47E-02 & $6.61 \mathrm{E}-02$ \\
\hline SO42. & 0.186 & $1.08 E+04$ & $3.42 \mathrm{E}+04$ & 0.139 & 0.161 & 0.211 & 0.233 \\
\hline $\mathrm{Si}(\mathrm{ns} \mathrm{SiO} 32-)$ & $7.03 \mathrm{E}-02$ & $1.19 \mathrm{E}+03$ & $3.79 \mathrm{E}+03$ & 5.82E-02 & 6.41E-02 & $7.64 \mathrm{E}-02$ & $8.24 E-02$ \\
\hline F. & $3.63 \mathrm{E}-02$ & 415 & $1.32 \mathrm{E}+03$ & $2.97 \mathrm{E}-02$ & $3.25 E-02$ & 3.95E-02 & 4.20E-02 \\
\hline C. & 0.249 & $5.32 \mathrm{E}+03$ & $1.69 \mathrm{E}+04$ & 0.220 & 0.234 & 0.266 & 0.267 \\
\hline C6HSOT- & $2.27 E-02$ & $2.99 \mathrm{E}+03$ & B.23E+03 & 2.10E-02 & $2.18 E-02$ & $2.36 \mathrm{E}-02$ & 2.44E-02 \\
\hline EDTA4 & $2.26 \mathrm{E}-03$ & 39 & $1.25 \mathrm{E}+03$ & $1.12 \mathrm{E}-03$ & $1.68 \mathrm{E}-03$ & 2.84E-03 & $3.41 \mathrm{E}-03$ \\
\hline HEDTA3. & $3.70 \mathrm{E}-03$ & 611 & $1.95 \mathrm{E}+03$ & 1.43E-03 & 2.54E-03 & $4.87 \mathrm{E}-03$ & $6.01 E-03$ \\
\hline gtyeolnite- & $3.75 \mathrm{E}-02$ & $1.20 \mathrm{E}+03$ & $5.40 \mathrm{E}+03$ & 2.12E-02 & $2.92 \mathrm{E}-02$ & $4.58 E-02$ & $5.38 \mathrm{E}-02$ \\
\hline ncetater. & $2.63 \mathrm{E}-03$ & 93.6 & $29 \%$ & 2.13E-03 & $2.39 \mathrm{E}-03$ & $2.89 E-03$ & $3.13 \mathrm{E}-03$ \\
\hline Oxalinte2- & $2.23 E-08$ & I.18E-03 & $3.776-03$ & 1.99E-08 & 2.11E-08 & $2.35 E-08$ & 2.47E-08 \\
\hline DBP & 1.51E-02 & $1.91 \mathrm{E}+03$ & $6.005+03$ & $1.21 \mathrm{E}-02$ & $136 \mathrm{E}-02$ & $1.66 \mathrm{E}-02$ & $1.80 \mathrm{E}-02$ \\
\hline buthinal & 1.51E-02 & 674 & $2.15 E+03$ & $1.21 \mathrm{E}-02$ & $1.36 \mathrm{E}-02$ & $1.66 \mathrm{E}-02$ & $1.80 \mathrm{E}-02$ \\
\hline $\mathrm{NH3}$ & $7.43 E-02$ & 762 & $2,2 E+03$ & 6.07E-02 & $6.61 \mathrm{E}-02$ & B $48 E-02$ & $9.70 \mathrm{E}-02$ \\
\hline $\mathrm{Fo}(\mathrm{CN}) 64$ & 0 & 0 & 0 & 0 & 0 & 0 & 0 \\
\hline
\end{tabular}

Unkonowns in tunk solids invemany ane eatigned by Tenk Lyering Model (TLM).

tWaler wt\% derived from the difference of density and total diseolved spocies. 
HNF-SD-WM-ER-352, Rev. 1

HDW Moded Rev. 4

\begin{tabular}{|c|c|c|c|c|c|c|c|}
\hline \multirow{4}{*}{ Physial } & \multicolumn{7}{|c|}{ Single-Shell Tank 241-S-109 } \\
\hline & \multicolumn{7}{|c|}{ TLM Solids Composite Inventory Estimate* } \\
\hline & & & & & & & \\
\hline & & & & $95 \mathrm{CI}$ & $-67 \mathrm{cl}$ & $+67 \mathrm{Cl}$ & $+95 \mathrm{Cl}$ \\
\hline Total TLM Waste & $8.71 \mathrm{E}+04(\mathrm{~kg})$ & (13.0 kgal) & $\ldots$ & - & $-=$ & - & - \\
\hline Heat Loud & $1.22 \mathrm{E}-03(\mathrm{~kW})$ & (4.17 BTU/hr) & - & $1.02 \mathrm{E}-03$ & $1.18 \mathrm{E}-03$ & $1.26 \mathrm{E}-03$ & $1,30 \mathrm{E}-03$ \\
\hline Bulk Density & $1.7(g / \infty)$ & $\cdots$ & - & 1.47 & 1.57 & 1.92 & 2.02 \\
\hline Void Fraction & 0.651 & $\ldots$ & - & 0.488 & 0.557 & 0.720 & 0.825 \\
\hline Water wt $\%$ & 24.5 & $\ldots$ & $\overline{-}$ & 4.37 & 12.2 & 45.5 & 55.1 \\
\hline TOC w\% $\mathrm{C}$ (wet & 0 & $=$ & - & 0 & 0 & 0 & 0 \\
\hline $\begin{array}{l}\text { Rowiologent } \\
\text { Constituents: }\end{array}$ & $\mathbf{C V}$, & $\mathrm{HC} / \mathrm{s}$ & $\mathbf{C}$ & $\begin{array}{l}9 \mathrm{cl} \\
\mathrm{CM})\end{array}$ & $\begin{array}{l}67 \mathrm{Cl} \\
(\mathrm{C} / \mathrm{L})\end{array}$ & $\begin{array}{c}-67 \mathrm{Cl} \\
(\mathrm{CuL})\end{array}$ & $\begin{array}{l}+95 \mathrm{Cl} \\
\text { (ch) }\end{array}$ \\
\hline $\mathrm{H}-3$ & $1.01 E-06$ & S.71E-04 & $4.98 \mathrm{E}-02$ & 6.33E-07 & 8.96E-07 & $1.13 E-06$ & $1.24 E-06$ \\
\hline $\mathrm{C}-14$ & 1.09E-07 & $6.16 \mathrm{E}-05$ & $5.37 \mathrm{E}-09$ & $9.15 \mathrm{E}-08$ & 1.05E-07 & $1.13 \mathrm{E}-07$ & $1.16 E-07$ \\
\hline $\mathrm{Ni}-59$ & $3.09 E-08$ & $1.758-05$ & $1.52 \mathrm{E}-03$ & $2.59 \mathrm{E}-08$ & $2.99 \mathrm{E}-08$ & $4.60 \mathrm{E}-08$ & 1.15E-07 \\
\hline $\mathrm{Ni}-63$ & $2.90 \mathrm{E}-06$ & 1.64E-03 & 0.143 & 2.013-06 & $2000-06$ & 4.31E-06 & $1.08 \mathrm{E}-05$ \\
\hline $\operatorname{Co} 60$ & $4.71 E-08$ & $2.66 \mathrm{E}-05$ & $2.32 E-03$ & $3.96 \mathrm{E}-08$ & 4.56E-08 & $4.87 \mathrm{E}-08$ & $5.02 \mathrm{E}-08$ \\
\hline Se-79 & $2.3 \pi-08$ & $1.34 \mathrm{E}-05$ & $1.17 \mathrm{E}-03$ & $1.99 \mathrm{E}-08$ & $2.29 \mathrm{E}-08$ & $2.45 E-08$ & $2.53 E-08$ \\
\hline Sr-90 & 20.0SE-03 & 1.16 & 101 & $1.72 \mathrm{E}-03$ & 1.98E-03 & $2.12 E-03$ & $2.18 E-09$ \\
\hline$Y-90$ & 2.05E-03 & 1.16 & 101 & $1.72 \mathrm{E}-03$ & 1.96E-03 & $2.12 E-03$ & 2.18E-03 \\
\hline $\mathrm{Z}_{\mathrm{r}-93}$ & 1.12E-07 & 6.34E-05 & $5.52 E-03$ & $9.42 E-00$ & $1.09 \mathrm{E}-07$ & $1.16 \mathrm{E}-07$ & 1.20E-07 \\
\hline $\mathrm{Nb}-93 \mathrm{~m}$ & $9.13 \mathrm{E}-08$ & $5.15 E-05$ & $4.49 E-03$ & $7.66 \mathrm{E}-\mathrm{GB}$ & B.82E-08 & $9.43 \mathrm{E}-0 \mathrm{~B}$ & 9.73E-08 \\
\hline Tc-99 & 78AE-07 & 4.42E-04 & $3.85 E-02$ & $6.57 \mathrm{E}-07$ & $7.57 \mathrm{E}-07$ & 8.10E-07 & $8.35 E-07$ \\
\hline Ru-106 & $2.76 \mathrm{E}-13$ & $1.56 \mathrm{E}-10$ & $1.36 \mathrm{E}-0 \mathrm{0}$ & 2.31E-13 & $2.66 \mathrm{E}-13$ & $2.8 S E-13$ & $2.94 \mathrm{E}-13$ \\
\hline Cd-113m & 3.5.E-07 & 1.99E-04 & $1.73 \mathrm{E}-02$ & $2.94 \mathrm{E}-07$ & $3.39 \mathrm{E}-07$ & $3.62 \mathrm{E}-07$ & $3.74 E-07$ \\
\hline$S \mathrm{~b}-125$ & $789 \mathrm{E}-0 \mathrm{~B}$ & 446E-05 & 3.88E-03 & $6.62 \mathrm{E}-08$ & $7.63 \mathrm{E}-0 \mathrm{~B}$ & $8.16 \mathrm{E}-08$ & 8.41E-08 \\
\hline$S n-126$ & 3.61E-08 & $2.04 E-05$ & I.7RE-03 & $3.03 E-08$ & $3.49 \mathrm{E}-08$ & 3.73E- 08 & 3.85E-08 \\
\hline I-129 & $1.50 E-09$ & C.4TE-07 & $7,318 E-05$ & $1.26 \mathrm{E}-09$ & $1.45 E-09$ & 1.55E-09 & $1.60 \mathrm{E}-\infty$ \\
\hline Cs-134 & $1.65 \mathrm{E}-09$ & $9.34 E-07$ & B.14E-05 & 1.39E-O9 & $1.60 \mathrm{E}-109$ & $1.71 \mathrm{E}-09$ & $1.76 \mathrm{E}-09$ \\
\hline Cs-137 & $2.35 E-03$ & 1,33 & 116 & $1.9 \pi-03$ & $2.27 \mathrm{E}-03$ & 2.43E-03 & 2.SOE -03 \\
\hline $\mathrm{Be}-137 \mathrm{~m}$ & $2.22 \mathrm{E}-03$ & 1.26 & 109 & $1.87 \mathrm{E}-03$ & $2.15 \mathrm{E}-03$ & $2.30 \mathrm{E}-03$ & 2.37E-03 \\
\hline$S_{m-151}$ & B.4TE-05 & 4.78E-02 & 4.17 & $7.10 \mathrm{E}-05$ & 8.18E-05 & $8.75 E-05$ & 9.02E-05 \\
\hline Eu-152 & $1.75 E-07$ & 9.89E-05 & 8.62E-03 & 1.74E-07 & $1.75 E-0 ?$ & $1.76 \mathrm{E}-07$ & $1.76 \mathrm{E}-07$ \\
\hline Eu-154 & 1.15E-06 & $6.48 \mathrm{E}-04$ & $5.65 \mathrm{E}-02$ & $9.63 \mathrm{E}-07$ & $1.11 E-\infty 6$ & $1.19 \mathrm{E}-06$ & 1.22E- 06 \\
\hline Eu-155 & $8.33 E-06$ & $471 \mathrm{E}-03$ & 0.410 & $8.27 \mathrm{E}-06$ & $8.32 E-06$ & c.35E-06 & $8.36 \mathrm{E}-06$ \\
\hline Ra-226 & $2.83 E-12$ & $1.60 E-09$ & $1.39 \mathrm{E}-07$ & $2.37 \mathrm{E}-12$ & $2.73 \mathrm{E}-12$ & $2.92 \mathrm{E}-12$ & $3.01 E-12$ \\
\hline Ra-228 & $2.92 \mathrm{E} \cdot 16$ & $1.65 \mathrm{E}-13$ & 1.44E-II & $2905-16$ & $2.918-16$ & $2.92 \mathrm{E}-16$ & $2.93 E-16$ \\
\hline Ac-227 & $1.45 \mathrm{E}-11$ & $8.19 \mathrm{E}-09$ & $7.13 \mathrm{E}-07$ & $1.22 \mathrm{E}-11$ & 1.40E-11 & 1.50E-1] & $1.55 \mathrm{E}-11$ \\
\hline $\mathrm{P}_{2-231}$ & 3.48E-11 & $1.94 \mathrm{E}-08$ & 1..9E-06 & $2.9 E-11$ & $3.32 \mathrm{E}-11$ & $3.55 \mathrm{E}-11$ & $3.6 \mathrm{E}-11$ \\
\hline Th-229 & $4.13 \mathrm{E}-14$ & $2.33 \mathrm{E}-11$ & $2.03 \mathrm{E}-09$ & $409 E-14$ & $4.12 \mathrm{E}-14$ & 4.13E-14 & 4.14E- 14 \\
\hline Th-232 & 4.04E-17 & $2.28 \mathrm{BE} \cdot 14$ & $1.99 \mathrm{E}-12$ & 3.39E-17 & $3.90 \mathrm{E}-17$ & $4.17-17$ & $4.30 \mathrm{E}-17$ \\
\hline U-232 & $0.4 \mathrm{E}-10$ & $3.65 \mathrm{E}-07$ & 3.18E-0S & $5.62 \mathrm{E}-10$ & $6.12 \mathrm{E}-10$ & $6.7 \mathrm{E}-10$ & $6.90 \mathrm{E} \cdot 10$ \\
\hline U.233 & $2.39 \mathrm{E}-11$ & 1.35E-08 & $1.18 E-06$ & $2.088-11$ & $2.26 \mathrm{E}-11$ & 2.96-11 & $255 \mathrm{E}-11$ \\
\hline U-234 & 1.48E-05 & .36E-03 & 0.78 & $1.29 \mathrm{E}-05$ & 1. $40 \mathrm{E}-05$ & 1.54E-05 & $1.58 E-05$ \\
\hline U.235 & $6.29 E-07$ & $3.55 E-04$ & $3.09 \mathrm{E}-02$ & $5,60 E-67$ & 5.95E-07 & $6.53 \mathrm{E}-07$ & 6.71E-07 \\
\hline $\mathrm{U}-236$ & $3.26 \mathrm{E}-07$ & $1 . B A E-M A$ & $1.60 \mathrm{E}-02$ & $2.83 \mathrm{E}-07$ & $3.08 E-07$ & $3.39 \mathrm{E}-07$ & 3.48E-07 \\
\hline U-238 & 1.44E-05 & B 14E-03 & 0.709 & 1.25E-0S & $1.36 \mathrm{E}-05$ & 1.50E-05 & $1.54 \mathrm{E}-05$ \\
\hline $\mathrm{Np}-237$ & 5.48E-09 & $3.09 \mathrm{E}-105$ & $2.70 \mathrm{E}-0 \mathrm{~A}$ & $4.60 \mathrm{E}-09$ & $5.30 \mathrm{E}-09$ & $5.67 \mathrm{TE}-09$ & 584E-09 \\
\hline Pu-238 & $2.51 E-05$ & 1.42E-02 & 1.23 & 2. $40 \mathrm{E}-05$ & 2.45E-05 & $2.5 \pi E-05$ & 2.62E-05 \\
\hline Pu-239 & $1.61 \mathrm{E}-03$ & 0.908 & 79.1 & 1.SAE-03 & $1.57 \mathrm{E}-03$ & 1.64E-03 & $1680-03$ \\
\hline Pu-240 & $2.27 \mathrm{E}-04$ & 0.128 & 11.2 & $2.1 \pi-04$ & 2.22E-01 & $2.32 \mathrm{E}-04$ & 2.37E-04 \\
\hline Pu-241 & $1.39 \mathrm{E}-03$ & 0.782 & 68.2 & $1.32 \mathrm{E}-03$ & $1.35 \mathrm{E}-03$ & $1.42 E-03$ & 1.4SE-03 \\
\hline$P u-242$ & 5.83E-199 & $3.32 E-06$ & 2.89E-04 & $5.62 \mathrm{E}-09$ & 5.7SE-09 & $6.01 E-09$ & $6.14 E-09$ \\
\hline Am-24l & $4.09 \mathrm{E}-07$ & $2.31 \mathrm{E}-04$ & $2.01 \mathrm{E}-02$ & $3.43 E-07$ & 3.95E-07 & 4.2nE-07 & 436E-07 \\
\hline Am-243 & $3.7 \mathrm{EE}-12$ & $2.30 \mathrm{E}-0.9$ & 1.83E-07 & $3.13 E-12$ & $3.00 E-12$ & 3.85E-12 & $397 \mathrm{E}-12$ \\
\hline $\mathrm{Cm}-242$ & $3.65 \mathrm{E}-09$ & $2.06 \mathrm{E}-06$ & $1.80 \mathrm{E}-04$ & $3.62 \mathrm{E}-09$ & 3.65E-09 & $3.66 \mathrm{E} \cdot 09$ & $3.67 \mathrm{e}-09$ \\
\hline $\mathrm{Cm}_{\mathrm{m}-243}$ & $8.34 E-11$ & $4.71 \mathrm{E}-08$ & 4.10E-06 & $8.2 \pi-11$ & $8.32 \mathrm{E}-11$ & 8.35E-11 & $8.36 \mathrm{E}-11$ \\
\hline $\mathrm{Cm}-244$ & 1.30E-10 & $7.33 \mathrm{E}-08$ & $6.392-06$ & $1.096-10$ & $1.25 \mathrm{E}-10$ & $1.34 E-10$ & $138 \mathrm{E}-10$ \\
\hline Totels: & $\mathbf{M}$ & yolst & $\mathrm{Lr}$ & $\begin{array}{l}95 \mathrm{Gl} \\
\text { No L }\end{array}$ & $\begin{array}{l}67 \mathrm{cr}(\mathrm{M} \\
\text { or } \mathrm{LL})\end{array}$ & $\begin{array}{r}+67 \mathrm{cl} \mathrm{M} \\
\mathrm{ar} / \mathrm{L})\end{array}$ & $\begin{array}{r}+95 \mathrm{Cl} \\
(\mathrm{M} \text { or } \mathrm{L})\end{array}$ \\
\hline $\mathrm{Pu}$ & $2.69 \mathrm{E}-02(\mathrm{~g} / \mathrm{L})$ & - & 1.32 & 2.57E-02 & $2.63 \mathrm{E}-02$ & $2.75 \mathrm{E}-02$ & $281 \mathrm{E}-02$ \\
\hline $\mathbf{U}$ & 0.181 & $2.44 E+04$ & $2.12 \mathrm{E}+03$ & 0.158 & 0.172 & 0.188 & 0.194 \\
\hline
\end{tabular}

"Unknowns in tenk solids invertory are assigned by Tenk Loyering Model (TLM) 
HDW Madel Aev. 4

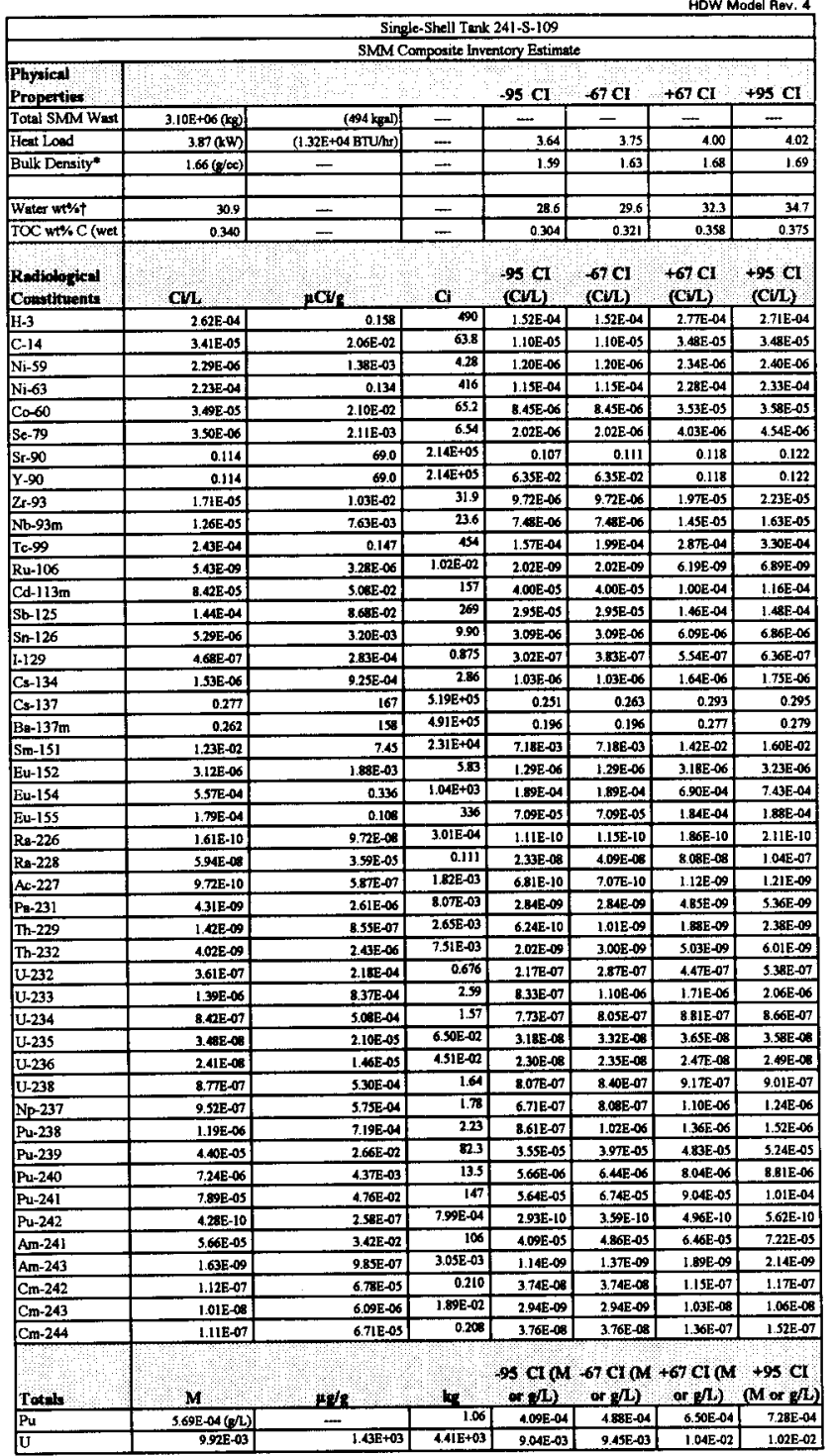

-Density is calculated besed on $\mathrm{Na}, \mathrm{OH}$-, and $\mathrm{AlO} 2$.

†Water wt\% derived from the difference of density and total dissolved species. 
HNF-SD-WM-ER-352, Rev. 1

\begin{tabular}{|c|c|c|c|c|c|c|c|}
\hline \multirow[b]{3}{*}{$\begin{array}{l}\text { Physical } \\
\text { Properties }\end{array}$} & \multicolumn{7}{|c|}{ Single-Shell Tark 24I-S-109 } \\
\hline & \multicolumn{7}{|c|}{ Total Inventory Estimate* } \\
\hline & & & & $95 \mathrm{CI}$ & $-67 \mathrm{CI}$ & $+67 \mathrm{CI}$ & $+95 \mathrm{CI}$ \\
\hline Total Weste & $3.18 E+06(60)$ & $(507 \mathrm{kgal})$ & $=$ & - & $\ldots$ & - & - \\
\hline Heat Loed & $3.87(\mathrm{kw})$ & $(1.32 \mathrm{E}+04 \mathrm{BTU} / \mathrm{MT})$ & - & 3.64 & 3.75 & 4.01 & 4.03 \\
\hline Bulk Densityt & $1.66(\mathrm{~g} / \mathrm{x})$ & $=$ & - & 1.60 & 1.63 & 1.68 & 1.69 \\
\hline Water wt\%t & 30.7 & $=$ & - & 28.4 & 29.4 & 32.1 & 34.4 \\
\hline TOC wt\% C (wet & 0.331 & $=$ & - & 0.295 & 0.313 & 0.348 & 0.365 \\
\hline 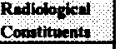 & $\mathrm{C} / \mathrm{L}$ & $\mathrm{BC} / \mathrm{s}$ & Q & $\begin{array}{l}95 \mathrm{Cl} \\
\text { (CVI) }\end{array}$ & $\begin{array}{l}67 \mathrm{Cl} \\
(\mathrm{Cn})\end{array}$ & $\begin{array}{l}+67 \mathrm{ct} \\
\text { (Ci/L) }\end{array}$ & $\begin{array}{l}+9 \mathrm{Cl} \\
\text { (CUL) }\end{array}$ \\
\hline $\mathrm{H}-3$ & 2.55E-04 & 0.154 & 400 & $1.485-04$ & 1.48E-04 & $2.70 \mathrm{E}-04$ & $2.64 E-04$ \\
\hline $\mathrm{C}-14$ & 3.32E-0s & $2.00 E-02$ & 63.8 & $1.08 E-05$ & $100 \mathrm{E}-05$ & 3.40E-05 & 339E-05 \\
\hline $\mathrm{Ni}-59$ & $2.23 E-06$ & $1.34 \mathrm{E}-03$ & 4.28 & $1.17 E-06$ & $1.17 E-06$ & 2.28E- -6 & $2.34 E-06$ \\
\hline $\mathrm{Ni}-63$ & $2.1 \pi-04$ & 0.131 & 416 & $1.12 E-04$ & $1.12 \mathrm{E}-0 \mathrm{~A}$ & $2.22 \mathrm{E}-04$ & $2.27 E-04$ \\
\hline $\cos 60$ & $3.40 E-03$ & 2.05E-02 & 65.2 & $8.24 E-06$ & $8.24 \mathrm{E}-06$ & $3.44 E-05$ & 3.49E-05 \\
\hline Se- -9 & $3.41 E-06$ & 2.05E-03 & 6.54 & $1.97 \mathrm{E}-06$ & $1.97 \mathrm{E}-06$ & 3.93E-06 & 4.42E-06 \\
\hline Sr-90 & 0.111 & 67.2 & $2.14 E+05$ & 0.104 & 0.108 & 0.115 & 0.118 \\
\hline Y.90 & 0.111 & 67.2 & $2.14 E+05$ & $6.19 \mathrm{E}-02$ & 6.19E- -02 & 0.115 & 0.119 \\
\hline $\mathrm{Z}-93$ & $1.66 \mathrm{E}-05$ & $1.00 E-02$ & 31.9 & $9.48 E-06$ & $9.48 \mathrm{E}-06$ & $1.92 \mathrm{E}-05$ & $2.17 E-05$ \\
\hline $\mathrm{Nb}-93 \mathrm{~m}$ & $1.23 \mathrm{E}-05$ & $7.42 E-03$ & 23.6 & $7.29 \mathrm{E}-06$ & $7.29 \mathrm{E}-06$ & 1.41E-05 & 1.50E-05 \\
\hline Tc-99 & $2.372-01$ & 0.143 & 454 & $1.53 E-04$ & $1.94 \mathrm{E}-04$ & $2.80 \mathrm{E}-04$ & $3.22 \mathrm{E}-04$ \\
\hline Ru-106 & $5.29 \mathrm{E}-09$ & $3.19 E-86$ & $1.02 E-02$ & $1.976-09$ & $1.97 E-09$ & $6.04 \mathrm{E}-09$ & $6.71 \mathrm{E}-09$ \\
\hline Cd-113m & $8.21 E-05$ & 4.95E -02 & 157 & $3.90 \mathrm{E}-05$ & $3.90 E-05$ & 9.76E-05 & $1.13 E-04$ \\
\hline$S b-125$ & 1. $.0 E-04$ & 8.4AE-02 & 269 & 2.87E-05 & $287 \mathrm{E}-05$ & $1.42 E-04$ & $1.442-04$ \\
\hline Sn-126 & $5.16 \mathrm{E}-06$ & 3.11E-03 & 9.90 & $3.01 E-16$ & 3.01E-06 & 5.94E-06 & $6.68 \mathrm{E}-06$ \\
\hline $\mathrm{I}-129$ & $456 \mathrm{E}-07$ & $2.75 \mathrm{E}-04$ & 0.875 & 2.94E-07 & 3.73E-07 & $5.40 E-97$ & $6.20 E-07$ \\
\hline Cs-134 & $1,49 E-\infty 6$ & $8.99 \mathrm{E}-04$ & 286 & $1.00 E-106$ & $1.00 \mathrm{E}-06$ & $1.60 \mathrm{E}-06$ & $1.71 \mathrm{E}-06$ \\
\hline Cs 137 & $0.2 \pi$ & 163 & $3.19 \mathrm{E}+05$ & 0.244 & 0.257 & 0.285 & 0.288 \\
\hline Bs $137 m$ & 0.256 & 154 & 491E+05 & 0.191 & 0.191 & 0.270 & 0.272 \\
\hline $\mathbf{S m}-151$ & $1.20 \mathrm{E}-12$ & 7.24 & $2.31 \mathrm{E}+04$ & 6.99E-03. & $6.99 \mathrm{E}-03$ & $1.38 E-02$ & $1.56 \mathrm{E}-02$ \\
\hline Eu-152 & $3.04 E-06$ & $1.83 E-03$ & 3.4 & $1.27 \mathrm{TE}-06$ & $1.27 \mathrm{E}-06$ & 3.10E-06 & 3.15E-06 \\
\hline Eu-154 & 3.42E-0A & 0.327 & $1.04 \mathrm{E}+03$ & 1.24E-04 & 1.MEE-04 & 6.72E-0.4 & $7.24 \mathrm{E}-04$ \\
\hline Eu-155 & 1.75E-0.4 & $\begin{array}{r}0.106 \\
\end{array}$ & 336 & $6.93 E-65$ & 6.935E-05 & $1.79 E-04$ & $1.83 \mathrm{E}-04$ \\
\hline Re-226 & $1.5 \pi=10$ & $9,45 E-08$ & $3.01 \mathrm{E}-04$ & $1.08 E-10$ & $1.12 \mathrm{E}-10$ & 1.82E-10 & $2.06 \mathrm{E}-10$ \\
\hline Ro-228 & 5.79E-68 & 3.4EE-05 & 0.111 & $2.27 \mathrm{E}-6 \mathrm{~S}$ & $3992-08$ & $7.87 \mathrm{E}-108$ & $1.01 \mathrm{E}-07$ \\
\hline Ac-227 & $9.48 \mathrm{E}-10$ & 5.n1E-07 & $1.82 \mathrm{E}-03$ & 6.64E-10 & 6.09E-10 & $1.09 \mathrm{E}-0.9$ & $1.18 E-09$ \\
\hline $\mathrm{Pg}-231$ & $1.20 \mathrm{E}-09$ & $2.53 \mathrm{E}-16$ & B.07E-03 & $2.77 \mathrm{E}-09$ & $2 \pi n-\infty$ & $4.72 \mathrm{E}-09$ & $3.22 \mathrm{E}-69$ \\
\hline Th-229 & 139E-09 & 8.31E-07 & $2.65 \mathrm{E}-03$ & 6.06E-10 & $9.85 \mathrm{EE}-10$ & $1.84 E-09$ & $2.32 \mathrm{E}-09$ \\
\hline Th-232 & 3.91E-09 & $2.36 E-16$ & $7.31 E-03$ & 1.97t-09 & $2.92 \mathrm{E}-09$ & 4.91E-09 & $5.86 \mathrm{E}-09$ \\
\hline U-232 & $3.52 E-07$ & 2.12E-04 & 0.676 & 2.11E-07 & 2:008:07 & $4.36 \mathrm{E}-07$ & $5.24 E-07$ \\
\hline U-233 & 1.35E-06 & $8.14 E-04$ & 2.5 & 8.12E-07 & $1.008-06$ & $1.67 \mathrm{E}-06$ & $2.01 \mathrm{E}-66$ \\
\hline U-234 & $1.20 E-06$ & $7.23 \mathrm{E}-04$ & 2.30 & 1.13E-96 & $1.10 E-\infty$ & $1.24 E-06$ & $1.22 \mathrm{E}-06$ \\
\hline $\mathrm{U}-235$ & S.00E-08 & 3.01E-05 & $9.60=-02$ & 4.71E-08 & 4.85E-AB & $5.17 \mathrm{E}-08$ & S.11E-08 \\
\hline U-236 & $3.19 E-00$ & $1.925-05$ & 6.11E-0? & $3.08 \mathrm{E}-\infty 8$ & $3.138-08$ & $3.25 \mathrm{E}-0.8$ & $3.26 \mathrm{E}-0$. \\
\hline U.238 & 1.22E-06 & 7.38E-04 & 2.35 & $1.16 \mathrm{E}-06$ & $1.19 E-\infty 6$ & $126 \mathrm{E}-06$ & $1.25 E-96$ \\
\hline Np-237 & $9.28 \mathrm{E}-07$ & $5.59 \mathrm{E}-04$ & 1.78 & 6.54E-07 & 7.APE-07 & $1.07 \mathrm{E}-06$ & $1.21 \mathrm{E}-06$ \\
\hline Pu-238 & 1.80E-06 & 1.09E-03 & 3.46 & 1.48E-O6 & $1.64=06$ & $197 \mathrm{E}-06$ & $2.13 E-06$ \\
\hline Pu-239 & EAIE-05 & $5.0 \pi E-12$ & 161 & 7.SBE-05 & $7.99 \mathrm{E}-05$ & $8.83 E-05$ & $9.23 \mathrm{E}-0 \mathrm{~S}$ \\
\hline Pu.240 & $1.29 \mathrm{E}-05$ & $7.76 \mathrm{E}-03$ & 24.7 & $1.13 E-05$ & $1.21 \mathrm{E}-05$ & $1.37 \mathrm{E}-05$ & $1.44 \mathrm{E}-05$ \\
\hline Pu-241 & $1.12 E-04$ & $6.77 \mathrm{E}-02$ & 216 & $9.05 E-05$ & 1.01E-04 & $1.24 E-04$ & $1.34 \mathrm{E}-04$ \\
\hline Pu=242 & $5.6 \pi \mathrm{E}-10$ & $3.20-07$ & $1.09 \mathrm{E}-03$ & 4.36E-10 & S.OOE. 10 & 6.36E-10 & $6.98 \mathrm{E}-10$ \\
\hline Am-241 & S.SIE-05 & $3.325-02$ & 106 & $3.99 E-05$ & 4.74E-05 & $6.29 \mathrm{E}-05$ & 7.04E-05 \\
\hline$A m-243$ & $1.59 \mathrm{E}-09$ & $9.58 E-07$ & 3.05E-03 & 1.11E-09 & $1.30 \mathrm{E}-09$ & 1.RAE-OS & 2.09E-09 \\
\hline $\mathrm{Cm}-242$ & $1,10 E-07$ & $6.60 E-05$ & 0.210 & $3.06 \mathrm{E}-0 \mathrm{E}$ & $3.60 \mathrm{E}-08$ & $1.12 E-97$ & 1.14E-07 \\
\hline $\mathrm{Cm}-243$ & $9.80 \mathrm{E}-09$ & 5.92E-06 & $1.80 \mathrm{E}-02$ & $2.87 E-09$ & $2.87 E-09$ & $1.01 \mathrm{E}-08$ & $1.03 E-08$ \\
\hline Cm-244 & 1.08E-07 & $6.53 E-05$ & 0.201 & $3.66 \mathrm{E}-08$ & $3.66 \mathrm{E}-0 \mathrm{E}$ & $1.33 \mathrm{E}-07$ & $1.48 \mathrm{E}-07$ \\
\hline Totel & M & $1 / / 8$ & to & $\frac{2010}{0+L}$ & $67 \mathrm{Cl}(\mathrm{d}$ & $+67 \mathrm{Cl}(\mathrm{M}$ & $\begin{array}{c}+95 \mathrm{Cl} \\
(\mathrm{M} \text { or } / \mathrm{L})\end{array}$ \\
\hline Pu & $1.24 \mathrm{E}-03(\mathrm{~L} / \mathrm{L})$ & - & 2.39 & $1.09 \mathrm{E}-03$ & $1.168-03$ & $1,32 \mathrm{E}-03$ & $\begin{array}{r}.40 E-03 \\
\end{array}$ \\
\hline $\mathrm{U}$ & $1.43 \pm-02$ & $2.05 \mathrm{E}+03$ & $6.54 E+03$ & 1.35E-02 & 1.39E- -22 & 1. $4 \mathrm{BE}-02$ & $1.46 \mathrm{E}-02$ \\
\hline
\end{tabular}




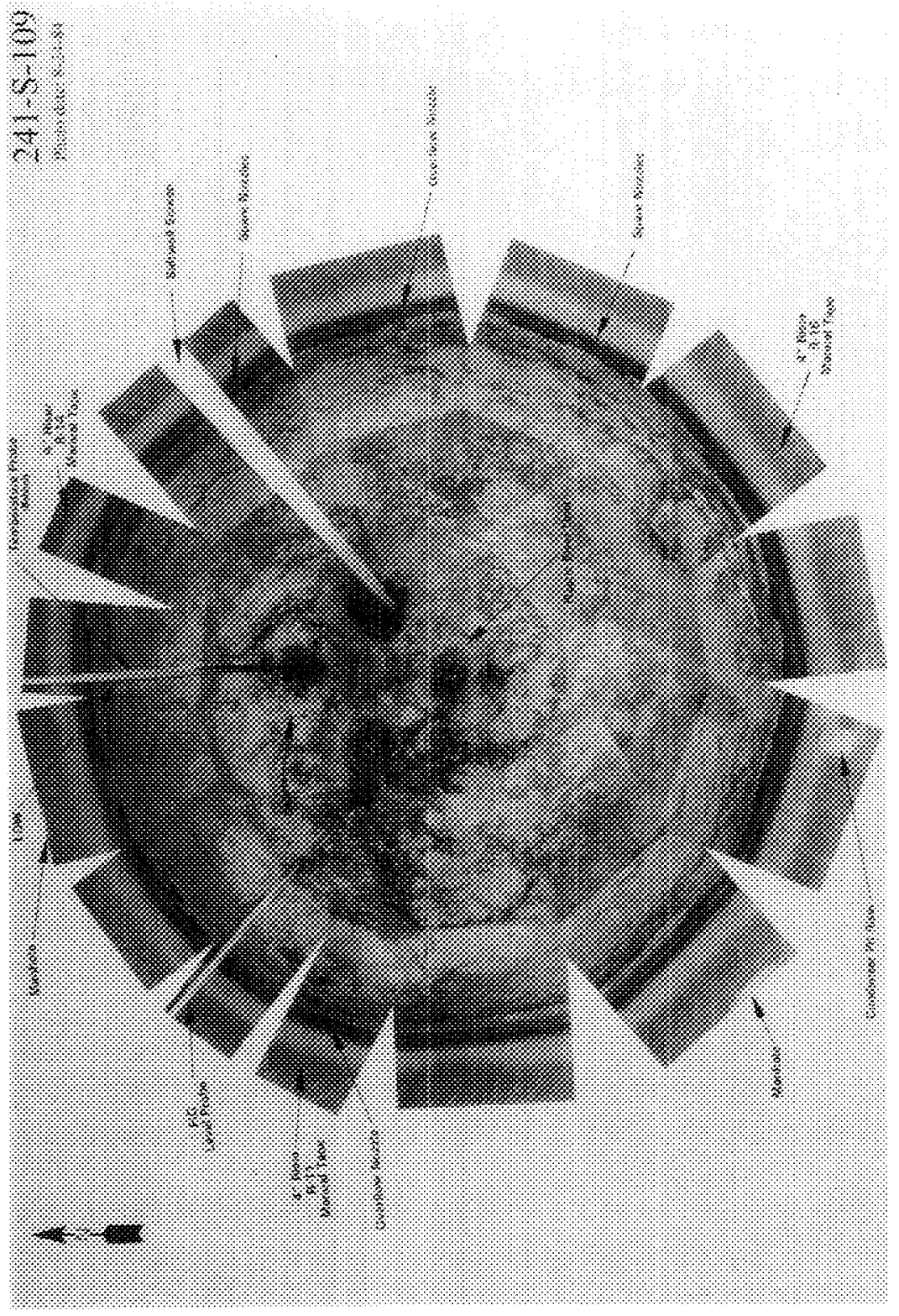




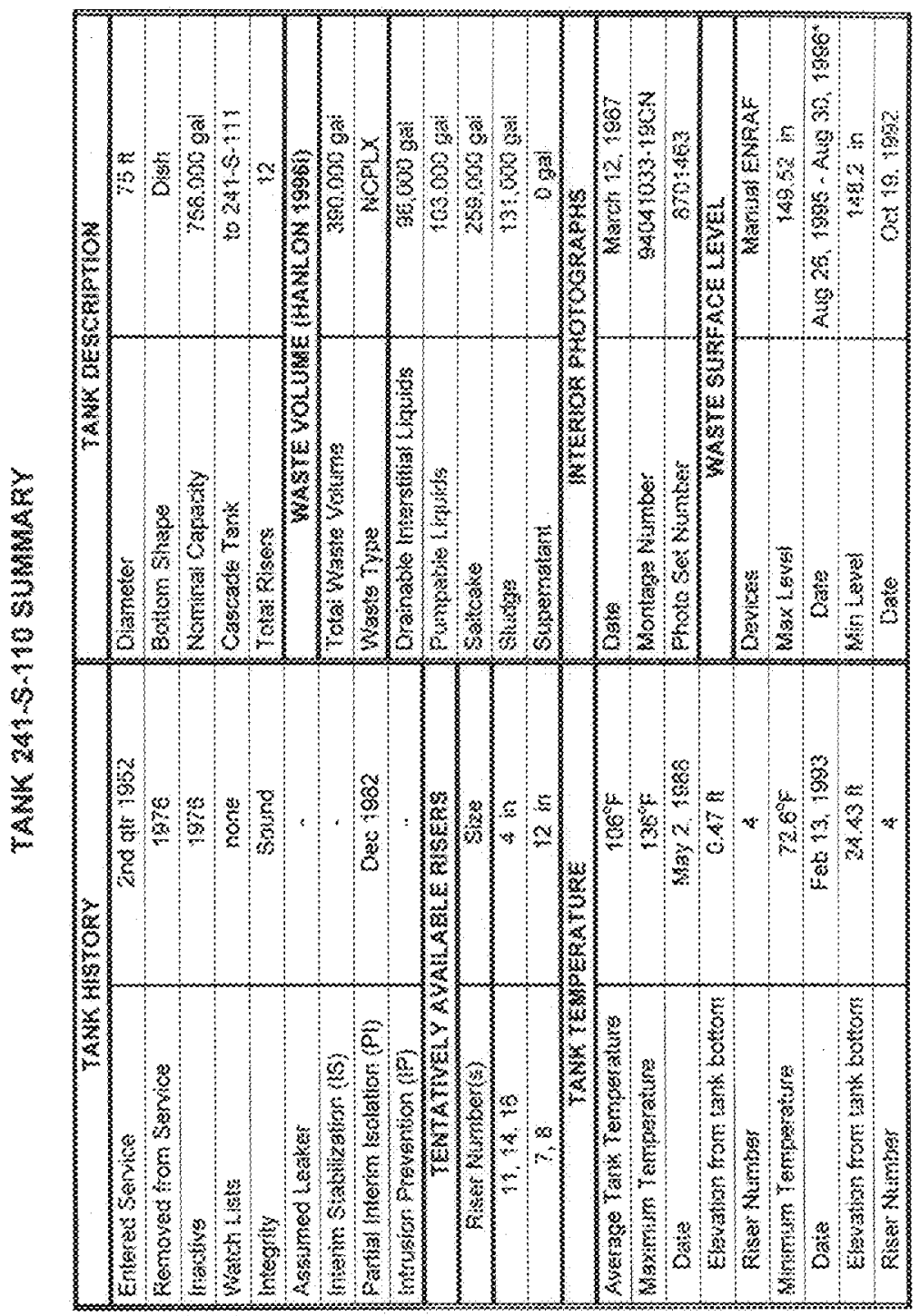

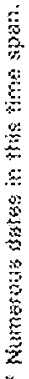




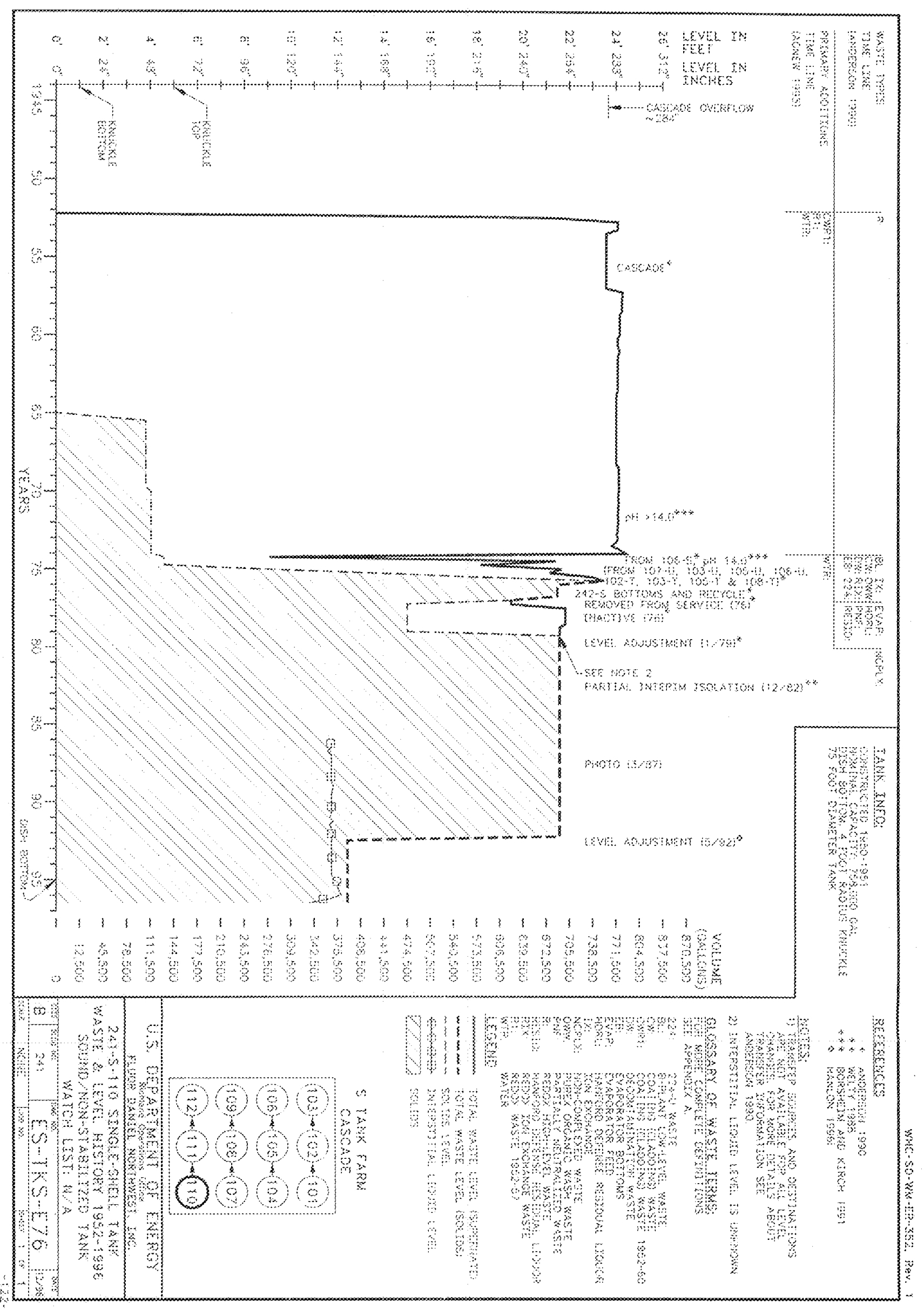




\section{$241-5-110$}

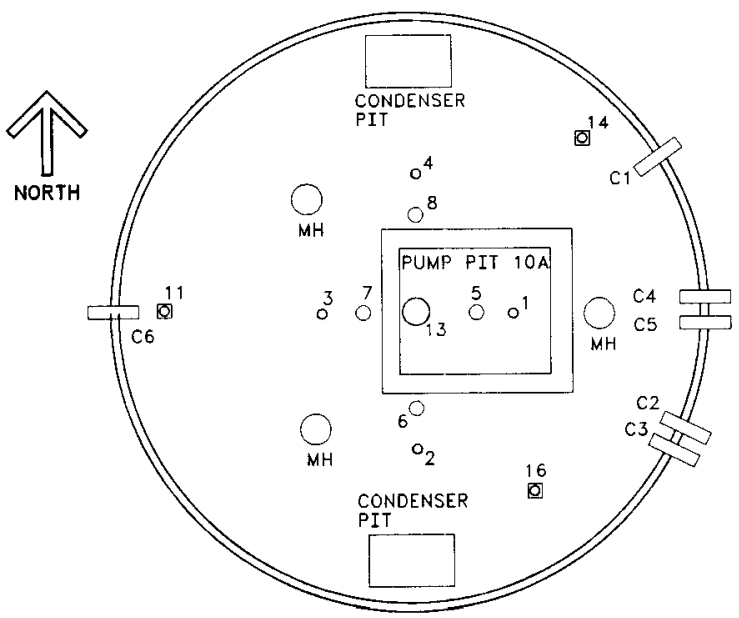

Ref: Alstad 1993

$\mathrm{H}-2-73189$, Rev. 4
$\mathrm{H}-2-37533$, Rev. 2

\section{TANK RISER LOCATION}

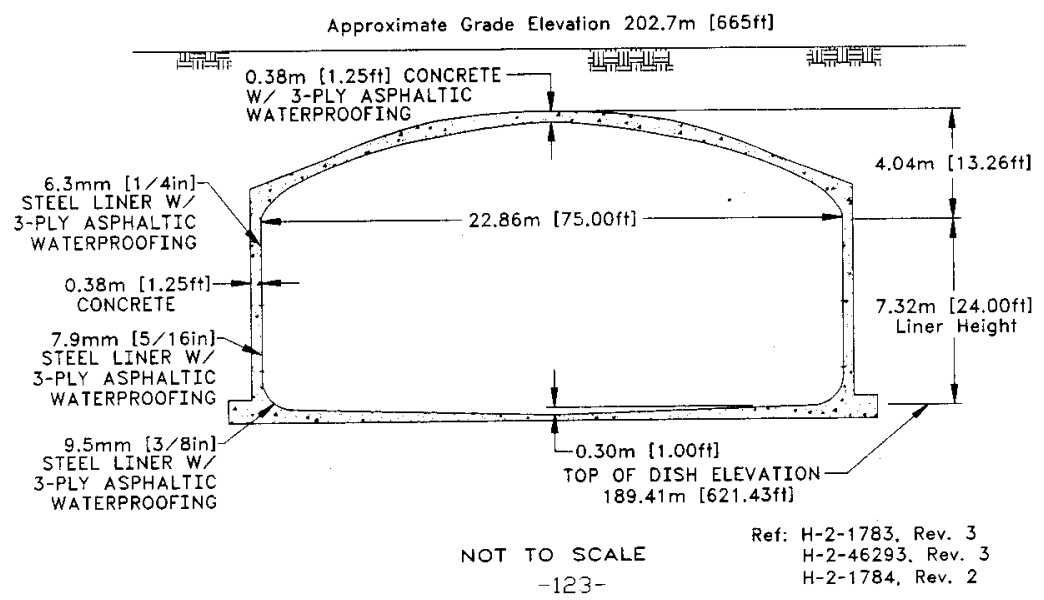


HNF-SD-WM-ER-352, Rev.

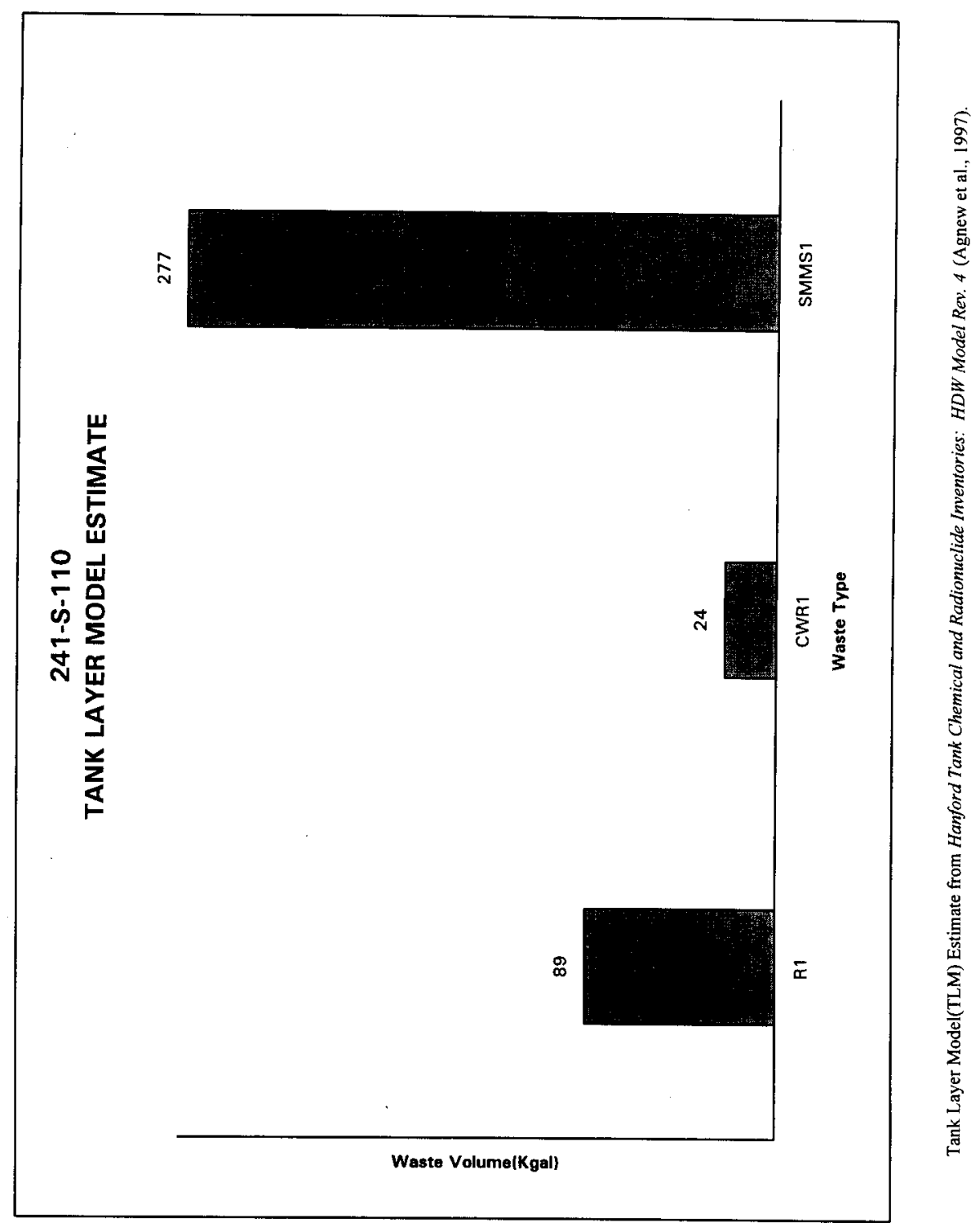

$-124-$ 


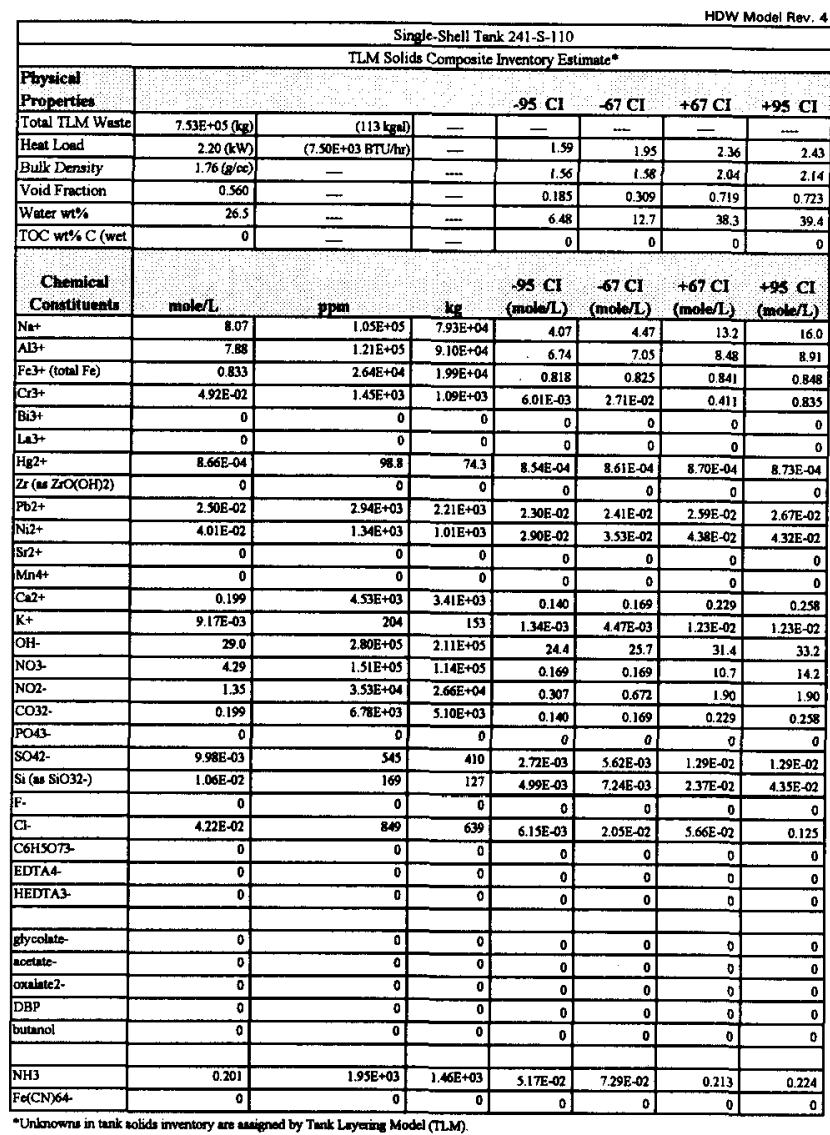

$-125$. 


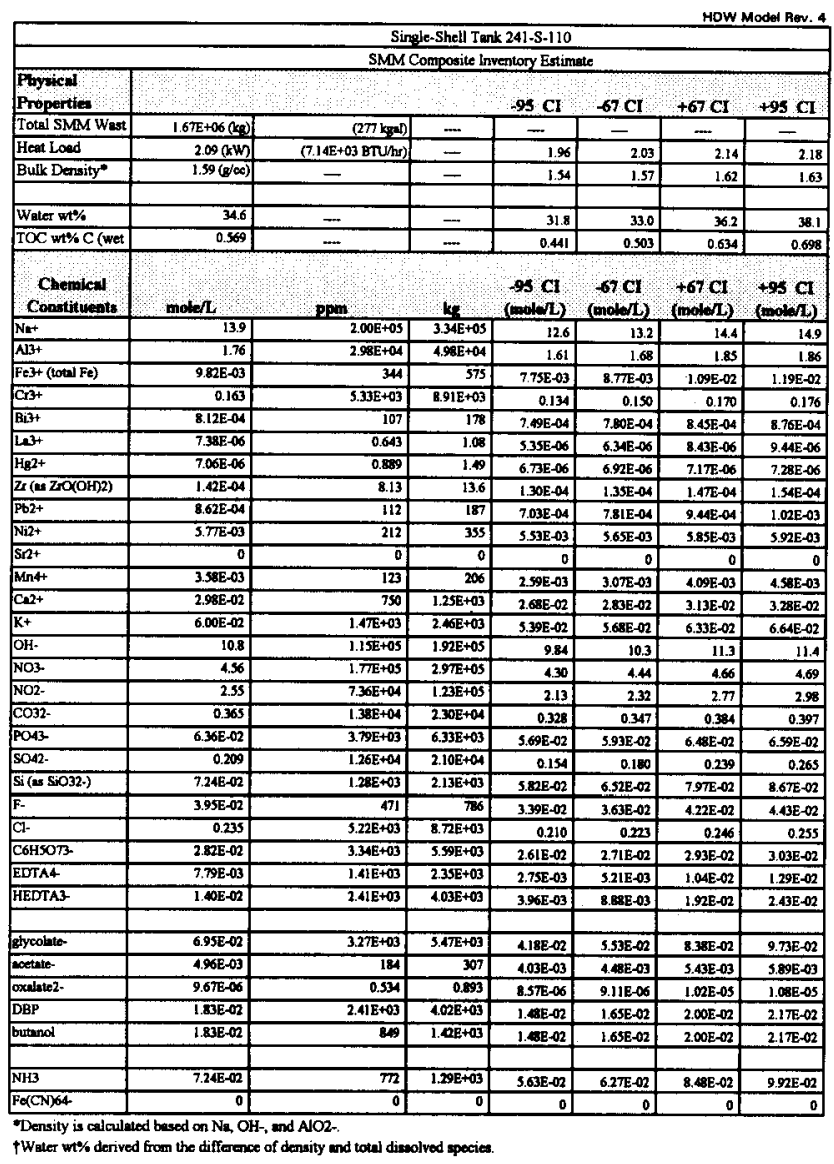




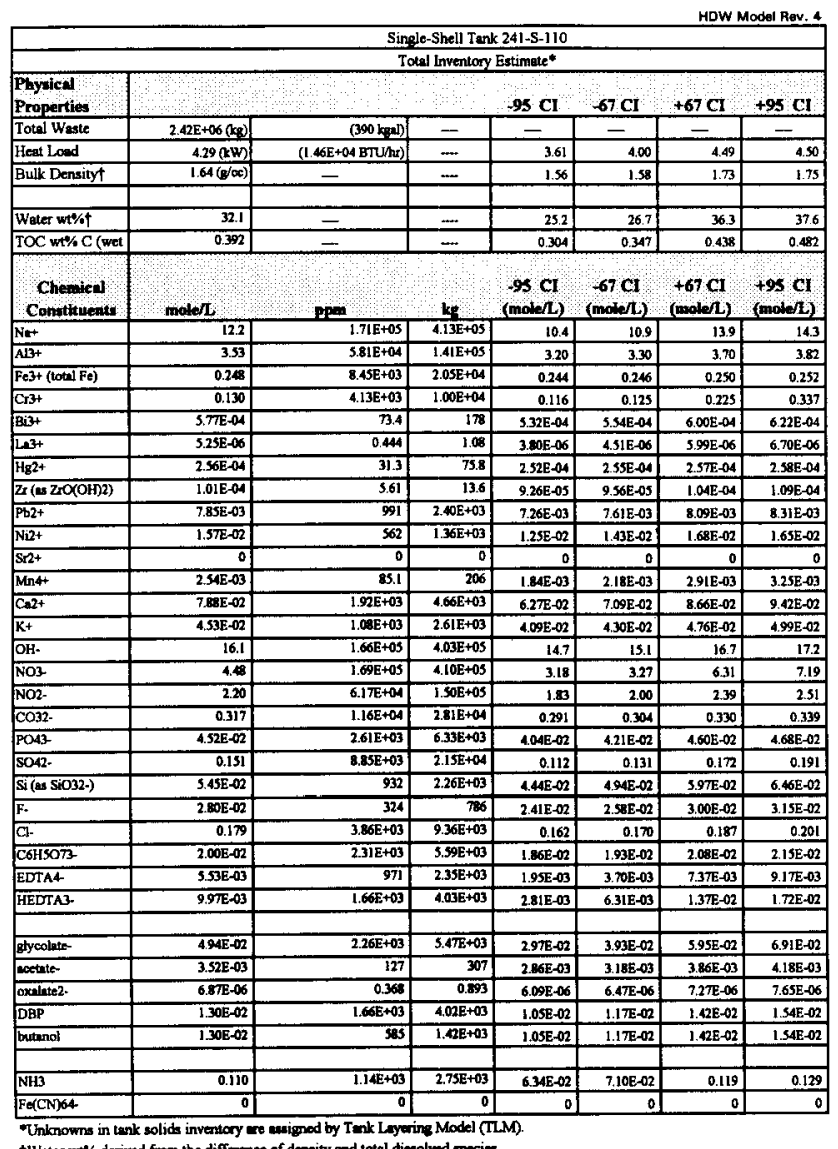

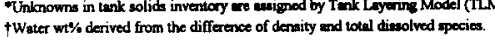




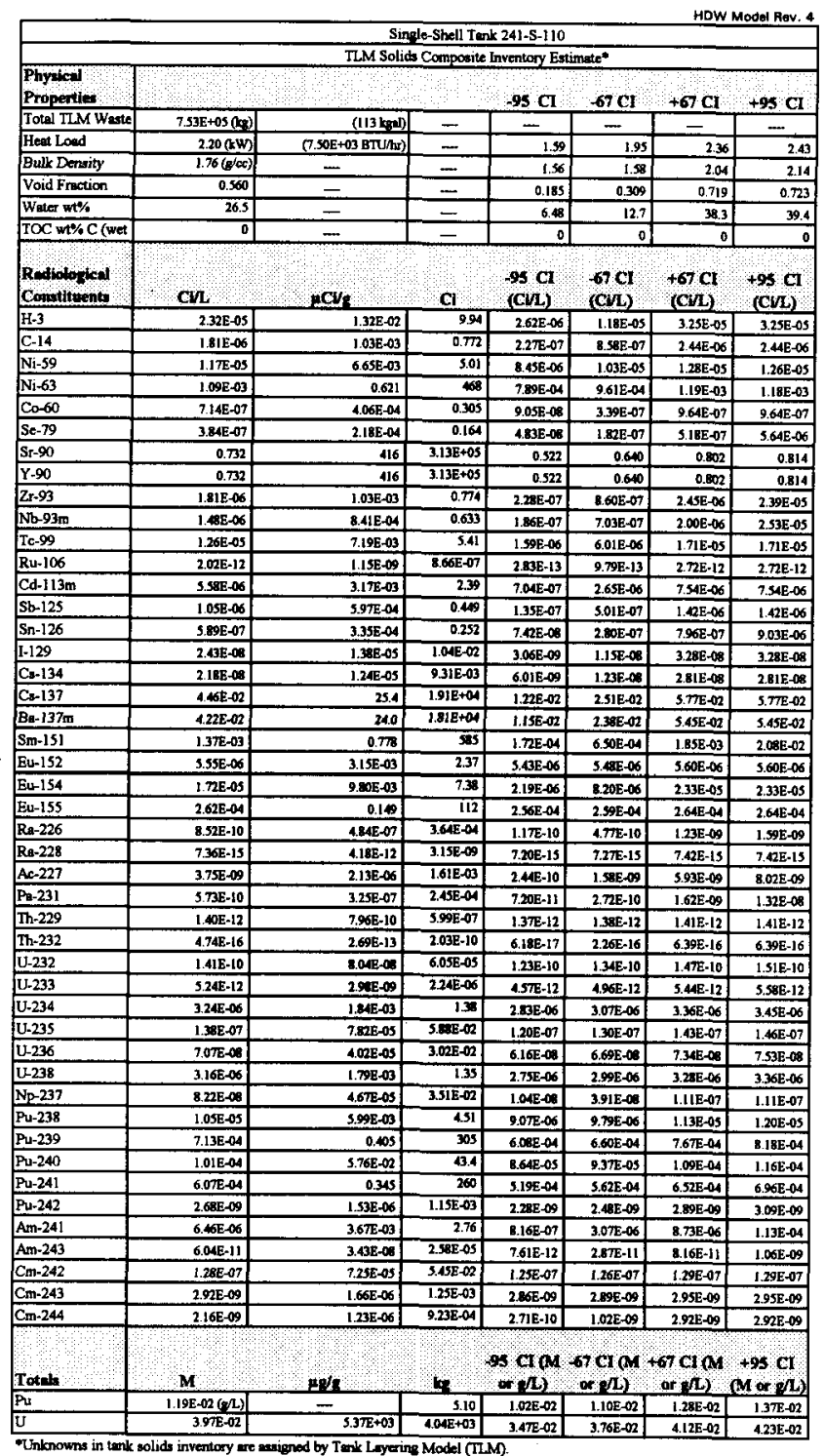




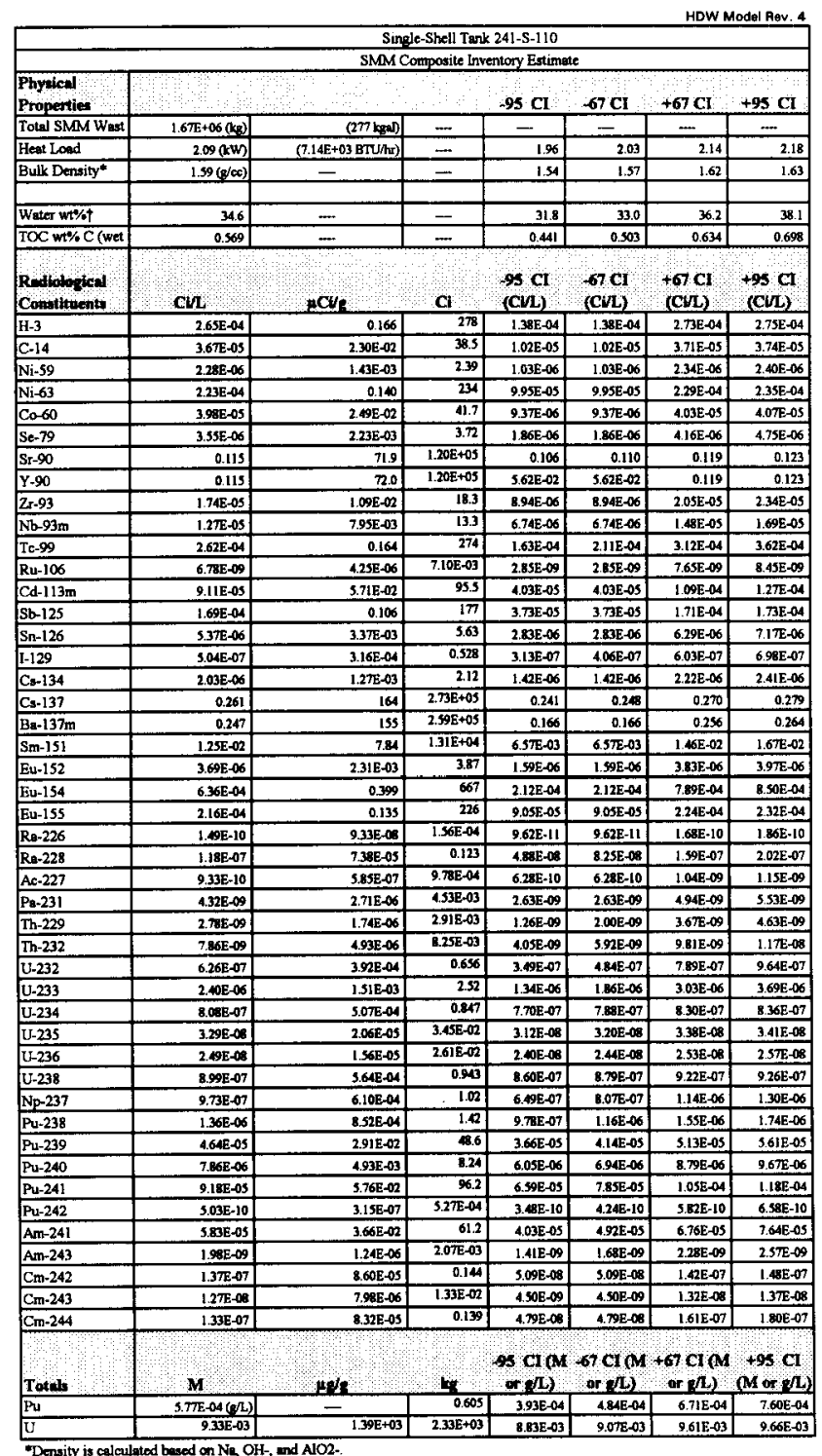

twater wto derived from the difference of denceity and total dissolved apeciea. 


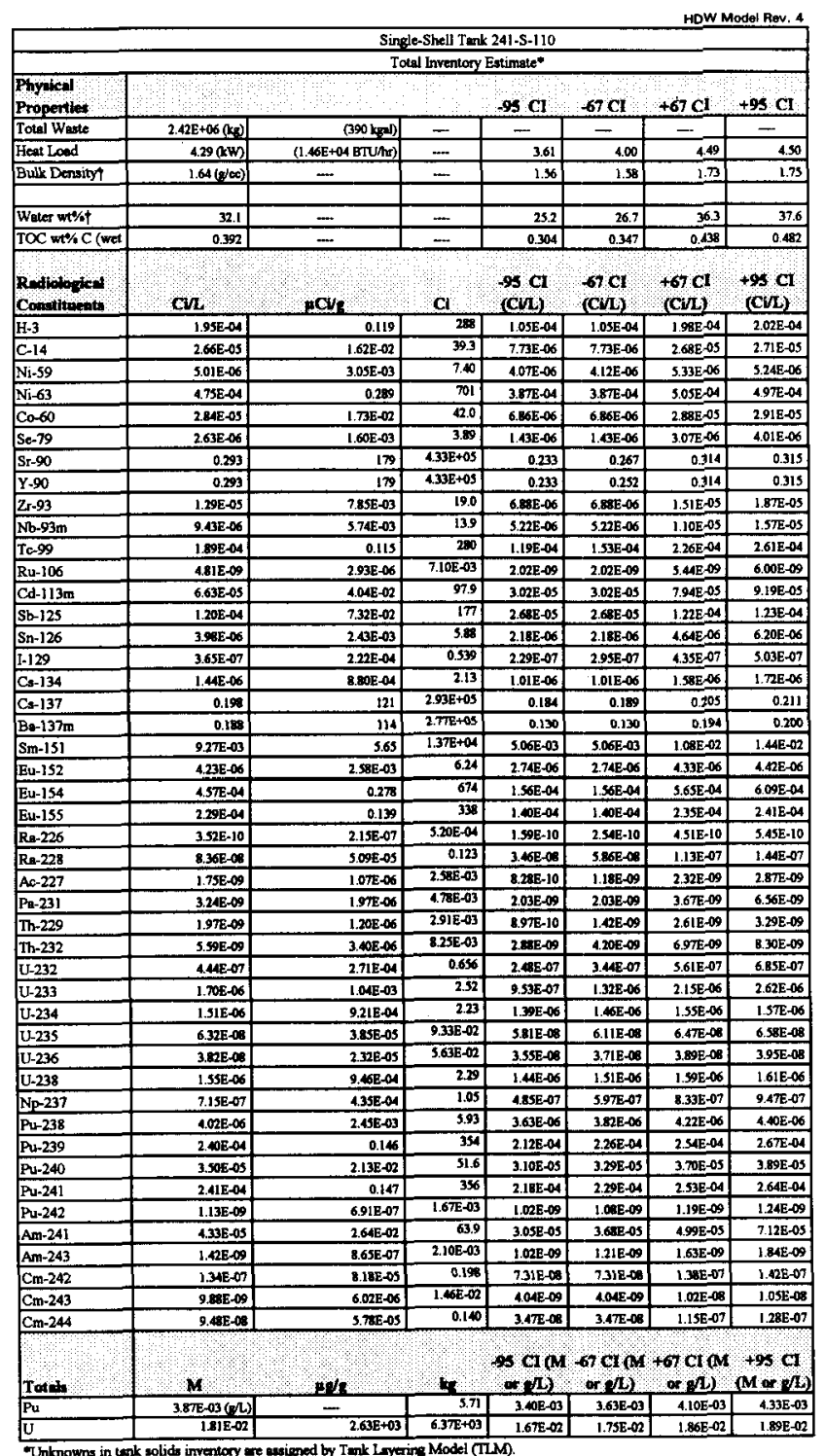

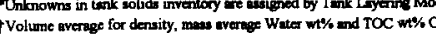




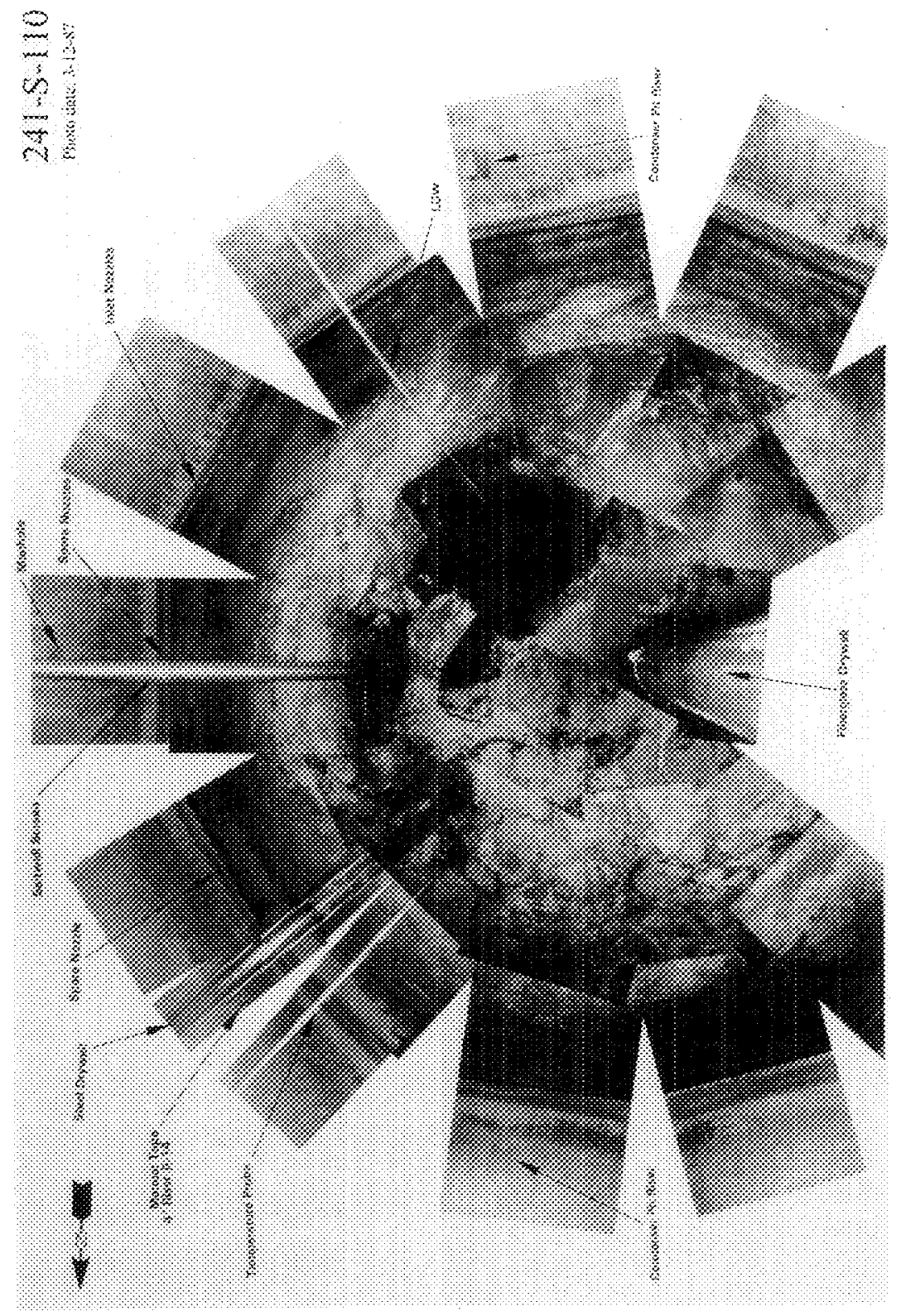

ni- 
TANK 241-S-111 SUMMARY

\begin{tabular}{|c|c|c|c|}
\hline \multicolumn{2}{|c|}{ TANK HISTORY } & \multicolumn{2}{|c|}{ TANK DESCRIPTION } \\
\hline Entered Service & 3rd qtr 1952 & Diameter & $75 \mathrm{ft}$ \\
\hline Removed from Service & 1976 & Bottom Shape & Dish \\
\hline Inactive & 1976 & Nominal Capacity & $758,000 \mathrm{gal}$ \\
\hline Watch Lists & Hydrogen \& Organics & Cascade Tank & to $241-\mathrm{S}-112$ \\
\hline Integrity & Sound & Total Risers & 12 \\
\hline Assumed Leaker & - & \multicolumn{2}{|c|}{\begin{tabular}{|l} 
WASTE VOLUME (HANLON 1996i) \\
\end{tabular}} \\
\hline Interim Stabilization (IS) & - & Total Waste Volume & $596,000 \mathrm{gal}$ \\
\hline Partial Interim Isolation (PI) & Dec 1982 & Waste Type & NCPLX \\
\hline Intrusion Prevention (IP) & - & Drainable Interstitial Liquids & $195,000 \mathrm{gal}$ \\
\hline \multicolumn{2}{|c|}{ TENTATIVELY AVAILABLE RISERS } & Pumpable Liquids & $134,000 \mathrm{gal}$ \\
\hline Riser Number(s) & Size & Saltcake & $447,000 \mathrm{gal}$ \\
\hline 11,14 & 4 in & Sludge & $139,000 \mathrm{gal}$ \\
\hline $6,7,8$ & 12 in & Supernatant & $10,000 \mathrm{gal}$ \\
\hline \multicolumn{2}{|c|}{ TANK TEMPERATURE } & \multicolumn{2}{|c|}{ INTERIOR PHOTOGRAPHS } \\
\hline Average Tank Temperature & $83^{\circ} \mathrm{F}$ & Date & Aug 10, 1989 \\
\hline Maximum Temperature & $97^{\circ} \mathrm{F}$ & Montage Number & $94041033-17 \mathrm{CN}$ \\
\hline Date & arch 9, 1991 - March 1, 1992 & Photo Set Number & 89081043 \\
\hline Elevation from tank bottom & $0.5 \mathrm{ft}, 2.5 \mathrm{ft}, 4.5 \mathrm{ft}$ & \multicolumn{2}{|c|}{\begin{tabular}{|c|} 
WASTE SURFACE LEVEL \\
\end{tabular}} \\
\hline Riser Number & 4 & Devices & Auto and Manual ENRAF \\
\hline Minimum Temperature & $65.2^{\circ} \mathrm{F}$ & Max Level & 204.255 in \\
\hline Date & April 18, 1992 & Date & Dec. 12,1995 \\
\hline Elevation from tank bottom & $30.50 \mathrm{ft}$ & Min Level & 202.5 in \\
\hline Riser Number & 4 & Date & Feb 11,1991 - March $13,1994^{*}$ \\
\hline
\end{tabular}

- Numerous dates in this time span. 


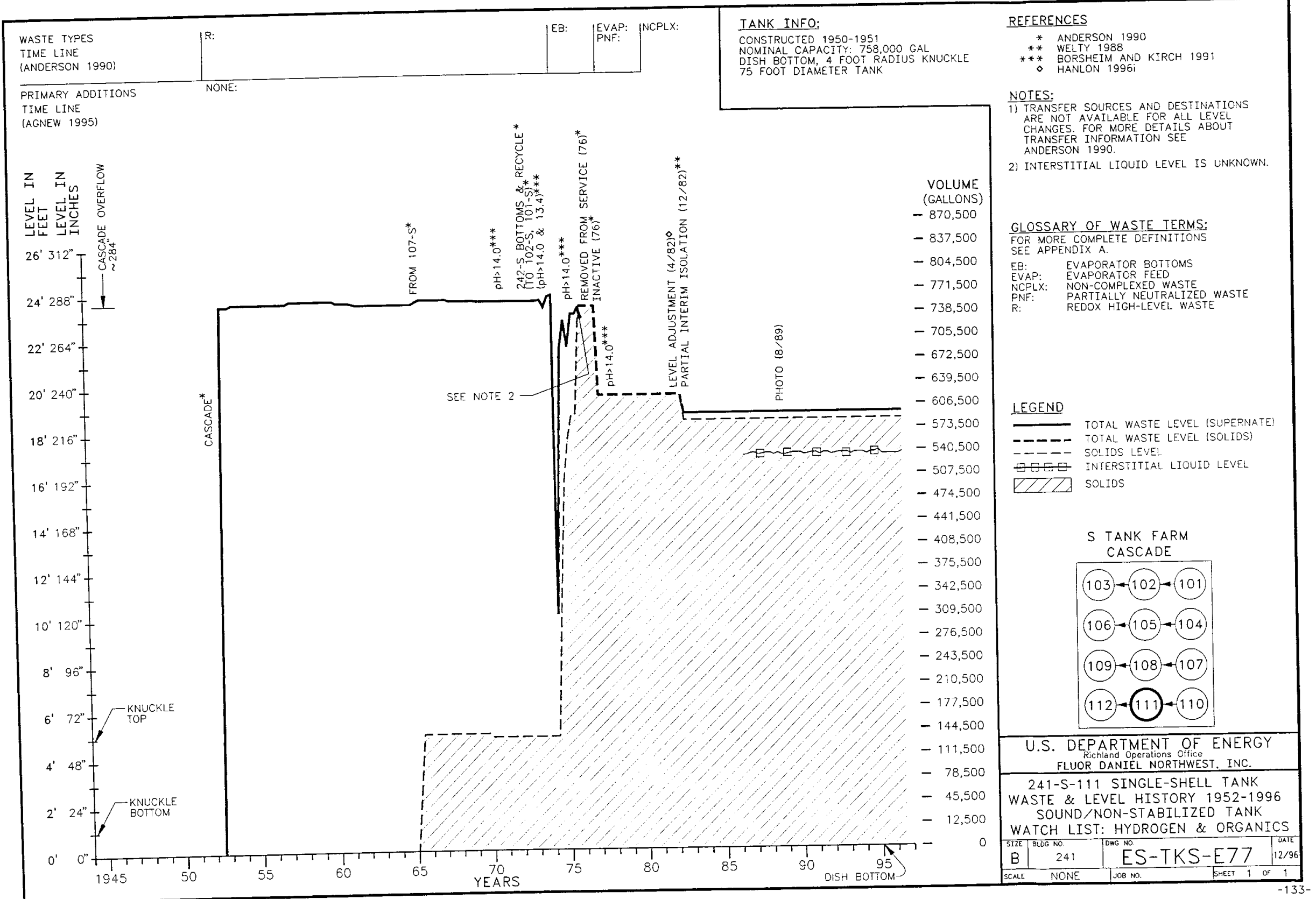




$$
241-5-111
$$

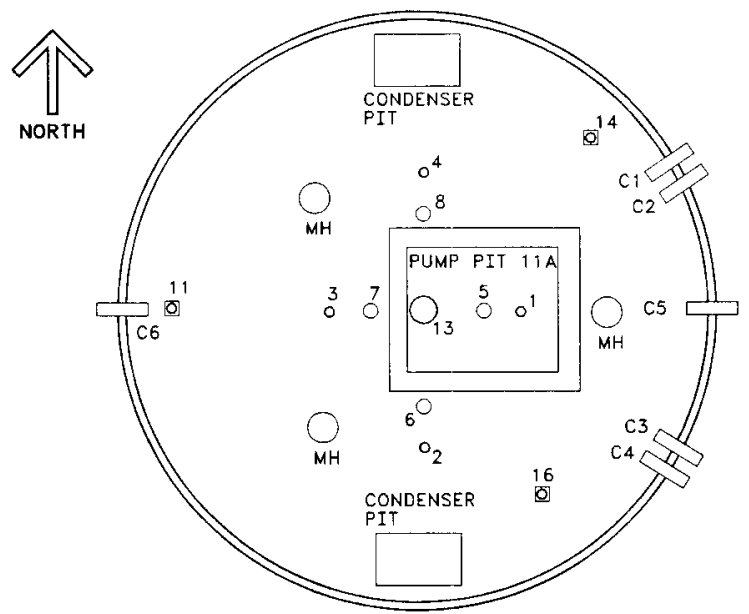

Ref: Alstad 1993

$\mathrm{H}-2-73190$, Rev. 4

$\mathrm{H}-2-37534$, Rev. 2

\section{TANK RISER LOCATION}

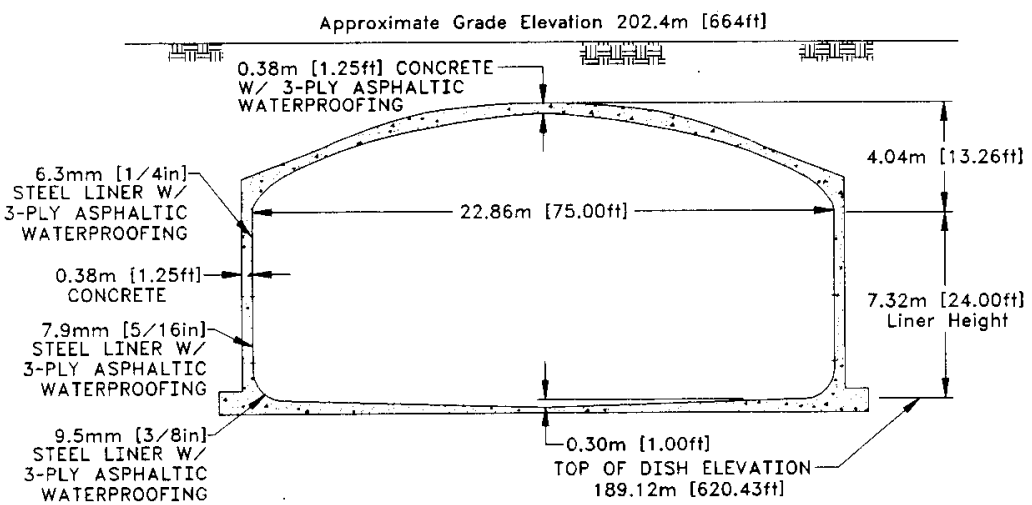

NOT TO SCALE $-134-$
Ref: $\mathrm{H}-2-1783$, Rev. 3 $\mathrm{H}-2-46293$. Rev, 3 $\mathrm{H}-2-1784$, Rev. 2 


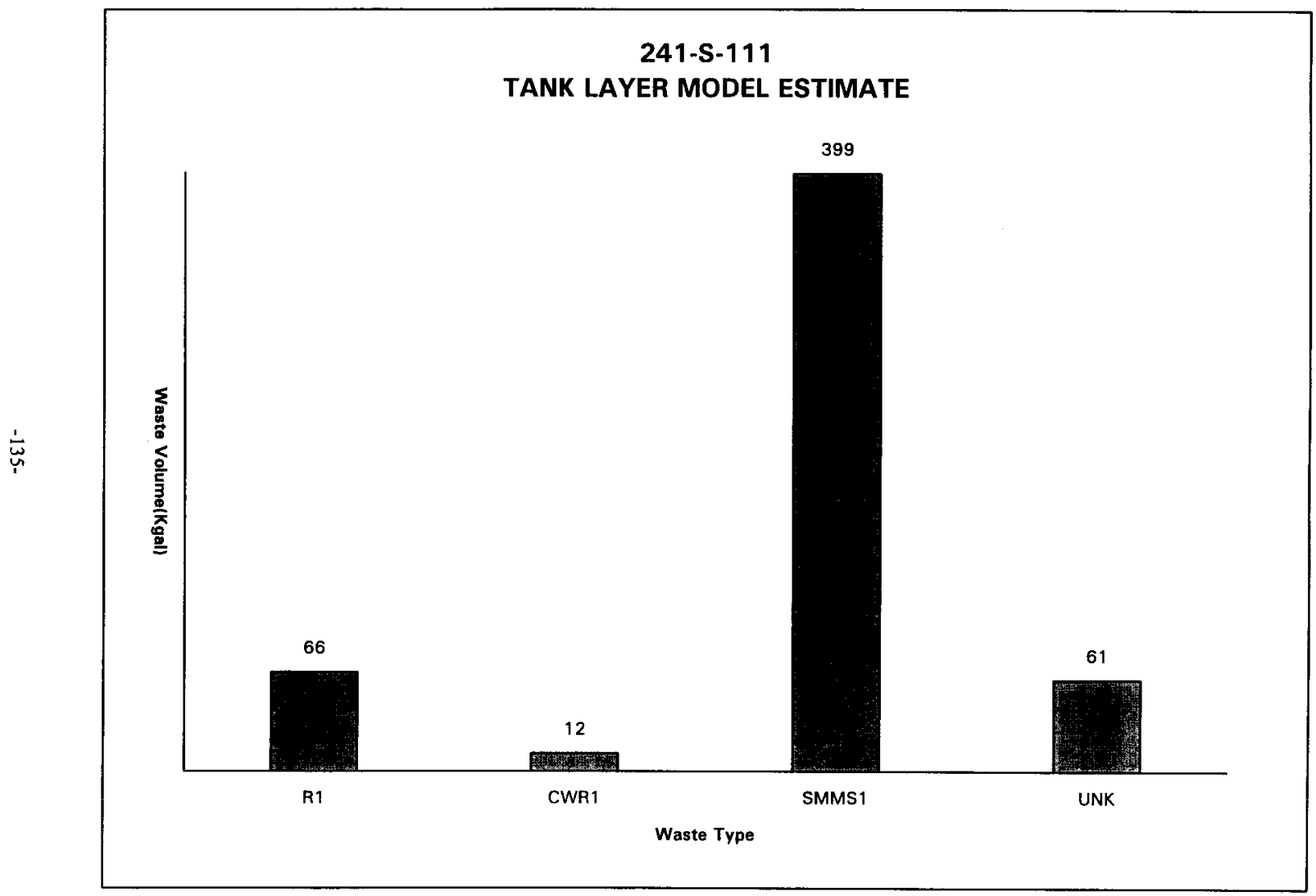


HNF-SD-WM-ER-352, Rev. 1

HOW Model Rev. 4

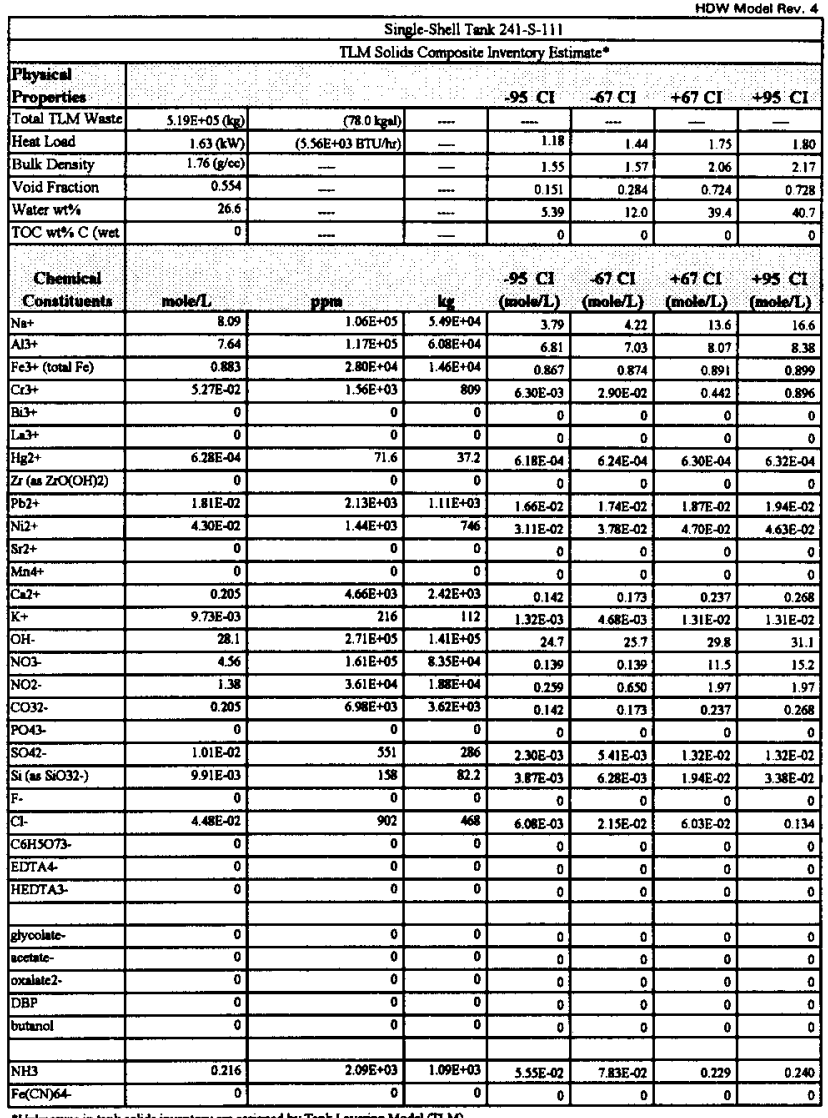

*Unirnowns in mank solids inventory we maigned by Tant Layering Model (TLM). 
HNF-SD-WM-ER-352, Rev. 1

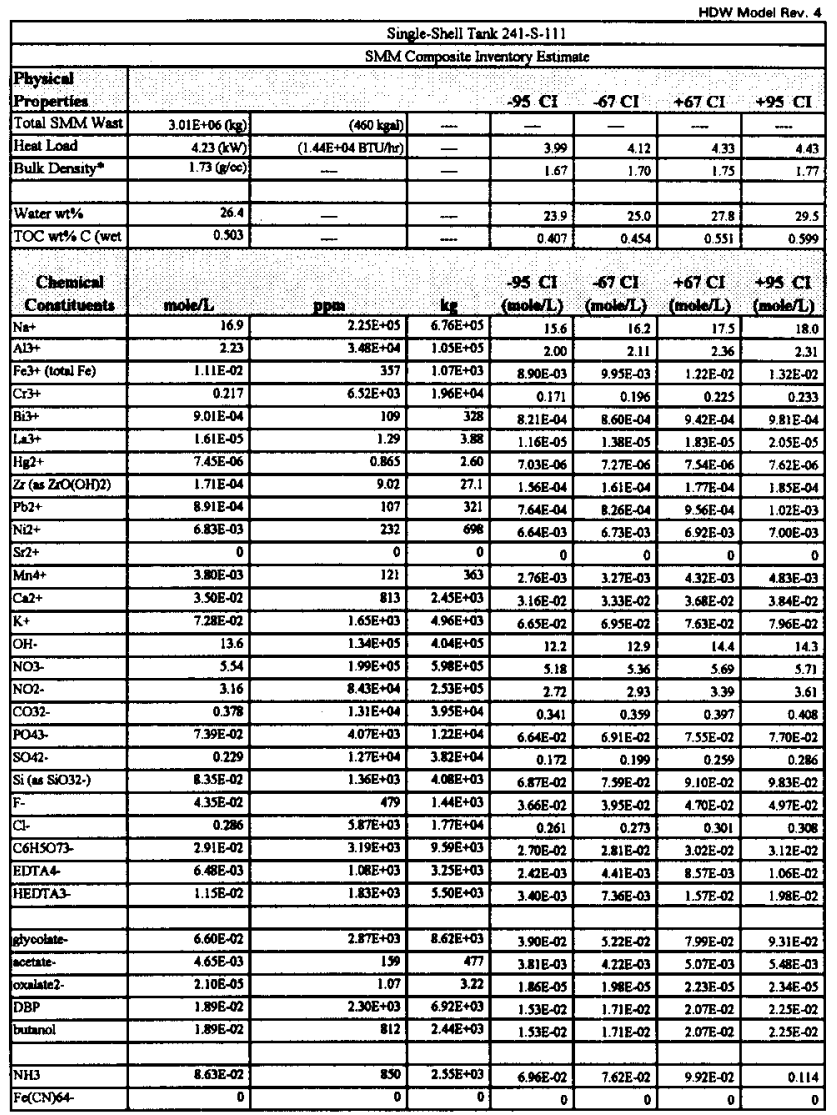

-Density is calculated baed on $\mathrm{Na}, \mathrm{OH}$, and $\mathrm{AlO} 2$.

+ Water wt\% derived from the difference of density and total diswolved apecies. 


\begin{tabular}{|c|c|c|c|c|c|c|c|}
\hline \multicolumn{8}{|c|}{ Single-Shell Tonk 241-S-111 } \\
\hline & \multicolumn{7}{|c|}{ Iotel Inventory Entimete* } \\
\hline $\begin{array}{l}\text { Phylicil } \\
\text { Properties. }\end{array}$ & & & & $95 \mathrm{CI}$ & $-67 \mathrm{CI}$ & $+67 \mathrm{CI}$ & $+95 \mathrm{CI}$ \\
\hline Total Waste & $3.53 \mathrm{E}+06(\mathrm{~kg})$ & (538 kgal $)$ & $\cdots$ & $\ldots$ & $\ldots$ & $\cdots$ & $\ldots$ \\
\hline Heat Load & $5.85(\mathrm{~kW})$ & (2.00E+04 BTU/hr) & 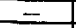 & 5.22 & 5.57 & 6.08 & 6.06 \\
\hline Bulk Dersityt & $1.73(0 / \infty)$ & $\ldots$ & - & 1.65 & 1.68 & 1.79 & 1.78 \\
\hline & & & & & & & \\
\hline Water wh\%t & 26.4 & - & - & 23.7 & 233 & 29.1 & 30.8 \\
\hline TOC wt\% C (wet & 0.429 & - & $=$ & 0.347 & 0.387 & 0470 & 0.511 \\
\hline $\begin{array}{l}\text { Chearicil } \\
\text { Constiknouts }\end{array}$ & nold $/ \mathrm{L}$ & $\mathrm{Pg}$ & tro & (os Cle $/ \mathrm{L})$ & $\begin{array}{l}67 \mathrm{Cl} \\
\text { (meled ) }\end{array}$ & $($ mol $/ 6)$ & $\begin{array}{l}+95 \mathrm{t} \\
(\mathrm{wol} / \mathrm{L})\end{array}$ \\
\hline Wat & 15.6 & $2.07 \mathrm{E}+05$ & $7.31 \mathrm{E}+0 \mathrm{05}$ & 13.9 & 14.6 & 16.8 & 16.5 \\
\hline $\mathrm{Al}^{\mathrm{B}+}$ & 3.01 & $4.09 \mathrm{E}+0.04$ & $1.65 \mathrm{E}+05$ & 2.82 & 292 & 3.09 & 3.10 \\
\hline Fe?+ (total Fe) & 0.137 & $4,43 \mathrm{E}+03$ & $1,56 \mathrm{E}+04$ & 0.135 & 0.136 & 0.138 & 0.139 \\
\hline $\mathrm{Cr} 3+$ & 0.193 & $5.79 \mathrm{E}+03$ & $204 E+04$ & 0.167 & 0.181 & 0.231 & 0.276 \\
\hline $\mathrm{Bi}+$ & $7.70 \mathrm{E}-\mathrm{04}$ & 93.0 & 328 & $7.02 \mathrm{E}-04$ & $7,36 \mathrm{E}-04$ & B.05E-04 & $8.39 \mathrm{E}-04$ \\
\hline Lest & $1.37 \mathrm{E}-05$ & 1.10 & 388 & 9.95E-06 & $1.18 \mathrm{~F}-05$ & $1.57 \mathrm{E}-0 \mathrm{~s}$ & 1.75E-05 \\
\hline $\mathrm{Hs} 2+$ & 973E-05 & 11.3 & 39.8 & $9.60 \mathrm{E}-05$ & $9.68 \mathrm{E}-05$ & 9.7TE-05 & $9.80 \mathrm{E}-05$ \\
\hline $\mathrm{Zr}(\mathrm{as} \mathrm{ZFO} \times \mathrm{OH}) 2)$ & $1.46 \mathrm{E}-04$ & 7.69 & 27.1 & $1.33 E-04$ & $1,38 E-04$ & I.SIE-04 & $1.58 \mathrm{E}-04$ \\
\hline $\mathrm{Pb} 2+$ & 3.38E-03 & 405 & $1.43 \mathrm{E}+03$ & $3.17 \mathrm{E}-03$ & $3.30 \mathrm{E}-03$ & $3.45 \mathrm{E}-03$ & 3. $49 \mathrm{E}-03$ \\
\hline $\mathrm{Ni2}+$ & $1.21 \mathrm{E}-62$ & 410 & $1.44 E+03$ & 1.03E- -02 & $1.13 \mathrm{E}-02$ & $1.27 \mathrm{TE}-02$ & $1.24 \mathrm{E}-02$ \\
\hline $\mathrm{Sr} \mathbf{2 +}$ & 0 & 0 & 0 & 0 & 0 & 0 & 0 \\
\hline Mn4t & $3.25 E-63$ & 103 & 363 & $2.36 \mathrm{E}-09$ & $2.79 \mathrm{E}-03$ & $3.70 \mathrm{E}-03$ & 4.13E-03 \\
\hline $\mathrm{Cn} 2+$ & $5.96 \mathrm{E}-02$ & $1.39 \mathrm{E}+03$ & 4. $\bar{B} 7 \mathrm{E}+03$ & $5.10 \mathrm{E}-02$ & $5.59 E-02$ & $6.28 \mathrm{E}-02$ & $6.59 \mathrm{E}-02$ \\
\hline $\mathrm{K}+$ & $6.37 \mathrm{E}-02$ & $1.44 E+03$ & $5.07 \mathrm{t}+03$ & $5.83 \mathrm{E}-02$ & $6.085-02$ & $6.66 \mathrm{E}-02$ & $6.94 E-02$ \\
\hline OH. & 15.7 & $1.54 \mathrm{E}+05$ & $3.45 \mathrm{E}+05$ & 14.5 & $\begin{array}{r}15.2 \\
\end{array}$ & 16.3 & 16.3 \\
\hline $\mathrm{NO3}$ & 5.40 & $1.93 \mathrm{E}+05$ & $6.02 \mathrm{E}+05$ & 4.54 & 4.71 & 6.34 & 6.64 \\
\hline $\mathrm{NO}$ - & 291 & $7.72 \mathrm{E}+04$ & $2.72 E+05$ & 2.53 & 2.70 & 3.10 & 3.29 \\
\hline Co32: & 0.353 & $1.22 \mathrm{E}+04$ & $4.31 E+04$ & 0.322 & 0.336 & 0.369 & 0.379 \\
\hline PO43 & $6.32 \mathrm{E}-02$ & $347 \mathrm{E}+03$ & $1.22 E+04$ & $5.68 \mathrm{E}-02$ & $5.90 \mathrm{E}-02$ & 6.45E-02 & $6.58 E-02$ \\
\hline SOA2- & 0.197 & $1.09 \mathrm{E}+04$ & $3.85 E+04$ & 0.148 & 0.171 & 0.223 & 0.246 \\
\hline $\mathrm{Si}\left(\mathrm{s} \mathrm{SiO}_{2} 2\right)$ & $7,28 \mathrm{E}-02$ & $1.18 E+03$ & 4.16E+03 & $6.02 \mathrm{E}-02$ & $6.64 \mathrm{E}-02$ & $7.93 E-02$ & 8.55E-02 \\
\hline F. & $372-02$ & $\$ 09$ & $1,4 E+03$ & 3.1]E-02 & $3,35 E-02$ & 4.02E-02 & 4.25E-02 \\
\hline $\mathrm{Cl}-$ & 0.251 & $5.14 E+03$ & $1,81 \mathrm{E}+0 \mathrm{04}$ & 0.230 & 0.240 & 0.261 & 0.270 \\
\hline C6HSOT3 & $2.482-02$ & $2.72 \mathrm{E}+03$ & $9.59 \mathrm{E}+03$ & $2,31 E-02$ & 2. $10 E-02$ & $2.58 \mathrm{E}-02$ & $2.67 \mathrm{E}-02$ \\
\hline EDTA4 & $5.54 E-03$ & 92 & $3.75 \mathrm{E}+03$ & 2.07E-03 & 3.77E-03 & $7.33 E-03$ & 9.07E-03 \\
\hline HEDTA3 & 9.85E-03 & $1.56 \mathrm{E}+03$ & $5.50 \mathrm{E}+03$ & $2.90 \mathrm{E}-03$ & $6.30 \mathrm{E}-03$ & $1.34 \mathrm{E}-02$ & 1.69E-02 \\
\hline gitycolate- & 5.64E-02 & $2.45 E+03$ & $8.62 \mathrm{E}+03$ & $3.33 \mathrm{E}-02$ & 4.46E-02 & $6.83 \mathrm{E}-02$ & $7.96 \mathrm{E}=02$ \\
\hline cotrite- & 3.97E-03 & 135 & 47 & $3.26 \mathrm{E}-03$ & $3.61 \mathrm{E}-0 \mathrm{~s}$ & 1.34E-03 & $4.69 \mathrm{E}-03$ \\
\hline oralnte?. & 1.0E-05 & 0.914 & 3.22 & $1.59 E-O S$ & $1.69 \mathrm{E}-0 \mathrm{~s}$ & $1.90 \mathrm{E}-05$ & $200 E-05$ \\
\hline DBP & $1.62 \mathrm{E}-02$ & $1.96 \mathrm{E}+03$ & $6.92 \mathrm{E}+03$ & 1.31E-0? & 1. $46 \mathrm{E}-02$ & $1.77 \mathrm{E}-02$ & $1.92 \mathrm{E}-02$ \\
\hline butanol & $1.62 E-02$ & 692 & $2.4 E+B 3$ & $1.31 E-02$ & I.46E-02 & $1.7 \mathrm{EE}-02$ & $1.92 \mathrm{E}-02$ \\
\hline $\mathrm{NH3}$ & 0.105 & $1.03 \mathrm{E}+03$ & $3.64 E+03$ & $7.64 E-02$ & & & \\
\hline Fo(CN)64 & 0 & 0 & 0 & $\frac{1.040-02}{0}$ & $\frac{8.24 E-02}{0}$ & $\frac{0.116}{0}$ & $\begin{array}{r}0.129 \\
0\end{array}$ \\
\hline
\end{tabular}

+ Water $w \%$ derived from the difference of dentity and total diesolved species. 
HNF-SD-WM-ER-352, Rev. 1

HDW Modal Rov. 4

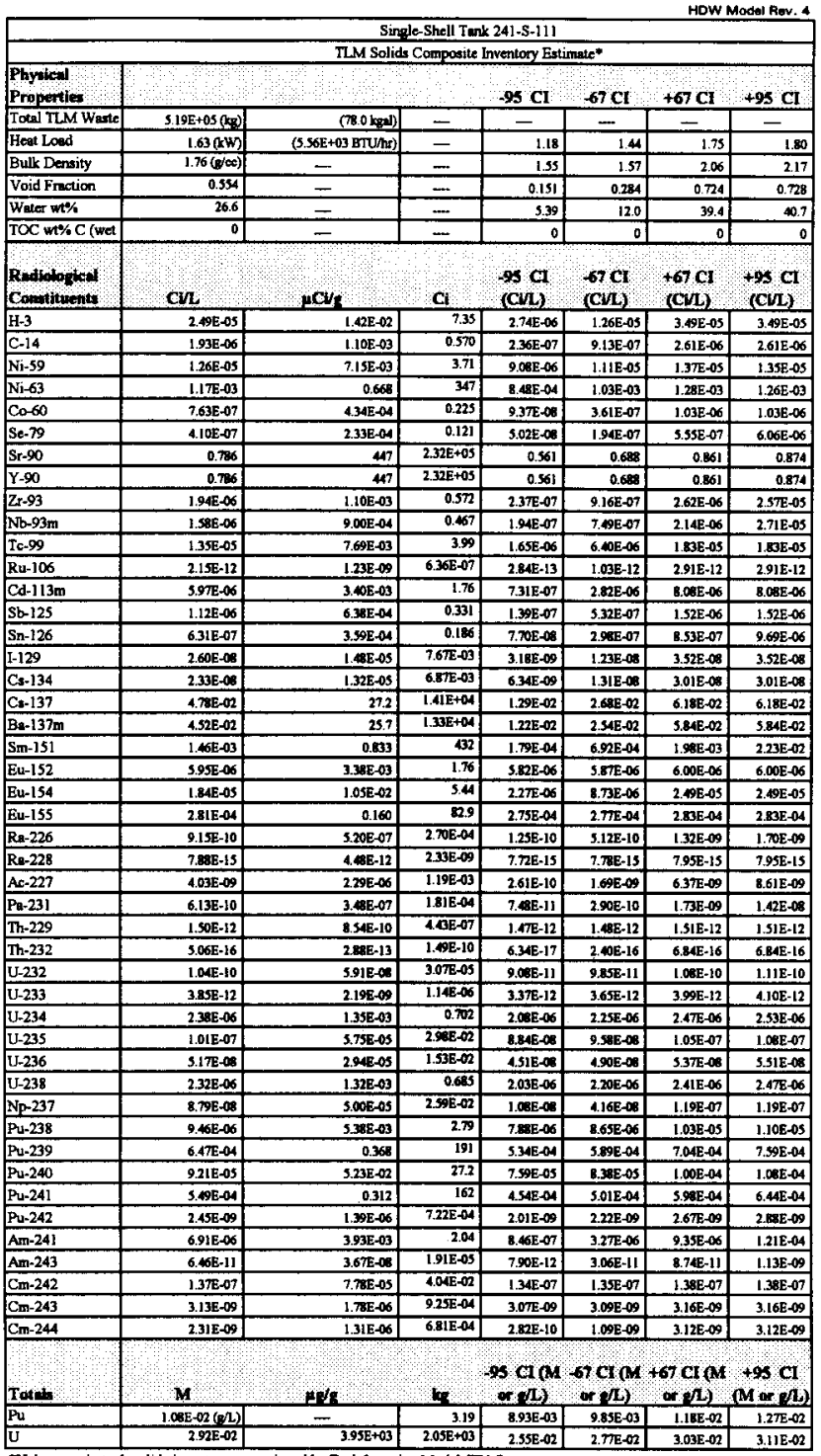

"Unknowns in terlk solids inventory are ensignod by Tank Layering Model (TLM) 
HDW Model Rav. 4

\begin{tabular}{|c|c|c|c|c|c|c|c|}
\hline \multirow{4}{*}{$\begin{array}{l}\text { Phyplical } \\
\text { Propertics }\end{array}$} & \multicolumn{7}{|c|}{ Single-Shell Tank 241-S-111 } \\
\hline & \multicolumn{7}{|c|}{ SMM Composite Inventory Estimate } \\
\hline & & & & & & & \\
\hline & & & & $-95 \mathrm{Cl}$ & $-67 \mathrm{CI}$ & $+67 \mathrm{CI}$ & $+9 \mathrm{SCl}$ \\
\hline Total SMM Wast & $3.01 E+06(\mathrm{~kg})$ & (460 kgal) & - & 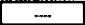 & - & - & - \\
\hline Heat Loed & $4.23(\mathrm{kw})$ & $(1.44 \mathrm{E}+04 \mathrm{BTU} / \mathrm{hr})$ & - & 3.99 & 4.12 & 4.33 & 4.43 \\
\hline Bulk Density* & $1,73(1 / \infty)$ & - & 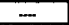 & 1.67 & 1.70 & 175 & 1.77 \\
\hline Water wt\% $\varphi^{+}$ & 26.4 & - & - & 239 & 250 & 278 & 29.5 \\
\hline TOC wt\% C (wet & 0.503 & - & - & 0.407 & 0.454 & 0.551 & 0.599 \\
\hline Condiolintestes & $\mathbf{C H}$ & $\mathrm{poy}_{\mathrm{s}}$ & $\mathbf{C l}$ & $\begin{array}{l}-9 \mathrm{C} \\
\text { (Qvi) }\end{array}$ & $\begin{array}{l}67 \mathrm{CI} \\
(\mathrm{CiL})\end{array}$ & $\begin{array}{c}+67 \mathrm{CI} \\
\text { (CVL) }\end{array}$ & $\begin{array}{r}+95 \mathrm{CI} \\
(\mathrm{Ci} / \mathrm{L})\end{array}$ \\
\hline $\mathrm{H}-3$ & $3.15 E-04$ & 0.182 & 548 & 1.83E-04 & $1.838-04$ & $3.28 \mathrm{E}-04$ & $3.25 \mathrm{E}-04$ \\
\hline C.14 & 4.0TE-05 & $2.36 \mathrm{E}-02$ & 70.9 & 1.31E-05 & 1.3IE-05 & 4.14E-05 & $415 E-05$ \\
\hline$\sqrt{1}-59$ & $2.62 \mathrm{E}-06$ & I.5IE-03 & 4.55 & $132 \mathrm{E}-06$ & $1.32 E .06$ & $2.68 \mathrm{E}-06$ & $2.74 E-06$ \\
\hline $\mathrm{Ni}-63$ & 2.55E-04 & 0.148 & 44 & $127 \mathrm{E}-04$ & $1.27 \mathrm{E}-04$ & 2.62E-04 & $2.68 \mathrm{E}-04$ \\
\hline $\mathrm{Co}-60$ & $4.29 \mathrm{E}-05$ & $2,48 \mathrm{E}-02$ & 74.7 & $1.13 \mathrm{E}-0 \mathrm{~S}$ & J.13E-05 & 4,34E-05 & $4.39 \mathrm{E}-05$ \\
\hline Sc-79 & 4.12E-06 & $2.39 \mathrm{E}-03$ & 7.17 & $2.35 \mathrm{E}-\mathrm{D}$ & $2.35 \mathrm{E}-06$ & $4.75 \mathrm{E}-06$ & $5.37 E-06$ \\
\hline Sr-90 & 0.131 & 76.0 & $2.28 \mathrm{E}+05$ & 0.122 & 0.127 & 0.136 & 0.140 \\
\hline$Y-90$ & 0.131 & 76.0 & $2.26 E+05$ & $7.03 E_{-02}$ & 2.03E-02 & 0.136 & 0.140 \\
\hline $2 \pi-93$ & $2.02 \mathrm{E}-05$ & $1.17 \mathrm{E}-02$ & 35.1 & 1.14E-05 & 1.14E-05 & $2.34 \mathrm{E}-05$ & $2.64 E-05$ \\
\hline Nb-93m & 1.4BE-05: & 8.55E-03 & 25.7 & B. $60 \mathrm{E}-06$ & 8.60E-06 & $1.70 \mathrm{E}-05$ & 1.91E-05 \\
\hline Te-99 & 2.91E-04 & 0.169 & 507 & 1.80E-04 & $2.395-04$ & 3.44E-04 & $3.95 E-04$ \\
\hline Ru- 106 & $7.19 \mathrm{E}-\infty$ & 4.17E- 06 & $1.25 \mathrm{E}-02$ & 3.11E-09 & $3.11 \mathrm{E}-09$ & B.11E.09 & 8.94E-09 \\
\hline $\mathrm{Cd}-113 \mathrm{~m}$ & $1.03 \mathrm{E}-04$ & SSAE-O2 & 180 & 5.04E-05 & $5.04 \mathrm{E}-05$ & $1.22 E-04$ & 1.41E-04 \\
\hline $\mathrm{Sb}-125$ & 1.79E-04 & 0.104 & 312 & $4.25 \mathrm{E}-05$ & 4.25E-05 & $1.82 \mathrm{E}-04$ & $184 E-04$ \\
\hline $5 n-126$ & $6.23 E-06$ & $3.61 E-03$ & 10.8 & 3.59E-06 & $3.59 \mathrm{E}-06$ & 7.19E- 06 & $8.10 \mathrm{E}-05$ \\
\hline $1-129$ & 5.61E-07 & 3.25E-04 & 0.976 & $3.62 \mathrm{E}-107$ & 4.95E-07 & $6.63 \mathrm{E}-07$ & $7.62 \mathrm{E}-07$ \\
\hline Cs-134 & $2.24 \mathrm{E}-06$ & $1.30 \mathrm{E}-03$ & $3 \mathbf{5 0}$ & $1.43 \mathrm{E}-06$ & $1.43 \mathrm{E}-06$ & $2.40 \mathrm{E}-06$ & 2.56E-06 \\
\hline Cs-137 & 0.330 & 191 & $5.74 E+05$ & 0.306 & 0.316 & 0.343 & 0.354 \\
\hline $\mathrm{Ba}-137 \mathrm{~m}$ & 0.312 & 181 & $5.43 E+05$ & 0.204 & 0.204 & 0.324 & 0.330 \\
\hline $\mathrm{Sm} \cdot 151$ & 1.45E-02 & 8.40 & $2.53 \mathrm{E}+04$ & 8.34E-03 & 8.34E-03 & $1.67 \mathrm{E}-02$ & 1.89E-02 \\
\hline Eu-152 & $3.906-06$ & $2,29 \mathrm{E}-03$ & 6.89 & $1.77 E-06$ & $1.77 \mathrm{E}-0.6$ & $407 \mathrm{E}-\infty$ & 4.18E-106 \\
\hline Eu-154 & 6.96E-04 & 0.403 & $1.21 E+03$ & $2.55 \mathrm{E}-04$ & $2.55 \mathrm{E}-04$ & B 55E-04 & $9.19 \mathrm{E}-0.4$ \\
\hline Eu-155 & $2.29 \mathrm{E}-04$ & 0.133 & 399 & 9.91E-05 & 9.91E-05 & 2.36E-04 & $2.43 \mathrm{E}-04$ \\
\hline Ra-226 & 1.78E-10 & $1.03 E-07$ & $3.10 \mathrm{E}-04$ & $1.23 \mathrm{E}-10$ & 1.23E-10 & $2.00 \mathrm{E}-10$ & $2.21 \mathrm{E}-10$ \\
\hline Ra-228 & B.85E- 0 : & $5.12 \mathrm{E}-05$ & 0.154 & 3.78E-08 & $6.26 \mathrm{E}-08$ & 1.19E-07 & 1.51E-07 \\
\hline Ac-227 & $1.09 \mathrm{E}-09$ & $6.30 \mathrm{E}-07$ & 1.6E- 03 & $7.71 \mathrm{E}-10$ & $7.71 \mathrm{E}-10$ & $1.21 \mathrm{E}-09$ & $1.31 \mathrm{E}-09$ \\
\hline Pe-231 & 4.95E-09 & $2.87 \mathrm{E}-06$ & 8.62E- 03 & $3.19 \mathrm{E}-09$ & $3.19 \mathrm{E}-09$ & 5.59E-09 & $6.20 \mathrm{E}-09$ \\
\hline Th-229 & 2.10E-09 & $1.22 \mathrm{E}-06$ & $3.66 \mathrm{E}-03$ & 9.20 - 10 & $1.53 E-09$ & $2.76 E-09$ & 3.46E-09 \\
\hline Th-232 & 6.18E-09 & $3.58 E-06$ & $1.00 E-42$ & $3.37 \mathrm{E}-09$ & $4.75 \mathrm{E}-09$ & 7.61E-09 & $8.99 \mathrm{E}-09$ \\
\hline $\mathrm{U}-232$ & 4.99E-07 & $2.89 \mathrm{E}-04$ & 0.09 & 2.96E-07 & $3.95 \mathrm{E}-07$ & 6.19E-07 & $7,48 \mathrm{E}-07$ \\
\hline U-233 & 1.91E-06 & 1.11E-03 & 3.33 & $1.14 E-\infty 6$ & $1.52 \mathrm{E}-06$ & 2.38E-06 & 2.87E-06 \\
\hline U-234 & 9.3]E-07 & $5395-04$ & 1.62 & B.7BE-07 & $9.00 \mathrm{E}-07$ & $9.65 \mathrm{E}-07$ & 9.61E-07 \\
\hline U.235 & 3.82E-08 & $2.21 E-05$ & 6.65E-02 & 35TE- & $3.69 \mathrm{E}-0 \mathrm{~B}$ & 3.97E-08 & 394E-08 \\
\hline $\mathrm{U}-236$ & $2.80 \mathrm{E}-08$ & $1.62 \mathrm{E}-05$ & $4.87 \mathrm{E}-02$ & $2.71 E-08$ & $2.75 E-08$ & 2.2SE-08 & $2.890-08$ \\
\hline L-238 & 9.94E-07 & 5.76E-AM & 1.73 & 9.35E-07 & 9.63E-07 & $1.03 \mathrm{E}-06$ & 1.02E-06 \\
\hline $\mathrm{Np}-237$ & $1.11 \mathrm{E}-06$ & $6.45 \pm-04$ & 194 & $7.77 \mathrm{E}-07$ & $9.41 E-07$ & 1.20E-06 & $1.45 \mathrm{E}-06$ \\
\hline Pu-238 & $1.46 \mathrm{E}-06$ & $8.46 E-64$ & 2.54 & 1.07E-06 & $1.26 \mathrm{E}-06$ & $1.66 \mathrm{E}-06$ & 1.B6E-06 \\
\hline Pu-239 & 5.15E-0S & $2.99 E-02$ & 89.7 & 4.14E-05 & $4.64 E-05$ & $5.66 \mathrm{E}-05$ & $6.16 \mathrm{E}-05$ \\
\hline Pu-240 & B. $62 \mathrm{E}-06$ & 499E-03 & 150 & 6.74E-06 & $7.66 \mathrm{E}-06$ & 9.58E- -06 & 1.05E-05 \\
\hline $\mathrm{Pu}-241$ & $9.76 \mathrm{E}-0 \mathrm{~S}$ & $5.65 \mathrm{E}-02$ & 17 & 7.0\%-05 & 895-05 & 1.11E-04 & $1.25 \mathrm{E}-04$ \\
\hline Pu-242 & $5.34 \mathrm{E}-10$ & 3.09E-07 & 9.20E-04 & 373E-10 & $4.52 \mathrm{E}=10$ & $6.16 \mathrm{E}-10$ & $695 \mathrm{E}-10$ \\
\hline Am-24l & $6.73 \mathrm{E}-0 \mathrm{~S}$ & 3.DVE-M & 117 & 485-05 & $5.7 \pi \mathrm{E}-0 \mathrm{~s}$ & 7.64E-05 & a.60E-05 \\
\hline Ann-243 & 2.14E-09 & 1.24E-A6 & 3.7BE-03 & 1.55E-0s & $1.83 E-09$ & 2.45E-09 & $275 \mathrm{E}-09$ \\
\hline $\mathrm{Cm}-242$ & $1,42 E-07$ & $8.205-05$ & 0.247 & $5.20 \mathrm{E}-0 \mathrm{~B}$ & $5.20 \mathrm{E}-08$ & 1.46E-07 & 1.S0E-07 \\
\hline $\mathrm{Cm}-243$ & $1.29 \mathrm{E}-08$ & $7.49 \mathrm{E}-06$ & $2.25 \mathrm{E}-02$ & $439 \mathrm{E}-09$ & 439E-09 & $1.33 \mathrm{E}-108$ & 1.37E-08 \\
\hline $\mathrm{Cm}-244$ & 1,39E-07 & 2.05E-05 & 0.242 & 5.08E-08 & $5.005-08$ & $1.69 \mathrm{E}-07$ & $1.88 \mathrm{E}-07$ \\
\hline Total & $\mathbf{M}$ & $t / \%$ & 40 & -9s $1 \mathrm{~L}$ & $\begin{array}{l}67 \mathrm{CH}(\mathrm{M} \\
\mathrm{or} / \mathrm{L})\end{array}$ & $\begin{array}{c}+67 \mathrm{Cl} \mathrm{M} \\
\text { or } \mathrm{AL})\end{array}$ & $\begin{array}{r}+95 \mathrm{CI} \\
\mathrm{M} \sim \mathrm{OL}\end{array}$ \\
\hline Pu & $6.50 \mathrm{E}-04(8 / \mathrm{L})$ & - & 1.13 & 4.SSE-OA & $5.53 \mathrm{E}-04$ & 7.48E-04 & 8.41E-04 \\
\hline $\mathrm{U}$ & $100 \mathrm{E}-\mathrm{O} 2$ & $1.508+03$ & $150 \mathrm{E}+03$ & 1.01E-02 & $1.05 \mathrm{E}-02$ & $1.13 \mathrm{E}-02$ & $1.12 \mathrm{E}-02$ \\
\hline
\end{tabular}

"Density is colculated bened on Na, OH-, and AlO2.

†Water wh\% derived from the difference of dentity and totel diwnolved species. 
HNF-SD-WM-ER-352, Rev. 1

HDW Model Rev. 4

\begin{tabular}{|c|c|c|c|c|c|c|c|}
\hline \multirow{4}{*}{$\begin{array}{l}\text { Phyied } \\
\text { Propertles }\end{array}$} & \multicolumn{7}{|c|}{ Single-Shell Tark 241-S-111 } \\
\hline & \multicolumn{7}{|c|}{ Total Inventory Estinme* } \\
\hline & & & & & & & \\
\hline & & & & $95 \mathrm{CI}$ & $-67 \mathrm{CI}$ & $+67 \mathrm{Cl}$ & $+95 \mathrm{CI}$ \\
\hline Total Waste & $3.53 \mathrm{E}+0 \mathrm{~s}(\mathrm{~kg})$ & $(538 \mathrm{kgal})$ & - & - & $m$ & $\cdots$ & - \\
\hline Heat Load & $5.85(\mathrm{CW})$ & $(2.00 E+04$ BTU/Ar) & - & 5.22 & 5.57 & 6.08 & 6.06 \\
\hline Bulk Densityt & $1 . \pi(g / \infty)$ & - & - & 1.65 & 1.68 & 1.79 & 1.78 \\
\hline & & & & & & & \\
\hline Water wot & 26.4 & - & $=$ & 23.7 & 23.3 & 29.1 & 30.8 \\
\hline TOC wt\% C (wet & 0.429 & - & $\ldots$ & 0.347 & 0.387 & 0.470 & 0.511 \\
\hline Rodoloulol & $\mathrm{CV}_{\mathrm{L}} \mathrm{N}_{\mathrm{H}}$ & $\mathrm{pCV} / \mathrm{s}$ & 9 & $\begin{array}{l}95 \mathrm{Cl} \\
(\mathrm{CLL})\end{array}$ & $\begin{array}{l}67 \mathrm{Cl} \\
(\mathrm{Cu})\end{array}$ & $\begin{array}{l}+6 \mathrm{Cl} \\
\text { (CUL) }\end{array}$ & $\begin{array}{l}+95 \mathrm{Cl} \\
\text { (CU) }\end{array}$ \\
\hline $\mathrm{H} \cdot 3$ & $2.73 E-04$ & 0.158 & 550 & $1.600-04$ & $1.60 \mathrm{E}-04$ & $2.82 E-04$ & 2.82E-04 \\
\hline C.14 & 3.51E-05 & 2.03E-02 & 71.3 & 1.15E-05 & $1.15 E-05$ & $3.55 E-05$ & $3.57 \mathrm{E}-05$ \\
\hline Ni-59 & 4.06E-06 & 2.34E-03 & 8.27 & $2.95 E-06$ & $2.95 E-06$ & $4.23 \mathrm{E}-06$ & $4.17 \mathrm{E}-06$ \\
\hline Ni-63 & 3.82E-04 & 0.224 & 791 & 2.79E-04 & 2.79E-A4 & 4.04E-04 & $3.99 E-04$ \\
\hline $\mathrm{Co}-60$ & $3.68 \mathrm{E}-0 \mathrm{~s}$ & $2.12 \mathrm{E}-02$ & 74.9 & $9.77 E-06$ & 9.77E- -16 & $3.72 E-05$ & $3.7 T E-05$ \\
\hline Se-79 & $3.58 E-06$ & 2.07E-03 & 7.29 & $2.07 \mathrm{E}-06$ & 2.07E-06 & $4.12 E-06$ & 4.65E-06 \\
\hline St-90 & 0.226 & 131 & $1.60 \mathrm{E}+05$ & 0.193 & 0.212 & 0.237 & 0.235 \\
\hline$Y-90$ & 0.226 & 131 & $4.60 E+05$ & 0.174 & 0.174 & 0.237 & 0.235 \\
\hline $2 x-93$ & 1.75E-05 & 1.01E-02 & 35.7 & $1.00 \mathrm{E}-05$ & 1.00E-05 & $2.03 \mathrm{E}-05$ & $2.29 \mathrm{E}-05$ \\
\hline $\mathrm{Nb}-93 \mathrm{~m}$ & $129 \mathrm{E}-05$ & $7.43 \mathrm{E}-03$ & 26.2 & $7.58 \mathrm{E}-06$ & $7.58 \mathrm{E}-06$ & 1.43E-05 & 1.66E-05 \\
\hline Te-99 & $2.51 \mathrm{E}-04$ & 0.145 & $\$ 11$ & 1.63E-04 & $2.06 \mathrm{E}-04$ & 2.96E-04 & 3.40E-04 \\
\hline Ru-106 & $6.15 E-09$ & 3.55E- 46 & $1.25 \mathrm{E}-02$ & $2.66 \mathrm{E}-09$ & 2.66E-09 & $6.93 \mathrm{E}-99$ & $7.64 \mathrm{E}-09$ \\
\hline $\mathrm{Cd}-113 \mathrm{~m}$ & $8.92 \mathrm{E}-05$ & $5.15 E-02$ & 182 & 4.0E-05 & 4.40E-05 & $1.06 \mathrm{E}-04$ & 1.21E-04 \\
\hline Sb-125 & $153 E-4$ & 8.86E 02 & 313 & 3.65E-05 & 3.65e.05 & I.SSE-04 & 1.57E-04 \\
\hline $5 n-126$ & $5.42 E-06$ & $3.13 E-03$ & 11.0 & $3.16 \mathrm{E}-06$ & $3.16 \mathrm{E}-06$ & $6.24 E-06$ & $7,02 \mathrm{E}-06$ \\
\hline I-129 & $4.83 \mathrm{E}-07$ & 2.79E-04 & 0.984 & 3.13E-07 & $3.96 \mathrm{t}-97$ & $5.71 \mathrm{E}-07$ & $6.56 \mathrm{E}-07$ \\
\hline $\mathrm{Cs}-134$ & 1.92E-06 & 1.11E-03 & 3.91 & 1.23E-06 & $1.23 E-\infty 6$ & $2.06 \mathrm{E}-06$ & $2.19 \mathrm{E}-06$ \\
\hline Cs-137 & 0.289 & 167 & $5.68 E+05$ & 0.269 & $0.2 \pi$ & 0.299 & 0.310 \\
\hline $\mathrm{Be}-137 \mathrm{~m}$ & 0.273 & 188 & $5566+05$ & 0.181 & 0.181 & 0.281 & 0.269 \\
\hline$S m-151$ & $1.26 \mathrm{E}-02$ & 7.20 & $25 \pi+a+$ & 7.34E-03 & 7,3ME-03 & 1.4SE-02 & 1.64E- -02 \\
\hline Eu-152 & 4.24E-06 & 2.45E-03 & 8.64 & $2.38 \mathrm{E}-06$ & $2.39 E-06$ & $4.34 E-06$ & 4.43E-06 \\
\hline Eu-154 & 5.98E-0A & 0.345 & $1.22 \mathrm{E}+03$ & $2.21 \mathrm{E}-04$ & $2.21 E-04$ & $734 E-04$ & $7.38 \mathrm{E}-04$ \\
\hline Eu-155 & $2.372-04$ & 0.137 & 42 & $1.25 \mathrm{E}-04$ & $1.25 \mathrm{E}-04$ & $2.42 \mathrm{E}-04$ & $2.48 E-04$ \\
\hline $\operatorname{Re}-226$ & 2.SSE-10 & $1.65 E-07$ & $5.80 E-04$ & $2.07 \mathrm{E}-10$ & $2.30 \mathrm{E}-10$ & $3.25 E-10$ & $3.63 \mathrm{E}-10$ \\
\hline $\mathrm{Re}-228$ & $7.57 \mathrm{E}-08$ & $4.37 \mathrm{E}-05$ & 0.154 & $3.23 E-08$ & 5.35E-06 & $1.01 E-07$ & $1.29 \mathrm{E}-07$ \\
\hline Ac-227 & $1.51 E-09$ & 8.75E-07 & $3.006-03$ & $1.14 \mathrm{E}-09$ & $1.240-09$ & $1.75 E-09$ & $1.97 \mathrm{E}-09$ \\
\hline $\mathrm{Pa}-231$ & $4.32 \mathrm{E}-09$ & $2.50 \mathrm{E}-06$ & 8.81E-03 & 2.25E-09 & $2.82 \mathrm{E}-09$ & 4.87E-09 & 5.67E-09 \\
\hline Th-229 & $100 E-10$ & 1.04E-06 & $3.66 \mathrm{E}-03$ & $2.45 E-10$ & $1,31 E-09$ & $2.36 \mathrm{E}-09$ & $2.96 \mathrm{E}-\infty$ \\
\hline Th-232 & $5.28 \mathrm{E}-09$ & $3.05 E-96$ & $1.06 E-02$ & 2.89E-09 & $4.065-09$ & $6.51 E-09$ & $7.68 \mathrm{E}-09$ \\
\hline $\mathrm{v}-232$ & $4.27 E-07$ & 2. $.6 \mathrm{E}-04$ & 0.869 & 2.53E-07 & $3,39 E-07$ & $5.30 \mathrm{E}-07$ & $6.39 \mathrm{E}-0 \mathrm{~T}$ \\
\hline U-233 & $1.64 \mathrm{E}-05$ & 9.45E-04 & 3.33 & 9.7E-07 & 1.30E-06 & $2.03 E-06$ & 2.45E- 06 \\
\hline U-234 & 1.14E-06 & $6.59 \mathrm{E}-0.19$ & 2.32 & $1.09 E-06$ & 1.12E-06 & $1.16 E-06$ & $1.17 \mathrm{E}-06$ \\
\hline $\mathrm{U}-235$ & 4.75E-08 & 2.73E- 05 & $9.64 \mathrm{E}-02$ & 4.52E- 08 & 4.63E-08 & $4.82 E-48$ & 4.84E-08 \\
\hline $\mathrm{U}-236$ & 3.14E-08 & $1.82 E-05$ & $6.40 \mathrm{E}-02$ & $3.05 E-08$ & $3.100-08$ & $3.18 E-08$ & $3.22 \mathrm{E}-08$ \\
\hline $\mathrm{U}-238$ & 1.19E- -06 & 6.83E-01 & 2.42 & 1.1.4E-06 & $1.16 E-06$ & $1.218-06$ & $1.21 E-06$ \\
\hline Np-237 & 9.64E.07 & $3.5 \pi-04$ & 1.96 & 6.7TE-07 & $8.1 \pi-07$ & $1.11 E-06$ & 1.26E-06 \\
\hline Pu-238 & $2.62 E-06$ & 1.51E-03 & 5.34 & 2.28E-06 & $2.45 E-06$ & 2.79E-06 & $2.96 \mathrm{E}-06$ \\
\hline Pu-239 & 1.38E-04 & $7.965-02$ & 281 & $1.27 \mathrm{E}-0 \mathrm{~A}$ & $1.32 E-04$ & $1.43 E-04$ & 1.49E-04 \\
\hline Pu-240 & $2.07 \mathrm{E}-05$ & $1.20 E-02$ & 42.2 & 1.91E-0S & $1.995-05$ & 2.15E-05 & $2.23 \mathrm{E}-05$ \\
\hline Pu-24l & $1.63 E-04$ & $9.0 \mathrm{E}-02$ & $\mathbf{3 3 2}$ & $1.00-04$ & $1.51 E-04$ & $1.75 E-04$ & D. \\
\hline$P_{11-242}$ & B.11E-10 & 4.68E-07 & $1.65 \mathrm{E}-03$ & $6.73 E-10$ & $7.41 E-10$ & $8.81 E-10$ & 9.49E-10 \\
\hline Am-241 & $5.85 E-05$ & $3,32 E-02$ & 119 & 1.25E-05 & $5.03 E-05$ & $6.67 \mathrm{E}-0 \mathrm{~S}$ & $7.45 E-05$ \\
\hline $\mathrm{Am}-243$ & $184 \mathrm{E}-09$ & $1.06 \mathrm{E}-06$ & 3.75E-03 & $1.34 \mathrm{E}-0 \mathrm{~g}$ & 1.58E-09 & $2.11 E-\infty$ & $2.36 \mathrm{E}-09$ \\
\hline $\mathrm{Cm}-242$ & 1.41E-07 & 8.14E-05 & 0.257 & 6.43E-06 & 6.43E-08 & 1.45E-07 & $1.48 \mathrm{E}-07$ \\
\hline $\mathrm{Cm}-243$ & $1.15 \mathrm{E}-66$ & $6.65 \mathrm{E}-06$ & 2.34E-02 & 4.21E-09 & $4.21 \mathrm{E}-09$ & $1.19 E-08$ & $1.22 \mathrm{E}-08$ \\
\hline $\mathrm{Cm}-244$ & 1.198-07 & 6.8BE-05 & 0.243 & 4.38E-08 & 4.38E-0B & 1.45E-07 & $1.61 \mathrm{E}-07$ \\
\hline Totals: & $\mathbf{M}$ & $y / t$ & F & $96 \mathrm{dg}$ & $\begin{array}{l}67 \mathrm{cl}(\mathrm{H} \\
\text { or } / 2)\end{array}$ & $+67 \mathrm{cl}(\mathrm{M}$ & $+95 \mathrm{dl}$ \\
\hline $\mathrm{Pu}$ & $2.12 \mathrm{E}-03(\mathrm{~g} / \mathrm{L})$ & - & 433 & $1.948-03$ & $203 \pm-03$ & $2.22 \mathrm{E}-03$ & $2.31 \mathrm{E}-03$ \\
\hline $\mathrm{U}$ & 1.35E-02 & $1.86 \mathrm{E}+03$ & $6.55 E+03$ & $1.29 \mathrm{E}-02$ & $1.32 E-02$ & $1.39 \mathrm{E}-02$ & $1.38 \mathrm{E}-02$ \\
\hline
\end{tabular}




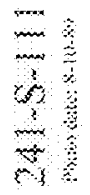
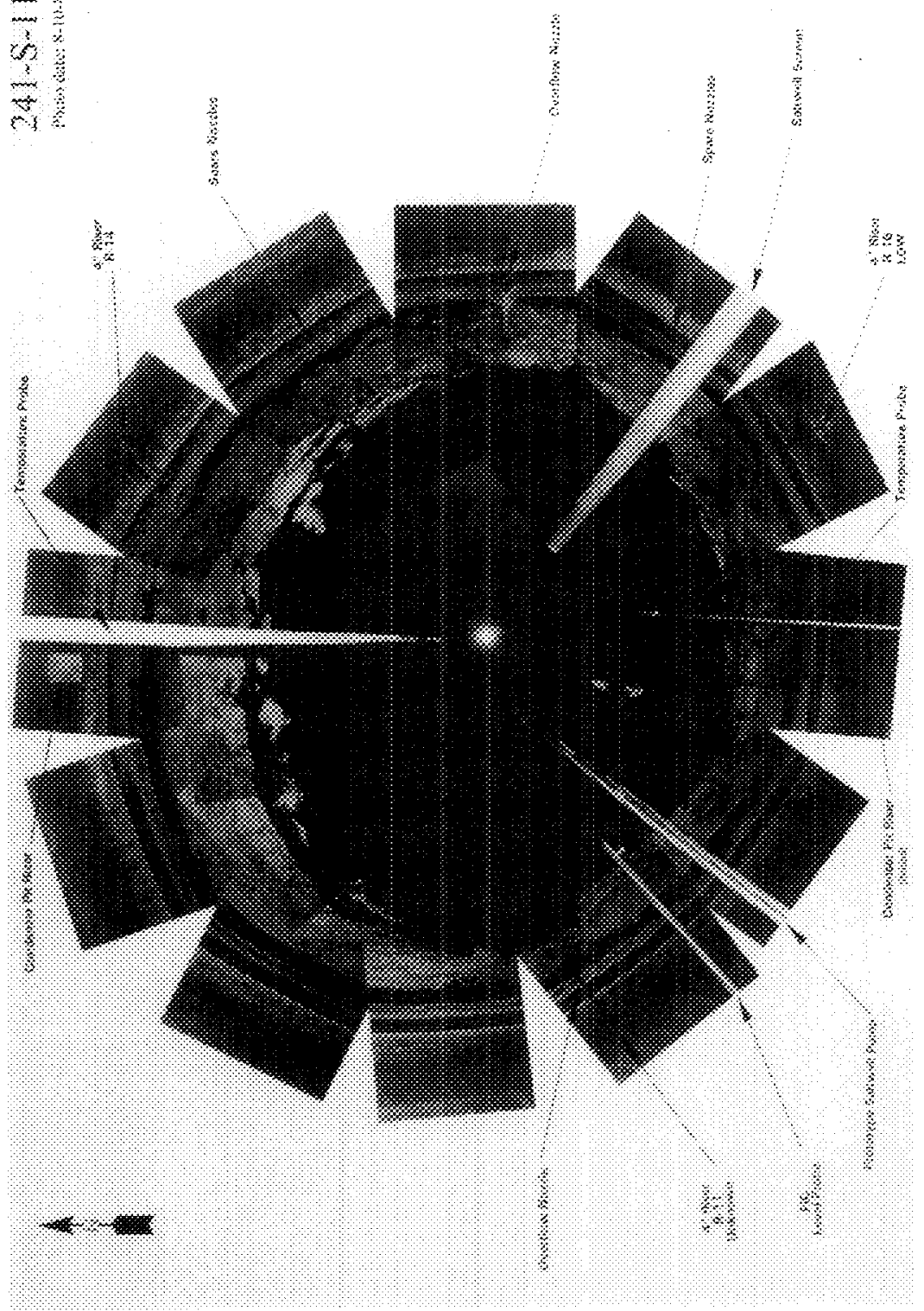
TANK 241-S-112 SUMMARY

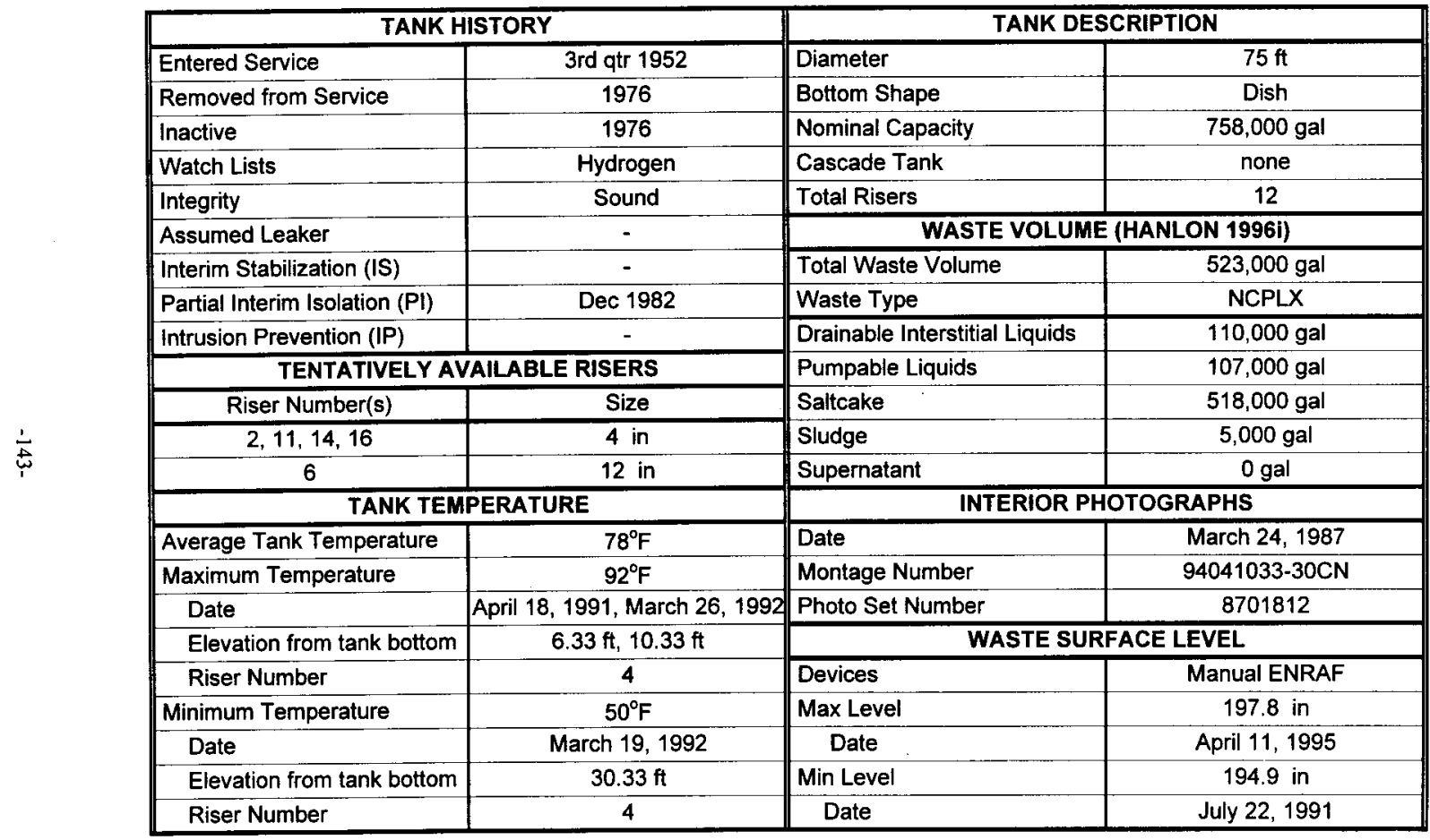




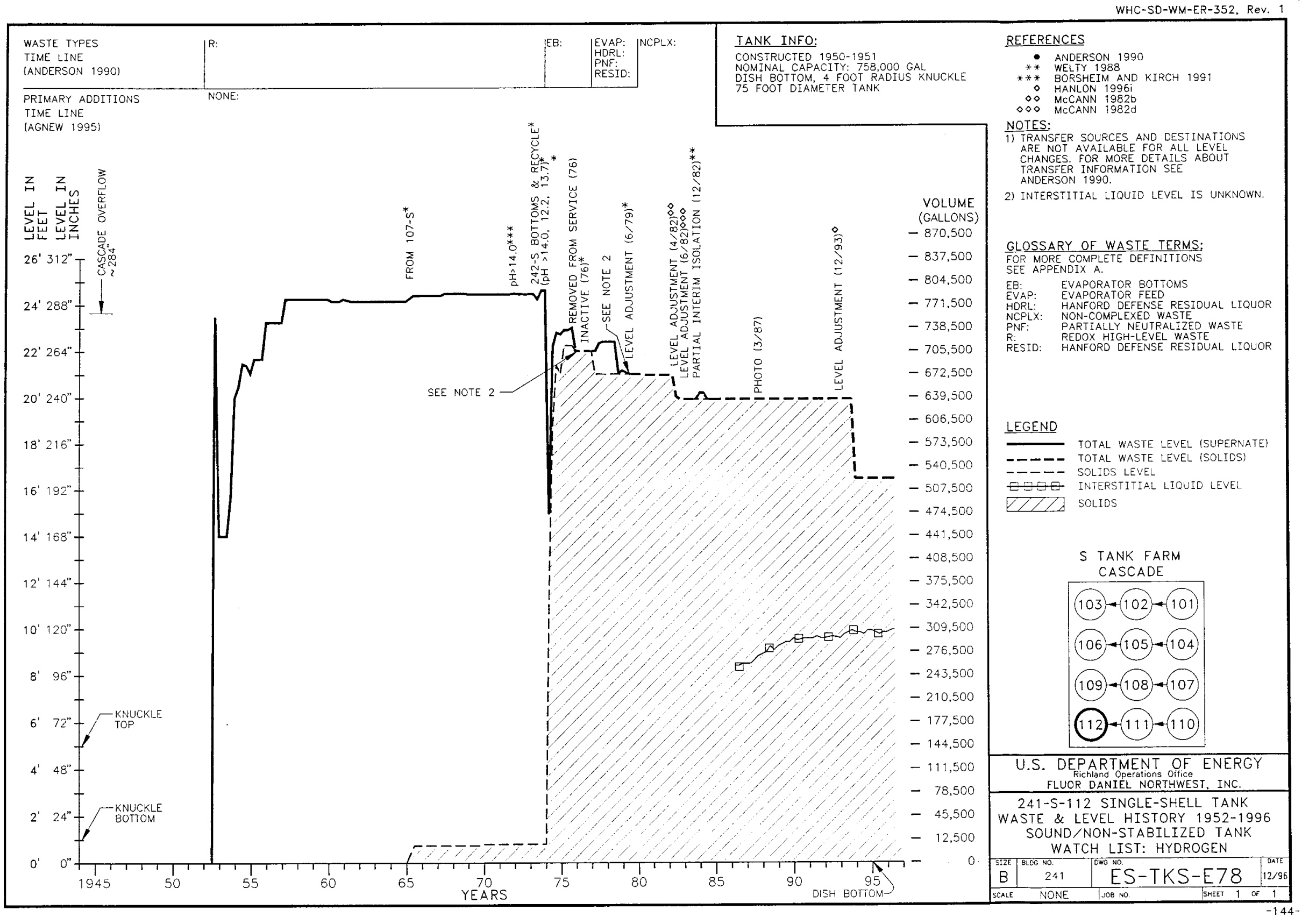




\section{$241-S-112$}

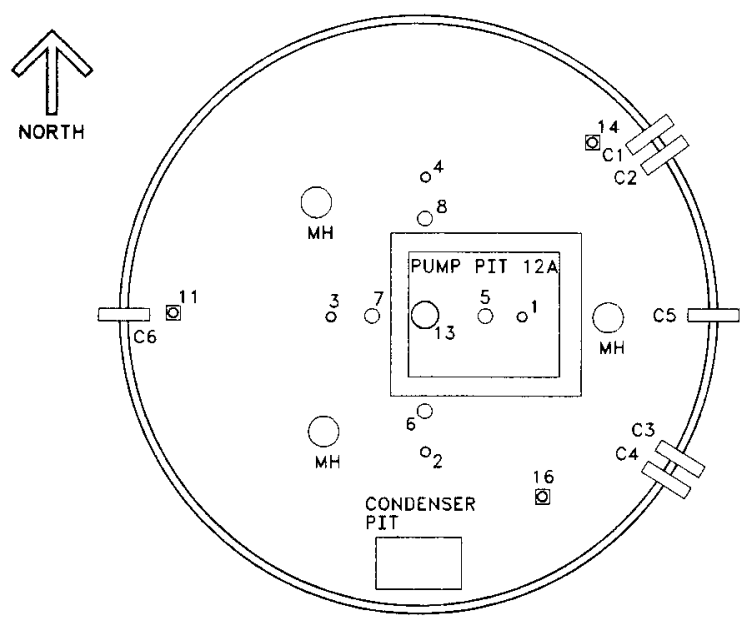

Ref: Alstad 1993

$\mathrm{H}-2-73191$, Rev. 4

$\mathrm{H}-2-37535$, Rev. 2

\section{TANK RISER LOCATION}

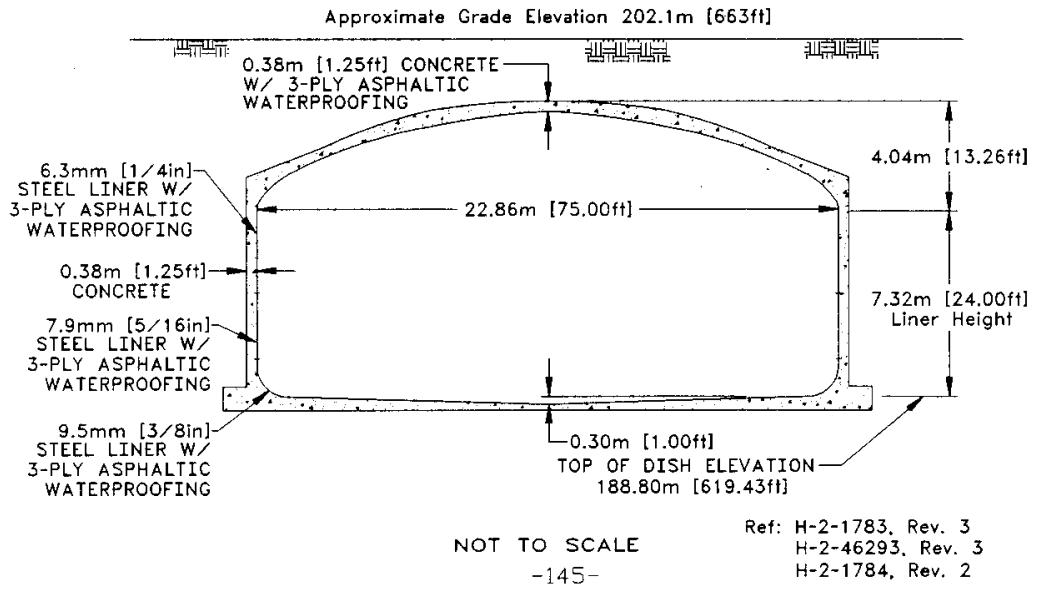


HNF-SD-WM-ER-352, Rev. 1

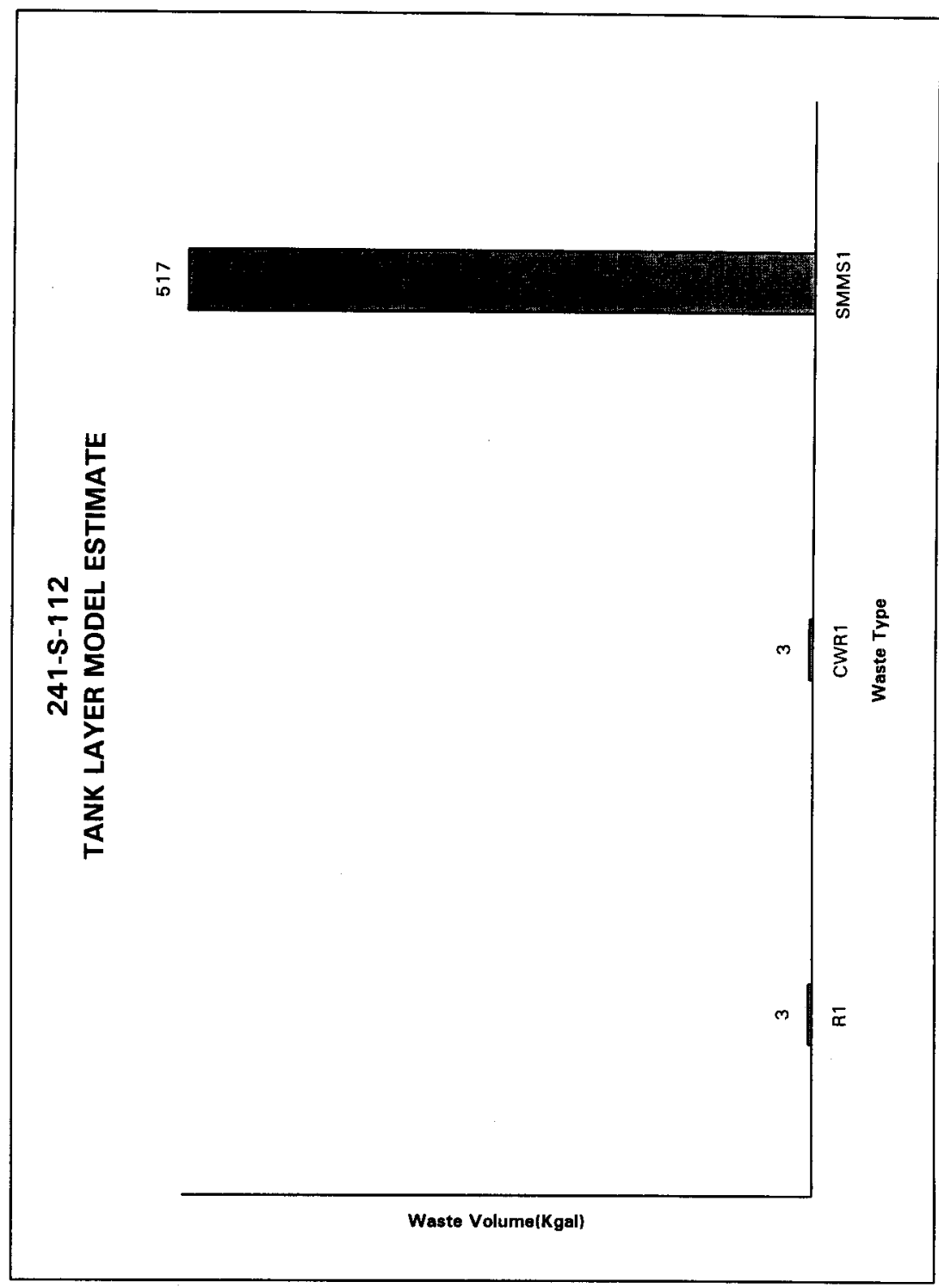

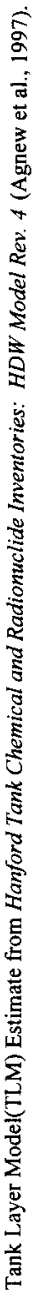


HNF-SD-WM-ER-352, Rev. 1

HDW Modei Rev. 4

\begin{tabular}{|c|c|c|c|c|c|c|c|}
\hline \multirow[b]{3}{*}{$\begin{array}{l}\text { Frypict } \\
\text { Propertios }\end{array}$} & \multicolumn{7}{|c|}{ Sinfle-Shell Tank 241-S-112 } \\
\hline & \multicolumn{7}{|c|}{ TLM Solids Composite Inventory Estimate" } \\
\hline & & & & $9 \mathrm{sct}$ & $-67 \mathrm{CI}$ & $+67 \mathrm{CI}$ & $+95 \mathrm{CI}$ \\
\hline Total TLM Waste & $4.00 \mathrm{E}+04(\mathrm{~kg})$ & $(6.00 \mathrm{kgal})$ & $\ldots$ & - & - & - & - \\
\hline Heat Load & $7.43 \mathrm{E}-02(\mathrm{~kW})$ & (2S4 BTUhY) & - & $5.30 \mathrm{E}-02$ & $6.58 E-02$ & $7.97 E-02$ & 8.22E-02 \\
\hline Bulk Density & $1.76(\mathrm{~g} / \mathrm{cc})$ & 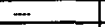 & $\cdots$ & 1.61 & 1.65 & 1.94 & 2.01 \\
\hline Void Fraction & 0.593 & $\Rightarrow$ & $\ldots$ & 0.355 & 0.434 & 0.694 & 0.697 \\
\hline Water wt\% & 25.8 & - & $\ldots$ & 12.3 & 16.7 & 35.7 & 39.8 \\
\hline TOC wt $\% \mathrm{C}$ (wet & 0 & - & - & 0 & 0 & 0 & 0 \\
\hline $\begin{array}{l}\text { Chento-1 } \\
\text { Contituents }\end{array}$ & $\mathrm{moll}_{\mathrm{L}} \mathrm{n}$ & $\mathrm{p}=\mathrm{N} \quad \mathrm{L}$ & k & $\begin{array}{l}95 \mathrm{CI} \\
(\mathrm{gol}) \mathrm{L}\end{array}$ & $\begin{array}{l}67 \mathrm{CI} \\
(\mathrm{mol})\end{array}$ & $\begin{array}{l}+67 \mathrm{CI} \\
\text { (10dele L) }\end{array}$ & $\begin{array}{l}+9 \mathrm{Cl} \\
(\text { molar) }\end{array}$ \\
\hline $\mathrm{Na}+$ & 7.99 & $1,04 \mathrm{E}+05$ & $4.17 \mathrm{E}+03$ & 5.18 & 3.71 & 11.3 & 13.0 \\
\hline $\mathbf{A} \mathbf{B}+$ & 9.10 & 1.39E+05 & $5.57 \mathrm{E}+03$ & 6.41 & 7.13 & 10.5 & 11.5 \\
\hline $\mathrm{Fe} 3+$ (total Fe) & 0.589 & $1.87 \mathrm{E}+04$ & 747 & 0.580 & 0.584 & 0.594 & 0.599 \\
\hline $\mathrm{Cr} 3+$ & $3.20 \mathrm{E}-02$ & 942 & 37.7 & 4.56E-03 & 1.80E-02 & 0.262 & 0.531 \\
\hline $\mathrm{Bi3+}$ & 0 & 0 & 0 & 0 & 0 & 0 & 0 \\
\hline $\mathrm{LeOH}$ & 0 & 0 & 0 & 0 & 0 & 0 & 0 \\
\hline $\mathrm{Hig}+$ & 204E-03 & 232 & 9.29 & 2.01E-0.3 & $203 E-03$ & 2.05E-03 & 2.05E-03 \\
\hline $\mathrm{Zr}(\mathrm{a} \mathrm{ZrO}(\mathrm{OH}) 2)$ & 0 & 0 & 0 & $\therefore \quad 0$ & 0 & 0 & 0 \\
\hline $\mathrm{Pb24}$ & $58:-02$ & $6.91 \mathrm{E}+03$ & 277 & $5.40 \mathrm{E}-12$ & $5.67 \mathrm{E}-\mathrm{M}$ & $6.09 \mathrm{E}-02$ & $6.29 \mathrm{E}-02$ \\
\hline Ni2t & $2595-02$ & 861 & 34.5 & $1.88 \mathrm{E}-02$ & $2.28 E-02$ & $2.82 E-02$ & $2.78 \mathrm{E}-02$ \\
\hline $\mathrm{sin}+$ & 0 & 0 & 0 & 0 & 0 & 0 & 0 \\
\hline Mn4t & 0 & 0 & 0 & 0 & 0 & 0 & 0 \\
\hline $\mathrm{Cn} 2+$ & 0.170 & $3.87 \mathrm{E}+03$ & 155 & 0.133 & 0.151 & 0.189 & 0.208 \\
\hline $\mathrm{K}+$ & $6.385-03$ & 141 & $\begin{array}{r}5.67 \\
\end{array}$ & 2.41E-03 & $340 \mathrm{E}-03$ & $8.38 E-03$ & 8.38E- 03 \\
\hline $\mathrm{OH}$ & $\begin{array}{r}33.7 \\
\end{array}$ & $3.24 \mathrm{E}+05$ & $1.30 \mathrm{E}+04$ & 22.8 & 23.8 & 39.3 & 43.4 \\
\hline $\mathrm{No3}$ & 2.93 & $0.03 \mathrm{E}+05$ & 4.12E+03 & 0.316 & 0.316 & 7.02 & 9.24 \\
\hline NO2- & 1.21 & $3.15 \mathrm{E}+04$ & $1.26 \mathrm{E}+03$ & 0.545 & 0.776 & 1.55 & 1.55 \\
\hline $\cos 32$ & 0.170 & $5.79 \mathrm{E}+03$ & 232 & 0.133 & 0.151 & 0.189 & 0.208 \\
\hline PO43- & 0 & 0 & 0 & 0 & 0 & 0 & 0 \\
\hline SO42. & $9.40 \mathrm{E}-03$ & 512 & 20.5 & 4.79E-03 & $6.63 \mathrm{E}-03$ & $1.12 \mathrm{E}-02$ & $1.12 \mathrm{E}-02$ \\
\hline Si (w St032-) & $1.41 \mathrm{E}-02$ & 224 & 8.97 & $1.05 E-02$ & $1.19 \mathrm{E}-02$ & $4.49 E-02$ & $9.16 \mathrm{E}-02$ \\
\hline F. & 0 & 0 & 0 & 0 & 0 & 0 & 0 \\
\hline $\mathrm{Cl}$ & $2.94 \mathrm{E}-02$ & 590 & 23.6 & $6.49 \mathrm{E}-03$ & $1.56 \mathrm{E}-02$ & 3.BSE-02 & $8.20 \mathrm{E}-02$ \\
\hline C6HSO73- & 0 & 0 & 0 & 0 & 0 & 0 & 0 \\
\hline EDTA4 & 0 & 0 & 0 & 0 & 0 & 0 & 0 \\
\hline HEDTA3- & 0 & 0 & 0 & 0 & 0 & 0 & 0 \\
\hline Wycointe- & 0 & 0 & of & 0 & 0 & 0 & 0 \\
\hline ecetate- & 0 & 0 & 0 & 0 & 0 & 0 & 0 \\
\hline oxollate2- & 0 & 0 & 0 & 0 & 0 & 0 & 0 \\
\hline DEP & 0 & 0 & 0 & 0 & 0 & 0 & 0 \\
\hline butanol & 0 & 0 & 0 & 0 & 0 & 0 & 0 \\
\hline $\mathrm{NH3}$ & 0.128 & $1.23 \mathrm{E}+03$ & 49.4 & 3.29E- -02 & $4.64 \mathrm{E}-02$ & 0.135 & 0.142 \\
\hline Fo(CN)64 & 0 & 0 & 0 & 0 & 0 & 0 & 0 \\
\hline
\end{tabular}

-Unkrowns in tank colids inventory are curiened by Tent Layering Model (TLM). 
HNF-SD-WM-ER-352, Rev. 1

HDW Model Rov. 4

\begin{tabular}{|c|c|c|c|c|c|c|c|}
\hline \multirow{4}{*}{$\begin{array}{l}\text { Phylical } \\
\text { Propertites }\end{array}$} & \multicolumn{7}{|c|}{ Single-Shell Tmk 241-S-I12 } \\
\hline & \multicolumn{7}{|c|}{ SMM Composite Laventory Entimete } \\
\hline & & & & & & & \\
\hline & & & & $-95 \mathrm{Cl}$ & $67 \mathrm{CI}$ & $+67 \mathrm{CI}$ & $+95 \mathrm{CI}$ \\
\hline Total SMM Wat & $3.29 \mathrm{E}+06(\mathrm{~kg})$ & $(517 \mathrm{ky}$ (z) $)$ & $\cdots$ & - & $\ldots$ & - & - \\
\hline Heat Load & $4.21(\mathrm{kw})$ & (l.44E+04 B7U/hr) & $=$ & 3.94 & 4.07 & 4.36 & 4.38 \\
\hline Bulk Dersity" & $1.68(\mathrm{~g} / \infty)$ & - & - & 1.61 & 1.65 & 1,71 & 1.72 \\
\hline Water w\% & 29.3 & $m$ & - & 27.1 & 28.0 & 30.8 & 33.3 \\
\hline TOC wt\% C (wet & 0.328 & $\ldots$ & - & 0.293 & 0.310 & 0.346 & 0.362 \\
\hline 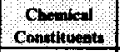 & hoth $/ \mathrm{L}$ & vow & kn & $\begin{array}{l}90 \mathrm{Cl} \\
(\text { nowh })\end{array}$ & $\begin{array}{l}67 \mathrm{cl} \\
(\mathrm{mol} / \mathrm{L})\end{array}$ & $\begin{array}{l}+67 \mathrm{CI} \\
(0,6 / \mathrm{L})\end{array}$ & $(2501$ \\
\hline Net & $\begin{array}{r}15.7 \\
\end{array}$ & $2.15 E+05$ & $7.06 E+05$ & 14.1 & 15.0 & 16.2 & 16.6 \\
\hline $\mathrm{Ab+}$ & 2.18 & $3.50 E+04$ & $1.15 E+05$ & 1.59 & 2.03 & 2.34 & 2.25 \\
\hline Fez+ (totel Fe) & 9.7TE-03 & 325 & $1.07 \mathrm{E}+03$ & B.02E-03 & $8.88 E-03$ & $1.07 \mathrm{E}-02$ & $1.15 \mathrm{E}-02$ \\
\hline $\mathrm{Cr} 3+$ & 0.224 & $6.92 \mathrm{E}+03$ & $2.285+04$ & 0.166 & 0.197 & 0.229 & 0.234 \\
\hline $\mathrm{Bi}+$ & 8.16E-04 & 101 & 334 & $7.35 \mathrm{E}-04$ & $7.75 \mathrm{E}-04$ & 8.58E-04 & $8.98 \mathrm{E}-04$ \\
\hline Let+ & $5.41 E-07$ & 4.47E-02 & 0.147 & $3,92 \mathrm{E}-07$ & 4.65E-07 & 6.JBE-07 & $6.91 \mathrm{E}-07$ \\
\hline Hg2+ & 6.84E-O6 & 0.916 & 2.68 & $6.41 \mathrm{E}-06$ & $6.65 \mathrm{E}-06$ & $6.93 \mathrm{E}-06$ & $7.02 \mathrm{E}-06$ \\
\hline $2 \mathrm{r}(\mathrm{s} \mathrm{ZOC}(\mathrm{OH}) 2)$ & 1.68E-04 & 9.14 & 30.1 & $1.532-04$ & $1.59 \mathrm{E}-04$ & $1.75 E-04$ & $1.83 \mathrm{E}-04$ \\
\hline Fb2+ & 8.08E-04 & 99.6 & 328 & $6.785-04$ & 7.42E-04 & 8.75E-04 & $9.39 \mathrm{E}-04$ \\
\hline $\mathrm{Ni2+}$ & $6.372-03$ & 222 & 731 & $6.140-03$ & $6.25 E-03$ & $6.495-03$ & 6.59E-03 \\
\hline 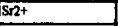 & 0 & 0 & 0 & 0 & 0 & 0 & 0 \\
\hline Mn4+ & $3.00 \mathrm{E}-03$ & 101 & 331 & $2.23 E-03$ & $2.65 \mathrm{E}-03$ & $3.51 \mathrm{E}-03$ & $3.92 \mathrm{E}-03$ \\
\hline $\mathrm{Con}+$ & 3.25E-02 & $\pi 5$ & $255 \mathrm{E}+03$ & $2.82 \mathrm{E}-02$ & $3.03 \mathrm{E}-02$ & 3.47E-02 & $3.68 \mathrm{~B}-02$ \\
\hline $\mathrm{K}+$ & $6.59 \mathrm{E}-02$ & $1.53 \mathrm{E}+03$ & $5.04 \mathrm{E}+03$ & $5.89 \mathrm{E}-02$ & $6.22 E-02$ & $6.99 \mathrm{E}-02$ & $7.14 \mathrm{E}-02$ \\
\hline $\mathrm{OH} \cdot$ & 13.2 & $1.33 E+05$ & $4.39 \mathrm{E}+05$ & 11.4 & 12.3 & 142 & 13.7 \\
\hline $\mathrm{NO3}$ & 5.25 & $1.94 \mathrm{E}+05$ & $6.37 \mathrm{E}+05$ & 4.79 & 5.02 & 5.4 & 5.44 \\
\hline NO2- & 2.97 & $8.11 E+04$ & $2.6 \pi+05$ & 2.59 & 2.7 & 3.15 & 3.33 \\
\hline $\mathrm{CO32}$ & 0.291 & $1.04 E+04$ & $3.42 \mathrm{E}+04$ & 0.262 & 0.275 & 0.311 & 0.314 \\
\hline $\mathrm{POA} 3$ & 6. $6 \mathrm{EE}-02$ & $3.65 E+03$ & $1.205+04$ & $5.76 \mathrm{E}-02$ & $6.00 \mathrm{E}-02$ & $6.61 E-02$ & $6.76 \mathrm{E}-02$ \\
\hline SO42- & 0.192 & $1.10 \mathrm{E}+0 \mathrm{a}$ & 161E+04 & 0.146 & 0.168 & 0.217 & 0.239 \\
\hline $\mathrm{Si}\left(\mathrm{s} \mathrm{SiO}_{2-}\right.$ ) & $7.31 \mathrm{E}-02$ & $1.22 E+03$ & $4.02 E+03$ & 6.10E-02 & $6.69 \mathrm{E}-02$ & $7.92 \mathrm{E}-02$ & C51E-02 \\
\hline F. & $3.90 \mathrm{E}-02$ & 41 & $1.45 E+03$ & $3.19 \mathrm{E}-02$ & 3.49E-02 & 4.25E-02 & 4.53E-0? \\
\hline $\mathrm{C}$ & 0.265 & $5.598+03$ & $1.84 E+04$ & 0.233 & 0.248 & 0.284 & 0.253 \\
\hline C6HSO73 & 2.25:-02 & $2.53 \mathrm{E}+03$ & 832E+03 & 2.05E-02 & $2.16 \mathrm{E}-02$ & 2.34E- 02 & 2.42E-03 \\
\hline EDTA4- & 2.49E-03 & 427 & $1.00 \mathrm{E}+03$ & $1.21 \mathrm{E}-03$ & 1.23E-03 & 3.15E-03 & $3.80 \mathrm{E}-03$ \\
\hline HEDTA 3 & 40.05-03 & 667 & $2.19 E+03$ & $1.52 \mathrm{E}-03$ & $27 E E-03$ & 3.41E-03 & 6.70E- -13 \\
\hline dyoolate. & $3.5 \mathrm{E}-02$ & $1.58 \mathrm{E}+03$ & $5.20 \mathrm{E}+03$ & 2.05E-02 & $2.7 \mathrm{TE}-02$ & 4.31E-02 & $5.04 E-02$ \\
\hline encetite- & $2.87 \mathrm{E}-03$ & 101 & 332 & 2.33E-03 & 2.59E-03 & 3.15E-03 & $3.42 \mathrm{E}-03$ \\
\hline oculate2- & 709E-O & $3.7 \mathrm{E}-02$ & 0.122 & $6.28 \mathrm{E}-07$ & $669 E-07$ & $7.50 \mathrm{E}-07$ & $7.90 \mathrm{E}-07$ \\
\hline DBP & $1.52 \mathrm{E}-02$ & $1.90 \mathrm{E}+03$ & $6.25 \mathrm{E}+03$ & $1.22 \mathrm{E}-02$ & $137 \mathrm{E}-92$ & $1.67 \mathrm{E}-02$ & 1.81E-02 \\
\hline butand & $1.52 \mathrm{E}-02$ & 639 & $2.20 E+03$ & 1.22E-02 & $137 \mathrm{E}-02$ & $1.67 \mathrm{E}-02$ & 1.81E-02 \\
\hline $\mathrm{NH} 3$ & 7.80E-02 & $\$ 7$ & $2.62 E+03$ & $6.53 \mathrm{E}-02$ & 7.07E- -2 & $8.93 \mathrm{E}-02$ & 0.102 \\
\hline $\mathrm{Fa}(\mathrm{CN}) 64$ & 0 & o & 0 & 0 & 0 & 0 & 0 \\
\hline
\end{tabular}

*Density is calculated besed on Na, OH, and $\mathrm{AlO2}$.

pWater wt\% derived from the difference of density and total diseolvod apecies. 
HNF-SD-WM-ER-352, Rev. 1

\begin{tabular}{|c|c|c|c|c|c|c|c|}
\hline & \multicolumn{7}{|c|}{ Single-Shell Tunk 241-S-112 } \\
\hline & \multicolumn{7}{|c|}{ Total inventory Estimate* } \\
\hline $\begin{array}{l}\text { Pryutical } \\
\text { Propertiea }\end{array}$ & & & & $9 \mathrm{~S} \mathrm{Cl}$ & $.67 \mathrm{CI}$ & $+67 \mathrm{CI}$ & $+95 \mathrm{CI}$ \\
\hline Total Waste & $3333 E+06(\mathrm{~kg})$ & $(523 \mathrm{~kg}$ & 一 & $\ldots$ & - & $\cdots$ & - \\
\hline Heat Loed & $4.28(\mathrm{~kW})$ & $(1.46 E+04$ BTU/Wr) & $\ldots$ & 4.00 & 4.13 & 4.44 & 4.45 \\
\hline Bulk Density & $1.69(g / \infty)$ & - & - & 1.61 & 1.65 & 1.71 & 1,72 \\
\hline Water wtopt & 29.2 & - & - & 27.1 & 28.0 & 30.8 & 33.3 \\
\hline TOC wt\%C (wet & 0.324 & $\ldots$ & $\ldots$ & 0.289 & 0.306 & 0.341 & 0.358 \\
\hline Constlturents & moled & pps & Le & (molal & $\begin{array}{r}-67 \mathrm{CI} \\
(\mathrm{mol} / \mathrm{L})\end{array}$ & $\begin{array}{l}+67 \mathrm{Cl} \\
\text { (nodeft) }\end{array}$ & $\begin{array}{c}+95 \mathrm{Cl} \\
\text { (moled) }\end{array}$ \\
\hline Nat & 15.6 & 2.13E+05 & $7.11 \mathrm{E}+05$ & 140 & 14.9 & 16.1 & 16.5 \\
\hline $\mathbf{A B} \mathbf{B}$ & 2.26 & $3.62 E+04$ & $1.21 E+05$ & 1.98 & 2.11 & 2.42 & 2.33 \\
\hline $\mathrm{Fe} 3+(\cot 1 \mathrm{~F} e)$ & $1.64 \mathrm{E}-02$ & 545 & $1.82 E+03$ & $1.47 \mathrm{E}-02$ & $1.55 E-02$ & $1.73 E-02$ & $1.82 \mathrm{E}-02$ \\
\hline Cr3+ & 0.222 & $6.85 E+03$ & $2.28 \mathrm{E}+04$ & 0.170 & 0.198 & 0.228 & 0.232 \\
\hline Biz+ & $8.07 \mathrm{E}-04$ & 100 & 334 & $7.27 \mathrm{E}-04$ & $7.66 \mathrm{E}-04$ & 8. $48 \mathrm{E}-04$ & $8.88 \mathrm{E}-04$ \\
\hline $16+$ & 5.35E-07 & 4.42E-02 & 0.54 & $3.88 \mathrm{E}-07$ & 4.60E-07 & 6.J1E-07 & $6.83 \mathrm{E}-07$ \\
\hline Hg2+ & 3.0IE-05 & 3.59 & 12.0 & 2.97E-0S & $3.00 \mathrm{E}-05$ & $3.02 E-05$ & $3.03 \mathrm{E}-05$ \\
\hline $\mathrm{Zr}$ (as ZrO(OH)2) & $1.66 \mathrm{E}-04$ & 9.03 & 30.1 & 1.52E-04 & $1.57 \mathrm{E}-04$ & 1.73E-04 & I.8IE-04 \\
\hline $\mathrm{Pb} 2+$ & $1.47 \mathrm{E}-03$ & 181 & 604 & $1.385-03$ & 1.43E-03 & $1.52 \mathrm{E}-03$ & 1.57E-03 \\
\hline $\mathrm{Ni24}$ & $6.59 \mathrm{E}-03$ & 230 & 766 & $6.39 \mathrm{E}-03$ & 6.49E-03 & $6.69 \mathrm{E}-03$ & $6.79 \mathrm{E}-03$ \\
\hline $\mathrm{Sr} 2+$ & 0 & 0 & 0 & 0 & 0 & 0 & $\mathbf{0}$ \\
\hline Mn4t & $3.04 E-03$ & 99.3 & 331 & $2.21 \mathrm{E}-03$ & $262 \mathrm{E}-03$ & $3.47 \mathrm{E}-03$ & 3.8TE-03 \\
\hline $\mathrm{Cln}_{2+}$ & $3.41 \mathrm{E}-02$ & 812 & $2.71 E+63$ & $3.02 \mathrm{E}-02$ & $3.21 \mathrm{E}-\mathrm{O2}$ & $3.6 \mid E-02$ & $3.80 E-02$ \\
\hline $\mathrm{K}+$ & $6.52 E-02$ & $1.52 \mathrm{E}+03$ & $5.05 E+03$ & $5.83 E-02$ & $6.16 \mathrm{E}-02$ & 6.92E-02 & 7.06E-02 \\
\hline $\mathrm{OH}$ - & 13.4 & $1.36 \mathrm{E}+0 \mathrm{~s}$ & $4.52 E+05$ & 11.7 & 12.5 & 14.4 & 14.0 \\
\hline NO3- & 5.23 & $1.93 \mathrm{E}+0 \mathrm{~s}$ & $6.41 E+05$ & 485 & 5.03 & 5.38 & 5.38 \\
\hline NO2- & 2.95 & $8.05 \mathrm{E}+04$ & $2.60 \mathrm{E}+05$ & 2.57 & 2.75 & 3.13 & 3.31 \\
\hline cos2. & 0.259 & $1.03 \mathrm{E}+04$ & $3.44 E+04$ & 0.261 & 0.274 & 0.309 & 0.312 \\
\hline POA3 & $6.38 E-02$ & $3.605+03$ & $1.20 E+04$ & $5.69 \mathrm{E}-02$ & $5.93 E-02$ & $6.54 E-02$ & $6.68 \mathrm{E}-02$ \\
\hline SO42- & 0.190 & $1.09 \mathrm{E}+04$ & $3.62 \mathrm{E}+0 \mathrm{~A}$ & 0.144 & 0.166 & 0.215 & 0.237 \\
\hline Si (at SO32-) & $7.24 E-02$ & $1.21 E+03$ & $4.03 E+03$ & 6.05E-02 & $6.63 \mathrm{E}-02$ & $7.85 E-02$ & 8.43E-02 \\
\hline$F-$ & $3.86 E-02$ & 456 & $1.45 E+03$ & $3.16 \mathrm{E}-02$ & 3.45E-02 & 4.20E-02 & $4.48 \mathrm{E}-02$ \\
\hline Cl- & 0.263 & $5.53 E+03$ & 1.84E+04 & 0.231 & 0.246 & 0.281 & 0.280 \\
\hline C6HSOT3- & 2.22802 & $2.50 \mathrm{E}+43$ & B.32E+03 & 2.05E-02 & 2.14E-02 & $231 E-02$ & $2.39 \mathrm{E}-02$ \\
\hline EDTA4 & $2.46 E-03$ & 422 & $1.40 \mathrm{E}+03$ & $1.195-03$ & $1.81 E-03$ & $3.12 \mathrm{E}-03$ & 3.75E-03 \\
\hline HEDTA3 & 104E-03 & 659 & $2.19 \mathrm{E}+03$ & $1.50 \mathrm{E}-03$ & 2.7AE-03 & $5.35 \mathrm{E}-03$ & $6.63 \mathrm{E}-03$ \\
\hline glycointe- & $3.50 E-02$ & $1.56 \mathrm{E}+03$ & $5.20 \mathrm{E}+03$ & 2.02E-02 & 2.75E-02 & 426E-02 & $4.98 \mathrm{AE}-02$ \\
\hline ecetute- & 2.84E-03 & 99.5 & 332 & $2,30 \mathrm{E}-03$ & 2.56E-03 & 3.11E-03 & 3.38E-03 \\
\hline oxalate2- & 7.01E-07 & $3.67 \mathrm{E}-02$ & 0.12 & 6.21E-07 & $6.60 \mathrm{E}-07$ & $7,42 \mathrm{E}-07$ & $7.81 \mathrm{E}-07$ \\
\hline DBP & $1.50 \mathrm{E}-02$ & $1.83+03$ & $6.25 \mathrm{E}+03$ & $1.2\} \mathrm{E}-02$ & $1.35 \mathrm{E}-02$ & 1.65E-02 & 1.79E-02 \\
\hline butanol & $1.505-02$ & 61 & $2.202+03$ & $1.21 \mathrm{E}-02$ & $1.35 \mathrm{E}-02$ & $1.65 \mathrm{E}-02$ & 1.79E-02 \\
\hline $\mathrm{NH3}$ & 7.94E-02 & 802 & $2.67 \mathrm{E}+03$ & $6.60 \mathrm{E}-02$ & 7.13E-02 & 8.99E-02 & 0.102 \\
\hline Fo(CN)64 & 0 & 0 & 0 & 0 & 0 & 0 & 0 \\
\hline
\end{tabular}

-Unknowns in tenk solids inventory are anjigred by Tank Loyaring Model (TLM)

+Water wt\% derived from the difference of density and total diesolvod species. 
HNF-SD-WM-ER-352, Rev. 1

HOW Model Rev. 4

\begin{tabular}{|c|c|c|c|c|c|c|c|}
\hline & & Sin: & C-Shell Tan & k 241-S-112 & & & \\
\hline & & TL.M Solid & Composite & Inventory Esti & mate* & & \\
\hline Phyich & & & & & & & \\
\hline Properties & & & & $95 \mathrm{CI}$ & $-67 \mathrm{CI}$ & $+67 \mathrm{CI}$ & $+95 \mathrm{CI}$ \\
\hline Total TLM Weste & $4.00 \mathrm{E}+04(\mathrm{~kg})$ & $(6.00 \mathrm{~kg})$ & - & - & - & $\ldots$ & - \\
\hline Heat Load & $7.43 \mathrm{E}-02(\mathrm{~kW})$ & (25d BTU/hr) & - & 5.39E-02 & $6.58 \mathrm{E}-02$ & $7.97 \mathrm{E}-02$ & $8.22 \mathrm{E}-02$ \\
\hline Bulk Density & $1.76\left(\sigma^{\prime} \propto c\right)$ & - & - & 1.61 & 1.65 & 1.94 & 2.01 \\
\hline Void Fraction & 0.593 & - & - & 0.355 & 0.434 & 0.694 & 0.697 \\
\hline Water wt\% & $25 . \bar{B}$ & $\ldots$ & - & 12.3 & 16.7 & $\mathbf{3 5 . 7}$ & 39.8 \\
\hline TOC wr\% C (wet & 0 & $\ldots$ & $=$ & 0 & 0 & 0 & \\
\hline $\begin{array}{l}\text { Rodiotogicel } \\
\text { Constituents }\end{array}$ & $\mathrm{Cl} / \mathrm{L}$ & $\mu C_{/}$, & ब & $\begin{array}{c}93 \mathrm{Cl} \\
\mathrm{CON})\end{array}$ & $\begin{array}{l}67 \mathrm{Cl} \\
(\mathrm{C}, \mathrm{L})\end{array}$ & $\begin{array}{l}+67 \mathrm{Cl} \\
\text { (CUL) }\end{array}$ & $\begin{array}{r}+95 \mathrm{ct} \\
\text { (cuL) }\end{array}$ \\
\hline $\mathrm{H} \cdot \mathbf{3}$ & $1.51 \mathrm{E}-05$ & $8.58 \mathrm{E}-03$ & 0.344 & $2.03 \mathrm{E}-06$ & $7.86 \mathrm{E}-06$ & $2.10 \mathrm{E}-0 \mathrm{~s}$ & $2.10 E-05$ \\
\hline C.14 & 1.19E-06 & $6.73 E-04$ & $2.69 \overline{0}-02$ & 1.84E-07 & 5.85E-07 & $1.59 E-06$ & $1.59 \mathrm{E}-06$ \\
\hline $\mathrm{Ni}-59$ & 7.45E-06 & 4.22E-03 & 0.169 & 5.385-06 & $6.59 E-06$ & 8.14E-06 & $8.01 \mathrm{E}-06$ \\
\hline $\mathrm{Ni}-63$ & 695E-04 & 0.394 & 15.8 & $3.02 E-04$ & $6.11 \mathrm{E}-04$ & $7.60 \mathrm{E}-04$ & $7.48 \mathrm{E}-04$ \\
\hline $\mathrm{Co}-60$ & 4.71E-07 & $2.67 \mathrm{E}-04$ & $1.07 \mathrm{E}-02$ & $7.4 \mathrm{TE}-0 \mathrm{~S}$ & 2.33E-07 & $6.30 \mathrm{E}-07$ & $6.30 \mathrm{E}-07$ \\
\hline Se-79 & 2.52E-07 & 1.43E-04 & $3.73 \mathrm{E}-03$ & 3.94E-08 & 1.24E-47 & 3.38E-07 & $3.59 E-106$ \\
\hline Sr-90 & 0.465 & 264 & $1.06 \mathrm{E}+04$ & 0.332 & 0.407 & 0.510 & 0.517 \\
\hline$Y-90$ & 0.465 & 264 & $1.06 \mathrm{E}+04$ & 0.332 & 0.407 & 0.510 & 0.518 \\
\hline $\mathrm{Zr} \cdot 93$ & 1.19E-06 & $6.75 E-04$ & $2.70 E-02$ & 1.86E-07 & S.87E-07 & 1.59E-06 & $1.52 \mathrm{E}-05$ \\
\hline $\mathrm{Nb}-93 \mathrm{~m}$ & $9.73 E-07$ & 5.52E-04 & $2.21 \mathrm{E}-02$ & 1.52E-07 & 4.80E-07 & $1.30 E-06$ & 1.61E-05 \\
\hline $\mathrm{Tc}-99$ & $8.32 \mathrm{E}-06$ & 472E-03 & 0.189 & $1.30 \mathrm{E}-06$ & $4.10 \mathrm{E}-\infty$ & 1.11E-05 & 1.11E-05 \\
\hline $\mathrm{Ru}-106$ & $1.39 \mathrm{E}-12$ & $7.86 E-10$ & 3.15E-08 & $2.91 \mathrm{E}-13$ & $7.22 \mathrm{E}-13$ & 1.83E-12 & $1.83 \mathrm{E}-12$ \\
\hline Cd-113m & $3.67 E-06$ & $2.08 E-03$ & B.3AE-02 & $5.75 E-07$ & 1.81E-06 & 4.92E-06 & 4.92E-66 \\
\hline Sb-125 & 6.96E-07 & 3.9AE-94 & $1.58 \mathrm{E}-02$ & $1.15 E-07$ & $3.4 \pi-07$ & $9.29 \mathrm{E}-07$ & $9.29 E-07$ \\
\hline$S n-126$ & 3.8TE-07 & $2.20 E-04$ & 8.00E-03 & $6.03 E-08$ & 1.91E-07 & $5.19 \mathrm{E}-07$ & $5.74 E-06$ \\
\hline $1-129$ & $1.60 \mathrm{E}-08$ & 906E-06 & $3.63 \mathrm{E}-04$ & 2.49E-09 & $7.85 E-09$ & $2.14 E-08$ & $2.14 E-08$ \\
\hline Cs-134 & $1.44 E-06$ & 8.13E-06 & $3.27 \mathrm{E}-04$ & 1.42E-09 & 8.42E-09 & 1.BAE-18 & 1.84E-06 \\
\hline$C_{s-137}$ & 2.92E-02 & 16.6 & 663 & 8.58E-03 & $1.68 \mathrm{E}-02$ & $3.75 E-02$ & $3.79 \mathrm{E}-02$ \\
\hline $\mathrm{Ba}-137 \mathrm{~m}$ & $2.76 \mathrm{E}-02$ & 19.7 & 627 & 8.12E-03 & $1.59 \mathrm{E}-02$ & $3.54 E-02$ & 3.54E-02 \\
\hline $\mathrm{sm}-151$ & $9.00 \mathrm{E}-04$ & 0.510 & 20.4 & $1.40 \mathrm{E}-0 \mathrm{~A}$ & 4.4AE-04 & $1.21 E-03$ & 1.32E-02 \\
\hline Eu-152 & $3.59 \mathrm{E}-06$ & 2.03E-03 & $8.14 \mathrm{E}-02$ & 3.SIE- 06 & $3.54 E-05$ & $3.62 \mathrm{E}-06$ & $3.62 \mathrm{E}-16$ \\
\hline Eu-154 & t.14E-05 & 6.45E-03 & 0.256 & 1.R1E- 06 & $3.63 E-06$ & 1.52E-05 & 1.52E-05 \\
\hline Eu-155 & 1.69E-04 & $9.60 \mathrm{E}-02$ & 3.85 & $1.66 \mathrm{E}-04$ & 1.6TE-04 & 1.71E-04 & $1.71 E-04$ \\
\hline Ra-226 & $3.42 \mathrm{E}-10$ & $3.07 E-07$ & $1.23 \mathrm{E}-05$ & $7.52 \mathrm{E}-11$ & 3.04E-10 & $7.20 \mathrm{E}-10$ & 1.01E-09 \\
\hline $\mathrm{Re}-228$ & $478 E-15$ & $271 E-12$ & $1.09 \mathrm{E}-10$ & $4.69 \mathrm{E}-15$ & $4.72 E-15$ & 4.83E-15 & $4.82 \mathrm{E}-15$ \\
\hline$A c-227$ & $2.39 \mathrm{E}-69$ & $1.35 E-06$ & 5.02E-05 & $1.60 \mathrm{E}-10$ & $1.01 E-09$ & $3.772-09$ & S.10E-09 \\
\hline $\mathrm{Pa}-231$ & $3.76 E-10$ & 2.13E-07 & 8.54E-06 & 5.83E-11 & $1.85 \mathrm{E}-10$ & $1.04 E-09$ & 8.41E- -99 \\
\hline Th-229 & $9.05 E-13$ & $5.13 E-10$ & $2.05 \mathrm{E}-08$ & 8.86E-13 & 8.93E-13 & 9.12E-13 & 9.12E-13 \\
\hline $\mathrm{Th}-232$ & 3.16E-16 & $1.79 \mathrm{E}-13$ & $7.172-12$ & S.40E-17 & $158 E-16$ & 4.218-16 & $4.21 E-16$ \\
\hline $\mathrm{U} .232$ & $3.26 \mathrm{E}-10$ & 1.85E-07 & $7.40 E-16$ & $283 E-10$ & 3.08E-10 & $3.38 \mathrm{E}-10$ & $3.47 \mathrm{E}-10$ \\
\hline U-233 & 1.21E-11 & 684E-99 & $2.74 E-07$ & $105 E-11$ & 1.14E-11 & 1.25E-11 & 1.29E-11 \\
\hline U-234 & 7.46E-06 & 4.23E-03 & 0.169 & 6.49E-06 & $7.06 E-06$ & $7.75 \mathrm{E}-06$ & $7,95 \mathrm{E}-06$ \\
\hline $\mathrm{U}-235$ & $3.17 \mathrm{E}-07$ & $1.800-04$ & $7,19 \mathrm{E}-03$ & $2.766-07$ & $3.00 \mathrm{E}-07$ & 3.29E-07 & 3.38E-07 \\
\hline U-236 & $1.64 \mathrm{E}-07$ & 9.29E-0s & $3.72 \mathrm{E}-03$ & 1.42E-07 & 1.55E-07 & 1.70E-07 & 1.75E-07 \\
\hline $\mathrm{U}-238$ & $7.27 E-06$ & 4.12E-03 & 0.165 & $6.32 \mathrm{E}-06$ & $6.87 \mathrm{E}-06$ & $7.54 E-06$ & $7.75 E-06$ \\
\hline $\mathrm{Np}-237$ & 5.42E-08 & 3.07E-05 & $1.231-03$ & $8.62 E-09$ & $2.00 \mathrm{E}-08$ & $7.25 E-08$ & $7.25 \mathrm{E}-08$ \\
\hline Pu-238 & $1.59 \mathrm{E}-05$ & $8.99 \mathrm{E}-03$ & 0.360 & 1.49E-05 & 1.SAE-05 & 1.63E-05 & $1.60 \mathrm{E}-05$ \\
\hline Pu-239 & $1.04 E-03$ & 0.590 & 23.6 & 9.73E-04 & 1.01E-03 & $1.07 \mathrm{E}-03$ & 1.11E-03 \\
\hline Pu-240 & $1.47 E-04$ & 8.34E-02 & 3.34 & $1.3 \mathrm{eE}-04$ & $1.42 \mathrm{E}-04$ & $1.52 \mathrm{E}-04$ & 1.57E-04 \\
\hline Pu-241 & 8.91E-04 & 0.505 & 20.2 & 8.35E-04 & 8.63E-04 & $9.20 \mathrm{E}-04$ & 9.47E-04 \\
\hline $\mathrm{Pu}-242$ & 3.85E-09 & $2.18 E-06$ & 8.74E-05 & $3.59 \mathrm{E}-09$ & $372 E-09$ & $398 \mathrm{E}-09$ & $4.11 \mathrm{E}-09$ \\
\hline Am-241 & 4.25E-06 & $2.41 E-03$ & $9.65 \mathrm{E}-02$ & $6.67 \mathrm{E}-07$ & $2.10 \mathrm{E}-06$ & $5.69 \mathrm{E}-\infty 6$ & $7,18 E-05$ \\
\hline $\mathrm{Am}-243$ & $3.97 \mathrm{E}-11$ & $2.25 E-08$ & 9.02E-07 & $6.19 \mathrm{E}-12$ & 1.96E-11 & 5.32E-11 & $6.71 \mathrm{E}-10$ \\
\hline $\mathrm{Cm}-242$ & 8.23E-68 & 4.67E-05 & $1.8 \pi-03$ & $8.06 \mathrm{E}-06$ & 8.13E-08 & $8.30 \mathrm{E}-98$ & $8.30 \mathrm{E}-08$ \\
\hline $\mathrm{Cm}-243$ & $1.89 \mathrm{E}-69$ & 1.07E-06 & 1.28E-0S & 185E-09 & $1.86 \mathrm{E}-09$ & $1.90 \mathrm{E}-09$ & $1.900-09$ \\
\hline $\mathrm{Cm}-244$ & $1,42 E-69$ & 8.04E-07 & 3.22E-0s & $2.20 \mathrm{E}-10$ & $6.98 \mathrm{E}-10$ & 1.90E-09 & $190 \mathrm{E}-09$ \\
\hline Totals: & $\mathbf{M}$ & ysts & $\mathrm{Lr}$ & $95 \mathrm{dr}(\mathrm{M}$ & $67 \mathrm{cla}$ & $\begin{array}{c}6 \mathrm{CI} M \\
\sigma \mathrm{ML}\end{array}$ & $\begin{array}{l}+95 \mathrm{Cl} \\
\mathrm{M} \circ \mathrm{L} \mathrm{L})\end{array}$ \\
\hline Pu & $1.74 \mathrm{E}-02(\mathrm{e} / \mathrm{L})$ & - & 0.393 & $1.63 \mathrm{E}-02$ & 1.66E-02 & $1.00 \mathrm{E}-02$ & $1.85 \mathrm{E}-02$ \\
\hline $\mathrm{U}$ & 9.14E-02 & $1.23 \mathrm{E}+04$ & 994 & $7.95 \mathrm{E}-02$ & 8.65E-02 & $9.50 \mathrm{E}-02$ & $9.75 E-02$ \\
\hline
\end{tabular}

"Crknowns in tenk solids inventory are assigned by Terle Layering Model (TLM) 
HNF-SD-WM-ER-352, Rev. 1

HDW Madel Rev, 4

\begin{tabular}{|c|c|c|c|c|c|c|c|}
\hline \multirow{4}{*}{$\begin{array}{l}\text { Phyoled } \\
\text { Properties }\end{array}$} & \multicolumn{7}{|c|}{ Single-Shell Tank 241-S-112 } \\
\hline & \multicolumn{7}{|c|}{ SMM Composite Inventory Estimate } \\
\hline & & & & & & & \\
\hline & & & & $.95 \mathrm{Cl}$ & $67 \mathrm{CI}$ & $+67 \mathrm{CI}$ & $+95 \mathrm{CI}$ \\
\hline Total SMM Wast & $3.29 \mathrm{E}+06(\mathrm{~kg})$ & (517 k kal) & - & - & - & - & $\ldots$ \\
\hline Heat Load & $4.21(\mathrm{~kW})$ & (1.44E+04 BTU/Ar) & $\ldots$ & 394 & 4.07 & 4.36 & 4.38 \\
\hline Bulk Density" & $1.68(\mathrm{~g} / c)$ & + & - & 1.61 & 1.65 & 1.71 & 17 \\
\hline & & & & & & & \\
\hline Water wt\%t & 29.3 & $-m$ & $=$ & 27.1 & 28.0 & 30.8 & 33.3 \\
\hline TOC we\% C (wet & 0.328 & - & - & 0.293 & 0.310 & 0.346 & 0.362 \\
\hline Rodioloodses & $\mathrm{Cn}, \mathrm{s}$ & $\mathrm{nC/} / \mathrm{s}$ & $\mathrm{Cl}$ & $\begin{array}{l}9 \mathrm{Cl} \\
\text { (Cin) }\end{array}$ & (Cin) & $\begin{array}{l}+67 \mathrm{CI} \\
\text { (CVL) }\end{array}$ & $\begin{array}{r}+95 \mathrm{Cl} \\
(\mathrm{Cu})\end{array}$ \\
\hline H.3 & $2.6 \pi-04$ & 0.159 & 523 & $1.60 \mathrm{E}-04$ & $1.60 \mathrm{E}-04$ & $2.84 E-0.9$ & $2.77 \mathrm{E}-04$ \\
\hline C-14 & 3.41E-05 & 2.03E-02 & 66.8 & $1.17 \mathrm{TE}-05$ & $1.17 \mathrm{E}-0 \mathrm{~S}$ & 3.49E-05 & $3.48 \mathrm{E}-0 \mathrm{~S}$ \\
\hline Ni-59 & $2.33 E-06$ & $1.36 E-03$ & 4.56 & $1.27 \mathrm{E}-06$ & $1.2 \pi-06$ & $2.38 E-06$ & $2.43 \mathrm{E}-0.6$ \\
\hline $\mathrm{Ni}-63$ & $2.26 \mathrm{E}-04$ & 0.134 & 443 & $1.22 \mathrm{E}-04$ & $1.22 \mathrm{E}-24$ & $2.31 E-04$ & $2.37 \mathrm{E}-04$ \\
\hline $\mathrm{Co}-60$ & 3.45E-05 & $2.05 E-02$ & 67.5 & $8.81 E-06$ & $881 E-06$ & 3.50E-05 & $3.55 \mathrm{E}-05$ \\
\hline Se-79 & $3.56 \mathrm{E}-00$ & 2.12E-03 & 6.96 & $2.13 E-16$ & $2.13 E-06$ & $408 \mathrm{E}-06$ & $4.57 \mathrm{E}-06$ \\
\hline Sr-90 & 0.117 & 69.3 & $2.28 E+05$ & 0.110 & 0.113 & 0.120 & 0.124 \\
\hline Y.90 & 0.117 & 69.3 & $2.28 \mathrm{E}+05$ & $6.71 E-02$ & 6.71E-02 & 0.120 & 0.124 \\
\hline $2 \mathrm{r}-93$ & $1.74 E-05$ & $1.03 E-02$ & 34.0 & $1.02 \mathrm{E}-05$ & $1.02 \mathrm{E}-05$ & $2.00 \mathrm{E}-05$ & $2.25 \mathrm{E}-05$ \\
\hline $\mathrm{Nb}-93 \mathrm{~m}$ & 1.29E-05 & $7.66 \mathrm{E}-03$ & 25.2 & $7.87 \mathrm{E}-06$ & $7.87 \mathrm{E}-06$ & $1.47 \mathrm{E}-05$ & $1.648-05$ \\
\hline Tc-99 & 2.43E-04 & 0.145 & 476 & $1.60 \mathrm{E}-04$ & $2.00 E-04$ & $2.86 \mathrm{E}-\mathrm{-04}$ & $3.285-04$ \\
\hline $\mathrm{Ru}-106$ & $5.31 E-09$ & $3.16 \mathrm{E}-06$ & $1.04 \mathrm{E}-02$ & $1.99 \mathrm{E}-99$ & 1.99E-OS & 6.0SE-09 & $6.73 E-09$ \\
\hline Cd-113m & $8.50 E-05$ & 5.05E-02 & 166 & 4.20E-05 & 4.20E-05 & $1.01 \mathrm{E}-04$ & 1.15E-04 \\
\hline $\mathrm{sb}-125$ & $1.41 \mathrm{E}-\mathrm{NA}$ & 8.41E-02 & 27 & $3.03 E-05$ & 3.03E-05 & $1.44 E-04$ & $1.46 \mathrm{E}-04$ \\
\hline$\$ n-126$ & 5.39E-06 & $3.21 E-03$ & 10.5 & $3.24 E-06$ & 3.24E-06 & $6.17 E-06$ & $6.91 \mathrm{E}-06$ \\
\hline-129 & $4.68 \mathrm{E}-07$ & 2.79E-04 & 0.917 & $3.06 \mathrm{E}-07$ & 3.86E-07 & $5.52 E-07$ & $6.32 E-07$ \\
\hline $\mathrm{Cs}-134$ & $1.59 \mathrm{E}-06$ & 9.44E-04 & 3.11 & $1.06 E-06$ & $1.06 E-06$ & 1.71E-06 & $1.82 \mathrm{E}-06$ \\
\hline Cs- 137 & 0.291 & 17 & 5.70E+05 & 0.762 & 0.276 & 0.308 & 0.310 \\
\hline $\mathrm{Ba}-137 \mathrm{~m}$ & 0.276 & 164 & $5.39 E+05$ & 0.206 & 0.206 & 0.291 & 0.293 \\
\hline Snt-151 & $1.26 \mathrm{E}-02$ & 7.46 & $2,46 \mathrm{E}+04$ & 7. S4E-03 & 7.5AE-03 & $1.44 E-02$ & $1.61 \mathrm{E}-02$ \\
\hline Eu-152 & $3.12 E-06$ & $1.85 \mathrm{SE}-03$ & 6.10 & $1.34 E-\infty$ & 1.34E-O6 & $3.18 \mathrm{E}-06$ & $3.24 \mathrm{E}-06$ \\
\hline Eu-154 & S.SAE-9M & 0.329 & $1.08 E+03$ & $1.962-04$ & 1.96E-04 & 6.BAE-A4 & 7.35E-04 \\
\hline Eu-155 & 1.79E- -04 & 0.106 & 330 & 7.34E-05 & 7.34E-05 & 1.BAE-04 & $1.88 \mathrm{E}-04$ \\
\hline RA-226 & $1.66 \mathrm{E}-10$ & $9.88 E-08$ & $3.25 E-04$ & $1.128-10$ & $1.22 \mathrm{E}-10$ & $1.94 \mathrm{E}-10$ & $2.21 \mathrm{E}-10$ \\
\hline$R a-228$ & $6.02 \mathrm{E}-08$ & 3.SRE-05 & 0.118 & 2.33E-08 & 4.14E-08 & B.19E-08 & $1.05 \mathrm{E}-07$ \\
\hline Ac-227 & $1.00 \mathrm{E}-09$ & 5.96-07 & $196 \mathrm{E}-03$ & 6.87E-10 & $7,45 \mathrm{E}-10$ & $1.16 \mathrm{E}-09$ & $1.26 \mathrm{E}-09$ \\
\hline $\mathrm{Pg}-231$ & 4.43E-09 & 2.63E- 06 & B.67E-03 & $3.00 \mathrm{E}-09$ & $3.00 \mathrm{E}-09$ & $4.95 \mathrm{E}-09$ & $5.45 \mathrm{E}-09$ \\
\hline Th-229 & $1.43 E-09$ & 8.52E-07 & $2.60 \mathrm{E}-03$ & $6.29 \mathrm{E}-10$ & $1.02 E-09$ & $1.91 \mathrm{E}-09$ & $2.42 \mathrm{E}-09$ \\
\hline Th-232 & 4.05E-09 & 2.41E-06 & $7.936-03$ & $2.03 \mathrm{E}-09$ & $3.02 \mathrm{E}-09$ & $5.09 \mathrm{E}-09$ & $6.08 \mathrm{E}-09$ \\
\hline U-232 & $3.63 \mathrm{E}-07$ & $2.165-04$ & 0.710 & $2.16 E-07$ & 2.EAE-07 & $4.50 \mathrm{E}-07$ & $5.42 \mathrm{E}-07$ \\
\hline U-233 & $1,39 E-06$ & 8.27E-04 & 2.72 & 8.29E-07 & $1.10 E-\infty 6$ & $1.72 \mathrm{E}-06$ & $2.06 \mathrm{E}-06$ \\
\hline U-234 & 8.70E-07 & 5.18E-04 & 1.70 & $7.96 \mathrm{E}-07$ & 8.31E-07 & 9.13E-07 & 8.95E-07 \\
\hline U.235 & $3.60 \mathrm{E}-08$ & 2.14E-05 & $7.0 \mathrm{se}-102$ & $3.2 \mathrm{gE}-08$ & $3.43 E-08$ & $3.78 \mathrm{E}-08$ & $3.70 \mathrm{E}-08$ \\
\hline U-236 & $2.4 \pi-08$ & 1.47-05 & $4.83 \mathrm{E}-02$ & 2.35E-OB & $2.41 E-08$ & $2.54 E-08$ & $2.54 \mathrm{E}-0 \mathrm{~s}$ \\
\hline U.238 & $9.068-07$ & $5.39 \mathrm{E}-0.4$ & 1.77 & 8.31E-07 & $8.66 \mathrm{E}-07$ & $9.50 \mathrm{E}-07$ & $9.29 \mathrm{E}-07$ \\
\hline Np-237 & $9.63 \mathrm{E}-07$ & $3.72 E-01$ & 1.88 & $6.09 \mathrm{E}-07$ & $8.23 E-07$ & $1,10 \mathrm{E}-06$ & $1.24 \mathrm{E}-06$ \\
\hline Pu-238 & 1.19E-06 & $7.05 E-04$ & 2.32 & $8.64 \mathrm{E}-07$ & $1.02 E-06$ & $1.35 \mathrm{E}-66$ & $1.51 \mathrm{E}-06$ \\
\hline Pu-239 & 4.45E-05 & $2.655-02$ & 87.1 & $3.63 E-05$ & 4.03E-05 & $4.87 \mathrm{TE}-05$ & $5.27 \mathrm{E}-05$ \\
\hline Pu-240 & $7.28 \mathrm{E}-06$ & $4.33 \mathrm{E}-03$ & 14.3 & $5.75 E-06$ & $6.50 E-06$ & $8.06 \mathrm{E}-06$ & $8.81 E-06$ \\
\hline Pu-241 & $7.22 E-05$ & 4.6SE-02 & 153 & $9.64 \mathrm{E}-05$ & $6.71 \mathrm{E}-0 \mathrm{~s}$ & $8.94 E-05$ & $1.00 E-04$ \\
\hline $\mathrm{Pu}-242$ & $4.23 \mathrm{E}-10$ & 2.51E-07 & E. $2 \pi-04$ & $292 \mathrm{E}-10$ & $3.56 \mathrm{E}-10$ & $4.89 \mathrm{E}-10$ & $5.54 E-10$ \\
\hline Am-241 & $5.75 E-05$ & $3.42 E-02$ & 112 & $4.23 \mathrm{E}-05$ & 4.97t-05 & $6.53 \mathrm{E}-05$ & $7.27 \mathrm{E}-05$ \\
\hline Am-243 & $1.61 \mathrm{E}-09$ & $9.55 \mathrm{E}-07$ & $3.14[-03]$ & $1.13 E-09$ & $1.36 \mathrm{E}-99$ & $186 E_{-19}$ & $2.11 \mathrm{E}-09$ \\
\hline $\mathrm{C}_{\mathrm{m}-242}$ & 1. LIE- -07 & 6.59E-05 & 0.217 & $3.808-08$ & $3.80 \mathrm{E}-\mathbf{0 8}$ & 1.13E-07 & 1.15E-07 \\
\hline $\mathrm{Cm}-243$ & $9.88 \mathrm{E}-09$ & $5.87 E-06$ & $1.93 \mathrm{E}-02$ & $2.93 E-09$ & $2.93 E-09$ & $1.01 E-\infty 8$ & $1.03 E-08$ \\
\hline $\mathrm{Cm}-244$ & $1.09 E-07$ & $6.46 \mathrm{E}-05$ & 0.213 & $3,205-08$ & 3.70E-08 & $1.33 \mathrm{E}-07$ & 1.48E-07 \\
\hline Totels & $\mathbf{M}$ & ends & lon & 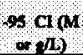 & $\begin{array}{c}67 \mathrm{Cl}(\mathrm{M} \\
\text { or } \% / L)\end{array}$ & $\begin{array}{c}+67 \mathrm{Cl}(\mathrm{M} \\
\text { orfol })\end{array}$ & $\begin{array}{c}+9 s \mathrm{Ct} \\
\mathrm{M} \text { ar }\end{array}$ \\
\hline $\mathrm{Pu}$ & $5.82 \mathrm{E}-\mathrm{OA}(\mathrm{N} / \mathrm{L})$ & - & 1.14 & $4.26 \mathrm{E}-04$ & S.03E-94 & $6.61 \mathrm{E}-0 \mathrm{~A}$ & $7.37 \mathrm{EA4}$ \\
\hline $\mathrm{U}$ & $1.03 \mathrm{E}-02$ & $1.458+03$ & $4.79 \mathrm{E}+03$ & $9.32 \mathrm{E}-03$ & $9.77 \mathrm{E}-03$ & $1.08 E-02$ & $1.06 \mathrm{E}-02$ \\
\hline
\end{tabular}

† Water wi\% derived from the difference of density and total dissolved species. 
HNF-SD-WM-ER-352, Rev. 1

HDW Model Rev. 4

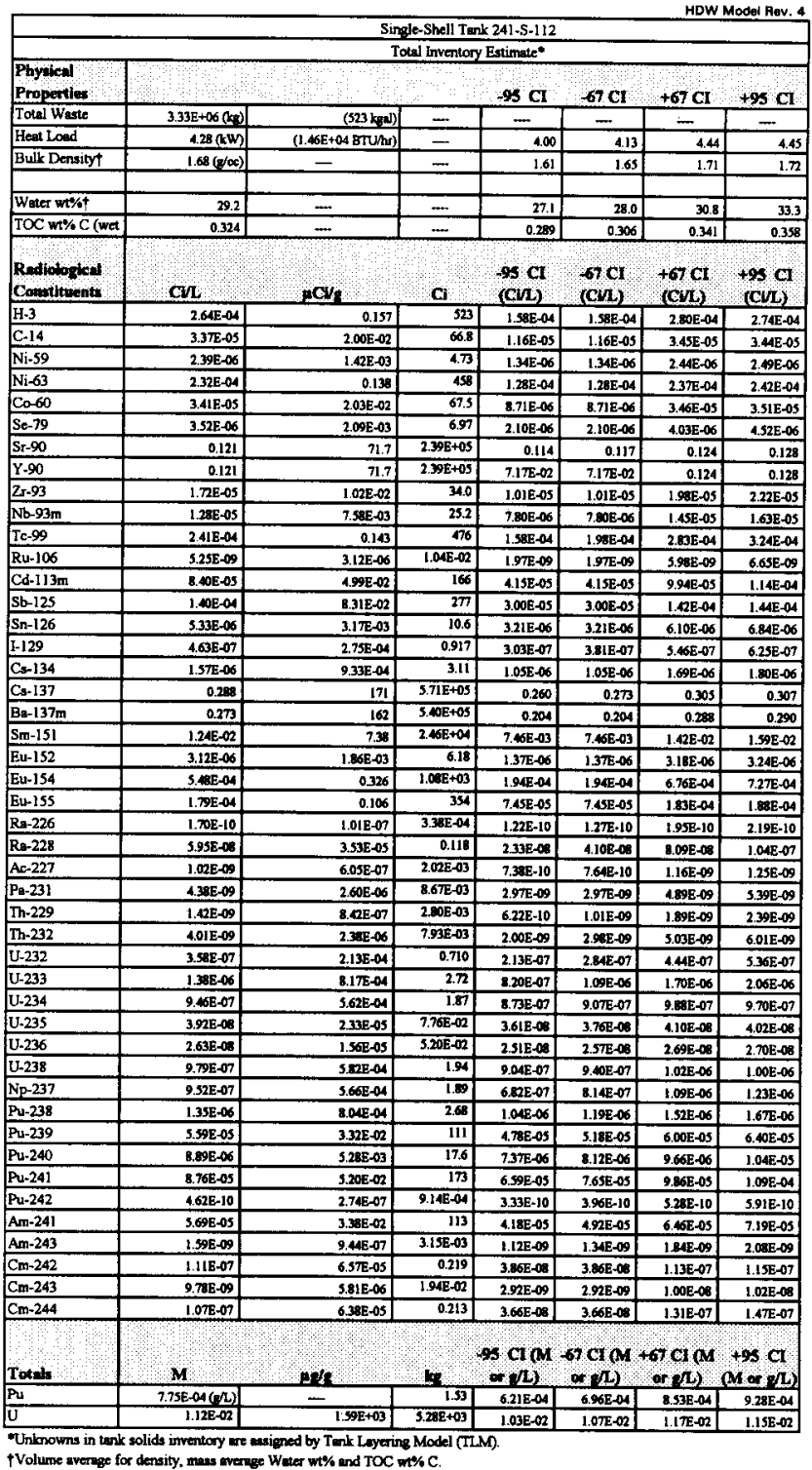




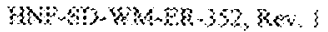

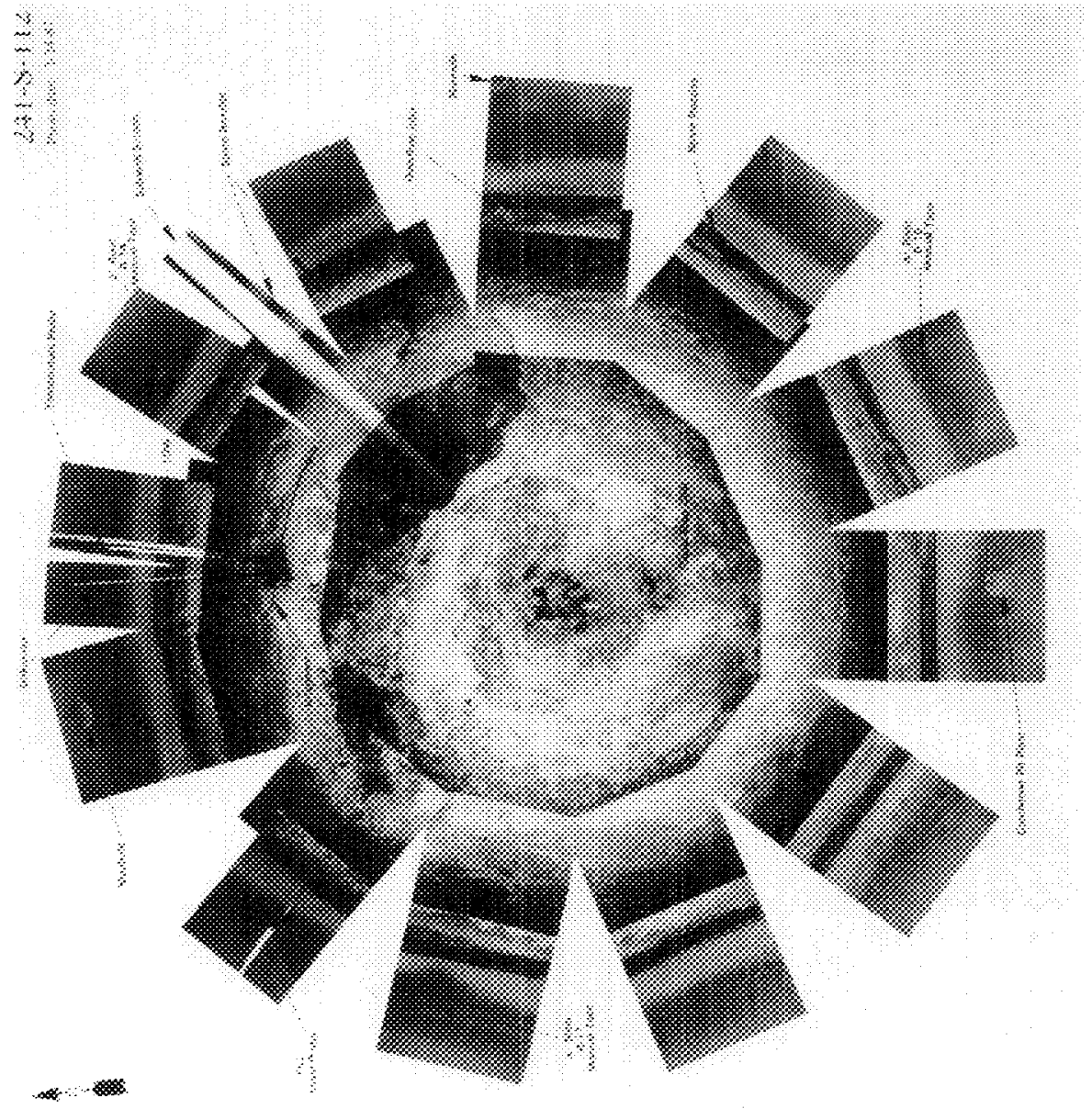




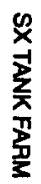


The SX Tank Farm is located in the 200 West Area west of Beliot Avenue and south of 13th Street. The farm contains fifteen 100 series, dish bottom design, single-shell tanks built in 1953 and 1954. The 100 series tanks are 75 feet in diameter with an operating capacity of $1,000,000$ gallons The tanks were designed to store boiling waste. The tanks contents were allowed to boil for one to five years at a fluid temperature up to $250^{\circ} \mathrm{F}$. The fifteen tanks were constructed at different elevations with connecting overflow lines that allowed waste to cascade from tank to tank. The tank farm has five cascades of three tanks each. For more information on the design, construction, and waste contents of the tanks, refer to the SX Tank Farm Supporting Document, HNF-SD-WM-ER-324, Rev. 1 (Brevick et al., 1997b).

The SX Tank Farm tanks were the first tanks designed to store self-boiling wastes. Tanks 241-SX-101 through -106 started operations in 1954 and began receiving REDOX (S Plant) plant salt waste and first-cycle condensate. The other nine tanks (241-SX-107 through -115) were not released for use until late 1955 when they began receiving REDOX boiling high-level waste and salt waste (Leach and Stahl, 1993). The primary additions to the tanks were REDOX high-level waste (Rl and R2), REDOX cladding waste (CWR1), REDOX saltcake (RsltCk), partial neutralization feed (NIT), diatomaceous earth (DE), and water (WTR) (Agnew et al., 1995).

Concrete or diatomaceous earth has been added to various tanks in the SX farm. Tank 241-SX107 contains 41 small bottles of neutralized waste from $100 \mathrm{~F}$, each bottle contained less than $1 \mathrm{~g}$ (gram) of ${ }^{239} \mathrm{Pu}$ (Plutonium 239). Tank 241-SX-110 contains 16 bottles totaling $204 \mathrm{~g}{ }^{239} \mathrm{Pu}$ and $6 \mathrm{~g}$ of enriched uranium (Leach and Stahl, 1993).

The following table presents a summary of the 241-SX Tank Farm. Refer to the glossary for waste type terminology.

\section{1-SX TANK FARM SUMMARY}

\begin{tabular}{|c|c|c|c|}
\hline \multicolumn{2}{|c|}{ TANK FARM DESCRIPTION } & \multicolumn{2}{|c|}{ WASTE VOLUME (HANLON 1996i) } \\
\hline Shell Type & Single & Total Waste Volume & $4,419,000 \mathrm{gal}$ \\
\hline Number of Tanks & 15 & Waste Type & DSSF, DC, NCPLX \\
\hline Construction Date & 1953.1954 & Drainable Interstitial Liquids & $1,298,000 \mathrm{gal}$ \\
\hline \multicolumn{2}{|c|}{ INTEGRITY } & Pumpable Liquids & $1,445,000 \mathrm{gal}$ \\
\hline \multirow[t]{2}{*}{ Sound } & $101,102,103,105$ & Saltcake & $3,102,000 \mathrm{gal}$ \\
\hline & 106 & Sludge & $1,254,000 \mathrm{gal}$ \\
\hline \multirow[t]{3}{*}{ Assumed Leaker } & $104,107,108,109$ & Supernatant & $63,000 \mathrm{gal}$ \\
\hline & $110,111,112,113$ & \multicolumn{2}{|c|}{ WATCH LISTS } \\
\hline & 114,115 & \multirow[t]{2}{*}{ Hydrogen } & $101,102,103,104$ \\
\hline \multicolumn{2}{|c|}{ TANK VOLUMES } & & $105,106,109$ \\
\hline \multirow{3}{*}{\multicolumn{2}{|c|}{$15 @ 1,000,000 \mathrm{gal}$}} & Organics & 103,106 \\
\hline & & High Heat & none \\
\hline & & Ferrocyanide & none \\
\hline
\end{tabular}


The historical characterization and waste inventory information for each tank is assembled into a set of tables, sketches, graphs, and interior tank montage. The set consists of the following for each tank in SX Tank Farm:

- Tank Summary table

- Waste and Level History sketch

- Riser Configuration sketch

- Tank Layer Model Estimate graph

- Total Inventory Estimate tables

- TLM Solids Composite Inventory Estimate tables

- $\quad$ SMM Composite Inventory Estimate tables

- Interior tank montage

The supporting document for SX Tank Farm provides backup data and further explanation of the above tables, sketches, and graphs. 


\section{SX TANK FARM}

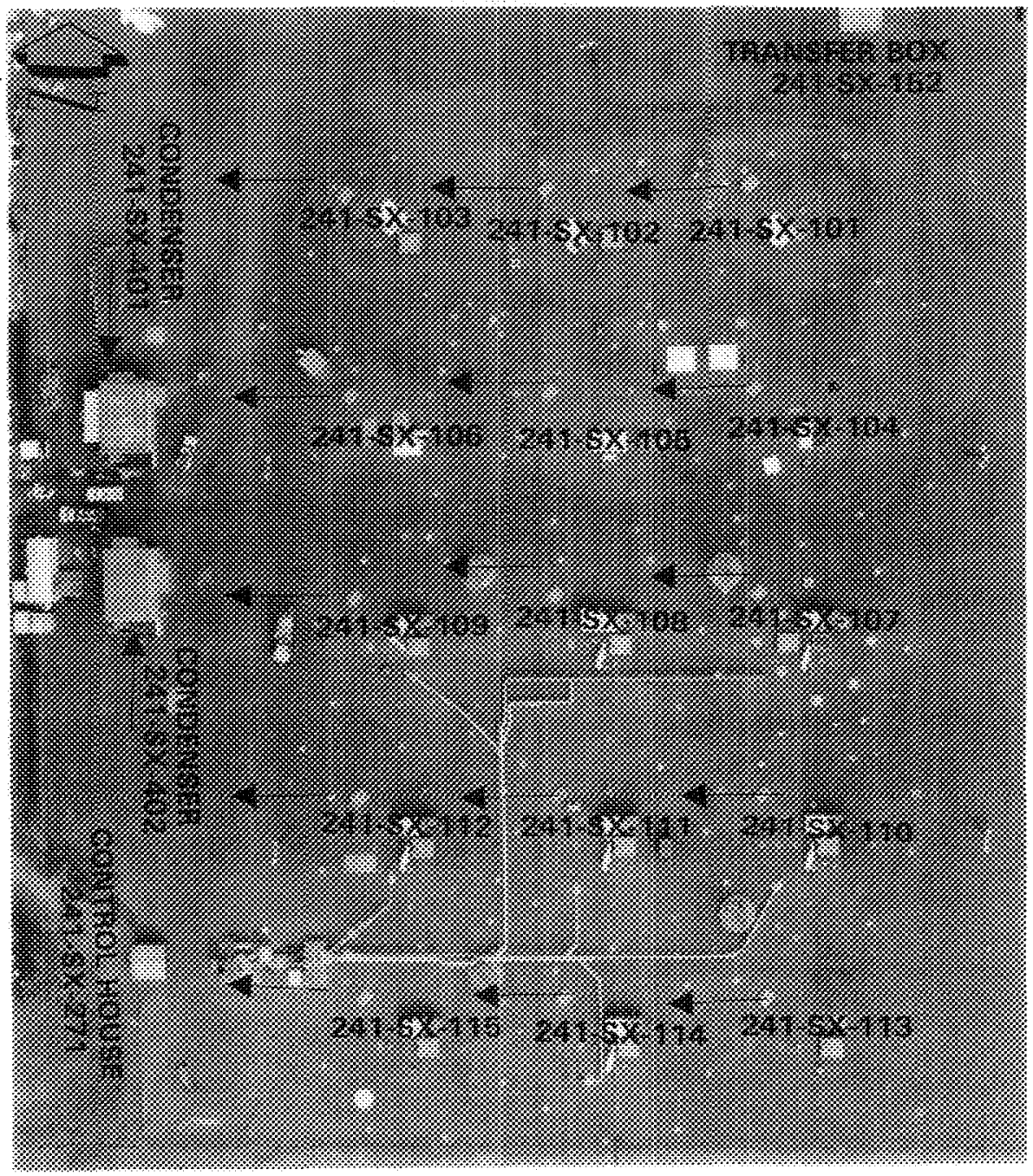


TANK 241-SX-101 SUMMARY

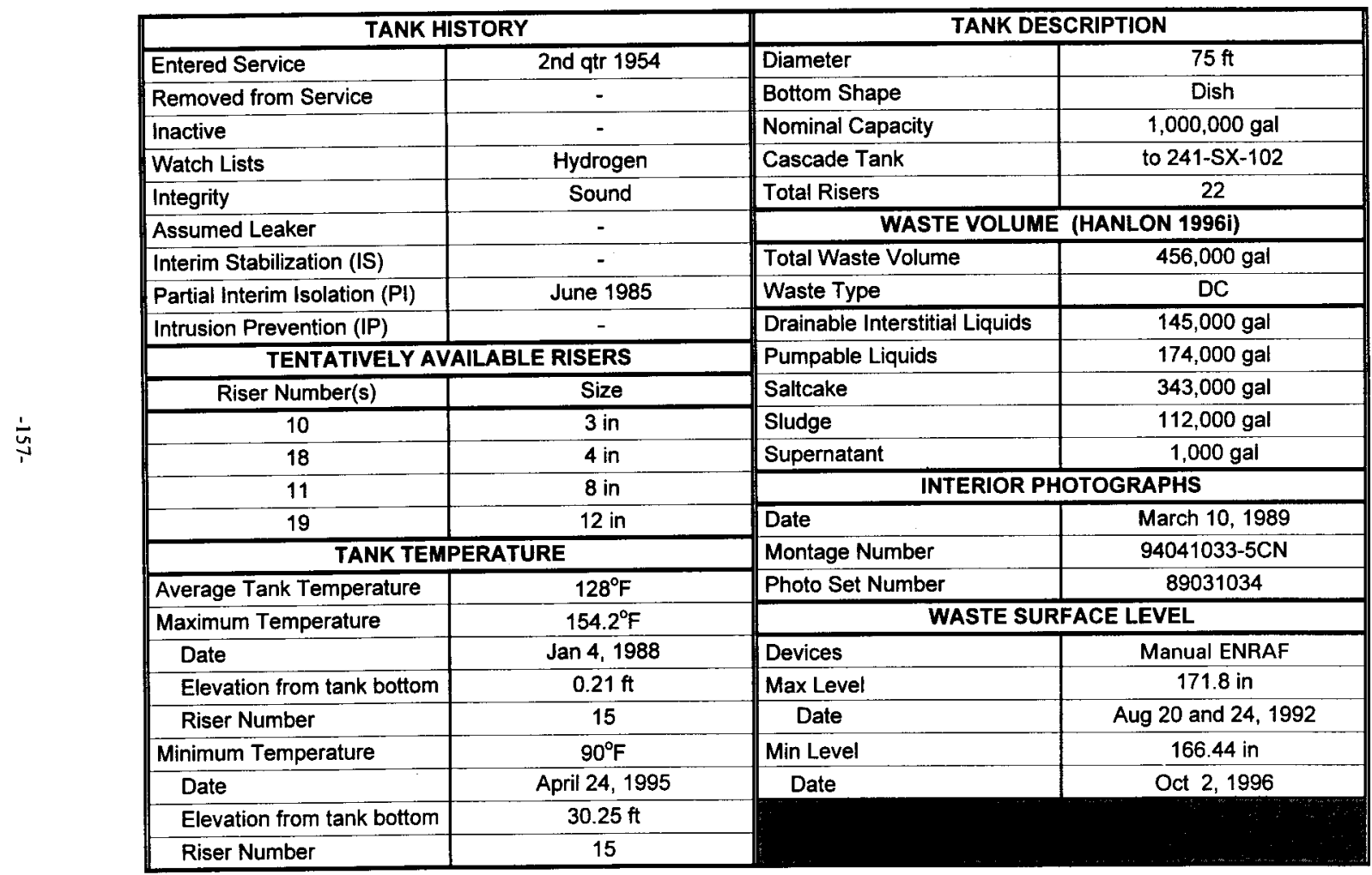




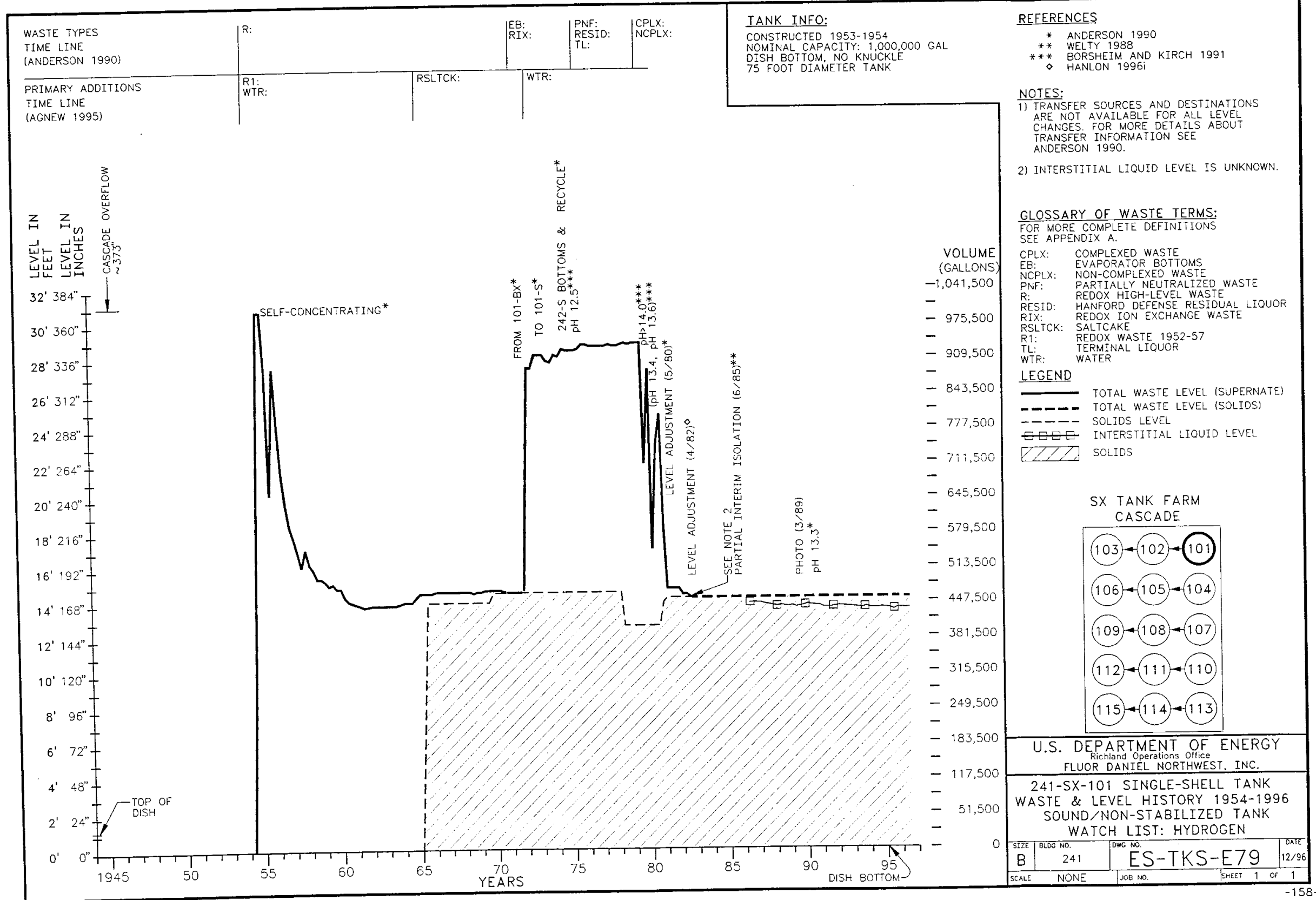



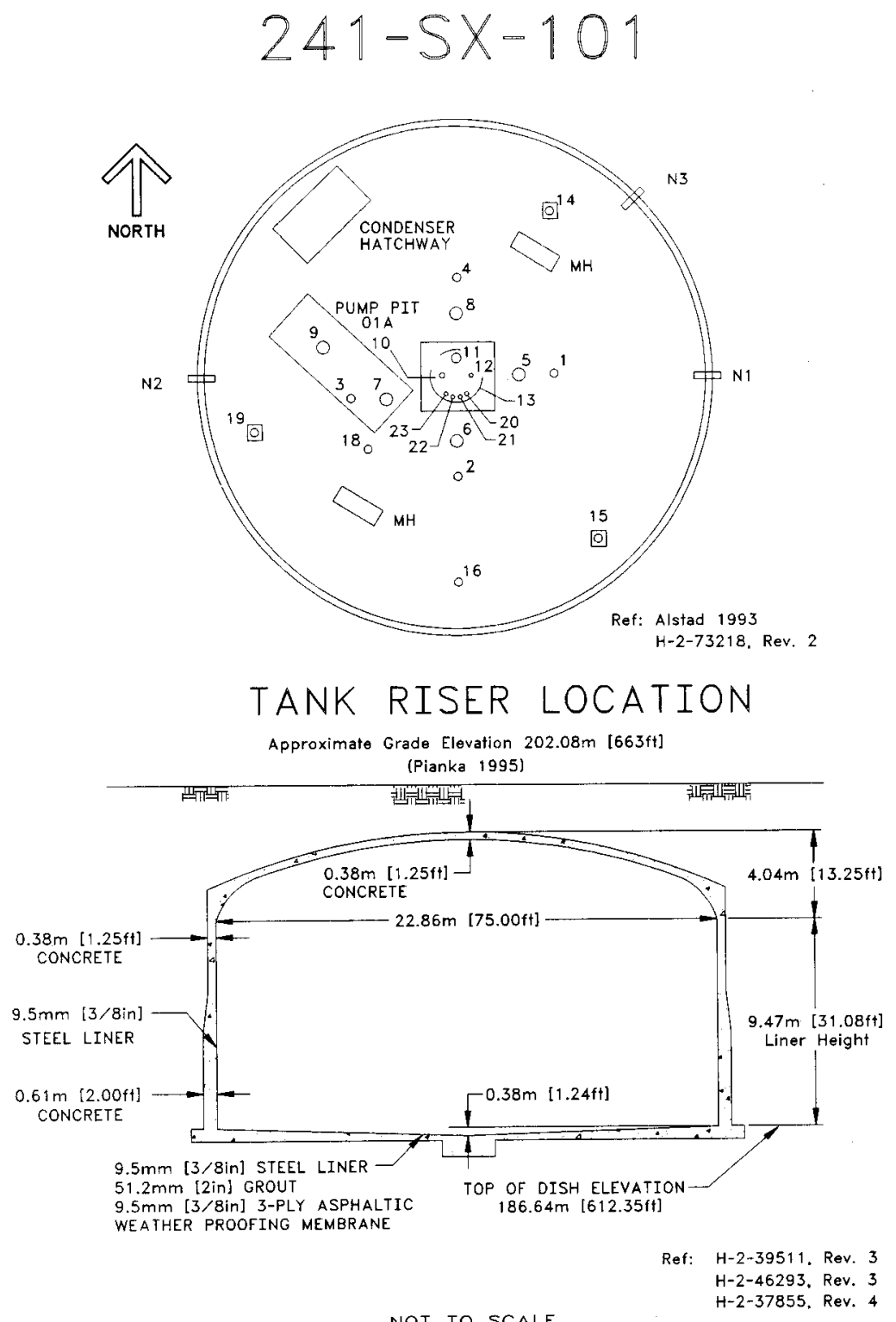

NOT TO SCALE

46293. Rev. 3

$-159-$ 


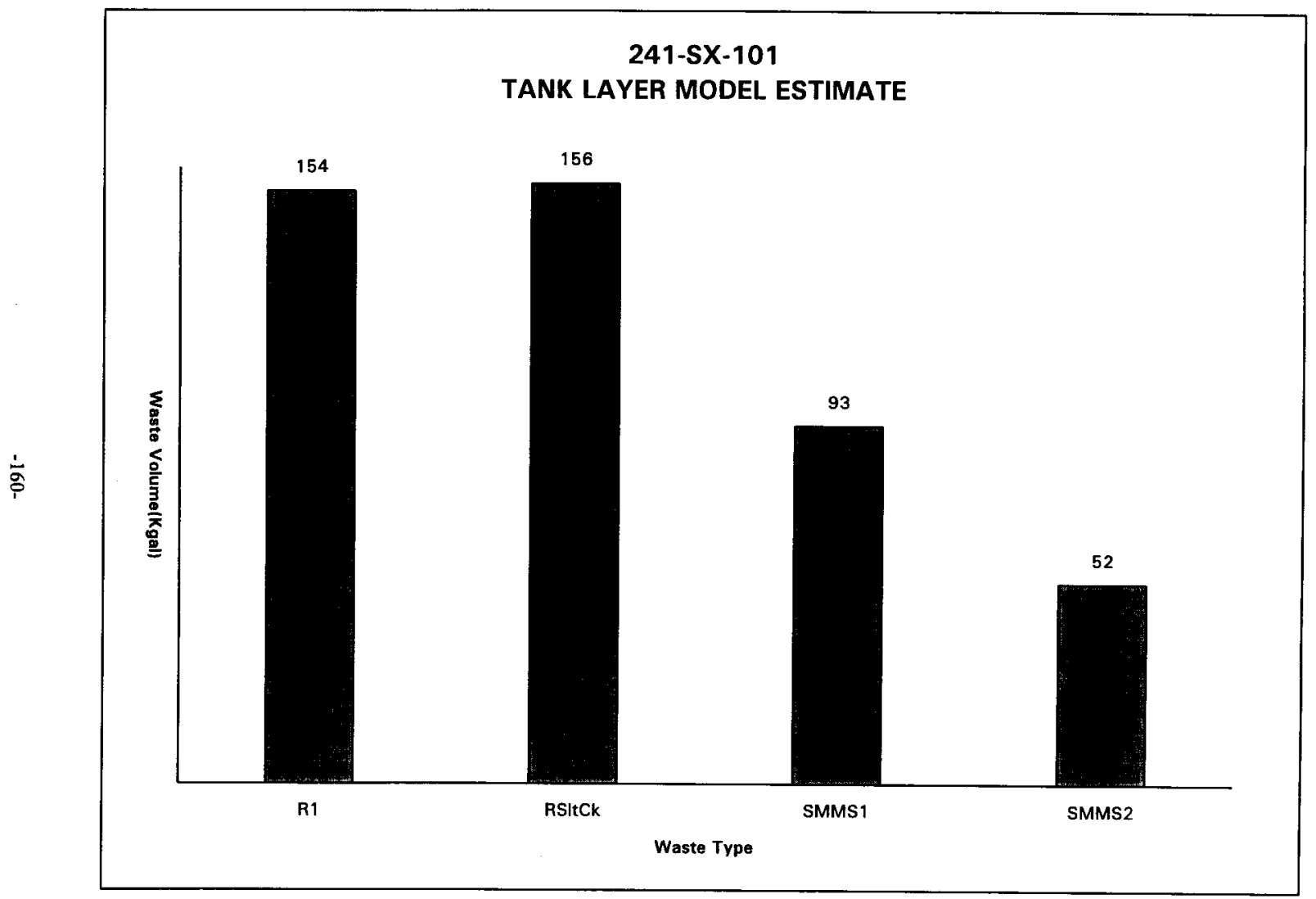


HNF-SD-WM-ER-352, Rev. 1

HDW Model Rov. 4

\begin{tabular}{|c|c|c|c|c|c|c|c|}
\hline & \multicolumn{7}{|c|}{ Single-Shell Tank 24!-SX-10! } \\
\hline . & \multicolumn{7}{|c|}{ TLM Solids Composite Inventory Estimate* } \\
\hline $\begin{array}{l}\text { Physical } \\
\text { Propertios }\end{array}$ & & & & $.95 \mathrm{Cl}$ & $-67 \mathrm{CI}$ & $+67 \mathrm{CI}$ & $+9 \mathrm{CH}$ \\
\hline Total TLM Wa & $2.04 \mathrm{E}+06(\mathrm{~kg})$ & $(310 \mathrm{~kg} a)$ & $\ldots$ & - & $\cdots$ & $\ldots$ & - \\
\hline Heat Load & $5.74(\mathrm{~kW})$ & (1.96E+04 BTU/Mr) & $=$ & 4.70 & 5.31 & 6.02 & 6.15 \\
\hline Bulk Density & $1.73(\mathrm{~g} / \mathrm{cc})$ & $-m$ & - & 1.60 & 1.62 & 1.91 & 1.98 \\
\hline Void Fraction & 0.661 & - & $=$ & 0.425 & 0.503 & 0.761 & 0.764 \\
\hline Water wt\% & 30.8 & - & $=$ & 16.6 & 21.2 & 38.4 & 40.9 \\
\hline TOC wt\%C $(w$ & $2.61 E-03$ & $\cdots$ & - & $2.29 \mathrm{E}-03$ & $2.37 \mathrm{E}-03$ & $2.81 \mathrm{E}-03$ & $3.04 \mathrm{E}-03$ \\
\hline $\begin{array}{l}\text { Chonaled } \\
\text { Constituents }\end{array}$ & mole/L & ppm : _ & k $\mathrm{g}$ & $\begin{array}{c}95 \mathrm{Cl} \\
\text { (inole/L) }\end{array}$ & $\begin{array}{l}67 \mathrm{Cl} \\
\text { (mole/L) }\end{array}$ & $\begin{array}{l}+67 \mathrm{CT} \\
(\mathrm{mol} / \mathrm{d})\end{array}$ & $\begin{array}{r}+95 \mathrm{CI} \\
\text { (mole/L) }\end{array}$ \\
\hline $\mathrm{Nat}$ & 10.8 & $1.43 \mathrm{E}+05$ & $2.92 \mathrm{E}+05$ & 8.29 & 8.54 & 14.1 & 15.8 \\
\hline $\mathrm{Alj}+$ & 5.18 & 8.06E+104 & $1.64 E+05$ & 419 & 4.48 & 5.93 & 6.69 \\
\hline Fe3+ (totn Fe) & 0.512 & $1.65 \mathrm{E}+04$ & $3.36 \mathrm{E}+04$ & 0.503 & 0.507 & 0.517 & 0.521 \\
\hline $\mathrm{Ct} 3+$ & 0.444 & $1.33 \mathrm{E}+0 \mathrm{~A}$ & $2.71 E+04$ & 0.272 & 0.354 & 0.673 & 0.940 \\
\hline Bi3+ & 3.46E-46 & 0.417 & 0.848 & $2.92 \mathrm{E}-06$ & $3,18 \mathrm{E}-06$ & $3.81 \mathrm{E}-06$ & $4.19 \mathrm{E}-06$ \\
\hline $\mathrm{La3+}$ & B.52E-12 & $6.82 \mathrm{E}-07$ & $1.39 \mathrm{E}-06$ & $7.46 \mathrm{E}-12$ & $8.06 \mathrm{E}-12$ & $9,42 \mathrm{E}-12$ & 1.03E-11 \\
\hline $\mathrm{Fg}_{2+}^{+}$ & 5.A3E-07 & $6.28 \mathrm{E}-02$ & 0.128 & $4.81 \mathrm{E}-07$ & 5.11E-07 & 5.98E- -07 & 6.56E-07 \\
\hline $\mathrm{Zr}(4 \mathrm{ZrO}(\mathrm{OH}) 2$ & 3.45E-07 & $1.82 \mathrm{E}-02$ & $3.69 \mathrm{E}-02$ & $3.17 \mathrm{E}-07$ & 3.31E-07 & 3.62E-07 & 3.90E-07 \\
\hline $\mathrm{Pb2+}$ & 8.61E-05 & 10.3 & 20.9 & 4.61E-0S & $6.56 \mathrm{E}-05$ & $1.07 \mathrm{E}-04$ & $1.27 \mathrm{E}-04$ \\
\hline $\mathrm{Ni24}$ & $3.28 \mathrm{E}-02$ & $1.11 \mathrm{E}+03$ & $2.26 \mathrm{E}+03$ & $258 \mathrm{E}-02$ & $2.97 \mathrm{E}-02$ & 3.51E-02 & $3.47 \mathrm{E}-02$ \\
\hline $5 \sqrt{2+}$ & 0 & 0 & 0 & 0 & 0 & 0 & 0 \\
\hline Mnst & 2.52E-05 & 0.797 & 1.62 & 1.80E-05 & 2.15E-05 & $2.89 \mathrm{E}-05$ & 3.25E-05 \\
\hline $\mathrm{C} 2+$ & 0148 & $3.42 \mathrm{E}+03$ & 6.95E+03 & 0.111 & 0.129 & 0.167 & 0.185 \\
\hline$\overline{\mathrm{K}+}$ & $2.17 \mathrm{E}-02$ & 49 & 995 & $1.6 \pi-02$ & $1.87 \mathrm{E}-02$ & 2.37E-02 & $247 \mathrm{E}-02$ \\
\hline $\mathrm{OH}-$ & 22.1 & $2.17 \mathrm{E}+05$ & 441E+05 & 17.6 & 19.0 & 25.4 & 28.6 \\
\hline NO3- & 5.27 & I. $.8 B E+05$ & 3.13E+05 & 2.67 & 2.67 & 933 & 11.5 \\
\hline NO2- & 1.67 & 4.42E+O4 & 8.99E+04 & 1.01 & 1.24 & 2.01 & 2.01 \\
\hline Co32. & 0.150 & $5.20 \mathrm{E}+03$ & $1.06 \mathrm{E}+04$ & 0.113 & 0.132 & 0.169 & 0.187 \\
\hline PO43- & 2.24E-04 & 12.3 & 24.9 & 2.08E-04 & $2.17 \mathrm{E}-04$ & $2.33 \mathrm{E}-0 \mathrm{-1}$ & 2.53E-04 \\
\hline SO42- & 2.55E-02 & $1.41 \mathrm{E}+03$ & $2818+03$ & 209E- 02 & 2.28E-02 & $2.74 E-02$ & 2.92E-02 \\
\hline Si (n SiO32-) & $6.94 \mathrm{E}-02$ & $1.12 \mathrm{E}+03$ & $229 \mathrm{E}+03$ & 4,54E-02 & $6.03 \mathrm{E}-02$ & $7.86 \mathrm{E}-02$ & 3.74E-02 \\
\hline $\mathrm{F}$ - & $1.78 \mathrm{E}-04$ & $1 . \% 5$ & 3.98 & $1.52 \mathrm{E}-04$ & 1.69E-O4 & 1.96E-04 & 2.11E-04 \\
\hline $\mathrm{Cl}-$ & $9.28 \mathrm{E}-02$ & $1.90 E+03$ & 3.86E+03 & 6.18E-02 & $7.69 \mathrm{E}-02$ & 0.102 & 0.145 \\
\hline C6H5O73- & $1.84 \mathrm{E}-04$ & 20.1 & 40.9 & $1.78 \mathrm{E}-04$ & 1.81E-04 & $1.92 \mathrm{E}-04$ & 2.09E-04 \\
\hline EDTA4 & $7.15 E-06$ & 119 & 2.43 & 5.05E-06 & $6.21 E-06$ & 8.45E-00 & $9.72 \mathrm{E}-06$ \\
\hline HEDTA3. & 5.96E-06 & 0.942 & 1.92 & $1.91 \mathrm{E}-06$ & $4.10 \mathrm{E}-06$ & $7.96 \mathrm{E}-06$ & $9.90 \mathrm{E}-06$ \\
\hline Blycolitite- & $2.60 \mathrm{E}-04$ & 11.3 & 22.9 & $1.41 \mathrm{E}-04$ & $206 \mathrm{E}-04$ & $3.23 \mathrm{E}-04$ & 3.84E-04 \\
\hline ecetute- & $2.70 \mathrm{E}-05$ & 0.919 & 1.87 & 2.61E-05 & $2.66 \mathrm{E}-05$ & $2.67 \mathrm{E}-05$ & $3.0 T \mathrm{E}-05$ \\
\hline oxalate?- & 1.12E-11 & $5.66 E-07$ & $1.15 \mathrm{E}-06$ & $9.89 \mathrm{E}-12$ & $1.05 \mathrm{E}-41$ & $1.23 \mathrm{E}-11$ & $1.35 \mathrm{E}-11$ \\
\hline DBP & $1,63 \mathrm{E}-04$ & 19.8 & 40.3 & $1.53 \mathrm{E}-04$ & $1.59 \mathrm{E}-04$ & 1.7TE-04 & $1,91 \mathrm{E}-04$ \\
\hline butanol & $1.63 \mathrm{E}-04$ & 6.99 & 14.2 & 1.53E-OA & $1.59 \mathrm{E}-04$ & $1.77 \mathrm{E}-04$ & $1.91 \mathrm{E}-04$ \\
\hline $\mathrm{NH3}$ & 0.145 & $1.42 \mathrm{E}+03$ & $2.89 \mathrm{E}+03$ & $5.07 \mathrm{E}-02$ & $6.41 E-02$ & 0.152 & 0.159 \\
\hline Fe(CN)64 & 0 & 0 & 0 & 0 & 0 & 0 & 0 \\
\hline
\end{tabular}

"Unknowns in tank solide inventory are ansigned by Tenk Layering Model (TLM). 


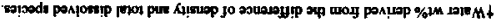

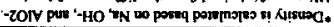

\begin{tabular}{|c|c|c|c|c|c|c|c|}
\hline 0 & 0 & 0 & 0 & 0 & 0 & 0 & $\rightarrow$ (ND) \\
\hline zo-gzes & $20-399$ & $20-959^{\prime} \mathrm{E}$ & 20-A9T: & res & LES & $20-360 t$ & [HN \\
\hline & & & & & & & \\
\hline $20-9501$ & co-70t 6 & $50-960 \%$ & EOFIOC 2 & 598 & ols & c0-506: & pounng \\
\hline 20-950? & 50-9016 & $50-960^{\circ}$ & CO-Fof'L & co+acol & $20+9 s+1$ & E0-906: & ded \\
\hline so-azt 2 & So 3622 & so-a10' & sotar6 I & sol & $|v|$ & so-alliz & $-2010=0$ \\
\hline c0-76⿷ ⿷ & E0-3et' & $50-309^{-2}$ & E0-G) $z$ & +16 & 151 & E0-59: 2 & $\rightarrow$ Intoos \\
\hline Z0-ats's & torazst & worate & 200507 & $\mathrm{CO}+\mathrm{BO} L 1$ & E0+בLEC $z$ & zo-golt & 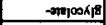 \\
\hline $20 \mathrm{gsz} 2$ & 207\% I & $50-1866$ & EO-Gatc & $50+356:$ & $\varepsilon 0+\mathrm{Bz} / z$ & torgst & -EYLOSHH \\
\hline $20 \mathrm{r}$ LII & รO-GEE' 6 & corant & to-3II $\tau$ & $60+360 !$ & $60+9551$ & co-aces & $\rightarrow V \perp a A$ \\
\hline to-artl & $20-3<51$ & $20 \cdot 3 a z !$ & $20-3121$ & $60+a 8 \varepsilon 1$ & $50+9561$ & 20 -get 1 & $-\angle \angle O S H 90$ \\
\hline szl'o & mio & 2110 & 0110 & $60+\operatorname{agc} z$ & $50+75 z: t$ & 6110 & $-[3$ \\
\hline 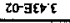 & $20-3 n t c$ & $20-926 z$ & zo-gIsZ & SIE & ow & zO-g00 I & -4 \\
\hline $20-365$ & $20-9<0$ & 20 aIt & $20-760 \mathrm{C}$ & Ins & 218 & $20-$ anc $c^{\prime} \varepsilon$ & 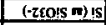 \\
\hline 0 trio & 9210 & 2010 & $20-966 \mathrm{E}$ & $60+3019$ & $60+3 E 58$ & SIIO & -zros \\
\hline $20-300 t$ & $20-30 e^{2}$ & zo-azac & 20-GI9' & $10+20, z$ & $\mathrm{EO}+300 \mathrm{C}$ & to-rox't & $-\mathrm{thOS}$ \\
\hline 6120 & 9120 & 1610 & ro & $60+F 2<9$ & $50+36 E 6$ & 2020 & $-2\{00$ \\
\hline Ort & $|c|$ & ol't & 101 & DO+FLOE & $6+36 z_{0}$ & $I z \mid$ & $20 N$ \\
\hline 962 & $n z$ & EEZ & $\pi z \pi$ & Dotafec ह & $50+3511$ & $0+z$ & $-\mathrm{EON}$ \\
\hline$t z s$ & $0 z ' s$ & $L t$ & $95+$ & $10+709+$ & $60+3+5 \cdot 9$ & 86t & $-\mathrm{HO}$ \\
\hline 20-astic & 20-a10E'E & $20-500 \mathrm{C}$ & Z0-3L8 2 & 189 & ZS6 & zo-gsIE & $+x$ \\
\hline $20-39 L^{\prime} \mathrm{I}$ & $20-3691$ & $20-35 s 1$ & zo- $36 t^{\circ} 1$ & 6Sf & $20 s$ & $20-329.1$ & +20 \\
\hline co-352? & $50-360 \tau$ & EO-79S1 & Co-3tE I & $\sin$ & $19 L$ & c0-76CI & $++4 W$ \\
\hline 0 & 0 & 0 & 0 & 0 & 0 & 0 & $+2 \pm S$ \\
\hline E0-7ulc & EOAET' & Co-grot & 50-7L6' & 001 & $0+1$ & EO-Zol't & $+Z ! N$ \\
\hline 60-395"s & bo-301's & 60-79z: & borate & ates & $\tau s L$ & wo-gocts & $+29 \mathrm{~d}$ \\
\hline 100-392I & Do- 3021 & 109601 & $00-390^{\circ} \mathrm{t}$ & ns & 912 & b0-391 1 & $2(\mathrm{HO}) \mathrm{OZ} \mathrm{m}) \mathrm{JZ}$ \\
\hline 9oralet & 90-71द & 90-3CI' & $90-796 \mathrm{E}$ & $1 \angle 60$ & 6590 & 90-352:0 & $+z^{2}$ \\
\hline $50-3712$ & 50-968 I & so-azr I & so-3ot' I & $\angle 21$ & $8 \angle 1$ & $50-9991$ & $+[7$ \\
\hline $60-9899$ & to-attig & 10-Bto's & woges's & $\angle O L$ & 846 & hosis 9 & $+\mathrm{q}$ \\
\hline $20-316 L$ & $20-969 \mathrm{~L}$ & $20-9989$ & $20-3519$ & $60+3+1 z$ & E0+700'E & $20-39+2$ & $+\mathbf{E}$ \\
\hline EO-glb19 & $\operatorname{cotalg} \mathrm{s}$ & 50-G/6) & $60-3120$ & 091 & 102 & co-36I's & $(0,1+79)+\left[P_{3}\right.$ \\
\hline I8E 0 & 1980 & $66 L^{\prime} 0$ & 59LO & $60+9 \Omega 2 I$ & $60+92<1$ & 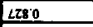 & $+\mathrm{EIV}$ \\
\hline$L \mathbf{F} L$ & +12 & 199 & 769 & $60+39 c$ & $50+3221$ & 689 & $\begin{array}{r}+\mathrm{N} \\
\end{array}$ \\
\hline (19tow) & (T) $19+$ & (1Plow) & $(1 / 9+1)$ & +1 & ndad & Trpot & Aponuptwo? \\
\hline$\angle 850$ & EISO & $09 \varepsilon^{\prime} 0$ & 4820 & 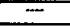 & - & $\angle E+0$ & м) $2 \%$ \\
\hline 029 & 809 & 985 & LLS & - & - & 965 & $\% 1 \mathrm{M} \pm 10 \mathrm{M}$ \\
\hline$|\varepsilon|$ & OE [ & 821 & $\angle Z 1$ & - & - & & Surtuact xाmg \\
\hline 0090 & MS'O & 0550 & BESO & 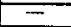 & (rq//2 $\left.\varepsilon 0+3 t^{\prime} 1\right)$ & $(A x) \angle 9 S O$ & 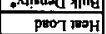 \\
\hline- & - & $\ldots$ & - & - & $(1$ wis 9p1) & (N) $50+3916$ & M WWS FOL \\
\hline \multicolumn{7}{|c|}{$1096+1049+1069$ 10 96} & 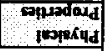 \\
\hline \multicolumn{8}{|c|}{ 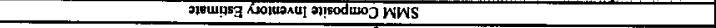 } \\
\hline & & & $1-x s^{-1+z}$ & 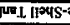 & Pou!s & & \\
\hline
\end{tabular}


HDW Model Rov. 4

\begin{tabular}{|c|c|c|c|c|c|c|c|}
\hline \multicolumn{8}{|c|}{ Single-Shel1 Tank 241-\$X-101 } \\
\hline & \multicolumn{7}{|c|}{ Total Inventory Estimate" } \\
\hline $\begin{array}{l}\text { Physical } \\
\text { Prepertics }\end{array}$ & & $\therefore \quad \because$ & & $-95 \mathrm{CI}$ & $-67 \mathrm{CI}$ & $+67 \mathrm{CI}$ & $+95 \mathrm{CI}$ \\
\hline Total Wiste & $2.75 \mathrm{E}+06(\mathrm{~kg})$ & (456 kgal) & $=$ & - & $\ldots$ & - & 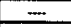 \\
\hline Heat Load & $6.30(\mathrm{~kW})$ & $(2.15 E+04 \mathrm{BTU} / \mathrm{hr})$ & $=$ & 5.25 & 5.86 & 6.60 & 6.71 \\
\hline Bulk Densityt & $1.59(\mathrm{~g} / \mathrm{cc})$ & + & $=$ & 1.50 & 1.52 & 1.71 & 1.76 \\
\hline Water wtot & 383 & & & & & & \\
\hline Water wher & 36.3 & - & $=$ & 26.9 & 30.4 & 41.3 & 46.2 \\
\hline TOC wt\% C (w) & 0.115 & - & $=$ & 7.65E-02 & $9.55 \mathrm{E}-02$ & 0.136 & 0.155 \\
\hline $\begin{array}{c}\text { Chentcol } \\
\text { Constituents }\end{array}$ & noled & ppm & k & $\begin{array}{l}-95 \mathrm{CI} \\
(\mathrm{mol} / \mathrm{L})\end{array}$ & $\begin{array}{l}67 \mathrm{Cl} \\
\text { (molel })\end{array}$ & $\begin{array}{l}+67 \mathrm{Cl} \\
\text { (mole/l) }\end{array}$ & $\begin{array}{l}+95 \mathrm{Cl} \\
\text { (moleth) }\end{array}$ \\
\hline Nat & 9.56 & $1382+05$ & $3.79 \mathrm{E}+05$ & 773 & 796 & 11.8 & 12.9 \\
\hline $\mathrm{Al} 3+$ & 3.78 & $6.41 E+04$ & $1.76 \mathrm{E}+0 \mathrm{~s}$ & 3.11 & 3.31 & 4.30 & 4.82 \\
\hline Fe3 $+($ toul $\mathrm{Fe})$ & 0.350 & $1.23 E+04$ & $3.37 \mathrm{E}+04$ & 0.143 & 0.347 & 0.353 & 0.356 \\
\hline $\mathrm{Cr} 3+$ & 0.326 & $1.06 \mathrm{E}+04$ & $2.93 \mathrm{E}+04$ & 0.209 & 0.265 & 0.479 & 0.658 \\
\hline $\mathrm{Bij+}$ & $1.98 \mathrm{E}-04$ & 26.0 & 71.6 & $1.80 \mathrm{E}-04$ & I.B9E-OA & $2.08 \mathrm{E}-04$ & $2.16 \mathrm{E}-04$ \\
\hline Les?+ & $5.30 \mathrm{E}-06$ & 0.462 & 1.27 & $3.84 E-06$ & 4.55E-06 & $6.05 \mathrm{E}-06$ & 6.77E- -06 \\
\hline $\mathrm{Hg} 2+$ & $1.73 E-06$ & 0.218 & 0,599 & 1.64E-06 & $1.69 \mathrm{E}-06$ & 1.77E-06 & $1.8 \pm E-06$ \\
\hline $\mathrm{Zr}$ (as $\mathrm{ZrO}(\mathrm{OH}) 2$ & $3.73 \mathrm{E}-05$ & 2.14 & 587 & $3.40 \mathrm{E}-05$ & $3.52 E-05$ & 3.87E-05 & $405 E-05$ \\
\hline $\mathrm{Pb2+}$ & $209 \mathrm{E}-04$ & 27.2 & 74.8 & $1.79 \mathrm{E}-04$ & $1.94 E-04$ & $2.25 \mathrm{E}-04$ & $2.40 \mathrm{E}-04$ \\
\hline $\mathrm{Ni2+}$ & 2.33E-02 & 858 & $2.36 \mathrm{E}+03$ & $1.85 \mathrm{E}-02$ & 2.12E-02 & $2.49 \mathrm{E}-02$ & $2.46 \mathrm{E}-02$ \\
\hline $\mathrm{sin} 2+$ & 0 & 0 & 0 & 0 & 0 & 0 & 0 \\
\hline Mndt & $\$ .91 \mathrm{E}-04$ & 20.4 & 56.1 & $4.46 \mathrm{E}-04$ & $5.17 \mathrm{E}-04$ & $6.66 \mathrm{E}-64$ & 7.37E-04 \\
\hline Cs.2t & 0.106 & $2.66 \mathrm{E}+03$ & $7.31 \mathrm{E}+03$ & 3.09E-02 & $9.31 \mathrm{E}-02$ & 0.118 & 0.131 \\
\hline$K^{+}$ & $2.48 \mathrm{E}-02$ & $\begin{array}{r}609 \\
\end{array}$ & $1,68 \mathrm{E}+03$ & 2. $15 \mathrm{E}-02$ & $2.31 \mathrm{E}-02$ & $2.62 \mathrm{E}-02$ & $2.72 \mathrm{E}-02$ \\
\hline OH- & 16.6 & $1.77 \mathrm{E}+05$ & $4.88 \mathrm{E}+05$ & 13.5 & 14.5 & 18.8 & 21.0 \\
\hline NO3- & 4.35 & $1.69 \mathrm{E}+05$ & $466 \mathrm{E}+03$ & 2.58 & 259 & 7.10 & 8,58 \\
\hline NO2- & 1.52 & $4.38 E+04$ & $1215+05$ & 1.06 & 1.23 & 1.76 & 1.76 \\
\hline CO32- & $0.16 ?$ & $6.29 \mathrm{E}+03$ & $1.735+04$ & 0.142 & 0.154 & $0.1 \% 0$ & 0.192 \\
\hline$P 043$. & $1,36 \mathrm{E}-02$ & 810 & $223 E+03$ & $1.17 \mathrm{E}-02$ & $1.24 \mathrm{E}-02$ & $1.39 \mathrm{E}-02$ & $1.42 \mathrm{E}-02$ \\
\hline S042- & 5.42E-02 & $3.26 \mathrm{E}+03$ & B.9e+03 & $4.61 \mathrm{E}-02$ & $5,00 \mathrm{E}-02$ & $5.85 \mathrm{E}-02$ & $6.23 \mathrm{E}-02$ \\
\hline Si (asio32-) & $5,92 \mathrm{E}-02$ & $1.04 E+03$ & $287 \mathrm{E}+03$ & $4.33 E-02$ & $5.30 \mathrm{E}-02$ & 6.54E-02 & 7. 14E- 02 \\
\hline F. & $9.72 E-03$ & 116 & 319 & $8.16 \mathrm{E}-03$ & 8.82E-03 & $1.05 \mathrm{E}-02$ & $1,11 E-02$ \\
\hline Cl. & 0.101 & $2.25 \mathrm{E}+03$ & $6.13 \mathrm{E}+03$ & $7,78 \mathrm{E}-02$ & 8.92E-02 & 0.107 & 0.136 \\
\hline C6HSO73- & 4.35E-03 & 316 & $1.42 E+03$ & $4.01 \mathrm{E}-03$ & 4.18E-OS & $4,53 \mathrm{E}-03$ & $4.69 \mathrm{E}-03$ \\
\hline EDTA4- & $2.21 \mathrm{E}-03$ & 399 & $1.10 \mathrm{E}+03$ & 6.90E-04 & $1.42 \mathrm{E}-03$ & 2.99E-03 & $3.76 \mathrm{E}-03$ \\
\hline HEDTA3- & $4.12 \mathrm{E}-03$ & 709 & $1.95 \mathrm{E}+03$ & $1.07 \mathrm{E}-03$ & $2.56 E-03$ & 5.69E-03 & $7.22 \mathrm{E}-03$ \\
\hline glycolate- & $1.33 \mathrm{E}-02$ & 626 & $1.72 \mathrm{E}+03$ & B. $75 \mathrm{E}-03$ & $1.10 \mathrm{E}-02$ & $156 \mathrm{E}-02$ & $1.78 \mathrm{E}-02$ \\
\hline acente- & $9,36 \mathrm{E}-04$ & 34.7 & 95.3 & $7.69 \mathrm{E}-04$ & B.51E-04 & $1.02 \mathrm{E}-03$ & $1.10 \mathrm{E}-03$ \\
\hline oxnlnte2. & 6.94E 206 & 0,314 & 1.05 & $6.15 \mathrm{E}-06$ & $6.54 E-06$ & $7.35 \mathrm{E}-06$ & $7.74 \mathrm{E}-06$ \\
\hline DAP & 2.96E-03 & 391 & $1.07 \mathrm{E}+03$ & $2.45 \mathrm{E}-03$ & $2.70 \mathrm{E}-03$ & 3.22E-03 & $3,46 E-03$ \\
\hline butanol & $2,96 E-03$ & 137 & 379 & 2.45E-03 & $270 \mathrm{E}-03$ & $3.22 \mathrm{E}-0 \mathrm{~g}$ & 3,46E-03 \\
\hline NhB & & & & & & & \\
\hline Fof & 0.112 & $1.19 \mathrm{E}+03$ & 3.2 $2 \mathrm{EE}+03$ & $4.70 \mathrm{E}-02$ & $5.64 \mathrm{E}-02$ & 0.417 & 0.121 \\
\hline $\mathrm{Fo}(\mathrm{CN}) 64$ & 0 & 0 & 0 & 0 & 0 & 0 & 0 \\
\hline
\end{tabular}

"Unknowns in tank solids inventory we assigned by Trak Lyyering Model (TLM)

+Water wro derived from the difference of dentity and total disuolved apecies. 


\begin{tabular}{|c|c|c|c|c|c|c|c|}
\hline & \multicolumn{7}{|c|}{ Single-Shell Tank 241-SX-101 } \\
\hline \multicolumn{8}{|c|}{ ILM Solids Composite Inventory Estimate* } \\
\hline Phyoleal & & & & & & & \\
\hline Propertis: & & & & $+95 \mathrm{Cl}$ & $67 \mathrm{CI}$ & $+67 \mathrm{Cl}$ & $+95 \mathrm{CI}$ \\
\hline Total TLM W/ & $2.04 \mathrm{E}+06(\mathrm{~kg})$ & $(310 \mathrm{kgal})$ & - & - & $\ldots$ & - & - \\
\hline Heat Load & $5.74(\mathrm{~kW})$ & (1 96E+04 BTU/hs) & - & 4.70 & 5.31 & 6.02 & 6.15 \\
\hline Bulk Density & $1.73(\mathrm{gcc})$ & - & - & 1.60 & 1.62 & 1.91 & 1.98 \\
\hline Void Fraction & 0.661 & - & $\ldots$ & 0.425 & 0.503 & 0.761 & 0.764 \\
\hline Woter wt\% & 30.8 & $=$ & - & 16.6 & 21.2 & 38.4 & 40.9 \\
\hline TOC wt\% C (w) & $2.61 \mathrm{E}-03$ & $-m$ & $=$ & $2.29 \mathrm{E}-03$ & $2.37 \mathrm{E}-03$ & $2.81 E-03$ & $3.04 E-03$ \\
\hline $\begin{array}{l}\text { Rodiolosited } \\
\text { Constituenti }\end{array}$ & CUL & $\mathrm{HCV} / \mathrm{s}$ & Ci & $\begin{array}{l}9 \mathrm{CC} \\
\text { (CVL) }\end{array}$ & $\begin{array}{l}67 \mathrm{CI} \\
(\mathrm{CM})\end{array}$ & $\begin{array}{l}+67 \mathrm{CI} \\
\text { (CUL) }\end{array}$ & $\begin{array}{l}+95 \mathrm{cl} \\
\text { (c)t })\end{array}$ \\
\hline H-3 & 1.27E-04 & $7.31 \mathrm{E}-02$ & 149 & $1.45 \mathrm{E}-05$ & $1.45 E-05$ & $133 \mathrm{E}-\mathrm{A}$ & $1.43 \mathrm{E}-04$ \\
\hline$c-14$ & $6.88 E-06$ & $3.9 \pi-03$ & 0.07 & $1.12 \mathrm{E}-06$ & $1.12 \mathrm{E}-00$ & $7.28 \mathrm{E}-06$ & $7.64 E-06$ \\
\hline Ni-59 & $1.08 \mathrm{E}-05$ & 6.23E-03 & 12.7 & $7,38 \mathrm{E}-06$ & $7.38 \mathrm{E}-06$ & 1.15E-05 & 1.14E-05 \\
\hline $\mathrm{Ni}-63$ & $1.02 E-03$ & 0.549 & $1.20 \mathrm{E}+03$ & $6.19 \mathrm{E}-04$ & $6.89 \mathrm{E}-04$ & $1.09 \mathrm{E}-03$ & $1.07 E-03$ \\
\hline Co-60 & $5.62 \mathrm{E}-06$ & $3.24 \mathrm{E}-03$ & 6.60 & 4.44E-07 & $4.445 ; 07$ & $3.85 \mathrm{E}-26$ & $6.32 \mathrm{E}-106$ \\
\hline Se-79 & $3.74 E-06$ & $2.15 E-03$ & 4.38 & $2.39 \mathrm{E}-07$ & $2.39 \mathrm{E}-07$ & $3.82 E-06$ & 7.05E-06 \\
\hline Sr-90 & 0.593 & 342 & $6.96 \mathrm{E}+05$ & 0.45 & 0.536 & 0.638 & 0.645 \\
\hline$Y .90$ & 0.594 & 342 & $6.96 \mathrm{E}+05$ & 0.461 & 0.461 & 0.638 & 0.645 \\
\hline $\mathrm{Zr}-93$ & 1.77E\&S & $1.02 \mathrm{E}-02$ & 207 & $1.13 \mathrm{E}-06$ & $1.13 \mathrm{E}-06$ & 1.81E-05 & 3.16E-0S \\
\hline$N \mathrm{~N}-93 \mathrm{~m}$ & $1.44 E-05$ & $8.28 \mathrm{E}-03$ & 16.8 & $9.21 \mathrm{E}-07$ & $9.21 \mathrm{E}-07$ & 1.47E-05 & 2.94E-05 \\
\hline Tc-99 & $5.26 \mathrm{E}-05$ & 3.03E- 02 & 61.7 & 4.56E-05 & 4 B4E-0S & S.60E-05 & 5.98E-05 \\
\hline Ru-106 & $1.20 \mathrm{E}-09$ & $6.91 \mathrm{E}-07$ & I.4[E-0\} & $1.24 \mathrm{E}-12$ & $1.24 E_{-12}$ & $1.25 \mathrm{E}-09$ & $1.36 E-09$ \\
\hline $\mathrm{Cd}-113 \mathrm{~m}$ & $2.68 E-05$ & $1.54 E-02$ & 31.4 & 34 EE- 06 & $3.47 \mathrm{E}-\infty 6$ & $3.52 \mathrm{E}-0 \mathrm{~s}$ & 5.35E-05 \\
\hline $5 \mathrm{~s}-12 \mathrm{~s}$ & $1.92 \mathrm{E}-05$ & $1,100-02$ & 22.5 & $6.52 \mathrm{E}-07$ & $6.52 \mathrm{E}-07$ & $2.00 E-05$ & 2.17E-05 \\
\hline $5 n-126$ & $3.73 E-06$ & $3.31 E-03$ & 6.73 & $3.67 \mathrm{E}-07$ & $3.6 \pi-07$ & $5.86 \mathrm{E}-06$ & L.11E-05 \\
\hline $1-129$ & 1.00E-07 & 5.77E-0s & 0.117 & B.66E-08 & $9.20 \mathrm{E}-08$ & $1.06 \mathrm{E}-07$ & $1.14 \mathrm{E}-07$ \\
\hline Cr-134 & $1.1 \pi-06$ & $6.75 \mathrm{E}-04$ & 1.37 & $1.35 \mathrm{E}-08$ & $1,35 E=08$ & $1.22 \mathrm{E}-06$ & I.31E-06 \\
\hline $\mathrm{C}-137$ & 0.191 & 110 & $2.24 E+03$ & 0.170 & 0.179 & 0.203 & 0.216 \\
\hline $\mathrm{Ba}_{\mathrm{a}-137_{m}}$ & 0.180 & 104 & $2.12 \mathrm{E}+05$ & 2.63E-02 & $2.63 \mathrm{E}-02$ & 0.188 & 0.198 \\
\hline $\mathrm{Sm}-151$ & $1.33 \mathrm{E}-02$ & 7.68 & $1.56 \mathrm{E}+04$ & $8.52 \mathrm{E}-04$ & $8.52 \mathrm{E}-04$ & $1.36 \mathrm{E}-02$ & $2.56 \mathrm{E}-02$ \\
\hline Eu-152 & $7.94 \mathrm{E}-06$ & $4.5 \mathrm{AE}-03$ & 9.32 & $3.48 \mathrm{E}-06$ & $3.48 \mathrm{E}-06$ & 7.97E-06 & $8.02 E-06$ \\
\hline$E x-154$ & 1.35E-04 & $7.76 \mathrm{E}-02$ & 158 & $1.07 \mathrm{E}-05$ & 1.07E-05 & 1.40E-04 & $1.74 \mathrm{E}-04$ \\
\hline Ep-155 & 3.91E-04 & 0.225 & 458 & $1.64 \mathrm{E}-04$ & $1.64 E_{0}-04$ & $3.92 \mathrm{E}-04$ & 3.95E-04 \\
\hline$\overline{R a-226}$ & $7.73 \mathrm{E}-10$ & 4.46E- 07 & $9.07 \mathrm{E}-04$ & $3.10 \mathrm{E}-10$ & $5.37=10$ & $1,016.09$ & $1.24 \mathrm{E}-09$ \\
\hline Rq-228 & $7.93 \mathrm{E}-09$ & $4.57 E-06$ & $9.30 \mathrm{E}-03$ & $4.60 \mathrm{E}-15$ & $4.60 \mathrm{E}-15$ & B.00E-09 & $8.09 E-09$ \\
\hline Ac-227 & 1.75E-09 & $2.16 E-06$ & A.40E-03 & 1.51E-09 & $2.37 \mathrm{E}-199$ & $5,12 E-09$ & $6.44 E-09$ \\
\hline $\mathrm{Pg}-231$ & $5.56 \mathrm{E}-09$ & $3.21 \mathrm{E}-06$ & $6.52 \mathrm{E}-03$ & $3.57 \mathrm{E}-10$ & $3.57 \mathrm{E}-10$ & $6.22 \mathrm{E}-099$ & $1.35 \mathrm{E}-0 \mathrm{~s}$ \\
\hline Th-229 & 1.9]E-10 & 1.10E-07 & $2.24 E-04$ & $8.7 \mathrm{BE}=13$ & $8.78 E_{-13}$ & $1.93 \mathrm{E}-10$ & $1.95 \mathrm{E}-10$ \\
\hline $\mathrm{Th}-232$ & $1.06 \mathrm{E}-10$ & 6.13E-06 & $1.25 \mathrm{E}-04$ & $2.93 \mathrm{E}-16$ & $2.93 \mathrm{E}-16$ & 1.34E-10 & $1.61 E-10$ \\
\hline $\mathrm{U}-232$ & 3.54E- -08 & 2.04E-0S & 4.16E-02 & $1.23 \mathrm{E}-0 t$ & $2.31 E-08$ & 5.05E-08 & $6.72 \mathrm{E}-08$ \\
\hline $\mathrm{U}-233$ & $1.36 \mathrm{E}-07$ & 7.62E-05 & 0.159 & 4.79E-08 & 8.84E-08 & $1.93 \mathrm{E}-07$ & $2.57 \mathrm{E}-07$ \\
\hline U.234 & 6.99E-07 & 4.03E-04 & 0.121 & 3.39E-07 & $\$ .15 \mathrm{E}-07$ & 8.99E-07 & $1.10 E-06$ \\
\hline $\mathrm{U}-235$ & 2ME-0 & 1.64E-05 & $3.33 \mathrm{E}-02$ & 1.38E-08 & $2.09 \mathrm{E}-08$ & $3.65 E-08$ & 4.47E-08 \\
\hline U. 236 & $2.74 \mathrm{E}-0 \mathrm{~B}$ & 1.5AE-05 & $3.22 \mathrm{E}-02$ & $1.30 \mathrm{E}-08$ & $2.02 \mathrm{E}-08$ & 3.5!E-08 & 4.26E-08 \\
\hline $\mathrm{U}-238$ & 6.22E-07 & 3.5NE-04 & 0.729 & $3.04 \mathrm{E}-07$ & 4.5tE-07 & 7.99E-07 & $9.79 \mathrm{E}-07$ \\
\hline$N_{p-237}$ & 2.46E-07 & 1.42E-04 & 0.259 & 2.01E-07 & $2.19 \mathrm{E}-07$ & 2.64E-07 & 2.81E-07 \\
\hline $\mathrm{Pu}-238$ & 4.47E-60 & $2.58 \mathrm{E}-03$ & 5.25 & $3.54 \mathrm{E}-06$ & 4.00E-06 & 4.94E-06 & 5.40E-06 \\
\hline Pu-239 & $2.73 \mathrm{E}-04$ & 0.157 & 320 & $2.07 \mathrm{E}-04$ & $2.39 \mathrm{E}-0 \mathrm{~A}$ & $3.07 E-04$ & 3.39E-04 \\
\hline $\mathrm{Pu}-240$ & $3.99 \mathrm{E}-05$ & $2.30 \mathrm{E}-02$ & 468 & 3.04E-05 & $3.51 \mathrm{E}-05$ & 4.47E-05 & 4.94E-05 \\
\hline$P_{u}=241$ & $2.58 E-\alpha$ & 0.149 & 303 & 203E-04 & $2.30 \mathrm{E}-0 \mathrm{~A}$ & 2.87E-04 & $3.14 E-04$ \\
\hline$P_{u}=242$ & $1.23 \mathrm{E}-09$ & $7.08 \mathrm{E}-07$ & $1.44 \mathrm{E}-03$ & $9.73 \mathrm{E}-10$ & $1.10 \mathrm{E}-09$ & 1.36E-09 & $1.48 \mathrm{E}-09$ \\
\hline Ann-241 & $6.27 \mathrm{E}-05$ & $3.62 \mathrm{E}-02$ & 73.6 & 3.74E-05 & $5,48 \mathrm{E}-05$ & 7.13E.0S & $1,30 \mathrm{E}-04$ \\
\hline Am-243 & 1.91E-09 & $1.10 E-06$ & $2.24 E-03$ & $9.00 E-10$ & $1.66 \mathrm{E}-09$ & $2.10 E-09$ & $2.54 \mathrm{E}-09$ \\
\hline $\mathrm{Cm}-242$ & $8.07 \mathrm{E}-08$ & 4.66E-05 & $9,47 E-02$ & $7.900-08$ & $7.9 \pi-08$ & $8.14 E-08$ & $8.14 \mathrm{E} .09$ \\
\hline $\mathrm{Cm}-243$ & $1.85 \mathrm{E}-09$ & $1.07 \mathrm{E}-06$ & 2.17E-03 & 1.81E-09 & $1.83 \mathrm{E}-09$ & 1.86E-09 & $1.86 \mathrm{E}-09$ \\
\hline$C_{m}-244$ & $1.44 E-09$ & $8.30 E-07$ & $1.696-03$ & $2.49 \mathrm{E}-10$ & $7.24 \mathrm{E}-10$ & $1,92 E-09$ & $1.92 \mathrm{E}-09$ \\
\hline Totals: & $\mathbf{M}$ & $\pi / \%$ & ks & $\begin{array}{l}\mathrm{CCl} \\
(\mathrm{M}) \\
(\mathrm{L})\end{array}$ & $\begin{array}{l}67 \mathrm{CI} \\
(\mathrm{M} \mathrm{or} \\
\text { oL) }\end{array}$ & $\begin{array}{l}7 \mathrm{Cl} \\
(\mathrm{Mor} \\
7 \mathrm{~A})\end{array}$ & $\begin{array}{l}\text { CT } \\
\text { (M or } \\
\text { I })\end{array}$ \\
\hline$\overline{P_{4}}$ & $4.56 \mathrm{E}-03(g / L)$ & - & 5.35 & 3.45E-03 & $4.00 \mathrm{E}-03$ & $5.12 E-03$ & $3.67 \mathrm{E}-03$ \\
\hline$\overline{\mathrm{U}}$ & $7.69 \mathrm{E}-03$ & $1.06 \mathrm{E}+03$ & $2.15 E+03$ & $3.76 \mathrm{E}-03$ & $5.66 \mathrm{E}-03$ & $9.90 E-03$ & $1.21 \mathrm{E}-02$ \\
\hline
\end{tabular}

"Unknowns in tark solids inventory are anaigned by Tank Layering Model (TLM). 
HNF-SD-WM-ER-352, Rev. 1

HDW Model Rer. 4

\begin{tabular}{|c|c|c|c|c|c|c|c|}
\hline & \multicolumn{7}{|c|}{ Single-Shell Tank 24I-SX-101 } \\
\hline & \multicolumn{7}{|c|}{ SMM Composite Inventory Estimate } \\
\hline Phyolical & & & & & & & \\
\hline Propertiea & & & & $+95 \mathrm{CI}$ & $-67 \mathrm{CI}$ & $+67 \mathrm{CI}$ & $+95 \mathrm{CI}$ \\
\hline Total SMM W & $7.16 \mathrm{E}+05(\mathrm{~kg})$ & (146 kgal) & 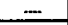 & $\ldots$ & $\ldots$ & - & - \\
\hline Feat Lond & $0.567(\mathrm{~kW})$ & (1.94E+03 BTU/hr) & - & 0.534 & 0.550 & 0.584 & 0.600 \\
\hline Bulk Density & $1.29(\mathrm{~g} / \mathrm{cc})$ & $=$ & - & 1.27 & 1.28 & 1.30 & 1.31 \\
\hline Water wh $\%$ & 59.6 & $\cdots$ & $\cdots$ & 57.7 & 58.6 & 60.8 & 62.0 \\
\hline TOC wt\% C (w & 0.437 & - & 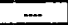 & 0.287 & 0.360 & 0.513 & $0.5 \%$ \\
\hline $\begin{array}{l}\text { Aedfolotisel } \\
\text { Censtltuent }\end{array}$ & $\mathbf{C V L}$ & $\mathrm{HCV} / \mathrm{s}$ & C1 & $\begin{array}{l}98 \mathrm{C} \\
(\mathrm{CVL})\end{array}$ & $\begin{array}{l}-7 \mathrm{Cl} \\
(\mathrm{CV} / \mathrm{L})\end{array}$ & $\begin{array}{l}+67 \mathrm{CI} \\
(\mathrm{Cl} / \mathrm{L})\end{array}$ & $\begin{array}{l}+15 \mathrm{CI} \\
(\mathrm{CUL})\end{array}$ \\
\hline $\mathrm{H}-3$ & $1.23 \mathrm{E}-04$ & $9.54 \mathrm{E}-02$ & 68.2 & $6.55 \mathrm{E}-05$ & $6.55 \mathrm{E}-05$ & $1.27 \mathrm{E}-04$ & $1.32 \mathrm{E}-04$ \\
\hline $\mathrm{C}-14$ & $1.7 \mathrm{SE}-05$ & $1.356-02$ & 9.67 & $3.38 \mathrm{E}-06$ & $5.38 \mathrm{E}-06$ & $1.78 \mathrm{E}-05$ & $1.80 \mathrm{E}-05$ \\
\hline Ni-59 & $1.13 \mathrm{E}-06$ & 8.74E-04 & 0625 & $5,61 E-07$ & $5.61 E-07$ & $1.16 \mathrm{E}-06$ & $1.19 \mathrm{E}-06$ \\
\hline $\mathrm{Ni}-63$ & $1.11 \mathrm{E}-0 \mathrm{~d}$ & $8.56 \mathrm{E}-02$ & 61.2 & $5.44 E-105$ & $5.44 \mathrm{E}-0 \mathrm{~S}$ & 1,14E-04 & $1,16 \mathrm{E}-04$ \\
\hline Co-60 & $1.92 \mathrm{E}-05$ & $1.49 \mathrm{E}-02$ & 10.6 & $5.37 \mathrm{E}-06$ & 5.37E-06 & $1.96 \mathrm{E}-05$ & 1.99E-05 \\
\hline Se-79 & $1.75 \mathrm{E}-06$ & $1.35 \mathrm{E}-03$ & 0.965 & $9.73 \mathrm{E}-07$ & 9.73E- 07 & $2.03 \mathrm{E}-066$ & 2.29E-06 \\
\hline St-90 & $5.80 \mathrm{E}-02$ & 448 & $3.20 \mathrm{E}+04$ & $5.42 \mathrm{E}-02$ & $560 \mathrm{E}-02$ & $599 \mathrm{E}-02$ & $6.18 E-02$ \\
\hline$Y-90$ & $5.80 \mathrm{E}-02$ & 44.8 & $3.20 \mathrm{E}+04$ & $3.13 \mathrm{E}-02$ & 3.13E-02 & $5.99 \mathrm{E}-02$ & 6.18E-02 \\
\hline 7r-93 & B.57E-O6 & $6.62 \mathrm{E}-03$ & 4.74 & $4,700-06$ & $4.70 €-06$ & $9.97 \mathrm{E}-06$ & $1.13 E_{0.05}$ \\
\hline $\mathrm{Nb}-93 \mathrm{~m}$ & 6.23E-06 & 4.81E-03 & 3.4 & 3.52E-06 & $3.52 \mathrm{E}-06$ & $7.21 \mathrm{E}-06$ & B.15E-06 \\
\hline Tc-99 & $1.25 \mathrm{E}-\mathrm{OA}$ & $9.63 \mathrm{E}-02$ & 68.9 & 7.95E-05 & $1.02 \mathrm{E}-04$ & $1.48 \mathrm{E}-04$ & $1.70 \mathrm{E}-04$ \\
\hline$R u-106$ & $3.41 \mathrm{E}-09$ & 2.63E-06 & $1.88 \mathrm{E}-03$ & $1.61 \mathrm{E}-09$ & $1.61 \mathrm{E}-09$ & $3.81 \mathrm{E}-09$ & 4. $17 \mathrm{E}-09$ \\
\hline $\mathrm{Cd}-113 \mathrm{~m}$ & 4.48E-05 & $3.46 \mathrm{E}-02$ & 24.8 & $2.16 \mathrm{E}-05$ & 2.16E-05 & $5.32 E-05$ & $6.13 \mathrm{E}-05$ \\
\hline Sb-125 & 8.25E-05 & 6.37E-02 & 45.6 & 2.24E-05 & $2.24 \mathrm{E}-0 \mathrm{~s}$ & 8.40E-05 & 8.55E-05 \\
\hline $5 n-126$ & $2.64 \mathrm{E}-06$ & 2.04E- 03 & 1.46 & $1.48 \mathrm{E}-06$ & $1.48 E-06$ & $3.06 \mathrm{E}-06$ & $3,46 \mathrm{E}-96$ \\
\hline $1-129$ & $2.40 \mathrm{E}-07$ & $1.96 \mathrm{E}-04$ & 0.133 & 1.53E-07 & $1.96 \mathrm{E}-07$ & $2.85 E_{-07}$ & 3.29E-07 \\
\hline $\mathrm{C}-134$ & $1.29 \mathrm{E}-06$ & $9.95 \mathrm{E}-\mathrm{AS}$ & 0.712 & 9.21E-07 & $1.06 \mathrm{E}-\infty 6$ & $1.47 \mathrm{E}-06$ & $1.65 \mathrm{E}-06$ \\
\hline C.- 137 & 0.136 & 105 & $7.50 E+04$ & 0.123 & 0.129 & 0.142 & 0.148 \\
\hline Bn-137m & 0.128 & 99.1 & $7.095+04$ & 9.4AE-02 & 9 MEE-02 & 0.135 & 0.140 \\
\hline Sm-151 & 6.15E-03 & 4.75 & $3.40 \mathrm{E}+03$ & 3.44E-03 & 3.44E-03 & $7.13 E-03$ & 8.07E-03 \\
\hline$E_{v}-152$ & $1.901 E-06$ & $1.53 \mathrm{E}-03$ & 1.09 & $1,02 \mathrm{E}-06$ & $1.02 \mathrm{E}-06$ & $2.11 \mathrm{E}-06$ & $2.24 \mathrm{E}-06$ \\
\hline Ex-154 & $3.13 \mathrm{E}-04$ & 0.242 & 173 & $1.20 E-04$ & $1.20 \mathrm{E}-04$ & 3.83E-04 & 4.11E-04 \\
\hline Eu-15s & 1.1TE-04 & $9.02 E-02$ & 64.5 & 5.97E-05 & $5.97 E-05$ & $1.25 \mathrm{E}-\mathrm{AA}$ & $1.33 \mathrm{E}-04$ \\
\hline $\mathrm{Ra}-226$ & $7.48 \mathrm{E}-11$ & $5.7 \mathrm{EE}-0 \mathrm{~B}$ & 4.13E-05 & 5.08E-11! & $5.08 \mathrm{E}-11$ & 8.35E-11 & $9.18 \mathrm{E}-11$ \\
\hline Ra-228 & $6.65 \mathrm{E}-01$ & $5.14 \mathrm{E}-05$ & $3.6 \mathrm{EE}-02$ & 2.76E-08 & $4.6 \pi-08$ & $8.96 \mathrm{E}-08$ & 1.14E-07 \\
\hline Ac- 227 & $4.70 \mathrm{E}-10$ & 3.63E-07 & $2.60 \mathrm{E}-04$ & $3.31 \mathrm{E}-10$ & 3.31E-10 & $5.2 \mathrm{E}-10$ & $5.69 \mathrm{E}-10$ \\
\hline $\mathrm{Pa}-23 \mathrm{I}$ & $2.16 \mathrm{E}-09$ & 1.66E-06 & 1.19E-03 & $1,340-09$ & 1.38E-09 & 2.44E-09 & $270 \mathrm{E}-09$ \\
\hline Th-229 & $1.56 \mathrm{E}_{-} 09$ & 1.21E-06 & 8.63E-04 & $709 \mathrm{E}-10$ & 1. $13 \mathrm{E}-09$ & 2.07E-09 & $2.6 ! \mathrm{E}, 09$ \\
\hline Th-232 & 4.43E- 09 & 3.42E-06 & 2.45E-03 & $2.26 \mathrm{E}-09$ & 3.33E-09 & 5.53E- 09 & $6.58 \mathrm{E}-09$ \\
\hline $\mathrm{U}-232$ & $3.47 \mathrm{E}-07$ & 2.60E-04 & 0.192 & 1.91E-07 & 2.67E-07 & 4.39E-07 & $5.38 E-07$ \\
\hline U-233 & $1,33 \mathrm{E}-06$ & $1.03 E-03$ & 0.735 & 7.33E- 07 & $1.02 \mathrm{E}-06$ & $1.68 \mathrm{E}-06$ & $2.06 \mathrm{E}-06$ \\
\hline $\mathrm{U}-234$ & 1.15E-07 & 1.21E-04 & 0.230 & 3.99E-07 & $4.07 \mathrm{E}-07$ & 4.25E-07 & $4.28 \mathrm{E}-07$ \\
\hline $\mathrm{U}-235$ & $1.69 \mathrm{E}-08$ & $1.30 \mathrm{E}-0 \mathrm{~s}$ & $9.33 \mathrm{E}-0]$ & $1.62 \mathrm{E}-04$ & $1.65 \mathrm{E}-08$ & 1.73E-08 & $1.74 \mathrm{E}-08$ \\
\hline U-236 & 1.29E_ $0 \mathrm{~B}$ & 9.96E-06 & $7.14 E-03$ & $1.25 \mathrm{E}-0 \mathrm{~B}$ & $1.27 \mathrm{E}-08$ & $1.3 \mathrm{JE}-08$ & $133 \mathrm{E}-0 \mathrm{~s}$ \\
\hline U-238 & $4.76 \mathrm{E}-07$ & $3.67 \mathrm{E}-04$ & 0.263 & 458E-07 & $4.66 \mathrm{E}-07$ & $4.85 \mathrm{E}-07$ & 4. $88 \mathrm{E}-07$ \\
\hline Np-237 & 4.51E-O7) & 3.54E-04 & 0.253 & 3.11E-0? & 3.83E- -07 & 5.35E-07 & $6.08 \mathrm{E}-07$ \\
\hline Pud-238 & 7.03E-07 & 5.43E-04 & 0.381 & 5.29E-07 & 6.14E-07 & 7.91E-07 & $8.76 \mathrm{E}-07$ \\
\hline $\mathrm{Pu}-239$ & $2.42 \mathrm{E}-05$ & 1.8TE-02 & 13.4 & 1.97E-05 & 2. ISE-05 & 2.64E-05 & $2.86 \mathrm{E}-0 \mathrm{~S}$ \\
\hline Pu-240 & 4.09E- 06 & 3.16E-03 & 2.26 & $3.27 \mathrm{E}-06$ & $3.6 \pi+06$ & $4.52 \mathrm{E}-06$ & 4. 2 E- 06 \\
\hline Pu-241 & $4.74 E-05$ & $3.66 \mathrm{E}-02$ & 26.2 & $3.96 \mathrm{E}-05$ & 4.14E,0S & 5.35E-05 & $5.93 \mathrm{E}-05$ \\
\hline Pu-242 & $2.60 \mathrm{E}-10$ & 2.01E-07 & 1.44E-04 & 1.90E-10 & $2.24 \mathrm{E}-10$ & $2.96 \mathrm{E}-10$ & $3.3 \mathrm{HE}-10$ \\
\hline Am-241 & $2.95 \mathrm{E}-05$ & 2.28E-02 & 16.3 & 2.13E-05 & 2.53E-0S & 3.37E-05 & $3.77 \mathrm{E}-0 \mathrm{~S}$ \\
\hline Am-243 & 1.01E-09 & 7.83E-07 & 5.60E-04 & $7.55 \mathrm{E}-10$ & 8.78E-10 & $1.15 E-09$ & $1.28 \mathrm{E}-09$ \\
\hline $\mathrm{Cm}+242$ & 7.46E-08 & $5.76 \mathrm{E}-05$ & 4.12E-02 & $3.52 \mathrm{E}-08$ & 3.52E-08 & $7.96 \mathrm{E}-08$ & 8.45E-03 \\
\hline $\mathrm{Cm}-243$ & 6.89E-09 & $5.32 \mathrm{E}-06$ & 3.81E-03 & 3.14E-09 & 3.14E- 09 & 7.34E-09 & $7.38 \mathrm{E}-09$ \\
\hline $\mathrm{Cm}-244$ & 6.85E-OA & $5.29 \mathrm{E}-2 \mathrm{~S}$ & $3.78 E-02$ & 2.9AE-00 & 2.98E-08 & $8.16 E-08$ & $8.99 E-08$ \\
\hline Totals & $\mathbf{M}$ & 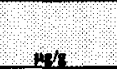 & 16 & 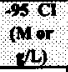 & $\begin{array}{l}67 \mathrm{CT} \\
\mathrm{Mor} \\
\text { /L) }\end{array}$ & $\begin{array}{l}+6 \mathrm{CI} \\
\mathrm{MOr} \\
1 \mathrm{~L})\end{array}$ & $\begin{array}{l}+9 \mathrm{Cl} \\
\mathrm{Mut} \\
(\mathrm{d})\end{array}$ \\
\hline Po & $3.03 \mathrm{E}-04(\mathrm{~g} / \mathrm{L})$ & $=$ & 0.167 & 2.19E-04 & 2.60t-04 & $3.45 \mathrm{E}-04$ & $3.46 \mathrm{E}-04$ \\
\hline $\bar{U}$ & $4.77 \mathrm{E}-03$ & 877 & 627 & 4.56E- 03 & $4.66 \mathrm{E}-03$ & 4.89E-03 & $4,92 \mathrm{E}-03$ \\
\hline
\end{tabular}

"Denaity is calculeted based an Na, OH-, and AJO2-

tWater wr\% derived from the difference of density and total dievolved species. 
HNF-SD-WM-ER-352, Rev. 1

HDW Model Rov. 4

\begin{tabular}{|c|c|c|c|c|c|c|c|}
\hline \multirow[b]{3}{*}{$\begin{array}{l}\text { Plyyileal } \\
\text { Propentite }\end{array}$} & \multicolumn{7}{|c|}{ Single-Shell Tank 241-SX-101 } \\
\hline & \multicolumn{7}{|c|}{ Total Inventory Estimate* } \\
\hline & $\mathrm{O}$ & & & $95 \mathrm{CI}$ & $67 \mathrm{Cl}$ & $+67 \mathrm{CI}$ & $+95 \mathrm{Cl}$ \\
\hline Total Waste & $2.75 \mathrm{E}+06(\mathrm{~kg})$ & (456 kgal) & - & - & $\ldots$ & $\rightarrow$ & $\ldots$ \\
\hline Heat Load & $6.30(\mathrm{~kW})$ & (2.15E+04 BTU/hr) & - & 5.25 & 5.86 & 6.60 & 0.71 \\
\hline Bulk Density' & $1.59(\mathrm{~g} / \mathrm{cc})$ & $m$ & - & 1.50 & 1.52 & 1.71 & 1.76 \\
\hline & & & & & & & \\
\hline Water wt $\%$ & 38.3 & $=$ & - & 26.9 & 304 & 44.3 & 46.2 \\
\hline $\mathrm{TOC} \mathrm{w} \% \mathrm{C}(\mathrm{w}$ & 0.115 & 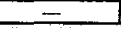 & 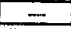 & $7.65 \mathrm{E}-02$ & $9,59 \mathrm{E}-02$ & 0,136 & 0.155 \\
\hline 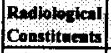 & $\mathrm{CV} / \mathrm{L}$ & $\mu \mathrm{CV} /$ & Cl & $\begin{array}{l}95 \mathrm{CA} \\
(\mathrm{CL} / \mathrm{L})\end{array}$ & $\begin{array}{l}-67 \mathrm{Cl} \\
(\mathrm{CV})\end{array}$ & $\begin{array}{l}+67 \mathrm{Cl} \\
(\mathrm{CH} / \mathrm{L})\end{array}$ & $\begin{array}{l}+95 \mathrm{CI} \\
(\mathrm{CUD})\end{array}$ \\
\hline $\mathrm{H}-3$ & $1.26 \mathrm{E}-04$ & 7.59E-02 & 217 & 4. ISE-05 & $4.15 \mathrm{E}-05$ & $1.30 \mathrm{E}-04$ & $1.36 \mathrm{E}-04$ \\
\hline C-14 & $1.03 E-05$ & $6.45 E-03$ & 17.7 & 9.96E-06 & $5.96 \mathrm{E}-06$ & $1.06 E-05$ & $1,00 E-0 S$ \\
\hline Ni-59 & 7.71E- $-\infty 6$ & 4.84E-03 & 13.3 & $5.36 \mathrm{E}-06$ & $5,36 \mathrm{E}-06$ & 8.18E- 06 & 8.09E-06 \\
\hline $\mathrm{Ni}-63$ & $7.30 \mathrm{E}-04$ & 0.458 & $1.26 \mathrm{E}+03$ & $5.02 \mathrm{E}-04$ & S.02E-04 & $7.74 \mathrm{E}-04$ & $7.65 \mathrm{E}-04$ \\
\hline $\mathrm{Co}-60$ & 9,99E-06 & $6.27 \mathrm{E}-03$ & 17.2 & $5.54 E-06$ & $5,54 \mathrm{E}-06$ & $1.01 \mathrm{E}_{0} 05$ & $1.05 \mathrm{E}-05$ \\
\hline Se-79 & $3.10 \mathrm{E}-06$ & $1.94 E-03$ & 5.35 & $6.66 \mathrm{E}-07$ & $6.66 E-07$ & $3.19 \mathrm{E}-06$ & 5.32E-06 \\
\hline $5 \mathrm{r}-90$ & 0.422 & 265 & $7.28 \mathrm{E}+05$ & 0.332 & 0.383 & 0.452 & 0.457 \\
\hline$Y-90$ & 0.422 & 265 & 7.28E+0S & $0.33 !$ & 0.331 & 0.452 & 0.457 \\
\hline $2 r-93$ & 1.48E-05 & 9.26E-03 & 25.5 & $3.23 \mathrm{E}-06$ & 1.23E- 06 & 1.52E-05 & $2.41 \mathrm{E}-05$ \\
\hline $\mathrm{Nb}-93 \mathrm{~m}$ & 1.18E-05 & $7.37 \mathrm{E}-03$ & 20.3 & $2.43 \mathrm{E}-06$ & 2.43E-06 & $1.21 \mathrm{E}-05$ & $2.18 \mathrm{E} \cdot 0 \mathrm{~S}$ \\
\hline Tc-99 & 7.57E-0S & $+75 \mathrm{E}-02$ & 131 & 6.12E-05 & $6.83 \mathrm{E}-05$ & $8.31 \mathrm{E}-0 \mathrm{~S}$ & $9.03 \mathrm{E}-05$ \\
\hline$R_{u-106}$ & $1.91 \mathrm{E}-09$ & $1.20 E-06$ & $3.29 \mathrm{E}-03$ & 1.01E-09 & $1.01 \mathrm{E}-09$ & 2.03E- 09 & 2.15E-09 \\
\hline $\mathrm{Cd}+113 \mathrm{~m}$ & $3.26 \mathrm{E}-05$ & 2.04E-02 & 56.2 & $1.51 \mathrm{E}-05^{-1}$ & $1.51 E-05$ & $5.13 E-05$ & 495E-05 \\
\hline Sb-12s & 3.94E-0S & $2.47 \mathrm{E}-02$ & 68.1 & 2.02E-05 & $202 \mathrm{E}-05$ & 4.00E-05 & $4.11 \mathrm{E}-05$ \\
\hline$S n-126$ & 4.74E-06 & 2.91E-03 & 8.19 & $1.01 \mathrm{E}-06$ & $1,01 \mathrm{E}-06$ & 4.81E-06 & 8.32E-06 \\
\hline $\mathrm{I}-129$ & 1.45E-07 & 9. LOE-05 & 0.250 & $1.17 E-07$ & $1.31 \mathrm{E}-07$ & $1.59 \mathrm{E}-07$ & $1.73 \mathrm{E}-07$ \\
\hline C:-134 & $1.21 \mathrm{E}-06$ & $7.58 \mathrm{E}-04$ & 2.09 & 3.50E-07 & $3.50 \mathrm{E}-07$ & $1.27 E-06$ & 1.33E-06 \\
\hline$C_{5} \cdot 137$ & 0.173 & 109 & $299 \mathrm{E}+05$ & 0.159 & 0.166 & 0.182 & 0.192 \\
\hline Ba-137m & 0.164 & 103 & $2.63 \mathrm{E}+05$ & $4.94 \mathrm{E}-02$ & $4.94 E-02$ & 0.169 & 0.176 \\
\hline $5 m-151$ & $1.10 \mathrm{E}-02$ & 6.92 & $1,90 E+04$ & $2.35 \mathrm{E}-03$ & $2.35 \mathrm{E}-03$ & 1.13E-02 & 1.92E-02 \\
\hline Eu-152 & $6.03 \mathrm{E}_{-}-06$ & 3.79:-03 & 104 & 2.97E-06 & $2.97 \mathrm{E}-06$ & $6.07 \mathrm{E}-06$ & 6. 12E-06 \\
\hline$E x-154$ & 1.92E-04 & 0.120 & 331 & 9.ASE-0S & 9.68E-0S & 2. $14 \mathrm{E}-04$ & $2.23 \mathrm{E}-04$ \\
\hline Eu+15s & $3.03 E-04$ & 0.190 & 523 & $1.4 \pi \mathrm{E}-04$ & 1.47E-04 & $3.05 \mathrm{E}-04$ & 3.0BE-04 \\
\hline $\operatorname{Ra}-226$ & 5. $49 \mathrm{E}-10$ & 3.45E- -07 & 9,48E-04 & 2.38E-10 & $3.87 \mathrm{E}-10$ & $7.08 \mathrm{E}-10$ & 8.61E-10 \\
\hline Rn.228 & $2.6 \pi-08$ & 1,69t-05 & 4.61E-02 & $1,42 E-08$ & $2.03 \mathrm{E}-0 \mathrm{~s}$ & $3.41 E-08$ & 4.19E-08 \\
\hline Ac-227 & 2.70E-09 & $1.69 \mathrm{E}-06$ & 4.66E-03 & $121 \mathrm{E}-09$ & $1.75 \mathrm{E}-09$ & $3.62 E-09$ & 4.50E-09 \\
\hline Pa-231 & 4.47E-09 & $2.81 \mathrm{E}-06$ & $7.72 E-03$ & 8.77E-10 & 8.T7E-10 & 4.91E- 09 & 9.83E-09 \\
\hline Th-229 & 6.30E-10 & $3.95 \mathrm{E}-07$ & $1.0 \mathrm{EE}-03$ & $3.57 \mathrm{E} \cdot 10$ & 4.90E-10 & $7.92 E-10$ & $9.65 \mathrm{E}-10$ \\
\hline $\mathrm{Th}-232$ & 1.49E-09 & 9,35E-07 & $2.57 \mathrm{E}-03$ & t.01E-10 & $1.14 E-09$ & 1.84E-09 & 2.18E-09 \\
\hline U-232 & $1.35 \mathrm{E}-07$ & 8.4SE-0S & 0.233 & 1.52E-08 & 1.10E-07 & $1.65 E-07$ & $1.96 \mathrm{E}-07$ \\
\hline $\mathrm{U}-233$ & 5. 18E-07 & $3.25 \mathrm{SE}-04$ & 0.294 & $3,37 \mathrm{E}-07$ & 1.20E-07 & 6.31E-07 & $7.52 \mathrm{E}-07$ \\
\hline $\mathrm{U}-234$ & 6.03E-07 & 3.E2E-OA & 1.05 & 1.64E-07 & 4.63E-07 & 7.4AE-07 & 8.81E-07 \\
\hline $\mathrm{U}-235$ & $2.47 E-08$ & $1.55 \mathrm{E}-05$ & $427 \mathrm{E}-02$ & 1.48E-0s & $1.96 \mathrm{E}-0 \mathrm{~s}$ & 3.02E-0: & $3.58 \mathrm{E}-0 \mathrm{~B}$ \\
\hline $\mathrm{U}-236$ & 2.265-0A & 1.43E-05 & $3.93 \mathrm{E}-02$ & $1.30 \mathrm{E}-0 \mathrm{~B}$ & $1.79 \mathrm{E}-08$ & 2.105-00 & $3.3 \mathrm{IE}-08$ \\
\hline $\mathrm{U}-238$ & 5.75E-OP & 3.61E-04 & 0.992 & $3.5 \%-07$ & $464 E-07$ & $6.965-07$ & $8.1 \mathrm{E}-\mathrm{m}$ \\
\hline$N p-237$ & 3. $14 \mathrm{E}-07$ & $1.97 \mathrm{E}-04$ & 0.542 & $2.6 \mathrm{TE}-07$ & 2.90E-07 & $3.39 \mathrm{E}-07$ & $3.62 \mathrm{E}-07$ \\
\hline Pu-238 & $3.26 \mathrm{E}-06$ & $2.05 \mathrm{E}-03$ & 5.64 & $2.64 \mathrm{E}-06$ & $2.95 \mathrm{E}-06$ & $3.58 \mathrm{E}-06$ & $3.89 \mathrm{E}-06$ \\
\hline Pu-239 & $1,93 \mathrm{E}-\mathrm{OA}$ & 0.121 & 333 & 1.49E-04 & $1.71 \mathrm{E}-04$ & $2.16 E-04$ & $2.38 \mathrm{E}-04$ \\
\hline Pu-240 & 2.84E-05 & 1.79E-02 & 49.1 & 2.21E-0S & 2.52E-05 & $3.17 \mathrm{E} 0 \mathrm{~S}$ & 3.48E-05 \\
\hline Pu-241 & $1.93 \mathrm{E}-04$ & 0.120 & 329 & 1.53E-04 & $1.72 \mathrm{E}-04$ & $2.10 \mathrm{E}-0.1$ & $2.25 E-04$ \\
\hline $\mathrm{Pu}-242$ & $9.18 \mathrm{E}-10$ & $5.76 \mathrm{E}-07$ & 1.STE-03 & $7,47 \mathrm{E}-10$ & 8.31E-10 & $1.01 \mathrm{E}-09$ & $1.09 \mathrm{E}-09$ \\
\hline Am-241 & S.21E-05 & $3.27 \mathrm{E}-02$ & 89.9 & $3.43 \mathrm{E}-05$ & $4.67 \mathrm{E}-05$ & $5.79 \mathrm{E}-05$ & $9.71 \mathrm{E}-0 \mathrm{~s}$ \\
\hline Am-243 & $1,625-09$ & $1.02 E-06$ & 2.20E-03 & $9.16 \mathrm{E}-10$ & $1.44 \mathrm{E}-09$ & $1.76 E-09$ & $2.05 \mathrm{E}-09$ \\
\hline $\mathrm{Cm}-242$ & $7.85 E-06$ & 4.9E-AS & 0.136 & 6.62E-00 & $6.62 \mathrm{E}-0 \mathrm{t}$ & B.04E-08 & 8.195-08 \\
\hline $\mathrm{Cm}-243$ & $3.46 \mathrm{E}-09$ & $2.17 \mathrm{E}-06$ & 59:E-03 & $2.26 \mathrm{E}-09$ & $2.26 \mathrm{E}-09$ & $3.61 E-09$ & 3.75E-09 \\
\hline $\mathrm{Cm}-244$ & $2.29 \mathrm{E}-0 \mathrm{~s}$ & 1.44E-OS & $3,95=-02$ & 1.05E-08 & $1.05 \mathrm{E}-08$ & $2.71 \mathrm{E}-08$ & $2.98 \mathrm{E}-08$ \\
\hline Totills & $\mathrm{M} \mathbf{M l}$ & $\operatorname{ton} \alpha$ & $k$, & $\begin{array}{l}-95 \mathrm{Cl} \\
\mathrm{am} / \mathrm{ar} \\
\mathrm{s} / \mathrm{L}) \mathrm{l}\end{array}$ & 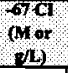 & 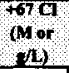 & 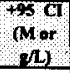 \\
\hline Pu & $3.20 \mathrm{E}-03(\mathrm{~g} / \mathrm{L})$ & - & 5.52 & 2.45E-03 & 2.82E-03 & $3.50 \mathrm{D}-03$ & $3.94 \mathrm{E}-03$ \\
\hline $\mathrm{v}$ & $0.70 \mathrm{E}-03$ & 1.01E+03 & $2.78 E+03$ & $4.00 \mathrm{E}-03$ & $5.38 \mathrm{E}-03$ & $0.26 \mathrm{E}-03$ & $9,78 \mathrm{E}-03$ \\
\hline
\end{tabular}

-Unknowns in tank solids inventory are asnigned by Tank Layering Model (TLM).

+Volume avertage for density, mass average Water wt\% and TOC wt\% C. 


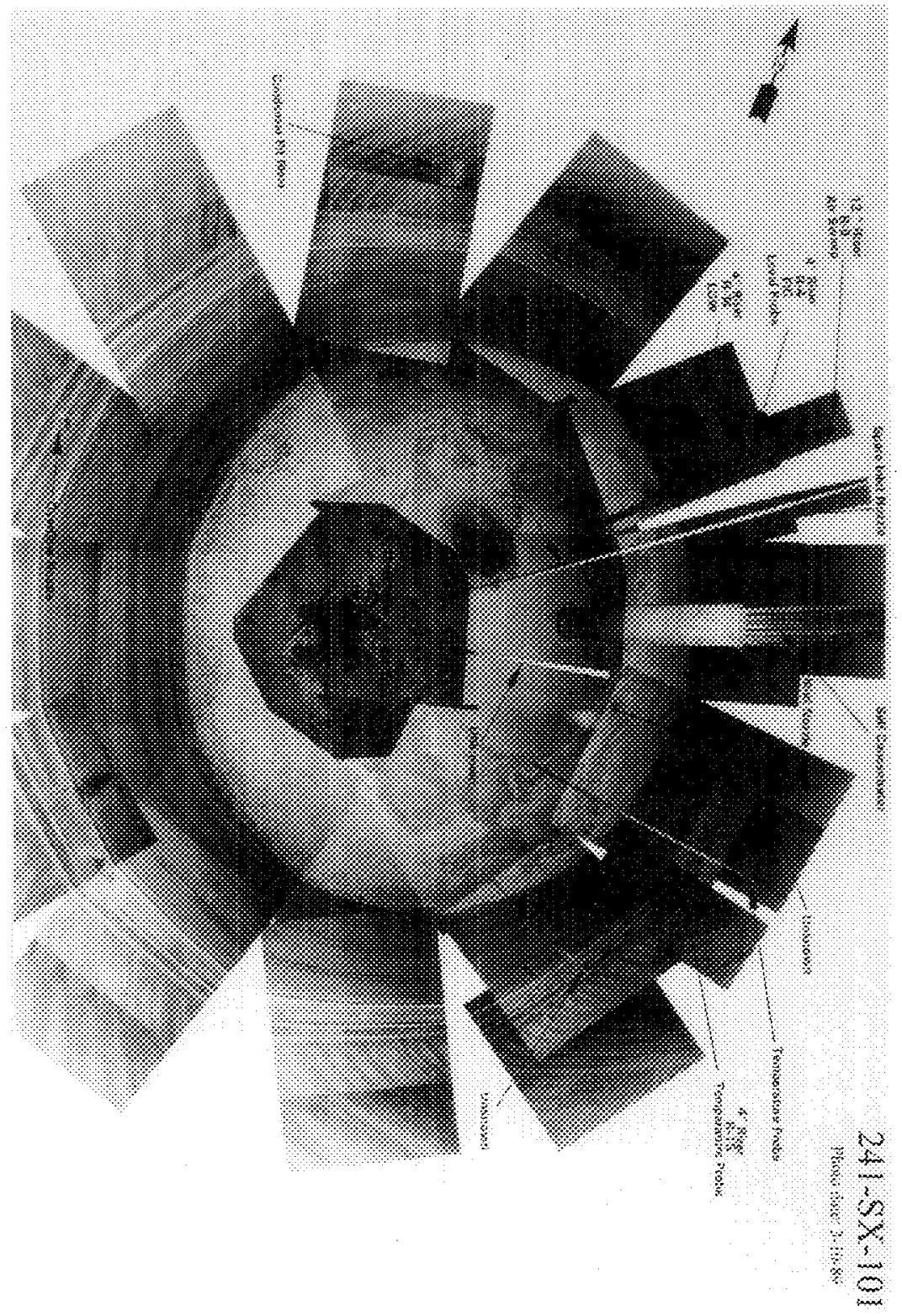


TANK 241-SX-102 SUMMARY

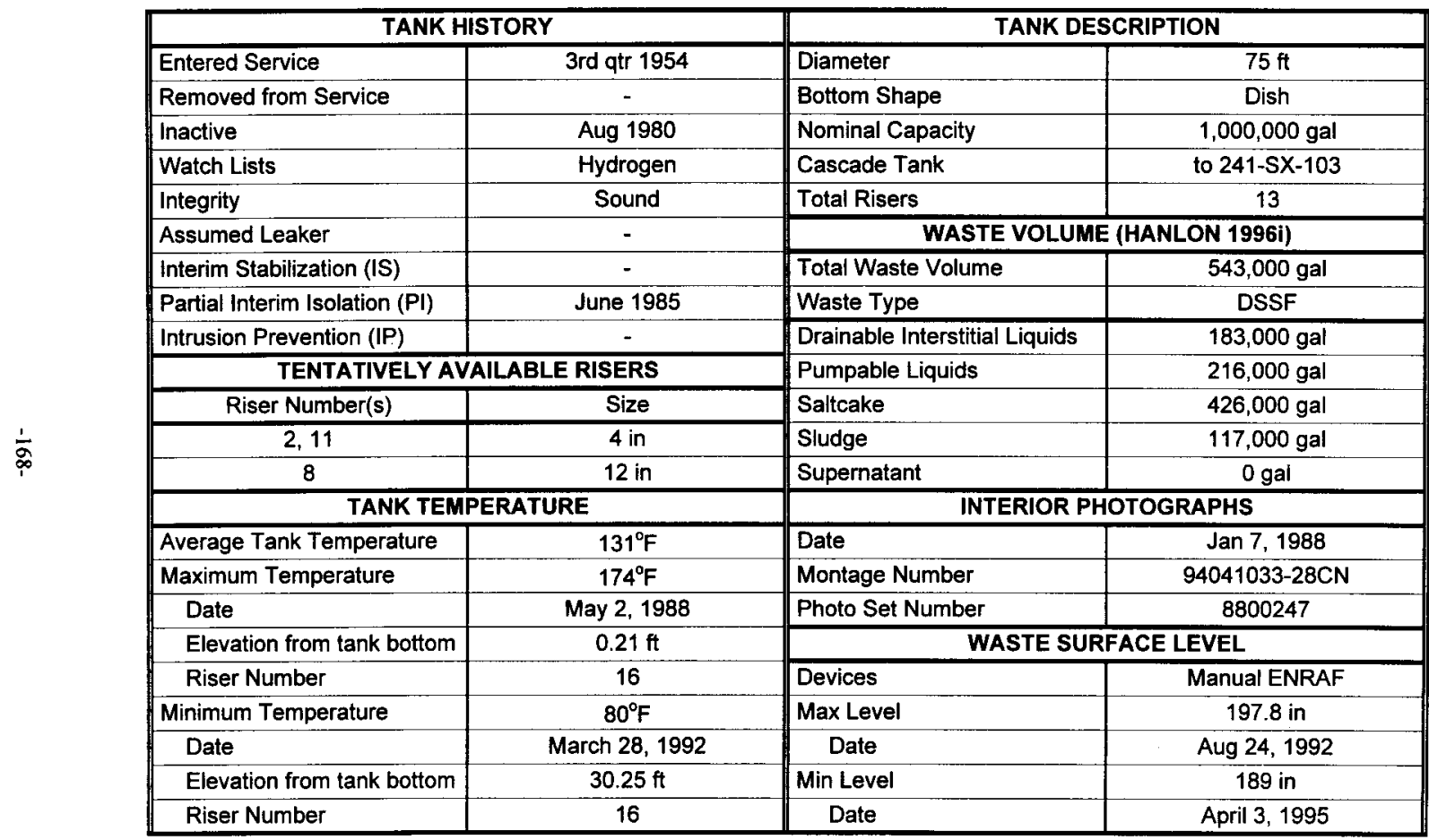




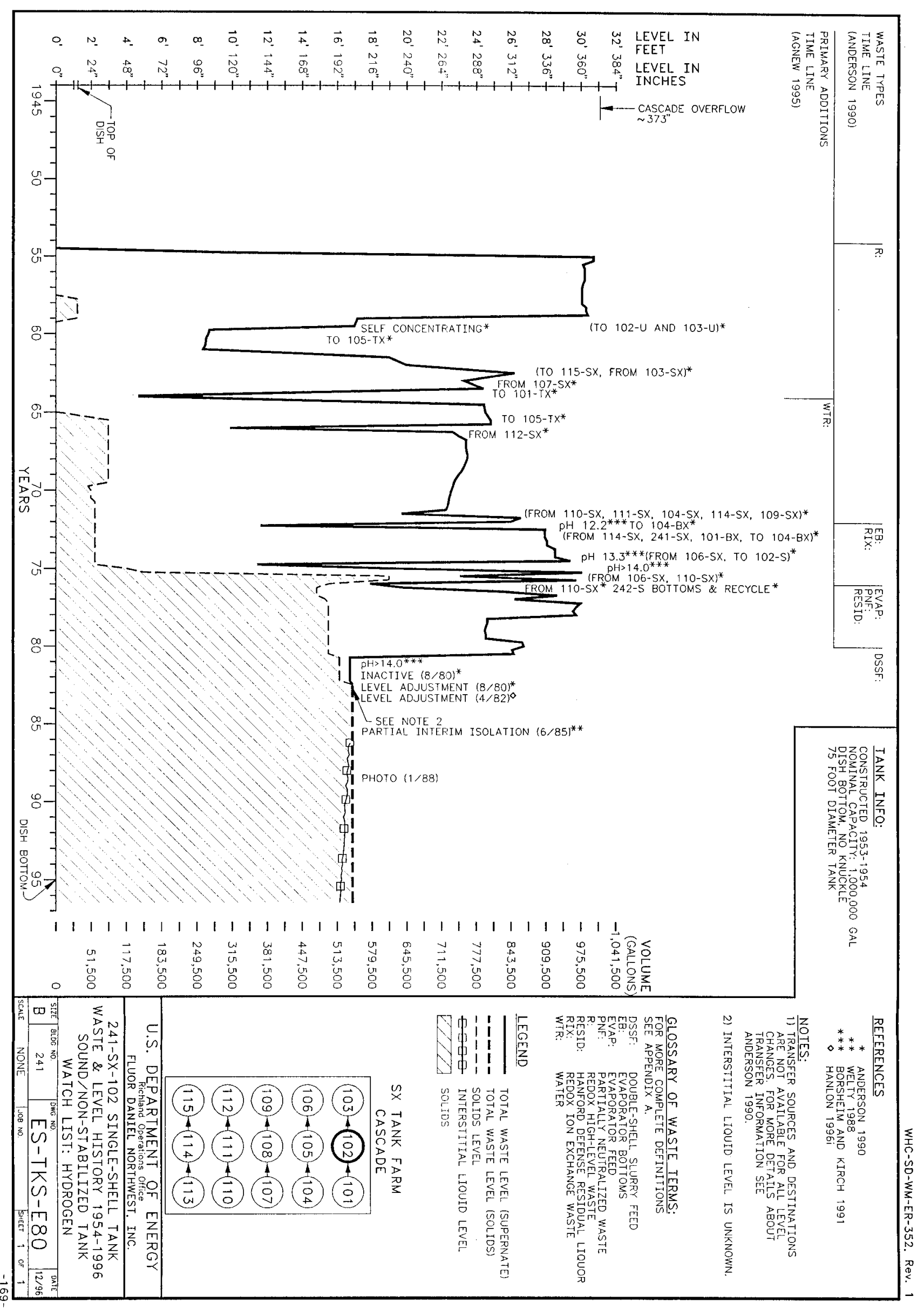



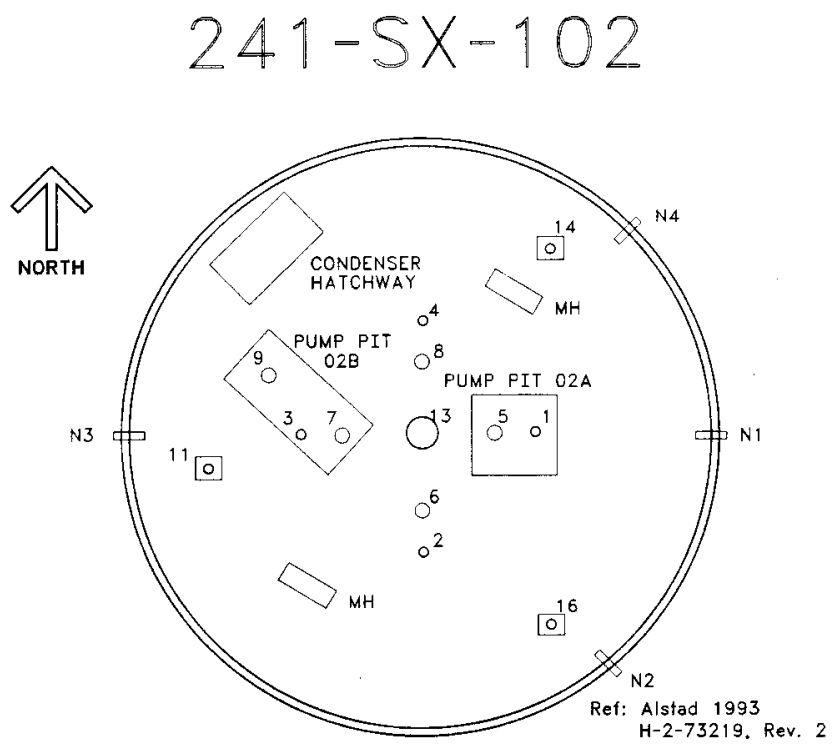

TANK RISER LOCATION

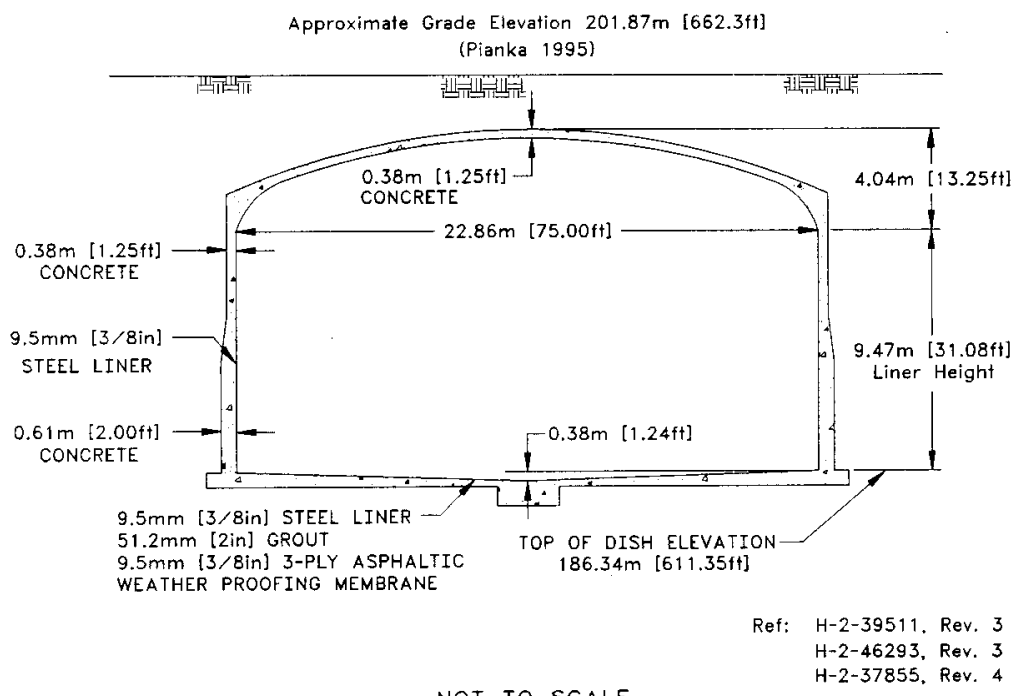

NOT TO SCALE

$-170-$ 


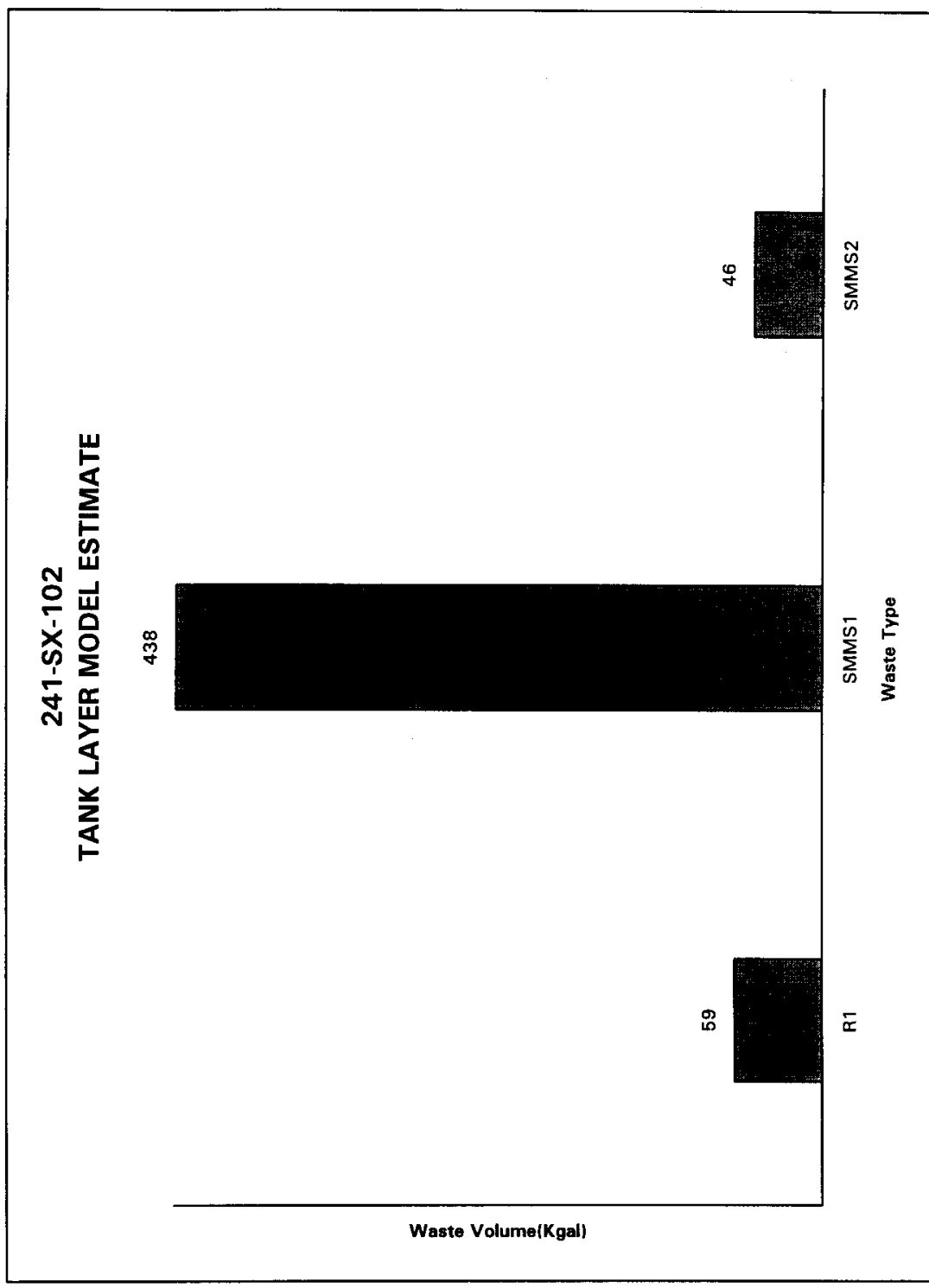


HDW Model Rev. 4

\begin{tabular}{|c|c|c|c|c|c|c|c|}
\hline \multicolumn{8}{|c|}{ Single-Shell Tank 241-SX-102 } \\
\hline & \multicolumn{7}{|c|}{ TLM Solids Composite Inventory Estimate" } \\
\hline $\begin{array}{l}\text { Phycical } \\
\text { Prepictitlas }\end{array}$ & & & & $-95 \mathrm{CI}$ & $-67 \mathrm{CI}$ & $+67 \mathrm{CI}$ & $+95 \mathrm{Cl}$ \\
\hline Total TLM Wo & $3.92 \mathrm{E}+05(\mathrm{~kg})$ & $(59.0 \mathrm{kgal})$ & - & $\ldots$ & - & $\ldots$ & - \\
\hline Heat Load & $1.45(\mathrm{~kW})$ & (4.97E+03 BTU/hr) & - & 1.00 & 1.29 & 1.56 & 1.61 \\
\hline Bulk Density & $1.76(\mathrm{~g} / \mathrm{cc})$ & $\cdots$ & - & 1.51 & 1.53 & 2.11 & 2.24 \\
\hline Void Fraction & 0.536 & - & $=$ & $6.00 \mathrm{E}-02$ & 0.217 & 0.737 & 0.743 \\
\hline Water wt $\%$ & 27.0 & - & - & 2.64 & 100 & 42.6 & 44.2 \\
\hline$T O C w T C(w)$ & 0 & $m$ & - & 0 & 0 & 0 & 0 \\
\hline $\begin{array}{l}\text { Chemical } \\
\text { Constituest }\end{array}$ & notel & $\mathrm{Pom}$ & ks & $\begin{array}{l}95 \mathrm{CI} \\
\text { (mole/L) }\end{array}$ & $\begin{array}{l}-67 \mathrm{Cl} \\
\text { (moled ) }\end{array}$ & $\begin{array}{l}+67 \mathrm{ct} \\
(\text { molelt })\end{array}$ & $\begin{array}{l}+9 \mathrm{C1} \\
\text { (mote/L) }\end{array}$ \\
\hline Nat & 8.13 & $1.06 \mathrm{E}+05$ & $4.17 \mathrm{E}+04$ & 3.05 & 3.56 & 14.7 & 18.2 \\
\hline$\overline{A 13+}$ & 6.99 & $1.07 \mathrm{E}+05$ & $4.21 \mathrm{E}+04$ & 6.58 & 6.74 & 7.15 & 7,15 \\
\hline $\mathrm{Fe} 3+($ tolal Fe $)$ & 1.01 & $3.22 \mathrm{E}+04$ & $1.26 \mathrm{E}+04$ & 0.994 & 1,00 & 1.02 & 1.03 \\
\hline $\mathrm{Cr}^{3}+$ & 6.19E-02 & $1.83 \mathrm{E}+03$ & 718 & $7.08 E-03$ & $3,39 \mathrm{E}-02$ & 0.522 & 1,06 \\
\hline Bi3+ & 0 & 0 & 0 & 0 & 0 & 0 & 0 \\
\hline Le3t & 0 & 0 & 0 & 0 & 0 & 0 & 0 \\
\hline $\mathrm{Hg} 2+$ & 0 & 0 & 0 & 0 & 0 & 0 & 0 \\
\hline $\mathrm{Zr}(\mathrm{MrOMOH}) 2$ & 0 & 0 & 0 & 0 & 0 & 0 & 0 \\
\hline Pb2+ & 0 & 0 & 0 & 0 & 0 & 0 & 0 \\
\hline $\mathrm{N} 2+$ & $3.07 E-02$ & $1.69 E+03$ & 665 & $3.66 \mathrm{E}-02$ & $4.45 \mathrm{E}-02$ & $5.54 \mathrm{E}-02$ & 5.45E-02 \\
\hline $\mathrm{Sr} 2+$ & 0 & 0 & 0 & 0 & 0 & 0 & 0 \\
\hline $\mathrm{Mn4t}$ & 0 & 0 & 0 & 0 & 0 & 0 & 0 \\
\hline $\mathrm{Cu} 2 \mathrm{t}$ & 0.220 & $5.02 \mathrm{E}+03$ & $1.97 \mathrm{E}+03$ & 0.146 & 0.182 & 0.258 & 0.294 \\
\hline $\mathbf{K +}$ & $1.12 \mathrm{E}-02$ & 250 & 98,0 & 1.28E-03 & $5.25 \mathrm{E}-03$ & $1.52 \mathrm{E}-02$ & $1.52 \mathrm{E}-02$ \\
\hline OH- & 25.6 & $2.48 \mathrm{E}+03$ & $9.72 \mathrm{E}+04$ & 23.1 & 24.3 & 26.9 & 28.4 \\
\hline NO3- & 5.29 & 1.8TE+05 & $7.32 \mathrm{E}+04$ & $6.03 \mathrm{E}-02$ & $6.03 \mathrm{E}-02$ & 13.5 & 17.9 \\
\hline NO2. & 1.46 & $3.82 \mathrm{E}+04$ & $1.50 \mathrm{E}+0 \mathrm{~A}$ & 0.132 & 0.595 & 2.15 & 2.15 \\
\hline CÖ2- & 0.220 & $7.51 \mathrm{E}+03$ & $295 E+03$ & 0.146 & 0.182 & 0.258 & 0.294 \\
\hline PO43: & 0 & 0 & 0 & 0 & 0 & 0 & 0 \\
\hline SO42- & 1.04E-02 & 569 & 223 & 1,19E-03 & 4.87E-03 & $1.41 \mathrm{E}-02$ & $1.41 \mathrm{E}-02$ \\
\hline $\mathrm{Si}$ (a $\mathrm{SiO32-)}$ & $8.06 \mathrm{E}-03$ & 129 & 50.5 & 9.21E-04 & $3.77 \mathrm{E}-03$ & 1.09E-02 & $100 E-02$ \\
\hline $\mathbf{F}-$ & 0 & 0 & 0 & $\mathbf{0}$ & 0 & 0 & 0 \\
\hline $\mathrm{Cl}$ - & $5.16 \mathrm{E}-02$ & 1.04E+03 & 409 & $591 \mathrm{E}-03$ & $2.42 \mathrm{E}-02$ & $7.00 \mathrm{E}-02$ & 0.157 \\
\hline C6HSO73- & 0 & 0 & 0 & $\mathbf{0}$ & 0 & 0 & 0 \\
\hline EDTA4 & 0 & 0 & 0 & 0 & 0 & 0 & 0 \\
\hline HEDTA3- & 0 & 0 & 0 & 0 & 0 & 0 & 0 \\
\hline glyoolnte- & 0 & 0 & 0 & 0 & 0 & 0 & 0 \\
\hline acetane- & 0 & 0 & 0 & 0 & 0 & 0 & 0 \\
\hline oxalate2- & 0 & 0 & 0 & 0 & 0 & 0 & 0 \\
\hline DBP & 0 & 0 & 0 & 0 & 0 & 0 & 0 \\
\hline butanol & 0 & 0 & 0 & 0 & 0 & 0 & 0 \\
\hline $\mathrm{NH3}$ & 0.256 & $2.47 E+03$ & 970 & 6.55E-02 & $9.25 \mathrm{E}-02$ & 0.270 & 0284 \\
\hline$F \propto(C N) 64$ & 0 & 0 & 0 & 0 & 0 & 0 & 0 \\
\hline
\end{tabular}

-Unknowns in tank solids inventory are tuigned by Tank Leyering Model (TLM) 
HOW Model Rev. 4

\begin{tabular}{|c|c|c|c|c|c|c|c|}
\hline & \multicolumn{7}{|c|}{ Single-She!l Tank 24!-SX-102 } \\
\hline & \multicolumn{7}{|c|}{ SMM Composite lnventory Estimate } \\
\hline $\begin{array}{l}\text { Phyoltal } \\
\text { Propertices }\end{array}$ & & & & $-95 \mathrm{CI}$ & $-67 \mathrm{CI}$ & $+67 \mathrm{CI}$ & $+95 \mathrm{Cl}$ \\
\hline Total SMM W & $3.20 \mathrm{E}+06(\mathrm{~kg})$ & $(484 \mathrm{kgal})$ & $\cdots$ & - & 一 & $\rightarrow$ & - \\
\hline Heat Lond & $477(\mathrm{kw})$ & (1.63E+04 BTUhr) & $=$ & 4.46 & 4.02 & 4.92 & 5.07 \\
\hline Bulk Density & $1.75(g / c c)$ & - & - & 1.68 & 1.72 & 1.77 & 180 \\
\hline Water wt\% & 24.0 & $\longrightarrow$ & ــ & 210 & 223 & 25.7 & 27.7 \\
\hline TOC wt\% C (w) & 0.932 & $\cdots$ & - & $0.6] \mathrm{B}$ & 0.772 & 1.09 & 1.25 \\
\hline Chomton 1 & nold 1 & pms & tre & $\begin{array}{l}-95 \mathrm{CI} \\
\text { (noled }\end{array}$ & $\begin{array}{l}-67 \mathrm{Ct} \\
\text { (wale/h) }\end{array}$ & $\begin{array}{l}+67 \mathrm{Cl} \\
(\text { noldt })\end{array}$ & (mole/t) \\
\hline Nat & 17,6 & 2.31E+05 & $7.39 \mathrm{E}+05$ & 15.9 & 16.7 & 18.2 & 18.9 \\
\hline Al3+ & 2.03 & 3.14E+04 & $1.00 E+05$ & 1.87 & 1.95 & 2.11 & 2.19 \\
\hline$F_{e j}^{3+}\left(\cot 1 \mathrm{~F}_{\theta}\right)$ & $1.39 \mathrm{E}-02$ & 445 & $1,42 E+03$ & $1.12 \mathrm{E}-02$ & $1.261-02$ & $1.53 \mathrm{E}-02$ & $1.66 \mathrm{E}-02$ \\
\hline $\mathrm{Cr}_{3+}$ & 0.177 & $5.26 \mathrm{E}+03$ & $1.62 E+04$ & 0.151 & 0.165 & 0.182 & 0.187 \\
\hline $\mathrm{Bi} 3+$ & $1.72 E-03$ & 205 & 657 & I.S7E-03 & $1.64 E-03$ & $1.79 \mathrm{E}-03$ & $1.86 \mathrm{E}-03$ \\
\hline Le3+ & $6.94 E-03$ & 5.51 & 17.7 & $5.03 E-05$ & $5.96 \mathrm{E}-05$ & $7.92 \mathrm{E} \_05$ & $1.26 \mathrm{E}-05$ \\
\hline $\mathrm{Hg} 2+$ & 1.17E-05 & 1.34 & 430 & 1.10E-0S & 1.14E-0S & 1.19E-05 & $1.21 \mathrm{E}-05$ \\
\hline $2 \mathrm{r}(\mathrm{arO}(\mathrm{OH}) 2$ & 3.04E-04 & 15.9 & 50.8 & 2.77E-04 & $287 \mathrm{E} O \mathrm{OA}$ & 3,15E-OA & 3.30E-04 \\
\hline $\mathrm{Pb2+}$ & I.JIE-03 & 155 & 495 & $1.05 \mathrm{E}-03$ & J.18E-03 & $1.44 E_{-03}$ & $1.56 \mathrm{E}-03$ \\
\hline $\mathrm{Ni24}$ & $8.08 \mathrm{E}-03$ & 271 & 869 & $7.69 \mathrm{E}-03$ & $7.88 E-03$ & C.16E-03 & 8.27E-03 \\
\hline $\mathrm{S} 2+$ & 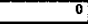 & 0 & 0 & $\mathbf{0}$ & 0 & 0 & 0 \\
\hline $\operatorname{Mn} 4+$ & 5.05E-03 & 159 & 508 & 3.76E-03 & $4,30 \mathrm{E}-03$ & $5.70 E-03$ & $6.33 \mathrm{E}-03$ \\
\hline $\mathrm{Ca2+}$ & $424 E-02$ & 973 & 3.11E+03 & 3.16E- 02 & 4.04E-02 & $4.44 E-02$ & $4.63 \mathrm{E}-02$ \\
\hline $\mathbf{K +}$ & 8. 30E-02 & 1. $86 \mathrm{E}+03$ & $5.95 E+03$ & $7.52 \mathrm{E}-02$ & $7.89 \mathrm{E}-02$ & $8.73 \mathrm{E}-02$ & $9.14 \mathrm{E}-02$ \\
\hline $\mathrm{OH}-$ & $\sqrt{2.2}$ & $1.19 E+05$ & 3.11E+0S & 11.4 & 118 & 12.7 & 130 \\
\hline No3. & 6.10 & 2.16E+0S & $6.93 \mathrm{E}+05$ & 5.64 & 5.91 & 6.19 & 6.27 \\
\hline NO2- & 3.03 & $7.97 \mathrm{E}+04$ & 2,55E+05 & 2.48 & 2.73 & 3.32 & 3.59 \\
\hline CO32- & 0.571 & $1,96 \mathrm{E}+04$ & $6.20 \mathrm{E}+04$ & 0.517 & 0,543 & 0.607 & 0.617 \\
\hline PO43- & 0.117 & $6.35 \mathrm{E}+03$ & $2.03 E+04$ & 0.101 & 0.107 & 0.119 & 0.122 \\
\hline SO42- & 0.318 & $1.75 E+04$ & $5.60 \mathrm{E} 404$ & 0.247 & 0.281 & 0.356 & 0.390 \\
\hline Si (as SiO32-) & 9.87E-02 & $1.59 \mathrm{E}+03$ & $5.08 E+03$ & 8.03E-02 & $8.93 \mathrm{E}-02$ & 0.108 & 0.117 \\
\hline F- & $8.46 \mathrm{E}-02$ & 920 & $2.94 E+03$ & $7.21 E-02$ & $7.73 \mathrm{E}-02$ & $9.07 \mathrm{E}-02$ & $9.56 \mathrm{E}-02$ \\
\hline $\mathrm{Cl}-$ & 0.304 & $6.16 E+03$ & $1.97 \mathrm{E}+04$ & 0.272 & 0.287 & 0.318 & 0.331 \\
\hline C6HSO73- & $3.81 E-02$ & $4.12 \mathrm{E}+03$ & $1.32 \mathrm{E}+04$ & $3.50 \mathrm{E}-02$ & $3.65 \mathrm{E}-02$ & $3.96 \mathrm{E}-02$ & 4.11E-02 \\
\hline EDTA4 & 1.99E-02 & $3.28 E+03$ & 1.05E+04 & $6.30 E-03$ & $1.30 \mathrm{E}-\mathrm{O} 2$ & $2.69 \mathrm{E}-02$ & $338 \mathrm{E}-02$ \\
\hline HEDTA 3- & $3.67 \mathrm{E}-02$ & $5.76 \mathrm{E}+03$ & 1.44E+04 & $9.50 E-03$ & $2,20 \mathrm{E}-02$ & $5.07 \mathrm{E}-02$ & $6.44 E-02$ \\
\hline glycolute- & 0.117 & $5.03 E+03$ & $1.61 \mathrm{E}+04$ & $7,67 \mathrm{E}-02$ & $9.65 \mathrm{E}-02$ & 0.138 & 0.158 \\
\hline scetniter & 9.9603 & 336 & $1.0 \mathrm{EE}+03$ & (29E-03 & $9.11 \mathrm{E}-03$ & $1.08 E-02$ & $1.16 \mathrm{E}-02$ \\
\hline onslate2- & 9.09E-05 & 4.58 & 14.7 & 1.05E-05 & C.56E-05 & $9.62 \mathrm{E}-05$ & $1.01 E-04$ \\
\hline DBP & $257 \mathrm{E}-02$ & 3.09E+03 & 9.85E+03 & 2. $12 \mathrm{E}-02$ & $2.34 \mathrm{E}-02$ & 2.79E-02 & 3.01E-02 \\
\hline butanol & $2.57 \mathrm{E}-02$ & $1.09 E+03$ & $3.49 \mathrm{E}+03$ & 2. $12 \mathrm{E}-02$ & $2.34 E-02$ & $2.79 E-02$ & $3.016-02$ \\
\hline NH3 & 0.106 & $1.04 E+03$ & $3.31 \mathrm{E}+03$ & E.575-02 & $9.39 E-02$ & 0.122 & 0.141 \\
\hline $\mathrm{Fe}(\mathrm{CN}) 64$ & 0 & 0 & 0 & 0 & 0 & 0 & 0 \\
\hline
\end{tabular}

-Density is calculated baned on Na, OH-, and ANO2.

+ Water wt\% derived from the difference of dentity and toth diusolved species. 
HDW Model Rov 4

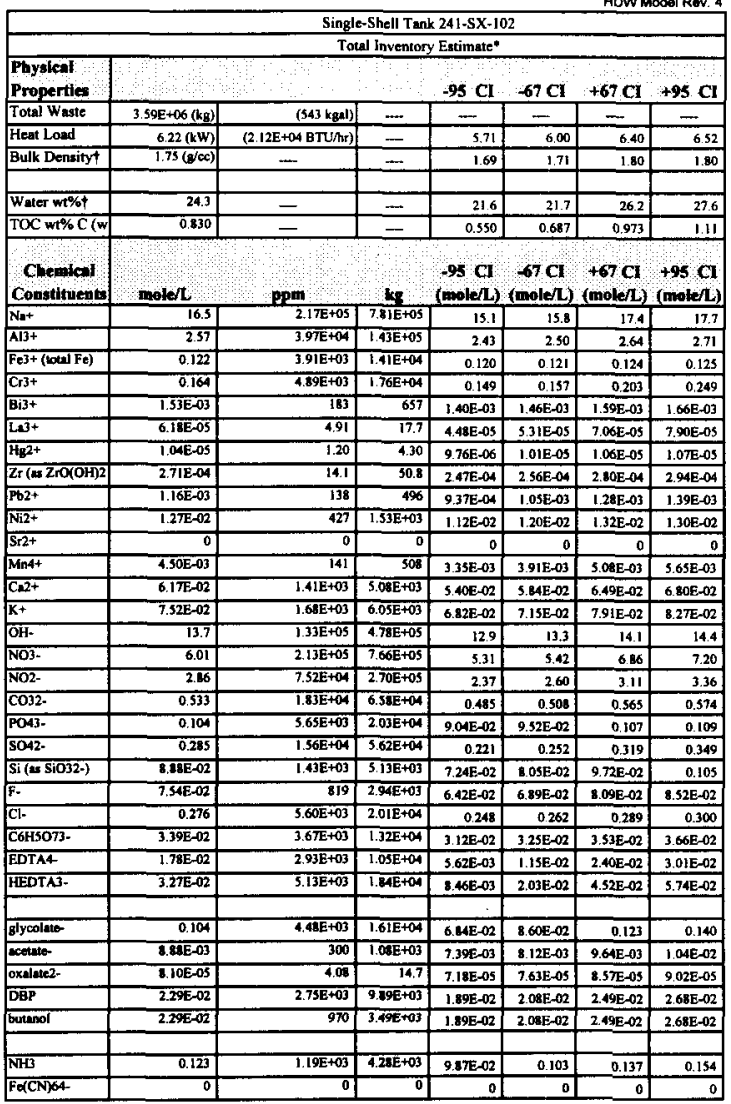

"Unknowns in tenk eolids inventory are asigned by Tenk Leyering Model (TLM).

$p$ Water wt\% derived from the difference of density and total dineolved epocies 
HDW Model Rey. 4

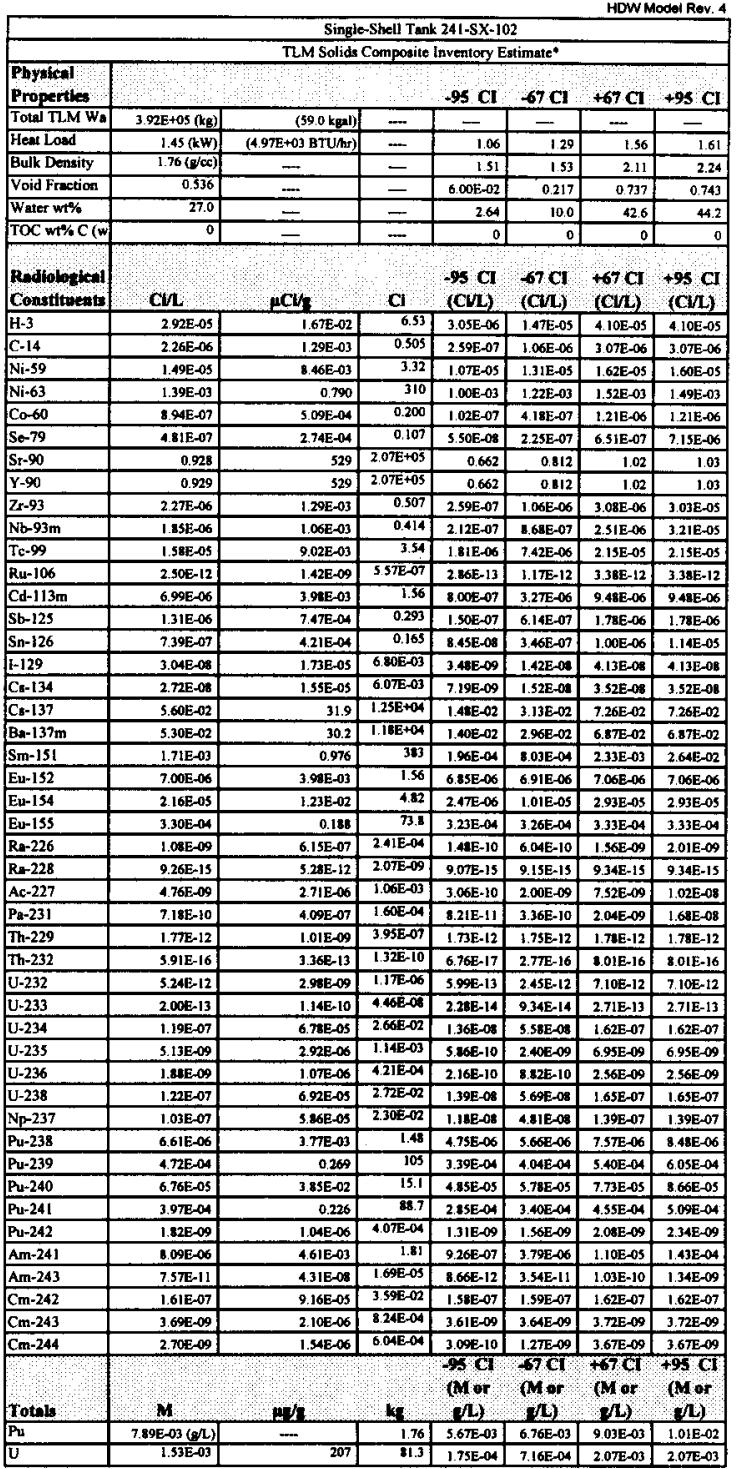

"Unknowns in tank solids inventory are assigned by Tenk Layering Model (TLM) 


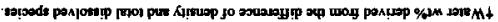

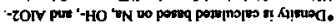

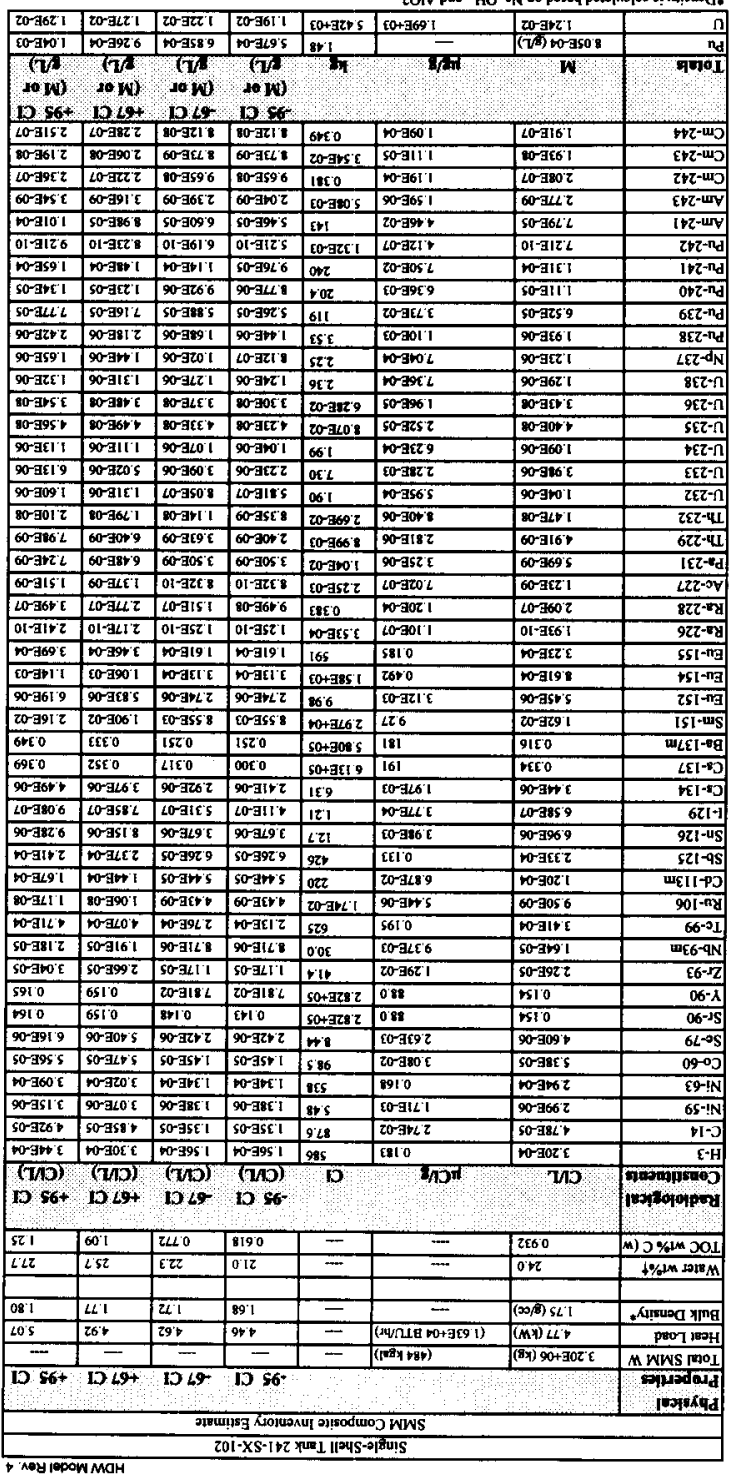


HNF-SD-WM-ER-352, Rev. 1

HDW Model Rev, 4

\begin{tabular}{|c|c|c|c|c|c|c|c|}
\hline \multirow{4}{*}{\begin{tabular}{|l|} 
\\
Phyacici \\
Propertics
\end{tabular}} & \multicolumn{7}{|c|}{ Single-Shell Tank 241-SX-102 } \\
\hline & \multicolumn{7}{|c|}{ Total Inventory Estimate* } \\
\hline & & & & & & & \\
\hline & & $\therefore$ & & $-95 \mathrm{Cl}$ & $+67 \mathrm{Cl}$ & $+67 \mathrm{CI}$ & $+9 s \mathrm{CI}$ \\
\hline Total Waste & $3.39 \mathrm{E}+06(\mathrm{~kg})$ & (543 kgal) & - & - & . & - & - \\
\hline Fent Load & $6.22(\mathrm{~kW})$ & (2.12E+04 BTU/hr) & - & 5.71 & 6.00 & 6.40 & 6.52 \\
\hline Bulk Density & $1.75(\mathrm{~g} / \mathrm{cc})$ & - & $\ldots$ & 1.69 & 1.71 & 1.80 & 1.80 \\
\hline & & & & & & & \\
\hline Water wt $\%+$ & 24.3 & - & - & 21.6 & 21.7 & 26.2 & 27,6 \\
\hline TOC wt\% C (w) & 0.830 & - & $\cdots$ & 0.550 & 0.657 & 0.973 & 1.11 \\
\hline 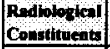 & $\mathrm{Cl} / \mathrm{L}$ & $\mathrm{nCl} / \mathrm{s}$ & $\mathrm{Cl}$ & $\begin{array}{l}95 \mathrm{CI} \\
(\mathrm{CL} / \mathrm{L})\end{array}$ & $\begin{array}{l}67 \mathrm{C} \\
\text { (C) }\end{array}$ & $\begin{array}{l}+67 \mathrm{C} \\
(\mathrm{C} / \mathrm{L})\end{array}$ & $\begin{array}{c}+4 \mathrm{C} \\
(\mathrm{C} / \mathrm{L})\end{array}$ \\
\hline $\mathrm{H}-3$ & $288 \mathrm{E}-\mathrm{-O4}$ & 0.165 & 392 & $1.42 \mathrm{E}-04$ & $1.42 \mathrm{E}-04$ & $2.98 \mathrm{~B}-04$ & $3.10 \mathrm{E}-04$ \\
\hline C-14 & $4.29 \mathrm{E}-05$ & $2.45 \mathrm{E}-02$ & 88.1 & $1.23 \mathrm{E}-05$ & $1.23 \mathrm{E}-05$ & 4.35E-05 & 441E-05 \\
\hline $\mathrm{Ni}-59$ & $4.28 \mathrm{E}-06$ & $2.45 E-03$ & 8.80 & 2.54E-06 & $2.84 \mathrm{E}-06$ & 4.43E-06 & $442 \mathrm{E}-06$ \\
\hline $\mathrm{Ni}-63$ & 4.12E-04 & 0.236 & 848 & $2.70 \mathrm{E}-04$ & $2.70 \mathrm{E}-04$ & $4.26 \mathrm{E}-04$ & $4.27 E-04$ \\
\hline $\mathrm{Co}-60$ & $480 \mathrm{E}-05$ & $2.75 \mathrm{E}-02$ & 98.7 & $130 E-05$ & $1.30 \mathrm{E}-0 \mathrm{~s}$ & 4,88E-05 & $4.97 \mathrm{E}-05$ \\
\hline Se-79 & 4. 16E-06 & $2.38 \mathrm{E}-03$ & 8.54 & 2.21E-06 & $2.21 \mathrm{E}-06$ & 4.86E-06 & 5.54E-06 \\
\hline Sr-90 & 0.238 & 136 & $4.89 \mathrm{E}+0 \mathrm{~S}$ & 0.209 & 0.225 & 0.248 & 0.247 \\
\hline $\bar{Y}-90$ & 0.238 & 136 & 4.89E+05 & 0.171 & 0.171 & 0.248 & 0.248 \\
\hline Zn.93 & 2.0AE-05 & $1.17 \mathrm{E}-02$ & 41.9 & 1.07E-05 & 1,07E-05 & 2.39E-05 & $2.73 \mathrm{E}-05$ \\
\hline $\mathrm{Nb}-93 \mathrm{~m}$ & $1.48 \mathrm{E}-0 \mathrm{~S}$ & $8.46 \mathrm{E}-0 \mathrm{3}$ & 30.4 & $7.97 \mathrm{E}-06$ & 7.97E-06 & $1.73 \mathrm{E}-05$ & 1.96E-05 \\
\hline Tc-99 & $3.06 \mathrm{E}-04$ & 0.175 & 629 & 1.92E-04 & $2.48 \mathrm{E}-04$ & 3.65E-04 & $4.21 \mathrm{E}-04$ \\
\hline$R u-106$ & 8.47E-09 & 4.84E-06 & $174 E-02$ & 3.95E-09 & 3.95E-09 & 9.48E-09 & $1.04 E-08$ \\
\hline Cd.113m & $1.08 \mathrm{E}-04$ & $6.17 \mathrm{E}-02$ & 222 & $4.93 \mathrm{E}-05$ & 4.93E-05 & $1.29 \mathrm{E}-0 \mathrm{4}$ & 1.49E-04 \\
\hline $\mathrm{Sb}-125$ & $2.07 \mathrm{E}-04$ & 0.119 & 426 & 5.59E-05 & 5.59E-05 & 2.11E-04 & $2.15 \mathrm{E}-04$ \\
\hline Sin-126 & 6.28E-06 & $3.59 \mathrm{E}-03$ & 12.9 & $3.36 \mathrm{E}-06$ & 3.36E-06 & 7.34E-06 & $8.36 \mathrm{E}-06$ \\
\hline $\mathrm{I}-129$ & $5.90 \mathrm{E}-07$ & $3.37 \mathrm{~T}-04$ & 1.21 & $369 \mathrm{E}-07$ & 4.7mE-07 & $7.03 \mathrm{E}-07$ & 8. 3]E-07 \\
\hline C.-134 & $3.07 \mathrm{E}-06$ & $1.76 \mathrm{E}-03$ & 0.31 & 2.15E- -6 & $2.60 \mathrm{E}-106$ & 3.54E-06 & $4,01 E-06$ \\
\hline Cs-137 & 0.304 & 174 & $6.25 E+05$ & 0.274 & 0.209 & 0320 & 0.335 \\
\hline Ba-137m & 0.258 & 165 & $5.91 E+05$ & 0.230 & 0.230 & 0.303 & 0317 \\
\hline $5 m-151$ & 1.46E-02 & 837 & $3.01 E+04$ & $7.80 \mathrm{E}-03$ & $7.80 \mathrm{E}-03$ & $1.71 \mathrm{E}-02$ & $1.95 \mathrm{E}_{-02} 02$ \\
\hline Eu-152 & $5.62 \mathrm{E}-06$ & $3.21 \mathrm{E}-03$ & 11.5 & $3.20 \mathrm{E}-06$ & $3.20 \mathrm{E}-06$ & $5.95 \mathrm{E}-06$ & $0.28 \mathrm{E}-06$ \\
\hline Ey-154 & $7.60 \mathrm{E}-04$ & 0.440 & $1.58 \mathrm{E}+03$ & 2.32E-04 & 2.12E-04 & 9.46E-C4 & $1.02 \mathrm{E}-03$ \\
\hline Eu-15s & $3.24 \mathrm{E}-04$ & 0.185 & 665 & $180 E_{-04}$ & 1.80E-04 & 3.44E- 04 & $3.64 \mathrm{E}-04$ \\
\hline R-226 & $2,19 \mathrm{E}-10$ & 1.65E-07 & 5.94E-04 & $2.30 E-10$ & $2.29 \mathrm{E}-10$ & $3.10 \mathrm{E}-10$ & $3.69 \mathrm{E}-10$ \\
\hline $\mathrm{Re-228}$ & 1.36E-07 & $1.07 E-04$ & 0.313 & 1.46E-0B & $1.34 \mathrm{E}-07$ & 2.47E-07 & 3.11E-07 \\
\hline Ac-227 & $1.61 \mathrm{E}-09$ & $9.21 E-07$ & $3.31 \mathrm{E}-03$ & $1.23 \mathrm{E}-09$ & $1.26 \pm-09$ & 1.65E-09 & $2.07 \mathrm{E}-09$ \\
\hline $\mathrm{Pa}-231$ & $5.15 \mathrm{E}-09$ & 2.94E-06 & 1.06E-02 & $3.20 \mathrm{E}-09$ & 3.20E-09 & $5.85 E-09$ & 6.53E-09 \\
\hline Th-229 & $4.38 \mathrm{E} .09$ & $2.50 \mathrm{e}-06$ & 1.99E-03 & $2.14 \mathrm{E}-09$ & $3.23 \mathrm{E}-60$ & $5.70 \mathrm{E}-09$ & $7.12 \mathrm{E}-09$ \\
\hline Th-232 & $131 \mathrm{E}_{0} \mathrm{a} 8$ & $7.48 \mathrm{E}-0.5$ & $260^{\circ}-02$ & $7.44 \mathrm{E}-09$ & 1.02E-98 & $1.60 \mathrm{E}-08$ & $1.87 \mathrm{E}-08$ \\
\hline U-232 & $9.265-07$ & $5.30 \mathrm{E}-04$ & 1.90 & 5.18E-07 & 7.1TE-07 & $1.17 E-06$ & $1,43 \mathrm{E}-06$ \\
\hline U-233 & $3.55 \mathrm{E}-06$ & $2.03 E-03$ & 7.30 & 199E-06 & 2.75E-06 & 4.48E-06 & $547 \mathrm{E}-\infty 6$ \\
\hline U-234 & $9.83 \mathrm{E}-07$ & $5.62 \mathrm{E}-04$ & 2.02 & $9.44 \mathrm{E}-07$ & $9.66 \mathrm{E}-07$ & $9.99 \mathrm{E}-07$ & $1.02 E-06$ \\
\hline U-235 & $3.98 \mathrm{E}-06$ & 2.2:E-05 & 8.18E-02 & 3.t2E-08 & $3.91 \mathrm{E}-08$ & $4.05 \mathrm{E}-08$ & 4.12E- 08 \\
\hline U-236 & $3.08 \mathrm{E}-08$ & $1.76 \mathrm{t}-0 \mathrm{~s}$ & $6.32 \mathrm{E}-02$ & $2.96 \mathrm{E}-00$ & 3.03E-01 & 3.12E-08 & $3.17 \mathrm{E}-08$ \\
\hline U-238 & $1.16 \mathrm{E}-06$ & $6.61 \mathrm{E}-04$ & 2.35 & $1.12 \mathrm{E}-06$ & I.14E-A6 & $1.17 \mathrm{E}-06$ & $1.19 \mathrm{E}-06$ \\
\hline Np-237 & 1,1 $1 \mathrm{E}-06$ & $6.33 \mathrm{E}-04$ & 2.28 & $7.35 \mathrm{E}-07$ & $9.17 \mathrm{E}-07$ & 1.30E-06 & $1.48 \mathrm{E}-06$ \\
\hline Pu-238 & $244 \mathrm{E}-06$ & 1.39E-03 & 5.01 & $2.00 \mathrm{E}-06$ & $2.21 E-06$ & $2.66 \mathrm{E}-06$ & 2.88E-06 \\
\hline Pu-239 & $1,09 \mathrm{E}-04$ & $6.26 \mathrm{E}-02$ & 225 & 9.11E-05 & $1.04 E_{-04}$ & $1.15 \mathrm{E}-04$ & $1.21 \mathrm{E}-04$ \\
\hline $\mathrm{Pu}-240$ & $1.72 \mathrm{E}-05$ & 9.36E-03 & 35.4 & $1.52 \mathrm{E}-05$ & $1.62 \mathrm{E}-05$ & 1.83E-05 & $1.93 \mathrm{E}-05$ \\
\hline Pu-241 & $1.60 \mathrm{E}-04$ & $9.15 E-02$ & 329 & $1.30 \mathrm{E}-04$ & 1.45E-04 & 1.75E-04 & 1.90E-04 \\
\hline Pu-242 & $8.40 \mathrm{E}-10$ & 4.11E-07 & 1.73E-03 & $6.62 \mathrm{E}-10$ & $7.50 \mathrm{E}-10$ & $9.31 \mathrm{E}-10$ & $1.02 E_{-09}$ \\
\hline Am-241 & $7.03 \mathrm{E}-05$ & $4.02 \mathrm{E}-02$ & 14 & 4.95E-05 & $5.97 \mathrm{E}-05$ & 3.09E-05 & $9.10 \mathrm{E}-05$ \\
\hline Am-243 & $2.48 \mathrm{E}-199$ & $1.42 \mathrm{E}-06$ & $5.10 E-03$ & 1. BIE-09 & 2.14E-09 & 2.83E-09 & 3.16E-09 \\
\hline $\mathrm{C}_{\mathrm{m}-242}$ & 2.03E-07 & 1.16E-04 & 0.417 & $1.03 \mathrm{E}-07$ & $1.03 \mathrm{E}-07$ & 2.15E-07 & $2.28 \mathrm{E}-07$ \\
\hline $\mathrm{Cm}-243$ & $1.76 \mathrm{E}-00$ & 1.01E-05 & $3.63 \mathrm{E}-02$ & 8. 1 PE-09 & $8.18 \mathrm{E}-09$ & 1.88E-08 & 1.99E-08 \\
\hline $\mathrm{Cm}_{\mathrm{m}}-244$ & 1.70E-07 & $9.73 \mathrm{E}-05$ & 0.350 & $7.26 \mathrm{E}^{-08}$ & $7.26 \mathrm{E}-0 \mathrm{9}$ & 2.03E-07 & $2.24 \mathrm{E}-07$ \\
\hline Totals & $\mathbf{M}$ & $v \%$ & 4, & $\begin{array}{l}-9 \mathrm{C} \\
\mathrm{Mor} \\
\mathrm{ML})\end{array}$ & $\begin{array}{l}-87 \mathrm{C} \\
(\mathrm{wor} \\
(1 \mathrm{~L})\end{array}$ & $\begin{array}{l}\text { GCP } \\
\text { Wor } \\
10)\end{array}$ & 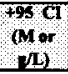 \\
\hline $\mathrm{Pu}$ & 1. StE-03 $(\pi / 2)$ & - & 3.24 & $1,36 \mathrm{E}-03$ & 1.47E- 03 & 1.60E-03 & $1.79 \mathrm{E}-03$ \\
\hline $\mathrm{U}$ & 1. I3E-03 & $1.53 \mathrm{E}+03$ & $5.50 \mathrm{E}+03$ & 1.08E-02 & 1.11E-02 & $1.14 \mathrm{E}-02$ & $1.16 \mathrm{E}-02$ \\
\hline
\end{tabular}

- Unknowns in tank solids inventory are astigned by Tank Layering Modet (TLM).

+ Volume average for denuity, mass average Water wt\% and TOC wt\% C. 


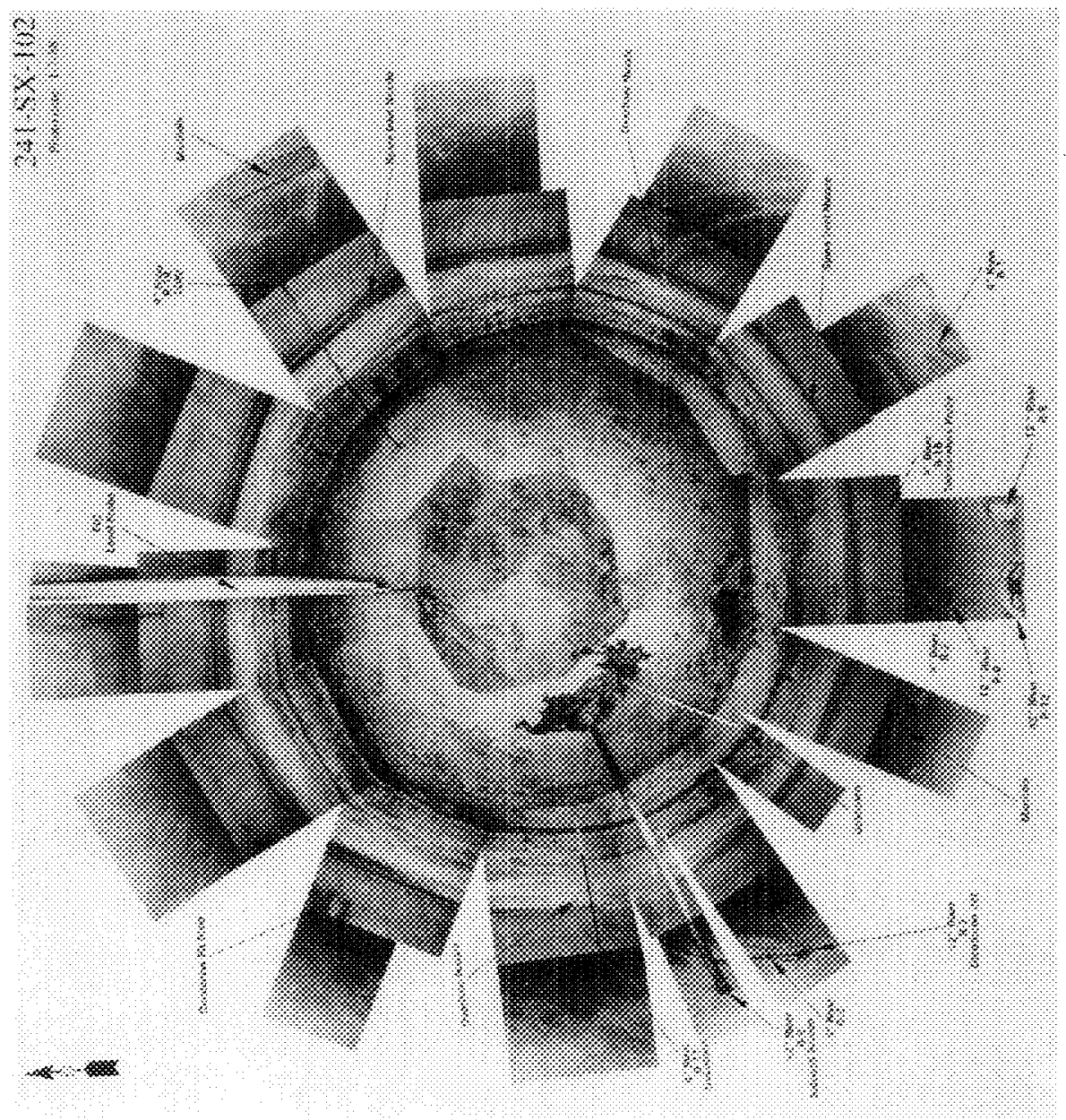




\begin{tabular}{|c|c|c|c|}
\hline $966 \mathrm{l}$ '9l l! Id $\forall$ pue $\rightarrow$ uer & әре0 & 2 & JəqunN Jəs!y \\
\hline$u ! 1+9 \varepsilon 2$ & |әләך U!W & HSS'OE & 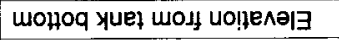 \\
\hline L66l'Zl uer & әेeव & $966 L^{\prime} \mathrm{g} q \partial_{\exists}$ & әpeg \\
\hline U! $S \varepsilon \triangleright Z$ & คคงәา XeW & $J_{0} \vdash<8$ & 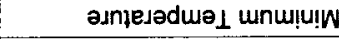 \\
\hline$\exists \forall \forall N \exists$ ןenuew & seगุıe0 & 2 & JequnN Jes!y \\
\hline \multicolumn{2}{|c|}{ 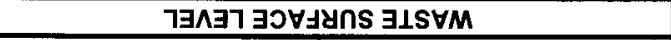 } & $H \varepsilon \mathcal{H}$ & woHoq ue? سод uо!jen키 \\
\hline $80 \angle \angle 0 \angle 8$ & JəqunN los $0104 d$ & $886 \mathrm{l}$ 'Z KeW & әңеव \\
\hline 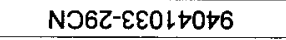 & JəqunN ə6ejuow & $J_{0} 96 \mathrm{l}$ & 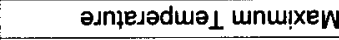 \\
\hline$\angle 86 L^{\prime} \angle L$ गə马 & әреg & $J_{0} 69 l$ & әлnłe \\
\hline \multicolumn{2}{|c|}{ SHd $\forall Y 9 O \perp O H d$ yOIY $3 \perp N I$} & \multicolumn{2}{|c|}{$\exists \forall n \perp \forall Y \exists d W \exists \perp$ Y $N \forall \perp$} \\
\hline 1e6 $000^{\prime} \mathrm{L}$ & fuepeusadns & $u !$ ZL & $L$ \\
\hline e6 000'sı & ə6pnis & u! $\downarrow$ & $91 '\llcorner$ \\
\hline je6 $000 \div 989$ & әxeoljes & ez!S & (s)」əqunN Jəs!y \\
\hline 1e6 000'ZLZ & sp!nb!̣ & \multicolumn{2}{|c|}{ SyヨSIy $\exists า g \forall า I \forall \wedge \forall \wedge า \exists \wedge I \perp \forall \perp N \exists \perp$} \\
\hline ןe6 000'حع乙 & 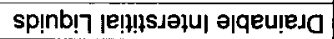 & - & (d) Uo!̣uәnә」d uo!̣snנlu| \\
\hline $\mathrm{X} 7 \mathrm{~d}$ ON & əd $K_{\perp}$ әlseM & S861 oun & (Id) uo!qejos| u!̣อ|u| |e!ped \\
\hline 1e6 000'Z99 & 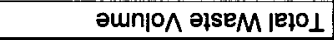 & - & (sı) uo!̣ez!!!qeıs u!ıәңu| \\
\hline \multicolumn{2}{|c|}{ 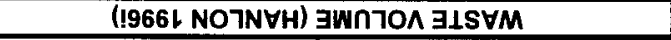 } & - & נәхеәา pəunss $\forall$ \\
\hline$\varepsilon l$ & 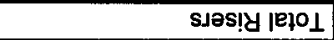 & punos & кұ!6อฺบ \\
\hline əuou & yue $\perp$ әpeoseว & so!ue6jO 8 uə6odpKH & SIS! 7 पગЕM \\
\hline ןe6 000'000' ᄂ & Kł!oedej jeu!moN & $8 \angle 6 L$ & วм!ฺวeu| \\
\hline us!a & ədeus wonog & - & 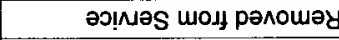 \\
\hline$\# S L$ & Jәңәше!̣ & $t S 6 \mathrm{~L}$ 」qb ult & 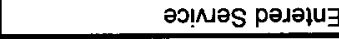 \\
\hline \multicolumn{2}{|c|}{ NOIIdIYכS } & \multicolumn{2}{|c|}{ AXO $\perp$ SIH YN $\forall \perp$} \\
\hline
\end{tabular}

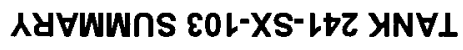




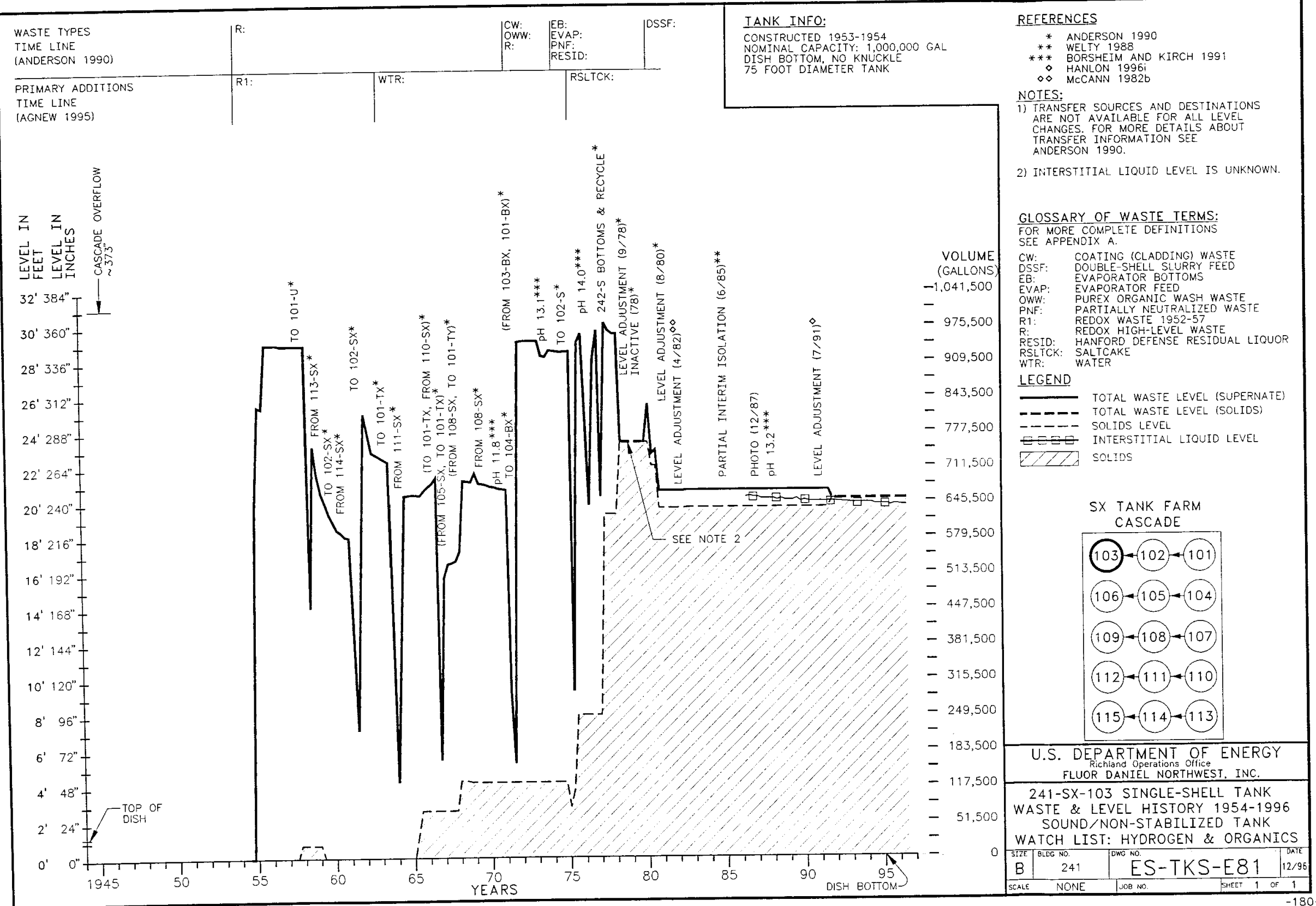




\section{$241-5 X-103$}

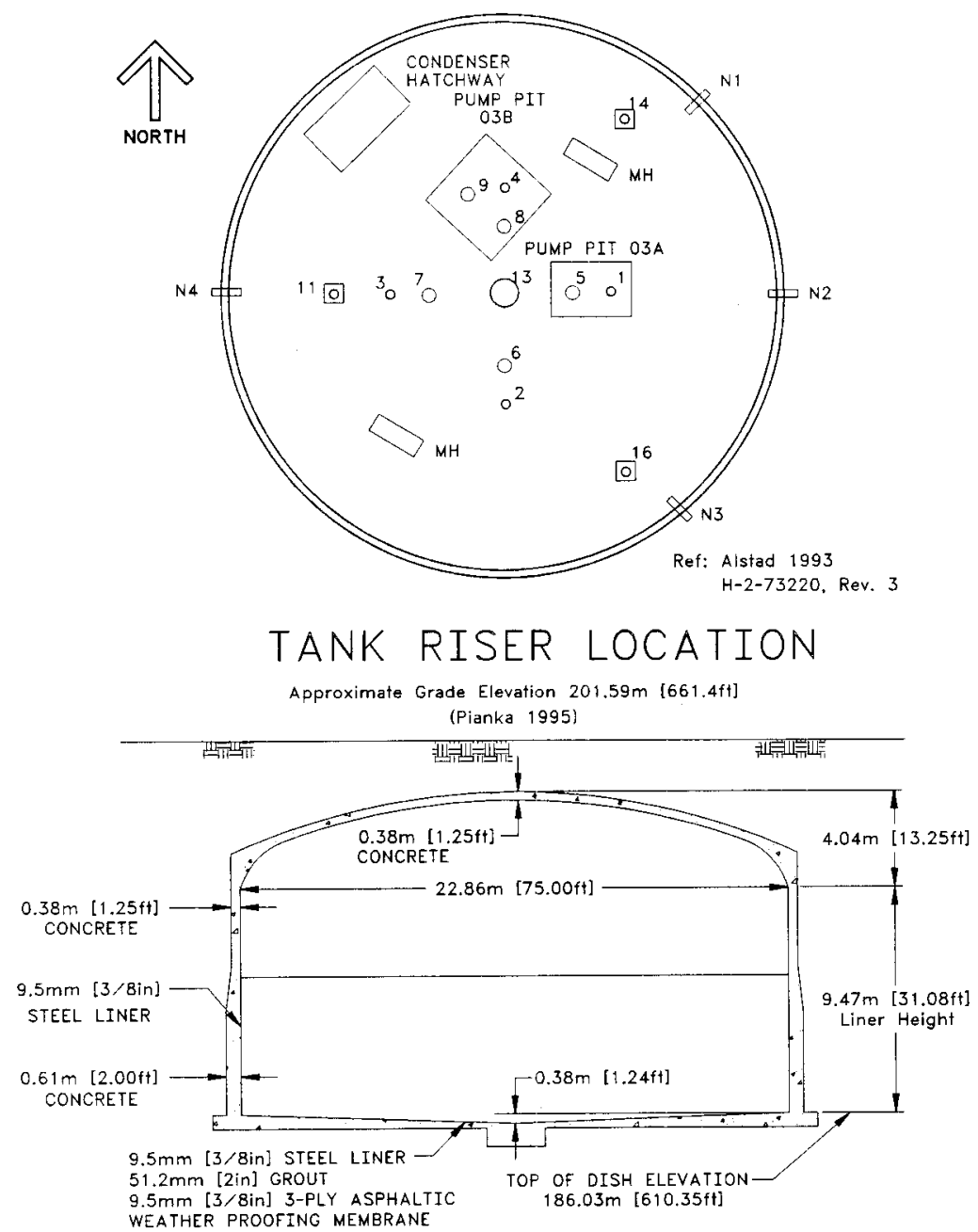

Ref: $\mathrm{H}-2-39511$, Rev. 3 H-2-46293. Rev. 3 NOT TO SCALE $\mathrm{H}-2-37855$, Rev. 4 


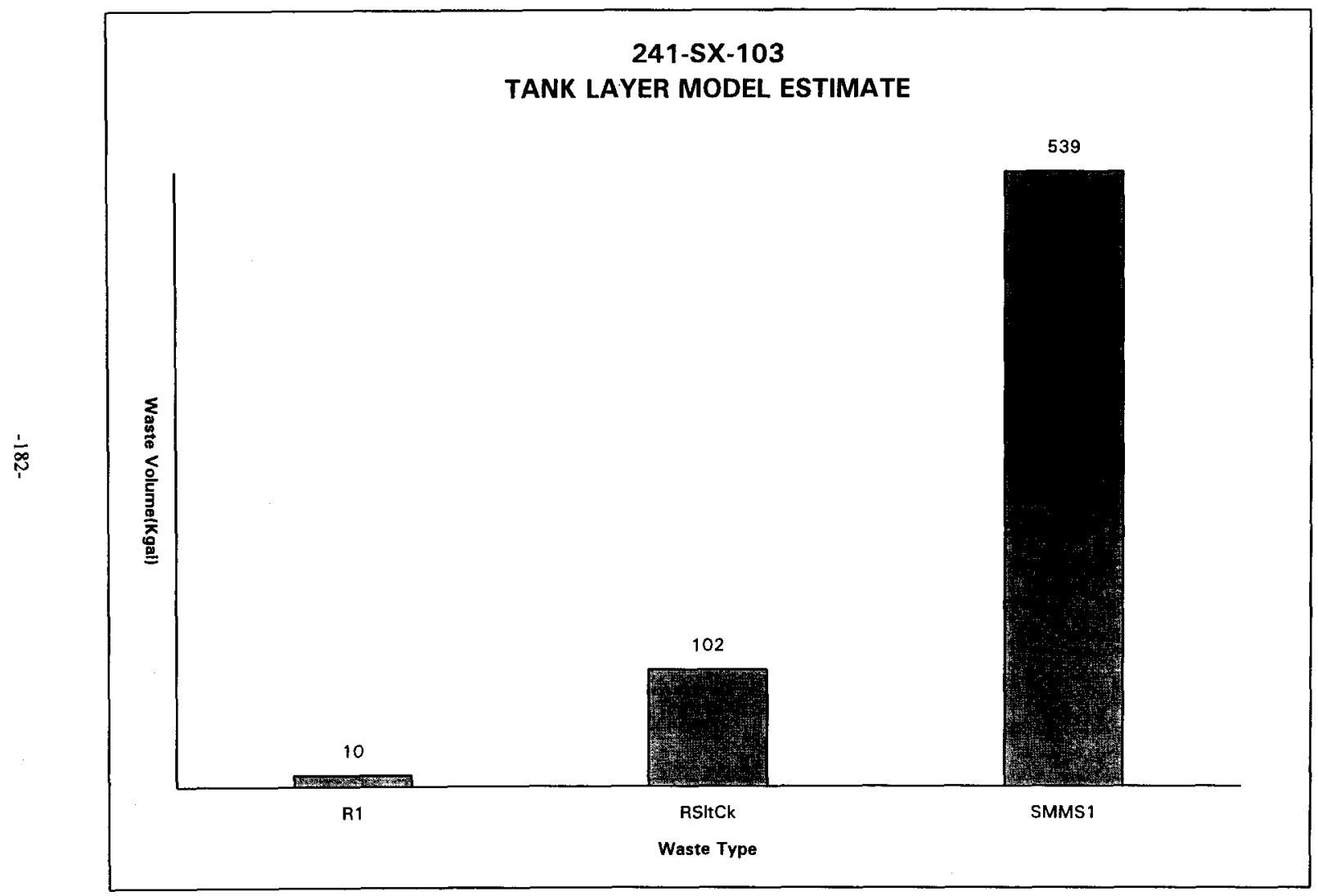

滎

Tank Layer Model(TLM) Estimate from Hanford Tank Chemical and Radionuclide Inventories: HDW Model Rev. 4 (Agnew et al., 1997). 
HDW Model Rev 4

\begin{tabular}{|c|c|c|c|c|c|c|c|}
\hline \multicolumn{8}{|c|}{ Single-Shell Tank 241-SX-103 } \\
\hline & \multicolumn{7}{|c|}{ TLM Solids Composite Inventory Estimate* } \\
\hline $\begin{array}{l}\text { Plyotical } \\
\text { Properties }\end{array}$ & & & $\therefore$ & $-95 \mathrm{CI}$ & $-67 \mathrm{CI}$ & $+67 \mathrm{CI}$ & $+95 \mathrm{CI}$ \\
\hline Total TLM Wa & $7.28 \mathrm{E}+05(\mathrm{~kg})$ & $(112 \mathrm{kgal})$ & - & $=$ & - & - & - \\
\hline Heat Load & $1.52(\mathrm{~kW})$ & $(5.18 E+01 \mathrm{BTU} / \mathrm{hr})$ & - & 1.41 & 146 & 1.57 & 1.62 \\
\hline Bulk Denity & $1.72(\mathrm{~g} / \mathrm{ec})$ & - & 一 & 1.46 & 1.60 & 1.77 & 1.82 \\
\hline Void Fraction & 0.763 & - & $=$ & 0.720 & 0.734 & 0.846 & 0.938 \\
\hline Water wo & 340 & 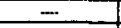 & $=$ & 24.5 & 28.8 & 43.8 & 543 \\
\hline TOC wt\% C (w) & 4.77E-03 & - & - & 4S6E 03 & $4.66 \mathrm{E}-03$ & $5.06 \mathrm{E}-03$ & $5.68 \mathrm{E}-03$ \\
\hline $\begin{array}{l}\text { Chemiat } \\
\text { Constituents }\end{array}$ & moledL & ppm : . & $\mathrm{kg}$ & $\begin{array}{l}95 \mathrm{Cl} \\
(\mathrm{n} \text { elelt) }\end{array}$ & $\begin{array}{l}67 \mathrm{CI} \\
\text { (mole/L) }\end{array}$ & $\begin{array}{l}+67 \mathrm{Cl} \\
(\mathrm{mol} / \mathrm{L})\end{array}$ & $\begin{array}{r}+96 \mathrm{C} \\
\text { (molef })\end{array}$ \\
\hline Nat & $\begin{array}{r}13.0 \\
\end{array}$ & 1.74E+05 & $1.27 \mathrm{E}+05$ & 831 & 109 & 14.1 & 15.0 \\
\hline $\mathrm{Al3+}$ & 3.7 & $5.34 E+04$ & $4.25 E+04$ & 1.93 & 2.45 & 5.07 & 6.46 \\
\hline 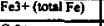 & 0.106 & $346 E+03$ & $2.52 \mathrm{E}+03$ & $9.92 \mathrm{E}-02$ & 0.103 & 0.110 & 0.113 \\
\hline $\mathrm{Cr}^{3+}$ & 0.754 & $2.28 \mathrm{E}+04$ & $1.66 \mathrm{E}+04$ & 0.443 & 0.591 & 0.820 & 0.843 \\
\hline $\mathrm{Bi3+}$ & 6.26E-06 & 0.762 & 0.554 & $5.29 \mathrm{E}-06$ & S.76E-06 & $6.89 \mathrm{E}-06$ & $7.58 \mathrm{E}-0.06$ \\
\hline $\mathrm{Le}+$ & $1.54 \mathrm{E}-11$ & $1.25 \mathrm{E}-06$ & 9.08E-07 & $1.35 \mathrm{E}-11$ & 1.46E-11 & $1.70 \mathrm{E}-11$ & $1.87 \mathrm{E}-11$ \\
\hline $\mathrm{Hg}_{\mathrm{g}+}$ & $9.83 \mathrm{E}-07$ & 0.115 & Q36E-012 & 8.71E-07 & 9.26E-07 & $1.08 \mathrm{E}-06$ & $1.19 \mathrm{E}-05$ \\
\hline $\mathrm{Zr}(\mathrm{ar} \mathrm{ZrO}(\mathrm{OH}) 2$ & $6.25 \mathrm{E}-07$ & $3.32 \mathrm{E}-02$ & $2.42 \mathrm{E}-02$ & 5.74E- -07 & $5.98 \mathrm{E}-07$ & 6.55E-07 & $7.06 \mathrm{E}-07$ \\
\hline $\mathrm{Pb2+}$ & I.56E- 04 & 18.8 & 11.7 & 8.35E-0S & $1.19 E-04$ & $1.93 \mathrm{E}-04$ & $2.30 \mathrm{E}-04$ \\
\hline $\mathrm{Ni2+}$ & $1.63 \mathrm{E}-02$ & 625 & 455 & $1.20 \mathrm{E}-02$ & $1.76 \mathrm{E}-02$ & 1 89E-02 & $1.95 \mathrm{E}-02$ \\
\hline Sr2+ & 0 & 0 & 0 & 0 & 0 & 0 & 0 \\
\hline Min4t & $4.55 \mathrm{E}-0 \mathrm{~S}$ & 1.46 & 1.06 & $3.26 \mathrm{E}-4 \mathrm{~S}$ & 3.89E-05 & $5.22 \mathrm{E}-0 \mathrm{~S}$ & 5.89E-05 \\
\hline C.2. & $8.96 \mathrm{E}-02$ & $2.09 \mathrm{E}+03$ & $1.52 \mathrm{E}+03$ & $6.23 \mathrm{E}-02$ & 7.56E-02 & 0.103 & 0.117 \\
\hline $\mathbf{K}+$ & $3.01 E-02$ & 687 & 500 & $2.86 \mathrm{E}-02$ & 2.94E-02 & $3.27 E-02$ & $3.56 \mathrm{E}-02$ \\
\hline OH- & 19.3 & $1.91 \mathrm{E}+05$ & 1.39E+0s & II.I & 13.7 & 25.2 & 31.0 \\
\hline $\mathrm{NO} 3$. & 5.25 & $1.90 \mathrm{E}+0 \mathrm{~S}$ & $1.38 E+03$ & 2.94 & 4.57 & 598 & 6.38 \\
\hline NO2- & 153 & $4.92 \mathrm{E}+04$ & $3.58 \mathrm{E}+04$ & 1.58 & 1.71 & 1.96 & 2.12 \\
\hline COJ2:- & $9.11 \mathrm{E}-02$ & $3.29 \mathrm{E}+03$ & $2.39 \mathrm{E}+03$ & $6.68 \mathrm{E}-02$ & B. 01E-02 & 0.108 & 0.121 \\
\hline PO43. & $4,05 \mathrm{E}-04$ & 22.4 & 16.3 & $3.76 \mathrm{E}-04$ & $3.93 \mathrm{E}-04$ & $4.22 E-04$ & $4.58 \mathrm{E}-04$ \\
\hline SO42- & $3.77 \mathrm{E}-02$ & $2.11 \mathrm{E}+03$ & $1.54 E+03$ & 3.58E-02 & $3.68 \mathrm{E}-02$ & $4.10 \mathrm{E}-02$ & 4.45E-02 \\
\hline Si (as SiO32-) & 0.119 & $1.95 E+03$ & 1.42E+03 & $7,56 E-02$ & 0.103 & 0.136 & 0.152 \\
\hline$F=$ & $3.23 \mathrm{E}-04$ & 3.57 & 2.60 & 2.75E-04 & 3.04E-04 & $3.55 \mathrm{E}-04$ & $3.82 \mathrm{E}-0 \mathrm{~d}$ \\
\hline $\mathrm{Cl} \cdot$ & 0.126 & $2.60 \mathrm{E}+03$ & $1.89 \mathrm{E}+03$ & $6,99 \mathrm{E}-02$ & $9.74 \mathrm{E}-02$ & 0.138 & 0.145 \\
\hline C6HSOT3- & $3.33 \mathrm{E}-04$ & 36.7 & 26.7 & 3.23E-04 & 3.28E-04 & $3.48 \mathrm{E}-04$ & $3.79 \mathrm{E}-04$ \\
\hline EDTA4 & $1.30 \mathrm{E}-05$ & 2.18 & 1.59 & $9.14 E-06$ & $1.12 \mathrm{E}-05$ & 1.53E-05 & $1.76 \mathrm{E}-05$ \\
\hline HEDTA3. & $1.08 \mathrm{E}-05$ & 1.72 & 1.25 & 3.46E-06 & $7.41 \mathrm{E}-06$ & 1.44E-OS & 1.79E-0S \\
\hline glyoolate & 4.71E-04 & 206 & 15.0 & 2.55E-04 & 3.72E- -04 & 5.85E-04 & $6.95 \mathrm{E}-04$ \\
\hline nocinte & 4.89E-05 & 1.68 & 1.22 & $4.73 \mathrm{E}-05$ & 4.81E-0s & $5.19 \mathrm{E}-05$ & S.SSE-0S \\
\hline oxulata2- & 2.02E-11 & I.04E-06 & $7,53 \mathrm{E}-07$ & 1.79E-11 & $1.90 \mathrm{E}-11$ & $2.22 \mathrm{E}-11$ & 2.44E-11 \\
\hline DAP & $2.96 \mathrm{E}-04$ & 36.2 & 25.4 & 2.76E-04 & $2.85 E-04$ & $3.20 \mathrm{E}-04$ & 3.45E-04 \\
\hline butanol & 2.96E-04 & 12.2 & 9.29 & $2.76 \mathrm{E}-04$ & 2.89E-OA & $3.20 \mathrm{E}-04$ & 3.45E-04 \\
\hline $\mathrm{NH3}$ & $5.56 \mathrm{E}-\overline{2} 2$ & 351 & 401 & $3.8 \pi-02$ & $4.11 \mathrm{E}-02$ & $6.02 \mathrm{E}-02$ & $6.46 \mathrm{E}-02$ \\
\hline $\mathrm{Fe}(\mathrm{CN}) 64-$ & 0 & 0 & 0 & 0 & 0 & 0 & 0 \\
\hline
\end{tabular}

"Unknowns in tank solids inveniary wre maigned by Tank Layering Model (TLM) 
HDW Model Rov 4

\begin{tabular}{|c|c|c|c|c|c|c|c|}
\hline & \multicolumn{7}{|c|}{ Single-Shell Tank 241-SX-103 } \\
\hline & \multicolumn{7}{|c|}{ SMM Composite Inventory Estimate } \\
\hline $\begin{array}{l}\text { Phyoled } \\
\text { Propertles }\end{array}$ & & & & $-95 \mathrm{CI}$ & $67 \mathrm{CI}$ & $+67 \mathrm{CI}$ & $+95 \mathrm{CI}$ \\
\hline Total SMM W & $3.45 \mathrm{E}+06(\mathrm{~kg})$ & $(540 \mathrm{kgal})$ & $\ldots$ & - & $\ldots$ & - & $=$ \\
\hline Heat Lond & $5.00(\mathrm{~kW})$ & $(1.71 \mathrm{E}+04 \mathrm{BTU} / \mathrm{hr})$ & - & 4.67 & 4.83 & 5.17 & 5.34 \\
\hline Bulk Density" & $1.69(\mathrm{~g} / \mathrm{cc})$ & - & - & 1.63 & 1.66 & 1.71 & 1.73 \\
\hline Water wt\% & 27.3 & - & $\therefore$ & 24.6 & 25.8 & 28.9 & 30.8 \\
\hline TOC wt\% C (w & 0.917 & - & $\ldots$ & 0.578 & 0.744 & 1.09 & 1.26 \\
\hline $\begin{array}{l}\text { Cherieal } \\
\text { Conitituetats }\end{array}$ & nolet L & ppm & It & $\begin{array}{l}94 \mathrm{Cl} \\
\text { (wale/L) }\end{array}$ & $\begin{array}{c}67 \mathrm{Cl} \\
\text { (noled) }\end{array}$ & $\begin{array}{l}+67 \mathrm{Cl} \\
(\mathrm{Hole} / \mathrm{L})\end{array}$ & $\begin{array}{l}+9 s \mathrm{CI} \\
\text { (nole/L) }\end{array}$ \\
\hline $\mathrm{Nat}$ & 16.2 & $2.20 \mathrm{E}+03$ & $7.59 \mathrm{E}+05$ & 148 & 15.5 & 16.8 & 17.3 \\
\hline $\mathrm{A} \sqrt{3}+$ & 1.84 & $2.99 \mathrm{E}+04$ & $1.01 E+05$ & 1,69 & 1.77 & 1.92 & 1.99 \\
\hline $\mathrm{Fe} .3+(\operatorname{total} \mathrm{Fe})$ & $1.29 \mathrm{E}-02$ & 427 & $1.47 \mathrm{E}+03$ & $1.06 E-02$ & $1.17 \mathrm{e}-02$ & $1.41 \mathrm{E}-02$ & 1.52E-02 \\
\hline $\mathrm{Cr} 3+$ & 0.159 & $4912+03$ & $1.69 E+04$ & 0.535 & 0.148 & 0.164 & 0.168 \\
\hline $\mathrm{BE} 3+$ & $1.73 E-03$ & 214 & 739 & $1.5 \pi-03$ & $1.65 \mathrm{E}-03$ & $1.81 E-03$ & $1.89 \mathrm{E}-03$ \\
\hline Lent+ & $6.48 \mathrm{E}-05$ & 534 & 78.4 & 4.70E-0S & $5.57 \mathrm{E}-05$ & 7.40E-05 & 8.28E-05 \\
\hline $\mathrm{Hg} 2+$ & $1.12 \mathrm{E}-0 \mathrm{~s}$ & 1.33 & 4.58 & $1.03 \mathrm{E}-05$ & $1.08 \mathrm{E}-05$ & 1.13E-0S & 1.19E-05 \\
\hline $\operatorname{Zr}(m \mathrm{ZrO}(\mathrm{OH}) 2$ & $3.23 \mathrm{E}-04$ & 17.5 & 603 & 2.94E-04 & $3.05 \mathrm{E}-04$ & 3.35E-04 & $3.52 E-04$ \\
\hline Pb2+ & $1.20 \mathrm{E}-03$ & 147 & 507 & $9.61 \mathrm{E}-04$ & $1.08 \mathrm{E}-03$ & $1.32 \mathrm{E}-03$ & $1.44 \mathrm{E}-0.3$ \\
\hline $\mathrm{Ni2+}$ & $7.62 \mathrm{E}-03$ & 265 & 914 & $7.26 \mathrm{E}-03$ & 7.43E-03 & $7.70 \mathrm{E}-03$ & $7.79 \mathrm{E}-03$ \\
\hline $\mathrm{Sr} 2+$ & 0 & 0 & 0 & 0 & 0 & 0 & 0 \\
\hline Mndt & $4.460-03$ & 143 & 49 & $3.30 \mathrm{E}-03$ & $3.84 \mathrm{E}-03$ & 4.96E-03 & 5.50E-03 \\
\hline $\mathrm{Con}_{2+}^{2+}$ & $4.01 E-02$ & 953 & $3.29 \mathrm{E}+03$ & 3.68E-02 & 3.85E-02 & 4.18E-02 & 4.34E-02 \\
\hline $\mathbf{K}+$ & $7.69 E-02$ & $1.78 E+03$ & $6.15 E+03$ & $7.02 \mathrm{E}-02$ & $7.34 E-02$ & $8.07 E-02$ & $8.41 \mathrm{E}-02$ \\
\hline OH- & 11.1 & 1.1IE+05 & $3.4 \mathrm{E}+05$ & 10.3 & 10.7 & 11.5 & 118 \\
\hline NO3. & 373 & $2.11 E+05$ & $7.26 \mathrm{E}+05$ & 5.22 & 5.52 & 5.81 & 5.89 \\
\hline NO2- & 2.73 & $7.43 E+04$ & $2.56 \mathrm{E}+05$ & 2.26 & 2.47 & 2.97 & 3.21 \\
\hline Cos2. & 0.531 & $189 \mathrm{E}+04$ & $6.52 E+04$ & 0.480 & 0.500 & 0.570 & 0.576 \\
\hline PO43- & 0.112 & $6.33 \mathrm{E}+03$ & $2.18 \mathrm{E}+04$ & $9.57 \mathrm{E}-02$ & 0.102 & 0.115 & 0.318 \\
\hline SO42- & 0.295 & $160+04$ & $5,7 \mathrm{E}+64$ & 0.234 & 0.263 & 0.328 & 0.356 \\
\hline Si (es SiÖ32-) & $9.15 \mathrm{E}-02$ & $1.52 \mathrm{E}+03$ & $5.25 \mathrm{E}+03$ & $7.57 \mathrm{E}-02$ & 8.35E-02 & $9.95 \mathrm{E}-02$ & 0.107 \\
\hline F- & B.SIE- 02 & 958 & $3.30 \mathrm{E}+03$ & $7.13 \mathrm{E}-02$ & $7.71 \mathrm{E}-02$ & 9.190-02 & $9.72 \mathrm{E}-02$ \\
\hline $\mathrm{Cl}-$ & 0.282 & $5.92 \mathrm{E}+03$ & $2.04 E+04$ & 0.254 & 0.264 & 0.294 & 0.305 \\
\hline C6H5073. & $3.27 \mathrm{E}-02$ & $3.67 \mathrm{E}+03$ & $1.26 \mathrm{E}+04$ & $2.98 \mathrm{E}-02$ & $3.13 \mathrm{E}-02$ & $3.42 \mathrm{E}-02$ & $3.56 \mathrm{E}-12$ \\
\hline EDTA4- & $2.02 \mathrm{E}-02$ & $3.45 E+03$ & $1.19 \mathrm{E}+04$ & $6.04 E-03$ & $1,30 \mathrm{E}-02$ & $2.75 E-02$ & $3.47 \mathrm{E}-02$ \\
\hline FEDTA3- & $3,20 \mathrm{E}-02$ & $6.18 E+03$ & $2.13 \mathrm{E}+04$ & $9.6 \mathrm{TE}-03$ & $2.35 \mathrm{E}-02$ & $5.26 \mathrm{E}-02$ & $6.69 \mathrm{~F}-02$ \\
\hline glycolinte- & 0.114 & $5.05 E+03$ & $1.74 \mathrm{E}+04$ & $7.52 \mathrm{E}-02$ & $9,390-02$ & 0.133 & 0.152 \\
\hline acetate- & $7.72 \mathrm{E}-03$ & 270 & 932 & $6.34 \mathrm{E}-03$ & 7,02E-03 & $8,43 E-03$ & $9.11 E-03$ \\
\hline oxalate- & 8.49E-05 & 4,41 & 15.3 & $7.52 \mathrm{E}-05$ & 8.00A-OS & 8.99E-05 & $9.46 \mathrm{E}-05$ \\
\hline DBP & $2.23 \mathrm{E}-02$ & $2.77 \mathrm{E}+03$ & $9.56 \mathrm{E}+03$ & J,84E-02 & $2.03 \mathrm{E}-02$ & $2.42 \mathrm{E}-02$ & $2.61 \mathrm{E}-02$ \\
\hline butanol & 2.23E-02 & 978 & $3.37 \mathrm{E}+03$ & 1,84E-02 & $2.03 \mathrm{E}-02$ & $2.42 \mathrm{E}-02$ & $2.61 \mathrm{E}-02$ \\
\hline NH3 & 0,102 & $1.03 E+03$ & $3.56 \mathrm{E}+03$ & S.47E-02 & $9.17 \mathrm{E}-02$ & 0.116 & 0.132 \\
\hline $\mathrm{Fo}(\mathrm{CN}) 64$ & 0 & 0 & 0 & 0 & 0 & 0 & 0 \\
\hline
\end{tabular}

-Density is calculated based on $\mathrm{Na}, \mathrm{OH}$-, and AIO2-

$+W$ ater $w t \%$ derived from the difference of density and total diwtolved upecies. 
HDW Modol Rev. 4

\begin{tabular}{|c|c|c|c|c|c|c|c|}
\hline \multicolumn{8}{|c|}{ Single-Shell Tank 241-SX-103 } \\
\hline & \multicolumn{7}{|c|}{ Totsil Inventory Estimate" } \\
\hline $\begin{array}{l}\text { Physicil } \\
\text { Properties }\end{array}$ & & & & $-95 \mathrm{CI}$ & $-67 \mathrm{Cl}$ & $+67 \mathrm{CI}$ & $+95 \mathrm{Cl}$ \\
\hline Total Waste & $4.18 \mathrm{E}+06(\mathrm{~kg})$ & $(652 \mathrm{kgg})$ & - & - & -- & - & - \\
\hline Heat Load & $6.52(\mathrm{~kW})$ & $(2.23 \mathrm{E}+04 \mathrm{BTU} / \mathrm{hr})$ & $m$ & $6.1 \mathrm{~B}$ & 6.35 & 6.69 & 685 \\
\hline Bulk Densityt & $169(g / c)$ & $\ldots$ & - & 1.64 & 1.67 & 171 & 1.73 \\
\hline & & & & & & & \\
\hline Water wt\%t & 28.5 & $=$ & - & 26.2 & 27.2 & 30.1 & 31.8 \\
\hline TOC $w t \% C(w$ & 0.758 & $\cdots$ & - & 0.477 & 0.615 & 0.901 & 1.04 \\
\hline $\begin{array}{c}\text { Cherical } \\
\text { Constituents }\end{array}$ & motert & Dpan & $\mathbf{k g}$ & $\begin{array}{r}-95 \mathrm{CI} \\
\text { (nnolo/L) }\end{array}$ & $\begin{array}{r}-67 \mathrm{Cl} \\
\text { (mole/t) }\end{array}$ & $\begin{array}{l}+67 \mathrm{Cl} \\
\text { (mole/L) }\end{array}$ & $\begin{array}{r}+95 \mathrm{CI} \\
\text { (molel })\end{array}$ \\
\hline $\mathrm{Na}+$ & 15,6 & $2.12 \mathrm{E}+05$ & $8.86 E+05$ & 14.5 & 15.0 & 16.1 & 16.6 \\
\hline Al3+ & 2.16 & $3,45 E+04$ & I.44E+05 & 1.84 & 1,95 & 240 & 2.63 \\
\hline$F \in 3+(t o t e d F e)$ & $2.89 \mathrm{E}-02$ & 955 & $3.99 \mathrm{E}+03$ & $2.70 \mathrm{E}-02$ & $2.60 \mathrm{E}-02$ & $2.99 \mathrm{E}-02$ & $3.08 \mathrm{E}-02$ \\
\hline $\mathrm{Cr}^{3}+$ & 0.261 & $8.03 \mathrm{E}+03$ & $3.35 \mathrm{E}+04$ & 0.206 & 0.233 & 0.273 & 0273 \\
\hline Bi3t & $1.43 E-03$ & 177 & 739 & $1.30 \mathrm{E}-03$ & $1.37 \mathrm{E}-03$ & $1.50 \mathrm{E}-03$ & $1.56 \mathrm{E}-03$ \\
\hline La3+ & $5.3 \mathrm{TE}-05$ & $4 . \overline{41}$ & 18.4 & $3.89 \mathrm{E}-05$ & 4.61E-05 & $6.13 \mathrm{E}-05$ & 6.06E-05 \\
\hline $\mathrm{Hg} 2+$ & $9.43 \mathrm{E}-06$ & 1.12 & 4.67 & 8.74E-06 & $9.13 E-06$ & $9.57 \mathrm{E}-06$ & 9.70E-06 \\
\hline $\mathrm{Zr}(\mathrm{as} \mathrm{ZrO}(\mathrm{OH}) 2$ & 2,68E-04 & 14.4 & 60.3 & $2.44 E-04$ & $2.52 \mathrm{Em}-4$ & $2.78 \mathrm{E}-04$ & $2.92 \mathrm{E}-04$ \\
\hline $\mathrm{Pb} 2+$ & 1.02E-03 & 125 & 521 & $8.23 E-04$ & 9.19E-04 & 1.12E-03 & $1.22 \mathrm{E}-03$ \\
\hline $\mathrm{Ni2+}$ & $9.45 \mathrm{E}-03$ & 328 & $1.37 \mathrm{E}+03$ & 9.15E-03 & $9.30 \mathrm{E}-03$ & 9.57E-03 & 9.69E-03 \\
\hline $\mathrm{sr} 2+$ & 0 & 0 & 0 & 0 & 0 & 0 & 0 \\
\hline $\operatorname{Mnd+}$ & $3.65 E-03$ & 119 & 495 & $2.74 \mathrm{E}-03$ & 3.19E-03 & $4.12 \mathrm{E}-03$ & $4.57 \mathrm{E}-03$ \\
\hline $\mathrm{C}_{2+}+$ & $4.86 \mathrm{E}-02$ & 1. $15 \mathrm{E}+03$ & $4.81 \mathrm{E}+03$ & 4.35E- 02 & 4. $50 \mathrm{E}-02$ & $5.12 \mathrm{E}-02$ & $5.38 \mathrm{E}-02$ \\
\hline $\mathbf{K +}$ & $6.89 \mathrm{E}-02$ & $159 \mathrm{E}+03$ & $6.65 E+03$ & $6.33 E-02$ & $6.60 \mathrm{E}-02$ & $7.201-02$ & $7.48 \mathrm{E}-02$ \\
\hline OH- & 12.5 & $1.25 \mathrm{E}+05$ & $5.23 \mathrm{E}+05$ & 10.9 & 11.5 & 13.5 & 14.6 \\
\hline $\mathrm{NO3}$ & 5.65 & $20 \overline{7 E+05}$ & 8.65E+05 & 5.23 & 5.48 & 5.74 & 5.78 \\
\hline NO2: & 2.57 & $7.00 E+04$ & $2.92 E+05$ & 2.18 & 2.36 & 2.78 & 2.97 \\
\hline $\cos 32$ & 0.456 & $162 \mathrm{E}+04$ & $6.76 \mathrm{E}+04$ & 0.414 & 0.430 & 0.489 & 0,493 \\
\hline PO43- & $9.32 E-02$ & $5.23 \mathrm{E}+03$ & $2.18 \mathrm{E}+04$ & $7.94 \mathrm{E}-02$ & 8.42E-02 & 9.57E-02 & $9.80 \mathrm{E}-02$ \\
\hline SO42- & 0.250 & $1.42 E+04$ & $5.94 \mathrm{E}+04$ & 0.200 & 0.224 & 0.278 & 0,301 \\
\hline Si (n SiO32-) & $9.62 \mathrm{E}-02$ & $1.60 \mathrm{E}+03$ & $6.67 \mathrm{E}+03$ & $8.32 E-02$ & $896 E-02$ & 0.103 & 0.109 \\
\hline$F$ & $7.05 \mathrm{E}-02$ & 792 & $3.3 \mathrm{ME}+03$ & $5.91 \mathrm{E}-02$ & 6.39E-02 & $7.61 \mathrm{E}-02$ & $8.06 \mathrm{E}-02$ \\
\hline $\mathrm{Cl}$ - & 0.255 & $5.34 E+103$ & $2.23 E+04$ & 0.232 & 0.240 & 0.265 & 0.274 \\
\hline C6H5073. & $2.72 E-02$ & $3.03 \mathrm{E}+03$ & $1.27 \mathrm{E}+04$ & $2.48 \mathrm{E}-02$ & $2.59 \mathrm{E}-02$ & $2.84 \mathrm{E}-02$ & $2,95 \mathrm{E}-02$ \\
\hline EDTA4 & $1,60 \mathrm{E}-02$ & $2.85 E+03$ & $1.19 \mathrm{E}+04$ & 5.01E-03 & $1.07 \mathrm{E}-02$ & $2.28 \mathrm{E}-02$ & $2.87 \mathrm{E}-02$ \\
\hline HEDTA3- & $3.15 \mathrm{E}-12$ & $3.10 \mathrm{E}+03$ & $2.13 \mathrm{E}+04$ & $0.01 E-03$ & $1.95 \mathrm{E}-02$ & $436 \mathrm{E}-02$ & $5.54 \mathrm{E}-02$ \\
\hline glycolute- & 9.41E-02 & $417 \mathrm{E}+0.3$ & $1.74 E+04$ & $6.23 \mathrm{E}-02$ & $7.79 \mathrm{E}-02$ & 0.110 & 0.126 \\
\hline acelinite- & $6.41 E-03$ & 223 & 933 & $5.26 \mathrm{E}-03$ & $5.82 E-03$ & $6.99 \mathrm{E}-03$ & $7.55 \mathrm{E}-03$ \\
\hline oxajate?- & $7.03 \mathrm{E}-05$ & 3.66 & is.3 & $6.23 \mathrm{E}-05$ & $6,62 E-05$ & 7.44E-0S & 7.84E-05 \\
\hline DBP & I. $55 \mathrm{E}-02$ & $2.30 E+03$ & $9.59 \mathrm{E}+03$ & 1.53E-02 & $1.69 \mathrm{E}-02$ & $2.01 \mathrm{E}-02$ & $2,16 \mathrm{E}-02$ \\
\hline butenol & 1.85E-02 & 810 & $3.38 \mathrm{E}+03$ & $1.53 \mathrm{E}-02$ & $1.69 \mathrm{E}-02$ & 2.01E-02 & $2.16 \mathrm{E}-02$ \\
\hline NH3 & $9.44 E^{-02}$ & 948 & $396 E+03$ & & & & \\
\hline $\mathrm{Fe}(\mathrm{CN}) 64$ & 0 & 0 & $\frac{3.50 E+43}{0}$ & $\frac{7.97 \mathrm{E}-02}{0}$ & $\frac{\text { B.55E-02 }}{0}$ & $\frac{0.106}{0}$ & 0.119 \\
\hline
\end{tabular}

$\uparrow$ Water wt\% derived from the difference of dentity and total diasolved apecies. 
HDW Modal Rew. 4

\begin{tabular}{|c|c|c|c|c|c|c|c|}
\hline \multicolumn{8}{|c|}{ Single-Shell Tank 241-SX-103 } \\
\hline & \multicolumn{7}{|c|}{ TLM Solids Composite Inventory Estimate* } \\
\hline $\begin{array}{l}\text { Physletl } \\
\text { Properties }\end{array}$ & & -0 & & $-95 \mathrm{Cl}$ & $-67 \mathrm{CI}$ & $+67 \mathrm{CI}$ & $+95 \mathrm{cl}$ \\
\hline Total TLM Wa & $7,28 \mathrm{E}+05(\mathrm{~kg})$ & (112 kgal) & 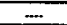 & - & 一 & $\ldots$ & - \\
\hline Heat Load & $1.52(\mathrm{~kW})$ & (5.18E+03 BTU/ht) & $\ldots$ & 1.41 & 1.46 & 1.57 & 1.62 \\
\hline Bulk Density & $1.72(g / c)$ & - & - & 1.46 & 1.60 & 1.77 & 1.82 \\
\hline Void Fraction & 0.763 & - & - & 0,720 & 0.734 & 0.846 & 0.938 \\
\hline Water wt\% & 34.0 & $m$ & - & 24.5 & 28.8 & 43.8 & 54.3 \\
\hline $\mathrm{TOC} w \mathrm{w} \% \mathrm{C}(\mathrm{w}$ & $477 \mathrm{E}-03$ & $\cdots$ & - & $4.56 \mathrm{E}-03$ & $4.66 \mathrm{E}-03$ & $5.06 \mathrm{E}-03$ & $5.68 \mathrm{E}-03$ \\
\hline $\begin{array}{l}\text { Rodiolosictil } \\
\text { Constlineth }\end{array}$ & CUL & $\mathrm{sev}$ & Cl & $\begin{array}{l}95 \mathrm{Cl} \\
(\mathrm{CVL})\end{array}$ & $\begin{array}{l}67 \mathrm{Ct} \\
\text { (CVI) }\end{array}$ & $\begin{array}{l}+67 \mathrm{CI} \\
\text { (c)L) }\end{array}$ & $\begin{array}{r}+95 \mathrm{C} \\
\text { (C) }\end{array}$ \\
\hline $\mathrm{H}-3$ & $2.06 \mathrm{E}-04$ & 0.120 & 87.2 & $2.61 E-06$ & 2.61E-06 & $2.16 \mathrm{E}-04$ & $2.34 \mathrm{E}-04$ \\
\hline $\mathrm{C}-14$ & $1.06 \mathrm{E}-05$ & $6.19 \mathrm{E}-0.03$ & 4.50 & 2.02E-07 & $2.02 \mathrm{E}-07$ & 1.10E-05 & $1.20 \mathrm{E}-05$ \\
\hline Ni-59 & $7.53 \mathrm{E}-06$ & $4.39 \mathrm{E}-03$ & 3.19 & $1.33 \mathrm{E}-06$ & $1.33 \mathrm{E}-06$ & 7.84E-06 & 8.13E-06 \\
\hline $\mathrm{Ni}-63$ & $7.27 \mathrm{E}-04$ & 0.423 & 308 & $1.24 \mathrm{E}-04$ & $1.24 E-04$ & $7.56 \mathrm{E}-04$ & $7.84 \mathrm{E}-04$ \\
\hline Co-60 & $9.46 \mathrm{E}-06$ & 5.5IE-03 & 4.01 & $7.98 E-0 B$ & $7.98 \mathrm{E}-08$ & $9.86 \mathrm{E}-06$ & $1.07 \mathrm{E}-05$ \\
\hline So-79 & $6.37 \mathrm{E}-06$ & $3.71 \mathrm{E}-03$ & 2.70 & 4.29E-08 & $4.29 \mathrm{E}-08$ & $6.45 \mathrm{E}-06$ & $6.97 \mathrm{E}-06$ \\
\hline $5 r-90$ & 0.322 & 188 & 1.37E+05 & 0.288 & 0.305 & 0.340 & 0.356 \\
\hline$Y-90$ & 0.322 & 188 & $1.3 \overline{\mathrm{E}}+05$ & $8.29 \mathrm{E}-02$ & 8.29E-02 & 0.340 & 0.356 \\
\hline Zr-93 & 3.02E-05 & $1,76 \mathrm{E}-02$ & 12.8 & $2.03 \mathrm{E}-07$ & 2.03E-07 & $3.05 \mathrm{E}-05$ & 3.27E-05 \\
\hline $\mathrm{Nb}-93 \mathrm{~m}$ & 2.45E-05 & 1.43E-02 & 10.4 & $1.66 \mathrm{E}-07$ & 1.66E-07 & $2.48 \mathrm{E}-05$ & 2.72E-05 \\
\hline Tc-99 & 8.24E-05 & 4.80E-02 & 349 & $7.9 \mathrm{sE}-05$ & $8.100-05$ & 8.85E-05 & 9.54E-05 \\
\hline $\mathrm{Ru}-106$ & $2.17 E-09$ & $1.26 \mathrm{E}-06$ & $9.19 E-04$ & $2.25 \mathrm{E}-13$ & $2.23 \mathrm{E}-13$ & $2.26 \mathrm{E}-09$ & $2.46 \mathrm{E}-09$ \\
\hline $\mathrm{Cd}-113 \mathrm{~m}$ & 4.28E-05 & 2.49E-02 & 18.2 & $6.24 E-07$ & $6.24 \mathrm{E}-07$ & 9.43E-05 & $9.11 E-05$ \\
\hline Sb-125 & $3.36 \mathrm{E}-05$ & $1.96 \mathrm{E}-02$ & 14.3 & 1.17E-07 & I.ITE-07 & 3.5LE-0S & $3.81 \mathrm{E}-05$ \\
\hline $5 n-126$ & $9.78 \mathrm{E}-06$ & $5.70 \mathrm{E}-03$ & 4.15 & 6.59E_- 08 & 6.59E-OB & 9.90E-06 & 1.07E-05 \\
\hline$I-129$ & 1.56E-07 & 9.1IE-05 & $6.63 \mathrm{E}-02$ & 1.51E-07 & 1.54E-07 & 1.68E-07 & $1.81 E-07$ \\
\hline $\mathrm{C}-134$ & $2.10 \mathrm{E}-06$ & 1.22E-03 & 0.839 & 2.43E- 09 & $243 \mathrm{E}-09$ & $2.18 E-06$ & 2.35E-06 \\
\hline Cs-137 & 0.300 & 175 & $1.27 \mathrm{E}+0 \mathrm{~S}$ & 0.290 & 0.296 & 0.321 & 0.346 \\
\hline$B a-137 m$ & 0.284 & 165 & $1.20 \mathrm{E}+0 \mathrm{~s}$ & 4.73E- 03 & $4.73 \mathrm{E}-03$ & 0.294 & 0,316 \\
\hline $5 m-151$ & $2.27 \mathrm{E}-02$ & 13.2 & $9.63 \mathrm{E}+03$ & 1.53E-44 & $1.53 \mathrm{E}-04$ & $2.30 \mathrm{E}-02$ & 2.49E-02 \\
\hline Eu- 152 & 1.70E-06 & $5.076-03$ & 3.69 & $6.25 \mathrm{E}-07$ & $6.25 \mathrm{E}-07$ & $8.76 E-06$ & 8.96E-06 \\
\hline Eu-154 & $2.26 \mathrm{E}-04$ & 0.132 & 93.8 & $1.93 \mathrm{E}-06$ & $1.93 \mathrm{E}-06$ & $2.36 \mathrm{E}-04$ & $2,97 \mathrm{E}-04$ \\
\hline Eu-155 & 4.39E-04 & 0.256 & 166 & 2.95E-05 & 2.95E-05 & 4.42E-04 & 4.47E-04 \\
\hline $\operatorname{Rn}-226$ & $5.24 E-10$ & $3.05 \mathrm{E}-07$ & $2.22 \mathrm{E}-\mathrm{OA}$ & $9.65 \mathrm{E}-11$ & $9.65 \mathrm{E}-11$ & $6.77 \mathrm{E}-10$ & 1.24E-10 \\
\hline $\mathrm{Ra}-228$ & 1.43E- -08 & $36 \mathrm{E}-06$ & $6.08 \mathrm{E}-03$ & 8.27E-16 & $8.27 \mathrm{E}-16$ & 1.45E-08 & $1.46 \mathrm{E}-08$ \\
\hline Ac- 227 & $2.93 \mathrm{E}-69$ & $1.71 E-06$ & $1.24 \mathrm{E}-03$ & $4.25 \mathrm{E}-10$ & $425 E-10$ & $3.3 \mathrm{E}-09$ & 4.38E-09 \\
\hline $\mathrm{Pa}-231$ & $9.48 E-69$ & $5.52 E-06$ & $4.02 \mathrm{E}-03$ & $6.41 \mathrm{E}-11$ & 6.41E-11 & $9.60 \mathrm{E}-09$ & $1.09 \mathrm{E}-08$ \\
\hline Th-229 & $3.44 \mathrm{E}-10$ & $200 \mathrm{E}-07$ & $1.46 \mathrm{E}-04$ & 1.5:E. 13 & 1.5UE-13 & 3,4TE-10 & 3.51E-10 \\
\hline $\mathrm{Th}-232$ & $1.92 \mathrm{E}-10$ & 1.12E-07 & 8.15E-05 & $5.27 \mathrm{E}-17$ & $5.27 \mathrm{E}-17$ & $2.42 \mathrm{E}-10$ & 2.92E-10 \\
\hline $\mathrm{v}-232$ & $6.41 \mathrm{E}-08$ & 3.73E-0S & $2.72 \mathrm{E}-02$ & $2.27 \mathrm{E}-0.98$ & $4.18 E-00$ & $9.13 \mathrm{E}-0 \mathrm{~B}$ & t. 22E-07 \\
\hline $\mathrm{U}-2,33$ & $2.45 \mathrm{E}-07$ & 1.43E-04 & 0.104 & $8.67 \mathrm{E}-06$ & $1.60 \mathrm{E}-07$ & 3.49E-07 & 4.65E-07 \\
\hline $\mathrm{U}-234$ & 1.17E-06 & 6.81E-04 & 0.496 & 5.18E-07 & 1.35E-07 & $1.53 \mathrm{E}-06$ & $1,89 \mathrm{E}-06$ \\
\hline U-235 & $4.72 \mathrm{E}-08$ & $2.75 \mathrm{E}-05$ & $2.00 \mathrm{E}-02$ & $2.09 \mathrm{E}-08$ & 3.37- -08 & $6.20 E-08$ & 7.68E-08 \\
\hline U-236 & 4.8IE-0:8 & 2. HoE-05 & $2.00 \mathrm{E}-02$ & $2.21 \mathrm{E}-0 \mathrm{~B}$ & $3.50 \mathrm{E}-08$ & $6.19 \mathrm{E}-0 \mathrm{~s}$ & $7.55 \mathrm{E}-08$ \\
\hline $\mathrm{U}-238$ & $1.03 \mathrm{E}-06$ & $5.98 \mathrm{E}-04$ & 0.435 & 4.52E-07 & $7.30 \mathrm{E}-07$ & 1.35E- 06 & $1.6 \mathrm{TE}-06$ \\
\hline Np-237 & $3.62 \mathrm{E}-07$ & $2.11 E-04$ & 0.154 & 3.47E-07 & 3.56E-07 & $3.92 \mathrm{E}-07$ & 4.24E-07 \\
\hline $\mathrm{Pu}-238$ & $2.74 \mathrm{E}-06$ & $1.59 E-03$ & 1.16 & $1.23 \mathrm{E}-06$ & $1.97 \mathrm{E}-06$ & $3.51 \mathrm{E}-06$ & $4.25 \mathrm{E}-06$ \\
\hline Pu-239 & $1.12 \mathrm{E}-\mathrm{04}$ & $6.50 \mathrm{E}-02$ & 47,3 & $6.29 \mathrm{E}-05$ & $8.6 \pi-05$ & $1.36 \mathrm{E}-04$ & $1,60 \mathrm{E}-04$ \\
\hline$P u-240$ & $1.75 \mathrm{E}-05$ & $1.02 E-02$ & 7.43 & $9.48 \mathrm{E}-06$ & 1.34E-0s & $2.16 \mathrm{E}-05$ & $2.56 \mathrm{E}-05$ \\
\hline$P(x-241$ & $1.46 \mathrm{E}-04$ & 8.48E-02 & 61.7 & $6.85 \mathrm{E}-0 \mathrm{~S}$ & $1.06 \mathrm{E}-04$ & $1.85 \mathrm{E}-0 \mathrm{~d}$ & $2.23 \mathrm{E}-04$ \\
\hline$P u-242$ & $7,47 \mathrm{E}-10$ & 4.35E-07 & $3.17 \mathrm{E}-04$ & $3,38 \mathrm{E}-10$ & $5.38 \mathrm{E}-10$ & $9.56 \mathrm{E}-10$ & $1.16 \mathrm{E}-09$ \\
\hline$A m-241$ & 1.07E-04 & $6.23 \mathrm{E}-02$ & 45.4 & $6.11 \mathrm{E}-05$ & $9.26 \mathrm{E}-05$ & $1.23 E-04$ & t.39E-S4 \\
\hline Am-243 & $3.40 \mathrm{E}-09$ & $1.98 \mathrm{E}-06$ & $1.44 \mathrm{E}-03$ & $1.57 \mathrm{E}-09$ & 2.94E-09 & $3.74 \mathrm{E}-09$ & 4.09E-09 \\
\hline $\mathrm{C} n-242$ & $1.58 \mathrm{E}-08$ & $9.21 \mathrm{E}-06$ & $6.70 \mathrm{E}-03$ & 1.44E-08 & 1.4AE-08 & 1.59E- -08 & 1.59E-08 \\
\hline $\mathrm{Cm}-243$ & 3.59E-10 & $2.09 \mathrm{E}-07$ & $1.52 \mathrm{E}-04$ & $3.29 \mathrm{E}-10$ & $3.29 \mathrm{E}-10$ & $3.62 \mathrm{E}-10$ & $3.62 \mathrm{E}-10$ \\
\hline $\mathrm{Cm}-244$ & 4.14E-10 & 2.41E-07 & $1.75 \mathrm{E}-04$ & $2,00 \mathrm{E}-10$ & $2.41 \mathrm{E}-10$ & $5.00 \mathrm{E}-10$ & $5.00 \mathrm{E}-10$ \\
\hline Totils & $\ldots \mathbf{M}$ & $n / \mathrm{s}$ & k & $\begin{array}{l}\text { gSC } \\
\text { (M) } \\
\text { / } 14)\end{array}$ & $\begin{array}{l}\text { SCI } \\
\text { (4) } \\
\text { II) }\end{array}$ & $\begin{array}{l}+30 \mathrm{C} \\
(\mathrm{Mlor} \\
(\mathrm{L})\end{array}$ & $\begin{array}{l}+\mathrm{CI} \\
(\mathrm{Nor} \\
(\mathrm{t})\end{array}$ \\
\hline Pu & $1.86 \mathrm{E}-03(\mathrm{~g} / \mathrm{L})$ & - & 0.788 & 1.04E-03 & $1.44 \mathrm{E}-03$ & 2.28E-03 & $2.68 E-03$ \\
\hline U & $1.27 \mathrm{E}-02$ & $1.76 \mathrm{E}+03$ & $1.28 \mathrm{E}+03$ & $5.56 E-03$ & $9.00 \mathrm{E}-03$ & $1.67 \mathrm{E}-02$ & $2.07 \mathrm{E}-02$ \\
\hline
\end{tabular}

"Unknowns in tank wolids inventory are aevigned by Tank Leyering Model (TLM). 
HDW Model Rev. 4

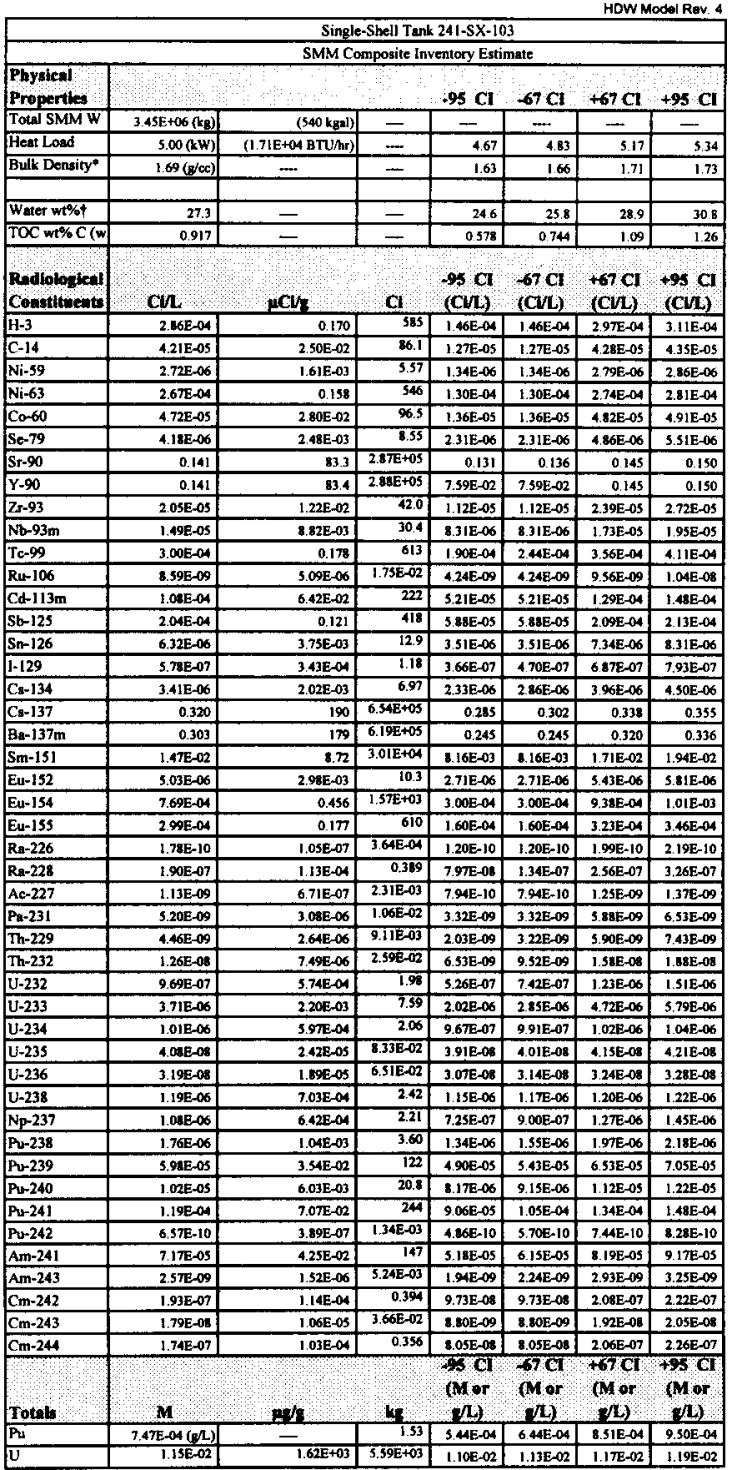

-Density is calculated based on $\mathrm{Na}, \mathrm{OH}-$, and $\mathrm{AJO2}$ -

tWater wt\% derived from the difference of density and total diwolved apeeies. 
HDW Model Rev. 4

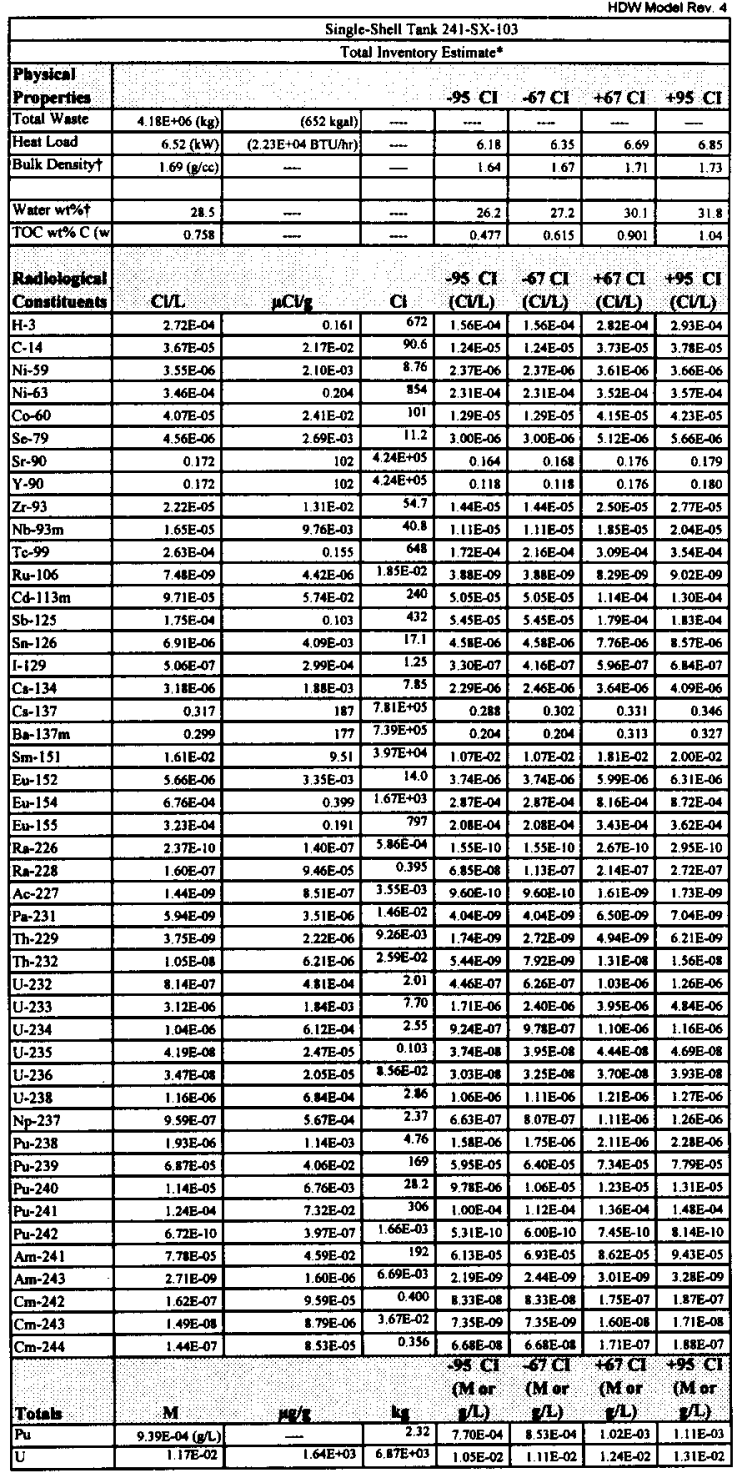

"Unknowns in tank colids inventory are ausigned by Tank Layering Model (TLM).

† Volurne average for denaity, mass sverage Water wt\% and toC wt\% C 


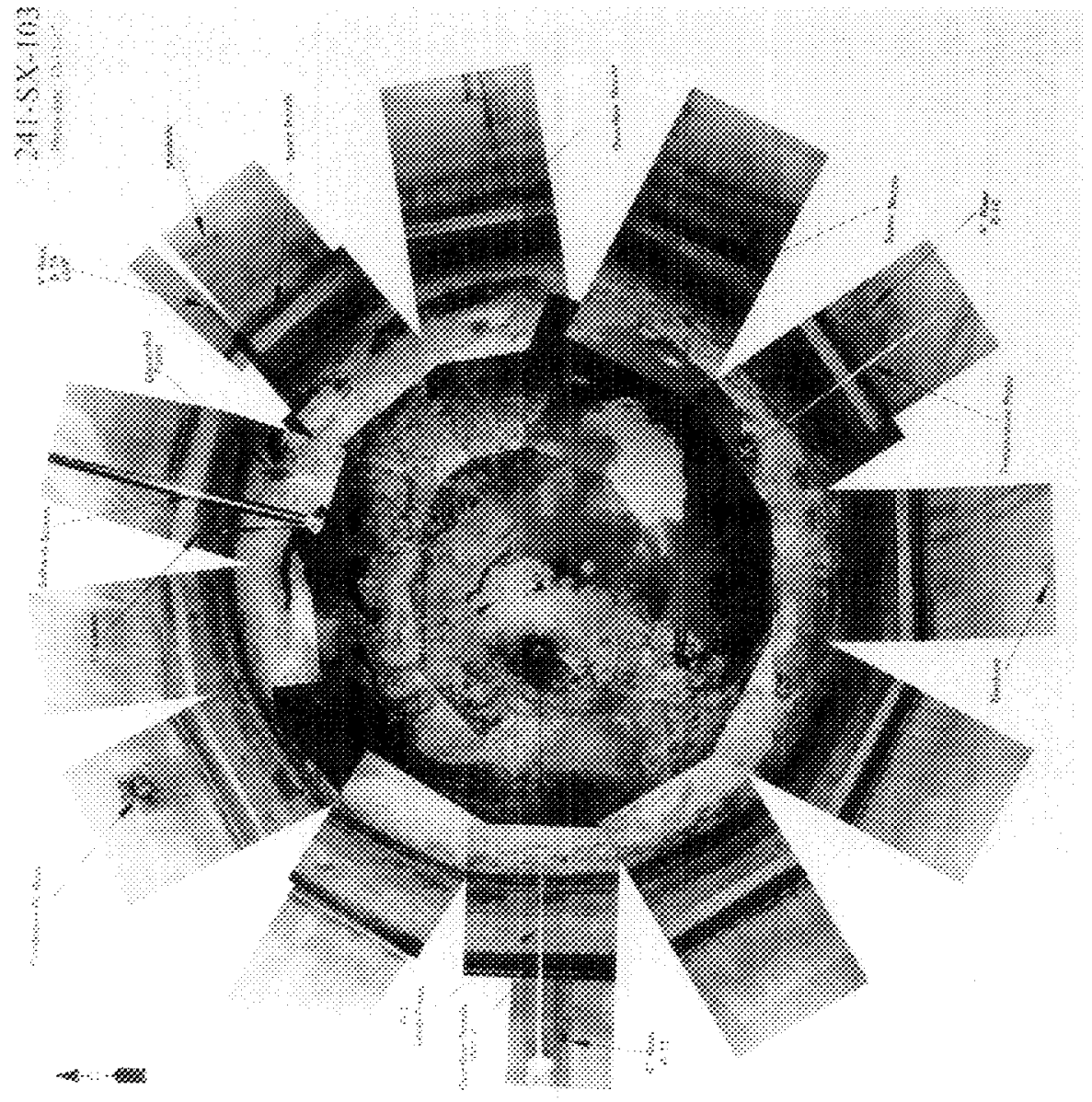


TANK 241-SX-104 SUMMARY

\begin{tabular}{|c|c|c|c|}
\hline \multicolumn{2}{|c|}{ TANK HISTORY } & \multicolumn{2}{|c|}{ TANK DESCRIPTION } \\
\hline Entered Service & 1st qtr 1955 & Diameter & $75 \mathrm{ft}$ \\
\hline Removed from Service & - & Bottom Shape & Dish \\
\hline Inactive & 1980 & Nominal Capacity & $1,000,000 \mathrm{gal}$ \\
\hline Watch Lists & Hyrdogen & Cascade Tank & to $241-S X-105$ \\
\hline Integrity & Assumed Leaker & Total Risers & 12 \\
\hline Assumed Leaker & 1988 & WASTE VOLUN & LON 1996i) \\
\hline Interim Stabilization (IS) & - & Total Waste Volume & $614,000 \mathrm{gal}$ \\
\hline Partial Interim Isolation (PI) & June 1985 & Waste Type & DSSF \\
\hline Intrusion Prevention (IP) & - & Drainable Interstitial Liquids & $201,000 \mathrm{gal}$ \\
\hline \multicolumn{2}{|c|}{$\begin{array}{l}\text { TENTATIVELY AVAILABLE RISERS } \\
\end{array}$} & Pumpable Liquids & $195,000 \mathrm{gal}$ \\
\hline Riser Number(s) & Size & Saltcake & $478,000 \mathrm{gal}$ \\
\hline 3,11 & 4 in & Sludge & $136,000 \mathrm{gal}$ \\
\hline Pे & 12 in & Supernatant & $0 \mathrm{gal}$ \\
\hline \multicolumn{2}{|c|}{ TANK TEMPERATURE } & \multicolumn{2}{|c|}{ INTERIOR PHOTOGRAPHS } \\
\hline Average Tank Temperature & $145^{\circ} \mathrm{F}$ & Date & Sept 9, 1988 \\
\hline Maximum Temperature & $200^{\circ} \mathrm{F}$ & Montage Number & $94030320-2 \mathrm{CN}$ \\
\hline Date & Aug 1, 1988 & Photo Set Number & 88090675 \\
\hline Elevation from tank bottom & $0.34 \mathrm{ft}$ & \multicolumn{2}{|c|}{\begin{tabular}{|l} 
WASTE SURFACE LEVEL \\
\end{tabular}} \\
\hline Riser Number & 2 & Devices & Manual ENRAF \\
\hline Minimum Temperature & $72.3^{\circ} \mathrm{F}$ & Max Level & 235.2 in \\
\hline Date & April 1, 1993 & Date & July 29,1991 \\
\hline Elevation from tank bottom & $30.55 \mathrm{ft}$ & Min Level & 219.52 in \\
\hline Riser Number & 2 & Date & Sept 17, 1996 \\
\hline
\end{tabular}




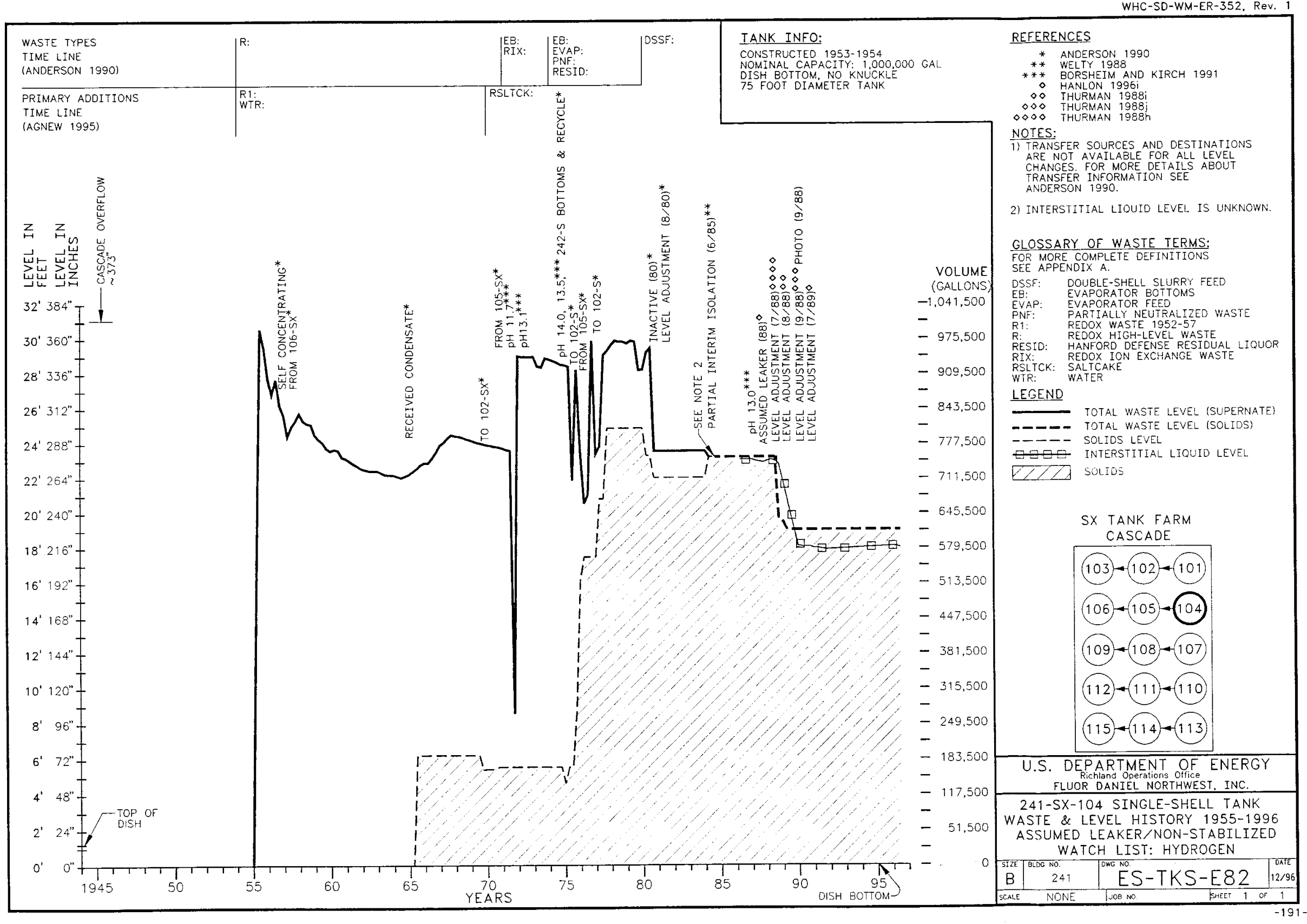




\section{$241-5 x-104$}

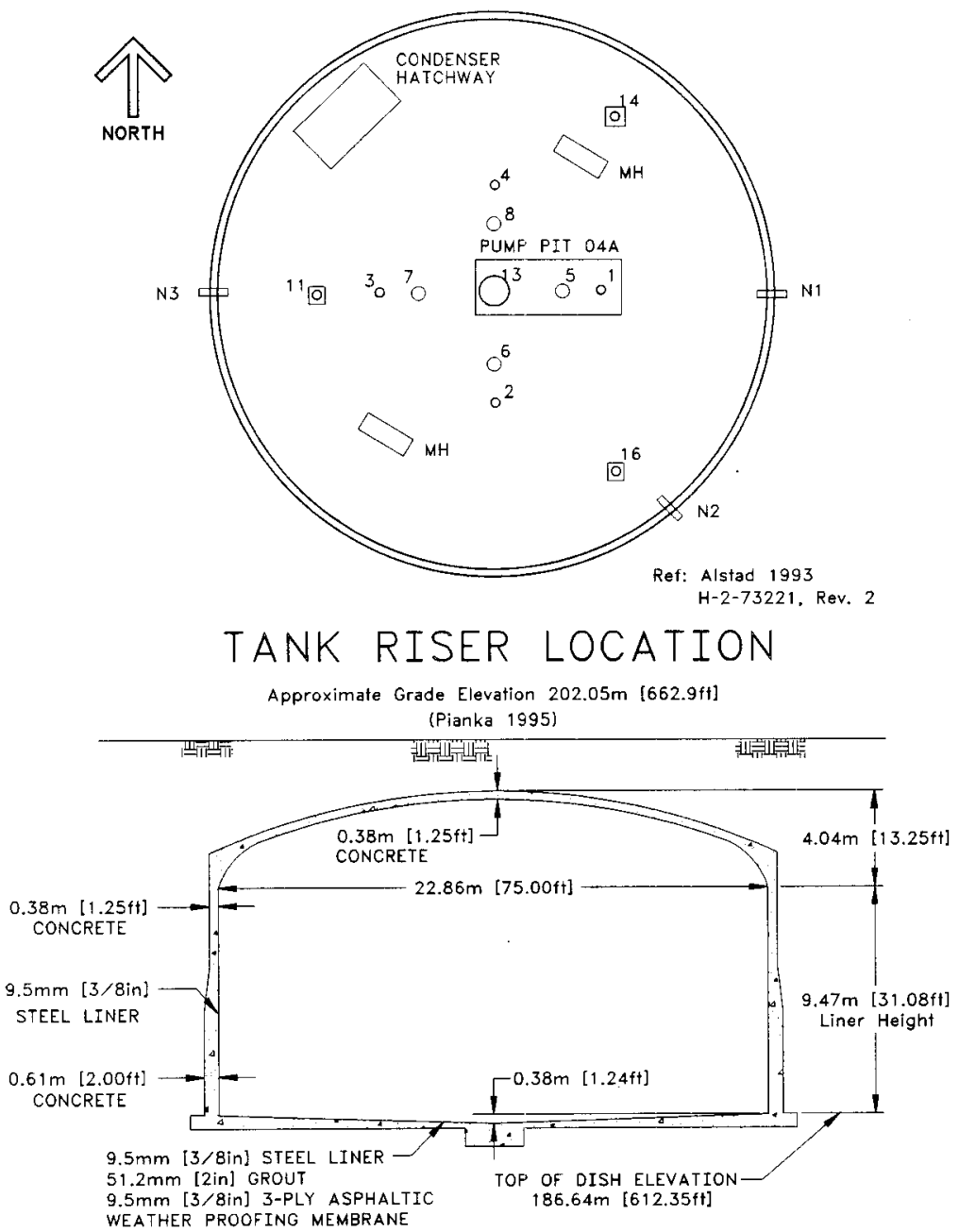

Ref: $\mathrm{H}-2-39511$, Rev, 3 H-2-46293, Rev. 3

NOT TO SCALE $\mathrm{H}-2-37855$, Rev. 4 
HNF-SD-WM-ER-352, Rev. 1

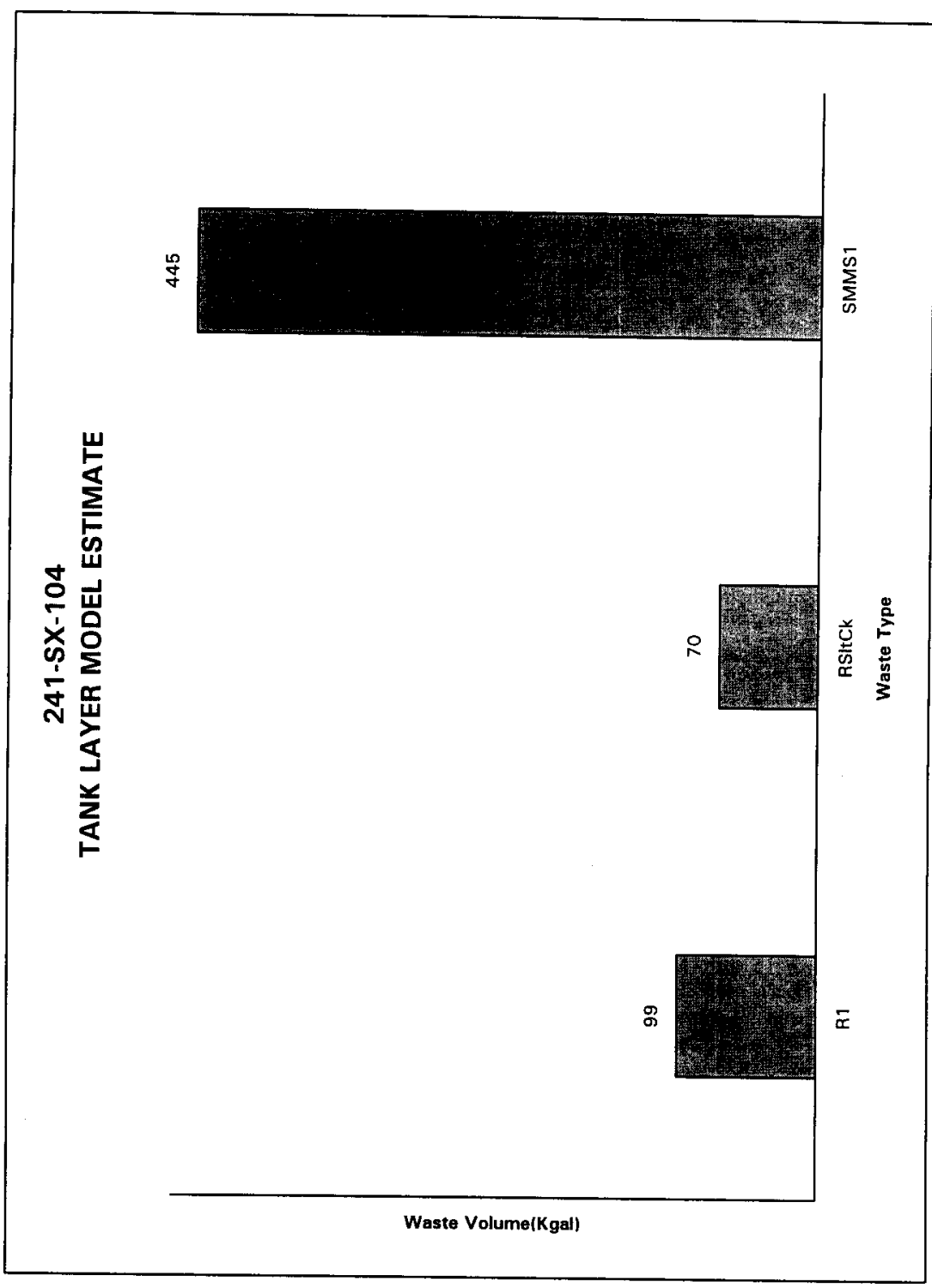

合 


\begin{tabular}{|c|c|c|c|c|c|c|c|}
\hline \multicolumn{8}{|c|}{ Single-Shell Tank 241-SX-104 } \\
\hline \multicolumn{8}{|c|}{ TLM Solids Composite inventory Estimate* } \\
\hline Phyilaten & & & & & & & \\
\hline Properties & & & & $95 \mathrm{CI}$ & $-67 \mathrm{CI}$ & $+67 \mathrm{Cl}$ & +950 \\
\hline Total TLM Wa & $1.11 \mathrm{E}+00(\mathrm{~kg})$ & $[169 \mathrm{kgal})]$ & - & - & - & - & - \\
\hline Heat Loed & $3.31(\mathrm{~kW})$ & (1.13E+04 BTU/hr) & - & 2.64 & 3.03 & 3.49 & 3.58 \\
\hline Bulk Density & $1.74(g / c)$ & $=$ & - & 1.59 & 1.61 & 1.94 & 2.02 \\
\hline Yoid Fraction & 0.639 & 二 & - & 0.360 & 0.452 & 0.757 & 0.760 \\
\hline Water wt\% & 30.1 & - & $=$ & 13.9 & 19.1 & 39.1 & 40.0 \\
\hline TOC wT\%C (w) & $2.14 \mathrm{E}-03$ & - & $=$ & $1.84 \mathrm{E}-03$ & $1.92 \mathrm{E}-03$ & $2.32 \mathrm{E}-03$ & $2.50 \mathrm{E}-03$ \\
\hline $\begin{array}{c}\text { Chemitent } \\
\text { Constifuents }\end{array}$ & mol/d/L , & Ppan _ _ & tg & $\begin{array}{r}95 \mathrm{Cl} \\
\text { (17ole/L) }\end{array}$ & $\begin{array}{r}67 \mathrm{Cl} \\
\text { (molen) }\end{array}$ & $\begin{array}{r}+67 \mathrm{Cl} \\
\text { (moleti) }\end{array}$ & $\begin{array}{r}98 \mathrm{Cl} \\
\text { (moled) }\end{array}$ \\
\hline \begin{tabular}{|l|l}
$\mathrm{Na+}+2+1$ \\
\end{tabular} & 10.3 & $1.37 \mathrm{E}+05$ & $1.52 \mathrm{E}+05$ & \begin{tabular}{r|}
7.36 \\
\end{tabular} & 7.66 & 14.2 & 16.2 \\
\hline $\mathrm{AB}+$ & 3.30 & $8.53 \mathrm{E}+04$ & $9.49 E+04$ & 4.69 & 4.93 & 6.12 & 6.75 \\
\hline $\mathrm{Fe} 3+(\operatorname{soth} \mathrm{Fe})$ & 0.601 & $1.93 E+04$ & $2.13 \mathrm{E}+04$ & 0.390 & 0.595 & 0.606 & 0.612 \\
\hline $\mathrm{Cr}^{3} 3+$ & 0.377 & $1.13 \mathrm{E}+04$ & $1.25 \mathrm{E}+04$ & 0.235 & 0.303 & 0.646 & 0.961 \\
\hline Bi3+ & $2.85 \mathrm{E}-06$ & 0.342 & 0.350 & $240 E-06$ & $2.62 E-06$ & $3.14 E-06$ & $3.45 \mathrm{E}-06$ \\
\hline 5 & $7.01 \mathrm{E}-12$ & 5.60E-07 & $6.23 \mathrm{E}-07$ & $6.14 E-12$ & 6.63E-12 & $7.75 \mathrm{E}-12$ & $8.52 \mathrm{E}-12$ \\
\hline $\mathrm{Hg}_{2+}+$ & $4.4 \bar{E}-07$ & $5.16 \mathrm{E}-02$ & $3.7 \mathrm{AE}-02$ & $3.96 E-07$ & 4.21E-07 & $4.92 E-07$ & 5.40E-07 \\
\hline $\mathrm{Zr}(\mathrm{N} 2 \mathrm{ZO}(\mathrm{OH}) 2$ & $2.84 E-07$ & 1.49E-02 & $1.66 \mathrm{E}-02$ & $2.61 \mathrm{E}-07$ & $2.72 \mathrm{E}-07$ & $2.98 \mathrm{E}-07$ & 3.21E-07 \\
\hline $\mathrm{Pb} 2+$ & 7.09E-05 & 8.45 & 939 & 3.80E-05 & $5.40 \mathrm{E}-05$ & 8.79E-05 & $1.05 E-04$ \\
\hline $\mathrm{Ni2+}$ & 339E-02 & $1.21 \mathrm{E}+03$ & $1.75 E+03$ & $2.77 \mathrm{E}-02$ & $3.23 \mathrm{E}-02$ & $3.87 \mathrm{E}-02$ & $382 E-02$ \\
\hline $5 \sqrt{2+}$ & 0 & 0 & 0 & 0 & 0 & 0 & 0 \\
\hline Mndt & $2.07 \mathrm{E}-05$ & 0.655 & 0.728 & $1.48 \mathrm{E}-0 \mathrm{~s}$ & $1.77 \mathrm{E}-05$ & $2.38 E-05$ & $2.68 \mathrm{E}-03$ \\
\hline $\mathrm{Cu}_{2+}+$ & 0.161 & $3.70 E+03$ & $412 E+03$ & 0.117 & 0.138 & 0.183 & 0.204 \\
\hline $\mathbf{K}+$ & $1.98 \mathrm{EE}-02$ & 446 & 496 & 1.40E-02 & $1.63 \mathrm{E}-02$ & 222E- 02 & $2.23 \mathrm{E}-02$ \\
\hline OH- & 22.7 & $222 \mathrm{E}+03$ & $2.47 \mathrm{E}+03$ & 19.0 & 20.2 & 25.4 & 28.0 \\
\hline NO3- & 5.27 & $1.88 \mathrm{E}+05$ & $2.09 E+05$ & 2.21 & 2.21 & 10.1 & 12.7 \\
\hline Nōo2- & $\mathbf{T . 6 3}$ & $.31 E+0 x$ & $4.79 E+04$ & 0.857 & 1.12 & 2.03 & 2.03 \\
\hline $\cos 2-$ & 0.163 & $5.62 \mathrm{E}+03$ & $6.24 \mathrm{E}+03$ & 0.119 & 0.140 & 0.185 & 0.206 \\
\hline PO43- & 184E-OA & 10.1 & 11.2 & $1.71 \mathrm{E}-04$ & $1.79 \mathrm{E}-04$ & $1.92 \mathrm{E}-04$ & $2.08 E-04$ \\
\hline SO42- & $2.28 \mathrm{E}-02$ & $1.26 \mathrm{E}+03$ & $1.40 E+03$ & $1.74 E-02$ & $1.96 \mathrm{E}-02$ & $2.50 E-02$ & $2.59 \mathrm{E} \sim-02$ \\
\hline $\mathrm{Si}(\boldsymbol{\mu} \mathrm{ST} 032-)$ & $3.86 \mathrm{E}-02$ & 97 & $1.05 E+03$ & $3.82 E-02$ & $5.11 E-02$ & $6.61 \mathrm{E}-02$ & 7.34E-02 \\
\hline $\mathbf{F}-$ & $1.47 \mathrm{E}-04$ & 160 & 1.78 & $1.25 \mathrm{E}-04$ & $1.38 \mathrm{E}-04$ & $1.61 \mathrm{E}-04$ & $1.74 E-04$ \\
\hline Cl- & 85SE-02 & $1.74 E+03$ & $1.94 E+03$ & 5.87E-02 & $6.94 \mathrm{E}-02$ & $9.63 \mathrm{E}-02$ & 0.147 \\
\hline C6HSO73- & $1.52 \mathrm{E}-04$ & 16.5 & 18.3 & $1.47 \mathrm{E}-\mathrm{A4}$ & $1.49 E-04$ & 1.5EE-04 & $1.72 \mathrm{E}-04$ \\
\hline EOTA4- & 5.91E-06 & 0.979 & 1.09 & $4.16 \mathrm{E}-06$ & S.IIE-06 & $6.96 \mathrm{E}-06$ & B.00E-06 \\
\hline HEDTA3- & 4.91E-06 & 0.774 & 0.860 & $1.57 \mathrm{E}-06$ & $3.37 \mathrm{E}-06$ & $6.55 E-06$ & 8. $19 \mathrm{E}-06$ \\
\hline & & & & & & & \\
\hline Elycolate- & $2.14 E-04$ & 9.24 & 10.3 & $1.16 \mathrm{E}-04$ & $1,69 \mathrm{E}-\mathrm{NA}$ & $2.66 \mathrm{E}-04$ & $3.16 \mathrm{E}-04$ \\
\hline acetonto- & $2.22 \mathrm{E}-05$ & 0.755 & 0.019 & 2.15E-05 & 2.19E-05 & 2.36E-0s & $232 \mathrm{E}-05$ \\
\hline oxallate2- & $9.18 E-12$ & 4.65E-07 & 3.17E-07 & 1.14E-12 & 8.65E-12 & $1.01 \mathrm{E}-11$ & 1.11E-11 \\
\hline DEP & 1.35E-04 & $\begin{array}{r}16.3 \\
\end{array}$ & 18.1 & $1.26 \mathrm{E}-04$ & $1.31 \mathrm{E}-04$ & 1.45E-04 & $1.57 \mathrm{E}-04$ \\
\hline butanol & 1.35E-04 & 5.74 & 6.38 & $1.26 \mathrm{E}-04$ & $4.31 \mathrm{E}-04$ & $1.45 E-04$ & $1.5 \pi \mathrm{E}-04$ \\
\hline & & & & & & & \\
\hline $\mathrm{NH3}$ & 0.163 & $1.61 E^{2}+03$ & $1.79 E+03$ & 5.33E-02 & $6.91 \mathrm{E}-02$ & 0.173 & 0.181 \\
\hline $\mathrm{Fe}(\mathrm{CN})^{64-}$ & 0 & 0 & 0 & 0 & 0 & 0 & 0 \\
\hline
\end{tabular}


HDW Model Rev. 4

\begin{tabular}{|c|c|c|c|c|c|c|c|}
\hline \multirow{4}{*}{$\begin{array}{l}\text { Phyoled } \\
\text { Properties }\end{array}$} & \multicolumn{7}{|c|}{ Single-Shell Tank 241-SX-104 } \\
\hline & \multicolumn{7}{|c|}{ SMM Composite Inventory Estimate } \\
\hline & & & & & & & \\
\hline & $\therefore \therefore$ & $\therefore$ & & $-95 \mathrm{CI}$ & $-67 \mathrm{Cl}$ & $+67 \mathrm{Cl}$ & $+95 \mathrm{Cl}$ \\
\hline Total SMM W & $2.85 \mathrm{E}+06(\mathrm{~kg})$ & (445 kgat) & - & - & 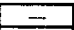 & - & - \\
\hline Heat Load & $4.05(\mathrm{~kW})$ & (1.38E+(04 BTU/hr) & $=$ & 3.80 & 3.93 & 4.17 & 4.29 \\
\hline Bulk Density" & $1.69(\mathrm{~g} / \mathrm{cc})$ & - & - & 1.64 & 1.67 & 1.72 & 1.74 \\
\hline & & & & & & & \\
\hline Wuter wt $\%$ & 27.3 & $=$ & $\cdots$ & 24.5 & 25.7 & 29.0 & 31.0 \\
\hline TOC wt $\% \mathrm{C}(w)$ & 0.859 & - & $\ldots$ & 0.568 & 0.711 & 1.01 & 1.15 \\
\hline $\begin{array}{l}\text { Chew } \\
\text { Constituents }\end{array}$ & molet & ppan : & k & $\begin{array}{l}95 \text { CI } \\
\text { (moledL) }\end{array}$ & $\begin{array}{r}67 \mathrm{Ct} \\
\text { (mole/t) }\end{array}$ & $\begin{array}{l}+67 \mathrm{Ct} \\
(\mathrm{unoth} / \mathrm{t})\end{array}$ & $\begin{array}{l}+95 \mathrm{CI} \\
\text { (mote/L) }\end{array}$ \\
\hline $\mathrm{Nat}$ & 16.3 & $2.21 \mathrm{E}+05$ & $6.30 \mathrm{E}+0 \mathrm{~S}$ & 14.8 & 155 & 16.9 & 17.5 \\
\hline $\mathrm{Al} 3+$ & 1.92 & $3,06 \mathrm{E}+04$ & $8.71 \mathrm{E}+04$ & 1.78 & 1.85 & 2.00 & 2.06 \\
\hline $\mathrm{Fe} 3+($ totel $\mathrm{Fe})$ & $1.27 \mathrm{E}-02$ & 418 & 1.19E+03 & $1.03 \mathrm{E}-02$ & 1.14E-02 & $1.39 \mathrm{E}-02$ & $1.51 \mathrm{E}-02$ \\
\hline $\mathrm{Cr} 3+$ & 0.170 & $5.22 \mathrm{E}+03$ & $1.49 \mathrm{E}+04$ & 0.143 & 0.157 & 0.175 & 0.180 \\
\hline $\mathrm{Bi}+$ & $1.45 \mathrm{E}-03$ & 178 & 309 & $1.32 \mathrm{E}-03$ & $1.38 \mathrm{E}-03$ & $1.51 \mathrm{E}-03$ & $1.57 \mathrm{E}-03$ \\
\hline Lnst+ & 4.57E-05 & 3.75 & 10.7 & $3.31 \mathrm{E}-05$ & $3.92 \mathrm{E}-05$ & $5.21 E-05$ & $5.83 \mathrm{E}-05$ \\
\hline \begin{tabular}{|l|} 
Hg2t \\
\end{tabular} & $1.02 \mathrm{E}-05$ & 1.21 & 3.46 & 9.58E-06 & $9.95 \mathrm{E}-06$ & $1.04 \mathrm{E}_{-0} 05$ & 1.05E-05 \\
\hline $\mathrm{Zr}(\approx \mathrm{ZrO}(\mathrm{OH}) 2$ & 2.64E-04 & 14.2 & 40.6 & $2.41 E-04$ & 2.49E-04 & $2.74 E-04$ & 2.87E-04 \\
\hline $\mathrm{Pb2+}$ & I.15E-03 & 141 & 403 & $9,34 E-04$ & 1.04E-03 & $1.27 \mathrm{E}-03$ & 1.37E-03 \\
\hline $\mathrm{Ni2+}$ & $7.39 E-03$ & 256 & 731 & $7,06 \mathrm{E}-03$ & $7.22 \mathrm{E}-03$ & $7.48 \mathrm{E}-03$ & 7.57E-03 \\
\hline $\mathrm{Sr} 2+$ & 0 & 0 & 0 & 0 & 0 & 0 & 0 \\
\hline $\mathrm{Mnd}^{+}$ & 4.47E-03 & 145 & 413 & $3.31 \mathrm{E}-03$ & 3.8SE-03 & $5.06 \mathrm{E}-03$ & 5.63E-03 \\
\hline $\mathrm{Con}+$ & 3.88E-02 & 917 & $262 \mathrm{E}+03$ & $3.53 \mathrm{E}-02$ & 3.70E- 02 & 4.05E-02 & $422 \mathrm{E}-02$ \\
\hline$\overline{k+}$ & $7.51 \mathrm{E}-02$ & $1,73 \mathrm{E}+03$ & $4.94 E+03$ & 6. $10 \mathrm{E}-02$ & $7.13 \mathrm{E}-02$ & $7.89 \mathrm{E}-02$ & $8.26 \mathrm{E}-02$ \\
\hline OH- & 11.6 & $1.16 \mathrm{E}+05$ & 3.32E+05 & 107 & 11.1 & 12.0 & 12.3 \\
\hline NO3- & 5,61 & $2.06 \mathrm{E}+0 \mathrm{~S}$ & $5.86 E+05$ & 5.21 & 5.45 & 5.70 & 5.77 \\
\hline NO2- & 25 & $7.68 \mathrm{E}+04$ & 2.19E+0S & 2.33 & 2.56 & 3.09 & 3.33 \\
\hline $\cos 2-$ & 0.506 & $1.79 \mathrm{E}+04$ & $5.11 \mathrm{E}+04$ & 0.456 & 0.480 & 0.537 & 0.548 \\
\hline POA3. & 0.103 & $5.76 \mathrm{E}+03$ & $1.64 \mathrm{E}+04$ & 8.94E-02 & 9.41E-02 & 0.105 & 0.107 \\
\hline SO42- & 0.281 & $1.60 E+04$ & 1.55E+04 & 0.217 & 0.24 & 0.316 & 0.346 \\
\hline $\mathrm{Si}(\mathrm{m} \mathrm{SiO32-})$ & $9.02 \mathrm{E}-42$ & $1.50 \mathrm{E}+03$ & $4.275+03$ & $7.36 \mathrm{E}-02$ & 8.18E-02 & $9.8 T \mathrm{E}-02$ & 0.107 \\
\hline $\mathrm{F}-$ & $7.0 \% E-02$ & 796 & $2.2 \pi+03$ & $6,00 \mathrm{E}-02$ & $6.46 \mathrm{E}-02$ & $7.63 \mathrm{E}-02$ & 8.06E-02 \\
\hline Cl- & 0.280 & $5.66 \pm+03$ & $1.6 \pi+04$ & 0.252 & 0.266 & 0.293 & 0.304 \\
\hline C6H5073- & $3.40 \mathrm{E}-02$ & 3. $20 E+03$ & T.08E+04 & 3.12E-02 & $3.26 \mathrm{E}-02$ & $3.54 E-02$ & $3.69 \mathrm{E}-02$ \\
\hline EDTA4- & 1.78E-02 & $3,03 \mathrm{E}+03$ & $8.63 \mathrm{E}+03$ & $5,58 E-03$ & $1.15 E-02$ & $2.41 E-02$ & $3.02 \mathrm{E}-02$ \\
\hline HEDTA3- & 3.29E-02 & $5.33 E+03$ & $1525+04$ & 8.51E-03 & $2.04 \mathrm{E}-02$ & 4.55E-02 & 5.7TE-02 \\
\hline glycointo- & 0.107 & $4.74 E+03$ & $1336+04$ & $6.95 \mathrm{E}-02$ & 8. $78 \mathrm{E}-02$ & 0.126 & 0.144 \\
\hline ecetute & 6.51E-03 & 296 & 846 & $6.9 \mathrm{TE}-03$ & $7.72 E-03$ & $9.29 \mathrm{E}-03$ & $1.00 \mathrm{E}-02$ \\
\hline oxalate2- & S.9E-0S & 3.11 & 68 & 5.30E-05 & $5.63 \mathrm{E}-05$ & $6.33 \mathrm{E}-05$ & 6.66F-05 \\
\hline DBP & $2.25 \mathrm{E}-02$ & $2.80 \mathrm{E}+03$ & $7.93 E+03$ & $1.85 \mathrm{E}-02$ & $2.05 \mathrm{E}-02$ & $2.46 \mathrm{E}-02$ & $2.65 \mathrm{E}-02$ \\
\hline buttunol & 2.25E-02 & 96 & $281 \mathrm{E}+03$ & $1.85 \mathrm{E}-02$ & $2.05 \mathrm{E}-102$ & 2.46E.02 & 2.65E-02 \\
\hline$\overline{N H 3}$ & $960 E_{-02}$ & 93 & $27515+43$ & & & & \\
\hline $\mathrm{Fo}(\mathrm{CN}) 64$ & 0 & 0 & 0 & $1.73 \mathrm{E}-12$ & $8.46 \mathrm{E}-02$ & 0.110 & 0.127 \\
\hline
\end{tabular}

-Density is calculated based on $\mathrm{Na}, \mathrm{OH}$-, and AlO2-.

tWater wh\% derived from the difference of denaity and total diswolved apocies. 
HNF-SD-WM-ER-352, Rev. 1

HDW Model Rev.

\begin{tabular}{|c|c|c|c|c|c|c|c|}
\hline \multirow{4}{*}{\begin{tabular}{|l|} 
\\
Physical \\
Propertics
\end{tabular}} & \multicolumn{7}{|c|}{ Single-Shell Tank 24I-SX-104 } \\
\hline & \multicolumn{7}{|c|}{ Total Inventory Estimate* } \\
\hline & & & & & & & \\
\hline & & $:$ & & $-95 \mathrm{CI}$ & $-67 \mathrm{Cl}$ & $+67 \mathrm{Cl}$ & $+95 \mathrm{CI}$ \\
\hline Total Waste & $3.96 E+06(\mathrm{~kg})$ & (614 kgal) & - & $\ldots$ & - & $=$ & - \\
\hline Heat Load & $7.36(\mathrm{~kW})$ & $(2.52 \mathrm{E}+04 \mathrm{BTU} / \mathrm{hr})$ & - & 6.59 & 2.03 & 7.61 & 760 \\
\hline Bulk Density ${ }^{\dagger}$ & $1.71(g / \infty)$ & - & - & 1.64 & 1.66 & 177 & 1.78 \\
\hline Water wt\%† & 28.1 & $\ldots$ & $\ldots$ & 23.6 & 24.4 & 30.9 & 32.0 \\
\hline TOC wr\% C (w & 0.619 & - & $\ldots$ & 0.409 & 0.512 & 0,726 & $0.83 !$ \\
\hline $\begin{array}{l}\text { Coentel } \\
\text { Constlifuent: }\end{array}$ & molen & Bom & ke & $\begin{array}{l}95 \mathrm{Cl} \\
\text { (mole/t) }\end{array}$ & $\begin{array}{l}-67 \mathrm{CI} \\
\text { (nolet })\end{array}$ & $\begin{array}{l}+67 \mathrm{Cl} \\
\text { (mole/L) }\end{array}$ & $\begin{array}{l}+98 \mathrm{Cl} \\
(\mathrm{moll} / \mathrm{L})\end{array}$ \\
\hline $\mathrm{Na4}$ & 14.6 & $1.97 \mathrm{E}+05$ & $7.22 \mathrm{E}+05$ & 13.3 & 13.7 & 15.9 & 16.1 \\
\hline $\mathbf{A} \mathbf{B}+$ & 2.90 & $4.59 \mathrm{E}+04$ & $1.82 \mathrm{E}+0 \mathrm{~S}$ & 2.66 & 2.75 & 3.07 & 3.25 \\
\hline Fes + (toul Fe) & 0.175 & $5.71 E+03$ & $2.26 E+04$ & 0.172 & 0.173 & 0.176 & 0.177 \\
\hline $\mathrm{Cr} 3+$ & 0.227 & $6.92 \mathrm{E}+03$ & $2.74 E+04$ & 0.184 & 0.207 & 0.292 & 0.368 \\
\hline Bi3+ & J,05E-03 & 120 & 509 & 9.57E-04 & $1.00 \mathrm{E}-03$ & $1,09 \mathrm{E}-03$ & 1.14E-03 \\
\hline Les+ & $3.31 \mathrm{E}-0 \mathrm{~S}$ & 2.69 & 10.7 & $2.40 \mathrm{E}-05$ & 2.84E-0S & $3.78 E-05$ & $4.23 \mathrm{E}-0 \mathrm{~S}$ \\
\hline $\operatorname{Hg} 2+$ & $7.54 E-06$ & 0.857 & 3.52 & $7,06 \mathrm{E}-06$ & $7.34 E-06$ & $7.65 \mathrm{E}-06$ & $7.76 \mathrm{E}-06$ \\
\hline $\mathrm{Zr}(\mathrm{ar} \mathrm{ZnO}(\mathrm{OH}) 2$ & 1.91E-04 & 10.2 & 40.6 & $1.75 \mathrm{E}-04$ & $1,81 E-04$ & $1.98 \mathrm{E}-\mathrm{OA}$ & $2.08 \mathrm{E}-04$ \\
\hline $\mathrm{Pb2+}$ & $8.56 \mathrm{E}-04$ & 104 & 412 & $6.96 E-04$ & $7.74 \mathrm{E}-04$ & $9.37 \mathrm{E}-04$ & $1.01 \mathrm{E}-03$ \\
\hline Ni2+ & $1.53 \mathrm{E}-02$ & 525 & $2.08 \mathrm{E}+03$ & 1.30E-02 & $1.43 \mathrm{E}-02$ & $1.60 \mathrm{E}-02$ & $1.58 \mathrm{E}-02$ \\
\hline $\mathrm{Sr} 2+$ & 0 & 0 & 0 & 0 & 0 & 0 & 0 \\
\hline Mndt & 3.24E-03 & 104 & 414 & $240 \mathrm{E}-03$ & $2.81 \mathrm{E}-03$ & $3.67 \mathrm{E}-03$ & $4.08 \mathrm{E}-03$ \\
\hline Cont & $7.23 \mathrm{E}-02$ & $1.70 \mathrm{E}+03$ & $6.73 \mathrm{E}+03$ & $6.09 \mathrm{E}-02$ & $6.69 \mathrm{E}-02$ & 7.7TE-02 & $8.28 \mathrm{E}-02$ \\
\hline $\mathrm{K}+$ & 5.99E-02 & $1.37 E+03$ & $5442+03$ & 5.47E-02 & $5.71 \mathrm{E}-02$ & $6.27 \mathrm{E}-02$ & $653 \mathrm{E}^{-02}$ \\
\hline OH- & 14.7 & $1.46 \mathrm{E}+05$ & $5.79 \pm+05$ & 13.5 & 13.9 & 15.4 & 16.2 \\
\hline NO3. & 5.52 & $2.01 E+05$ & $7.95 E+05$ & 4.57 & 4.66 & 6.80 & 7.40 \\
\hline NO2- & 250 & $6.73 \mathrm{E}+04$ & $2.67 \mathrm{E}+0 \mathrm{~S}$ & 2.14 & 2.30 & 2.63 & 2.86 \\
\hline $\cos 2-$ & 0.411 & $1.45 \mathrm{E}+04$ & $5.74 \mathrm{E}+04$ & 0.375 & 0.393 & 0.434 & 0.442 \\
\hline POA- & $7.46 \mathrm{E}-02$ & $4.15 E+03$ & T.65E+04 & $6.48 E-02$ & 6.82E-02 & $7.6 \mathrm{JE}-02$ & 7.79E-02 \\
\hline SOA2- & 0.210 & T.JEE+04 & $4.69 \mathrm{E}+04$ & 0.164 & 0.196 & 0.235 & 0.257 \\
\hline Si (ns Sí32-) & 8.15E-02 & $1.34 \mathrm{E}+03$ & $532 E+03$ & $6,95 \mathrm{E}-02$ & $7.54 \mathrm{E}-02$ & 8.77E-02 & 9.36E-02 \\
\hline F- & $5.15 E-02$ & 573 & $2,27 \mathrm{E}+03$ & 4.35E-02 & $4.69 \mathrm{E}-02$ & $5.53 \mathrm{E}-02$ & $5.84 E-02$ \\
\hline $\mathrm{Cl}$ & 0.227 & $4.70 \mathrm{E}+03$ & $1.87 \mathrm{E}+04$ & 0.206 & 0.216 & 0.236 & 0.24 \\
\hline C6H5073. & $2.47 \mathrm{E}-02$ & $2.74 E+03$ & $1.08 E+04$ & $2.27 \mathrm{E}-02$ & $2.36 \mathrm{E}-02$ & $2.57 \mathrm{E}-02$ & $2.67 \mathrm{E}-02$ \\
\hline EDTA4- & $1.296-02$ & 2.1BE+03 & $863 E+03$ & $4.05 \mathrm{E}-03$ & 8.37E-03 & $1.74 \mathrm{E}-02$ & 2. 19E-02 \\
\hline HEDTA3- & $2.3 \%-02$ & $3.03 \mathrm{E}+03$ & $1.525+04$ & $6.17 \mathrm{E}-03$ & $1.48 \pm-02$ & $3.30 \mathrm{E}-02$ & $4.196-02$ \\
\hline Glycolate- & $7.76 \mathrm{E}-02$ & $3.41 \mathrm{E}+03$ & J.35E+04 & $5.04 E-02$ & $6.3 \pi-02$ & $9.19 E-02$ & 0.105 \\
\hline ncerate & $6.17 \mathrm{E}-03$ & 214 & 4 & $5.06 \mathrm{E}-03$ & $5.60 \mathrm{E}-03$ & $6.74 \mathrm{E}-03$ & $7.29 \mathrm{E}-03$ \\
\hline oxusintez- & 4.34E-05 & 2.24 & 8.77 & 3.84E-05 & 4.08E-05 & $4.59 \mathrm{E}-05$ & $4.83 E-05$ \\
\hline DAP & 1.64E-02 & $2.02 E+03$ & $8.00 \mathrm{E}+03$ & 1.34E-02 & 1.49E-02 & $1.78 \mathrm{E}-02$ & 1.93E 02 \\
\hline bertanol & 1.64E-02 & 711 & $2 . \overline{22 E+03}$ & $1,34 \mathrm{E}-02$ & $1,49 \mathrm{E}-02$ & $1.78 E-02$ & $1.93 \mathrm{E}-02$ \\
\hline NE3 & 0.115 & $1.14 E+03$ & $4 \overline{54 E+03}$ & 8.14E-02 & $8.72 E-02$ & 0.125 & 0.138 \\
\hline Fe(CN)64: & 0 & 0 & 0 & 0 & 0 & 0 & 0 \\
\hline
\end{tabular}

- Unknowns in tank eolids inventory are assigred by Twak Layering Model (TLM).

tWater we\% derived from the difference of density and total dissolved apecies. 
HDW Model Rev. 4

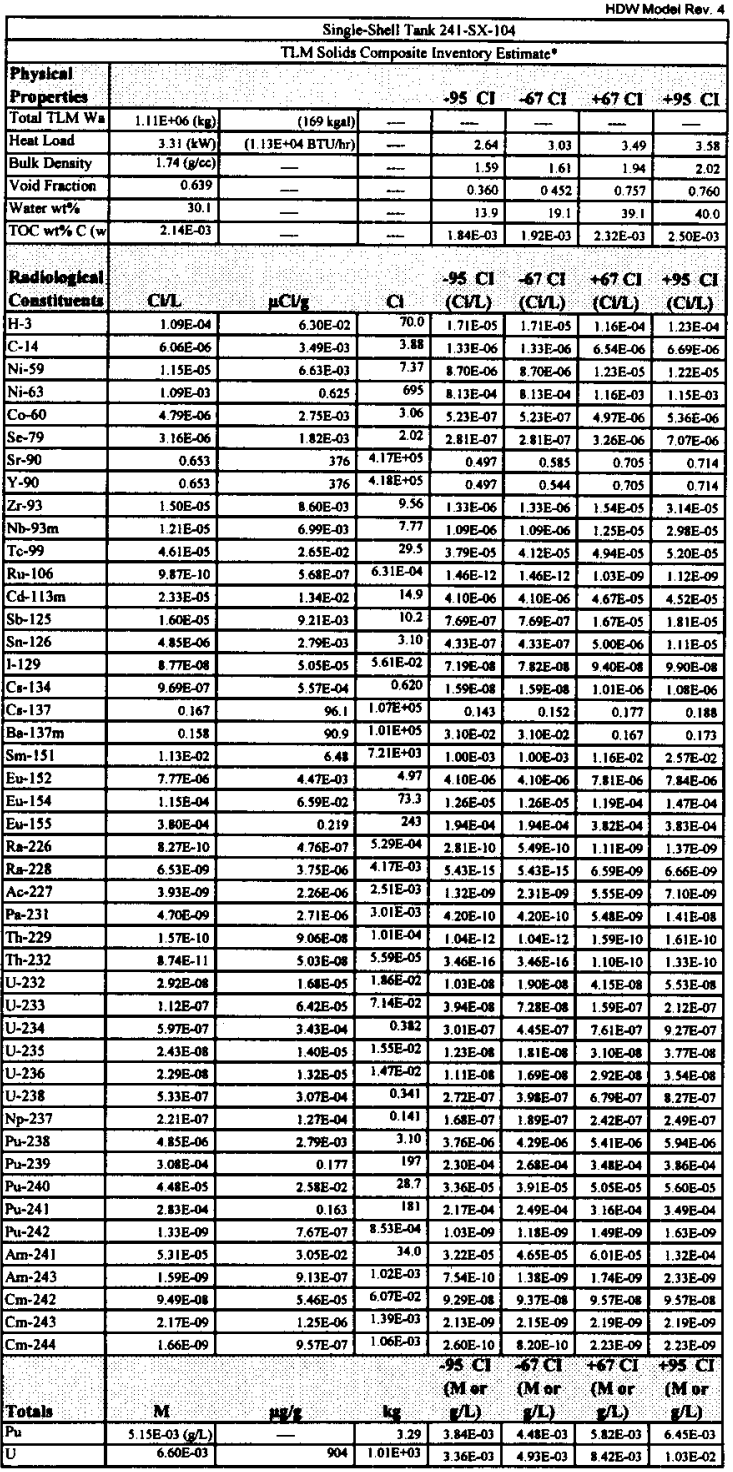

-Unknowns in tank molids inventory are axigned by Tank Layering Model (TLM). 
HNF-SD-WM-ER-352, Rev. 1

HOW Model Rev. 4

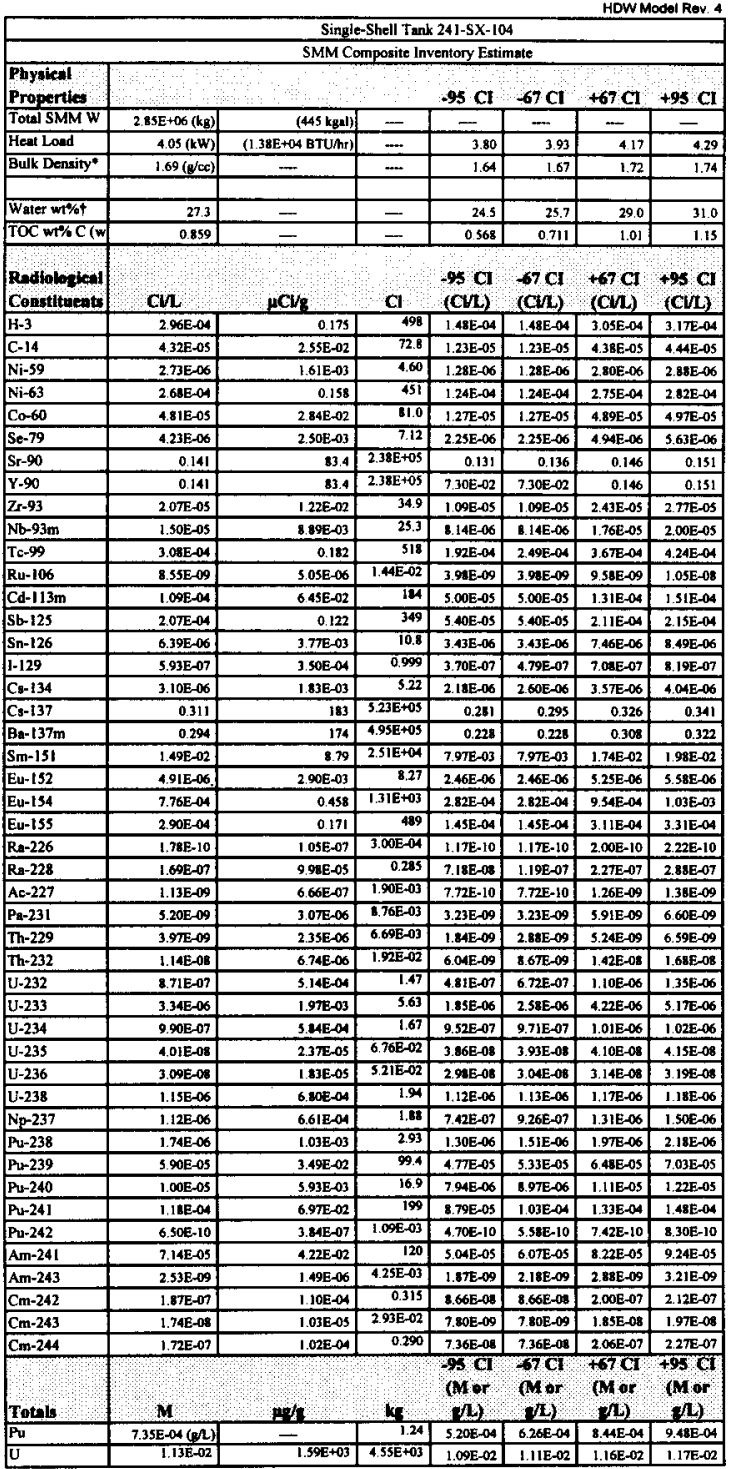

-Dengity is calculeted based on $\mathrm{Na}, \mathrm{OH}$-, and AlO2-

+Water w1\% derivod from the difference of density and totol disaolved apocies. 
HNF-SD-WM-ER-352, Rev. 1

\begin{tabular}{|c|c|c|c|c|c|c|c|}
\hline \multicolumn{8}{|c|}{ Single-Shell Tank 241-SX-104 } \\
\hline & \multicolumn{7}{|c|}{ Total Inventary Estimate* } \\
\hline Phyquical & & & & & & & \\
\hline Properties & & & & $95 \mathrm{CI}$ & $-67 C 1$ & $+67 \mathrm{CI}$ & $+95 \mathrm{CI}$ \\
\hline Total Waste & $3.96 \mathrm{E}+06(\mathrm{~kg})$ & $(614 \mathrm{kgal})$ & - & - & - & - & - \\
\hline Heat Load & $7.36(\mathrm{~kW})$ & $(2.52 \mathrm{E}+04 \mathrm{BTT} / \mathrm{hr})$ & $\ldots$ & 6.59 & 7.03 & 7.61 & 7.60 \\
\hline Bulk Density' & $1.71(y / c)$ & $\cdots$ & $\ldots$ & 1.64 & 1.66 & 1.77 & 1.78 \\
\hline & & & & & & & \\
\hline Water wt\%† & 28.1 & - & - & 23.6 & 24.4 & 30.9 & 32.0 \\
\hline $\mathrm{TOC} w \% \mathrm{C}(\mathrm{w}$ & 0.619 & - & - & 0.409 & 0.512 & 0.726 & 0.831 \\
\hline Constituentes & $\mathrm{CV}_{\mathrm{L}}$ & $\mu \mathrm{Cu} / v_{5}$ & $\mathrm{cl}$ & $\begin{array}{l}-95 \mathrm{Cl} \\
(\mathrm{Cu})\end{array}$ & $\begin{array}{l}67 \mathrm{CI} \\
(\mathrm{CnL})\end{array}$ & $\begin{array}{l}67 \mathrm{Cl} \\
(\mathrm{Cl} / \mathrm{L})\end{array}$ & $\begin{array}{l}+9 \mathrm{Cl}^{2} \\
\text { (CUL) }\end{array}$ \\
\hline $\mathrm{H}-3$ & $2.4 \mathrm{E}-04$ & 0.143 & 568 & $1.37 E-04$ & $1,37 \mathrm{E}-04$ & $2.51 \mathrm{E}-04$ & $2.60 \mathrm{E}-04$ \\
\hline C-14 & $3.30 \mathrm{E}-05$ & $1.93 \mathrm{E}-02$ & 76.7 & $1.06 \mathrm{E}-05$ & $1.06 \mathrm{E}-05$ & $3.34 \mathrm{E}-05$ & 3.38E-05 \\
\hline Ni-59 & 5.1SE-06 & 3.02E-03 & 12.0 & 4.10E-06 & 4. $10 \mathrm{E}-06$ & $3.37 \mathrm{E}-06$ & 5.31E-06 \\
\hline Ni-63 & $4.93 \mathrm{E}-04$ & 0.289 & $1.15 \mathrm{E}+03$ & 3.69E-04 & $3.99 \mathrm{E}-04$ & 3.14E-04 & 5.08E-04 \\
\hline $\mathrm{C}_{0}-60$ & $3.62 \mathrm{E}-05$ & $2.12 \mathrm{E}-02$ & 840 & 1.05E-05 & 1.05E-05 & $3.69 \mathrm{E}-05$ & $3.73 \mathrm{E}-05$ \\
\hline Se-79 & 3.93E-06 & $2.31 E-03$ & 9.14 & $2.50 \mathrm{E}-06$ & $2.50 E-06$ & $4.45 \mathrm{E}-06$ & 4.95E-06 \\
\hline Sr-90 & 0.282 & 165 & $6.55 E+05$ & 0.239 & 0.263 & 0.296 & 0.297 \\
\hline Y-90 & 0.212 & 165 & $6.55 \mathrm{E}+05$ & 0.233 & 0.233 & 0.296 & 0.297 \\
\hline$Z r-93$ & $1.91 \mathrm{E}-05$ & $1.12 \mathrm{E}-02$ & 4.5 & $1.20 \mathrm{E}-05$ & $1.20 \mathrm{E}-05$ & $2.17 \mathrm{E}-0 \mathrm{~s}$ & $242 \mathrm{E}-05$ \\
\hline $\mathrm{Nb}-93 \mathrm{~m}$ & 1.42E-05 & $835 \mathrm{E}-03$ & 33.1 & $9.24 \mathrm{E}-06$ & $9.24 \mathrm{E}-06$ & 1.61E-05 & 1.8SE-05 \\
\hline Tc-99 & $2.36 \mathrm{E}-04$ & 0.138 & 548 & 1.52E-04 & $1.91 \mathrm{E}-04$ & $2.79 \mathrm{E}-04$ & $3.20 \mathrm{E}-04$ \\
\hline Ru-106 & 6.47E-09 & 3.79E- -16 & $1.50 \mathrm{E}-02$ & 3.1SE-09 & 3.1 SE- 09 & 7.21E-09 & $7,89 \mathrm{E}-09$ \\
\hline $\mathrm{Cd}-113 \mathrm{~m}$ & $8.56 \mathrm{E}-05$ & 5.02E-02 & 199 & $4.27 \mathrm{E}-0 \mathrm{~S}$ & $4.27 \mathrm{E}-05$ & $1.01 \mathrm{E}-04$ & $1.16 E-04$ \\
\hline Sb-125 & $1.55 \mathrm{E}-04$ & $9.06 \mathrm{E}-02$ & 359 & 4,35E-05 & $4.35 \mathrm{E}-05$ & 1,57t-04 & $1.60 \mathrm{E}-04$ \\
\hline $5 n-126$ & $5.97 \mathrm{E}-06$ & $3,50 \mathrm{E}-03$ & 13.9 & $3.82 \mathrm{E}-06$ & 3.82E-06 & $6.74 E-06$ & 7.49E-06 \\
\hline 1.129 & 4.54E-07 & $2.66 \mathrm{E}-04$ & 1.06 & $2.92 E-07$ & $3.7 \mathrm{E}-07$ & $5.37 \mathrm{E}-07$ & 6. 18E-07 \\
\hline Ca-134 & $2.51 \mathrm{E}-06$ & $1.4 \pi \mathrm{E}-03$ & 3.84 & 1. SAE-06 & $1.89 E-06$ & 2.860 .06 & $3.19 \mathrm{E}-06$ \\
\hline$C_{s-137}$ & 0.271 & $\begin{array}{r}159 \\
\end{array}$ & $6.30 \mathrm{E}+05$ & 0.249 & 0.260 & 0.282 & 0.293 \\
\hline $\mathrm{B} s-137 \mathrm{~m}$ & 0.256 & 150 & $5.96 \mathrm{E}+05$ & 0.174 & 0.174 & 0.267 & $0.2 \pi 7$ \\
\hline Sm-151 & 1.39E-02 & 8.14 & $3.23 \mathrm{E}+04$ & $8.88 \mathrm{E}-03$ & B.88E-03 & $1.57 \mathrm{E}-02$ & $1.74 E-02$ \\
\hline Eu-152 & 5.70E-06 & $3.34 \mathrm{E}-03$ & i3.2 & $3.93 \mathrm{E}-06$ & $3.93 \mathrm{E}-06$ & 5.94E-06 & $6.18 \mathrm{E}-06$ \\
\hline$E_{u-154}$ & $5.94 \mathrm{E}-04$ & 0.348 & 1.3LE+01 & $2.36 E_{-04}$ & $2.36 \mathrm{E}-04$ & $7.23 E-04$ & $7.75 E-04$ \\
\hline Eu-155 & 3.15E-OA & 0.185 & 732 & $2.09 \mathrm{E}-04$ & $2.09 \mathrm{E}-04$ & $3.30 \mathrm{E}-04$ & $3.45 \mathrm{E}-04$ \\
\hline Ra-226 & $3.57 \mathrm{E}-10$ & $2.09 \mathrm{E}-07$ & $8.29 \mathrm{E}-04$ & $2.25 \mathrm{E}-10$ & $2.90 \mathrm{E}-10$ & $4.24 \mathrm{E}=10$ & 4.89E-10 \\
\hline $\operatorname{Ra}-228$ & 1.24E-07 & 7.29E-0S & 0.289 & $5.39 \mathrm{E}-08$ & $8.83 \mathrm{E}-0 \mathrm{~s}$ & $1,66 \mathrm{E}-07$ & $2.11 \mathrm{E}-07$ \\
\hline Ac-227 & 1.90E-09 & $1.11 \mathrm{E}-06$ & 4.4IE-03 & $1.2 \pi \mathrm{E}-09$ & $1.51 \mathrm{E}-09$ & $2.29 \mathrm{E}-09$ & $2.66 \mathrm{E}-09$ \\
\hline Pa-231 & $5.06 \mathrm{E}-09$ & $2.97 \mathrm{E}-06$ & I.18E-02 & $3.61 \mathrm{E}-09$ & $3.61 E-09$ & 5.58E-09 & $7.3 \mathrm{3E}-09$ \\
\hline Th-229 & 2.92E-09 & $1.71 E-06$ & $6.79 \mathrm{E}-03$ & $1388-09$ & $2.13 E-09$ & 3.84E-09 & 4,82E-09 \\
\hline Th-232 & 8. $30 \mathrm{E}-09$ & $4.8 \pi \mathrm{E}-06$ & $1.93 \mathrm{E}-02$ & $4,40 E-09$ & 6.31E-09 & $1.03 \mathrm{E}-08$ & $1.22 \mathrm{E}-08$ \\
\hline U-232 & 6.39E-07 & $3.75 E-04$ & 1.49 & $3.5 \pi \mathrm{E}-07$ & 4.93E,-07 & $8.07 \mathrm{E}-07$ & 9.85E-07 \\
\hline U.233 & $2.45 E-06$ & $144 E-03$ & 5.70 & $1.37 \mathrm{E}-06$ & $1.90 \mathrm{E}-00$ & $3.09 \mathrm{E}-06$ & $3.7 \mathrm{EE}-06$ \\
\hline U-234 & B.A1E-07 & S.1TEr.04 & 2.05 & S.01E-07 & $8.40 \mathrm{E}-07$ & $9.26 \mathrm{E}-07$ & $9.72 E-07$ \\
\hline U-235 & $3.58 E-08$ & $2.10 E-05$ & $8.32 \mathrm{E}-02$ & $3.25 \mathrm{E}-08$ & $3.41 E-08$ & $3.76 \mathrm{E}-08$ & $3.95 \mathrm{E}-08$ \\
\hline U-236 & $2.87 \mathrm{E}-0 \mathrm{~s}$ & $1.68 E-05$ & 6.66E-02 & $2.56 \mathrm{E}_{-08}$ & $2.71 \mathrm{E}-08$ & 3.04E-08 & $3.21 \mathrm{E}-08$ \\
\hline U-238 & $9.81 \mathrm{E}-07$ & $5.75 \mathrm{E}-04$ & 2.28 & $9.10 \mathrm{E}-07$ & $9.44 \mathrm{E}-07$ & $1.02 \mathrm{E}-06$ & $1.06 \mathrm{E}-06$ \\
\hline $\mathrm{Np}-237$ & 72E-07 & $5.11 E-04$ & 2.03 & 5.99E-07 & 7.32E-07 & $1.01 \mathrm{E}-06$ & $1.15 \mathrm{E}-06$ \\
\hline Pu-238 & $2.60 \mathrm{E}-106$ & $1.52 E-03$ & 6.03 & $2.28 \mathrm{E}-06$ & $2.43 \mathrm{E}-06$ & $2.76 E-06$ & $2.92 \mathrm{E}-06$ \\
\hline$P u-239$ & 1.2UE-04 & $7.48 E-02$ & 296 & $1.09 \mathrm{E}-04$ & $1.18 \mathrm{E}-04$ & $1.37 \mathrm{E}-\mathrm{AN}$ & $1.46 \mathrm{E}-04$ \\
\hline Pu-240 & $1.96 \mathrm{E}-0 \mathrm{~s}$ & $1.15 E-02$ & 45.6 & $1.69 t-05$ & $1.82 E-05$ & 2.10E-05 & $2.23 \mathrm{E}-05$ \\
\hline Pu-24I & $1.63 \mathrm{E}-04$ & $9.52 \mathrm{E}-02$ & 380 & $1.42 \mathrm{E}-04$ & $1.52 \mathrm{E}-04$ & $1.75 \mathrm{E}-04$ & $1.85 E-04$ \\
\hline Pu-242 & 8.38E-10 & 4.91E-07 & $1.95 \mathrm{E}-03$ & $7.07 \mathrm{E}-10$ & $7.71 \mathrm{E}-10$ & $9.04 \mathrm{E}-10$ & $9.69 \mathrm{E}-10$ \\
\hline$A m-241$ & 6.64E-05 & $3.89 \mathrm{E}-02$ & 134 & $5.12 \mathrm{E}-05$ & 5.86E-05 & $7.42 E-05$ & 8.54E-05 \\
\hline $\mathrm{Am}=243$ & $2.2 \pi-09$ & $1.33 \mathrm{E}-06$ & $5.27 \mathrm{E}-03$ & $1.79 \mathrm{E}-09$ & $2.02 E-09$ & $2.53 \mathrm{E}-09$ & $2.77 E-09$ \\
\hline $\mathrm{Cm}-242$ & $1.62 \mathrm{E}-07$ & $9.48 E-05$ & 0.376 & 8.59E-08 & 8. $.996-08$ & $1.71 \mathrm{E}-0 ?$ & $1.80 E-07$ \\
\hline $\mathrm{Cm}_{\mathrm{m}-243}$ & $1.32 E-08$ & 7.73E-06 & $3.07 \mathrm{E}-02$ & $6.25 \mathrm{E}-09$ & $6.25 \mathrm{E}-09$ & 2.40E-08 & 1,48E-09 \\
\hline $\mathrm{Cm}-244$ & $1.25 \mathrm{E}-07$ & $7.35 \mathrm{E}-05$ & 0291 & $3.38 \mathrm{E}-08$ & $5.38 \mathrm{E}-09$ & 1.50E-07 & $1,65 E-0 ?$ \\
\hline Totolls: & $\mathbf{M}$ & eivs & ks: & $\begin{array}{l}\text { ss } \\
\text { (Mar } \\
\text { r) }\end{array}$ & $\begin{array}{l}\text { SCI } \\
(\mathrm{Mor} \\
\mathrm{H})\end{array}$ & $\begin{array}{l}67 \mathrm{CI} \\
\text { (Mor } \\
\text { LL) }\end{array}$ & $\begin{array}{c}9 \mathrm{CC} \\
(\mathrm{Mar}\end{array}$ \\
\hline $\mathrm{Pu}$ & $1.95 E-03(\Omega / 2)$ & - & 4.53 & $1.64 \mathrm{E}-03$ & $1.79 \mathrm{E}-03$ & $2.11 \mathrm{E}-03$ & $2.26 E-03$ \\
\hline $\mathbf{U}$ & $1.00 \mathrm{E}-02$ & $1,40 E+03$ & $5.55 E+03$ & $9.15 \mathrm{SE}-03$ & $9.58 \mathrm{E}-03$ & $1.05 \mathrm{E}-02$ & I. 10E-02 \\
\hline
\end{tabular}




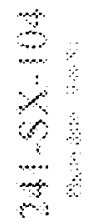




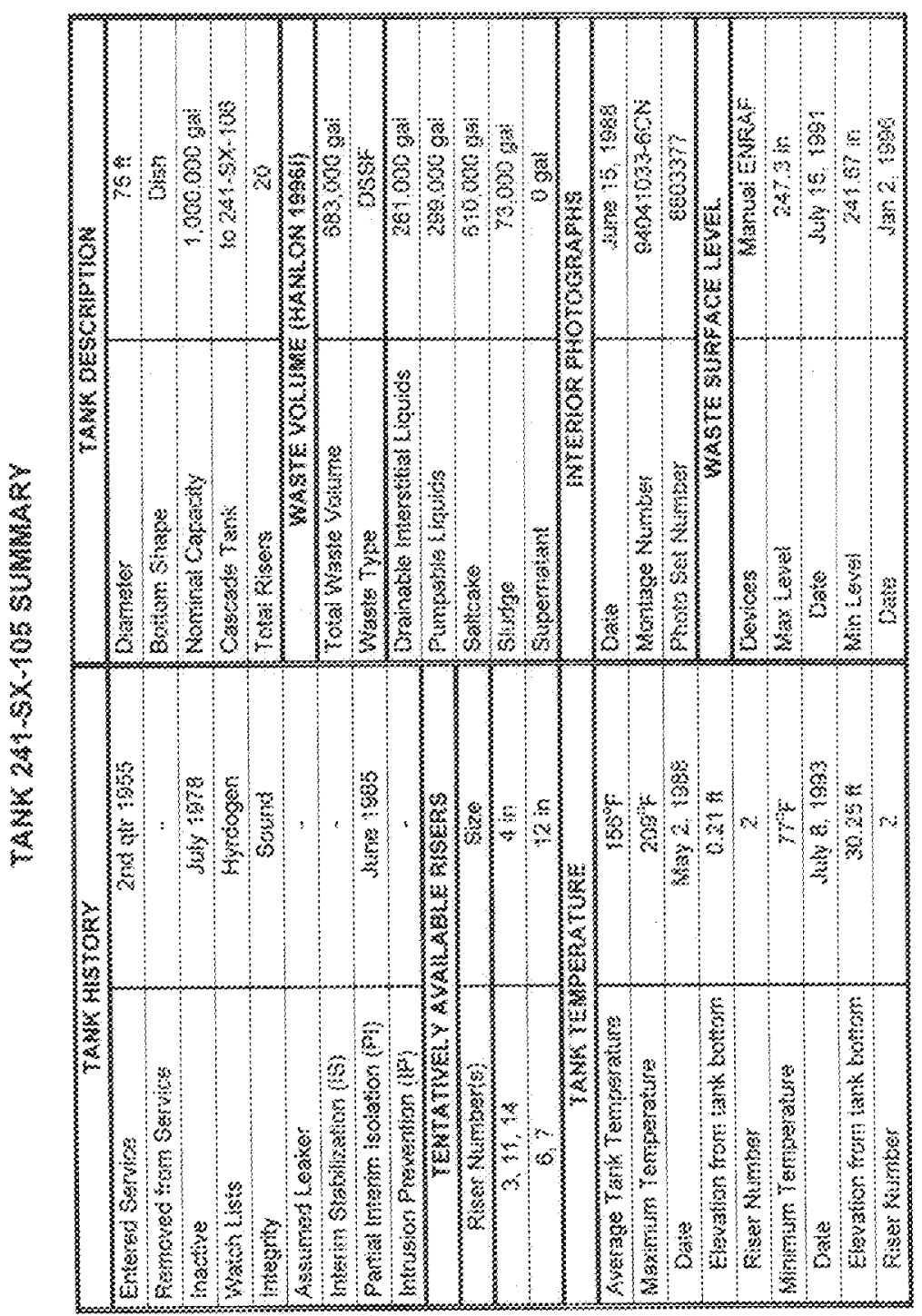




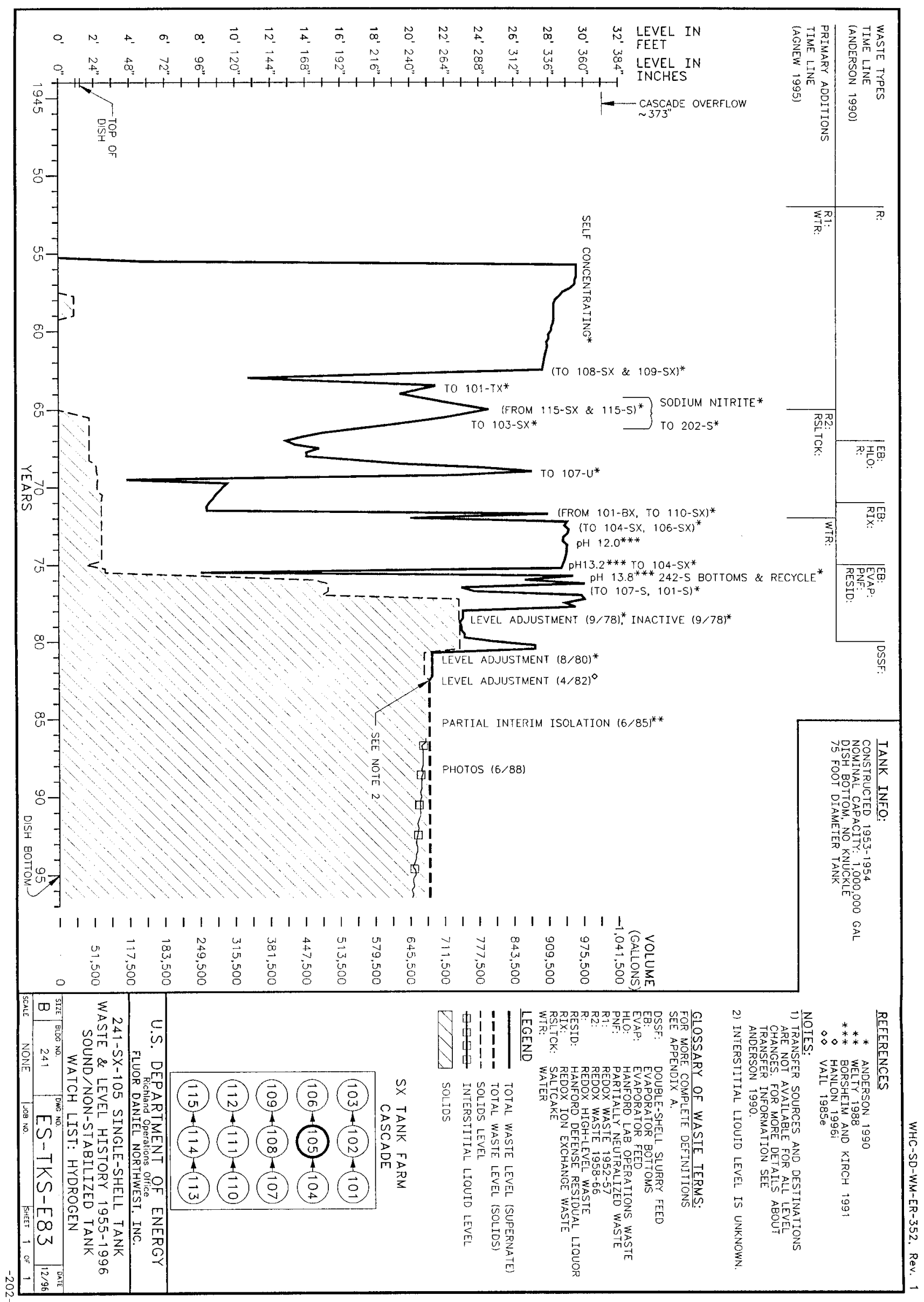



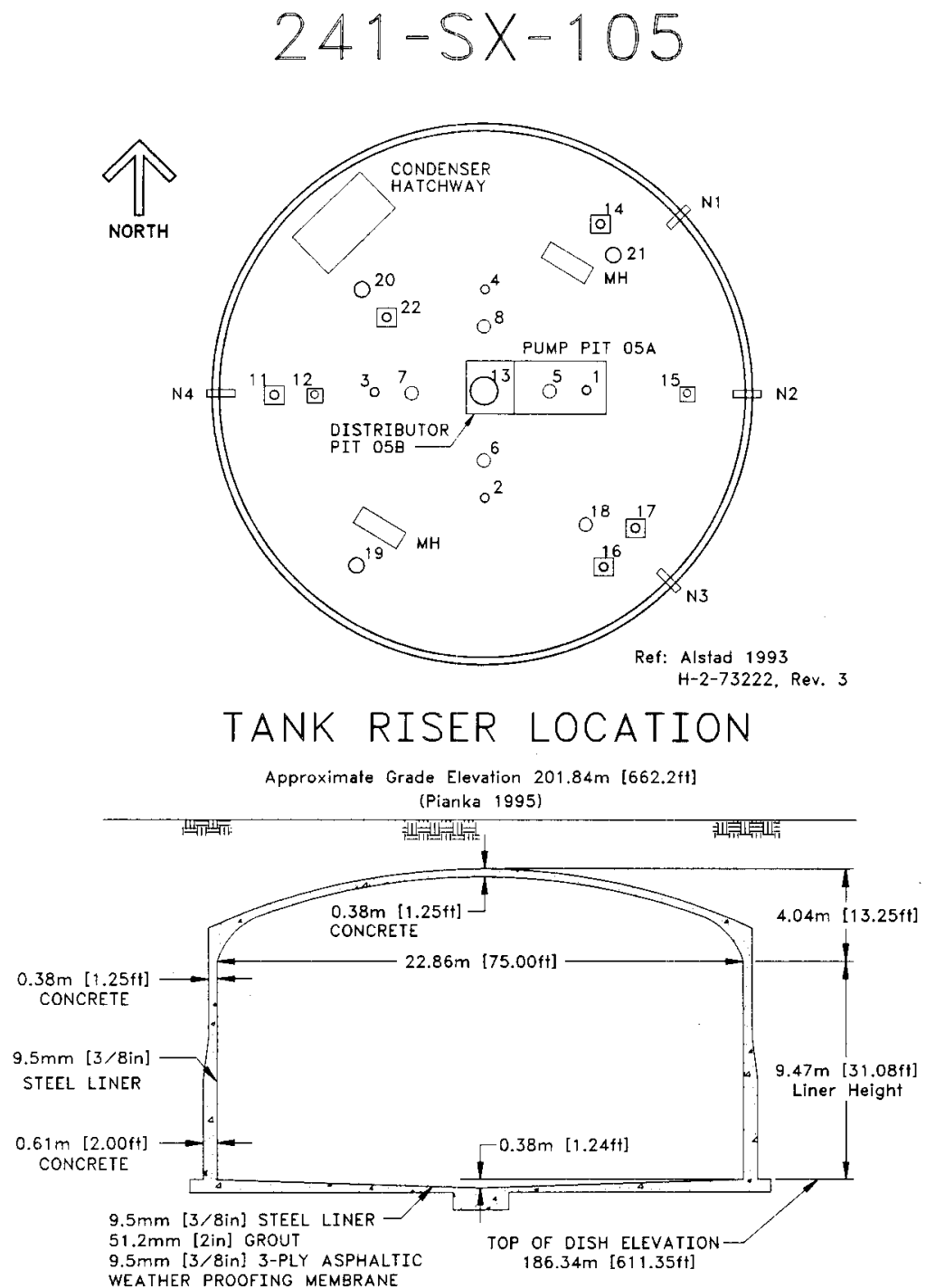

Ref: $\mathrm{H}-2-39511$. Rev. 3 H-2-46293. Rev. 3 NOT TO SCALE H-2-37855, Rev, 4 


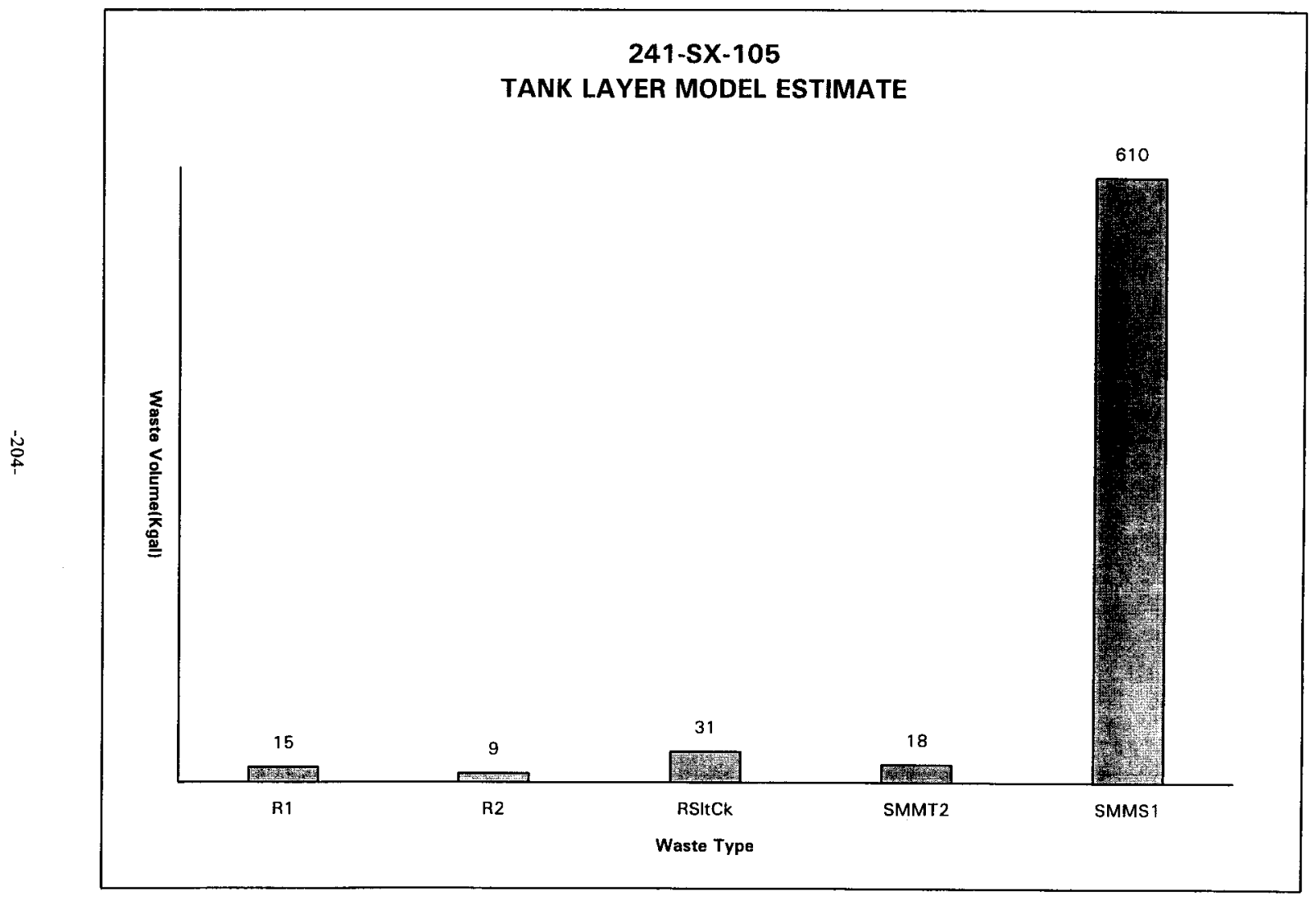

Tank Layer Model(TLM) Estimate from Hanford Tank Chemical and Radionuclide Inventories: HDW Model Rev. 4 (Agnew et al., 1997). 
HDW Model Rov. 4

\begin{tabular}{|c|c|c|c|c|c|c|c|}
\hline \multirow[b]{3}{*}{$\begin{array}{l}\text { Phyoles } \\
\text { Propertices }\end{array}$} & \multicolumn{7}{|c|}{ Single-Shell Tank 241-SX-105 } \\
\hline & \multicolumn{7}{|c|}{ TLM Solids Composite Inventory Estimatc* } \\
\hline & & & & $95 \mathrm{CI}$ & $-67 \mathrm{CI}$ & $+67 \mathrm{CI}$ & $+9 \mathrm{Cl}$ \\
\hline Total TLM Wa & $3.60 \mathrm{E}+05(\mathrm{~kg})$ & $(55.0 \mathrm{kgal})$ & $=$ & - & $=$ & $=$ & 7 \\
\hline Heat Losd & $3.66(\mathrm{~kW})$ & (1.25E+04 BTU/hr) & - & 3.56 & 3.62 & 3.69 & 3.72 \\
\hline Bulk Density & $1 . n(\alpha / \infty)$ & - & E & 1.57 & 1.66 & 1.83 & 1.86 \\
\hline Void Fraction & 0.685 & - & - & 0.555 & 0.598 & 0.739 & 0793 \\
\hline Water wt $\%$ & 32.1 & $=$ & $=$ & 23.7 & 26.5 & 37.9 & 43.6 \\
\hline TOC wt $\% C(w$ & 2.93E-03 & $\ldots$ & $=$ & $2.72 \mathrm{E}-03$ & 2.78E-03 & 3. $15 \mathrm{SE}-03$ & $3+2 \mathrm{E}-03$ \\
\hline Conolitisetw & molel & ppm & kr & (a) Cl & $\begin{array}{l}-67 \mathrm{CI} \\
\text { (noler) }\end{array}$ & $\begin{array}{l}+67 \mathrm{CI} \\
\text { (noleht }\end{array}$ & $\begin{array}{c}+9 \mathrm{C} \\
\text { (molefl })\end{array}$ \\
\hline $\mathrm{Na}+$ & 0,5 & $1.39 \mathrm{E}+05$ & $3,02 \mathrm{E}+04$ & 7.71 & 9.18 & 12.3 & 13.2 \\
\hline $\mathrm{Al}^{2+}$ & 5.18 & 1.08E+04 & $2.91 E+04$ & 4.08 & 4.40 & 6.02 & 6.88 \\
\hline $\mathrm{Fe} 3+($ Lotal Fe $)$ & 0725 & $2.3 \mathrm{AE}+04$ & $8.43 \mathrm{E}+03$ & 0.718 & 0.722 & 0.729 & D.733 \\
\hline $\mathrm{Cr} 3+$ & 0491 & $1.48 E+04$ & $5 . \overline{32 E}+03$ & 0.298 & 0.390 & 0.661 & 0.875 \\
\hline$\overline{B i 3+}$ & $3,87 \mathrm{E}-06$ & 0.468 & 0168 & $3.27 \mathrm{E}-06$ & 3.5TE-06 & $4.27 \mathrm{E}-06$ & 1.69E-06 \\
\hline $1+3+$ & 954E-12 & $7.66 \mathrm{E}-07$ & $2.76 E-07$ & $8.36 \mathrm{E}-12$ & $9.02 \mathrm{E}-12$ & $105 \mathrm{E} \cdot 11$ & 1.16E-11 \\
\hline He2+ & $6.08 \mathrm{E}-07$ & 7.05E-02 & $2.54 \mathrm{E}-02$ & 5. $39 \mathrm{E}-07$ & $5.73 E-07$ & 6.70E-07 & $7.35 \mathrm{E}-07$ \\
\hline $\operatorname{Zr}(\mathrm{MrO} / \mathrm{OH})^{2}$ & $3.8 \mathrm{TE}-07$ & 2.04E.02 & $7,34 \mathrm{E}-03$ & $3.55 \mathrm{E}-07$ & 3.70E-07 & 4.05E- -7 & $437 \mathrm{E}-07$ \\
\hline $\mathrm{Pb}_{2+}$ & 9.65E-05 & 11.6 & 4.16 & $5.17 \mathrm{E}-05$ & $7,35 \mathrm{E}-05$ & $1.30 \mathrm{E}-04$ & $1.42 E-04$ \\
\hline $\mathrm{Ni2+}$ & 4. 16E-02 & $1.41 \mathrm{E}+03$ & 508 & 3.77E-02 & $3,99 \mathrm{E}-02$ & 4.29E-02 & $4,31 \mathrm{E}-02$ \\
\hline $\mathrm{Sn} 2+$ & -0 & 0 & 0 & 0 & - 0 & a & 0 \\
\hline Mnt+ & $2.82 E-05$ & 0.695 & 0,322 & $2.01 E-05$ & $241 E-05$ & $3.23 \mathrm{E}-05$ & $3.64 \mathrm{E}-05$ \\
\hline $\mathrm{Cot}+$ & 0.160 & $432 E+03$ & $1.35 E+03$ & 0.157 & 0.171 & 0.201 & 0.216 \\
\hline$x+$ & $2.30 \mathrm{E}-02$ & 521 & 187 & $2.03 \mathrm{E}-02$ & $2.14 \mathrm{E}-02$ & 246E-02 & 2.64E-02 \\
\hline OH- & 23.2 & $2.28 E+05$ & $8.22 \mathrm{E}+04$ & 18.2 & 19.8 & 26.9 & 30.4 \\
\hline NO3- & 440 & $158 \mathrm{E}+03$ & $5.68 \mathrm{E}+04$ & 2.97 & 2.98 & 6.63 & 7.84 \\
\hline NO2- & 1.71 & $4.55 \mathrm{E}+04$ & $1.64 E+04$ & 1.35 & 1.48 & 1.90 & 1.90 \\
\hline Coj2- & 0.189 & $6.56 \mathrm{E}+03$ & $236 \mathrm{E}+03$ & 0.160 & 0.174 & 0,204 & 0.219 \\
\hline PO43- & 2.5IE-0A & 13.8 & 1.93 & $2.33 \mathrm{E}-04$ & $2.43 E-04$ & $261 \mathrm{E}-04$ & 2.84E-04 \\
\hline $5042-$ & $2.85 E-02$ & $1.52 E+02$ & 570 & $2,60 E-02$ & $2.67 \mathrm{E}-02$ & $3.05 E-02$ & $3.27 E-02$ \\
\hline $\mathrm{Si}\left(\mathrm{s} \mathrm{SiCr}_{2}\right.$ ) & 0.153 & $2.49 \mathrm{E}+03$ & 897 & 7.98E-02 & 8.13E-02 & 0.226 & 0.295 \\
\hline F- & $2000-04$ & $2 . \overline{19}$ & 0.790 & $1.70 \mathrm{E}-04$ & 1.88E-04 & $2.20 \mathrm{E}-0 \mathrm{~A}$ & $2.37 \mathrm{E}-04$ \\
\hline $\mathrm{Cl}-$ & $9.82 \mathrm{E}-02$ & $2.01 E+03$ & 724 & $6.34 \mathrm{E}-02$ & 3.04E-02 & 0.106 & 0.127 \\
\hline C615073- & $2.06 \mathrm{E}-04$ & 22.6 & 8.12 & $200 \mathrm{E}-04$ & $203 \mathrm{E}-04$ & 2.15E-04 & $234 \mathrm{E}-04$ \\
\hline EDTA4- & 64a-06 & $\mathbf{1 . 3 4}$ & 0.482 & $5.65 \mathrm{E}-06$ & $6.96 \mathrm{E}-06$ & $947 \mathrm{E}-06$ & $1.09 \mathrm{E}-05$ \\
\hline HEDTA3- & 6.68E-06 & 1.06 & 0.381 & $2.14 \mathrm{E}-06$ & 4.59E-06 & 8.91E-06 & 1.11E-05 \\
\hline glyoolate- & 2.91E-04 & 12.6 & 4.55 & 1.50E-04 & $2.30 \mathrm{E}, 04$ & $3.62 \mathrm{E}-04$ & $4.30 \mathrm{E}-04$ \\
\hline acetute- & 302200 & 1.03 & 037 & 2.93E-05 & $297 \mathrm{E}-05$ & $3.21 \mathrm{E}-05$ & 3.43E-05 \\
\hline Oxalinte:2- & $1.25 \mathrm{E}-11$ & $6.36 E-07$ & $2.29 \mathrm{E}-07$ & 1.11E-11 & $1.18 \mathrm{E}-11$ & 1.38E-11 & $1.51 \mathrm{E}-11$ \\
\hline$\overline{D B P}$ & $1.83 \mathrm{E}-04$ & 22.2 & 8.01 & $1.71 \mathrm{E}-04$ & 1.78E-04 & $1.98 \mathrm{E}-04$ & $2.14 \mathrm{E}-04$ \\
\hline butanol & TISE-04 & 784 & 2.82 & 1.71E-04 & $1.78 \mathrm{E}-04$ & $1.99 \mathrm{E}-04$ & 2.14E-0A \\
\hline Nस3 & 0.160 & $1.64 E+03$ & 589 & 0.115 & 0.122 & 0.289 & 0.174 \\
\hline$F(C N) 64$ & 임 & 0 & 0 & 0 & 0 & 0 & 0 \\
\hline
\end{tabular}

"Unknowns in tunk solids inventory we tutigned by Tank Layeriag Model (TLM). 


\begin{tabular}{|c|c|c|c|c|c|c|c|}
\hline \multirow[b]{3}{*}{$\begin{array}{l}\text { Plypileal } \\
\text { Properties }\end{array}$} & \multicolumn{7}{|c|}{ Single-Shell Tark 241-SX-105 } \\
\hline & \multicolumn{7}{|c|}{ SMM Composite Inventory Estimate } \\
\hline & & & & & & & \\
\hline Total SMM W & $406 \mathrm{E}+06(\mathrm{~kg})$ & $6028 \mathrm{k}=1 \mathrm{C}$ & 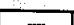 & $95 \mathrm{CI}:$ & $-67 \mathrm{CI}$ & $+67 \mathrm{Cl}$ & $+95 \mathrm{CI}$ \\
\hline Heat Lood & $5.83(\mathrm{~kW})$ & 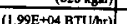 & - & $=$ & - & $=$ & $=$ \\
\hline Bulk Density" & $1.71(\mathbb{1} / \infty)$ & $(1.99 \mathrm{E}+04 \mathrm{BT}(\mathrm{h} / \mathrm{h})$ & & 5.45 & 3.65 & 6.00 & 6.16 \\
\hline & & $=$ & & 1.65 & 1.68 & 1.73 & 1.76 \\
\hline Watet w1\% & 26.5 & & & & & & \\
\hline TOCWt\% C (w & 0.887 & $=$ & $=$ & 23.5 & 24.9 & 28.3 & 30.3 \\
\hline & & & $=$ & 0590 & 0.736 & 1.04 & 1.19 \\
\hline Chemiral & moleh $\mathrm{L}$ & ppm & n: & 95 C1 & $67 \mathrm{CI}$ & $\begin{array}{c}+67 \mathrm{Cl} \\
(\text { mololet) }\end{array}$ & $\begin{array}{r}+9 \mathrm{Cl} \\
\text { (molet })\end{array}$ \\
\hline \begin{tabular}{l|l}
$\mathrm{Na}+$ \\
+
\end{tabular} & 16.6 & $2.23 \mathrm{E}+05$ & $9.07 \mathrm{E}+05$ & 15,0 & 15.8 & 17.3 & 17.9 \\
\hline $\mathrm{Al} 3+$ & 1.95 & $308 E+04$ & $1.25 \mathrm{E}+0 \mathrm{~S}$ & 1.81 & 1.88 & 2.03 & 2.09 \\
\hline $\mathrm{Fe} 3+($ otal $\mathrm{FE})$ & I.31E-02 & 427 & $1.73 E+03$ & 1.05E-02 & $1.18 \mathrm{E}-02$ & 14E-02 & 1.56E-02 \\
\hline $\mathrm{Cr} 3+$ & 0.173 & $5.26 E+03$ & $2.14 E+04$ & 0.146 & 0.161 & 0.179 & 0.184 \\
\hline Bijt & 1.43E-03 & 176 & 712 & $1.31 E-03$ & $1.37 \mathrm{TE}-03$ & 1. $50 \mathrm{E}-03$ & 1.55E-03 \\
\hline Lo3+ & $5.3] \mathrm{E}-65$ & 4.32 & 17.5 & 3.85E-05 & 4.56E-03 & 606E-05 & 6.78E-05 \\
\hline $\mathrm{Hg} 2+$ & 1.03E-05 & 1.21 & 4.90 & $9.63 \mathrm{E}-06$ & $1.00 \mathrm{E}-05$ & 1.04E-05 & $1.06 \mathrm{E}-05$ \\
\hline $\mathrm{Zr}(\mathrm{OU} 2 \mathrm{rO}(\mathrm{OH}) 2$ & $2.57 \mathrm{E}-04$ & 13.t & 55.8 & 2.35E-04 & $2,43 \mathrm{E}-04$ & $2.66 \mathrm{E}-04$ & $2.79 \mathrm{E}-04$ \\
\hline $\mathrm{Ph} 2+$ & $1.17 \mathrm{E}-03$ & 142 & 376 & 9.46E-04 & $106 \mathrm{E}-03$ & $1.28 \mathrm{E}-03$ & $1,39 \mathrm{E}-03$ \\
\hline Ni2+ & 7.54E-03 & 259 & $1.05 \bar{E}+03$ & $7.20 \mathrm{E}-03$ & $7.36 \mathrm{E}-03$ & $7.63 \mathrm{E}-03$ & $7.72 \mathrm{E}-03$ \\
\hline Sr2+ & 0 & 0 & 0 & 0 & o & 0 & 0 \\
\hline Mint+ & $469 \mathrm{E}-03$ & 151) & 611 & $3.45 \mathrm{E}-03$ & $4.05 \mathrm{E}-03$ & $5,30 E-03$ & $5.91 \mathrm{E}-03$ \\
\hline C.2+ & $3.95 \mathrm{E}-02$ & 928 & $3.76 \mathrm{E}+03$ & 3.58E-02 & $3.76 \mathrm{E}-02$ & $4.14 \mathrm{E}-02$ & $432 \mathrm{E}-02$ \\
\hline$\overline{\mathbf{K}+}$ & 7.69E-02 & $1.76 \mathrm{E}+0 \mathrm{0}$ & $7,15 \mathrm{E}+03$ & 6.99E-02 & $7.29 \mathrm{E}-02$ & B. $10 \mathrm{E}-02$ & 3.49E-02 \\
\hline OH- & iI.8 & T.18E+0s & $4.7 \%+05$ & 11,0 & 11.4 & 12.3 & 126 \\
\hline NO3. & 5.68 & $2.07 \mathrm{E}+05$ & $8.38 \mathrm{E}+05$ & 5.29 & 5.52 & 5.77 & 5.85 \\
\hline NO2- & 2.89 & $7.79 E+04$ & $3.16 \mathrm{E}+05$ & 2.37 & 2.61 & 3.16 & 3.42 \\
\hline $\cos 2$. & 0.520 & $1.83 E+04$ & $7.42 \mathrm{E}+04$ & 0.468 & 0.494 & 0.550 & 0.565 \\
\hline PO43- & 0.104 & $5.81 E+03$ & $2.36 \mathrm{E}+04$ & $9.13 \mathrm{E}-02$ & $9.59 \mathrm{E}-02$ & 0.107 & 0.109 \\
\hline SO42- & 0.288 & $1.62 E+04$ & $6.58 \mathrm{E}+04$ & 0.220 & 0.253 & 0.325 & 0.357 \\
\hline $\mathrm{Si}(\mathrm{n} \mathrm{SiO} 32-)$ & $9.23 \mathrm{E}-02$ & $1.52 \mathrm{E}+03$ & 6.18E+03 & $7.49 \mathrm{E}-02$ & 8.35E-02 & 0.101 & 0.110 \\
\hline F. & $3.042,02$ & 784 & $3.18 E+03$ & $5.99 \mathrm{E}-02$ & $6.43 \mathrm{E}-02$ & $7.56 E-02$ & $7.97 \mathrm{E}-02$ \\
\hline Cl- & 0.285 & $5.922+03$ & $2.40 E+04$ & 0.255 & 0.270 & 0.299 & 0.311 \\
\hline C6 6 HO 73. & $3.39 \mathrm{E}-02$ & $3.97 \mathrm{E}+03$ & $1.61 \mathrm{E}+04$ & $3.29 \mathrm{E}-02$ & $3.4 \mathrm{E}-02$ & 3.74E- 02 & $3.88 E-02$ \\
\hline EDTA4 & 1.84E-02 & $3.11 E+03$ & $1.26 \mathrm{E}+04$ & $5.83 \mathrm{E}-03$ & $1,20 \mathrm{E}-02$ & $2.49 \mathrm{E}-02$ & 3.12E-02 \\
\hline HEDTA3. & $3,40 \mathrm{E}-02$ & $5.46 \mathrm{E}+03$ & $2.21 \mathrm{E}+0 \mathrm{~A}$ & $8.80 \mathrm{E}-03$ & $2.11 E-02$ & 4.69:-02 & $5.96 E-02$ \\
\hline & & & & & & & \\
\hline glycolate & 0.111 & $4.89 \mathrm{E}+03$ & $198 \mathrm{E}+04$ & $7.22 \mathrm{E}-02$ & $9.12 E-02$ & 0.131 & 0.150 \\
\hline scetnle- & $9.18 E-03$ & 317 & $1.29 \mathrm{~g}+03$ & $7.50 \mathrm{E}-03$ & 8.32E-03 & $1.00 E_{-02}$ & $1.09 \mathrm{E}-02$ \\
\hline oxtilate2. & 6.96E-05 & 3.59 & 146 & 6.16E-05 & 6.55E-05 & $7.36 \mathrm{E}-05$ & $7.75 E-05$ \\
\hline DBP & $2.33 E-D_{2}$ & $2.90 \mathrm{E}+0 \mathrm{O}$ & 1.1BE+04 & $1.92 E-02$ & $2.13 \mathrm{E}-02$ & $2.57 \mathrm{E}-02$ & $2.77 \mathrm{E}-02$ \\
\hline butunel & $2,3 \mathrm{SE}-\Omega 2$ & 1.02E+03 & C14E+03 & $192 \mathrm{E}-02$ & 2.13E-02 & $2.57 \mathrm{E}-02$ & $2.77 E-02$ \\
\hline & & & & & & & \\
\hline $\mathrm{NH3}$ & $9.69 \mathrm{E}-02$ & 965 & $3.91 \mathrm{E}+03$ & $7.71 \mathrm{E}-02$ & 8.49E-02 & 0.112 & 0.130 \\
\hline $\mathrm{F} \alpha(\mathrm{CN}) \mathrm{G4}$ & 0 & 0 & 0 & 0 & 0 & 0 & 0 \\
\hline
\end{tabular}

"Density in calculated based on Na, OH-, and AlO2.

tWater wt\% derived from the difference of density and totul diasolved species. 
HDW Model Rer 4

\begin{tabular}{|c|c|c|c|c|c|c|c|}
\hline \multicolumn{8}{|c|}{ Single-Shell Tank 24I-SX-105 } \\
\hline & \multicolumn{7}{|c|}{ Total Inventory Estimate" } \\
\hline Physical & & & & & & & \\
\hline Propertie & & & & $95 \mathrm{CI}$ & $-67 \mathrm{CI}$ & $+67 \mathrm{Cl}$ & $+9 \mathrm{sc}$ \\
\hline Total Waste & $4.42 \mathrm{E}+06(\mathrm{~kg})$ & $(683 \mathrm{kgal})$ & - & 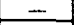 & $\ldots$ & 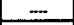 & $=$ \\
\hline Hest Load & $9.48(\mathrm{~kW})$ & $(3.24 E+04 \mathrm{BTU} / \mathrm{hc})$ & - & 9.11 & 9.31 & 9.66 & 9.82 \\
\hline Bulk Densityt & $1.71(\mathbf{g} / \mathbf{c})$ & $=$ & $\cdots$ & 1.05 & 1.68 & 1.73 & 1.75 \\
\hline Wht & 270 & & & & & & \\
\hline Walt witol & 2.0 & $=$ & $\cdots$ & 24.2 & 25.5 & 28.6 & 30.5 \\
\hline TOC wt\% C (w) & 0.815 & $=$ & 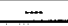 & 0.542 & 0.676 & 0.955 & 1.09 \\
\hline Chenpiont: & & & & $-9 \mathrm{CI}$ & $67 \mathrm{CT}$ & $+67 \mathrm{Cl}$ & +950 \\
\hline Constituent: & nooled & $\mathrm{pmin}$ & ki & (mole/L) & (molet) & (molea) & (molel ) \\
\hline $\mathrm{Na}+$ & 16.1 & 2.17E+05 & $9.57 \mathrm{E}+05$ & 14.7 & 15.4 & 16.7 & 17.3 \\
\hline $\mathrm{Al3+}$ & 2.21 & $3.49 \mathrm{E}+04$ & $1.54 E+05$ & 2.08 & 2.14 & 2.28 & 2.35 \\
\hline 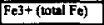 & $7.04 \mathrm{E}-02$ & $2.30 \mathrm{E}+03$ & $1.02 \mathrm{E}+04$ & $6 \mathrm{B1E}-1) 2$ & $6.92 \mathrm{E}-02$ & $7.16 \mathrm{E}-02$ & $7.2 \mathrm{BE}-02$ \\
\hline $\mathrm{Cr}^{3+}$ & $\begin{array}{r}0.199 \\
\end{array}$ & $6.06 \mathrm{E}+03$ & $2.67 \mathrm{E}+04$ & 0.176 & 0.189 & 0.211 & 0.226 \\
\hline $\mathrm{Bi3+}$ & $1,32 \mathrm{E}-03$ & 161 & 712 & 1.21E-03 & $126 \mathrm{E}-03$ & $1.38 \mathrm{E}-03$ & $143 \mathrm{E}-03$ \\
\hline Le3t & $4.88 \mathrm{E}-0 \mathrm{~S}$ & 3.97 & 17.5 & $3.54 \mathrm{E}-05$ & $4.19 \mathrm{E}-05$ & $5.57 \mathrm{E}-0 \mathrm{~S}$ & $6.24 \mathrm{E}-05$ \\
\hline $\mathrm{Hg} 2+$ & 9.49E-06 & 1.11 & 4.92 & $8.91 E-06$ & $9.24 \mathrm{E}-96$ & $9.64 \mathrm{E}-06$ & $9,7 \mathrm{BE}-06$ \\
\hline $\mathrm{Zr}(\mathrm{arO}(\mathrm{OH}) 2$ & 2.37E-04 & 12.6 & 55.8 & $2.16 \mathrm{E}-04$ & $2.23 \mathrm{E}-04$ & 2.45E-04 & $2.57 \mathrm{E}-04$ \\
\hline Po2t+ & $1.08 E-03$ & 131 & 580 & 8.77E-04 & $9.78 \mathrm{E}-04$ & $1.19 \mathrm{E}-03$ & $1.29 \mathrm{E}-03$ \\
\hline $\mathrm{Ni2+}$ & $1.03 \mathrm{E}-02$ & 353 & $1.56 \mathrm{E}+03$ & 9.97E-03 & 1.0tE-02 & $1.04 \mathrm{E}-02$ & 1.04E-02 \\
\hline $\mathbf{S r 2 +}$ & 0 & 0 & 0 & 0 & 0 & 0 & 0 \\
\hline MnAt & 4,30E-03 & 138 & 611 & $3,17 \mathrm{E}-03$ & $3.73 \mathrm{E}-03$ & 4.88E- 03 & 5.43E-03 \\
\hline $\mathrm{Co} 2+$ & $5.13 \mathrm{E}-02$ & $1.20 \mathrm{E}+03$ & $5.32 \mathrm{E}+03$ & $4.80 E-02$ & $4,96 \mathrm{E}-02$ & $5.31 \mathrm{E}-02$ & $5.4 \pi \mathrm{E}-02$ \\
\hline $\mathrm{K}+$ & $7.26 \mathrm{E}-02$ & $1.66 E+03$ & $7.33 E+03$ & $6.56 \mathrm{E}-02$ & $6.89 \mathrm{E}-02$ & $7.63 \mathrm{E}-02$ & $7.99 \mathrm{E}-12$ \\
\hline OH- & 12.7 & $1.27 E+05$ & $5.60 E+05$ & 12.0 & 12.3 & 13.1 & 13.5 \\
\hline No3. & 5.58 & $2.03 \mathrm{E}+05$ & 8.95E+05 & 5.22 & 5.43 & 5.72 & 5.73 \\
\hline NO2- & 2.80 & $7.53 \mathrm{E}+04$ & $3.33 \mathrm{E}+05$ & 2.31 & 2.54 & 3.05 & 3.29 \\
\hline $\cos 2$. & 0.49 & $1.73 E+04$ & $7.66 \mathrm{E}+04$ & 0.446 & 0.469 & 0.521 & 0.534 \\
\hline P043. & $9,60 \mathrm{E}-02$ & $5.34 \mathrm{E}+03$ & $2.36 \mathrm{E}+04$ & $8.40 \mathrm{E}-02$ & 8.82E-02 & 9.81E-02 & 0.100 \\
\hline SO42: & 0.267 & $1.50 \mathrm{E}+04$ & $6.64 E+04$ & 0.205 & 0.235 & 0.301 & 0.330 \\
\hline Si (ns SiO32-) & 9.74E-02 & $1.60 E+03$ & $7.07 E+03$ & 8.13E-02 & $8.92 E-02$ & 0.106 & 0.114 \\
\hline$F$. & $6,47 E-02$ & 720 & $3.18 E+03$ & $5.50 \mathrm{E}-02$ & 5.91E-02 & $6.95 E-02$ & $7.33 \mathrm{E}-02$ \\
\hline Cl- & 0.270 & $5.60 \mathrm{E}+03$ & $2.47 \mathrm{E}+04$ & 0.242 & 0.256 & 0.283 & 0.294 \\
\hline C6HSO73. & $3.30 \mathrm{E}-02$ & $3.65 \mathrm{E}+03$ & $1.61 \mathrm{E}+04$ & $3.03 \mathrm{E}-02$ & 3. $16 \mathrm{E}-02$ & 3.44E- 02 & $3.57 \mathrm{E}-02$ \\
\hline EDTA4 & $169 \mathrm{E}-02$ & 2.86E+03 & $1.26 \mathrm{E}+04$ & $5.36 \mathrm{E}-03$ & $1.10 \mathrm{E}-02$ & $2.29 \mathrm{E}-02$ & $2.87 \mathrm{E}-02$ \\
\hline HEDTA3- & $3.12 \mathrm{E}-02$ & $5.01 \mathrm{E}+03$ & $2.21 \mathrm{E}+04$ & $8.09 \mathrm{E}-03$ & 1.94E-02 & $4.31 \mathrm{E}-02$ & 5,4:BE-02 \\
\hline glycolate & 0.102 & $4.49 \mathrm{E}+03$ & $1.98 E+04$ & 6.64E-02 & 8. $39 \mathrm{E}-012$ & 0.121 & 0.138 \\
\hline acetale- & B.44E-03 & 292 & $1.29 \mathrm{E}+03$ & $6.90 \mathrm{E}-03$ & $7.66 \mathrm{E}-0.03$ & $9.23 \mathrm{E}-03$ & $9.98 \mathrm{E}-03$ \\
\hline oxulate?- & $6.40 \mathrm{E}-0 \mathrm{5}$ & 520 & 14.6 & $5.6 \%-05$ & 6.02E-05 & $677 \mathrm{E}-05$ & $7.12 \mathrm{E}-0 \mathrm{~s}$ \\
\hline DBP & $2.16 \mathrm{E}-02$ & $2.66 E+03$ & $1.18 E+04$ & $1.77 \mathrm{E}-02$ & $1.96 \mathrm{E}-02$ & $2.36 \mathrm{E}-02$ & $2.55 \mathrm{E}-02$ \\
\hline butumol & $2.16 \mathrm{E}-02$ & 939 & $4.15 \mathrm{E}+03$ & $1.77 \mathrm{E}-02$ & $1.96 \mathrm{E}-02$ & $236 \mathrm{E}-02$ & $255 \mathrm{E}-02$ \\
\hline NH3 & 0.102 & $1.02 \mathrm{E}+03$ & $4.50 \mathrm{E}+03$ & $8.43 \mathrm{E}-02$ & $9.15 \mathrm{E}-02$ & 0.117 & 0.133 \\
\hline Fe(CN)64: & 0 & 0 & 0 & 0 & 0 & 0 & 0 \\
\hline
\end{tabular}

"Unknowne in tark solide inventory are assigned by Tank Leyering Model (TLM)

+Water wt\% derived from the difference of dencity and total disaolved epecies. 
HDW Model Rew. 4

\begin{tabular}{|c|c|c|c|c|c|c|c|}
\hline \multirow[b]{3}{*}{$\begin{array}{l}\text { Phyticl } \\
\text { Propertics }\end{array}$} & \multicolumn{7}{|c|}{ Single-Shell Tank 241-SX-105 } \\
\hline & \multicolumn{7}{|c|}{ TLM Solids Composite Inventory Estimate* } \\
\hline & & & & $295 \mathrm{CI}$ & $-67 \mathrm{CI}$ & $+67 \mathrm{Cl}$ & $-95 \mathrm{Cl}$ \\
\hline Total TLM Wa & $3.60 \mathrm{E}+05(\mathrm{~kg})$ & [55.0 kgal] & 二 & $=$ & - & - & - \\
\hline Heat Losd & $3.66(\mathrm{~kW})$ & $(1.25 \mathrm{E}+04 \mathrm{BTU} / \mathrm{hr})$ & - & 3.56 & 3.62 & 3.69 & 3.72 \\
\hline Bulk Density & $1.73(\sigma / \infty)$ & - & - & 1.57 & 1.66 & 1.83 & 1.86 \\
\hline Void Fraction & 0.685 & - & $=$ & 0.555 & 0.598 & 0.739 & 0.793 \\
\hline Water wt\% & 32.1 & - & - & 23.7 & 26.5 & 37.9 & 43.6 \\
\hline TOC wi\% C (w) & 293E-03 & - & - & $2.72 \mathrm{E}-03$ & $2.78 \mathrm{E}-03$ & $3.15 \mathrm{E}-03$ & $3.42 \mathrm{E}-03$ \\
\hline 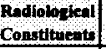 & $\mathrm{C} / \mathrm{L}$ & $\mathrm{nCl} / \mathrm{s}$ & C & $\begin{array}{l}-95 \mathrm{Cl} \\
\text { (C)/l) }\end{array}$ & $\begin{array}{l}67 \mathrm{Cl} \\
(\mathrm{C} / \mathrm{L})\end{array}$ & $\begin{array}{l}+67 \mathrm{CI} \\
(\mathrm{cul})\end{array}$ & $\begin{array}{l}+8 \mathrm{CI} \\
\text { (CUt) }\end{array}$ \\
\hline \begin{tabular}{l|l}
$\mathrm{H}-3$ \\
\end{tabular} & $1.58 \mathrm{E}-04$ & $9.12 \mathrm{E}-02$ & 32.8 & $3.20 E-05$ & $3.20 \mathrm{E}-0 \mathrm{~S}$ & 1.64E-04 & $1.75 \mathrm{E}-04$ \\
\hline C-14 & 8.10E-06 & 4.68E-03 & 1.69 & $1.65 \mathrm{E}-06$ & $1.65 E-106$ & $8.36 \mathrm{E}-06$ & 8.95E-06 \\
\hline Ni-59 & $2.12 \mathrm{E}-05$ & $1.22 \mathrm{E}-02$ & 4.40 & $1.73 \mathrm{E}-05$ & $1.73 \mathrm{E}-05$ & 2.19E-05 & 2.22E-05 \\
\hline Ni-63 & $2.06 \mathrm{E}-03$ & 1.19 & 429 & $1.69 \mathrm{E}-03$ & $1.69 \mathrm{E}-03$ & 2.14E-03 & $2.17 E-03$ \\
\hline Co-60 & $7.20 \mathrm{E}-06$ & $4.16 \mathrm{E}-03$ & 1.50 & $1.40 \mathrm{E}-06$ & $1.40 \mathrm{E}-06$ & $7,45 E-06$ & $7.98 \mathrm{E}-06$ \\
\hline Se-79 & 9.75E-06 & 5.64E-03 & 2.03 & $430 \mathrm{E}-06$ & 4.30E-06 & 1.59E-05 & 2.17E-0S \\
\hline Sr-90 & 244 & $1.41 E+03$ & 5.06E+0S & 2.37 & 2.41 & 2.47 & 2.48 \\
\hline$Y-90$ & 24 & $1.41 E+03$ & $5.08 \mathrm{E}+0 \mathrm{OS}$ & 2.20 & 2.29 & 2.47 & 2.48 \\
\hline$Z s-93$ & $4.30 \mathrm{E}-0 \mathrm{~s}$ & $2.49 \mathrm{E}-02$ & 8.95 & $2.04 E-05$ & 2.04E-05 & 7.35E-0S & 1.03E-04 \\
\hline $\mathrm{Nb}-93 \mathrm{~m}$ & 3.82E-05 & $2.21 \mathrm{E}-02$ & 7.94 & $1.65 \mathrm{E}-05$ & $1.68 E-05$ & $5.96 \mathrm{E}-05$ & $8.01 \mathrm{E}-0 \mathrm{~s}$ \\
\hline Tc-99 & $6.29 E-05$ & $3.64 \mathrm{E}-02$ & 13.1 & $5.91 \mathrm{E}-05$ & 5.76E-05 & $6.67 \mathrm{E}-0 \mathrm{~S}$ & 2. 10E-05 \\
\hline Ru- 106 & $1.66 \mathrm{E}-09$ & $9.57 \mathrm{E}-07$ & 3.45E-04 & $3.15 \mathrm{E}-10$ & $3.15 \mathrm{E}-10$ & $2.37 \mathrm{E}-09$ & 1. $200-08$ \\
\hline Cd-113m & $3.28 \mathrm{E}-05$ & $1.89 \mathrm{E}-02$ & 6.82 & $6.66 \mathrm{E}-06$ & $6.66 \mathrm{E}-06$ & 1.21E-04 & 3.04E-04 \\
\hline$S b-125$ & $2.96 \mathrm{E}-0.05$ & 1.40E-02 & 5.32 & 4.83E-06 & 4.83E-06 & $2.65 \mathrm{E}-05$ & 2.84E-0S \\
\hline $5 n-126$ & 1.34E-05 & $7.75 \mathrm{E}-03$ & 2.79 & $6.51 E-06$ & $6.58 \mathrm{E}-06$ & 2.26E-05 & 3.14E-05 \\
\hline$[-129$ & $1.19 \mathrm{E}-07$ & $690 \mathrm{E}-05$ & $2.48 E-02$ & $1.12 E-07$ & $1.092-07$ & $1.2 \pi \mathrm{E}-07$ & $1.35 E-07$ \\
\hline $\mathrm{Cs}-134$ & $1.70 \mathrm{E}-06$ & 9,84E-04 & 0.354 & 4.0SE-0? & 405E-07 & 1.75E-06 & 1,96E-06 \\
\hline $\mathrm{Cs}-137$ & 0.241 & 139 & $5,01 E+04$ & 0.229 & 0.222 & 0.254 & 0.269 \\
\hline $\mathrm{Ba}-137 \mathrm{~m}$ & 0.228 & 132 & $4.74 \mathrm{E}+04$ & $5.50 E-02$ & $5.50 E-02$ & 0.234 & 0.248 \\
\hline Sm-151 & $3.49 \mathrm{E}-02$ & 20.2 & $7.26 \mathrm{E}+03$ & $1.53 \mathrm{E}-02$ & $1.53 E-02$ & $5.63 \mathrm{E}-02$ & $7.66 \mathrm{E}-02$ \\
\hline Eur-152 & 2.52E-05 & 1. $46 \mathrm{E}-102$ & 5.25 & 2.02E-05 & $202 \mathrm{E}-05$ & $2.53 \mathrm{E}-05$ & 2.53E-05 \\
\hline Eu-154 & $1.73 E-04$ & 9.99E-02 & 36.0 & $3.41 \mathrm{E}-05$ & $3.41 \mathrm{E}-05$ & 1.79E-04 & 1.42E-03 \\
\hline Eu-155 & $1.26 E-03$ & 0.740 & 267 & $1.03 \mathrm{E}-03$ & $1.03 \mathrm{E}-03$ & $1.28 E-03$ & $1.29 \mathrm{E}-03$ \\
\hline $\mathrm{Ra}+226$ & $1.77 \mathrm{E}-09$ & 1.02E-06 & $3.69 \mathrm{E}-04$ & $1.40 \mathrm{E}-09$ & $1.51 \mathrm{E}-09$ & $1.96 \mathrm{E}-09$ & 2.14E-09 \\
\hline Ra-228 & B. ASE 00 & $513 E-06$ & I,ESE-03 & $3.47 \mathrm{E}-14$ & $3.4 \mathrm{E}-14$ & 8.96E-09 & 9.07E-09 \\
\hline Ac-227 & 8.26E-09 & $477 \mathrm{E}-06$ & $1.72 \mathrm{E}-03$ & $6.10 \mathrm{E}-09$ & $6.71 \mathrm{E}-09$ & $9.36 \mathrm{E}-09$ & $1.04 \mathrm{E}-08$ \\
\hline Pa-231 & $1.20 \mathrm{E}-08$ & $6.93 E-166$ & $2.50 \mathrm{E}-03$ & $6.16 \mathrm{E}-09$ & $6.16 \mathrm{E}-09$ & 1,81E-08 & $240 E-08$ \\
\hline Th-229 & $2.15 \mathrm{E}-10$ & 1.24E-07 & 4.4EE-65 & 2.46E-12 & $2,46 \mathrm{E}-12$ & $2.17 \mathrm{E}-10$ & 2. $19 \mathrm{E}-10$ \\
\hline Th-232 & $1.19 \mathrm{E}-10$ & 6.83E-08 & 2.48E-05 & 1.1EE-15 & $1,14 E-15$ & 1.50E-10 & $1.81 \mathrm{E}-10$ \\
\hline $\mathrm{U}-232$ & 3.97E-08 & 2.29E-05 & 8.26E-03 & 1. $40 \mathrm{E}-08$ & $2.59 \mathrm{E}-08$ & $5.65 E-08$ & $7.53 \mathrm{E}-08$ \\
\hline U.233 & $1.52 \mathrm{E}-07$ & 8.78E-05 & $3.16 \mathrm{E}-02$ & 5.37E-08 & $9.90 E-08$ & 2.16E-07 & $2.88 \mathrm{E}-07$ \\
\hline U-234 & $7.73 \mathrm{E}-07$ & 1.4TE-04 & 0.161 & 3.70E-07 & $5.66 \mathrm{E}-07$ & $9.96 E-07$ & $1.22 \mathrm{E}-06$ \\
\hline U-239 & $3.12 \mathrm{E}-08$ & 1.01E-05 & $6.50 \mathrm{E}-03$ & 1.49E-018 & $2.28 \mathrm{E}-0 \mathrm{~s}$ & $4.03 \mathrm{E}-0 \mathrm{~s}$ & $4.95 \mathrm{E}-08$ \\
\hline $\mathrm{U}-236$ & 3.18E-0:s & 1.AAE-05 & $6.61 \mathrm{E}-03$ & $1.56 \mathrm{E}-0 \mathrm{~s}$ & $2.36 \mathrm{E}-0 \mathrm{~B}$ & $4.03 \mathrm{E}-08$ & $4.87 \mathrm{E}-08$ \\
\hline U-238 & $6.78 \mathrm{E}-07$ & $3.92 \mathrm{E}-04$ & 0.141 & $322 \mathrm{E}-07$ & $4.95 \mathrm{E}-07$ & 8.77E-07 & $1.00 \mathrm{E}-\infty 6$ \\
\hline $\mathrm{Np}-237$ & $2.77 \mathrm{E}-07$ & $1.60 \mathrm{E}-04$ & $3.76 \mathrm{E}-02$ & $2.52 \mathrm{E}-07$ & 2.58E-07 & 2.95E-07 & 3.15E-07 \\
\hline Pu-238 & 1.54E-05 & $\$, 93 E-03$ & 3.21 & $1.27 \mathrm{E}-05$ & 1.41E-05 & $1.68 E-05$ & 1.81E-05 \\
\hline Pu-239 & 4. $10 \mathrm{E}-04$ & 0.237 & 85.3 & $3.58 \mathrm{E}-04$ & $3.83 \mathrm{E}-04$ & 4.37E-04 & $462 \mathrm{E}-04$ \\
\hline Pu-240 & $7.17 \mathrm{E}-05$ & $4.14 \mathrm{E}-02$ & 14.9 & $6.16 \mathrm{E}-05$ & $6.65 \mathrm{E}-05$ & 7.68E-05 & 8.18E-05 \\
\hline Pu-241 & $7.67 \mathrm{E}-04$ & 0.444 & 160 & 6.38E-04 & $7.01 \mathrm{E}-04$ & D.34E-04 & 8.97E-04 \\
\hline Pu-242 & $4.25 \mathrm{E}-09$ & $2.46 E-06$ & 8.84E-04 & $3.50 \mathrm{E}-09$ & $3.87 \mathrm{E}-09$ & $4.63 E-09$ & $4.99 \mathrm{E}-09$ \\
\hline Am-241 & 4.28E-04 & 0.247 & 89.1 & 2.48E-04 & $3.36 \mathrm{E}-04$ & $5.20 \mathrm{E}-04$ & $6.07 \mathrm{E}-\mathrm{S4}$ \\
\hline Am-243 & 1.83E-08 & $1.06 E-05$ & $3,82 \mathrm{E}-03$ & $1.02 \mathrm{E}-0 \mathrm{~s}$ & 1.42E-08 & $2.25 \mathrm{E}-08$ & 2.64E-08 \\
\hline $\mathrm{Cm}-242$ & $9.12 \mathrm{E}-07$ & $5.27 \mathrm{E}-04$ & 0.190 & 9.11E-07 & $9.06 \mathrm{E}-07$ & 9.13E-07 & $9.13 \mathrm{E}-07$ \\
\hline $\mathrm{Cm}-243$ & 8.89E-08 & 5. 14E-05 & $1.85 \mathrm{E}-02$ & 8. 88E-0B & $8.83 \mathrm{E}-08$ & $9.89 \mathrm{E}-08$ & 8.89E-68 \\
\hline $\mathrm{Cm}-244$ & $2.66 \mathrm{E}-06$ & $1.54 \mathrm{E}-03$ & 0.554 & $2.05 \mathrm{E}-06$ & $2.36 \mathrm{E}-06$ & 2.97E-06 & $3.26 \mathrm{E}-06$ \\
\hline Totbla & $\mathrm{M}$ & $\ln / \mathrm{t}$ : & ko: & $\begin{array}{l}9 \mathrm{Cl} \\
\mathrm{Mnt} \\
\mathrm{AL})\end{array}$ & $\begin{array}{l}67 \mathrm{CT} \\
\mathrm{A} \mathrm{pr} \\
\mathrm{dL}_{\mathrm{L}}\end{array}$ & $\begin{array}{l}+67 \mathrm{C} \\
(\mathrm{Mq} \\
(\mathrm{l})\end{array}$ & $\begin{array}{l}+95 \mathrm{CI} \\
(\mathrm{Mor} \\
\mathrm{L})\end{array}$ \\
\hline Pu & $6.91 \mathrm{E}-03(\mathrm{~g} / \mathrm{L})$ & - & 1.44 & 6.02E-03 & $6.46 \mathrm{E}-03$ & $7.36 \mathrm{E}-03$ & $7.80 \mathrm{E}-03$ \\
\hline $\mathrm{U}$ & 8.39E-03 & I.15E+03 & 415 & 3.91E-03 & $6.11 \mathrm{E}-03$ & $1.09 \mathrm{E}-02$ & $1.34 E-02$ \\
\hline
\end{tabular}

*Unknowrs in tank solids inventory are assigned by Tank Leyering Model (TLM). 
HDW Model Rey, 4

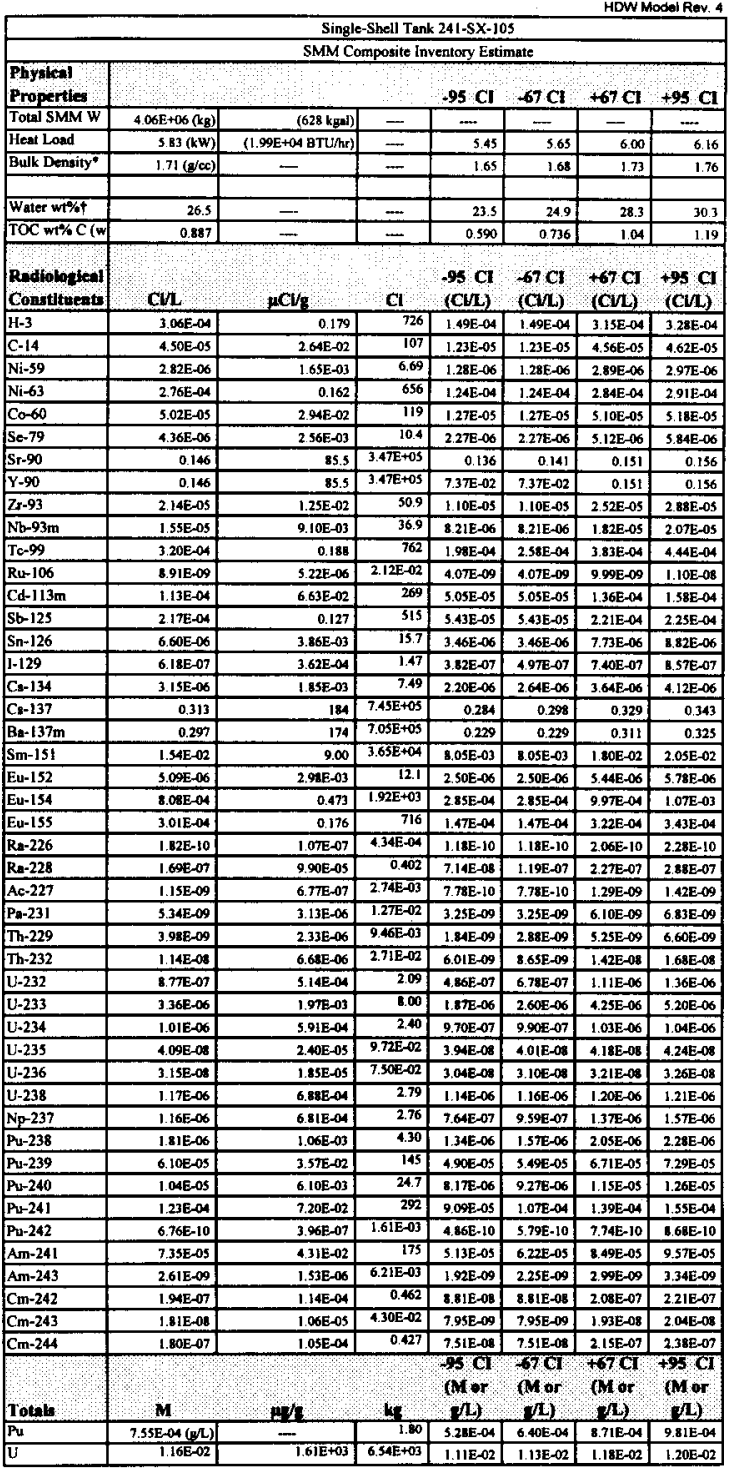

"Dennity is collealated baead on $\mathrm{Na}, \mathrm{OH}_{4}$ and $\mathrm{AlO} 2$ -

† Water wt\% derived from the difference of dentity and total diseolved epocios. 


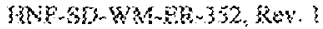
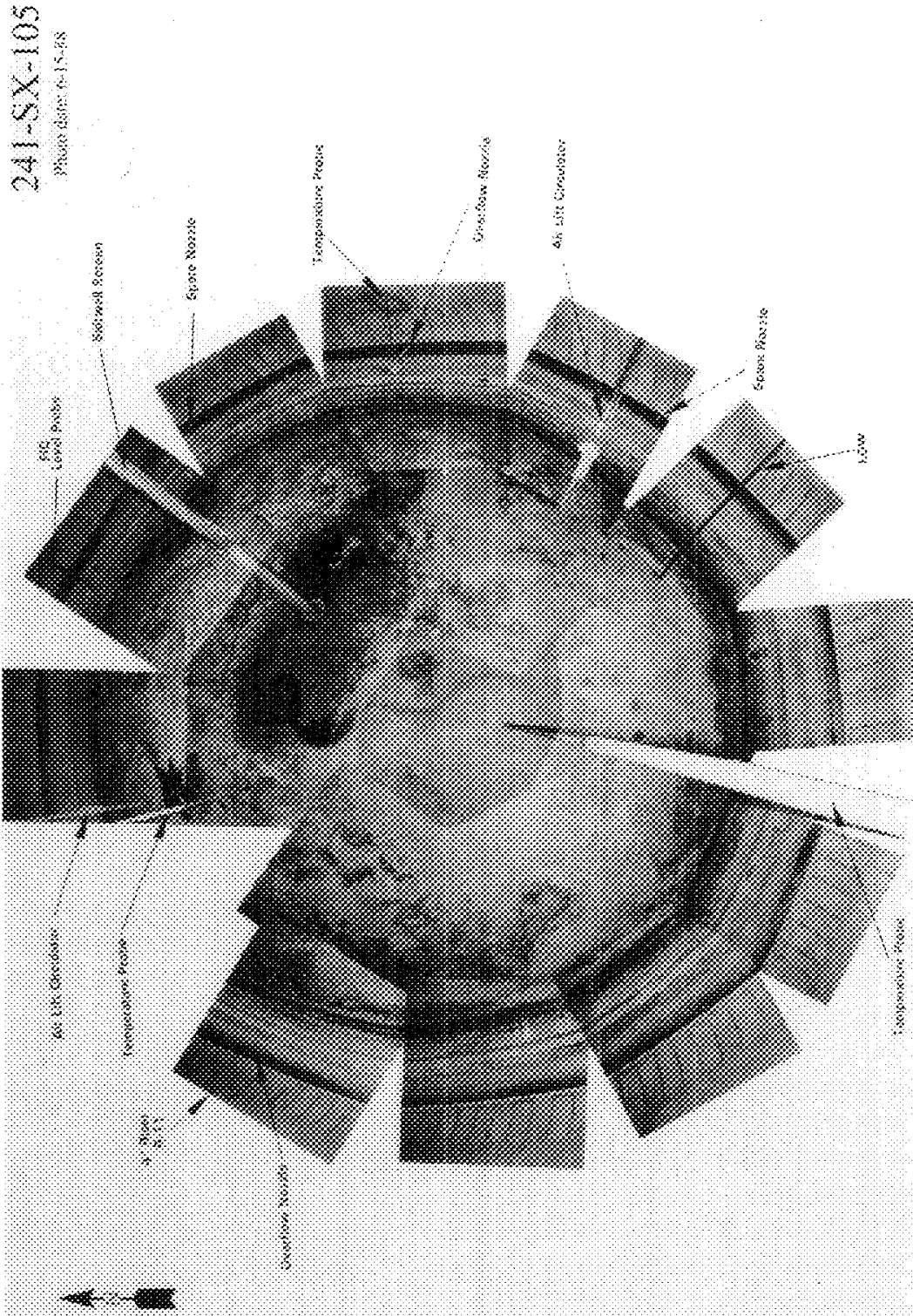
TANK 241-SX-106 SUMMARY

\begin{tabular}{|c|c|c|c|}
\hline \multicolumn{2}{|c|}{ TANK HISTORY } & \multicolumn{2}{|c|}{ TANK DESCRIPTION } \\
\hline Entered Service & 3rd qtr 1954 & Diameter & $75 \mathrm{ft}$ \\
\hline Removed from Service & - & Bottom Shape & Dish \\
\hline Inactive & 1980 & Nominal Capacity & $1,000,000 \mathrm{gal}$ \\
\hline Watch Lists & Hydrogen and Organics & Cascade Tank & none \\
\hline Integrity & Sound & Total Risers & 12 \\
\hline Assumed Leaker & - & \multicolumn{2}{|c|}{ WASTE VOLUME (HANLON 1996i) } \\
\hline Interim Stabilization (IS) & - & Total Waste Volume & $538,000 \mathrm{gal}$ \\
\hline Partial Interim Isolation (PI) & June 1985 & Waste Type & NCPLX \\
\hline intrusion Prevention (IP) & - & Drainable Interstitial Liquids & $194,000 \mathrm{gal}$ \\
\hline \multicolumn{2}{|c|}{$\begin{array}{l}\text { TENTATIVELY AVAILABLE RISERS } \\
\end{array}$} & Pumpable Liquids & 264,000 gal \\
\hline Riser Number(s) & Size & Saltcake & 465,000 gai \\
\hline 11 & 4 in & Sludge & $12,000 \mathrm{gal}$ \\
\hline 6 & 12 in & Supernatant & $61,000 \mathrm{gal}$ \\
\hline \multicolumn{2}{|c|}{ TANK TEMPERATURE } & \multicolumn{2}{|c|}{ INTERIOR PHOTOGRAPHS } \\
\hline Average Tank Temperature & $102^{\circ} \mathrm{F}$ & Date & June 1, 1989 \\
\hline Maximum Temperature & $124.8^{\circ} \mathrm{F}$ & Montage Number & $94041033-20 \mathrm{CN}$ \\
\hline Date & Feb 7, 1989 & Photo Set Number & 89060146 \\
\hline Elevation from tank bottom & $0.21 \mathrm{ft}$ & \multicolumn{2}{|c|}{ WASTE SURFACE LEVEL } \\
\hline Riser Number & 16 & Devices & Manual and Auto ENRAF \\
\hline Minimum Temperature & $63.8^{\circ} \mathrm{F}$ & Max Level & 209.9 in \\
\hline Date & Feb 16, 1991 & Date & May 17, 1991 \\
\hline Elevation from tank bottom & $14.83 \mathrm{ft}$ & Min Level & 198.06 in \\
\hline Riser Number & 16 & Date & Sept 23, 1996 \\
\hline
\end{tabular}




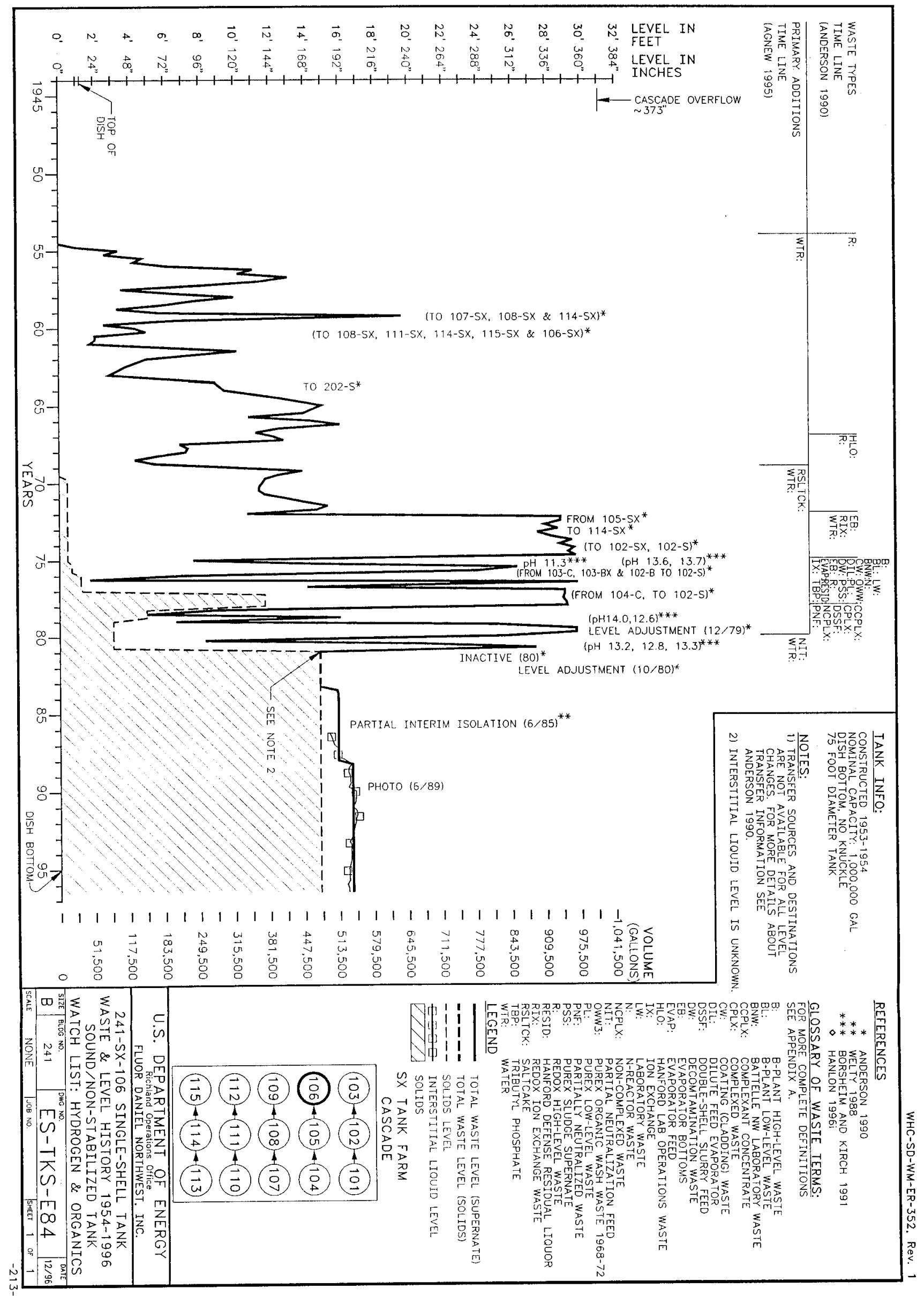




\section{$241-5 X-106$}

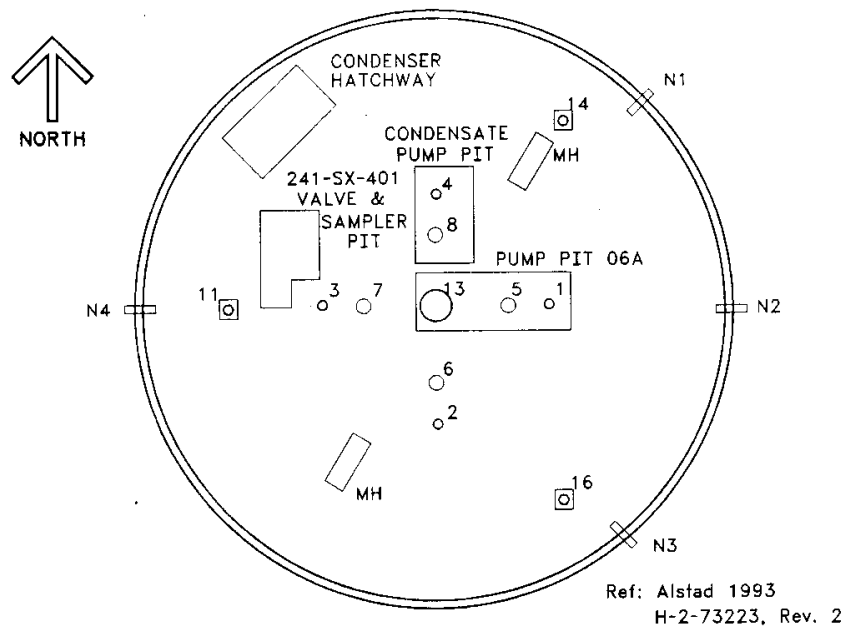

\section{TANK RISER LOCATION}

Approximate Grade Elevation $201.63 \mathrm{~m}$ [661.5ft]

(Pianka 1995)

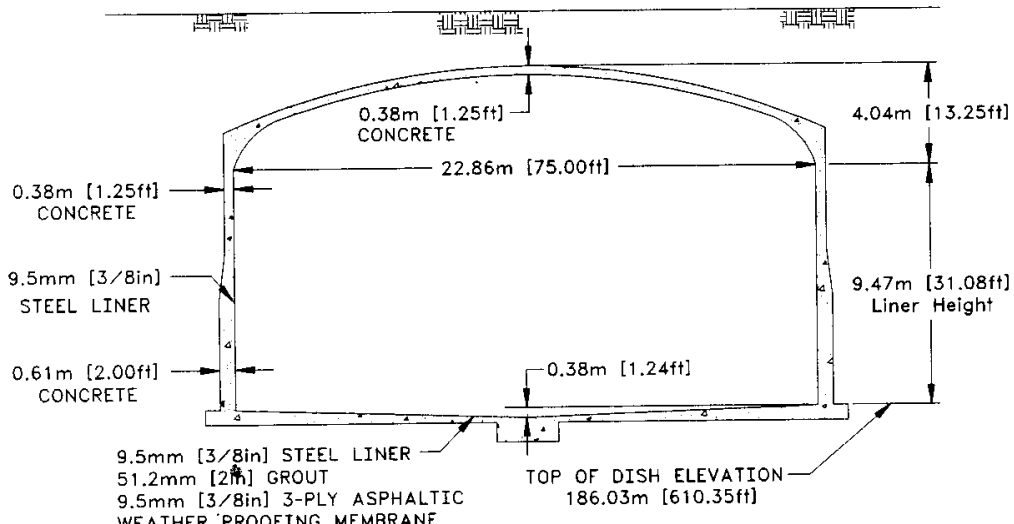

WEATHER PROOFING MEMBRANE

NOT TO SCALE

Ref: $H-2-39511$, Rev. 3

$\mathrm{H}-2-46293$, Rev. 3

H-2-37855, Rev. 4 
HNF-SD-WM-ER-352, Rev. 1

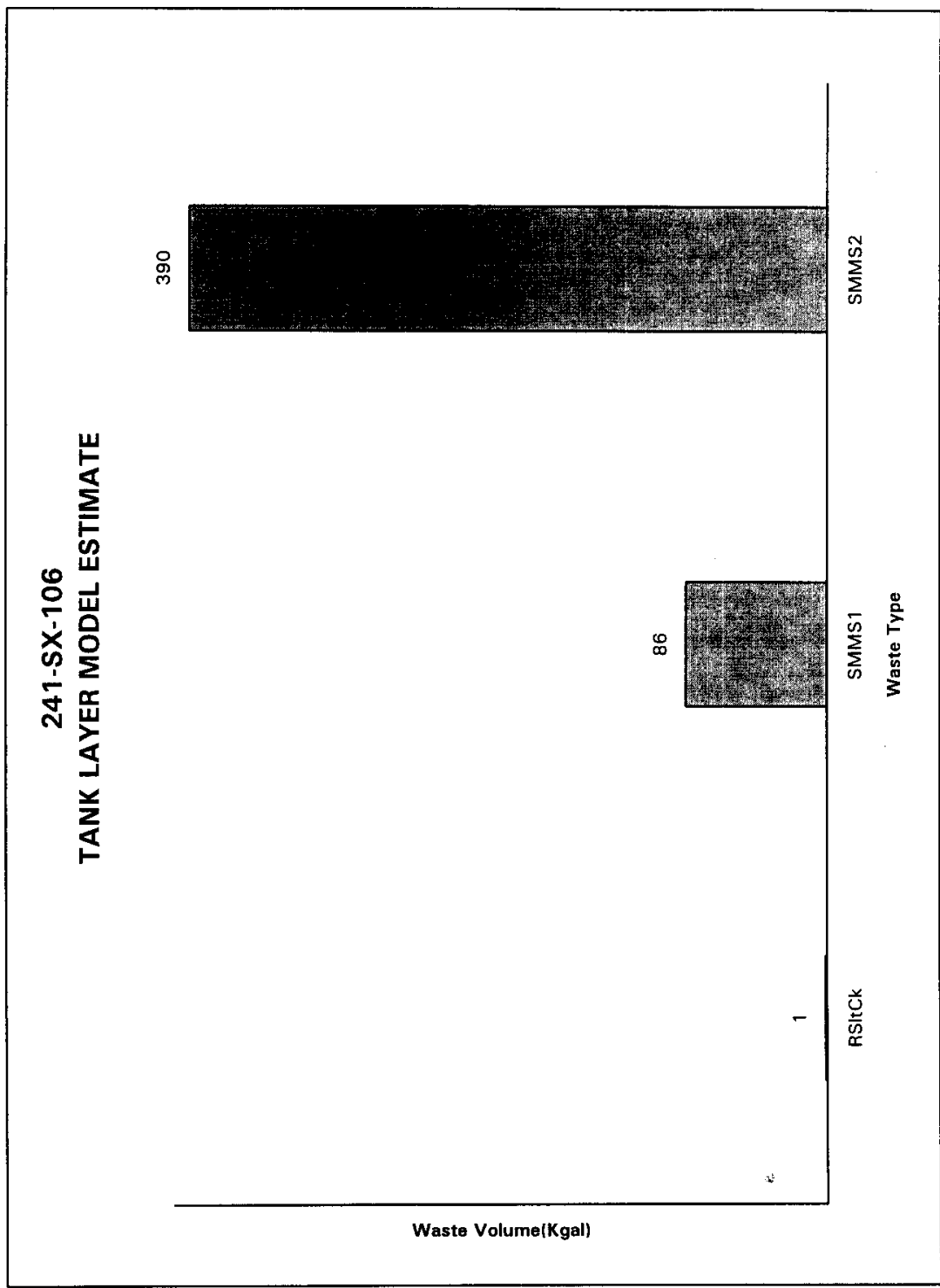

总 
HDW Model Rey. 4

\begin{tabular}{|c|c|c|c|c|c|c|c|}
\hline \multirow[b]{3}{*}{$\begin{array}{l}\text { Phyoled } \\
\text { Propertics }\end{array}$} & \multicolumn{7}{|c|}{ Single-Shell Tank 24I-SX-106 } \\
\hline & \multicolumn{7}{|c|}{ TLM Solids Composite Inventory Estimate* } \\
\hline & & & & $95 \mathrm{Cl}$ & $+67 \mathrm{CI}$ & $+67 \mathrm{Ct}$ & $\$ 9 \mathrm{SCI}$ \\
\hline Total TLM Wa & $6.4 \mathrm{BE}+03(\mathrm{~kg})$ & $(1.00 \mathrm{kgal})$ & 一 & + & - & $=$ & - \\
\hline Heak Load & $1.24 \mathrm{E}-02(\mathrm{~kW})$ & (42.5 BTU/hr) & - & $1.14 \mathrm{E}-02$ & 1.19E-02 & $1.30 E-02$ & $1.35 \mathrm{E}-02$ \\
\hline Bulk Density & $1.71(g / \alpha)$ & $m$ & - & 1.44 & 1,58 & 1.78 & 1.82 \\
\hline Void Fraction & 0.785 & $\ldots$ & - & 0.741 & 0.757 & 0.876 & 0.978 \\
\hline Wrter wt\% & 34.7 & $\cdots$ & - & 24.3 & 29,0 & 45.7 & 57.6 \\
\hline $\mathrm{TOC} w \% \mathrm{C}(\mathrm{w}$ & $5.25 \mathrm{E}-03$ & $\ldots$ & $\cdots$ & 5.01E-03 & $5.13 \mathrm{E}-03$ & $5.56 \mathrm{E}-03$ & 6.28E-03 \\
\hline Chentic & noted & $\mathrm{ppm}$ & lis & $\begin{array}{l}95 \mathrm{Cl} \\
\text { (moleth) }\end{array}$ & $\begin{array}{l}67 C 1 \\
\text { (moleiL) }\end{array}$ & $\begin{array}{l}+67 \mathrm{CI} \\
\text { (uolel } \mathrm{L})\end{array}$ & $\begin{array}{r}+95 \mathrm{CI} \\
(\mathrm{mole} / \mathrm{L}) \\
\end{array}$ \\
\hline $\mathrm{Nan+}$ & 13.5 & $1.81 \mathrm{E}+05$ & $1.17 \mathrm{E}+03$ & 8.55 & 11.2 & 146 & 15.6 \\
\hline Al3+ & 3.39 & $5.34 \mathrm{E}+0.4$ & 346 & 1.44 & 2.01 & 4.89 & 0.41 \\
\hline Fes+ (lotel Fe) & $1.74 \mathrm{E}-02$ & 568 & 3.68 & 9.62E-03 & $1.34 E-02$ & 2.14E-02 & 2.52E-02 \\
\hline $\mathrm{Cr}^{3+}$ & 0.822 & $2.50 \mathrm{E}+04$ & $\begin{array}{r}162 \\
\end{array}$ & 0.480 & 0.643 & 0.894 & 0.897 \\
\hline $\mathrm{Bi3+}$ & 6.87E-06 & 0.838 & $5.43 \mathrm{E}-03$ & $5.80 \mathrm{E}-06$ & $6.32 \mathrm{E}-06$ & $757 E-06$ & $8.32 \mathrm{E}-\infty 6$ \\
\hline L.13+ & $1.69 \mathrm{E}-11$ & $1.37 \mathrm{E}-06$ & $8.90 \mathrm{E}-09$ & $1,48 \mathrm{E}-11$ & $1.60 \mathrm{E}-11$ & $1.87 \mathrm{E}-11$ & $2.06 \mathrm{E}-11$ \\
\hline $\mathrm{Hg} 2+\mathrm{i}$ & $1.08 E-06$ & 0.126 & 8.19E-04 & $9.56 \mathrm{E}-07$ & $1.02 \mathrm{E}-06$ & $1.19 \mathrm{E}-06$ & $1.30 \mathrm{E}-06$ \\
\hline $\mathrm{Zr}$ (14 Zro(OH)2 & $6.86 \mathrm{E}-07$ & $3.65 \mathrm{E}-02$ & $2.37 \mathrm{E}-04$ & 6.30E-07 & 6.57E-07 & $7.19 \mathrm{E}-07$ & 7.75E-07 \\
\hline $\mathrm{Pb} 2+$ & $1.71 \mathrm{E}-04$ & 20.7 & 0.134 & $9.17 \mathrm{E}-05$ & $1.30 E-04$ & $2.12 \mathrm{E}-04$ & $2.53 \mathrm{E}-04$ \\
\hline $\mathrm{Ni2+}$ & T.51E-02 & 518 & 3.36 & $1.37 \mathrm{E}-02$ & $1.44 E-02$ & $1.58 \mathrm{E}-02$ & $1.65 \mathrm{E}-02$ \\
\hline $\mathrm{Sr} 2+$ & $\begin{array}{r}\mathbf{0} \\
\end{array}$ & 0 & 0 & 0 & 0 & 0 & 0 \\
\hline Mn4t & 5.00E-05 & 1.60 & $1.04 \mathrm{E}-02$ & $3.57 \mathrm{E}-0 \mathrm{~S}$ & 4.27E-0S & S.74E-05 & $6.46 \mathrm{E}-05$ \\
\hline $\mathrm{C}+2+$ & $7.6 . E-02$ & $1.40 \mathrm{E}+03$ & 11.6 & $4.68 \mathrm{E}-02$ & 6.15E- -02 & 9. 19E-02 & 0.106 \\
\hline $\mathbf{K +}$ & $3.20 \mathrm{E}-02$ & 731 & 4.74 & $3.03 \mathrm{E}-02$ & 3.12E-02 & $3.48 \mathrm{E}-02$ & $3.80 \mathrm{E}-02$ \\
\hline $\mathrm{OH} \cdot$ & 18.7 & $1.86 \mathrm{E}+05$ & $1.20 \mathrm{E}+03$ & 9.70 & 126 & 25.1 & 31.5 \\
\hline NO3- & 5.25 & $1.90 \mathrm{E}+05$ & $1.23 \mathrm{E}+03$ & 2.71 & 4.50 & 5.40 & 5.56 \\
\hline NO2:- & 1.87 & $5.03 E+04$ & 326 & 1.59 & 1.73 & 2.01 & 2.18 \\
\hline $\cos 2$ & $8.17 \mathrm{E}-02$ & $2.86 \mathrm{E}+03$ & 18.6 & $5.10 E-02$ & 6.64E-02 & $9.69 \mathrm{E}-02$ & 0.111 \\
\hline PO43. & 4.45E-04 & 24.7 & 0.160 & 4.13E-04 & $4.31 \mathrm{E}-04$ & 4.63E-04 & S.03E-04 \\
\hline $5042-$ & 4.04E-02 & $2.27 E+03$ & 14.7 & $3,83 \mathrm{E}-02$ & $3.94 \mathrm{E}-02$ & $4.40 \mathrm{E}-02$ & $4.78 \mathrm{E}-02$ \\
\hline Si (as Sion2-) & 0.130 & $2.13[+03$ & 13.8 & $8.22 \mathrm{E}-02$ & 0.112 & 0.148 & 0.166 \\
\hline F- & 3.54E-04 & 3.93 & 2.55E 02 & $3.02 \mathrm{E}-04$ & $3.34 \mathrm{E}-04$ & $3.90 \mathrm{E}-04$ & 4.20E-04 \\
\hline $\mathrm{Cl}-$ & 0.133 & $2.76 \mathrm{E}+03$ & 17.9 & $7.17 \mathrm{E}-02$ & 0.102 & 0.147 & 0.154 \\
\hline C6HSÖ73- & $3.66 \mathrm{E}-04$ & 40.4 & 0.252 & $3.55 \mathrm{E}-04$ & $3.60 \mathrm{E}-0 \mathrm{~A}$ & 3.82E- 04 & 4.16E-04 \\
\hline EDTA4- & 1.43E-05 & 2.40 & $1,565-02$ & 1.00E-05 & $1.23 \mathrm{E}-0.05$ & 1.68E-05 & 1.93E-0S \\
\hline HEDTA3- & $1.18 \mathrm{E}-05$ & 1.90 & $1,2315-02$ & 3.20E-06 & B. $14 \mathrm{E}-106$ & 1.5tE-0S & $1.97 \mathrm{E}-05$ \\
\hline glycolato- & $5.17 \mathrm{E}-04$ & 22.6 & 0.147 & $2.80 E-04$ & $4.09 \mathrm{E}-04$ & 6.43E- 04 & 1.63E-04 \\
\hline acevale & $5.36 \mathrm{E}-05$ & 1.85 & $1.20 \mathrm{E}-02$ & $5.19 \mathrm{E}-05$ & $5.28 \mathrm{E}-05$ & $5.70 \mathrm{E}-05$ & 6.09E-05 \\
\hline Oxalute?- & $2.22 \mathrm{E}-11$ & T.14E-06 & 7.39E-09 & $1.96 \mathrm{E}-11$ & 209E-11 & $2.44 \mathrm{E}-11$ & $2.68 \mathrm{E}-11$ \\
\hline DBP & $3.25 \mathrm{E}-04$ & 39.9 & 0.251 & $3.09 \mathrm{E}-04$ & $3.16 E-04$ & $3.51 \mathrm{E}-04$ & 3.79E-04 \\
\hline butunol & $3.25 \mathrm{E}-04$ & 14.1 & 9. IIE-02 & $3.03 \mathrm{E}-04$ & $3.16 \mathrm{E}-04$ & $3.51 \mathrm{E}-04$ & $3.79 \mathrm{E}-04$ \\
\hline $\mathrm{NH3}$ & $3.60 \mathrm{E}-02$ & $35:$ & 2.32 & 2.78E-02 & $3.21 \mathrm{E}-02$ & $4.11 \mathrm{E}-02$ & 4. $59 \mathrm{E}-02$ \\
\hline Fo(CN)64- & 0 & $\mathbf{0}$ & 0 & 0 & 0 & 0 & 0 \\
\hline
\end{tabular}

*Unknowns in tunk solids inventory tre meigned by Tank Layering Model (TLM) 
HDW Model Rev. 4

\begin{tabular}{|c|c|c|c|c|c|c|c|}
\hline \multirow[b]{3}{*}{$\begin{array}{l}\text { Phybical } \\
\text { Properties }\end{array}$} & \multicolumn{7}{|c|}{ Single-Shell Tank 241-SX-106 } \\
\hline & \multicolumn{7}{|c|}{ SMM Composite Inventory Estimate } \\
\hline & & & & $.95 \mathrm{CI}$ & $-67 \mathrm{CI}$ & $+67 \mathrm{Cl}$ & $+95 \mathrm{CI}$ \\
\hline Total SMM W & $3.27 \mathrm{E}+06(\mathrm{~kg})$ & (537 kgal) & - & n... & $\ldots$ & - & - \\
\hline Heat laad & $4.30(\mathrm{~kW})$ & $(1.47 \mathrm{E}+04 \mathrm{BTU} / \mathrm{hr})$ & $-\ldots$ & 4.03 & 4.16 & 4.44 & 4.57 \\
\hline Bulk Density ${ }^{*}$ & $1.61(\mathrm{~g} / \mathrm{cc})$ & - & -- & 1.56 & 1.58 & 1.63 & 1.65 \\
\hline Water wt\% & 32.7 & $\rightarrow$ & $m$ & 30.1 & 31.3 & 342 & 36.0 \\
\hline TOC wt\%C (w & 0.750 & $\ldots$ & - & 0.491 & 0.618 & 0.883 & $1.0]$ \\
\hline $\begin{array}{c}\text { Clienteil } \\
\text { Consilituents }\end{array}$ & moled & Epan : : & ry & $\begin{array}{l}-95 \mathrm{CI} \\
\text { (nimoled) }\end{array}$ & $\begin{array}{l}67 \mathrm{Cl} \\
\text { (mole/L) }\end{array}$ & $\begin{array}{l}+67 \mathrm{Cl} \\
(\mathrm{nin} / \mathrm{k} / \mathrm{L})\end{array}$ & $\begin{array}{l}+8 \mathrm{Cl} \\
\text { (mole/t) }\end{array}$ \\
\hline $\mathrm{Na}+$ & 14.2 & $2.03 \mathrm{E}+05$ & $6.64 \mathrm{E}+05$ & 13.0 & 13.6 & 14.7 & 15.2 \\
\hline $\mathbf{A} \mid \mathbf{3}+$ & 1.67 & 2.EOE+OA & $9.16 \mathrm{E}+04$ & 1.55 & 1.61 & 1.74 & 1,79 \\
\hline $\mathrm{Fe}+(\operatorname{tode} \mathrm{Fe})$ & $1.09 E-02$ & 378 & $1.24 \mathrm{E}+03$ & $8.93 \mathrm{E}-03$ & $9.89 \mathrm{E}-03$ & $1.19 \mathrm{E}-02$ & 1.29E-02 \\
\hline $\mathrm{Cr} 3+$ & 0.148 & $4,79 \mathrm{E}+03$ & $1.56 \mathrm{E}+04$ & 0.123 & 0.137 & 0.152 & 0.157 \\
\hline Bi3+ & 1.3BE-03 & 179 & 586 & $1.25 \mathrm{E}-03$ & $1.32 \mathrm{E}-03$ & $1.45 \mathrm{E}-03$ & $1.51 \mathrm{E}-03$ \\
\hline Le.3+ & 4.14E-05 & 3.57 & 11.7 & $3.00 E-05$ & $3.55 \mathrm{E}-0 \mathrm{~S}$ & $4.72 \mathrm{E}-05$ & $5.29 \mathrm{E}-03$ \\
\hline $\mathrm{H}_{\mathrm{Q} 2+}$ & $9.27 \mathrm{E}-06$ & 1.16 & 3.78 & 8.60E-06 & $8.98 \mathrm{E}-06$ & $9.40 \mathrm{E}-06$ & $9.53 \mathrm{E}-06$ \\
\hline $\mathrm{Zr}(\omega \mathrm{ZrO}(\mathrm{OH}) 2$ & $2.60 \pm-04$ & 14.8 & 48.2 & $2.37 \mathrm{E}-04$ & $2.45 \mathrm{E}-04$ & $270 E-04$ & $2.83 \mathrm{E}-04$ \\
\hline PB2+ & 1.01E-03 & 130 & 425 & 8.17E-04 & $9.11 \mathrm{E}-04$ & 1.11E-03 & $1.20 \mathrm{E}-03$ \\
\hline $\mathrm{Ni2+}$ & $6.50 \mathrm{E}-03$ & 237 & 776 & $6.21 E-03$ & $6.35 \mathrm{E}-03$ & 6.58E-03 & $6.65 \mathrm{E}-03$ \\
\hline Srat & 0 & 0 & 0 & 0 & $\begin{array}{r}0 \\
\end{array}$ & 0 & 0 \\
\hline Mndt & $3.896-03$ & 133 & 435 & 2.95E-03 & 3.41E-03 & 4.37E-03 & 4.14E- -03 \\
\hline C.2+ & 3.41E-02 & 850 & 2.78E+03 & 3.13E-02 & 3.27E-02 & 3.55E-02 & $3.69 \mathrm{E}-02$ \\
\hline $\mathbf{K}+$ & $6.60 \mathrm{E}-02$ & $1.60 \mathrm{E}+03$ & $5.24 E+03$ & $6.02 \mathrm{E}-02$ & $6.29 \mathrm{E}-02$ & 6.91E-02 & $7.21 \mathrm{E}-02$ \\
\hline OH- & 997 & $1.05 \mathrm{E}+05$ & $3.45 \mathrm{E}+05$ & $\begin{array}{r}9.21 \\
\end{array}$ & $\begin{array}{r}9.57 \\
\end{array}$ & $\begin{array}{r}10.4 \\
\end{array}$ & 10.6 \\
\hline No3- & 5.09 & $1.96 \mathrm{E}+05$ & $6.41 \mathrm{E}+05$ & 4.68 & 4.92 & 5.17 & 5.22 \\
\hline NO2- & 2.45 & $7.00 \mathrm{E}+04$ & $2.29 \mathrm{E}+05$ & 2.04 & 2.23 & 2.66 & 2.86 \\
\hline Co32- & 0.435 & $1.62 \mathrm{E}+04$ & $5.31 E+04$ & 0.395 & 0,410 & 0.466 & 0.471 \\
\hline PO43. & $9.16 \mathrm{E}-02$ & $5.41 \mathrm{E}+03$ & $1.77 \mathrm{E}+04$ & $7.83 \mathrm{E}-02$ & 8.30E-02 & 9.40E-02 & $9.62 \mathrm{E}-02$ \\
\hline $\mathrm{SO} 2-$ & 0.245 & $1.4 \pi+04$ & $4.79 \mathrm{E}+04$ & 0.193 & 0.218 & 0.273 & 0.298 \\
\hline Si (as SiO32-) & $7.80 \mathrm{E}-02$ & $136 \mathrm{E}+03$ & $4.45 \mathrm{E}+03$ & 6.45E-02 & $7.11 \mathrm{E}-02$ & B.49E-02 & 9.15E-02 \\
\hline $\mathbf{F}$ & $6.78 \mathrm{E}-02$ & 501 & $2.62 E+03$ & $5.67 \mathrm{E}-02$ & $6.14 \mathrm{E}-02$ & $7.320-02$ & $7.74 E-02$ \\
\hline $\mathrm{Cl}-$ & 0.246 & $3.42 \mathrm{E}+03$ & $1.77 \mathrm{E}+04$ & 0.223 & 0.231 & 0257 & 0.263 \\
\hline C6HSO73- & $2.76 \mathrm{E}-02$ & $3.24 \mathrm{E}+03$ & $1.06 \mathrm{E}+04$ & $2.53 \mathrm{E}-02$ & 2.64E-02 & $2.87 \mathrm{E}-02$ & $2.99 \mathrm{E}-02$ \\
\hline EDTA4- & 1.49E-02 & $2.66 \mathrm{E}+03$ & $8.70 E+03$ & 4.55E-03 & 9.59E-03 & $202 \mathrm{E}-02$ & 2.54E-02 \\
\hline FEDTA3. & $2.78 \mathrm{E}-02$ & $4.74 E+03$ & $1.55 E+04$ & $7.16 \mathrm{E}-03$ & $1.72 \mathrm{E}-02$ & $3 . \$ 4 \mathrm{E}-02$ & $4.83 \mathrm{E}-02$ \\
\hline glycolinte- & $8.75 E-02$ & 4.09E+03 & $1,33 \mathrm{E}+04$ & $5.74 \mathrm{E}-02$ & $7.21 \mathrm{E}-02$ & 0.103 & 0.118 \\
\hline acestate- & $6.21 \mathrm{E}-03$ & 221 & 745 & S.09E-03 & 5.64E-03 & 6.7HE-03 & $7.32 \mathrm{E}-03$ \\
\hline oxaluter- & 5.42E-05 & 2.97 & 9,20 & $4.80 \mathrm{E}-05$ & $5.10 \mathrm{E}-0 \mathrm{~s}$ & $5.73 \mathrm{E}-05$ & 6.04E-05 \\
\hline DBP & $1.81 \mathrm{E}-02$ & $2.46 \mathrm{E}+03$ & $8.03 \mathrm{E}+0.3$ & $1.55 \mathrm{E}-02$ & $1.71 \mathrm{E}-02$ & $2.05 \mathrm{E}-02$ & $2.20 \mathrm{E}-02$ \\
\hline butanol & $1.81 E-02$ & 865 & $2.83 \mathrm{E}+03$ & $1.55 \mathrm{E}-02$ & $1.71 \mathrm{E}-02$ & $2.05 E-02$ & $2.20 \mathrm{E}-02$ \\
\hline NH3 & B.69E-02 & 919 & $3.00 \mathrm{E}+03$ & $7.17 \mathrm{E}-02$ & $7.77 \mathrm{E}-02$ & $9.67 \mathrm{E}-02$ & 0.112 \\
\hline Fe(CN)64- & 0 & 0 & 0 & 0 & 0 & 0 & 0 \\
\hline
\end{tabular}

"Density is calculated bued on N2, OH-, and ANO2-,

+Water wT\% derived from the difference of density and total dissolved apecies. 
HNF-SD-WM-ER-352, Rev. 1

HDW Model Rev

\begin{tabular}{|c|c|c|c|c|c|c|c|}
\hline \multirow[b]{3}{*}{$\begin{array}{l}\text { Phythol } \\
\text { Properties }\end{array}$} & \multicolumn{7}{|c|}{ Single-Sheil Tank 241-SX-106 } \\
\hline & \multicolumn{7}{|c|}{ Total Inventory Estimate* } \\
\hline & & & & $95 \mathrm{Cl}$ & $.67 \mathrm{CI}$ & $+67 \mathrm{CI}$ & $+95 \mathrm{Cl}$ \\
\hline Total Waste & $3.27 \mathrm{E}+06(\mathrm{~kg})$ & (538 kgal) & $\ldots$ & $-\cdots$ & -- & -6 & $\ldots$ \\
\hline Heat Load & $4.31(\mathrm{kw})$ & $(1.47 \mathrm{E}+04 \mathrm{BTU} / \mathrm{hr})$ & - & 4.05 & 418 & 4.45 & 4.58 \\
\hline Bulk Densityt & $1.61(\mathrm{~g} / \mathrm{c})$ & $\ldots$ & - & 1.56 & 1.58 & 1.63 & 1.65 \\
\hline Water wt\% $\%$ & 32.7 & - & $\ldots$ & 301 & 31.3 & 34.2 & 36.0 \\
\hline $\mathrm{TOC} w \mathrm{w} \% \mathrm{C}(\mathrm{w})$ & 0.749 & $m$ & $\ldots$ & 0.490 & 0.617 & 0.881 & 1.01 \\
\hline $\begin{array}{l}\text { Chemileal } \\
\text { Constltuent: }\end{array}$ & mole $\mathrm{L}$ : & pem & 12 & $\begin{array}{l}9 S \mathrm{CI} \\
\text { (mole } \mathrm{L} \text { ) }\end{array}$ & $\begin{array}{r}67 \mathrm{Cl} \\
\text { (nole/t) }\end{array}$ & $\begin{array}{l}+67 \mathrm{CI} \\
\text { (mole/L) }\end{array}$ & $\begin{array}{l}+9 s \mathrm{CI} \\
(\mathrm{anol} / \mathrm{L})\end{array}$ \\
\hline $\mathrm{Na}+$ & 14.2 & $2.03 \mathrm{E}+03$ & $6.66 \mathrm{E}+05$ & 130 & 13,6 & 14.7 & 15.2 \\
\hline $\mathrm{A} / 3+$ & 1.67 & $2.81 \mathrm{E}+04$ & $9.19 E+04$ & 1.55 & 1.61 & 1.74 & 1.79 \\
\hline Fe3+ (total Fe) & $100 \mathrm{E}-02$ & 379 & $1.24 \mathrm{E}+03$ & $895 E-03$ & 9.91E-03 & $1.19 \mathrm{E}-02$ & $1.29 \mathrm{E}-02$ \\
\hline $\mathrm{Cr} 3+$ & 0.149 & $4.83 \mathrm{E}+03$ & TSEE+04 & 0.125 & 0.138 & 0.153 & 0.157 \\
\hline Bi3+ & $1,38 E-03$ & 179 & 526 & $1.25 \mathrm{E}-03$ & 1.31E-03 & $1.44 \mathrm{E}-03$ & 1.50E-03 \\
\hline L.34 & 4I3E-05 & 3.57 & 11.7 & 2.99E-05 & $3,55 \mathrm{E}-05$ & $4.71 \mathrm{E}-0 \mathrm{~S}$ & $5.28 \mathrm{E}-0.5$ \\
\hline $\mathrm{Hg}^{2}+$ & 9.25E-06 & 1.15 & 3.78 & 8.59E-06 & $8.97 \mathrm{E}-06$ & $9.39 \mathrm{E}-06$ & $9.52 \mathrm{E}-06$ \\
\hline $\mathrm{Zr}(\mathrm{m} \mathrm{ZrO}(\mathrm{OH}) 2$ & $2.60 \mathrm{E}-04$ & 14.7 & 48.2 & $236 \mathrm{E}-04$ & $2.45 \mathrm{E}-0.4$ & $2.69 \mathrm{E}-04$ & $2.82 E-04$ \\
\hline $\mathrm{Pb} 2+$ & $1.01 \mathrm{E}-03$ & 130 & 425 & C.16E-04 & 9.106 .04 & $1.10 \mathrm{E}-03$ & 1.20E-03 \\
\hline $\mathrm{Ni2t}$ & 6.52E-03 & 238 & 779 & 6.23E- -03 & $6.37 \mathrm{E}-03$ & 6.59E-03 & 6.66E-03 \\
\hline $5 r 2+$ & 0 & 0 & 0 & 0 & 0 & 0 & 0 \\
\hline Mr4t+ & $3.88 E-03$ & 133 & $\begin{array}{r}435 \\
\end{array}$ & 2.94E-03 & $3.40 \mathrm{E}-03$ & $4.36 \mathrm{E}-03$ & $183 \mathrm{E}-03$ \\
\hline $\mathrm{C} \Omega 2+$ & $3.42 \mathrm{E}-02$ & 852 & $2.79 \mathrm{E}+03$ & $3.14 \mathrm{E}-02$ & $3.27 \mathrm{E}-02$ & $3,56 \mathrm{E}-02$ & $3.70 \mathrm{E}-02$ \\
\hline $\mathbf{K +}$ & 6.39E-02 & $1.60 \mathrm{E}+03$ & $5.25 E+03$ & 6.02E-02 & $6.29 \mathrm{E}-02$ & $6.91 \mathrm{E}-02$ & $7.20 \mathrm{E}-02$ \\
\hline $\mathrm{OH}-$ & 9,99 & $1.06 \mathrm{E}+05$ & $3.46 \mathrm{E}+05$ & 9.23 & 959 & 10.4 & 10.6 \\
\hline No3- & 5.09 & $1.96 \mathrm{E}+05$ & $6.42 E+05$ & 4.68 & 492 & 5.17 & 5.22 \\
\hline NO2- & 2.45 & $7.00 \mathrm{E}+04$ & $2.29 \mathrm{E}+0 \mathrm{~S}$ & 2.04 & 2.23 & 2.66 & 2.85 \\
\hline $\cos 2$ & 0.434 & $1.62 E+04$ & $33 \mathrm{HE}+04$ & 0.394 & 0.409 & 0.466 & 0.470 \\
\hline $\mathrm{PO43-}$ & 9.14E-02 & $5.40 \mathrm{E}+03$ & $1,77 \mathrm{E}+04$ & $7.82 \mathrm{E}-02$ & $8.28 \mathrm{E}-02$ & $9.38 \mathrm{E}-02$ & 9.61E-02 \\
\hline $\mathrm{SOA2-}$ & 0.245 & $1.46 \mathrm{E}+04$ & $479 E+04$ & 0.193 & 0.218 & 0.273 & 0.297 \\
\hline Si (ns SiO032) & $7.81 \mathrm{E}-02$ & $1 . \overline{36} \mathrm{E}+03$ & $4,47 \mathrm{E}+03$ & $6.46 \mathrm{E}-02$ & $9.12 \mathrm{E}-02$ & $0.500-02$ & $9.16 \mathrm{E}-02$ \\
\hline F- & $6.76 \mathrm{E}-02$ & 799 & $2.62 \mathrm{E}+03$ & $5.66 \mathrm{E}-02$ & $6.12 \mathrm{E}-02$ & $7.30 \mathrm{E}-02$ & $7.73 \mathrm{E}-02$ \\
\hline $\mathrm{Cl}$ & 0.246 & $5.91 E+03$ & $1.77 \mathrm{E}+04$ & 0.223 & 0.231 & 0.256 & 0.265 \\
\hline C6HSO73. & $2.73 \mathrm{E}-02$ & $3.24 \mathrm{E}+03$ & $1.06 E+04$ & $2.53 \mathrm{E}-02$ & 2.64E-02 & $2.87 \mathrm{E}-02$ & 298E-02 \\
\hline EDTAA4- & 1.48E-02 & $2.66 \mathrm{E}+03$ & E70E+03 & 4.54E- 03 & $9.57 \mathrm{E}-03$ & 2.0JE-02 & $2.53 \mathrm{E}-02$ \\
\hline HEDTA3- & $2.77 \mathrm{E}-02$ & $4.73 \mathrm{E}+03$ & $1.55 E+04$ & $7.14 \mathrm{E}-03$ & $1.72 \mathrm{E}-02$ & 3.83E-02 & $4.87 \mathrm{E}-02$ \\
\hline glycoleter & 74E-02 & $408 E+03$ & $1.33 E+04$ & $5.72 \mathrm{E}-02$ & $7.20 \mathrm{E}-02$ & 0.103 & 0.118 \\
\hline acetato- & $6.20 E-03$ & 227 & 745 & $5.09 \mathrm{E}-03$ & $5.63 \mathrm{E}-03$ & $6.76 \mathrm{E}-03$ & $7.31 \mathrm{E}-03$ \\
\hline oxinuez- & $5,41 E-05$ & 2.96 & 9.70 & 1.79E-05 & $5.09 \mathrm{E}-05$ & $5.72 \mathrm{E}-05$ & $6.03 \mathrm{E}-05$ \\
\hline DBP & $1.28 \mathrm{E}-02$ & $2.45 \mathrm{E}+03$ & B.03E+03 & 1.S4E-02 & $1.71 \mathrm{E}-02$ & $2.04 E-02$ & $2.20 \mathrm{E}-02$ \\
\hline butanol & $1.88 E-02$ & 365 & 2.83E+03 & 1.54E-02 & $1.71 \mathrm{E}-02$ & 2.04E-02 & $2.20 \mathrm{E}-02$ \\
\hline $\mathrm{NH3}$ & 89E-02 & 918 & $3.01 E+03$ & 7.1TE-02 & $7.77 \mathrm{E}-02$ & $9.86 \mathrm{E}-02$ & 0.112 \\
\hline $\mathrm{Fo}(\mathrm{CN}) 64$ & 0 & 0 & 0 & 0 & 0 & 0 & 0 \\
\hline
\end{tabular}

"Unknowns in tank wolids inventory are assigned by Tank Leyering Model (TLM).

tWater wt\% derived from the difference of density and total disuolved species. 


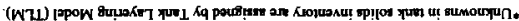

\begin{tabular}{|c|c|c|c|c|c|c|c|}
\hline zo-3927 & $20-928^{\prime} 1$ & $50-\mathrm{An}: 6$ & 20-756:5 & b2! & $60+9161$ & $20-385 !$ & $\Omega$ \\
\hline co-ALI2 & co-36: & 203018 & po-369' & c003084 & - & $\left(7^{\prime a}\right) 0^{\circ}-3 L z$ & $n_{d}$ \\
\hline (7) & , & $(T /)$ & $(\nabla /)$ & पा & $\delta / 7$ & $\mathbf{N}$ & 9जol \\
\hline $1055+$ & $0<5+$ & $10<$ & $19 \%$ & & & & \\
\hline $01-\mathrm{gzt} 2$ & $01-3561$ & 0 & 0 & $\angle 0-39 I^{\prime} L$ & Loall'1 & 0 ['-968 I I & $p q \tau-\omega]$ \\
\hline II-SIE & $\mathrm{It}-\bar{g} L \tau^{\prime} \mathcal{\varepsilon}$ & 0 & 0 & $\angle 0-3 z Z_{1}$ & $20-9601$ & II-get's & $\{v 2-400$ \\
\hline 6009721 & $60-309 \mathrm{I}$ & 0 & 0 & $90-3665$ & Lo'anc 6 & 6007851 & $2+t^{-w 10} 0$ \\
\hline $60-984+$ & $60-30 I^{\prime} 6$ & $60-327 \varepsilon$ & $60-31 C 1$ & sor $3[v]$ & 90-3012 & 60-9u' $\mathrm{x}$ & $\varepsilon v z-u v v$ \\
\hline wosis I & poste' 1 & pors10 & $50-3699$ & $2+10$ & $20-3289$ & $00 \mathrm{~B} \angle \mathrm{I} T$ & Int-urv \\
\hline $60-960^{\prime} 1$ & $01-91 L^{\prime} 8$ & OI-3SIt & O1-9261 & $90-9 e r z$ & $\angle 0-9 S L^{\prime} \varepsilon$ & $01-3269$ & $z t z-n d d$ \\
\hline por $390 t$ & Worgrg' & SOF 3826 & SOन-G59t & Asvo & to-390 L & no:3iz I & $I p Z^{-1 n_{d}}$ \\
\hline $50-9512$ & so-3lct & 90-kका ह & $90-76<t<$ & $20-38<>$ & CO-BBCL & $50-392^{\circ} 1$ & otz $2-2 d$ \\
\hline to- 3001 & noratot & $500700 \%$ & $50-9827$ & $\operatorname{Bg} z_{0} 0$ & zoossin & so-gzgl & $6 \varepsilon z^{-n_{\mathrm{d}}}$ \\
\hline $90-310^{\circ} t$ & 90-30t $\varepsilon$ & 90-315'i & $\angle 0-9104$ & 60-9268 & Eo-age'1 & $90-396 z$ & sfz-nd \\
\hline LOEMS P & LOF-30zt & LOAIIIE & $\angle 0$-3lL' $\mathrm{t}$ & so- $3\langle<|$ & no-39z $\tau$ & $\angle 0-998 \mathrm{E}$ & $\angle E Z^{-d} \mathrm{~N}$ \\
\hline 90-3zz' & $90-3 \angle+11$ & $\angle 0-706 L$ & co-ant & forgzz t & wals' & 90-azi' & $8 E Z-\Omega$ \\
\hline $80-a<210$ & roras's & poraze 6 & $80-\mathrm{al} z$ & $k-3661$ & $50-300^{\prime} \mathrm{C}$ & $80-3 \angle 255$ & $9 \varepsilon Z=\Omega$ \\
\hline 80-9he & sor-3SL'9 & 80-359' $\mathrm{c}$ & $90-9 k z z$ & Ho-ati I & $50-300^{2} \varepsilon$ & $20-9+1 / 5$ & $s \in \mathbb{Z}-\Omega$ \\
\hline $90 \mathrm{~B} L 0 \mathrm{z}$ & 90-3<91 & LOGSOG & LOFILS'S & co-a:8; & notath & 90:aLt 1 & $\hbar \varepsilon z+n$ \\
\hline L0-atis & Lo'ah't & $\angle 0-39 L^{\prime} \mathrm{T}$ & 10-9ts'6 & $\varepsilon \theta-3 z a^{\prime \prime}$ & noacsit & $\angle 0 \exists G 9 \sigma^{\prime}$ & $\varepsilon \varepsilon z-\Omega$ \\
\hline LOFAR'1 & $\angle 0700^{\prime} 1$ & $80-365 \%$ & $00-36+\tau$ & wa-399\% & sorall' & $90-\mathrm{g} 10 \mathrm{~L}$ & $2 \varepsilon 2-\Omega$ \\
\hline or-30z $\varepsilon$ & $01-\bar{g} 99^{\prime} z$ & 0 & 0 & $\angle 0-766 \mathrm{~L}$ & LO-3iz' 1 & ot-911'z & $25 z-4 t$ \\
\hline $0 t^{-3}-358 \varepsilon$ & 01-918E & 0 & 0 & goraE' I & Lo-3izz & 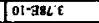 & $6 z z-41$ \\
\hline $80-3901$ & $80-350^{\circ} T$ & 0 & 0 & $50-3165$ & $90-3609$ & 100-3EO'1 & $1 \varepsilon Z^{-8} d$ \\
\hline 60-95Et & 60-3ELE & 0 & 0 & soratol & 907091 & $60-\overline{B S L Z}$ & $62 Z+\circ V$ \\
\hline 80-glo' & $80-365^{1} 1$ & 0 & 0 & sor-396s & $90-30 z^{6} 6$ & $20-88511$ & $82 z^{-84}$ \\
\hline $01-9662 \mathrm{~L}$ & $01-9829$ & 0 & 0 & sor-38L'1 & $\angle 0-\operatorname{an} C z$ & $01-90<+$ & $9 z z^{-k} y$ \\
\hline 100-385t & wo-anst & 0 & 0 & $a x+1$ & $\varepsilon 970$ & 10-70s't & $5 s 1^{-n} \mathrm{~g}$ \\
\hline to-anz't & rotalsz & 0 & 0 & 11660 & $n 10$ & nor-39t'z & $\phi S t^{-n g}$ \\
\hline 900-3006 & $90-350^{\circ} t$ & 0 & 0 & 20-39e $\mathrm{E}$ & E0-38।'s & $90-3 \angle 88$ & tsting \\
\hline 20-aEs 2 & 20-3ist & 0 & 0 & $8\{6$ & $5+1$ & $20-936 z$ & ISI-wS \\
\hline ZDE 0 & BIE0 & 0 & 0 & $60+791)$ & 061 & 9060 & 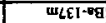 \\
\hline$B L E O$ & $\angle t E^{\prime} 0$ & 6150 & EIE० & cotasz। & 681 & neco & (E1-8) \\
\hline 90-als' & $90-36 E z$ & 0 & 0 & so-aic:s & 60-30C I & 90-30C & $m[1-5]$ \\
\hline 40-9951 & $20-3191$ & $20-399^{1}$ & $\angle 0-369]$ & 10-3ec9 & $50-358$ ' 6 & $\left.20-969^{\prime}\right]$ & $621-1$ \\
\hline $500-3601$ & $50-380^{\circ} 1$ & 0 & 0 & $20-3 \times 0 \%$ & 20-3529 & SOA 2001 & $g z I^{-0} \mathrm{~S}$ \\
\hline so-aLIt & $50-3+r \varepsilon$ & 0 & 0 & $651^{\circ} 0$ & zo-3sitz & 50-389'C & stI-qS \\
\hline 50rac6 6 & toratol & 0 & 0 & sLI0 & 20031L2 & SO-GE9 + & $w_{\mathcal{E}}[[-\mathrm{P}]$ \\
\hline $60-90<\bar{z}$ & $60-93+2$ & 0 & 0 & $90-3006$ & 90-3651 1 & 6o-asciz & $901-n y$ \\
\hline toragoI & $50-3956$ & soranc & $50-9198$ & 9r.'0 & $20-361 \mathrm{~s}$ & $50-768 \mathrm{~B}$ & $66-31$ \\
\hline 50-aEL $z$ & 5o-3iLz & 0 & 0 & 1010 0 & $20-3951$ & $5003 \angle 9 ' z$ & ш\{6-9N \\
\hline SO-G9E'E & 50-3LE E & 0 & 0 & bzio & $200-3261$ & 50-36Z' $\mathrm{E}$ & $\varepsilon 6^{-} \mathbf{Z}$ \\
\hline $00 \varepsilon^{\circ} 0$ & $z s z 0$ & 0 & 0 & $\$ 66$ & ESI & $29 Z^{\circ}$ & $06-\lambda$ \\
\hline $00 E 0$ & 2820 & 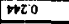 & stzo & 566 & ES! & $292^{\prime \prime}$ & $86^{-15}$ \\
\hline 90-alic 6 & $90-310^{\circ} \mathrm{L}$ & 0 & 0 & $20-359 \tau$ & E⿱ $0^{-} \exists 900^{\prime \prime}$ & $90-3569$ & $6 l^{-3} S$ \\
\hline 50-3LI I & 50-3201 & 0 & 0 & $20 \mathrm{r} 306 \mathrm{E}$ & E0-3109 & soracol & $09-0.0$ \\
\hline b0-Gsz'L & $00-3669$ & 0 & 0 & IS & LaE'O & ros 299 & $\varepsilon 9-! \mathrm{N}$ \\
\hline $90-\exists L r L$ & $90-35$ i 2 & 0 & 0 & $20-385 z$ & $50-386 \mathrm{C}$ & $90-3289$ & $65 \cdot 1 \mathrm{~N}$ \\
\hline $50-36 \tau^{2} 1$ & $50-3611$ & 0 & 0 & zo-afet & $50-3899$ & so-ghl 1 & $\nabla[-\tau$ \\
\hline notatsz & porabc z & 0 & 0 & mo & ost'o & ro- $3 \varepsilon^{2} z$ & $\varepsilon-\mathrm{H}$ \\
\hline $19 / 10)$ & $\begin{array}{l}\text { (M) } \\
\text { D } 29+\end{array}$ & 10 & $\begin{array}{l}(1 \%) \\
15 \%\end{array}$ & 0 & $8 / 19$ & ins & nwomproes \\
\hline 60.98z 9 & Eor-39s $\mathrm{S}$ & gOGE ' S & Eorg10's & $\cdots$ & $\cdots$ & co-asts & m) $2 \%^{1 n} 201$ \\
\hline $9<5$ & cst & $0.6 z$ & 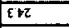 & + & - & $\angle R$ & $\%$ IN IDEM \\
\hline $8 \angle 60$ & $9 \angle \mathrm{A}^{\prime} 0$ & $\angle S \angle O$ & $1+<0$ & - & $=$ & 5820 & 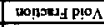 \\
\hline 281 & 821 & 951 & $t+1$ & - & - & $(x ; f)\left|c^{\prime}\right|$ & Kitpusg ying \\
\hline 20-FSE $t$ & $20-3001$ & $20-3611$ & $20-3+111$ & 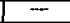 & $(x+10152 t)$ & $(\mathrm{M} y) 20-\exists b z^{\prime} \mid$ & ProT 180 \\
\hline-5 & $=$ & $=$ & 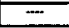 & $\cdots$ & $(103 \times 001)$ & (89) $\varepsilon 0+381 \div 9$ & 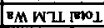 \\
\hline \multirow[t]{3}{*}{$1996+$} & $D<9$ & 1049 & 15 $56^{+}$ & & & & $\begin{array}{r}\text { oppodoud } \\
\text { jopisatqd }\end{array}$ \\
\hline & & & & (200da & & & \\
\hline & & & & $I I^{\circ}$ & & & \\
\hline
\end{tabular}




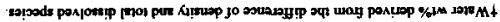

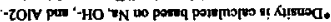

\begin{tabular}{|c|c|c|c|c|c|c|c|}
\hline $20-9501$ & $20-920 \mathrm{I}$ & co-ght6 & c0.75s' 6 & so+alt' & $(0+2)+1$ & $50-956.6$ & n \\
\hline DO-9LOE & $10-a z z L$ & worasts & $00-9656$ & 621 & - & (12) toatec 9 & $\mathrm{nd}$ \\
\hline 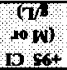 & 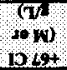 & 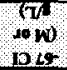 & $\begin{array}{l}(1 /) \\
10) \\
10\end{array}$ & 47 & $7 / 7$ & क $\mathbf{N}$ & Afol \\
\hline$\angle 00^{-368}$ & $\angle 0^{-}-\mathrm{G} I L^{\circ} \mathrm{I}$ & 80 & 20-76f 9 & 6620 & SOF $116 \%$ & $20-3+1$ & $t b 2$-40 \\
\hline $80-3591$ & $80-3951$ & $60-900$ & 60-3009 9 & $20-3 \angle 62$ & $90-3906$ & $80-39 b^{\prime} 1$ & chz-ш0 \\
\hline$\angle 0-96 \angle 1$ & $40-9891$ & $80-2652$ & $80-365 \mathrm{~L}$ & ozs:o & 50-50t 6 & Lo-ass 1 & $2 \not z-\omega_{2}$ \\
\hline $60-7692$ & $60-3162$ & 60-ass' I & $60-3651$ & co-gze't & 90-Gec1 & 60-7EI'Z & $\Sigma b \tau-\omega \forall$ \\
\hline $50-3 \times 2$ & So-gto't & forages & so-gitt & stl & 20-ate' & SO-JEI9 & $16 z$-ury \\
\hline $01-9669$ & or-aieg & $01-31 \angle b$ & $01-700 \%$ & EOFIII & Lo-aot $\varepsilon$ & $01-3965$ & $z+2-n d$ \\
\hline tosing & nogzt 1 & $50-9698$ & $50035+2$ & 202 & $20-3019$ & $50-3966$ & $\operatorname{lt} t-n_{d}$ \\
\hline $50-7501$ & $90-35 b^{\prime} 6$ & $90-90 L L$ & $90-9589$ & +21 & Eogle's & $90-$ GL6 8 & orz-nd \\
\hline $50.916 \mathrm{~s}$ & 50-ats 5 & $50-965 \%$ & so-geI t & 801 & Zo-atic & $50-390 \mathrm{~s}$ & $6 \leqslant \tau-n_{d}$ \\
\hline 90-358'1 & $90-7997$ & $90-3621$ & $90-3117$ & $66 z$ & 60-ast 6 & $90-a b l$ & $8 \varepsilon 2-\pi$ \\
\hline $90-35 \pi$ & 90-30I 1 & Lo-agth & Lorace 9 & 26.1 & noracs's & LO-gevo & $L \varepsilon Z-\mathrm{d} N$ \\
\hline 90-9160'! & 90-GEO'I & $10-3066$ & COASL6 & 502 & wo-act 9 & $90-3101$ & 8EZ- $\Omega$ \\
\hline 90-908' $\tau$ & $80-3922$ & 80-3092 & 00-5297 & 20-ges's & SO-369' & $80-3 u z$ & $9 E Z-\cap$ \\
\hline $80-119 \mathrm{E}$ & $80-909^{\circ} \mathrm{E}$ & 80-gst' & $80-\mathrm{G} 5 \mathrm{C}^{\prime} \mathrm{C}$ & $20-9 L I L$ & $50-7652$ & 90-GESC & SEZ-ก \\
\hline$\angle 0-3968$ & LOALB 8 & co-acs? & $\angle 0$-3LE & $L L i$ & walt's & $40-7698$ & $\operatorname{bez}=\Omega$ \\
\hline 90-769' & $90-3 C^{-} \varepsilon$ & $50-962 ?$ & 90-arg I & sos & to-asei & $90-4867$ & $\varepsilon \varepsilon Z-n$ \\
\hline 90-AIZ I & LO-ASB 6 & co-49s 5 & L0-3ntt & 851 & wostes & $40-4946$ & $z \xi z-\pi$ \\
\hline 80-36t I & eto-gst I & $60-3851$ & 60-30z's & $20-7102$ & 90-asz'9 & +0-910 I & $\tau \varepsilon \tau-q L$ \\
\hline $60-3065$ & $60-789 \%$ & 60-35s 2 & 60-9191 & co-g0z 6 & $90-302 \tau$ & 60 ats $c$ & $622-41$ \\
\hline 60-3I9's & 60-350's & 60-4les? & 60 319 & (50-9606 & $90-78 L Z$ & 60-albt & $152-8 d$ \\
\hline $60-9811$ & $60-9301$ & $01-1989$ & 0l-3989 & c0-9ะ6 1 & $40-3909$ & $01-95<6$ & $\angle Z Z-5 V$ \\
\hline$\angle 0-7652$ & Lo-aEOZ & Lo-190't & 80-Azt' 9 & $\angle O E O$ & $50-9656$ & L0-GIS'1 & $8 z 2-5 y$ \\
\hline $01-968$ I & $01-3 z L 1$ & $019+01$ & ol-G10 l & to-gsI & $80-3656$ & $0 \mathrm{f}-\mathrm{ak} \mid$ & $9 Z 2-8 y$ \\
\hline $60-7082$ & 100-gtg 2 & $10-3 i 2 t$ & no-zㅔㄴ? & $\cos$ & Est'o & 10-39+2 & SSI-ng \\
\hline to-ass 8 & $100-3 \angle 62$ & no-30s 2 & $60-\cos z$ & co+azc I & 5000 & wo-gts 9 & t5I In] \\
\hline $900 \mathrm{GZL}$ & $90 \mathrm{rath}$ & $90-3<11^{\circ}$ & $90-341 \mathrm{Z}$ & st's & $60-365 \tau$ & 90-391 ' & tsI-ng \\
\hline $20-329 \mathrm{i}$ & $20-912 t$ & $60-390 \mathrm{~L}$ & $50-790 \mathrm{~L}$ & $10+3152 \tau$ & 682 & $20-3 e \pi i$ & ISI-US \\
\hline 1620 & 820 & $\cot 0$ & $50 z^{\prime} 0$ & $50+79 \varepsilon^{\prime} s$ & 191 & 590 & 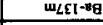 \\
\hline $\cos 0$ & 5620 & $592^{\circ}$ & 1520 & $50+7 \angle 9 S$ & $D C l$ & $6 L 20$ & $\angle \varepsilon[-8]$ \\
\hline $90-35 S E$ & 90-301t & 90-ale? & $90 \mathrm{as6} 1$ & gs: & $50-3021$ & $90-\mathbf{Z I} \ell \tau$ & $\forall E I-80$ \\
\hline $200 \arg 9$ & 203165 & Lo-95or & LOA91 2 & $10 \pi$ & rostol E & Lo-7966 & $621-1$ \\
\hline 90-351 2 & 90-azE'9 & so-atot & $90-3 \times 0^{\prime} \varepsilon$ & 111 & โ0-76£ \& & $90-3 b+5$ & $921-45$ \\
\hline t)-H6L' I & 60-39L I & $50-32 L$ & So-78 + & ISE & 2010 & to-35Li & $521-9 S$ \\
\hline wate & Wo-30I' I & $50-39 t^{\circ}$ & so-59t: & 601 & $20-311 \mathrm{~s}$ & so-ant 6 & wEI I PJ \\
\hline $60^{-9}=\angle 8$ & $60-9162 \mathrm{~L}$ & 60-3zot & $60-92+\varepsilon$ & $z_{0}-35 \theta^{-1}$ & $90-31+b$ & $60-3+12$ & $90 \mathrm{I}^{-\pi} \mathrm{y}$ \\
\hline WOGES'E & 10-3 $\angle 0$ \& & 60301t & $00 \mathrm{~s} 9 \mathrm{I}$ & sts & 1910 & roges 2 & $66^{-01}$ \\
\hline 50.789 i & $50-36 b^{-1}$ & $90-3 \mid \tau^{2} L$ & 90-alz'L & 192 & $50-386 \mathrm{~L}$ & 50-982' & IIE6-9N \\
\hline 50-Ales? & $50-\mathrm{g} 90 \mathrm{z}$ & $90-3596$ & $90-9596$ & $65 \varepsilon$ & $20-3011$ & So-slul & $56-\pi$ \\
\hline 8210 & nzIO & 20asts & 20 -1St 9 & sotan 2 & $9+2$ & 0210 & $06-\lambda$ \\
\hline Szlo & 1210 & 9110 & $\tau \pi 10$ & $50+9 t b 2$ & 96 & 0210 & $06^{-15}$ \\
\hline 90-ADLt & 90-381 t & $90-900 \mathrm{Z}$ & $90-300 \mathrm{z}$ & reL & co- $-9+z z$ & 90-909 C & $6 t-3 S$ \\
\hline 50-39: $\mathrm{t}$ & $50-3600$ & $50-3 \mathrm{el}$ & $50-9611$ & 918 & $20-3002$ & SOद्वा0 & $09-00$ \\
\hline torazt $z$ & No-g9e' 2 & WoBet & no-sel 1 & $89 t$. & $\cos 0$ & $60-305 z$ & {$[9-1 \mathrm{~N}$} \\
\hline $90-\mathrm{a} c+2$ & $90-a+b z$ & $907 \times 11$ & $90-3411$ & $L E$ & $50-39+1$ & 90-356 2 & $6 s-1 \mathrm{~N}$ \\
\hline SOAEL & 50-789 E & so-3iti & so-alli & $L E$ & $20-352 z$ & so-acet & $D I-3$ \\
\hline $10-30 L \tau$ & $10-3092$ & 60-GzE' I & rosze I & 215 & $\angle S 10$ & to-azs 2 & E-H \\
\hline $\begin{array}{l}\text { (1/D) } \\
15 \text { s }\end{array}$ & $\begin{array}{l}(\mathrm{TO}) \\
1 \mathrm{~L})\end{array}$ & $\begin{array}{l}(1 / 9) \\
10 \angle 9\end{array}$ & $\begin{array}{l}\text { (1/9) } \\
1096\end{array}$ & $\mathrm{DO}$ & n/137 & $\mathrm{T} / \mathrm{A}$ & Anmunfores \\
\hline 101 & tge'0 & 8190 & $16 \% 0$ & $=$ & - & OSLO & m) $2 \% 14$ \\
\hline $09 \varepsilon$ & $2+\varepsilon$ & $E I E$ & IOE & $\cdots$ & - & $L \tau \varepsilon$ & $+\% 1 \mathrm{~m}$ soje $\mathrm{M}$ \\
\hline 591 & E9 I & 851 & 951 & - & - & $(\infty / 6) 191$ & 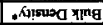 \\
\hline $25 \%$ & Dip & $91 \%$ & $20 \%$ & - & (14/И) $00+3<<1)$ & $(n \geqslant 0$ DE & PEOT JEeH \\
\hline- & $=$ & 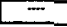 & $=$ & 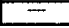 & $(\operatorname{Rof} ; \in[S)$ & $(8 y) 90+$ ILZ' $\mathrm{f}$ & M WWS $1810 \mathrm{~L}$ \\
\hline \multicolumn{7}{|c|}{ ID $96+1969+10<9+1256$} & 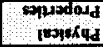 \\
\hline \multicolumn{8}{|c|}{ 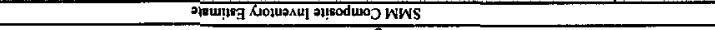 } \\
\hline & & & $01-x S^{-I t z}$ & 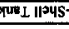 & plints & & \\
\hline
\end{tabular}


HDW Model Rev. 4

\begin{tabular}{|c|c|c|c|c|c|c|c|}
\hline \multicolumn{8}{|c|}{ Single-Shell Tank 241-SX-106 } \\
\hline & \multicolumn{7}{|c|}{ Total Invertory Estimate" } \\
\hline $\begin{array}{l}\text { Physicat } \\
\text { Properties }\end{array}$ & 18 & & & $-95 \mathrm{CI}$ & $-67 \mathrm{Cl}$ & $+67 \mathrm{CI}$ & $+95 \mathrm{Cl}$ \\
\hline Total Waste & $3.27 \mathrm{E}+06(\mathrm{~kg})$ & (938 kgal) & $\cdots$ & $\ldots$ & $=$ & - & $\ldots$ \\
\hline Heat Loed & $4.31(\mathrm{~kW})$ & (1.4TE+04 BTU/hr) & $\cdots$ & 405 & 4.18 & 4.45 & 4.58 \\
\hline Bulk Density & $1.61(\mathrm{~g} / \mathrm{cc})$ & $\ldots$ & $\ldots$ & 1.56 & 1.58 & 1.63 & 1.65 \\
\hline & & & & & & & \\
\hline Water wt\% & 32.7 & - & $\ldots$ & 30.1 & 31.3 & 34.2 & 36.0 \\
\hline TOC w $\% C(w$ & 0.749 & - & $\ldots$ & 0.490 & 0.617 & 0.881 & 1.01 \\
\hline $\begin{array}{l}\text { Rodioleries } \\
\text { Conitituests }\end{array}$ & $\mathrm{Cln}$ & LCV $\quad:$ & Cl & $\begin{array}{l}95 \mathrm{CI} \\
(\mathrm{C} / \mathrm{L})\end{array}$ & $\begin{array}{l}67 \mathrm{CI} \\
\text { (CVt) }\end{array}$ & $\begin{array}{l}+67 \mathrm{Cl} \\
(\mathrm{Cl} / \mathrm{L})\end{array}$ & $\begin{array}{l}+8 \mathrm{Cl} \\
\text { (c) }\end{array}$ \\
\hline $\mathrm{H}-3$ & $2.52 \mathrm{E}-04$ & 0157 & 513 & 1.32E-04 & $1.32 \mathrm{E}-04$ & $2.60 \mathrm{E}-04$ & 2.70E-04 \\
\hline C.14. & $3,62 \mathrm{E}-05$ & 2.25E-02 & 73.7 & I.11E-05 & I.11E-0s & $3.68 \mathrm{E}-05$ & 1.73E-05 \\
\hline $\mathrm{Ni}-59$ & 2.36E-06 & $1.47 \mathrm{E}-0,3$ & 4,80 & $1.18 E-06$ & $1.10 \mathrm{E}-06$ & $2.42 E-06$ & $247 \mathrm{E}-06$ \\
\hline $\mathrm{Ni}-63$ & 2.31E-04 & 0.144 & 470 & $1,14 \mathrm{E}-04$ & $1.14 E-04$ & $2.37 \mathrm{E}-\mathrm{A4}$ & $2.43 \mathrm{E}-04$ \\
\hline $\mathrm{Co}-60$ & $4.01 \mathrm{E}-05$ & $2.49 \mathrm{E}-02$ & 81.6 & $1.13 \mathrm{E}-05$ & $1.13 \mathrm{E}-05$ & 4.08E-05 & $4.16 \mathrm{E}-05$ \\
\hline Se-79. & $3.61 \mathrm{E}-0.0$ & 2.24E-03 & 7.35 & $2.00 \mathrm{E}-06$ & $2.00 \mathrm{E}-06$ & $4.19 E-06$ & $4.74 \mathrm{E}-06$ \\
\hline Sr-90 & 0.120 & 74.8 & $2.45 E+05$ & 0.112 & 0.116 & 0.124 & 0.128 \\
\hline$Y-90$ & 0.120 & 74.8 & $2.45 \mathrm{E}+05$ & 6.49E-02 & $6.49 \varepsilon-02$ & 0.124 & 0.128 \\
\hline $\mathrm{Zr}-93$ & $177 \mathrm{E}-0 \mathrm{~S}$ & $1.10 \mathrm{E}-02$ & 36.1 & $9.69 \mathrm{E}-06$ & $9.695-06$ & 2.06E-05 & $234 \mathrm{E}-05$ \\
\hline $\mathrm{Nb}-93 \mathrm{~m}$ & $1.29 \mathrm{E}-05$ & $8.00 \mathrm{E}-03$ & 262 & $7.25 \mathrm{E}-06$ & $7.25 \mathrm{E}-06$ & 1.49E-0S & $160 \mathrm{E}-05$ \\
\hline Te-99 & 25:E-OA & 0.160 & 525 & 1.64E-04 & $2.10 E-04$ & $3.06 \mathrm{E}-04$ & $3.53 \mathrm{E}-04$ \\
\hline Ru-106 & $7.13 \mathrm{E}-09$ & 4.44E-06 & $1.45 \mathrm{E}-02$ & 3.42E-09 & $3.42 \mathrm{E}-09$ & 7.96E-09 & 8.72E- -09 \\
\hline $\mathrm{Cd}-113 \mathrm{~m}$ & 9.27E-05 & $5.76 E-02$ & 189 & $4,46 E-05$ & $4.46 \mathrm{E}-05$ & $1.10 \mathrm{E}-04$ & 1.27E-04 \\
\hline$S b-125$ & $1.72 \mathrm{E}-04$ & 0.107 & 351 & 4.78E-05 & $4.78 \mathrm{E}-03$ & $1.76 \mathrm{E}-04$ & $1.79 \mathrm{E}-04$ \\
\hline$S n-126$ & $5,45 E-06$ & $3,39 \mathrm{E}-03$ & 11.1 & $3.05 \mathrm{E}-06$ & $3.05 \mathrm{E}-06$ & 6.32E-06 & $7.16 \mathrm{E}-06$ \\
\hline $1-129$ & $497 \mathrm{E}-07$ & $3.09 \mathrm{E}-\mathrm{A}$ & 1.01 & $3.16 \mathrm{E}-07$ & 4.05E-07 & $5.91 \mathrm{E}-07$ & $6.81 \mathrm{E}-07$ \\
\hline$C_{2}-134$ & $2.73 \mathrm{E}-06$ & $1.70 \mathrm{E}-03$ & 5.57 & 1.95E-06 & 2.30E-06 & $3.13 \mathrm{E}-06$ & $3.53 \mathrm{E}-06$ \\
\hline Cs-137 & 0.279 & 174 & $5.68 \mathrm{E}+05$ & 0.251 & 0.265 & 0.293 & 0.307 \\
\hline Ba-137m & 0.264 & 164 & $5.38 \mathrm{E}+05$ & 0.207 & 0.207 & 0278 & 0.291 \\
\hline $\mathrm{Sm}=151$ & $1.2 \mathrm{E}-02$ & 790 & $2.59 \mathrm{E}+04$ & $7,00 \mathrm{E}-03$ & $7.09 E-03$ & $1.47 \mathrm{E}-02$ & $1.67 \mathrm{E}-02$ \\
\hline Ev-152 & $4.17 \mathrm{E}-06$ & 2.59E-03 & 8.49 & $2.18 \mathrm{E}-06$ & 2.18E-06 & 4.45E-06 & 4,73E-06 \\
\hline Eu-154 & $651 \mathrm{E}-04$ & 0.405 & $1.33 E+03$ & $2.50 \mathrm{E}-04$ & 2.50E-04 & $7.96 \mathrm{E}-04$ & 8.53E-04 \\
\hline En-155 & $2.46 \mathrm{E}-04$ & 0.153 & \$0I & $1.2 \pm E-04$ & 1.28E-04 & $2.64 \mathrm{E}-04$ & $2.81 \mathrm{E}-04$ \\
\hline Re-226 & $1.55 \mathrm{E}-10$ & 9.62E-08 & 3. ISE-0A & $1,05 \mathrm{E}-10$ & $1.05 \mathrm{E}-10$ & $1.73 \mathrm{E}-10$ & $1,90 \mathrm{E}-10$ \\
\hline $\mathbf{R a - 2 2 8}$ & $1.51 E-07$ & $9.37 \mathrm{E}-05$ & 0.307 & $6.31 \mathrm{E}-0 \mathrm{~s}$ & $1.06 \mathrm{E}-07$ & $203 \mathrm{E}-07$ & 2.58E-07 \\
\hline Ac-227 & $9.73 E-10$ & $6,08 \mathrm{E}-07$ & $1.99 \mathrm{E}-03$ & $6.90 \mathrm{E}-10$ & $6.90 \mathrm{E}-10$ & $1.08 \mathrm{E}-09$ & I.18E-09 \\
\hline $\mathrm{Pu}-23 !$ & 4.4ME-09 & 2.79E-06 & 9.13E- 33 & $288 \mathrm{E}-09$ & 2.88E-09 & S.06E-09 & $5.62 \mathrm{E}-09$ \\
\hline Th-229 & $3.53 \mathrm{E}-69$ & $2.20 \mathrm{E}-06$ & $7.20 \mathrm{E}-03$ & $1.61 \mathrm{E}-09$ & 2.55E-09 & 4.60:-09 & $5.89 \mathrm{E}-09$ \\
\hline Th-232 & $1.00 \mathrm{E}-08$ & $6.24 \mathrm{E}-\mathrm{-06}$ & 20.01E-02 & $5.19 \mathrm{E}-09$ & $7.56 \mathrm{E}-09$ & $1.25 \mathrm{E}-08$ & $1.49 \mathrm{E}-08$ \\
\hline U.232 & $7.75 \mathrm{E}-07$ & 4.82E-04 & 1.58 & $4.24 E_{-07}$ & 5.95E-07 & $9.83 \mathrm{E}-07$ & 1.21E-06 \\
\hline $\mathrm{U}-233$ & $2.97 \mathrm{E}-06$ & I. BSE-03 & 6.05 & $1.63 \mathrm{E}-06$ & $228 \mathrm{E}-06$ & $3.77 \mathrm{E}-\infty$ & $4.62 \mathrm{E}-06$ \\
\hline $\mathrm{U}-\overline{234}$ & 8.70E-07 & 5.4JE-04 & 1.7 & $8.38 \mathrm{E}-07$ & 8.\$3E-0T & 8.88E-07 & $8,97 \mathrm{E}-07$ \\
\hline U-235 & $3.53 \mathrm{E}-0 \mathrm{~s}$ & 2.19E-0S & $7.192-02$ & 3.39E-08 & $346 E-08$ & $3.61 \mathrm{E}-0 \mathrm{~B}$ & $3.64 E-04$ \\
\hline $\mathrm{U}-236$ & $2.73 \mathrm{E}-08$ & $1,70=-05$ & 5.55E-02 & $2.63 \mathrm{E}-08$ & $2.68 E-08$ & 2.77E-0s & 2.81E-08 \\
\hline U-238 & I.01E-06 & $6.27 \mathrm{E}-04$ & 2.05 & 9.7SE-07 & 9.91E-07 & $1.03 \mathrm{E}-06$ & $1.03 \mathrm{E}-06$ \\
\hline$N_{p}-237$ & $9.42 \times-07$ & $5.86 \mathrm{E}-04$ & 1.92 & $6.36 \mathrm{E}-07$ & $7.85 E-07$ & 1.10E-06 & $1.25 \mathrm{E}-06$ \\
\hline Pu-238 & $1.47 \mathrm{E}-06$ & $9.16 \mathrm{E}-04$ & 3.00 & 1.11E-06 & $129 \mathrm{E}-06$ & $1.66 \mathrm{E}-06$ & $1,83 \mathrm{E}-06$ \\
\hline$P_{u v-239}$ & 5.06E-05 & 3.15E-02 & 103 & 4.14E-05 & $459 \mathrm{E}-05$ & $5,53 \mathrm{E}-0 \mathrm{~s}$ & $5.98 \mathrm{E}-05$ \\
\hline$P u-240$ & 8.5EE-06 & $5.34 \mathrm{E}-0 \mathrm{3}$ & 17.5 & $6.86 \mathrm{E}-06$ & $7.71 E-06$ & $945 E-06$ & $1.03 \mathrm{E}-05$ \\
\hline $\mathrm{Pu}-241$ & $9.95 \mathrm{E}-05$ & 6.19E-02 & 203 & $7.50 \mathrm{E}-05$ & $70 \mathrm{E}-0 \mathrm{~S}$ & $1.12 \mathrm{E}-04$ & $1.24 E-04$ \\
\hline$P u-242$ & $3.46 \mathrm{E}-10$ & $3.40 \mathrm{E}-07$ & $1.11 \mathrm{E}-03$ & $4.00 \mathrm{E}-10$ & $472 \mathrm{E}-10$ & $6.21 \mathrm{E}-10$ & $6.93 \mathrm{E}-10$ \\
\hline Am-241 & $0.14 \mathrm{E}-0 \mathrm{~s}$ & $3.82 \mathrm{E}-02$ & 125 & 4.44E-05 & $5.27 \mathrm{E}-05$ & $7.01 \mathrm{E}-05$ & $7.85 \mathrm{E}-05$ \\
\hline Am-243 & $2.13 \mathrm{E}-09$ & $1.32 \mathrm{E}-06$ & 4.34E-03 & $1.59 \mathrm{E}-99$ & $1.85 \mathrm{E}-09$ & $2.42 \mathrm{E}-09$ & 2.69E-09 \\
\hline $\mathrm{Cm}-242$ & $1.5 \% \mathrm{E}-07$ & $9.78 \mathrm{E}-05$ & 0.320 & $7.58 \mathrm{E}-0 \mathrm{0}$ & $7.58 \mathrm{E}-08$ & $1.69 \mathrm{E}-07$ & $1.79 \mathrm{E}-07$ \\
\hline Cm-243 & $1.46 \mathrm{E}-08$ & $9.06 \mathrm{E}-06$ & $2.97 \mathrm{E}-02$ & $6.79 \mathrm{E}-49$ & $679 \mathrm{E}-09$ & $1.55 \mathrm{E}-08$ & $1.65 \mathrm{E}-08$ \\
\hline $\mathrm{Cm}_{\mathrm{m}-244}$ & $1,44 E-07$ & 9.95E-05 & 0.293 & $6.3 \pm E-0.5$ & 6.38E-08 & 1.গ1E-07 & $1.88 \mathrm{E}-07$ \\
\hline Totol: & $\mathbf{M}$ & 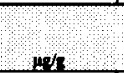 & $\mathrm{k}$ & $\begin{array}{l}9 \mathrm{CC} \\
\mathrm{Mpr} \\
(\mathrm{CH})\end{array}$ & $\begin{array}{l}\text { SC } \\
(\mathrm{Mor} \\
(/ \mathrm{l})\end{array}$ & $\begin{array}{l}+67 \text { Ct } \\
\text { (Aor } \\
\text { /4) }\end{array}$ & $\begin{array}{l}+95 \mathrm{Cl} \\
(\mathrm{Mms} \\
\mathrm{aL})\end{array}$ \\
\hline Pu & $6.35 \mathrm{E}-04(\mathrm{~g} L)$ & - & 1.29 & 4.61E-04 & $5.46 \mathrm{E}-04$ & $7.23 \mathrm{E}-04$ & 8.08E-04 \\
\hline V & $9.96 \mathrm{E}-03$ & $1.47 \mathrm{E}+03$ & $4.83 \mathrm{E}+03$ & 9.55E-03 & $9.75 E-03$ & $1.02 \mathrm{E}-02$ & 1.03E-02 \\
\hline
\end{tabular}




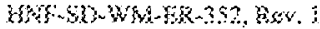

$\infty$
$\frac{\infty}{3}$ ?
$\frac{1}{4}$ ?
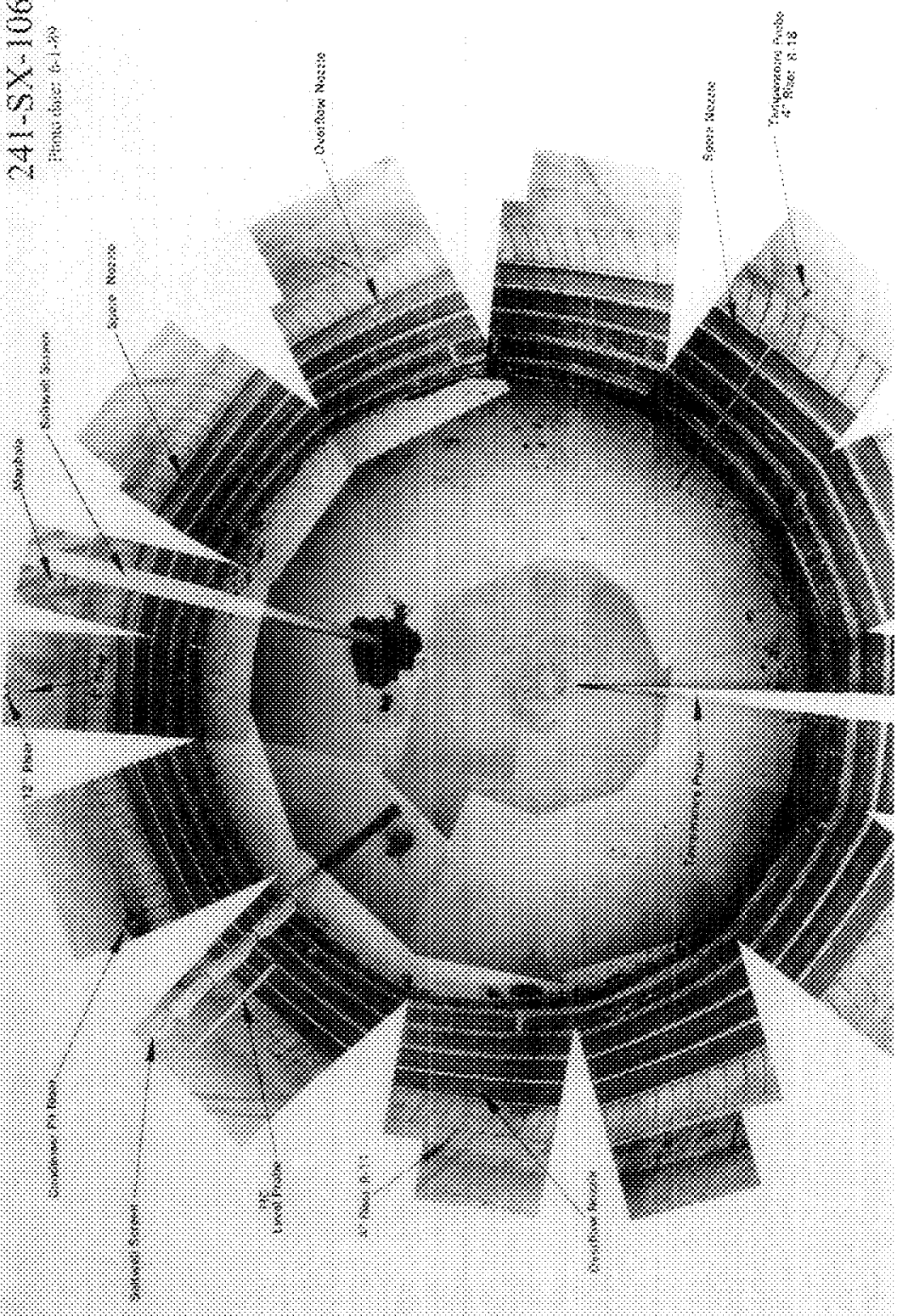
TANK 241-SX-107 SUMMARY

\begin{tabular}{|c|c|c|c|}
\hline \multicolumn{2}{|c|}{ TANK HISTORY } & \multicolumn{2}{|c|}{ TANK DESCRIPTION } \\
\hline Entered Service & 2nd qtr 1956 & Diameter & $75 \mathrm{ft}$ \\
\hline Removed from Service & - & Bottom Shape & Dish \\
\hline Inactive & - & Nominal Capacity & $1,000,000 \mathrm{gal}$ \\
\hline Watch Lists & none & Cascade Tank & to $241-S X-108$ \\
\hline Integrity & Assumed Leaker & Total Risers & 29 \\
\hline Assumed Leaker & 1964 & \multicolumn{2}{|c|}{$\begin{array}{l}\text { WASTE VOLUME (HANLON 1996i) } \\
\end{array}$} \\
\hline Interim Stabilization (IS) & Oct 1979 & Total Waste Volume & $104,000 \mathrm{gal}$ \\
\hline Partial Interim Isolation (PI) & - & Waste Type & NCPLX \\
\hline Intrusion Prevention (IP) & Sept 1982 & Drainable Interstitial Liquids & $5,000 \mathrm{gal}$ \\
\hline \multicolumn{2}{|c|}{\begin{tabular}{|c|} 
TENTATIVELY AVAILABLE RISERS \\
\end{tabular}} & Pumpable Liquids & $0 \mathrm{gal}$ \\
\hline Riser Number(s) & Size & Saltcake & $0 \mathrm{gal}$ \\
\hline $6,16,17$ & 12 in & Sludge & $104,000 \mathrm{gal}$ \\
\hline $13 \mathrm{~A}$ & 18 in & Supernatant & $0 \mathrm{gal}$ \\
\hline \multicolumn{2}{|c|}{ TANK TEMPERATURE } & \multicolumn{2}{|c|}{ INTERIOR PHOTOGRAPHS } \\
\hline Average Tank Temperature & $145^{\circ} \mathrm{F}$ & Date & March 6, 1987 \\
\hline Maximum Temperature & $177.4^{\circ} \mathrm{F}$ & Montage Number & $94041033-34 \mathrm{CN}$ \\
\hline Date & Nov 7, 1994 & Photo Set Number & 8701420 \\
\hline Elevation from tank bottom & $0.5 \mathrm{ft}$ & \multicolumn{2}{|c|}{\begin{tabular}{|l} 
WASTE SURFACE LEVEL \\
\end{tabular}} \\
\hline Riser Number & 10 & Devices & Manual Tape \\
\hline Minimum Temperature & $109^{\circ} \mathrm{F}$ & Max Level & 41.75 in \\
\hline Date & $\operatorname{Dec} 4,1992$ & Date & April 4, 1994 \\
\hline Elevation from tank bottom & $4.33 \mathrm{ft}, 5.0 \mathrm{ft}, 5.66 \mathrm{ft}$ & Min Level & 35 in \\
\hline Riser Number & 10 and 14 & Date & Oct 2, 1996 \\
\hline
\end{tabular}




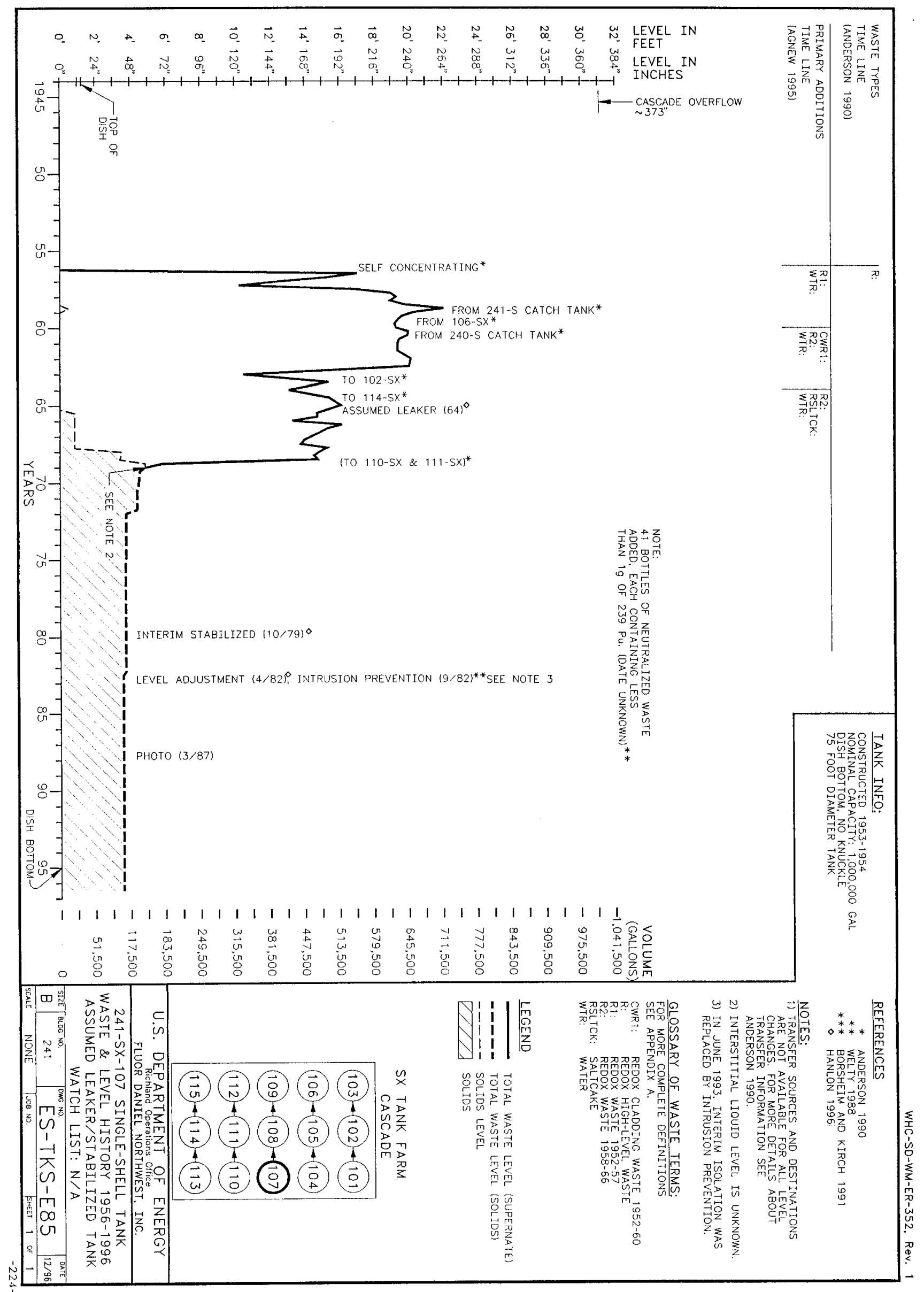



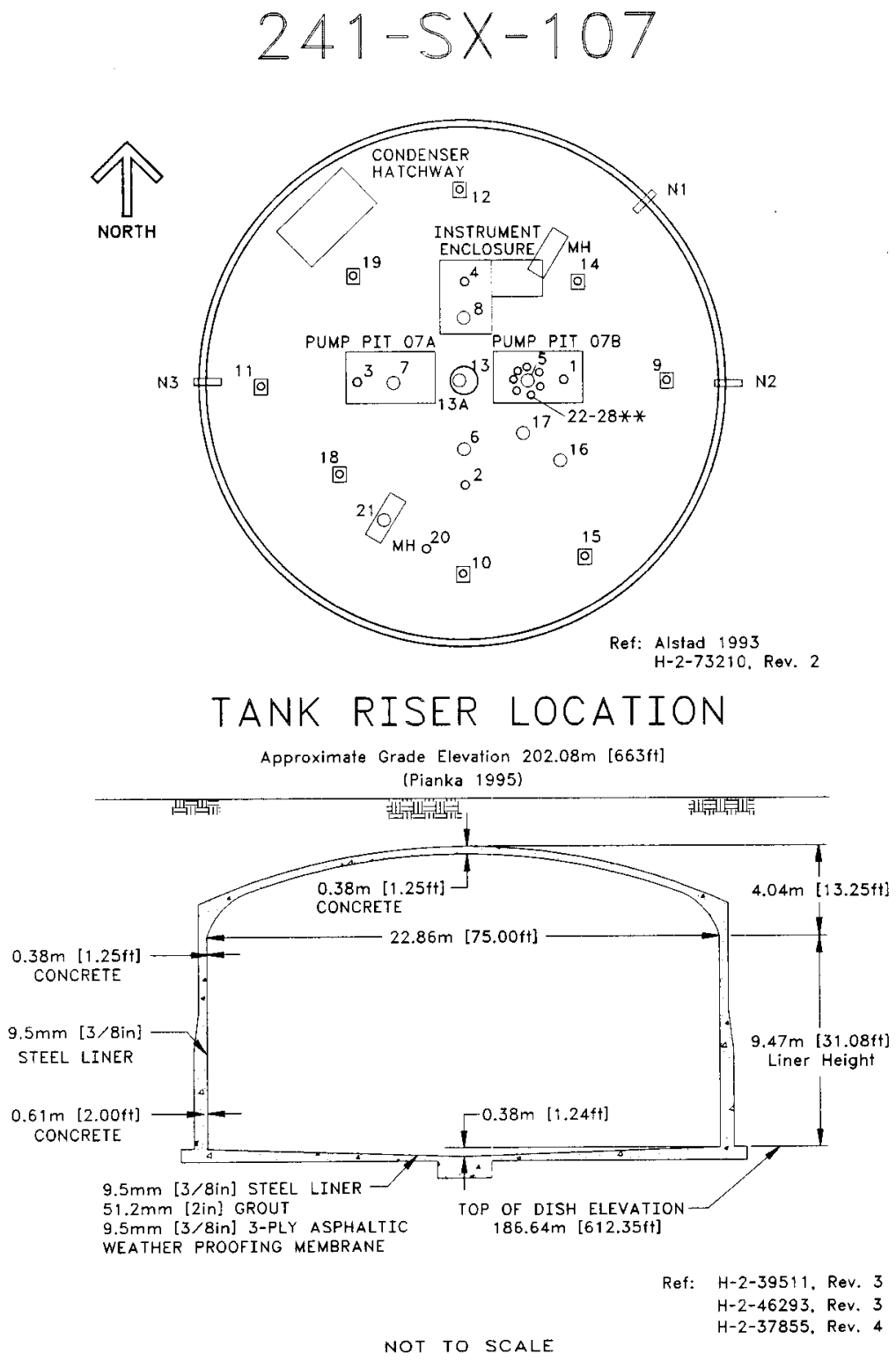
HNF-SD-WM-ER-352, Rev. 1

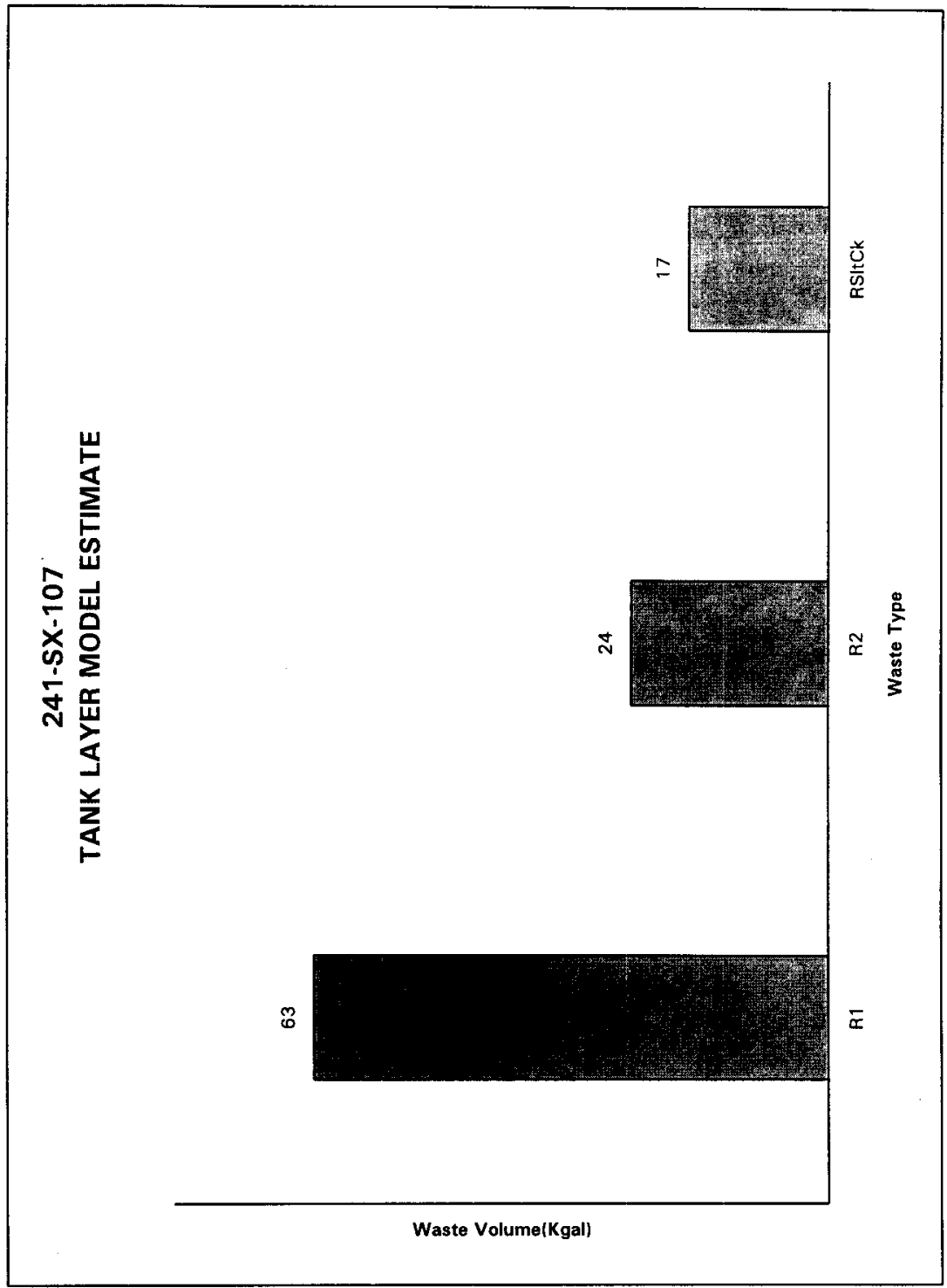




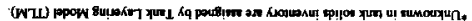

\begin{tabular}{|c|c|c|c|c|c|c|c|}
\hline 0 & 0 & 0 & 0 & 0 & 0 & 0 & $t g(N))_{1}$ \\
\hline 9820 & $16+0$ & 0410 & Esi'o & $50+3081$ & $z 0+119 z$ & $69 \sigma^{\prime} 0$ & $\mathrm{CHN}$ \\
\hline 50-9029 & so-abls & s0-a9l's & so-796 & s5l & $s z z$ & g0-GIES S & jouming \\
\hline $50-4029$ & so-ates & $50-791$ is & 50-996 & GE: & 659 & 50-aIEs & d80 \\
\hline$z 1-36 \varepsilon t$ & $21-766 \mathrm{C}$ & zl-gtb's & 21-aIz' & co-go'I & LOACOI & $z I-g z 9 \varepsilon$ & 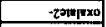 \\
\hline $90-9966$ & $90-3266$ & $90-3 E 98$ & $90-36+8$ & $\cos 0$ & 9620 & $90-a L C$ & -2010000 \\
\hline$\hbar 0-\operatorname{ss} 2 t$ & $100-3501$ & $50-9899$ & s0-385' & $6 r 2$ & $69:$ & so-aspig & эมा \\
\hline 90 -azz' & $90-3857$ & 90-ate'1 & 40-7129 & 6020 & noro & 90-abs I & -CYLABH \\
\hline $90-39 t^{2} \mathrm{E}$ & 9003527 & $90-1202$ & $90-\mathrm{al} 9 \mathrm{I}$ & +920 & SPE 0 & go-ace $z$ & -bY.Lda \\
\hline so-sos 9 & so-ast'9 & so-368's & 50-70s's & stit & $8+9$ & $50-366.5$ & $-\angle \operatorname{COSH} 9 \mathrm{O}$ \\
\hline 6210 & $20-389 L$ & 20.9064 & $20-36<\varepsilon$ & S16 & co+gef I & $20-9959$ & -10 \\
\hline $50-9989$ & so-iut? & so- $39 \mathrm{p}$ ' & s0-356t & EEto & 0690 & so-964's & -3 \\
\hline $9 \varepsilon 0_{0}$ & 8220 & $20-3 c t c$ & 20-GzZ E & cot 305 I & $20+76 I^{\prime} z$ & 9810 & (-Zro!s w) !s \\
\hline $20-9261$ & $20-326.1$ & $20-2151$ & $20-\mathrm{abl} 1$ & tos & 526 & $20-30<1$ & $-z+o s$ \\
\hline $50-a E z 8$ & $50-3 L 5 L$ & $50-950 \%$ & $50-35<9$ & $z L z$ & $56 \mathrm{E}$ & $S O-B L Z L$ & $-c t+0 d$ \\
\hline 6050 & $\angle 820$ & $1+20$ & 6120 & co+3itis & $50+390^{\circ} 6$ & 1920 & $-2 \varepsilon 00$ \\
\hline 461 & 261 & 501 & 1520 & $10+318 z$ & $10+7600$ & $\$ 51$ & $-20 \mathrm{~N}$ \\
\hline $4 I 1$ & 106 & 5690 & 5610 & $60+3166$ & so+goti & 906 & $-\mathrm{EON}$ \\
\hline$\angle 8 Z$ & $L L z$ & 9.52 & ist & SO+BSLI & $50+3652$ & g'st & $-\mathrm{HO}$ \\
\hline $20-9241$ & $20-a z L t$ & $20-911$ I & co-ants & $\angle \pi$ & OEE & $20-3981$ & $+x$ \\
\hline 2080 & 9020 & $0 b z 0$ & $9 t 20$ & CO+asit & $20+3 \varepsilon_{0} 9$ & $\operatorname{csz} 0$ & +2 \\
\hline 50-3901 & 90-38c'6 & $90-3869$ & $90 \mathrm{aths}$ & Cato & $\angle S O O$ & $90-9818$ & $+t+0 w$ \\
\hline 0 & 0 & 0 & 0 & 0 & 0 & 0 & +215 \\
\hline $20-39 r^{\prime} 9$ & $20-a 2 E 9$ & $20-399 \mathrm{~s}$ & $20-3815$ & $60+36 \mathrm{C}_{1}$ & $20+320 z$ & $z 0-3 \subseteq 09$ & $+2 ! N$ \\
\hline so-get to & so- $3 \angle t \varepsilon$ & 50-G612 & So-zos' 1 & $m ; z$ & ZE'E & $50-308 \mathrm{Z}$ & $+29 \mathrm{~d}$ \\
\hline$\angle \theta-3 i z \mid$ & 60-381' T & $\angle 0-3 \angle 0 !$ & $40-3601$ & 60-750\% & c0-a98's & $40-g Z I I$ & $z\left(\mathrm{HO}_{\mathrm{O}} \mathrm{O} \mathrm{Z} \mathrm{X}\right) \mathrm{Z}$ \\
\hline co-aEI $z$ & $40-3+61$ & LO-399'I & $\angle 0-995 \mathrm{I}$ & $20-36 E^{\prime} 1$ & zo-gro'z & $\angle 0-79 L^{\circ} 1$ & $+\tau^{-1} \mathrm{H}$ \\
\hline$z 1-39 \varepsilon^{\prime} \varepsilon$ & $\tau 1^{-390^{\prime} \varepsilon}$ & ZI- $329 \mathrm{z}$ & $z 1 \cdot 3 z=z$ & $L 0-3|s|$ & $\angle 0 \circ g 0 z z$ & $21-3<L 2$ & $+\mathrm{ENT}$ \\
\hline $90-3961$ & $90-$ abz I & $90-9501$ & $40-760^{\circ} 6$ & $20-3+26$ & tEIO & 90-3zI'I & $+\$ ! g$ \\
\hline $16 L^{\circ} 0$ & 9900 & 8510 & IEI 0 & $50+358\}$ & $60+3855$ & $\angle 8 I 0$ & $+\varepsilon 10$ \\
\hline s21 & 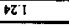 & 271 & $2 z 1$ & $70+\operatorname{gec} L z$ & $10+3 \$ 65$ & $b z^{\prime} !$ & $\left(e_{d} \mid m a\right)+e_{i}$ \\
\hline ozt & 569 & 809 & 659 & $n+321<$ & $50+310[$ & 119 & + EIV \\
\hline$\tau b 1$ & 121 & $1 E \mathrm{~s}$ & $00 ' s$ & $0+B i \varepsilon c$ & $50+3901$ & 10.8 & $+\pi N$ \\
\hline (1/Profi) & $(1 / 0<401)$ & (1904) & $\begin{array}{l}(\mathrm{T} / \mathrm{P} 0 \mathrm{in}) \\
196\end{array}$ & 84 & atadd & Tayotu & spenpres \\
\hline $70-9566$ & $10-3<16$ & $10-3052$ & $00-302 L$ & - & - & $00-a z+8$ & A) $2 \%$ a 201 \\
\hline$\nabla 6 \mathbf{6}$ & $58 E$ & 0 . & 821 & - & - & $\varepsilon 62$ & $\% 1 n$ sOlE M \\
\hline$\varepsilon \angle 0$ & $0 \mathrm{t} \angle 0$ & $56 \varepsilon^{\prime} 0$ & $00 \varepsilon 0$ & $\square$ & - & 8850 & wopores $p ! O \Lambda$ \\
\hline boz & 961 & 191 & 651 & - & - & $(\infty / A) S L I$ & 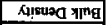 \\
\hline 896 & 596 & ES 6 & 806 & - & 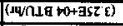 & $(m \geq 156$ & p107 1EOH \\
\hline+ & - & - & $+\infty$ & - & (IDfy to1) & $89050+7889$ & EM WTI MOI \\
\hline \multicolumn{7}{|c|}{$1096+10 \angle 9+10 \angle 9$ 19S6 } & 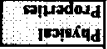 \\
\hline \multicolumn{8}{|c|}{ 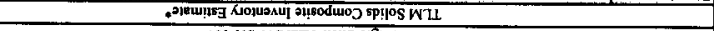 } \\
\hline & & & $1-x s-1+2$ & 708 I llous- & 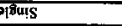 & & \\
\hline
\end{tabular}


HNF-SD-WM-ER-352, Rev. 1

\begin{tabular}{|c|c|c|c|c|c|c|c|}
\hline & \multicolumn{7}{|c|}{ Single-Shell Tank 241-SX-107 } \\
\hline & \multicolumn{7}{|c|}{ SMM Composite Inventory Estimate } \\
\hline $\begin{array}{l}\text { Phypleal } \\
\text { Properties }\end{array}$ & & & & $.95 \mathrm{Cl}$ & $-67 \mathrm{CI}$ & $+67 \mathrm{CI}$ & $\cos \mathrm{CI}$ \\
\hline Total SMM W & $0(\mathrm{~kg})$ & (1. 10E-02 kgal) & - & - & -- & $\cdots$ & $\cdots$ \\
\hline Fieal Lotd & $O(\mathrm{kw})$ & (OBTU/hr) & - & 0 & 0 & 0 & 0 \\
\hline Bulk Density* & $O\left(\mathrm{~g}^{\prime} \mathrm{cc}\right)$ & $\ldots$ & - & 0. & 0 & 0 & 0 \\
\hline & & & & & & & \\
\hline Water wi\% & 0 & - & $\ldots$ & 0 & 0 & 0 & 0 \\
\hline $\mathrm{TOC} w \mathrm{~W} \% \mathrm{C}$ & 0 & - & $\ldots$ & 0 & 0 & 0 & 0 \\
\hline Conentituents & notelL & ppm & ks: & $\begin{array}{r}85 \mathrm{CL} \\
(\mathrm{col} / \mathrm{L})\end{array}$ & $\begin{array}{l}-67 \mathrm{Ct} \\
(\text { molet })\end{array}$ & $\begin{array}{l}+67 \mathrm{CI} \\
(\mathrm{mol} / \mathrm{L})\end{array}$ & $\begin{array}{r}195 \mathrm{C1} \\
\text { (nobleft) }\end{array}$ \\
\hline Nat+ & 0 & 0 & 0 & 0 & 0 & 0 & 0 \\
\hline$\sqrt{3+}$ & 0 & 0 & 0 & 0 & 0 & 0 & 0 \\
\hline $\mathrm{Fe}^{3}+($ total $\mathrm{Fe})$ & 0 & 0 & 0 & 0 & 0 & 0 & 0 \\
\hline $\mathrm{Cr} 3+$ & 0 & 0 & 0 & 0 & 0 & 0 & 0 \\
\hline Bi3+ & 0 & 0 & 0 & 0 & 0 & 0 & 0 \\
\hline Los+ & 0 & 0 & 0 & 0 & 0 & 0 & 0 \\
\hline $\mathrm{Hg} 2+$ & 0 & 0 & 0 & 0 & 0 & 0 & 0 \\
\hline $\mathrm{Zr}$ ( $\mathrm{Ar} \mathrm{ZrO}(\mathrm{OH}) 2$ & 0 & 0 & 0 & 0 & 0 & 0 & 0 \\
\hline $\mathrm{Pb}_{2+}$ & 0 & 0 & 0 & 0 & 0 & 0 & 0 \\
\hline $\mathrm{Ni2+}$ & 0 & 0 & a & 0 & 0 & 0 & 0 \\
\hline $5 \sqrt{2+}$ & 0 & 0 & 0 & 0 & 0 & 0 & 0 \\
\hline Mndt & 0 & 0 & 0 & 0 & 0 & 0 & 0 \\
\hline $\mathrm{C}=2+$ & 0 & 0 & 0 & 0 & 0 & 0 & 0 \\
\hline $\mathbf{K +}$ & 0 & 0 & 0 & 0 & 0 & 0 & 0 \\
\hline $\mathrm{OH}$ - & 0 & 0 & 0 & 0 & 0 & 0 & 0 \\
\hline Non- & 0 & 0 & 0 & 0 & 0 & 0 & 0 \\
\hline NO2- & 0 & 0 & 0 & 0 & 0 & 0 & 0 \\
\hline CO32- & 0 & 0 & 0 & 0 & 0 & 0 & 0 \\
\hline PO43. & 0 & 0 & 0 & 0 & 0 & 0 & 0 \\
\hline SO42. & 0 & 0 & 0 & 0 & 0 & 0 & 0 \\
\hline $\mathrm{Si}\left(\boldsymbol{u}_{\mathrm{STO}} \mathrm{Si}_{2}\right)$ & 0 & 0 & 0 & 0 & 0 & 0 & 0 \\
\hline$F$ & 0 & 0 & 0 & 0 & 0 & 0 & 0 \\
\hline CI- & 0 & 0 & 0 & 0 & 0 & 0 & 0 \\
\hline C6H5073- & 0 & 0 & 0 & 0 & 0 & 0 & 0 \\
\hline EDTA4 & 0 & 0 & 0 & 0 & 0 & 0 & 0 \\
\hline HEDTA3- & 0 & 0 & 0 & 0 & 0 & 0 & 0 \\
\hline glycolave- & 0 & 0 & 0 & 0 & 0 & 0 & 0 \\
\hline scetato- & 0 & 0 & 0 & 0 & 0 & 0 & 0 \\
\hline oxilete2. & 0 & 0 & 0 & 0 & 0 & 0 & 0 \\
\hline DEP & 0 & 0 & 0 & 0 & 0 & 0 & 0 \\
\hline butanol & 0 & 0 & 0 & 0 & 0 & 0 & 0 \\
\hline $\mathrm{NHH}$ & 0 & 0 & 0 & 0 & 0 & 0 & 0 \\
\hline $\mathrm{Fc}(\mathrm{CN}) 64$ & 0 & 0 & 0 & 0 & 0 & 0 & 0 \\
\hline
\end{tabular}

"Density is calculated based on Ne, OH-, and AlO2-

+ Water wt\% derived froan the difference of denaity and total diswolved apecies. 
HDW Model Rev, 4

\begin{tabular}{|c|c|c|c|c|c|c|c|}
\hline \multicolumn{8}{|c|}{ Single-Shell Tank 241-SX-107 } \\
\hline & \multicolumn{7}{|c|}{ Total Inventory Eutimate* } \\
\hline $\begin{array}{l}\text { Phythenl } \\
\text { Properties }\end{array}$ & & & & $-95 \mathrm{CL}$ & $-67 \mathrm{Cl}$ & $+67 \mathrm{CI}$ & $+95 \mathrm{Cl}$ \\
\hline Total Waste & $6.28 \mathrm{E}+05(\mathrm{~kg})$ & (104 kgal) & $m$ & - & $\rightarrow$ & - & - \\
\hline Heat Load & $9.51(\mathrm{~kW})$ & (3.25E+04 BTU/hr) & - & 9.08 & 933 & 9.63 & 9.68 \\
\hline Bulk Density 4 & $1.75(\mathrm{~g} / \mathrm{cc})$ & -7 & - & 1.59 & 1.61 & 1.96 & 2.04 \\
\hline Water wt\% $\%$ & 29.3 & - & 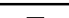 & $12=$ & & & \\
\hline TOC wt\% C (w & $\$ .42 \mathrm{E}-04$ & - & - & $7.20 \mathrm{E}-04$ & $7.50 \mathrm{E}-04$ & $9.17 \mathrm{E}-04$ & $9.95 \mathrm{E}-04$ \\
\hline $\begin{array}{l}\text { Coetulot } \\
\text { Congtituents }\end{array}$ & moled & Pegn _ _ & $\therefore$ wo & $\begin{array}{l}95 \mathrm{CI} \\
(\mathrm{mol} / \mathrm{L})\end{array}$ & $\begin{array}{l}-67 \mathrm{CI} \\
\text { (mole/t) }\end{array}$ & 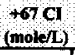 & $\begin{array}{l}+9 s \mathrm{CI} \\
\text { (molid) }\end{array}$ \\
\hline $\mathrm{Na}+$ & 8.08 & $1.06 \mathrm{E}+05$ & $7.31 \mathrm{E}+04$ & 5.00 & 5.31 & 12.1 & 14.2 \\
\hline$A \sqrt{3+}$ & 6.70 & 1.04E+05 & $7.12 \mathrm{E}+04$ & 6.39 & 645 & 6.95 & 7.20 \\
\hline $\mathrm{Fe}^{3}+(\mathrm{pote} \mathrm{Fe})$ & 1.24 & $3.95 \mathrm{E}+04$ & $2.72 \mathrm{E}+04$ & 1.22 & 1.23 & 1.24 & 1.25 \\
\hline $\mathrm{Cr}^{3}+$ & 0.187 & $5.58 \mathrm{E}+03$ & $3.83 \mathrm{E}+03$ & 0.131 & 0.158 & 0.466 & 0.791 \\
\hline Bi3+ & $1.12 \mathrm{E}-06$ & 0.134 & $9.24 E-02$ & $9.49 \mathrm{E}-07$ & $1.03 E-06$ & $1.24 \mathrm{E}-06$ & $130 \mathrm{E}-06$ \\
\hline $\mathrm{LSO}+$ & $2.77 \mathrm{E}-12$ & $2.20 \mathrm{E}-07$ & $1.51 \mathrm{E}-07$ & $242 \mathrm{E}-12$ & $2.62 \mathrm{E}-12$ & $3.06 \mathrm{E}-12$ & $3,36 E-12$ \\
\hline $\mathrm{Hg} 2+$ & $1.76 \mathrm{E}-07$ & $2.03 \mathrm{E}-02$ & $1.39 \mathrm{E}-02$ & $1,56 \mathrm{E}-07$ & $1.66 \mathrm{E}-07$ & $1.94 \mathrm{E}-07$ & 2.13E-07 \\
\hline $\operatorname{Zr}(\omega \mathrm{ZrO}(\mathrm{OH}) 2$ & $1.12 \mathrm{E}-07$ & $5.86 \mathrm{E}-03$ & 4.03E 03 & $1,03 \mathrm{E}-07$ & 1.07E-07 & 1.18E-07 & $1.27 \mathrm{E}-07$ \\
\hline $\mathrm{Pb} 2+$ & $2.80 \mathrm{E}-0 \mathrm{~S}$ & 3.32 & 2.28 & $1.50 \mathrm{E}-05$ & 2.13E-05 & 3.47E-05 & $4.13 \mathrm{E}-0 \mathrm{~S}$ \\
\hline $\mathrm{Ni2+}$ & 6.03E-02 & $203 E+03$ & $1.39 \mathrm{E}+0.3$ & $5.18 \mathrm{E}-02$ & $5.66 \mathrm{E}-02$ & $6.32 \mathrm{E}-02$ & $6.26 \mathrm{E}-02$ \\
\hline$\$ 12+$ & 0 & 0 & 0 & 0 & 0 & 0 & 0 \\
\hline $\operatorname{Mndt}$ & $8.17 \mathrm{E}-06$ & 0.257 & 0.177 & 5. $4 \mathrm{E}-0 \mathrm{~S}$ & $6.98 \mathrm{E}-06$ & 9.138-06 & $1.06 \mathrm{E}-05$ \\
\hline $\mathrm{C} 2+$ & 0.263 & $6.03 \mathrm{E}+03$ & 4.15E+03 & 0.218 & 0.240 & 0.286 & 0.308 \\
\hline $\mathbf{K t}$ & 1.4EE-02 & 330 & 227 & B. $74 E-03$ & $1.11 \mathrm{E}-02$ & $1.72 \mathrm{E}-02$ & $1.72 \mathrm{E}-02$ \\
\hline OH- & 25.6 & $2.59 \mathrm{E}+05$ & $1.78 \mathrm{E}+05$ & 25.1 & 25.6 & 27.7 & 28.7 \\
\hline NO3. & 4.06 & $1.44 \mathrm{E}+0 \mathrm{~S}$ & $9.91 \mathrm{E}+04$ & 0.895 & 0.895 & 9.01 & 11.7 \\
\hline NO2- & 1.55 & $4.09 E+04$ & $2.81 \mathrm{E}+04$ & 0.751 & 1.03 & 1.97 & 1.97 \\
\hline $\cos 2 \cdot$ & 0.264 & $9,06 E+03$ & $6.23 E+03$ & 0.219 & 0.241 & 0287 & 0.309 \\
\hline PO43- & 7.27E-05 & 3.95 & 2.72 & 6,75E-05 & $7.05 \mathrm{E}-05$ & $7.57 \mathrm{E}-0.5$ & $8.23 \mathrm{E}-05$ \\
\hline SO42- & 1.70E-02 & 935 & 643 & $1.14 \mathrm{E}-02$ & $1.37 \mathrm{E}-02$ & $1.92 \mathrm{E}-02$ & $1.92 \mathrm{E}-02$ \\
\hline Si (n SiO32-) & 0.136 & $2.19 \mathrm{E}+03$ & $1.50 \mathrm{E}+03$ & $3.22 E-02$ & 3.43E-02 & 0.238 & 0.336 \\
\hline F. & $5.79 \mathrm{E}-05$ & 0.630 & 0.433 & $4.93 \mathrm{E}-05$ & 5.46E-05 & $6.37 \mathrm{E}-0 \mathrm{~S}$ & $6.8 \mathrm{E}-05$ \\
\hline $\mathrm{Cl}-$ & $6.56 \mathrm{E}-02$ & $1.33 \mathrm{E}+03$ & 915 & $3.79 \mathrm{E}-02$ & 4.90E-02 & $7.68 \mathrm{E}-02$ & 0.129 \\
\hline C6H3O73- & 5.99E-05 & 6.48 & 4.45 & 5.,00E-05 & 5. $89 \mathrm{E}-05$ & $6.25 \mathrm{E}-03$ & $6.80 \mathrm{E}-05$ \\
\hline EDTA4 & $2.33 \mathrm{E}-06$ & 0.385 & 0.264 & $1.64 \mathrm{E}-06$ & $2.02 \mathrm{E}-06$ & 2.75E-06 & $3.16 \mathrm{E}-1 \mathrm{6}$ \\
\hline FIEDTA3. & $1.94 E-06$ & 0,304 & 0.209 & $6.21 E-07$ & $1,336-06$ & $2.58 \mathrm{E}-06$ & $3.22 \mathrm{E}-06$ \\
\hline glycolate- & 8.45E-0S & 3.63 & 2.49 & 4.58E-0S & 6.68E-05 & 1.05E-04 & $1.25 \mathrm{E}-\mathrm{OA}$ \\
\hline woctule- & $8.77 \mathrm{E}-06$ & 0.296 & 0.204 & $8.49 E-06$ & 8.63E-06 & $9,32 \mathrm{E}-06$ & $9.96 \mathrm{E}-06$ \\
\hline oxulater- & $3.62 \mathrm{E}-12$ & $1.83 \mathrm{E}-07$ & $1.26 \mathrm{E}-07$ & $3.21 \mathrm{E}-12$ & $3.41 E-12$ & $3,99 \mathrm{E}-12$ & 4.39E- 12 \\
\hline DBP & $5.31 E-05$ & 6.39 & 439 & 4.96E-05 & $5.16 \mathrm{E}-05$ & $5.74 E-05$ & $6.20 \mathrm{E}-0 \mathrm{5}$ \\
\hline butanol & $5.31 \mathrm{E}-05$ & 2.25 & 1.55 & 4.96E-05 & $5.16 E-05$ & $5.74 E-05$ & $6.20 \mathrm{E}-0 \mathrm{5}$ \\
\hline NH3 & 0.269 & $2.61 E+03$ & $1.00 E+03$ & 0.153 & 0.170 & 0.441 & 0.286 \\
\hline $\mathrm{Fo}(\mathrm{CN}) 64$ & 0 & 0 & $\overline{0}$ & 0 & 0 & 0 & 0 \\
\hline
\end{tabular}

-Unknowns in tenk solidn inventory are angigned by Tank Lnyering Model (TLM).

tWater wt\% derived from the difference of density and total diseolved species. 
HNF-SD-WM-ER-352, Rev. 1

HDW Model Rev. 4

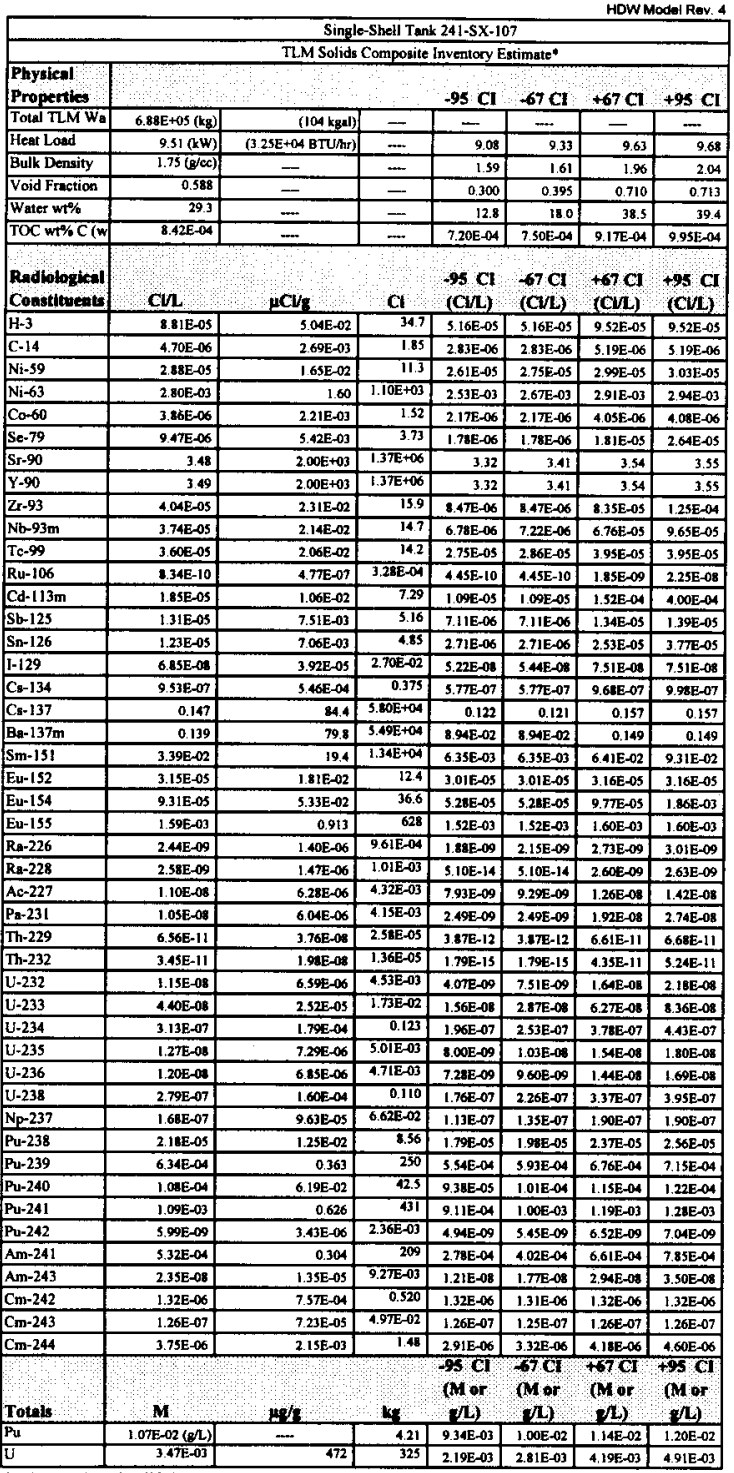

"Unknowns in tank volids inventory are assigned by Tunk Layering Model (TLM). 
HDW Model Rev. 4

\begin{tabular}{|c|c|c|c|c|c|c|c|}
\hline \multicolumn{6}{|c|}{ Single-Shell Tank 24i-SX-107 } & \multicolumn{2}{|c|}{ HDW Model Rev. 4} \\
\hline \multicolumn{8}{|c|}{ SMM Composite Inventory Estomate } \\
\hline $\begin{array}{l}\text { Phyaleal } \\
\text { Properties }\end{array}$ & & & .. & $-95 \mathrm{CI}$ & $67 \mathrm{CI}$ & $+67 \mathrm{CI}$ & $+9 \mathrm{CI}$ \\
\hline Totenl SMM W & $0(\mathrm{~kg})$ & (1.10E-02 kgal) & 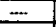 & - & - & $m$ & - \\
\hline Heat Load & $o(\mathrm{~kW})$ & (O BTU/hr) & $\ldots$ & 0 & 0 & 0 & 0 \\
\hline Bulk Density" & $O(g / c c)$ & 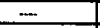 & 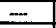 & 0 & 0 & 0 & 0 \\
\hline Water wt\% $\%$ & 0 & & & & & & \\
\hline TOC wt\%C $(w)$ & 0 & $\ldots$ & $\rightarrow$ & 0 & 0 & 0 & $\frac{0}{0}$ \\
\hline $\begin{array}{l}\text { Rediolojicd } \\
\text { Constitueds }\end{array}$ & Cin & $\mu C V / s$ & CI: & $\begin{array}{l}-95 \mathrm{CI} \\
(\mathrm{CV})\end{array}$ & $\begin{array}{l}67 \mathrm{C} \\
(\mathrm{CH})\end{array}$ & $\begin{array}{r}+67 \mathrm{CI} \\
\text { (CעL) }\end{array}$ & $\begin{array}{l}+95 \mathrm{GI} \\
\text { (CVL) }\end{array}$ \\
\hline \begin{tabular}{|l|l|l|}
$\mathrm{H}-3$ \\
\end{tabular} & 0 & 0 & 0 & 0 & 0 & 0 & 0 \\
\hline C-14 & 0 & 0 & 0 & 0 & 0 & 0 & 0 \\
\hline $\mathrm{Ni}-59$ & 0 & 0 & $\underline{0}$ & 0 & 0 & 0 & $\theta$ \\
\hline $\mathrm{Ni}-63$ & 0 & 0 & 0 & 0 & 0 & 0 & 0 \\
\hline $\operatorname{Cos} 60$ & 0 & 0 & 0 & 0 & 0 & 0 & 0 \\
\hline Se-79 & 0 & 0 & 0 & 0 & 0 & 0 & $\mathbf{0}$ \\
\hline Sr-90 & 0 & 0 & 0 & 0 & 0 & 0 & $\mathbf{0}$ \\
\hline$Y-90$ & 0 & 0 & 0 & 0 & 0 & 0 & 0 \\
\hline $2 x-93$ & 0 & 0 & 0 & 0 & 0 & 0 & 0 \\
\hline $\mathrm{Nb}-93 \mathrm{~m}$ & 0 & 0 & $\mathbf{0}$ & 0 & 0 & 0 & 0 \\
\hline$T c-99$ & 0 & 0 & 0 & 0 & 0 & 0 & 0 \\
\hline $\mathbf{R u}-106$ & 0 & 0 & 0 & 0 & 0 & 0 & 0 \\
\hline $\mathrm{Cd}-113 \mathrm{~m}$ & 0 & 0 & 0 & 0 & 0 & 0 & 0 \\
\hline Sb-125 & 0 & 0 & 0 & 0 & 0 & 0 & 0 \\
\hline $5 n-126$ & 0 & 0 & 0. & 0 & 0 & 0 & 0 \\
\hline $1-129$ & 0 & 0 & 0 & 0 & 0 & 0 & 0 \\
\hline $\mathrm{Cg}-134$ & 0 & 0 & 0 & 0 & 0 & 0 & 0 \\
\hline $\mathrm{Cs}=137$ & 0 & 0 & 0 & 0 & 0 & 0 & 0 \\
\hline $\mathrm{Ba}-137 \mathrm{~m}$ & 0 & 0 & 0 & 0 & 0 & 0 & 0 \\
\hline $5 m-151$ & 0 & 0 & 0 & 0 & 0 & 0 & 0 \\
\hline Ex-152 & 0 & 0 & 0 & 0 & 0 & 0 & 0 \\
\hline$E t=154$ & 0 & 0 & 0 & 0 & 0 & 0 & 0 \\
\hline Eur-155 & 0 & 0 & 0 & 0 & 0 & 0 & 0 \\
\hline Ra-226 & 0 & 0 & 0 & 0 & 0 & 0 & 0 \\
\hline $\mathrm{Ra}-228$ & 0 & 0 & 0 & 0 & 0 & 0 & 0 \\
\hline Acc-227 & 0 & 0 & 0 & 0 & 0 & 0 & 0 \\
\hline $\mathrm{Pa}-231$ & 0 & 0 & 0 & 0 & 0 & 0 & 0 \\
\hline Th-229 & 0 & 0 & 0 & 0 & 0 & 0 & 0 \\
\hline Th-232 & 0 & 0 & 0 & 0 & 0 & 0 & 0 \\
\hline $\mathrm{U}-232$ & 0 & 0 & $\dot{0}$ & 0 & 0 & 0 & 0 \\
\hline $\mathrm{U}-233$ & 0 & 0 & 0 & 0 & 0 & 0 & 0 \\
\hline $\mathrm{U}-234$ & a & 0 & 0 & 0 & 0 & 0 & 0 \\
\hline $\mathrm{U}-235$ & 0 & 0 & 0 & 0 & 0 & 0 & 0 \\
\hline $\mathrm{U}-236$ & 0 & 0 & 0 & 0 & 0 & 0 & 0 \\
\hline $\mathrm{U}-238$ & 0 & 0 & 0 & 0 & 0 & 0 & 0 \\
\hline $\mathrm{Np}-237$ & 0 & 0 & 0 & 0 & 0 & 0 & 0 \\
\hline P11-238 & 0 & 0 & 0 & 0 & 0 & 0 & 0 \\
\hline Pu-239 & 0 & 0 & 0 & 0 & 0 & 0 & 0 \\
\hline $\mathrm{Pu}-240$ & 0 & 0 & 0 & 0 & 0 & 0 & 0 \\
\hline Pu-24! & 0 & 0 & 0 & 0 & 0 & 0 & 0 \\
\hline \begin{tabular}{|l|}
$\mathrm{Pu}-242$ \\
\end{tabular} & 0 & 0 & 0 & 0 & 0 & 0 & 0 \\
\hline Am-24! & 0 & 0 & 0 & 0 & 0 & 0 & 0 \\
\hline Am-243 & 0 & 0 & 0 & 0 & 0 & 0 & 0 \\
\hline Cm-242 & 0 & 0 & 0 & 0 & 0 & 0 & 0 \\
\hline $\mathrm{Cm}-243$ & 0 & 0 & 0 & 0 & 0 & 0 & 0 \\
\hline Cm-244 & 0 & 0 & 0 & 0 & 0 & 0 & 0 \\
\hline Totols & $\mathbf{M}$ & $\mathrm{n} / \mathrm{s}$ & $\mathbf{k}=\mathbf{s}$ & $\begin{array}{l}05 \mathrm{CT} \\
\mathrm{Mor} \\
\mathrm{AO}\end{array}$ & $\begin{array}{l}67 \mathrm{CT} \\
(\mathrm{Mor} \\
8 \mathrm{~A})\end{array}$ & $\begin{array}{l}+67 \mathrm{CT} \\
\mathrm{M} \text { or } \\
\mathrm{N} / \mathrm{L})\end{array}$ & $\begin{array}{r}+95 \mathrm{CI} \\
\mathrm{Mur} \\
\mathrm{N} / \mathrm{L}\end{array}$ \\
\hline Pu & $O(g / L)$ & $\cdots$ & 0 & 0 & 0 & 0 & 0 \\
\hline$U$ & 0 & 0 & 0 & 0 & 0 & 0 & 0 \\
\hline
\end{tabular}

Ditusity is calculated based on $\mathrm{Na}, \mathrm{OH}$ - and $\mathrm{AlO2}$ -

$\uparrow$ Water $w+\%$ derived from the difference of dencity and total diswolved species. 
HNF-SD-WM-ER-352, Rev. 1

HDW Model Rav 4

\begin{tabular}{|c|c|c|c|c|c|c|c|}
\hline \multicolumn{8}{|c|}{ Single-Shell Tank 241-SX-107 } \\
\hline & \multicolumn{7}{|c|}{ Total Inventory Estimate* } \\
\hline Physteal & & & & & & & \\
\hline Properties: & & & & $.95 \mathrm{CI}$ & $-67 \mathrm{CI}$ & $+67 \mathrm{CI}$ & $+95 \mathrm{CI}$ \\
\hline Total Waste & $6.88 \mathrm{E}+05(\mathrm{~kg})$ & $(104 \mathrm{kgal})$ & - & - & $\cdots$ & - & $=$ \\
\hline Heat Load & $9.51(\mathrm{~kW})$ & $(3.25 E+04 \mathrm{BTU} / \mathrm{hr})$ & - & 9.08 & 9.33 & 9.63 & 9.68 \\
\hline Bulk Density' & $1.75(\mathrm{~g} / \mathrm{cc})$ & - & - & 1.59 & 1.61 & 1.96 & 2.04 \\
\hline Water wt\%t & & & & & & & \\
\hline 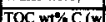 & 29.3 & $=$ & - & 12.8 & 18.0 & 38.5 & 39.4 \\
\hline & $8.42 E-04$ & - & - & $7.20 \mathrm{E}-04$ & $7.50 \mathrm{E}-04$ & $9.17 E-04$ & 9.95E-04 \\
\hline Rodiological & $\mathrm{CVL}$ & $\mu C V_{\varepsilon}$ & Cl & $\begin{array}{l}-95 \mathrm{CI} \\
\text { (CUL) }\end{array}$ & $\begin{array}{l}67 \mathrm{CI} \\
\text { (CUL) }\end{array}$ & $\begin{array}{l}67 \mathrm{CI} \\
(\mathrm{CV})\end{array}$ & $\begin{array}{l}+95 \mathrm{Cl} \\
\text { (ch) }\end{array}$ \\
\hline $\mathrm{H}-3$ & 8.81E-05 & $5.04 \mathrm{E}-02$ & 34.7 & $5.16 \mathrm{E}-05$ & $5.16 \mathrm{E}-95$ & $9.52 \mathrm{E}-0.5$ & $9.52 \mathrm{E}-05$ \\
\hline C-14 & $4.70 \mathrm{E}-06$ & $2.69 \mathrm{E}-03$ & 1.85 & $2.83 \mathrm{E}-06$ & 2.83E-06 & $5.19 \mathrm{E}-06$ & $5.19 \mathrm{E}-06$ \\
\hline $\mathrm{Ni}-59$ & $2.88 \mathrm{E}-05$ & $1.65 \mathrm{~S}-02$ & 11.3 & 2.61E-05 & $2.75 E-05$ & 2.99E-05 & $3.03 \mathrm{E}-0.5$ \\
\hline $\mathrm{Ni}-63$ & $2.80 \mathrm{E}-03$ & 1.60 & $1.10 \mathrm{E}+03$ & 2.53E-03 & $2.67 E-03$ & 2.91E-03 & 2.94E-03 \\
\hline $\mathrm{C} 0-60$ & $3.86 \mathrm{E}-06$ & $2.21 \mathrm{E}-03$ & 1.52 & $2.17 \mathrm{E}-06$ & $2.17 E-06$ & $4.05 \mathrm{E}-06$ & $4.08 \mathrm{E}-06$ \\
\hline Se-79 & 9.47E-06: & $5.42 E-03$ & 3.73 & $1.78 E-06$ & $1.78 E-06$ & 1.81E-0S & 2.64E-05 \\
\hline$S r-90$ & 3.48 & $2.00 \mathrm{E}+03$ & $1.37 E+\infty 6$ & 3.32 & 3.41 & 3.34 & 3.55 \\
\hline Y.90 & 3.48 & $2.00 \mathrm{E}+03$ & $1.37 \mathrm{TE}+\infty$ & 3.32 & 3.41 & 3.54 & 3.55 \\
\hline$Z s-93$ & 4.04E-05 & 2.31E-02 & 15.9 & B.47E-06 & $8.47 \mathrm{E}-06$ & 8.35E-05 & 1.25E-04 \\
\hline $\mathrm{Nb}-93 \mathrm{~m}$ & 3.74E-05 & $2.14 \mathrm{E}-02$ & 14.7 & $6.78 E-06$ & $7.22 \mathrm{E}-06$ & 6.76E-05 & 9.65E-05 \\
\hline$T e-99$ & 3.60E-05 & 206E-02 & 14.2 & 2.75E-05 & $2.86 \mathrm{E}-05$ & 3.95E-05 & 3.95E-05 \\
\hline$R u-106$ & 8.34E-10 & 4.77E- -07 & $3.28 \mathrm{E}-04$ & 4.45E-10 & $4.45 \mathrm{E}-10$ & $1.85 E-09$ & $2.25 \mathrm{E}-08$ \\
\hline Cd-113m & $1.85 E-05$ & $1.06 \mathrm{E}-02$ & 7.29 & $1.09 E .05$ & $1.09 \mathrm{E}-03$ & $1.52 \mathrm{E}-04$ & $4.00 E-04$ \\
\hline sb-125 & $1.31 E-05$ & $7.51 E-03$ & 5.16 & $7.11 E-06$ & $7.11 \mathrm{E}-06$ & $1.34 E-05$ & 1.39E-05 \\
\hline $5 \pi-126$ & $1.23 \mathrm{E}-05$ & $7.06 E-03$ & 4.85 & $2.71 E-06$ & 2.71E-06 & $2.53 E-05$ & $3.7 \pi \mathrm{E}-05$ \\
\hline$[-129$ & 6.85E-08 & 3.92E-05 & $2.70 \mathrm{E}-02$ & $5.22 \mathrm{E}-08$ & $5.44 \mathrm{E}-08$ & 7.5IE-08 & $7.51 E-08$ \\
\hline Ca-134 & $9.53 E-07$ & $3,46 \mathrm{E}-04$ & 0.375 & $3.77 \mathrm{E}, 07$ & 5.7) -07 & 9.68E-07 & $9.98 E-07$ \\
\hline C8-137 & 0.147 & 84.4 & 5.80E+04 & 0.122 & 0.121 & 0.157 & 0.157 \\
\hline Ba-137m & 0.139 & 79.8 & $3.49 \mathrm{E}+0 \mathrm{~A}$ & 8.94E-02 & $294 \mathrm{E}-02$ & 0.149 & 0.149 \\
\hline Sm-151 & $3.39 \mathrm{E}-02$ & 19.4 & $1.34 E+04$ & $6.35 E-03$ & 6.35E-03 & $6.41 \mathrm{E}-02$ & 9.31E-02 \\
\hline Eu-152 & 3.15E-05 & $1.81 \mathrm{E}-02$ & 12.4 & 3.01E-0S & 3.01E. 05 & 3.16E-05 & 3.16E-05 \\
\hline Eu-154 & 9.30E-05 & 5.33E-02 & 36.6 & $5.28 E-05$ & $5.28 \mathrm{E}-05$ & $9.77 \mathrm{E}-0 \mathrm{~s}$ & 1.86E-03 \\
\hline Eu-155 & 1.59E-03 & 0.913 & 628 & $1.52 \mathrm{E}-03$ & 1.52E-03 & $1.60 \mathrm{E}-03$ & I.60E-03 \\
\hline Ra-226 & 2.44E-09 & $1.40 E-06$ & 9.6IE-04 & 1.88E-09 & 2. $15 \mathrm{E}-09$ & $2.73 \mathrm{E}-09$ & $3.01 \mathrm{E}-09$ \\
\hline$R_{*-228}$ & $2.58 E-09$ & $1.47 \mathrm{E}-06$ & 1.01E-03 & $5.10 \mathrm{E}-14$ & 5.10E-14 & $2.60 \mathrm{E}-09$ & $2.63 \mathrm{E}-69$ \\
\hline Ac-227 & $1.10 \mathrm{E}-08$ & $6.28 E-06$ & 4.32E-03 & $7.93 \mathrm{E}-09$ & 9.29E-09 & $1.26 \mathrm{E}-08$ & $1.42 \mathrm{E}-0 \mathrm{~B}$ \\
\hline \begin{tabular}{|l|}
$a-231$ \\
\end{tabular} & $1.05 \mathrm{E}-08$ & 6.04E-06 & 4.15E-03 & 2.49E-09 & $2.49 \mathrm{E}-09$ & $1,922-a p$ & 2.74E-08 \\
\hline Th-229 & $6.56 \mathrm{E}-11$ & $3.76 \mathrm{E}-08$ & 2.58E-05 & 3.1.7E-12 & $3.87 E-12$ & 6.61E-11 & $6.68 \mathrm{E}-11$ \\
\hline Th-232 & $3.45 \mathrm{E}-11$ & 1.94e-ses & 1.36E-05 & $1.79 \mathrm{E}-15$ & $1.79 \mathrm{E}-15$ & 4.35E-11 & $5.24 E-11$ \\
\hline U-232 & 1.1SE-0 & 6.59e-06 & 4.53E-03 & 4.07E-09 & 7.51E-09 & 1.64E-08 & $2.18 E-08$ \\
\hline U-233 & $4.40 E-08$ & $2.52 \mathrm{E}-05$ & $1.73 \mathrm{E}-02$ & $1.56 \mathrm{E}-08$ & 2.17E-08 & $6.2 \pi-08$ & 8.36E-01 \\
\hline U-234 & 3.13E--07 & $1.79 \mathrm{E}-04$ & 0.123 & $1.96 E-07$ & 2.53E-07 & $3.78 E-07$ & 4.43E-07 \\
\hline U. & $1.2 \mathrm{E}-08$ & $7.29 \mathrm{E} .06$ & $5.01 \mathrm{E}-03$ & 1.00E-09 & $1.03 \mathrm{E}-08$ & $1,54 E-08$ & $1,80 \mathrm{E}-08$ \\
\hline U-236 & $1.20 \mathrm{E}-08$ & $6.85 \mathrm{E}-06$ & 4.71E- -63 & $7.28 \mathrm{BE}-09$ & 9.60E- -9 & 1.44E-0. & 1.69E-08 \\
\hline U-238 & 2.79E-07 & $1.60 \mathrm{E}-04$ & 0.110 & $1.76 E-07$ & $2.26 \mathrm{E}-07$ & 3,37E-07 & 3.95E-07 \\
\hline$N p-237$ & $1.68 E-07$ & 9.63E-05 & $6.62 \mathrm{E}-02$ & 1.13E-07 & 1.35E-07 & $1.90 \mathrm{E}-07$ & $1.90 \mathrm{E}-07$ \\
\hline Pu-238 & 2.17E-05 & $1.25 \mathrm{E}-02$ & 8.56 & 1.79E-0s & 1.98E-0s & $2.37 \mathrm{E}-05$ & $2.56 \mathrm{E}-05$ \\
\hline Pt-239 & $6.34 \mathrm{E}-04$ & 0.363 & 250 & $5.54 \mathrm{E}-04$ & $5.93 \mathrm{E}-04$ & $6.76 \mathrm{E}-04$ & 7. $15 \mathrm{E}-04$ \\
\hline$P v-240$ & $1.08 \mathrm{E}-04$ & 6. $19 \mathrm{E}-02$ & 42.5 & 9.38E-05 & $1.01 E-04$ & 1.15E-04 & $1.22 \mathrm{E}-04$ \\
\hline Py-241 & $1.09 \mathrm{E}-03$ & 0.626 & 431 & $9.11 \mathrm{E}-04$ & $1.00 E-03$ & 1.19E-03 & $1.28 \mathrm{E}-03$ \\
\hline Pu-242 & $3.99 \mathrm{E}-09$ & 3.43E- -06 & $2.36 \mathrm{E}-03$ & 4.94E-09 & 5.45E-09 & $6.52 \mathrm{E}-09$ & $7.04 \mathrm{E}-09$ \\
\hline Am-24] & $5.32 \mathrm{E}-04$ & 0.304 & 209 & $2.78 \mathrm{E}-04$ & $4.02 \mathrm{E}-04$ & $6.61 \mathrm{E}-04$ & $7.85 \mathrm{E}-04$ \\
\hline Am-243 & 2.35E -08 & 1.35E-05 & 9.27E- 03 & 1.21E-08 & $1.77 \mathrm{E}-08$ & $2.94 \mathrm{E}-09$ & $3.50 \mathrm{E}-08$ \\
\hline $\mathrm{Cm}_{\mathrm{m}}-242$ & $1.32 \mathrm{E}-06$ & $7.57 \mathrm{E}-04$ & 0.520 & $1.32 E-106$ & $1.31 \mathrm{E}-06$ & 1.32E-06 & $1.32 E-06$ \\
\hline $\mathrm{Cm}_{\mathrm{m}-243}$ & $1.26 \mathrm{E}-07$ & 7.23E-05 & 4.97E-02 & 1.26E-07 & 1.25E-07 & 1.26E-07 & 1.26E-07 \\
\hline Cm-244 & 3.75E- 06 & $2.15 E-03$ & 1.48 & 2.91E-06 & $3.32 E-06$ & $4.18 E-06$ & 4.50E-06 \\
\hline Totals & M & $\pi / 3$ & ro & $\begin{array}{l}98 \mathrm{CI} \\
\mathrm{M} / \mathrm{L})\end{array}$ & $\begin{array}{l}7 \mathrm{C} \\
\mathrm{Mor} \\
\mathrm{NL}\end{array}$ & $\begin{array}{c}690 \\
(\mathrm{Mor} \\
\mathrm{M})\end{array}$ & $\begin{array}{l}98 \mathrm{Cl} \\
(\mathrm{M} / \mathrm{pr})\end{array}$ \\
\hline Pu & $1,0 \pi \mathrm{E}-02(g / 2)$ & - & 4.21 & 9.34E-03 & $1.00 E-02$ & 1,14E-02 & $1.20 \mathrm{E}-02$ \\
\hline $\mathrm{U}$ & 1.97: -03 & 472 & 325 & 2.19E-03 & 2.81E-03 & 4.19E-03 & 4.91E-03 \\
\hline
\end{tabular}

+Volume avernge for density, mass average Water wt\% and TOC wi\% C. 


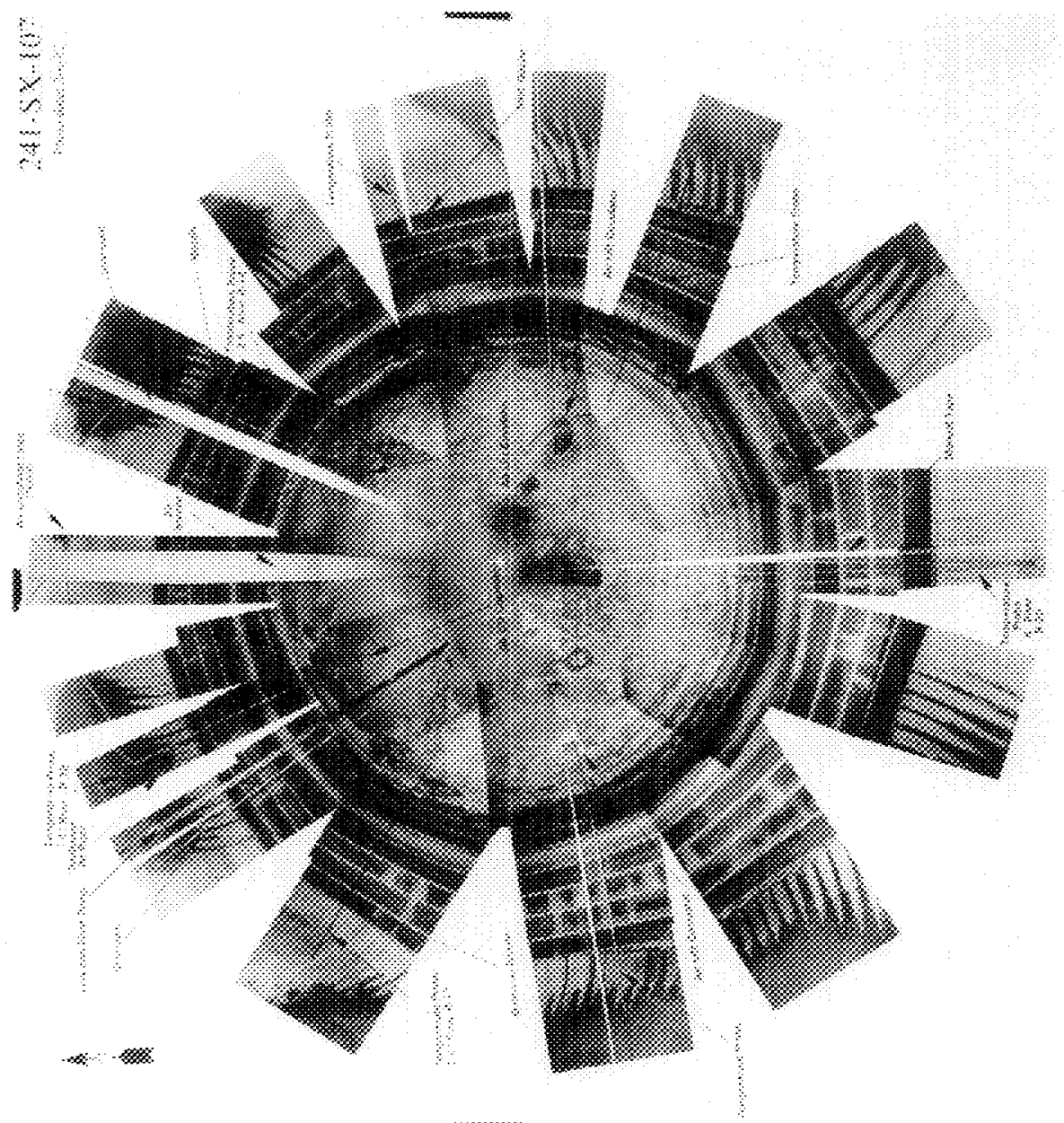


TANK 241-SX-108 SUMMARY

\begin{tabular}{|c|c|c|c|}
\hline \multicolumn{2}{|c|}{ TANK HISTORY } & \multicolumn{2}{|c|}{ TANK DESCRIPTION } \\
\hline Entered Service & 4th qtr 1955 & Diameter & $75 \mathrm{ft}$ \\
\hline Removed from Service & - & Bottom Shape & Dish \\
\hline Inactive & - & Nominal Capacity & $1,000,000 \mathrm{gal}$ \\
\hline Watch Lists & none & Cascade Tank & to $241-S X-109$ \\
\hline Integrity & Assumed Leaker & Total Risers & 22 \\
\hline Assumed Leaker & 1962 & WASTE VOLUI & LON 1996i) \\
\hline Interim Stabilization (IS) & Aug 1979 & Total Waste Volume & $87,000 \mathrm{gal}$ \\
\hline Partial Interim Isolation (PI) & - & Waste Type & NCPLX \\
\hline Intrusion Prevention (IP) & Dec 1982 & Drainable Interstitial Liquids & $5,000 \mathrm{gal}$ \\
\hline \multicolumn{2}{|c|}{$\begin{array}{l}\text { TENTATIVELY AVAILABLE RISERS } \\
\end{array}$} & Pumpable Liquids & $0 \mathrm{gal}$ \\
\hline Riser Number(s) & Size & Saltcake & 0 gal \\
\hline \multirow[t]{2}{*}{$7,16,17$} & 12 in & Sludge & $87,000 \mathrm{gal}$ \\
\hline & & Supernatant & 0 gal \\
\hline \multicolumn{2}{|c|}{ TANK TEMPERATURE } & \multicolumn{2}{|c|}{ INTERIOR PHOTOGRAPHS } \\
\hline Average Tank Temperature & $158^{\circ} \mathrm{F}$ & Date & March 6, 1987 \\
\hline Maximum Temperature & $204^{\circ} \mathrm{F}$ & Montage Number & $94041033-31 \mathrm{CN}$ \\
\hline Date & Oct 31,1994 & Photo Set Number & 8701429 \\
\hline Elevation from tank bottom & $0.5 \mathrm{ft}$ & \multicolumn{2}{|c|}{\begin{tabular}{|c|} 
WASTE SURFACE LEVEL \\
\end{tabular}} \\
\hline Riser Number & 19 & Devices & Manual Tape \\
\hline Minimum Temperature & $129.74^{\circ} \mathrm{F}$ & Max Level & 37.75 in \\
\hline Date & March 25 - April 5, 1996* & Date & Jan 3,1994 \\
\hline Elevation from tank bottom & $4.67 \mathrm{ft}$ & Min Level & 32 in \\
\hline Riser Number & 10 & Date & July 2,1994 \\
\hline
\end{tabular}

- Numerous dates in this time span 


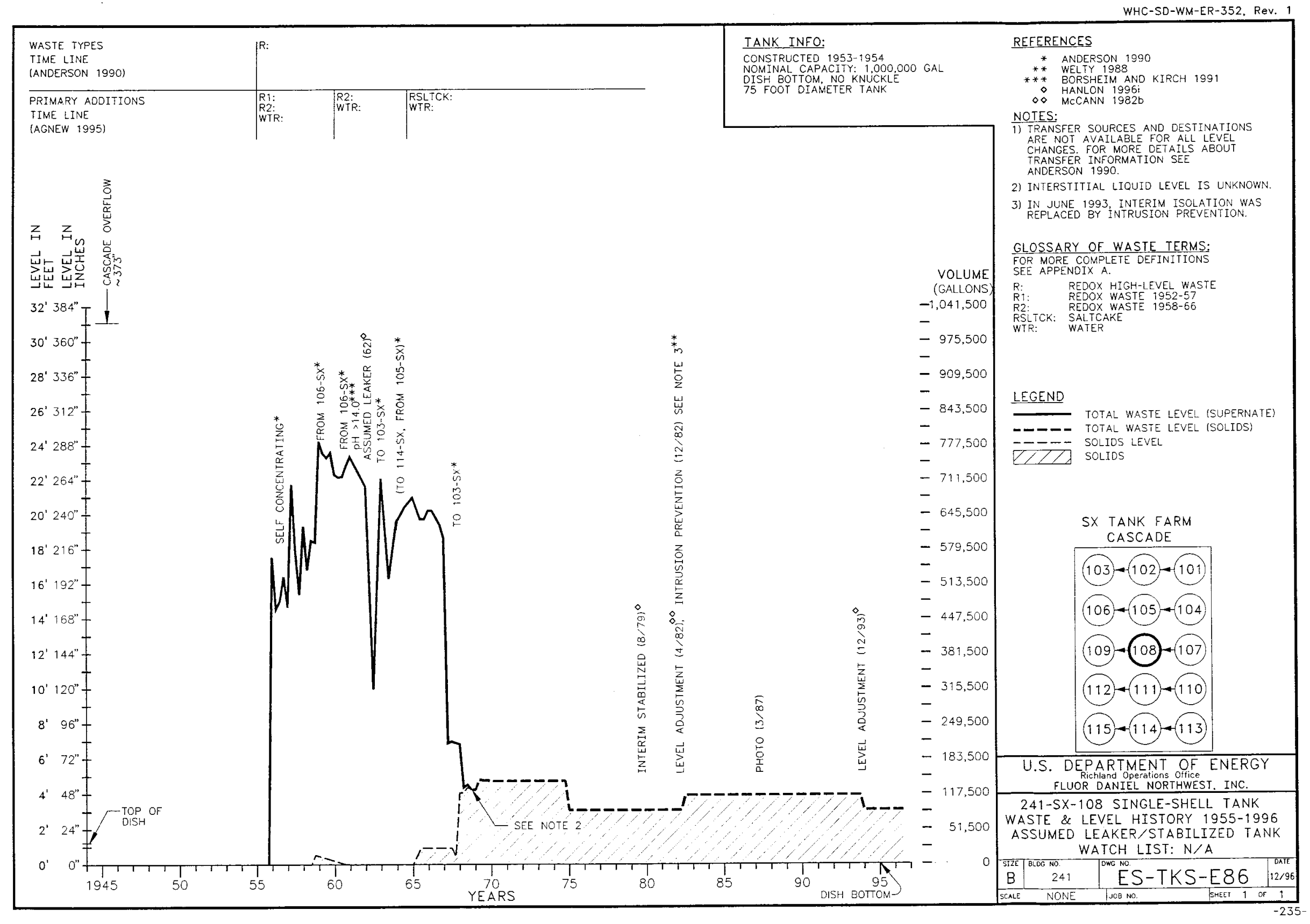




$$
241-5 X-108
$$
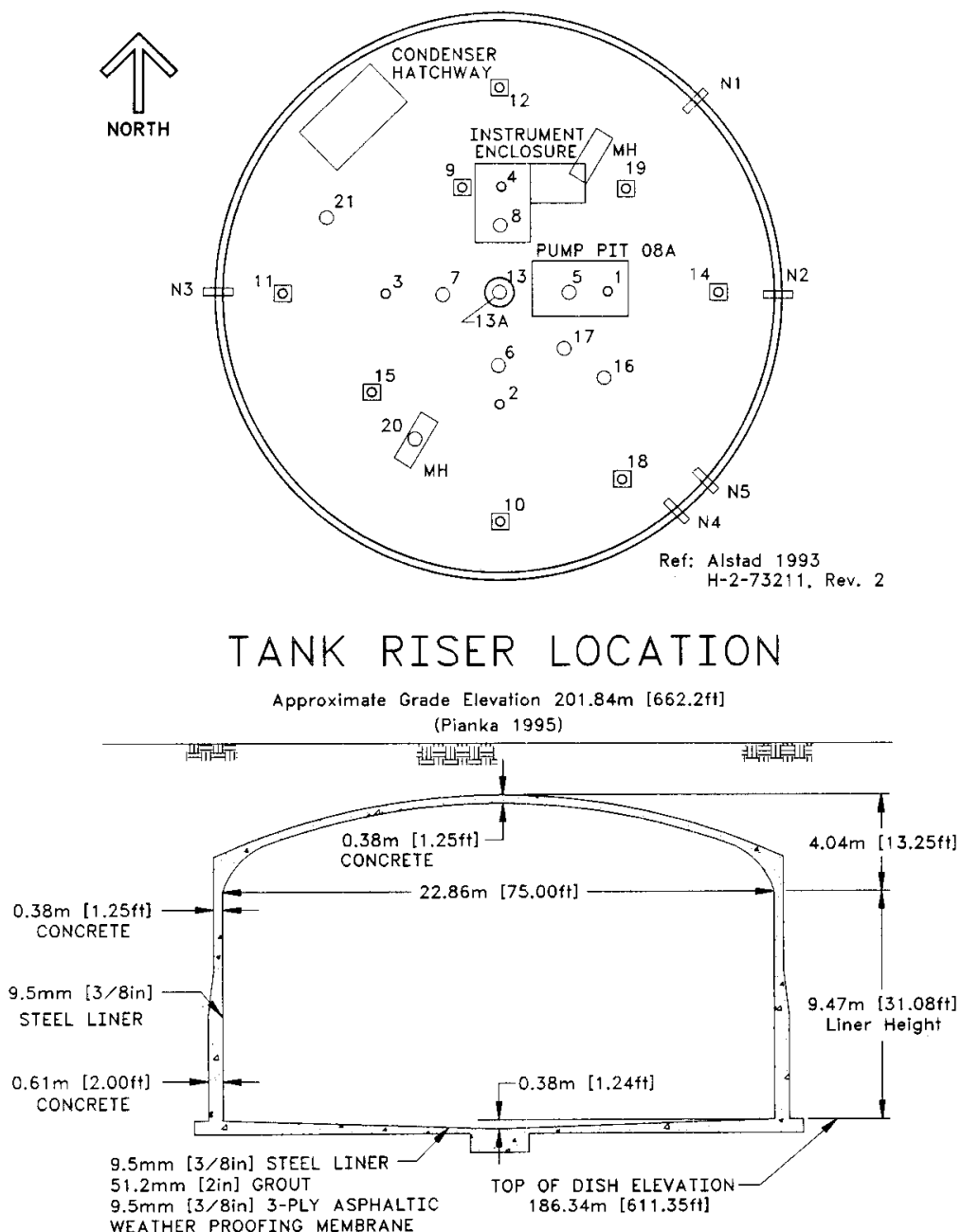

WEATHER PROOFING MEMBRANE 
HNF-SD-WM-ER-352, Rev. 1

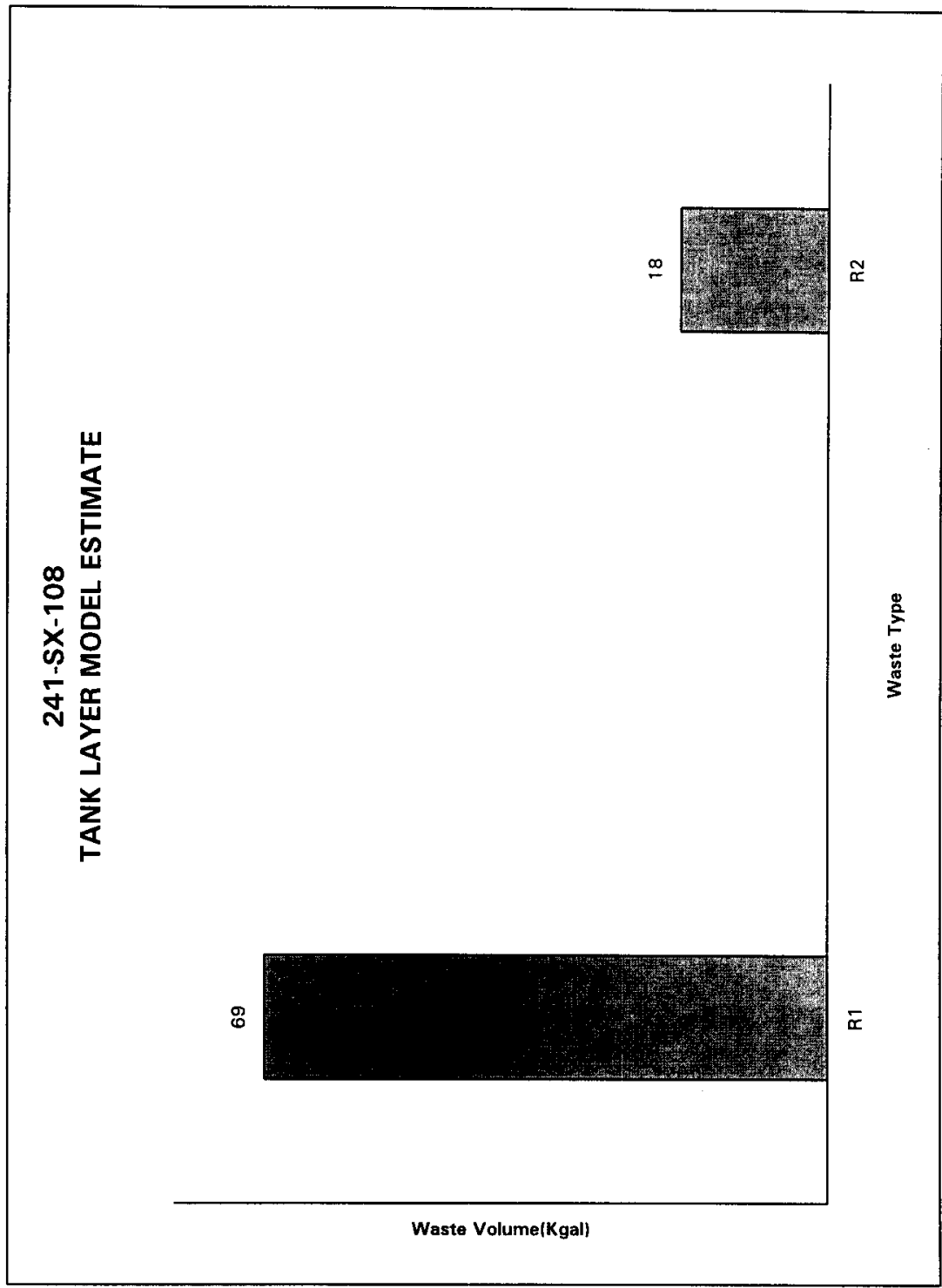

है 
HNF-SD-WM-ER-352, Rev. 1

HDW Model Rev. 4

\begin{tabular}{|c|c|c|c|c|c|c|c|}
\hline \multirow{4}{*}{$\begin{array}{l}\text { Physteal } \\
\text { Propertics }\end{array}$} & \multicolumn{7}{|c|}{ Single-Shell Tank 24I-SX-108 } \\
\hline & \multicolumn{7}{|c|}{ TLM Solids Comporite Inventory Estimate" } \\
\hline & & & & & & & \\
\hline & & & & $95 \mathrm{CI}$ & $.67 \mathrm{CI}$ & $+67 \mathrm{Cl}$ & $\operatorname{tgs} \mathrm{CI}$ \\
\hline Total TLM Wa & $5.78 E+05(\mathrm{~kg})$ & $(87.0 \mathrm{kgal})$ & $=$ & $\ldots$ & - & $\infty$ & $=$ \\
\hline Heat Losd & $7.51(\mathrm{~kW})$ & $(2.56 \mathrm{E}+04 \mathrm{BTU} / \mathrm{hr})$ & $=$ & 7.04 & 7.32 & 7.64 & 7.69 \\
\hline Bulk Density & $1.75(\mathrm{~g} / \mathrm{cc})$ & - & $=$ & 1.56 & 158 & 2.03 & 2.14 \\
\hline Void F raction & 0.547 & $\ldots$ & $\ldots$ & 0.169 & 0.294 & 0.706 & 0.710 \\
\hline Water wt\% & 28.0 & $\cdots$ & - & 7.54 & 13.9 & 40.1 & 41.3 \\
\hline TOC w $\%$ C (w) & 0 & $=$ & $\cdots$ & 0 & 0 & 0 & 0 \\
\hline $\begin{array}{c}\text { Chenifeal } \\
\text { Consulfuents }\end{array}$ & mole/L. & Pom _ : : & lo & $\begin{array}{l}95 \mathrm{Cl} \\
\text { (moled) }\end{array}$ & $\begin{array}{l}67 \mathrm{Cl} \\
\text { (mole/L) }\end{array}$ & $\begin{array}{l}+67 \mathrm{CI} \\
\text { (noblelt) }\end{array}$ & $\begin{array}{l}+95 \mathrm{CI} \\
(\mathrm{mole} / \mathrm{L})\end{array}$ \\
\hline \begin{tabular}{|l|}
$\mathrm{N} \boldsymbol{x}+$ \\
\end{tabular} & 7.30 & $9.57 \mathrm{E}+04$ & $5.53 E+04$ & 3.27 & 3.67 & 12.5 & 15.3 \\
\hline Al3t+ & 7.26 & $1.12 \mathrm{E}+05$ & $6.45 E+04$ & 6.94 & 7.07 & 7.39 & 7.39 \\
\hline $\mathrm{Fe}^{3+}+($ total $\mathrm{Fe})$ & 1.36 & 4.33E+04 & $2.50 E+04$ & 1.34 & 1.35 & 1.37 & 1.37 \\
\hline Cr3+ & $6.29 \mathrm{E}-02$ & $1.86 \mathrm{E}+03$ & $1.08 \mathrm{E}+03$ & $1.94 \mathrm{E}-02$ & $4.07 \mathrm{E}-02$ & 0.428 & 0.854 \\
\hline $\mathrm{Bi} 3+$ & 0 & 0 & 0 & 0 & 0 & 0 & 0 \\
\hline Le3t & 0 & 0 & 0 & 0 & 0 & 0 & 0 \\
\hline $\mathrm{Hg}+$ & 0 & 0 & 0 & o & 0 & 0 & 0 \\
\hline $\mathrm{Zr}(\mathrm{ns} \mathrm{ZrO}(\mathrm{OH}) 2$ & 0 & 0 & 0 & 0 & 0 & 0 & 0 \\
\hline $\mathrm{Pb2+}$ & $\begin{array}{r}0 \\
\\
\end{array}$ & 0 & 0 & 0 & 0 & 0 & 0 \\
\hline $\mathrm{Ni2+}$ & 6.45E-02 & $2.16 \mathrm{E}+03$ & $1.25 \mathrm{EE}+03$ & $5.33 \mathrm{E}-02$ & $3,97 \mathrm{E}-02$ & $0.83 \mathrm{E}-02$ & 6.76E-02 \\
\hline Sr2t+ & 0 & 0 & 0 & 0 & 0 & 0 & 0 \\
\hline Mn4t & 0 & 0 & 0 & 0 & 0 & 0 & 0 \\
\hline $\mathrm{Ca} 2 \mathrm{t}$ & 0.279 & $6.38 \mathrm{E}+03$ & $369 \mathrm{E}+03$ & 0.220 & 0.249 & 0.310 & 0.339 \\
\hline $\mathrm{K}+$ & $1.13 \mathrm{E}-02$ & 253 & 146 & $3.46 \mathrm{E}-03$ & $6.61 \mathrm{E}-03$ & $1.45 \mathrm{E}-02$ & $3.45 E-02$ \\
\hline OF- & 27.5 & $267 \mathrm{E}+05$ & ISAE+OS & 25.5 & 26.5 & 286 & 29.8 \\
\hline NO3. & 4.19 & $1.48 \mathrm{E}+05$ & $5.56 \mathrm{E}+04$ & 4.78E-02 & $4.78 \mathrm{E}-02$ & 10.7 & 14.2 \\
\hline NO2- & 1.48 & $3.89 \mathrm{E}+04$ & $2.25 \mathrm{E}+04$ & 0.432 & 0.799 & 2.03 & 2.03 \\
\hline $\cos 2$ & 0.279 & $9.56 \mathrm{E}+03$ & $5.52 \mathrm{E}+03$ & 0.220 & 0.249 & 0.310 & 0.339 \\
\hline PO43: & 0 & 0 & 0 & 0 & 0 & 0 & 0 \\
\hline SO42- & $1.19 E-02$ & 653 & 377 & $4.61 \mathrm{E}-03$ & $7.53 \mathrm{E}-03$ & $1.49 \mathrm{E}-02$ & $1.49 \mathrm{E}-02$ \\
\hline Si (usio32-) & 0.105 & $1.68 \mathrm{E}+03$ & 971 & $1.18 \mathrm{E}-02$ & $1.37 \mathrm{E}-02$ & 0.197 & 0.284 \\
\hline$F-$ & 0 & 0 & 0 & 0 & 0 & 0 & 0 \\
\hline c. & $5225-02$ & $1.05 \mathrm{E}+03$ & 609 & $1,596-02$ & 3.04E-02 & $6.68 \mathrm{E}-02$ & 0.136 \\
\hline C6HSON3- & 0 & 0 & 0 & 0 & 0 & 0 & 0 \\
\hline EDTA4: & 0 & 0 & 0 & 0 & 0 & 0 & 0 \\
\hline HEDTK & 0 & 0 & 0 & 0 & 0 & 0 & 0 \\
\hline glycolate- & 0 & 0 & 0. & 0 & a & 0 & 0 \\
\hline scetate- & 0 & 0 & 0 & 0 & 0 & 0 & 0 \\
\hline oxalate2- & 0 & 0 & 0 & 0 & 0 & D & 0 \\
\hline DBP & 0 & 0 & 0 & 0 & 0 & 0 & 0 \\
\hline butanal & 0 & 0 & 0 & o & 0 & 0 & 0 \\
\hline & 0299 & $2.90 E+03$ & $168 \mathrm{E}+03$ & 0 & I7רו & 0 & 0709 \\
\hline Fe(CN)64- & 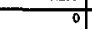 & 0 & 0 & $\frac{0.149}{0}$ & 0 & $\frac{0.454}{0}$ & $\frac{0.322}{0}$ \\
\hline
\end{tabular}

"Unknowns in tank solida inventory are ausigned by Tank Layering Model (TLM) 
HNF-SD-WM-ER-352, Rev. 1

HOW Madel Rov. 4

\begin{tabular}{|c|c|c|c|c|c|c|c|}
\hline \multicolumn{8}{|c|}{ Single-Shell Tank 241-SX-108 } \\
\hline & \multicolumn{7}{|c|}{ SMM Composite Inventory Estimate } \\
\hline $\begin{array}{l}\text { Phyuicat } \\
\text { Properties }\end{array}$ & & & & $\$ 95 \mathrm{Cl}$ & $-67 \mathrm{CI}$ & $+67 \mathrm{CI}$ & $+95 \mathrm{CI}$ \\
\hline Total SMM W & $0(\mathbf{k g})$ & (1.50E-02 kgal) & $\cdots$ & - & - & $\cdots$ & - \\
\hline Heat Load & $0(\mathrm{~kW})$ & (0 BTU/hr) & $\ldots$ & 0 & 0 & $\mathbf{0}$ & 0 \\
\hline Bulk Density" & $0\left(y^{\prime} \infty\right)$ & - & $\ldots$ & 0 & 0 & 0 & 0 \\
\hline & & & & & & & \\
\hline Water wt\% & 0 & $\ldots$ & 一 & 0 & 0 & 0 & 0 \\
\hline TOC w\% C (w) & 0 & معث. & - & 0 & 0 & 0 & 0 \\
\hline $\begin{array}{l}\text { Qhemingil } \\
\text { Constifien }\end{array}$ & $n a l d L$ & pron & $\mathbf{k g}$ & $\begin{array}{l}-95 \mathrm{CI} \\
\text { (nolid) }\end{array}$ & $\begin{array}{l}67 \mathrm{CT} \\
\text { (moled ) }\end{array}$ & $\begin{array}{l}+67 \mathrm{Cl} \\
\text { (noled }\end{array}$ & $\begin{array}{r}+95 \mathrm{CI} \\
\text { (mole/t) }\end{array}$ \\
\hline $\mathrm{Nat}$ & 0 & 0 & 0 & 0 & 0 & 0 & 0 \\
\hline $\mathrm{A} / 3+$ & 0 & 0 & 0 & 0 & 0 & 0 & 0 \\
\hline $\mathrm{Fe3}+($ total $\mathrm{Fe})$ & 0 & 0 & 0 & 0 & 0 & 0 & 0 \\
\hline $\mathrm{Cr} 3+$ & 0 & 0 & $\overline{0}$ & 0 & 0 & 0 & 0 \\
\hline Bist & 0 & 0 & 0 & 0 & $\overline{0}$ & 0 & 0 \\
\hline Lest & 0 & 0 & 0 & 0 & 0 & 0 & 0 \\
\hline Hg2t & 0 & 0 & 0 & 0 & 0 & 0 & 0 \\
\hline Zr (a ZrOKOH)2 & 0 & 0 & 0 & 0 & 0 & 0 & 0 \\
\hline $\mathrm{Pb} 2+$ & 0 & 0 & 0 & 0 & 0 & 0 & 0 \\
\hline $\mathrm{Ni2+}$ & 0 & 0 & 0 & 0 & 0 & 0 & 0 \\
\hline $512+$ & 0 & 0 & 0 & 0 & 0 & 0 & 0 \\
\hline Mnst & 0 & 0 & 0 & 0 & 0 & 0 & 0 \\
\hline $\mathrm{Ca} 2+$ & 0 & $\mathbf{0}$ & 0 & 0 & 0 & 0 & 0 \\
\hline $\mathrm{K}+$ & 0 & 0 & 0 & 0 & 0 & 0 & 0 \\
\hline $\mathrm{OH}-$ & 0 & $\mathbf{0}$ & 0 & 0 & 0 & 0 & 0 \\
\hline NO3- & 0 & 0 & 0 & 0 & 0 & 0 & 0 \\
\hline $\mathrm{NO2}-$ & 0 & 0 & 0 & 0 & 0 & 0 & 0 \\
\hline Co32- & 0 & 0 & 0 & 0 & 0 & 0 & 0 \\
\hline PO43- & 0 & 0 & 0 & 0 & 0 & 0 & 0 \\
\hline SO42: & 0 & $\overline{0}$ & 0 & 0 & 0 & 0 & 0 \\
\hline $5 i$ (4s S1032-) & 0 & 0 & 0 & 0 & 0 & 0 & 0 \\
\hline$F_{*}$ & 0 & 0 & 0 & 0 & 0 & 0 & 0 \\
\hline $\mathrm{Cl}-$ & 0 & 0 & 0 & 0 & 0 & 0 & 0 \\
\hline C6H5073- & 0 & 0 & 0 & 0 & 0 & 0 & 0 \\
\hline EDTA4- & 0 & 0 & 0 & 0 & 0 & 0 & 0 \\
\hline HEDTA3- & 0 & 0 & 0 & 0 & 0 & 0 & 0 \\
\hline glycalinte- & 0 & 0 & 0 & 0 & 0 & 0 & 0 \\
\hline acetine- & 0 & 0 & 0 & 0 & 0 & 0 & 0 \\
\hline axalate2- & 0 & 0 & 0 & 0 & 0 & 0 & 0 \\
\hline DBP & 0 & 0 & 0 & 0 & 0 & 0 & 0 \\
\hline butanol & 0 & 0 & 0 & 0 & 0 & 0 & 0 \\
\hline $\mathrm{NH}^{3}$ & 0 & 0 & 0 & $\theta$ & 0 & 0 & 0 \\
\hline Fe(CN)64 & 0 & 0 & 0 & 0 & 0 & 0 & 0 \\
\hline
\end{tabular}

"Density is calculated based on Ne, OH-, and AlO2-.

- Water wt\% derived from the difference of density and total distolvod epecies. 


\begin{tabular}{|c|c|c|c|c|c|c|c|}
\hline \multicolumn{8}{|c|}{ Single-Shell Tark 241-SX-108 } \\
\hline & \multicolumn{7}{|c|}{ Total Inventory Estimate* } \\
\hline $\begin{array}{l}\text { Physienl } \\
\text { Propertics }\end{array}$ & & & & $.95 \mathrm{CI}$ & $-67 \mathrm{CI}$ & $+67 \mathrm{CI}$ & $+95 \mathrm{Cl}$ \\
\hline Tótal Waste & $5.78 \mathrm{E}+05(\mathrm{~kg})$ & $(87.0 \mathrm{kgn})$ & $\cdots$ & $m$ & 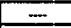 & $\ldots$ & $\ldots$ \\
\hline Heat Load & $7.51(\mathrm{~kW})$ & $(2.56 \mathrm{E}+04 \mathrm{BTU} / \mathrm{hr})$ & - & 704 & 7.32 & 7.64 & 7.69 \\
\hline Bulk Densityt & $1.75(\mathrm{~g} / \mathrm{cc})$ & $=$ & - & 1.56 & 1.58 & 2.03 & 2.14 \\
\hline Water wt\% $\%$ & 28.0 & - & - & 7.54 & 13.9 & 40.1 & 413 \\
\hline $\mathrm{TOC} w \mathrm{wt} \%(\mathrm{w}$ & 0 & $=$ & - & 0 & 0 & 0 & 0 \\
\hline $\begin{array}{l}\text { Chemicil } \\
\text { Constituents }\end{array}$ & moled 1 & ppm : : & $\mathrm{kg}$ & $\begin{array}{l}-95 \mathrm{Cl} \\
\text { (mole/L) }\end{array}$ & $\begin{array}{l}-67 \mathrm{Cl} \\
\text { (mole/L) }\end{array}$ & $\begin{array}{l}+67 \mathrm{CT} \\
\text { (mole/L) }\end{array}$ & $\begin{array}{l}+95 \mathrm{CT} \\
\text { (moled })\end{array}$ \\
\hline Net & 7.30 & $9.57 E+04$ & $5.53 \mathrm{E}+04$ & 3.27 & $\begin{array}{r}3.67 \\
\end{array}$ & 12.5 & 15.3 \\
\hline $\mathrm{AB}+$ & 7.26 & $1.12 \mathrm{E}+05$ & $6.45 \mathrm{E}+04$ & 6.94 & 2.07 & 7.39 & 7.39 \\
\hline $\mathrm{Fe} 3+(\operatorname{cotal} \mathrm{Fe})$ & 1.36 & $433 \mathrm{E}+04$ & $2.50 \mathrm{E}+0 \mathrm{~A}$ & 134 & 1.35 & 1.37 & 1,37 \\
\hline $\mathrm{Cr} 3+$ & $6.29 \mathrm{E}-02$ & $1.86 \mathrm{E}+03$ & $1.08 \mathrm{E}+03$ & $1.94 \mathrm{E}-02$ & 4.07E-02 & 0.428 & 0.854 \\
\hline Bijt+ & 0 & 0 & 0 & 0 & 0 & 0 & 0 \\
\hline $\mathrm{LE+}$ & 0 & 0 & 0 & 0 & 0 & 0 & 0 \\
\hline $\mathrm{Hg}^{2+}$ & 0 & 0 & 0 & 0 & 0 & 0 & 0 \\
\hline $\mathrm{Zr}(\mathrm{as} \mathrm{ZrO}(\mathrm{OH}) \mathrm{2}$ & 0 & 0 & 0 & 0 & 0 & 0 & 0 \\
\hline Pb2+ & 0 & 0 & 0 & 0 & 0 & 0 & 0 \\
\hline Ni2t & $6.45 \mathrm{E}-02$ & $2.16 \mathrm{E}+03$ & $1.25 \mathrm{E}+03$ & $5.33 \mathrm{E}-02$ & $5.97 \mathrm{E}-02$ & $6.83 \mathrm{E}-02$ & $6.76 \mathrm{E}-02$ \\
\hline Sr2+ & 0 & 0 & 0 & 0 & 0 & 0 & 0 \\
\hline $\mathrm{Mndt}$ & 0 & $\overline{0}$ & 0 & 0 & 0 & 0 & 0 \\
\hline $\mathrm{Cn} 2+$ & 0.279 & $6.32 E+03$ & $3.69 \mathrm{E}+03$ & 0.220 & 0.249 & 0.310 & 0,339 \\
\hline $\mathbf{K}+$ & $1.13 \mathrm{E}-02$ & 253 & 146 & 3.46E-03 & 6.61E-03 & 1.45E-02 & 1.45E-02 \\
\hline OH- & 27.5 & $2.67 \mathrm{E}+05$ & $1.54 E+05$ & 25.5 & 26.5 & 28.6 & 29.8 \\
\hline No3- & 4.19 & $1.48 E+05$ & $8.36 E+04$ & 4. $7 \mathrm{UE}-02$ & $47 \mathrm{gE}-02$ & 10.7 & 14.2 \\
\hline $\mathrm{NO}_{2-}$ & 1.48 & 3.69E+04 & $2.25 \mathrm{E}+04$ & 0.432 & 0.799 & 2.03 & 2.03 \\
\hline Co32. & 0.279 & $9.56 \mathrm{E}+03$ & $5.52 \mathrm{E}+03$ & 0.220 & 0.249 & 0.310 & 0.339 \\
\hline PO43- & 0 & 0 & 0 & 0 & 0 & 0 & 0 \\
\hline SOA2- & $1.19 \mathrm{E}-02$ & 653 & 377 & 4.61E-03 & $7.53 \mathrm{E}-03$ & 1.49E-02 & $1.49 \mathrm{E}-02$ \\
\hline Si (u SiO32-) & 0.105 & $1.68 \mathrm{E}+03$ & 971 & 1.18E-02 & $1.3 \%-02$ & 0.197 & 0.284 \\
\hline F: & 0 & 0 & 0 & 0 & o & 0 & 0 \\
\hline $\mathrm{Cl}-$ & $5.22 \mathrm{E}-02$ & $1.05 \mathrm{E}+03$ & 609 & 1.59E-02 & $3.04 \mathrm{E}-02$ & $6.68 \mathrm{E}-02$ & 0136 \\
\hline C6HSO73- & 0 & 0 & 0 & 0 & 0 & 0 & 0 \\
\hline EDTA4- & 0 & 0 & 9 & 0 & 0 & 0 & 0 \\
\hline HEDTA3. & 0 & 0 & 0 & 0 & 0 & 0 & 0 \\
\hline Blycolinte- & 0 & 0 & 0 & 0 & 0 & 0 & 0 \\
\hline coetale- & 0 & 0 & 0 & 0 & 0 & 0 & 0 \\
\hline oxnifuce?- & $\overline{0}$ & 0 & 0 & 0 & 0 & 0 & 0 \\
\hline DBí & 0 & 0 & 0 & 0 & 0 & 0 & 0 \\
\hline burtursol & 0 & 0 & 0 & 0 & 0 & 0 & 0 \\
\hline NH3 & 0.299 & $2.90 \mathrm{E}+03$ & $1.68 E+03$ & 0,149 & 0.170 & 0.454 & 0.322 \\
\hline $\mathrm{Fa}(\mathrm{CN}) 64$ & 0 & 0 & 0 & 0 & 0 & 0 & 0 \\
\hline
\end{tabular}

"Unknowns in tank solide inventory tre asnigned by Tank Layering Model (TLM)

+ Water wt\% derived from the difference of density and total dissolved species. 
HDW Model Rov. 4

\begin{tabular}{|c|c|c|c|c|c|c|c|}
\hline \multicolumn{8}{|c|}{ Single-Shell Tank 241-\$X-108 } \\
\hline & \multicolumn{7}{|c|}{ TLM Solids Composite Inventory Estimate" } \\
\hline $\begin{array}{l}\text { Phylleal } \\
\text { Properties }\end{array}$ & & & & $.95 \mathrm{CI}$ & $-67 \mathrm{CI}$ & $+67 C 1$ & $+98 \mathrm{Cl}$ \\
\hline Total TLM Wa & $5.78 \mathrm{E}+05(\mathrm{~kg})$ & $(87.0 \mathrm{kgal})$ & - & - & - & - & - \\
\hline Hest Lood & $751(\mathrm{kw})$ & $(2.56 \mathrm{E}+04 \mathrm{BTU} / \mathrm{hr})$ & $=$ & 7.04 & 7.32 & 7.64 & 7.69 \\
\hline Bulk Density & $1.75(\$ / \infty)$ & $\ldots$ & $=$ & 1.56 & 1.58 & 2.03 & 2.14 \\
\hline Void Fraction & 0.547 & 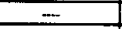 & $=$ & 0.169 & 0.294 & 0.706 & 0.710 \\
\hline Water $w t \%$ & 280 & $\ldots$ & - & 7.54 & 13.9 & 40.1 & 41.3 \\
\hline TOC $w t \% C(w$ & 0 & - & - & 0 & 0 & 0 & 0 \\
\hline $\begin{array}{l}\text { Rodiolod foil } \\
\text { Constiltueats }\end{array}$ & Cri. & $\mathrm{\mu Cl} / \mathrm{s}$ & Cl & $\begin{array}{l}95 \mathrm{C} \\
\text { (cit) }\end{array}$ & $\begin{array}{l}67 \mathrm{CI} \\
\text { (C/L) }\end{array}$ & $\begin{array}{l}+67 \mathrm{CI} \\
(\mathrm{Cl} / \mathrm{h})\end{array}$ & $\begin{array}{r}+93 \mathrm{CI} \\
(\mathrm{CVI})\end{array}$ \\
\hline H-3 & $5.36 \mathrm{E}-05$ & $3.06 \mathrm{E}-02$ & 17,7 & 3.28E-0S & $3.43 \mathrm{E}-05$ & $6.30 \mathrm{E}-05$ & $6,30 \mathrm{E}-05$ \\
\hline C-14. & 3. IOE-06 & $1.77 \mathrm{E}-03$ & 1.02 & I.51E-06 & $2.15 E-06$ & $3.74 E-06$ & $3.74 \mathrm{E}-06^{-06}$ \\
\hline $\mathrm{Ni}+59$ & $2.86 \mathrm{E}-05$ & $1.63 \mathrm{E}-02$ & 940 & $2.53 \mathrm{E}-05$ & $2.71 E-05$ & $2.96 \mathrm{E}-05$ & $2.99 \mathrm{E}-05$ \\
\hline $\mathrm{Ni}-63$ & $276 \mathrm{E}-03$ & 1.57 & 908 & 2.45E-03 & $2.63 \mathrm{E}-03$ & $2.86 \mathrm{E}-03$ & $2.89 \mathrm{E}-03$ \\
\hline Co-60 & 2.17E-06 & 1.24E-03 & 0.715 & $1.54 \mathrm{E}-06$ & $1.26 \mathrm{E}-06$ & $2.42 \mathrm{E}-06$ & 2.42E-06 \\
\hline Se-79 & $7.59 \mathrm{E}-06$ & $433 \mathrm{E}-03$ & 2.50 & $7.02 \mathrm{E}-07$ & $7.02 \mathrm{E}-07$ & $1.53 \mathrm{E}-05$ & $2.27 \mathrm{E}-05$ \\
\hline $\mathrm{Sr}-90$ & 3.32 & 1. $19 \mathrm{E}+03$ & $1.09 \mathrm{E}+06$ & 3.11 & 3,23 & 3.39 & 3.40 \\
\hline Y-90 & 3.32 & $1.89 E+03$ & $1.09 \mathrm{E}+06$ & 3.11 & 3.23 & 3.39 & 3.40 \\
\hline $\mathrm{Zr}-93$ & 3.19E-05 & $1.82 \mathrm{E}-02$ & 10.5 & $3,34 \mathrm{E}-06$ & $3.34 \mathrm{E}-06$ & 7.06E-05 & $1.08 \mathrm{E}-04$ \\
\hline $\mathrm{Nb}-93 \mathrm{~m}$ & 3.01E-05 & $1.71 \mathrm{E}-02$ & 9,90 & $2.63 \mathrm{E}-06$ & $3.02 E-06$ & $5.71 \mathrm{E}-05$ & 8.31E-05 \\
\hline Tc-99 & $2.32 \mathrm{E}-0 \mathrm{~S}$ & $1.33 \mathrm{E}-02$ & 7.65 & 1.21E-05 & $166 \mathrm{E}-0 \mathrm{~s}$ & $2.77 \mathrm{E}-05$ & 2.77E-05 \\
\hline Ru-106 & $3.99 \mathrm{E}-10$ & 2.2:E-07 & $1,32 \mathrm{E}-04$ & $3.53 \mathrm{E}-10$ & $1.51 \mathrm{E}-10$ & $1.31 \mathrm{E}-09$ & $1,99 \mathrm{E}-08$ \\
\hline Cd-113m & $1.16 E-05$ & 6.59E-03 & 3.80 & 6.64E-06 & $7,79 \mathrm{E}-06$ & $1.31 E-04$ & $3.54 \mathrm{E}-\mathrm{OH}$ \\
\hline $\mathrm{Sb}-125$ & 6.70E- 06 & $3.82 \mathrm{E}-03$ & 2,21 & $578 E-06$ & $3.16 \mathrm{E}-06$ & $7.07 E-06$ & $7.07 \mathrm{E}-06$ \\
\hline $5 n-126$ & $9.67 \mathrm{E}-06$ & $5.52 \mathrm{E}-03$ & 3.19 & $1.05 \mathrm{E}-06$ & 1.05E-06 & $2.13 \mathrm{E}-05$ & $324 E-05$ \\
\hline $1-129$ & $443 E A 8$ & 2.53E-OS & $1.46 \mathrm{E}-02$ & $2205-08$ & 3.15E-08 & $5.29 \mathrm{E}-00$ & $5.29 \mathrm{e}-08$ \\
\hline C8-134 & $5.24 \mathrm{E}-07$ & $2.99 \mathrm{E}-04$ & 0.173 & 4.84E-07 & $3.07 \mathrm{E}-07$ & $5.38 \mathrm{E}-07$ & $5.38 \mathrm{E}-07$ \\
\hline Cs-137 & $9.7 \mathrm{E}-02$ & 56.3 & $3.25 \mathrm{E}+04$ & $6.60 \mathrm{E}-02$ & $7.52 \mathrm{E}-02$ & 0.112 & 0.112 \\
\hline$B a-137 m$ & 9,34E-02 & 53.2 & $3.07 \mathrm{E}+04$ & 6.24E-02 & $7.12 \mathrm{E}-02$ & 0.106 & 0.106 \\
\hline $5 \mathrm{~m}-151$ & 2.72E-02 & 15.5 & $8,96 \mathrm{E}+03$ & 2.49E-03 & $2.49 \mathrm{E}-03$ & $5.43 E-02$ & $8.02 \mathrm{E}-02$ \\
\hline Eu-152 & $2.87 \mathrm{E}-05$ & 1.64E-02 & 9.46 & 2. A6E-05 & 2.6EE-05 & 2.88E-05 & $288 \mathrm{E}-05$ \\
\hline Eu-154 & $5.28 \mathrm{E}-05$ & $3.01 \mathrm{E}-02$ & 17.4 & $3.76 \mathrm{E}-05$ & 3.04E-05 & $5.89 \mathrm{E}-0 \mathrm{~S}$ & $1.64 \mathrm{E}-03$ \\
\hline Eu-155 & 1.45E-03 & 0.825 & 476 & $1.44 \mathrm{E}-03$ & $1.44 E-03$ & 1.45E-03 & 1.45E-03 \\
\hline $\mathrm{R}=226$ & $2.39 \mathrm{E}-09$ & $1.36 \mathrm{E}-06$ & $7.87 \mathrm{E}-04$ & 1.65E-09 & $2.01 \mathrm{E}-09$ & $2.77 \mathrm{E}-09$ & 3. 13E-09 \\
\hline Ra-228 & 4 BOE-14 & 2.74E-11 & $1.58 \mathrm{E}-08$ & 4.78E-14 & $4.7 \mathrm{EE}-14$ & $4.81 \mathrm{E}-14$ & $4.81 E-14$ \\
\hline Ac-227 & $1.06 \mathrm{E}-08$ & $6.05 E-00$ & 3.50E-03 & 7.09E-09 & $8.43 \mathrm{E}-09$ & 1.28E-08 & $1,49 \mathrm{E}-0 \mathrm{~s}$ \\
\hline Pa-231 & S. $12 E-09$ & $4.63 \mathrm{E}_{-106}$ & 2,67E-03 & SAE-10 & 8.94E-10 & $1.58 \mathrm{E}-08$ & $2.33 \mathrm{E}-0.8$ \\
\hline Th-229 & $3,91 \mathrm{E}-12$ & $2.23 \mathrm{E}-09$ & $1.29 \mathrm{E}-06$ & 3.89E-12 & $3.89 \mathrm{E}-12$ & $3.92 \mathrm{E}-12$ & $3.92 \mathrm{E}-12$ \\
\hline Th-232 & $1.75 \mathrm{E}-15$ & $999 \mathrm{E}-13$ & 5.7它-10 & $1.34 \mathrm{E}-15$ & $9.41 E-16$ & $1.92 \mathrm{E}-15$ & $1.92 \mathrm{E}-15$ \\
\hline U-232 & $7,05 \mathrm{E}-12$ & 4.02E-09 & $2.32 \mathrm{E}-06$ & $3.37 \mathrm{E}-12$ & $4.84 \mathrm{E}-12$ & 1.52E-12 & $852 \mathrm{E}-12$ \\
\hline U-233 & $2.25 \mathrm{E}-13$ & $1.26 \mathrm{E}-10$ & $7.42 E-08$ & 8.52E-14 & 1.41E-13 & $2.82 \mathrm{E}-13$ & $2.82 \mathrm{E}-13$ \\
\hline U-234 & 1.24E-07 & $7.06 \mathrm{E}-05$ & 403E-02 & $4.01 E-08$ & 7.35E-03 & $1.57 \mathrm{E}-07$ & 1.5TE-0? \\
\hline U.235 & $5,1 \pi-09$ & 2.95E-06 & 1. $\%$ EE-03 & $1.5 \pi-09$ & $3.01 E-09$ & $6.62 \mathrm{E}-09$ & 6.62E-09 \\
\hline $\mathrm{U}-236$ & $347 E-09$ & 1.95E-06 & 1.14E-03 & 2.15E- 09 & 2.24E-09 & 4.01E-09 & 4 O1E-09 \\
\hline U-238 & $1.1 \pi-07$ & $6.67 \mathrm{E}-05$ & 3.95E-02 & 3.15E-08 & $6.56 \mathrm{E}-0 \mathrm{~d}$ & $1.51 \mathrm{E}-07$ & $1.51 E-07$ \\
\hline$N_{p}-237$ & $1.20 E-07$ & $6.82 E-05$ & 3.94E-02 & 4.74E-08 & $7,62 \mathrm{E}-0 \mathrm{8}$ & 1.49E-07 & $1.49 \mathrm{E}-07$ \\
\hline Pu-238 & $2.085-05$ & 1. 19E-02 & 0.85 & $1.74 \mathrm{E}-0 \mathrm{~S}$ & 1.91E-05 & 2.25E-0s & $242 \mathrm{E}-03$ \\
\hline Pu-239 & $6.76 \mathrm{E}-04$ & 0.335 & 222 & 5.70E-04 & 6.22E-04 & 7.30E-04 & $781 \mathrm{E}-104$ \\
\hline Pu-240 & $1.12 E-04$ & $6.38 \mathrm{E}-02$ & 36.6 & $9,68 E_{-}-05$ & $1.04 E-04$ & 1.20E-04 & $1.2 \pi \mathrm{E}-04$ \\
\hline$P_{t-241}$ & 1.06E-03 & 0.606 & 350 & 8.99E-04 & $9.79 \mathrm{E}-04$ & 1.15E-03 & $1.23 \mathrm{E}-03$ \\
\hline$P_{u-242}$ & $5.73 \mathrm{E}-09$ & $3.27 \mathrm{E}-06$ & 1.69E-03 & $4.79 E-09$ & $3.25 \mathrm{E}-09$ & $6.21 E-09$ & $6.6 \mathrm{TE}-09$ \\
\hline Am-241 & $4.62 \mathrm{E}-04$ & 0.263 & 152 & 2.34E-04 & $3.46 \mathrm{E}-04$ & 5.T7E-04 & $6.89 E-04$ \\
\hline Am-243 & $2.06 \mathrm{E}-0 \mathrm{~B}$ & 1.17E-05 & $6.78 E-03$ & 1.03E-08 & I.S4E-08 & 2.5se-0s & 3.08E-08 \\
\hline $\mathrm{Cm}-242$ & $122 \mathrm{E}-06$ & $6.98 \mathrm{E}-04$ & 0.403 & $1.22 \mathrm{E}-06$ & $1.22 \mathrm{E}-0.06$ & 1.23E-06 & $1.23 \mathrm{E}-06$ \\
\hline $\mathrm{Cm}-243$ & $1.14 E-07$ & 6.50E-05 & $3.76 \mathrm{E}-02$ & 1.14E-07 & $1.13 \mathrm{E}-07$ & 1.14E-07 & 1.14E-07 \\
\hline $\mathrm{Cm}-244$ & $337 \mathrm{E}-06$ & $1,92 \mathrm{E}-03$ & 1.11 & $2.61 E-06$ & $2.98 \mathrm{E}-06$ & $3.75 \mathrm{E}-06$ & $412 \mathrm{E}-06$ \\
\hline Totals: & $\mathbf{M} \quad \ldots$ & ints & ne & $\begin{array}{l}98 \mathrm{C} \\
(\mathrm{Mer} \\
\text { (it) }\end{array}$ & $\begin{array}{l}6 \mathrm{CT} \\
(\mathrm{Mror} \\
(1)\end{array}$ & $\begin{array}{l}+67 \mathrm{CT} \\
(\mathrm{Mot} \\
\mathrm{p} / \mathrm{l})\end{array}$ & $\begin{array}{l}+9 \mathrm{Cl} \\
(\mathrm{M} \mathrm{or} \\
(\mathrm{A})\end{array}$ \\
\hline Pu & $1.14 E-02(g / L)$ & - & 3.74 & $961 \mathrm{E}-03$ & 1,05E-02 & $1.23 \mathrm{E}-02$ & $1,31 \mathrm{E}-02$ \\
\hline $\mathrm{U}$ & $1.47 \mathrm{E}-03$ & 200 & 115 & $3.97 \mathrm{E}-04$ & $8.26 \mathrm{E}-04$ & 1.90E-03 & $1.90 \mathrm{E}-03$ \\
\hline
\end{tabular}

*Unknowns in tank solids inventory are nasigned by Tank Loyering Model (TLM). 
HNF-SD-WM-ER-352, Rev. 1

HDW Model Rey. 4

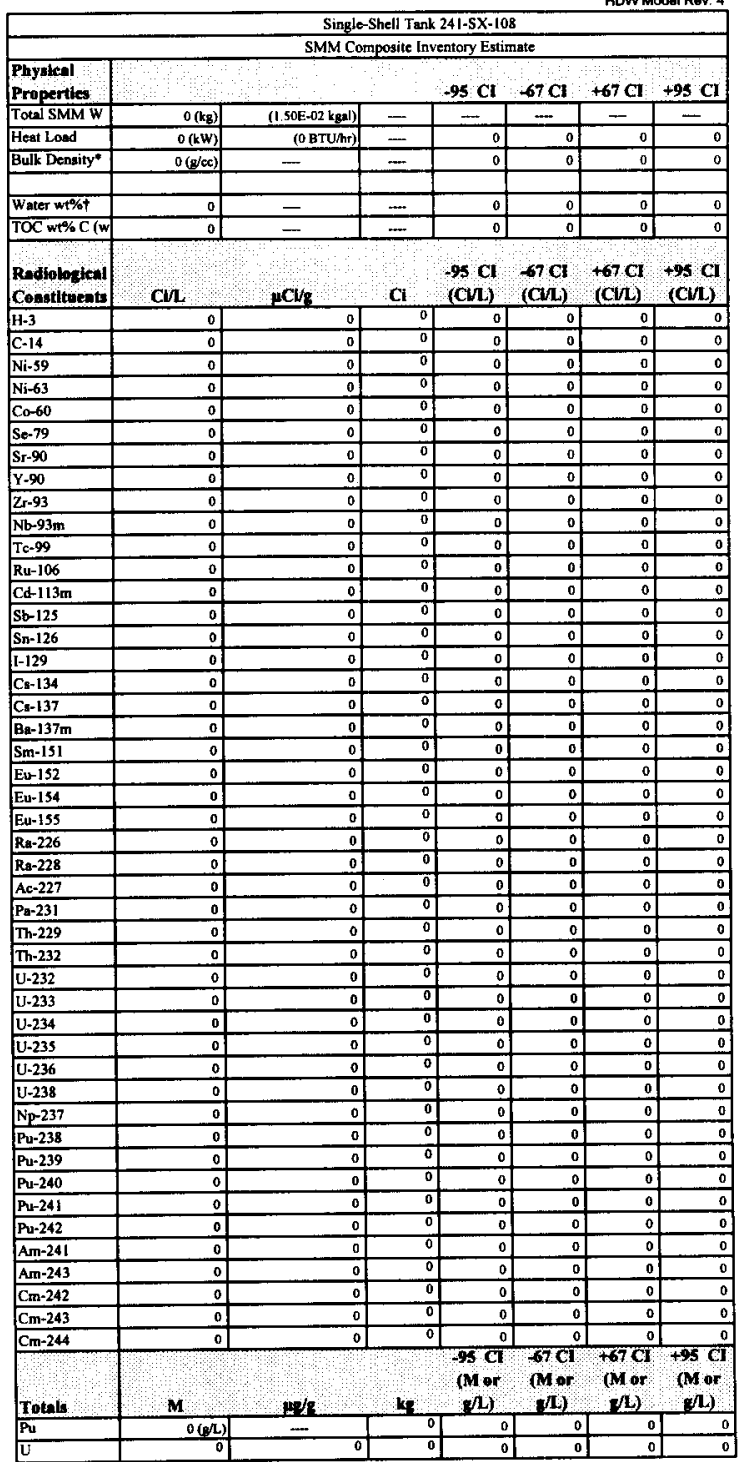

-Density is caiculated haeed on $\mathrm{Na}, \mathrm{OH} \cdot$, and $\mathrm{AlO2}$ -

tWater wt\% derived from the difference of density and total dinsolved species. 
HNF-SD-WM-ER-352, Rev. 1

HDW Modal Rev. 4

\begin{tabular}{|c|c|c|c|c|c|c|c|}
\hline & \multicolumn{5}{|c|}{ Single-Shell Tank 241-SX-108 } & \multicolumn{2}{|c|}{ HDW Model Rev. 4} \\
\hline & \multicolumn{7}{|c|}{ Total Inventory Estimate" } \\
\hline $\begin{array}{l}\text { Physletal } \\
\text { Propertics }\end{array}$ & & & & $95 \mathrm{Cl}$ & $-67 \mathrm{CI}$ & $+67 \mathrm{CI}$ & $+95 \mathrm{CL}$ \\
\hline Total Waste & $5.78 \mathrm{E}+05(\mathrm{~kg})$ & $(87.0 \mathrm{kgal})$ & - & $\ldots$ & - & $=$ & - \\
\hline Heat Lond & $7.51(\mathrm{~kW})$ & (2.56E +04 BTU $/ \mathrm{hr})$ & - & 7.04 & 7.32 & 7.64 & 7.69 \\
\hline Bulk Density ${ }^{\dagger}$ & $1.75(g / c c)$ & - & $-\cdots$ & 1.56 & 1.58 & 2.03 & 2.14 \\
\hline Water wt\%t & 28.0 & -- & $\ldots$ & 7.54 & 139 & 40.1 & 41.3 \\
\hline TOC wt\% C (w) & 0 & $\rightarrow$ & - & 0 & 0 & 0 & 0 \\
\hline $\begin{array}{l}\text { Rallolesical } \\
\text { Constituents }\end{array}$ & CVI & $\mathrm{nCU}, \mathrm{s}$ & $\mathrm{Cl}$ & $\begin{array}{l}-95 \mathrm{CI} \\
\text { (CUL) }\end{array}$ & $\begin{array}{l}67 \mathrm{CI} \\
(\mathrm{Clat})\end{array}$ & $\begin{array}{l}+67 \mathrm{CI} \\
(\mathrm{Cu} / \mathrm{L})\end{array}$ & $\begin{array}{l}+95 \mathrm{Cl} \\
\text { (CVL) }\end{array}$ \\
\hline $\mathrm{H}-3$ & 5.36E-0.5 & $3.06 \mathrm{E}-02$ & 17.7 & $3.28 \mathrm{E}-05$ & 3.43E-05 & $6.30 \mathrm{E}-0 \mathrm{~S}$ & $6.30 \mathrm{E}-05$ \\
\hline $\mathrm{C}-14$ & $3.10 \mathrm{E}-06$ & $1.77 \mathrm{E}-03$ & 1.02 & $1.51 \mathrm{E}-06$ & 2.15E-06 & $3.74 \mathrm{E}-06$ & 3.74E- 06 \\
\hline $\mathrm{Ni}-59$ & 2.65E-05 & $1.63 \mathrm{E}-02$ & 9,40 & 2.53E-05 & $2.71 \mathrm{E}-05$ & $2.96 \mathrm{E}-05$ & $2.99 \mathrm{E}-05$ \\
\hline $\mathrm{Ni}-63$ & $2.76 E-03$ & 1.57 & 908 & $2.45 \mathrm{E}-03$ & $2.63 \mathrm{E}-03$ & 2.86E-03 & $2.89 \mathrm{E}-03$ \\
\hline Co-60 & $2.17 E-06$ & $1,24 \mathrm{E}-03$ & 0.715 & 1.54E-06 & $1.26 \mathrm{E}-06$ & $2.42 \mathrm{E}-06$ & 2.42E-06 \\
\hline Se-79 & $7.59 \mathrm{E}-06$ & $433 \mathrm{E}-03$ & 2.50 & $7.02 \mathrm{E}-07$ & $7.02 \mathrm{E}-07$ & $1.53 \mathrm{E}_{0} 05$ & $2.27 \mathrm{E}-05$ \\
\hline Sr-90 & 3.32 & $1.89 \mathrm{E}+03$ & $1.09 \mathrm{E}+06$ & 3.11 & 3.23 & 3.39 & 3.40 \\
\hline$Y-90$ & 3.32 & $1.89 \mathrm{E}+03$ & $109 \mathrm{E}+06$ & 3.11 & 3.23 & 3.39 & 3.40 \\
\hline$Z r-93$ & $3.19 \mathrm{E}-0 \mathrm{~s}$ & $1.82 \mathrm{E}-02$ & 10.5 & $3.34 \mathrm{E}-06$ & $3.345-06$ & $7.06 \mathrm{E}-05$ & $1.08 E-04$ \\
\hline $\mathrm{Nb}-93 \mathrm{~m}$ & 3.01E-05 & $1.71 \mathrm{E}-02$ & 950 & $2.63 \mathrm{E}-06$ & $302 \mathrm{E}-\infty 6$ & $5.71 \mathrm{E}-0 \mathrm{~s}$ & B.31E-05 \\
\hline Te-99 & 2.32E-05 & 1.33E-02 & 7.65 & $1.21 E-0 S$ & $1.66 \mathrm{E}-05$ & $2.77 \mathrm{E}-05$ & $2.77 \mathrm{E}-05$ \\
\hline Ru-106 & 3.99E-10 & 2.28E-07 & T.32E-04 & $3.53 \mathrm{E}-10$ & $1.51 \mathrm{E}-10$ & $1,31 \mathrm{E}-00$ & 1.99E-08 \\
\hline$C d-113 \mathrm{~m}$ & $1.16 \mathrm{E}-05$ & $6.596-03$ & 3.80 & 6.64E-06 & $7.79 \mathrm{E}-06$ & $1.31 \mathrm{E}-04$ & 3.54E-04 \\
\hline$s b-125$ & $6.70 E-06$ & 3 S2E -03 & 2.21 & 5.78E-06 & $3.16 \mathrm{E}-06$ & 7.07E-06 & 7.07E-06 \\
\hline $5 n-126$ & $9.67 \mathrm{E}-06$ & $552 E-03$ & 3.19 & $1.05 \mathrm{E}-06$ & 1.05E-06 & 2.13E-05 & 3.24E-05 \\
\hline$[-129$ & 4.43E-08 & 2.53E-0s & $1.46 \mathrm{E}-02$ & $2.29 \mathrm{E}-08$ & $3.15 \mathrm{E}-0 \mathrm{~B}$ & 5.29E-08 & $5.29 \mathrm{E}-08$ \\
\hline Cs-134 & 5.24E-07 & $2.99 \mathrm{E}-04$ & 0.173 & 484E-07 & 3.07E-07 & $5.38 \mathrm{E}-07$ & $53 \mathrm{BE}-07$ \\
\hline $\mathrm{C} s-137$ & 9.87E-02 & 56.3 & $3.25 E+04$ & $6.60 \mathrm{E}-02$ & $7.52 \mathrm{E}-02$ & 0.112 & 0.112 \\
\hline Ba-137m & $9.33 \mathrm{E}-02$ & 53.2 & $307 \mathrm{E}+04$ & $6.24 \mathrm{E}-02$ & $7.12 \mathrm{E}-02$ & 0.106 & 0.106 \\
\hline Sm-151 & $2.72 \mathrm{E}-02$ & 15.5 & 2.96E+03 & $2.49 \mathrm{E}-03$ & 2.49E-03 & 5.43E-02 & 8.02E-02 \\
\hline Eu-152 & 2.87E-05 & 1.64E-02 & 9.46 & $2.86 \mathrm{E}-05$ & $2.86 \mathrm{E}-05$ & 2.88E-05 & 2.88E-05 \\
\hline Eu-154 & $5.28 \mathrm{E}-05$ & $3.01 \mathrm{E}-02$ & 17.4 & $3.76 \mathrm{E}-05$ & 3.04E-05 & $5,89 \mathrm{E}-05$ & $1.64 \mathrm{E}-03$ \\
\hline Eu-155 & $1.45 \mathrm{E}-03$ & 0.825 & 476 & 1.4E-03 & $1.44 \mathrm{E}-03$ & $1.45 \mathrm{E}-03$ & 1.45E-03 \\
\hline Ra-226 & 2.39E-69 & $1.36 \mathrm{E}-06$ & 7.87E-04 & $1.65 \mathrm{E}-09$ & $2.01 E-09$ & $2.77 \mathrm{E}-09$ & $3.13 \mathrm{E}-69$ \\
\hline Re-228 & 4.80E-14 & 2.74E-11 & 1.58E-08 & 4.7BE-14 & $4.77 \mathrm{E}-14$ & $4.81 \mathrm{E}_{-14}$ & 4BIE-14 \\
\hline$A c-227$ & 1.06E-08 & $6.05 \mathrm{E}-06$ & $3.50 \mathrm{E}-03$ & $7.09 E-09$ & $8.43 \mathrm{E}-09$ & $1.28 \mathrm{E}-08$ & $1,49 \mathrm{E}-0 \mathrm{~B}$ \\
\hline Pa-231 & $8.12 \mathrm{E}-09$ & $463 \mathrm{E}-06$ & $2.67 \mathrm{E}-03$ & $8.945-10$ & 8.94E-10 & 1.58E-08 & $233 \mathrm{E}-98$ \\
\hline Th-229 & $3.91 \mathrm{E}-12$ & $2.23 \mathrm{E}-09$ & $1.29 \mathrm{E}-06$ & $3.88 \mathrm{E}-12$ & $3.06-12$ & $3.92 \mathrm{E}-12$ & $392 \mathrm{E}-12$ \\
\hline Th-232 & 1.75E-15 & $9.996-13$ & $5.77 \mathrm{E}-10$ & $1,34 \mathrm{E}-15$ & $9.48 \mathrm{E}-16$ & $1.92 \mathrm{E}-15$ & $1.92 \mathrm{E}-15$ \\
\hline U-232 & $7.05 \mathrm{E}-12$ & 4.02E-09 & 2.32E-06 & $3.37 \mathrm{E}-12$ & $4.84 \mathrm{E}-12$ & $8.52 \mathrm{E}-12$ & 8.52E-12 \\
\hline $\mathrm{U}-233$ & 2.25E-13 & $121 \mathrm{E}_{-10}$ & $7.42 \mathrm{E}-08$ & $52 \mathrm{E}-14$ & $1.41 \mathrm{E}-13$ & 2.22E-13 & $2.82 \mathrm{E}-13$ \\
\hline U-234 & $1.24 \mathrm{E}-07$ & $7,06 E-05$ & 4.08E-02 & 4.01E-08 & 7.35E -08 & 1.57E-07 & $1.57 \mathrm{E}-07$ \\
\hline U.235 & $5.17 \mathrm{E}-09$ & $295 \mathrm{E}-06$ & $1.70 \mathrm{E}-03$ & $1.57 \mathrm{E}-99$ & $3.01 E-09$ & $6.62 \mathrm{E}-09$ & 6.62E-09 \\
\hline $\mathrm{U}-236$ & 3.47E-09 & 1.91E_-06 & $1.14 \mathrm{E}-03$ & 2.15E-09 & 2.24E-09 & 4.01E-09 & 4.01E-09 \\
\hline U-238 & 1.17F-07 & 6.67E-0S & 3.85E-02 & $3.15 \mathrm{E}-08$ & $6.56 \mathrm{E}-08$ & $1.51 \mathrm{E}-07$ & $1,51 \mathrm{E}-07$ \\
\hline $\mathrm{Np}-237$ & $1.20 \mathrm{E}-07$ & $6.82 \mathrm{E}-05$ & 3.94E-02 & $4.74 \mathrm{E}-08$ & $7.62 \mathrm{E}-0 \mathrm{0}$ & 1.49E-07 & $1.49 \mathrm{E}-07$ \\
\hline$P u-238$ & $2.05 E-05$ & $1.19 \mathrm{E}-02$ & 6.15 & 1.74E-05 & 1.91E-05 & $2.25 \mathrm{E}-05$ & $2.42 \mathrm{E}-05$ \\
\hline Pu-239 & $6.76 \mathrm{E}-04$ & $0.3 \mathrm{ss}$ & 222 & $5.70 \mathrm{E}-04$ & 6.22E-04 & $7,30 \mathrm{E}-04$ & $7.81 \mathrm{E}-04$ \\
\hline Pur-240 & $1.12 \mathrm{E}-04$ & 6.38E- -02 & 36.8 & 9.68E-05 & 1.04E-OA & $1.206-04$ & $1.2 \pi \mathrm{E}-\mathrm{A}$ \\
\hline Pu-241 & $1.06 \mathrm{E}-03$ & 0.606 & 350 & $699 \mathrm{E}-04$ & $9.79 \mathrm{E}-04$ & 1. ISE-03 & $1.23 E-03$ \\
\hline Put-242 & $5.73 \mathrm{E}-09$ & $3.27 \mathrm{E}-106$ & $1.89 \mathrm{E}-03$ & $4.79 \mathrm{E}-099$ & $5.25 \mathrm{E}-09$ & $6.21 \mathrm{E}-09$ & $6.67 \mathrm{E}-09$ \\
\hline Am-241 & 4.61E-04 & 0.263 & 152 & $2.34 \mathrm{E}-04$ & $3.46 \mathrm{E}-04$ & $5.77 \mathrm{TE}-04$ & $6.89 \mathrm{E}-04$ \\
\hline$A m-243$ & 2.06E-08 & $1.17 \mathrm{E}-0 \mathrm{~s}$ & $6.78 E-03$ & $1,03 \mathrm{E}_{-08}$ & $1.54 \mathrm{E}-08$ & 2.58E-AB & $3.09 \mathrm{E}-08$ \\
\hline $\mathrm{Cm}-242$ & $1.23 \mathrm{E}-06$ & $6.91 \mathrm{E}-04$ & 0.403 & $1.22 \mathrm{E}-06$ & $1.22 E-06$ & $1.23 \mathrm{E}-06$ & $1.23 \mathrm{E}-06$ \\
\hline $\mathrm{C}_{\mathrm{m}}-243$ & 1.14E-0? & 6 soet-os & $3.76 \mathrm{E}-02$ & 1.14E-07 & $1.13 \mathrm{E}-07$ & 1.14E-07 & 1.14E-07 \\
\hline $\mathrm{Cm}-244$ & 3.37E-06 & $1.92 e_{-03}$ & 1.11 & $2.61 \mathrm{E}-06$ & 2.94E-06 & $3.75 \mathrm{E}-06$ & 4.12E-06 \\
\hline Tothls: & $\mathbf{M}$ & sed & 4 & 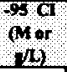 & 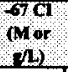 & 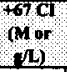 & $\begin{array}{l}+9 \mathrm{CT} \\
\mathrm{Mer} \\
\mathrm{dl}\end{array}$ \\
\hline $\mathrm{Pu}$ & $1.14 E-02(\phi L)$ & $\cdots$ & 3.74 & $9.61 \mathrm{E}-03$ & $1.05 E-02$ & $1.23 \mathrm{E}-02$ & $131 \mathrm{E}-02$ \\
\hline $\mathrm{U}$ & $1.47 \mathrm{E}-03$ & 200 & 115 & $3.97 \mathrm{E}-04$ & $8.26 \mathrm{E}-04$ & 1.90E-03 & $190 \mathrm{E}-03$ \\
\hline
\end{tabular}

"Unknowrs in tank solida inventory are assigned by Tenk Layering Model (TLM).

+ Volume average for density, mase average Water wt\% and TOC wr\% C. 


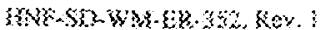

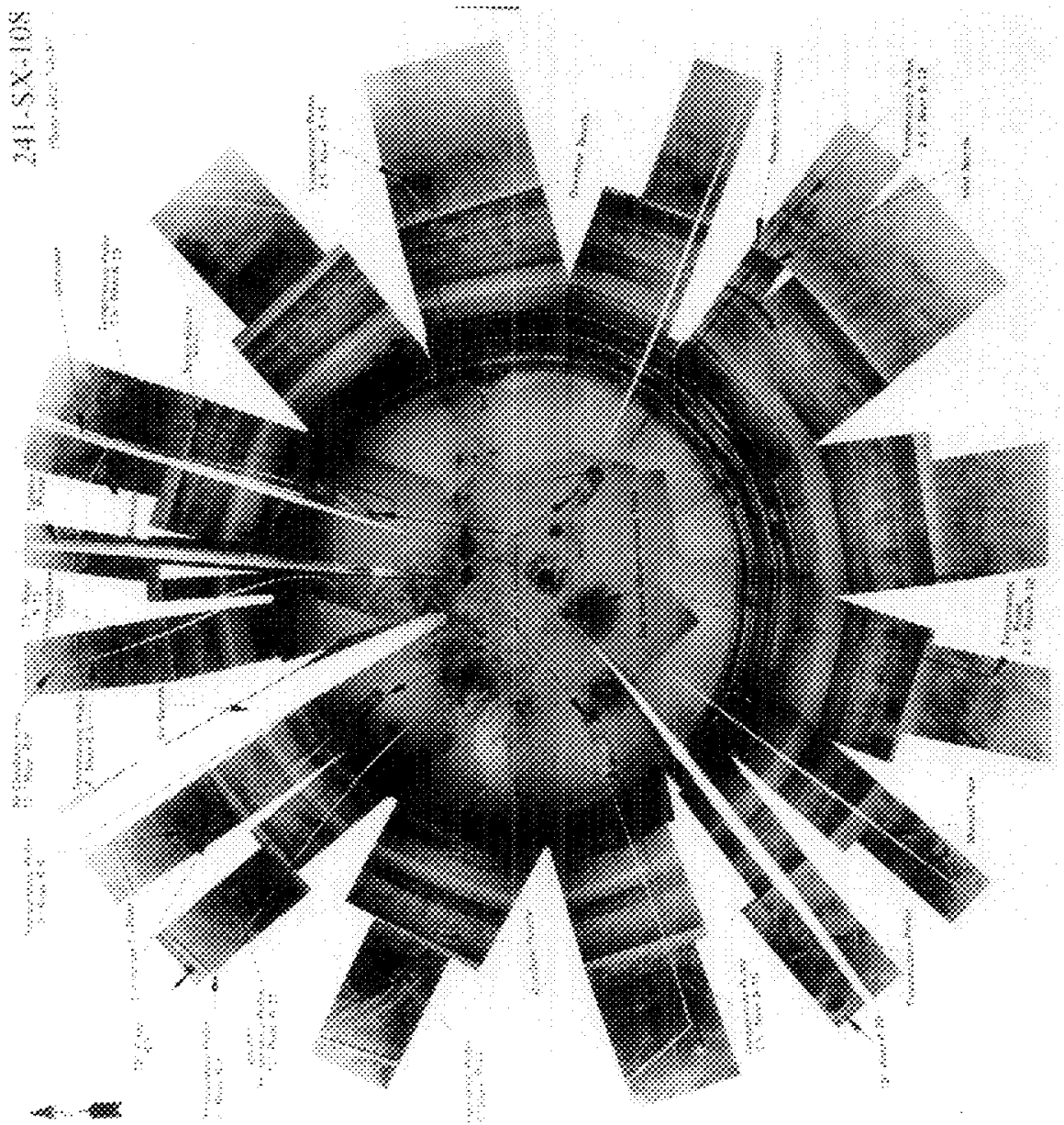


TANK 241-SX-109 SUMMARY

\begin{tabular}{|c|c|c|c|}
\hline \multicolumn{2}{|c|}{ TANK HISTORY } & \multicolumn{2}{|c|}{ TANK DESCRIPTION } \\
\hline Entered Service & 3rd qtr 1955 & Diameter & $75 \mathrm{ft}$ \\
\hline Removed from Service & - & Bottom Shape & Dish \\
\hline Watch Lists & Hydrogen & Cascade Tank & none \\
\hline Integrity & Assumed Leaker & Total Risers & 21 \\
\hline Assumed Leaker & 1965 & \multicolumn{2}{|c|}{ WASTE VOLUME (HANLON 1996i) } \\
\hline Partial Interim Isolation (PI) & - & Waste Type & NCPLX \\
\hline Intrusion Prevention (IP) & Sept 1982 & Drainable Interstitial Liquids & $48,000 \mathrm{gal}$ \\
\hline \multicolumn{2}{|c|}{ TENTATIVELY AVAILABLE RISERS } & Pumpable Liquids & $25,000 \mathrm{gal}$ \\
\hline Riser Number(s) & Size & Saltcake & $244,000 \mathrm{gal}$ \\
\hline 6 & 12 in & Sludge & $0 \mathrm{gal}$ \\
\hline Maximum Temperature & $153.9^{\circ} \mathrm{F}$ & Montage Number & $94041033-21 \mathrm{CN}$ \\
\hline Date & Feb 28, 1995 & Photo Set Number & 8603308 \\
\hline Elevation from tank bottom & unknown & \multicolumn{2}{|c|}{\begin{tabular}{|c|} 
WASTE SURFACE LEVEL \\
\end{tabular}} \\
\hline Riser Number & 19 & Devices & Manual Tape \\
\hline Minimum Temperature & $115.5^{\circ} \mathrm{F}$ & Max Level & 96 in \\
\hline Date & Nov 6, 1994 & Date & Oct 2, 1993 - Jan 2, 1994* \\
\hline Elevation from tank bottom & unknown & Min Level & 92 in \\
\hline Riser Number & 10 & Date & Jan 4, 1991 - July 1, 1991* \\
\hline
\end{tabular}

- Numerous dates in this time span 


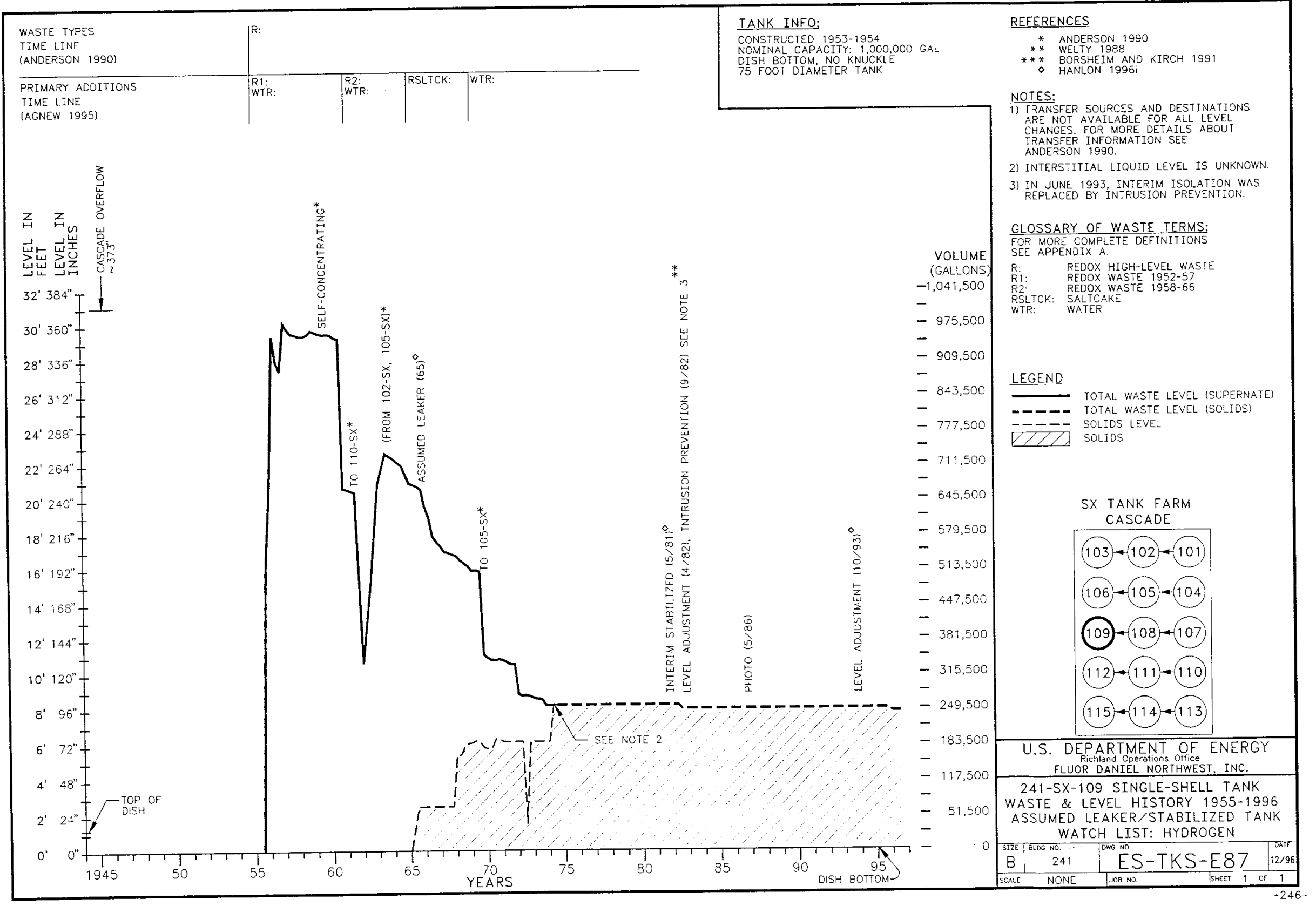




\section{$241-5 x-109$}
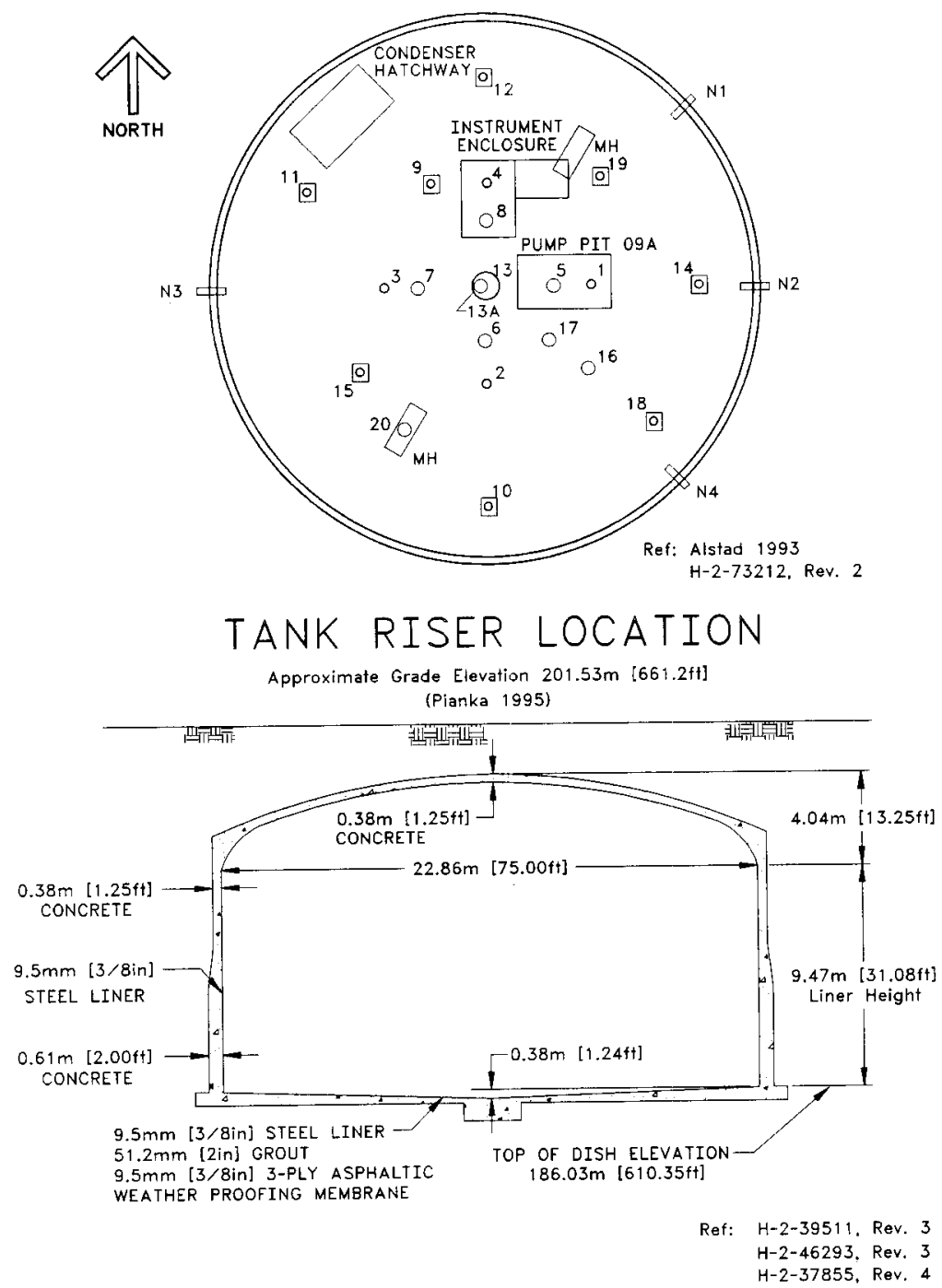

NOT TO SCALE

H-2-46293. Rev, 3

$-247-$ 
HNF-SD-WM-ER-352, Rev. 1

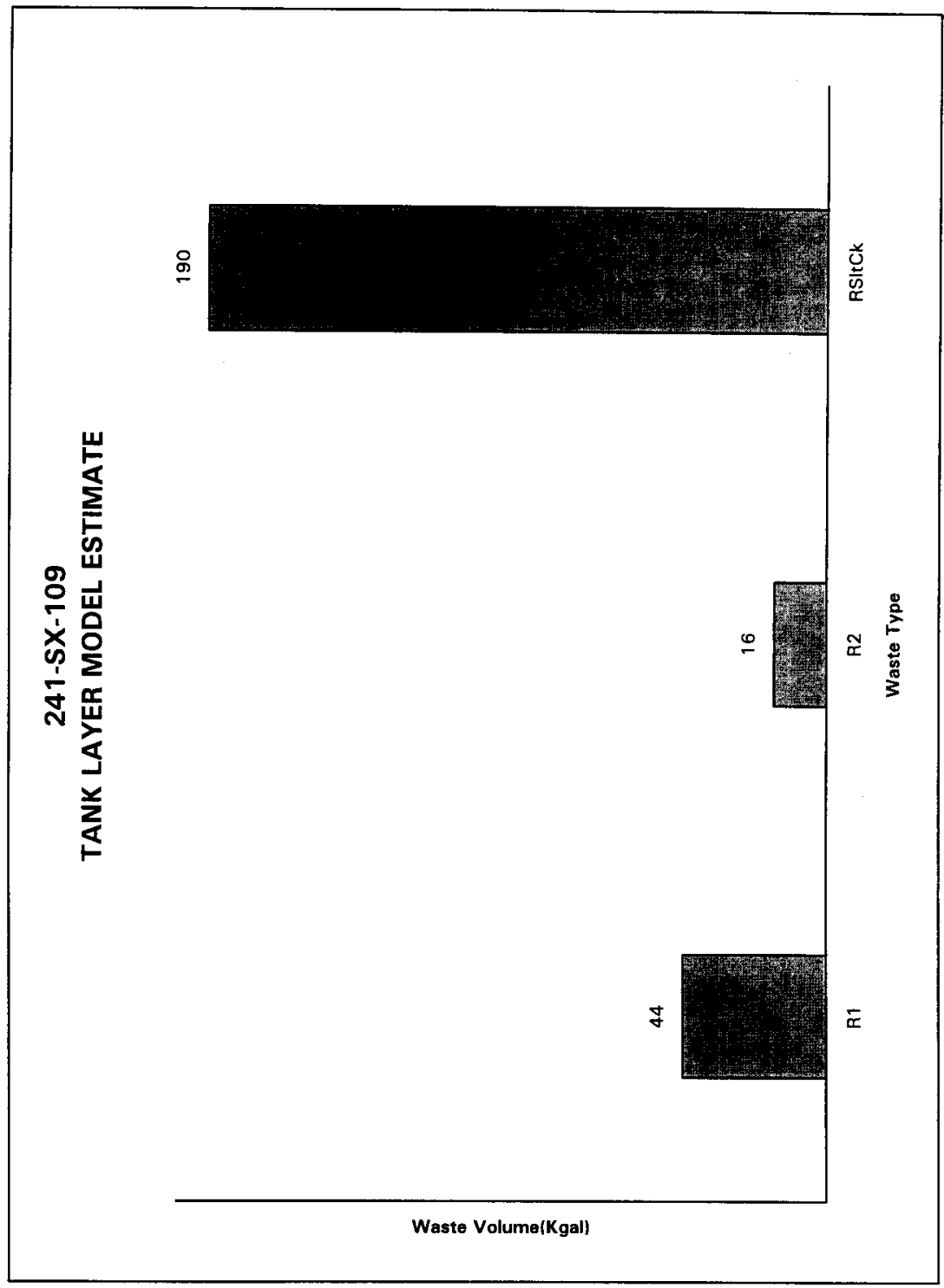

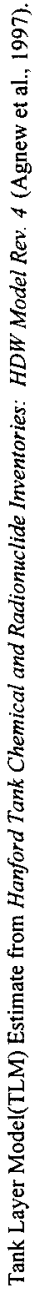


HPW Model Rey. 4

\begin{tabular}{|c|c|c|c|c|c|c|c|}
\hline & \multicolumn{5}{|c|}{ Single-Sheil Tank 241-\$X-109 } & \multicolumn{2}{|c|}{ HPW Model Rey. 4} \\
\hline & \multicolumn{7}{|c|}{ TLM Solids Composite Inventory Estimate* } \\
\hline Phytical & & & & & & & \\
\hline Properties: & & : & & $.95 \mathrm{CI}$ & $-67 \mathrm{Cl}$ & $+67 \mathrm{Cl}$ & $+98 \mathrm{Cl}$ \\
\hline Total ILM Wa & $1.63 \mathrm{E}+06(\mathrm{~kg})$ & $(250 \mathrm{kgal})$ & - & - & $\ldots$ & $m$ & - \\
\hline Heat Load & $8.61(\mathrm{~kW})$ & $(2.94 \mathrm{E}+04 \mathrm{gTU} / \mathrm{hr})$ & - & 8.32 & 8.49 & 8.71 & 8.81 \\
\hline Bulk Density & $1.72(\mathrm{~g} / \mathrm{cc})$ & - & - & 1.51 & 1.62 & 1.78 & 1.81 \\
\hline Void fraction & 0728 & - & - & 0.645 & 0.672 & Q798 & 0,875 \\
\hline Water wt\% & 33.1 & - & $=$ & 25.2 & 28.8 & 412 & 49.4 \\
\hline roC wt\% C $(w$ & 3.97E-03 & - & $=$ & $3.78 E-03$ & 3.83E-03 & $4.23 \mathrm{E}-03$ & 4.69E-03 \\
\hline $\begin{array}{l}\text { Chentical } \\
\text { Conitituents }\end{array}$ & moledL : & ppm & kg & $\begin{array}{l}95 \mathrm{CI} \\
\text { (inole/L) }\end{array}$ & $\begin{array}{l}-67 \mathrm{CL} \\
(\mathrm{mole} / \mathrm{L})\end{array}$ & $\begin{array}{r}+67 \mathrm{Cl} \\
\text { (maik/t) }\end{array}$ & $\begin{array}{l}+95 \mathrm{Cl} \\
\text { (mole/t) }\end{array}$ \\
\hline $\mathrm{Nat}$ & 11.9 & $1.59 E+05$ & $2.59 \mathrm{E}+05$ & 819 & 10.2 & 13.1 & 13.7 \\
\hline $\mathrm{Al} 3+$ & 4.34 & $6.80 \mathrm{E}+04$ & $1.11 \mathrm{E}+05$ & 2.86 & 3.29 & 5.48 & 6.63 \\
\hline Fe3+(total Fe) & 0.363 & $1.18 \mathrm{E}+04$ & $1.92 \mathrm{E}+04$ & 0.358 & 0.360 & 0.366 & 0.369 \\
\hline $\mathrm{Cr} 3+$ & 0.640 & $1,93 E+04$ & $3.15 E+04$ & 0.380 & 0.504 & 0.721 & 0.815 \\
\hline Bi3+t & $522 \mathrm{E}-06$ & 0.634 & 1,03 & $4.41 \mathrm{E}-06$ & 4.91E-06 & $5.75 \mathrm{E}-06$ & $6.32 \mathrm{E}-06$ \\
\hline $\mathrm{Le}+$ & 1.29E-11 & 1.04E-06 & $1.69 E-06$ & I.13E-11 & $1.22 \mathrm{E}+11$ & $1.42 \mathrm{E}-11$ & $1.56 \mathrm{E}-11$ \\
\hline $\mathrm{Hg}^{2+}$ & $8.20 \mathrm{E}-07$ & $9.55 \mathrm{E}-02$ & 0.156 & $7.27 \mathrm{E}-07$ & $7.72 \mathrm{E}-07$ & 9.03E-07 & $9.91 \mathrm{E}-07$ \\
\hline $\mathrm{Zr}$ (as $\mathrm{Zr} \overline{\mathrm{O}}(\mathrm{OH}) 2$ & $5.21 \mathrm{E}-07$ & $2.76 \mathrm{E}-02$ & $450 \mathrm{E}-02$ & 4.79E-07 & 4.99E-07 & $5.476-07$ & 5.89E-0? \\
\hline Pb2t & $1.30 \mathrm{E}-04$ & 15.6 & 25.5 & $6.97 \mathrm{E}-05$ & 9.91E-05 & $1.61 \mathrm{E-04}$ & $1.92 \mathrm{E}-04$ \\
\hline $\mathrm{Ni} 2+$ & $2.79 \mathrm{E}-02$ & 932 & $1.55 \mathrm{E}+03$ & $2.54 E-02$ & $268 E-02$ & $2.88 E-02$ & $2.90 \mathrm{E}-02$ \\
\hline Sr2+ & 0 & 0 & 0 & 0 & 0 & 0 & 0 \\
\hline $\mathrm{Mn} 4+$ & $380 \mathrm{E}-05$ & 121 & 198 & $2.72 \mathrm{E}-05$ & 3.25E-05 & 4.36E-05 & 4.91E-05 \\
\hline $\mathrm{C}_{2}+$ & 0.130 & $3.01 \mathrm{E}+03$ & $4.91 \mathrm{E}+03$ & 0.107 & 0.118 & 0.141 & 0.152 \\
\hline $\mathbf{K +}$ & $2.71 \mathrm{E}-02$ & 614 & $1.00 \mathrm{E}+03$ & $2.53 E-02$ & $260 \mathrm{E}-02$ & $2.92 \mathrm{E}-02$ & $3.16 \mathrm{E}-02$ \\
\hline OH- & 21.0 & $2.07 \mathrm{E}+05$ & $3.37 \mathrm{E}+05$ & 14.1 & 16.3 & 25.8 & 307 \\
\hline NO3- & 4.92 & $1.77 \mathrm{E}+0 \mathrm{~s}$ & $2.89 E+05$ & 2.99 & 4.00 & 6.36 & 7.14 \\
\hline $\mathrm{NO2-}$ & 1.78 & $4.75 \mathrm{E}+04$ & $7.75 \mathrm{E}+04$ & 1.55 & 1.63 & 1.90 & 2.02 \\
\hline $\cos 2=$ & 0.133 & $4.65 E+03$ & $7.57 \mathrm{E}+03$ & 0.111 & 0.122 & 0.145 & 0.156 \\
\hline PO43- & $3.38 \mathrm{E}-04$ & 18.6 & 30.4 & 3.14E-04 & $3.28 \mathrm{E}-04$ & $3.52 \mathrm{E}-04$ & $3.83 \mathrm{E}-04$ \\
\hline SO42. & $3.37 \mathrm{E}-02$ & $1.68 E+03$ & $3.06 \mathrm{E}+03$ & $3.20 \mathrm{E}-02$ & $3.27 \mathrm{E}-02$ & $3,64 \mathrm{E}-02$ & $3.93 \mathrm{E}-02$ \\
\hline Si (as Siö32-) & 0.131 & $2.13 E+03$ & $3.48 \mathrm{E}+03$ & 9.44E-02 & 0.103 & 0.159 & 0.186 \\
\hline F. & $2695-04$ & 2.97 & 4.84 & $2.29 \mathrm{E}-04$ & $254 E-04$ & $2.96 \mathrm{E}-04$ & 3.19E-04 \\
\hline $\mathrm{Cl}-$ & 0.114 & $2.34 \mathrm{E}+03$ & $3.82 \mathrm{E}+03$ & $6.71 \mathrm{E}-02$ & 9.00E-0? & 0.124 & 0.133 \\
\hline CGHSO7?. & $2.78 \mathrm{EA}-04$ & 30.5 & 49.8 & $2.69 \mathrm{E}-04$ & $2.74 E-04$ & $2.91 \mathrm{E}-04$ & $3.16 \mathrm{E}-04$ \\
\hline EDTA4- & $1.08 \mathrm{E}-05$ & 1.81 & $2 \overline{96}$ & $7.62 \mathrm{E}-06$ & $9.38 \mathrm{E}-06$ & $1.28 \mathrm{E}-05$ & $1.47 \mathrm{E}-05$ \\
\hline HEDTA3. & 9.00E-06 & 1.43 & 2.33 & $2.89 \mathrm{E}-06$ & $6.19 \mathrm{E}-06$ & $1.20 \mathrm{E}-05$ & $1.50 \mathrm{E}-0 \mathrm{~S}$ \\
\hline glycolato & $3.93 \mathrm{E}-04$ & 17.1 & 27.9 & $2.13 E-04$ & 3.JIE-04 & 4. B8E-04 & $5.80 \mathrm{E}-04$ \\
\hline eccetate- & 4.08E-05 & 1.40 & 2.28 & $3.95 \mathrm{E}-05$ & 4.01E-05 & $433 \mathrm{E}-05$ & $4.63 \mathrm{E}-05$ \\
\hline oxalnte2- & 1.68E-11 & B.61E-07 & $1.40 \mathrm{E}-06$ & 1.49E-11 & $1.59 \mathrm{E}-11$ & $1.86 \mathrm{E}-11$ & 2.04E-11 \\
\hline DBP & $2.47 \mathrm{E}-04$ & 30.1 & 49.1 & 2.30E-04 & $2.40 \mathrm{E}-04$ & $267 \mathrm{E}-04$ & $288 \mathrm{E}-04$ \\
\hline buisnol & $2.47 \mathrm{E}-0 \mathrm{~A}$ & 10.6 & 17.3 & 2.30E-04 & $2.40 \mathrm{E}-09$ & $2.67 \mathrm{E}-04$ & $2.88 \mathrm{E}-04$ \\
\hline $\mathrm{NH3}$ & 0.102 & $1.01 \mathrm{E}+03$ & $165 \mathrm{~F}+03$ & & & & \\
\hline Fe(CN)64- & 0 & Cots & & $6.8 \Delta \mathrm{E}-02$ & $7.36 \mathrm{E}-02$ & 0.150 & 0.110 \\
\hline & ( & 0 & 0 & 0 & 0 & 0 & 0 \\
\hline
\end{tabular}

"Unknowns in tank solich inventory are essigned by Tank Layeribg Model (TMM) 


\begin{tabular}{|c|c|c|c|c|c|c|c|}
\hline & \multicolumn{7}{|c|}{ Single-Shell Tank 241-\$X-109 } \\
\hline & \multicolumn{7}{|c|}{ SMM Composite Inventory Estimate } \\
\hline $\begin{array}{l}\text { Phyleal } \\
\text { Properties }\end{array}$ & & & & $95 \mathrm{C}$ & $-67 \mathrm{CI}$ & $+67 \mathrm{CI}$ & $+95 \mathrm{CI}$ \\
\hline Total SMM W & $0(\mathbf{k g})$ & $(2.00 \mathrm{E}-03 \mathrm{~kg} \mathrm{~kg})$ & - & - & $\cdots$ & $\ldots$ & $-\ldots$ \\
\hline Heat Loud & $0(\mathrm{~kW})$ & (0 BTU/hr) & $=$ & 0 & 0 & 0 & 0 \\
\hline Butk Density & $0(g / c)$ & $\ldots$ & - & 0 & 0 & 0 & 0 \\
\hline & & & & & & & \\
\hline Water w1\% & 0 & 一 & - & 0 & 0 & 0 & 0 \\
\hline TOC wt\% C (w & 0 & 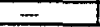 & $\ldots$ & 0 & 0 & 0 & 0 \\
\hline Coneintrit & mole/L & ppin & 4 & (mole/t) & $\begin{array}{r}\$ 7 \mathrm{Cl} \\
\text { (molel })\end{array}$ & $\begin{array}{r}+679 \\
\text { (1noles) }\end{array}$ & $\begin{array}{l}+95 \mathrm{CI} \\
\text { (nole } / \mathrm{t})\end{array}$ \\
\hline \begin{tabular}{|l|}
$\mathrm{Na}+$ \\
\end{tabular} & 0 & 0 & 0 & 0 & 0 & 0 & 0 \\
\hline $\mathrm{A}] 3+$ & $\overline{0}$ & 0 & 0 & 0 & 0 & 0 & 0 \\
\hline $\mathrm{Fe}^{3+}(\mathrm{tot}=\mathrm{Fe})$ & 0 & 0 & 0 & 0 & 0 & 0 & 0 \\
\hline $\mathrm{Cr} 3+$ & 0 & 0 & 0 & 0 & 0 & 0 & 0 \\
\hline Bij+ & 0 & 0 & 0 & 0 & 0 & 0 & 0 \\
\hline Lost & 0 & 0 & 0 & 0 & 0 & 0 & 0 \\
\hline $\mathrm{He}_{\mathrm{g}+}$ & 0 & 0 & 0 & 0 & 0 & 0 & 0 \\
\hline $\mathrm{Zr}(\mathrm{zrO}(\mathrm{OH}) 2$ & 0 & 0 & $\overline{0}$ & 0 & 0 & 0 & 0 \\
\hline Pb2t & 0 & 0 & 0 & 0 & 0 & 0 & 0 \\
\hline $\mathrm{Ni2+}$ & 0 & 0 & 0 & 0 & 0 & 0 & 0 \\
\hline Sr2t+ & 0 & 0 & 0 & 0 & 0 & 0 & $\overline{0}$ \\
\hline Mn4t & 0 & 0 & 0 & 0 & 0 & 0 & 0 \\
\hline $\mathrm{Cn} 2+$ & 0 & 0 & 0 & 0 & 0 & 0 & 0 \\
\hline $\mathbf{K}+$ & 0 & 0 & 0 & 0 & 0 & 0 & 0 \\
\hline OH- & 0 & 0 & 0 & 0 & 0 & 0 & $\overline{0}$ \\
\hline No3- & $\overline{0}$ & 0 & 0 & 0 & 0 & 0 & 0 \\
\hline Non- & 0 & $\overline{0}$ & 0 & 0 & 0 & 0 & 0 \\
\hline COJ2- & 0 & 0 & 0 & 0 & 0 & 0 & 0 \\
\hline PO43- & 0 & 0 & 0 & 0 & 0 & 0 & 0 \\
\hline $5042-$ & 0 & 0 & 0 & 0 & 0 & 0 & 0 \\
\hline Si (a SiO32-) & $\dot{0}$ & 0 & 0 & 0 & 0 & 0 & 0 \\
\hline F- & 0 & 0 & 0 & 0 & 0 & 0 & 0 \\
\hline Cl. & 0 & 0 & 0 & 0 & 0 & 0 & 0 \\
\hline C6HSOT3 & 0 & 0 & 0 & 0 & 0 & 0 & 0 \\
\hline EDTA4- & 0 & 0 & 0 & 0 & 0 & 0 & 0 \\
\hline HEDTA3- & 0 & 0 & 0 & 0 & 0 & 0 & 0 \\
\hline Elycolate- & 0 & 0 & 0 & 0 & 0 & 0 & 0 \\
\hline ncetinte- & 0 & 0 & 0 & 0 & 0 & 0 & 0 \\
\hline oxalate?. & 0 & 0 & 0 & 0 & 0 & 0 & 0 \\
\hline DBP & 0 & 0 & 0 & 0 & 0 & 0 & 0 \\
\hline butunol & 0 & 0 & 0 & 0 & 0 & 0 & 0 \\
\hline & & & & & & & \\
\hline $\mathrm{NH3}$ & 0 & 0 & 0 & 0 & 0 & 0 & 0 \\
\hline Fa(CN)64: & 0 & 0 & 0 & 0 & 0 & 0 & 0 \\
\hline
\end{tabular}

-Density is calcutated baned on Na, OH-, und AlO2-

+ Water wt\% derived from the difference of dentity and total disualved species. 
HDW Model Rev. 4

\begin{tabular}{|c|c|c|c|c|c|c|c|}
\hline \multicolumn{8}{|c|}{ Single-Shell Tank 241-SX-109 } \\
\hline & \multicolumn{7}{|c|}{ Total Inventory Estimate* } \\
\hline $\begin{array}{l}\text { Physical } \\
\text { Propertica }\end{array}$ & & & & $-95 \mathrm{CI}$ & $-67 \mathrm{CI}$ & $+67 \mathrm{CI}$ & $+95 \mathrm{Cl}$ \\
\hline Total Waste & $1.63 E+06(\mathrm{~kg})$ & (250 kgal) & $\ldots$ & - & $\ldots$ & - & $=$ \\
\hline Heat Loed & $8.61(\mathrm{~kW})$ & (2.94E +04 BTU/hr) & $\ldots$ & B.32 & 8.49 & 8.71 & 8.81 \\
\hline Bulk Densityt & $1.72(g / c c)$ & $\ldots$ & - & 1.51 & 1.62 & 1.78 & 1.81 \\
\hline & & & & & & & \\
\hline Water wet\% & 33.1 & - & - & 25.2 & 28.8 & 41.2 & 49.4 \\
\hline TOC wt\% C (w) & $3.97 \mathrm{E}-03$ & - & - & $3.78 \mathrm{E}-03$ & $3.83 \mathrm{E}-03$ & $423 \mathrm{E}-03$ & 4.69E-03 \\
\hline $\begin{array}{l}\text { Oherital } \\
\text { Constlthents }\end{array}$ & molet & Ppm : & $\mathbf{4 g}$ & $\begin{array}{l}95 \mathrm{CI} \\
\text { (moledL) }\end{array}$ & $\begin{array}{r}-67 \mathrm{CI} \\
\text { (mole/t) }\end{array}$ & $\begin{array}{l}+67 \mathrm{CI} \\
\text { (nole/L) }\end{array}$ & $\begin{array}{l}495 \mathrm{CI} \\
\text { (mole/L) }\end{array}$ \\
\hline $\mathrm{Nat}$ & 11.9 & $1.59 \mathrm{E}+05$ & $259 \mathrm{E}+05$ & 8.19 & 10.2 & 13.1 & 13.7 \\
\hline $\mathrm{A} / 3+$ & 4.34 & $6.80 E+04$ & $1.11 \mathrm{E}+05$ & 2.86 & 3.29 & 5.48 & 6.63 \\
\hline $\mathrm{Fe} 3+(\operatorname{cotal} \mathrm{Fe})$ & 0.363 & T. $18 E+04$ & $1.92 E+04$ & 0.358 & 0.360 & 0.366 & 0.369 \\
\hline $\mathrm{Cr} 3+$ & 0.640 & $1.93 \mathrm{E}+04$ & $3.15 \mathrm{E}+04$ & 0.380 & 0.504 & 0.721 & 0.815 \\
\hline Bi3+ & $5.22 \mathrm{E}-06$ & 0.634 & 103 & 4.41E-06 & $4.8 \mathrm{tE}-06$ & $5.75 \mathrm{E}-06$ & 6.32E-06 \\
\hline $1.23+$ & 1.29E-11 & $1.04 \mathrm{E}-06$ & $1,69 \mathrm{E}-106$ & 1. I3E-11 & $1.22 \mathrm{E}-11$ & $1.42 \mathrm{E}-11$ & 1.56E-11 \\
\hline $\mathrm{Hg} 2+$ & $8.20 \mathrm{E}-07$ & 9.55E-02 & 0.136 & $7.27 \mathrm{E}-07$ & $7.72 \mathrm{E}-07$ & $9.03 \mathrm{E}-07$ & 991E-07 \\
\hline $\mathrm{Zr}(\mathrm{nZ \textrm {ZC }}(\mathrm{OH}) 2$ & $5.21 \mathrm{E}-07$ & $2.76 \mathrm{E}-02$ & $4.50 \mathrm{E}-02$ & $4.79 \mathrm{E}-07$ & $4,99 \mathrm{E}-07$ & 5.47E-07 & 5. $99 \mathrm{E}-07$ \\
\hline $\mathrm{Pb2+}$ & $1.30 \mathrm{E}-04$ & 15.6 & 25.5 & $6.97 \mathrm{E}-05$ & $9.91 \mathrm{E}-0 \mathrm{~S}$ & $1.61 \mathrm{E}-04$ & $1.92 \mathrm{E}-04$ \\
\hline $\mathrm{Ni2+}$ & $2.79 \mathrm{E}-02$ & 952 & $1.55 \mathrm{E}+03$ & $2.54 \mathrm{E}-02$ & $2.68 E-02$ & 2.88E-02 & $2.90 \mathrm{E}-02$ \\
\hline $5 r 2+$ & 0 & 0 & 0 & 0 & 0 & 0 & \\
\hline Mndt & $3.80 \mathrm{E}-05$ & 1.21 & 1.98 & $2.72 \mathrm{E}-0 \mathrm{~S}$ & $3.25 \mathrm{E}-05$ & $436 \mathrm{E}-0 \mathrm{~S}$ & 4.91E-05 \\
\hline $\mathrm{Ca} 2+$ & 0.130 & $301 \mathrm{E}+03$ & $4912+03$ & 0.107 & 0.118 & 0.141 & 0.152 \\
\hline $\mathbf{K}+$ & $2.7 \mathrm{IE}-02$ & 614 & $1.00 \mathrm{E}+03$ & $2.53 \mathrm{E}-02$ & $2.60 \mathrm{E}-02$ & $2.92 \mathrm{E}-02$ & $3.16 \mathrm{E}_{-102}$ \\
\hline OH- & 21.0 & $207 \mathrm{E}+05$ & $3.37 \mathrm{E}+05$ & 14.1 & 16.3 & 25.8 & 30.7 \\
\hline No3- & 4.92 & $1.77 \mathrm{E}+05$ & $2.89 \mathrm{E}+05$ & 2.99 & 4.00 & 6.36 & 7.14 \\
\hline NO2- & 1.78 & $4.75 E+04$ & $7.75 \mathrm{E}+04$ & 1.59 & 1.63 & 1.90 & 2.02 \\
\hline $\cos 2$ & 0.133 & $4.65 \mathrm{E}+03$ & $7.57 \mathrm{E}+03$ & 0.111 & 0.122 & 0.145 & 0.156 \\
\hline PO43. & $3.38 \mathrm{E}-\mathrm{OM}$ & 18.6 & 30.4 & $3.14 \mathrm{E}-04$ & $3.28 \mathrm{E}-04$ & $3.525-04$ & 3.83E-04 \\
\hline 5042- & $3,37 \mathrm{E}-02$ & $3.88 E+03$ & $3.06 \mathrm{E}+03$ & $3.20 \mathrm{E}-02$ & $3.27 \mathrm{E}-02$ & $3.64 \mathrm{E}-02$ & 3.93E-02 \\
\hline Si (u SiO32-) & 0.131 & $2.13 \mathrm{E}+03$ & $3.48 \mathrm{E}+03$ & $9.44 E-02$ & 0.103 & 0.159 & 0.186 \\
\hline$F-$ & 2.69E-04 & 2.97 & 4.64 & $2.29 \mathrm{E}-04$ & $254 \mathrm{E}-04$ & $296 \mathrm{E}-04$ & 3.19E-04 \\
\hline $\mathrm{C}$. & 0.114 & $2.34 E+03$ & $3.82 \mathrm{E}+03$ & 6.71E-02 & $9,00 E-02$ & 0.124 & 0.133 \\
\hline C6H5073- & $2.78 E-04$ & 305 & 49.8 & $2.69 \mathrm{E}-04$ & $2.74 \mathrm{E}-04$ & $2.91 \mathrm{E}-04$ & $3.16 \mathrm{E}-04$ \\
\hline EDTA4 & 1.08E-05 & 1.81 & 2.96 & $7,62 E-06$ & 9.38E-06 & $1.28 \mathrm{E}-05$ & $1.4 \%-05$ \\
\hline HEDTA3- & $9.00 \mathrm{E}-06$ & 1.43 & 2,33 & 2. $89 \mathrm{E}-96$ & $6.19 \mathrm{E}-06$ & $1.20 \mathrm{E}-05$ & $150 \mathrm{E}-0 \mathrm{~S}$ \\
\hline & & & & & & & \\
\hline glycolate- & 3.93E-04 & 17.1 & 27.9 & 2.13E-04 & 3. $11 \mathrm{E}-04$ & 4.88E-OA & $5.80 \mathrm{E}-04$ \\
\hline scetale- & $4.08 \mathrm{E}-05$ & 1.40 & 2.28 & $3.95 \mathrm{E}-05$ & $4.01 E-05$ & $4.33 \mathrm{E}-05$ & 4.63E-05 \\
\hline oxtate? & T.68E-11 & E.6IEA7 & 1.40E-06 & $1.49 \mathrm{E}-11$ & $1.59 \mathrm{E}-11$ & 1.86E- 11 & $2.04 \mathrm{E}-11$ \\
\hline DAP & $2.47 \mathrm{E}-04$ & 30.1 & 49.1 & $230 \mathrm{E}-04$ & $2.40 \mathrm{E}-04$ & $2.67 \mathrm{E}-04$ & $2.83 \mathrm{E}-04$ \\
\hline butanol & $2.47 \mathrm{E}-04$ & 10.6 & 17.3 & $2.30 E-04$ & $2.40 \mathrm{E}-04$ & $2.67 \mathrm{E}-04$ & $2.88 \mathrm{E}-04$ \\
\hline & & & & & & & \\
\hline $\mathrm{NH3}$ & 0.102 & $1.01 \mathrm{E}+03$ & $165 \mathrm{E}+03$ & 6. B8E- 02 & $7.36 E-02$ & 0.150 & 0.110 \\
\hline Fe(CN)64 & 0 & 0 & 0 & 0 & 0 & 0 & 0 \\
\hline
\end{tabular}

- Uaknowns in tank solids inventory are asigned by Tank Lyering Model (TLM)

tWater wt\% derived from the difference of density and total dissolved species. 
HNF-SD-WM-ER-352, Rev. 1

HOW Madel Rov. 4

\begin{tabular}{|c|c|c|c|c|c|c|c|}
\hline & \multicolumn{7}{|c|}{ Single-Shell Tank 241-SX-109 } \\
\hline \multicolumn{8}{|c|}{ TLM Solids Composite Inventory Estimatc* } \\
\hline $\begin{array}{l}\text { Phyoletal } \\
\text { Properties }\end{array}$ & & & & $.95 \mathrm{CI}$ & $-67 \mathrm{CI}$ & $+67 \mathrm{CI}$ & $+9 s \mathrm{CI}$ \\
\hline Total TLM Wa & $1.63 E+06(\mathrm{~kg})$ & $(250 \mathrm{kgal})$ & - &..- & - & - & $\ldots$ \\
\hline Heat Load & $8.61(\mathrm{~kW})$ & (2.94E+04 BTU/hr) & $m$ & 8.32 & 8.49 & 8.71 & 8.81 \\
\hline Bulk Density & $1.72(1 / \infty)$ & 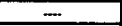 & - & 1.51 & 1.62 & 1.78 & 1.81 \\
\hline Void Fraction & 0.728 & 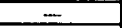 & - & 0.645 & 0.672 & 0.798 & 0.375 \\
\hline Water w1\% & 33.1 & - & - & 25.2 & 28.8 & 41.2 & 49.4 \\
\hline TOC wr\% C (w & $3.97 \mathrm{E}-03$ & - & $m$ & 3.78E-03 & $3.83 \mathrm{E}-03$ & 4.23E-03 & $4.69 E_{-03}$ \\
\hline $\begin{array}{l}\text { Roilotopleal } \\
\text { Constituents }\end{array}$ & $\mathrm{CUL}$ & $H(1 / 2)$ & $\mathrm{CI}$ & $\begin{array}{l}-95 \mathrm{CI} \\
\text { (CVI) }\end{array}$ & $\begin{array}{l}67 \mathrm{Cl} \\
(\mathrm{C} / \mathrm{C})\end{array}$ & $\begin{array}{l}+67 \mathrm{Cl} \\
(\mathrm{CH})\end{array}$ & $\begin{array}{r}+9 \mathrm{CI} \\
(\mathrm{C} / \mathrm{L})\end{array}$ \\
\hline $\mathrm{H}-3$ & $1.84 \mathrm{E}-04$ & 0.107 & 174 & $1.46 \mathrm{E}-05$ & 1.46E-05 & $1,92 \mathrm{E}-0 \mathrm{~A}$ & 2.08E-04 \\
\hline C-14 & $9.50 E-06$ & $5.51 \mathrm{E}-03$ & 8.99 & $8.03 \mathrm{E}-07$ & $8.03 \mathrm{E}-07$ & 9.85E-06 & $1.06 \mathrm{E}-05$ \\
\hline Ni-59 & $1.30 \mathrm{E}-05$ & $7.54 \mathrm{E}-03$ & 12.3 & $7.80 E-06$ & 7.80E-06 & 1.33E-0S & 1,35E-05 \\
\hline $\mathrm{Ni}-63$ & $1.26 \mathrm{E}-03$ & 0,732 & $1.19 E+03$ & 7.5TE-04 & $7.57 \mathrm{E}-04$ & 3.29E-03 & $1.31 \mathrm{E}-03$ \\
\hline $\operatorname{Co-} 60$ & 8.43E- -06 & 4.90E-03 & 7.98 & 6. 10E-07 & 6.10E-07 & 8.7TE-06 & $9.48 \mathrm{E}-06$ \\
\hline Se-79 & $7.60 \mathrm{E}-06$ & 4.41E-03 & 7.19 & 2.31E-06 & $2.31 \mathrm{E}-06$ & $9.99 \mathrm{E}-06$ & $1.23 \mathrm{E}-05$ \\
\hline Sr-90 & 1.16 & 675 & $1.10 \mathrm{E}+06$ & 1,11 & 1.14 & 1.18 & 1.19 \\
\hline$Y-90$ & 1.16 & 675 & $1.10 E+06$ & 0.962 & 0.962 & 1.18 & 1.19 \\
\hline $2 r-93$ & 3.47E-05 & $2.02 \mathrm{E}-02$ & 32.9 & $9.73 \mathrm{E}-06$ & $9.73 \mathrm{E}-06$ & $46 \pi-05$ & $5.81 \mathrm{E}-05$ \\
\hline $\mathrm{Nb}-93 \mathrm{~m}$ & 2.95E-05 & 1.71E-02 & 27.9 & $9.17 \mathrm{E}-06$ & $9.17 \mathrm{E}-06$ & 3.78E-05 & 4.59E-05 \\
\hline Tc-99 & $7.37 \mathrm{E}-05$ & $4.28 \mathrm{E}-02$ & 69.7 & 7.12E-05 & 7.16E-05 & $7.87 \mathrm{E}-05$ & 1.45E-05 \\
\hline Ru-106 & $1.93 \mathrm{E}-09$ & $1.12 \mathrm{E}-06$ & 1.83E 203 & $1.23 \mathrm{E}-10$ & 1.23E-10 & $2.21 E-09$ & 7.95E-09 \\
\hline Cd-113m & $3.83 \mathrm{E}-05$ & $2.22 \mathrm{E}-02$ & 36.2 & $3.09 \mathrm{E}-06$ & $3.09 \mathrm{E}-06$ & Q.13E-05 & $1.44 \mathrm{E}-04$ \\
\hline $\mathrm{Sb}-125$ & 2.996-05 & $1.74 \mathrm{E}-02$ & 28.3 & 1.98E-06 & $1.98 \mathrm{E}-06$ & $3.12 \mathrm{E}-05$ & $3.37 \mathrm{E}-05$ \\
\hline Sn-126 & 1.10E-05 & $6.41 \mathrm{E}-03$ & 10.5 & 2.94E-06 & $2.94 \mathrm{E}-06$ & $1.46 \mathrm{E}-05$ & $1.81 E-05$ \\
\hline I-129 & $1.40 \mathrm{E}-07$ & B. 12E-0S & 0.132 & 1.35E-07 & $1.36 \mathrm{E}-07$ & 1.49E,07 & 1.61E-07 \\
\hline $\mathrm{Cs}-134$ & 1.9]E-06 & $1.11 \mathrm{E}-03$ & 1.81 & $1.60 \mathrm{E}-07$ & $1.60 \mathrm{E}-0 ?$ & $1,90 \mathrm{E}-06$ & $2.12 \mathrm{E}-1 \mathrm{~B}$ \\
\hline Cs-137 & 0.273 & 158 & $2.58 \mathrm{E}+03$ & 0.265 & 0.266 & 0.291 & 0.311 \\
\hline B.-137m & 0.258 & 150 & $2,4 \mathrm{E}+0 \mathrm{~S}$ & $2.52 \mathrm{E}-02$ & $2.52 \mathrm{E}-02$ & 0.267 & 0.285 \\
\hline$S m-151$ & $2.71 \mathrm{E}-02$ & 15.8 & $2.57 \mathrm{E}+04$ & 8.30€-03 & 8.30E- 03 & $3.55 \mathrm{E}-02$ & 4.35E-02 \\
\hline Eu-152 & $1.51 \mathrm{E}-05$ & (69)-03. & 14.3 & D. $40 €-06$ & 8.40E-06 & 1.52E-0s & 1.53E-05 \\
\hline Eu-154 & $2.02 \mathrm{E}-04$ & 0.117 & 191 & 1.4EE-05 & 1.48E-05 & $2.10 \mathrm{E}-\mathrm{OA}$ & $6,92 \mathrm{E}-04$ \\
\hline Eu-155 & 7,67E-0. & 0.445 & 725 & 4.25E-04 & 4.25E-04 & $7.69 \mathrm{E}-04$ & $7.73 \mathrm{E}-04$ \\
\hline Ra.226 & $1.02 \mathrm{E}-09$ & $5.93 \mathrm{E}-07$ & $9.67 \mathrm{E}-04$ & $6.65 \mathrm{E}-10$ & $6.65 \mathrm{E}-10$ & $1.15 \mathrm{E}-\infty 9$ & $1.27 \mathrm{E}-09$ \\
\hline Ra-228 & $1.20 \mathrm{E}-08$ & 6.95E-06 & $1.13 \mathrm{E}-02$ & $1,42 \mathrm{E}-14$ & 1.12E-14 & I.2IE-08 & $1.22 E-08$ \\
\hline$A c-227$ & $5.04 E-09$ & $2.93 \mathrm{E}-06$ & $4.7 \mathrm{E}-03$ & $2.96 \mathrm{E}-09$ & $2.96 \mathrm{E}-09$ & S.79E- 09 & $6.26 \mathrm{E}-09$ \\
\hline Pa-231 & $1.03 E-08$ & 5.99E-06 & $9.7 \mathrm{E}-03$ & $2.46 \mathrm{E}-09$ & $2.46 \mathrm{E}-09$ & $1.27 \mathrm{E}-0 \mathrm{t}$ & $1.50 \mathrm{E}-08$ \\
\hline Th-229 & 2.88E-10 & 1.67E-07 & $2.73 \mathrm{E}-04$ & $1.09 \mathrm{E}-12$ & $1.09 \mathrm{E}-12$ & $2.91 E-10$ & $2.94 \mathrm{E}-10$ \\
\hline Th-232 & $1.60 \mathrm{E}-10$ & $9.32 E-08$ & $1.52 \mathrm{E}-04$ & 5.01E-16 & $5.01 \mathrm{E}-16$ & $2.02 \mathrm{E}-10$ & $2.43 \mathrm{E}-10$ \\
\hline $\mathrm{U}-232$ & 5.35E-05 & 3.11E-05 & $5.066-02$ & 1. & 3 49E-OB & 7.62E- 08 & 1.01E-07 \\
\hline $\mathrm{U}-233$ & 2.05E-07 & $1.19 \mathrm{E}-04$ & 0.194 & 7.24E-08 & 1.34E-07 & $2.92 E-07$ & $3.88 \mathrm{E}-07$ \\
\hline U-234 & $9,97 \mathrm{E}-07$ & 57 SE-A4 & 0.943 & 4.53E- 07 & $7.18 \mathrm{E}-07$ & $1.30 \mathrm{E}-06$ & $1.60 \mathrm{E}-06$ \\
\hline U.235 & $4.03 \mathrm{E}-0.5$ & 2.34E-05 & $3 . \mathrm{BIE-02}$ & 1.103E-08 & $2.90 \mathrm{E}-08$ & $5.26 \mathrm{E}-0 \mathrm{~B}$ & $6.49 \mathrm{E}-08$ \\
\hline $\mathrm{U}-236$ & $4.10 \mathrm{E}-08$ & $2.38 \mathrm{E}-05$ & 3,68E-02 & $1.92 \mathrm{E}-08$ & $3.00 \mathrm{E}-0 \mathrm{~A}$ & 1.25E-08 & 6.38E- $0 \mathrm{~g}$ \\
\hline $\mathrm{U}-238$ & $8.75 E-07$ & 5.08E-04 & 0.828 & 3.96E-07 & 6.28E-07 & $1.14 \mathrm{E}-06$ & 1.41E-06 \\
\hline $\mathrm{Np}-237$ & 3.25E-07 & 1.Bte-04 & 0.307 & 3.09E-OT & $3.15 \mathrm{E}-07$ & $3.49 \mathrm{E}_{-}-07$ & $3.76 \mathrm{E}-07$ \\
\hline Pu-238 & 7.77E-06 & 1.51E-03 & 7.35 & 6.51E-06 & $7.13 \mathrm{E}-06$ & $8,41 \mathrm{E}-06$ & $9.03 \mathrm{E}-06$ \\
\hline Pu-239 & $2.34 \mathrm{E}-04$ & 0.136 & 222 & 1.94E-04 & $2.14 \mathrm{E}-04$ & $2.55 \mathrm{E}-\mathrm{OA}$ & $2.75 \mathrm{E}-04$ \\
\hline Pu-240 & 3.95E-05 & $2.30 E-02$ & 37.4 & $3.28 \mathrm{E}-0 \mathrm{~S}$ & 3.61E-05 & $4.30 \mathrm{E}-05$ & 4.63E- 05 \\
\hline $\mathrm{Pu}-241$ & $3.93 \mathrm{E}-04$ & 0.228 & 372 & 129E-04 & $3.60 \mathrm{E}-04$ & 4.26E-04 & 4,5TE-04 \\
\hline $\mathrm{Pu}-242$ & 2.13E-09 & 1.24E-06 & $2.02 \mathrm{E}-03$ & 1.79E-09 & 1.960-09 & $2.31 \mathrm{E}-09$ & $2.48 \mathrm{E}-69$ \\
\hline Am-241 & 2.31E-A4 & 0.134 & 219 & $161 \mathrm{E}-04$ & $1.95 \mathrm{E}-04$ & $267 \mathrm{E}-04$ & $301 \mathrm{E}-04$ \\
\hline Am-243 & $9.19 \mathrm{E}-09$ & 5.34E-06 & $8.70 \mathrm{E}-03$ & $6.02 E-09$ & $7.58 \mathrm{E}-09$ & 1.08E-08 & $1.24 \mathrm{E}-08$ \\
\hline $\mathrm{Cm}-242$ & $3.69 \mathrm{E}-07$ & $2.14 \mathrm{E}-04$ & 0.349 & 3.68E-0? & $367 \mathrm{E}-07$ & $3.69 \mathrm{E}-07$ & $3.69 \mathrm{E}-07$ \\
\hline $\mathrm{Cm}-243$ & 3.51E-08 & 2.04E-05 & $3.32 \mathrm{E}-02$ & $3.50 \mathrm{E}-08$ & 3.48E-08 & $3.51 \mathrm{E}-06$ & $3.51 \mathrm{E}-08$ \\
\hline $\mathrm{Cm}-244$ & 1.04E-06 & $6.05 \mathrm{E}-04$ & 0.95 & 8.07E-07 & 9.22E-07 & $1.16 \mathrm{E}-06$ & 1.28E-06 \\
\hline Totels & $\mathbf{M}$ & $\mathrm{n} / \mathrm{s}$ & ks & 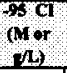 & $\begin{array}{l}-6 \mathrm{CP} \\
\mathrm{atr} \\
(\mathrm{L})\end{array}$ & $\begin{array}{l}+6701 \\
(\mathrm{Nor} \\
\mathrm{g} / \mathrm{L})\end{array}$ & 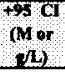 \\
\hline $\mathrm{Pu}$ & $3.93 \mathrm{E}-03(\mathrm{~g} / \mathrm{L})$ & - & 3.72 & 3.25E-03 & 3.59E-03 & 4.28E-03 & 4.62E-03 \\
\hline $\mathrm{U}$ & 1.08E-02 & $1,49 \mathrm{E}+03$ & $2.44 \mathrm{E}+03$ & 4.17E-03 & $7.75 \mathrm{E}-03$ & 1.42E-02 & $1.75 \mathrm{E}-02$ \\
\hline
\end{tabular}

-Unkmowns in tank solids inventory are astignod by Tank Layering Model (TLM). 
HDW Model Rev. 4

\begin{tabular}{|c|c|c|c|c|c|c|c|}
\hline \multirow[b]{3}{*}{$\begin{array}{l}\text { Physical } \\
\text { Properties }\end{array}$} & \multicolumn{7}{|c|}{ Single-Shell Tank 241-5X-109 } \\
\hline & \multicolumn{7}{|c|}{ SMM Composite Inventory Estimate } \\
\hline & & & & $.95 \mathrm{Cl}$ & $.67 \mathrm{CI}$ & $\begin{array}{r}+67 C I \\
\end{array}$ & $+95 \mathrm{CI}$ \\
\hline Total SMM W & $0(\mathbf{k g})$ & $(2.00 \mathrm{E}-03 \mathrm{kgal})$ & 一 & - & - & $\ldots$ & - \\
\hline Heat Load & $0(\mathrm{kw})$ & (o BTU/hr) & $=$ & 0 & 0 & 0 & 0 \\
\hline Bulk Density" & $0(\mathrm{~g} / \mathrm{cc})$ & - & 二 & 0 & 0 & 0 & 0 \\
\hline Water wt\%t & 0 & - & - & 0 & 0 & 0 & 0 \\
\hline TOC wt\% C (w & 0 & - & $\because$ & 0 & 0 & 0 & 0 \\
\hline $\begin{array}{l}\text { Rediologitenl } \\
\text { Censtltuenits }\end{array}$ & CIL & $1 \mathrm{CV} / \mathrm{g}$ & Cl & $\begin{array}{l}95 \mathrm{Cl} \\
(\mathrm{CIL})\end{array}$ & $\begin{array}{l}-67 \mathrm{Cl} \\
(\mathrm{Ch} / \mathrm{C}\end{array}$ & $\begin{array}{l}6701 \\
(\mathrm{Cl} / \mathrm{t})\end{array}$ & $\begin{array}{r}+9 \mathrm{Cl} \\
\text { (CVL) }\end{array}$ \\
\hline $\mathrm{H}-3$ & 0 & 0 & 0 & 0 & 0 & 0 & 0 \\
\hline C-14 & 0 & 0 & 0 & 0 & 0 & 0 & 0 \\
\hline Ni-59 & 0 & 0 & 0 & 0 & 0 & 0 & 0 \\
\hline $\mathrm{Ni}-63$ & 0 & 0 & 0 & 0 & 0 & 0 & 0 \\
\hline Co-60 & 0 & 0 & 0 & 0 & 0 & 0 & 0 \\
\hline Se-79 & 0 & 0 & 0 & 0 & 0 & 0 & 0 \\
\hline $\mathrm{Sr}=90$ & 0 & 0 & 0 & 0 & 0 & 0 & 0 \\
\hline$Y-90$ & 0 & 0 & 0 & 0 & 0 & 0 & 0 \\
\hline$Z x-93$ & 0 & 0 & 0 & 0 & 0 & 0 & 0 \\
\hline Nb-93m & 0 & 0 & 0 & 0 & 0 & 0 & 0 \\
\hline Tc-99 & 0 & 0 & 0 & 0 & 0 & 0 & 0 \\
\hline$R \mathrm{~N}-106$ & 0 & 0 & 0 & 0 & 0 & 0 & 0 \\
\hline $\mathrm{Cd}-113 \mathrm{~m}$ & 0 & 0 & 0 & 0 & 0 & 0 & 0 \\
\hline$S b-125$ & 0 & 0 & 0 & 0 & 0 & 0 & 0 \\
\hline$S_{n-126}$ & 0 & 0 & 0 & 0 & 0 & 0 & 0 \\
\hline $1-129$ & 0 & 0 & 0 & 0 & 0 & 0 & 0 \\
\hline C $=134$ & 0 & 0 & 0 & 0 & 0 & 0 & 0 \\
\hline Cs- 137 & 0 & 0 & 0 & 0 & 0 & 0 & 0 \\
\hline $\mathrm{Ba}+137 \mathrm{~m}$ & 0 & 0 & 0 & 0 & 0 & 0 & 0 \\
\hline $5 m-151$ & 0 & 0 & 0 & 0. & 0 & 0 & 0 \\
\hline Eu-152 & 0 & 0 & 0 & 0 & 0 & 0 & 0 \\
\hline Eu-154 & 0 & 0 & 0 & 0 & 0 & 0 & 0 \\
\hline Eu-155 & 0 & 0 & 0 & 요 & 0 & 0 & 0 \\
\hline Ra-226 & 0 & 0 & 0 & 0 & 0 & 0 & 0 \\
\hline Ra-228 & 0 & 0 & 0 & 0 & 0 & 0 & 0 \\
\hline$A c-227$ & 0 & 0 & 0 & 0 & 0 & 0 & 0 \\
\hline $\mathrm{Pa}-231$ & 0 & 0 & 0 & 0 & 0 & 0 & 0 \\
\hline Th-229 & 0 & 0 & 0 & 0 & 0 & 0 & 0 \\
\hline Th-232 & $\theta$ & 0 & 0 & 0 & 0 & 0 & 0 \\
\hline U-232 & 0 & 0 & 0 & 0 & 0 & 0 & 0 \\
\hline U-233 & 0 & 0 & 0 & 0 & 0 & 0 & 0 \\
\hline U-234 & 0 & 0 & 0 & 0 & 0 & 0 & 0 \\
\hline $\mathrm{U} \cdot 235$ & 0 & 0 & 0 & 0 & 0 & 0 & 0 \\
\hline $\mathrm{U}=236$ & 0 & 0 & 0 & 0 & 0 & 0 & 0 \\
\hline U-238 & 0 & 0 & 0 & 0 & 0 & 0 & 0 \\
\hline $\mathrm{Np}-237$ & 0 & 0 & 0 & 0 & 0 & 0 & 0 \\
\hline Pu-238 & 0 & 0 & 0 & 0 & 0 & 0 & 0 \\
\hline Pu-239 & 0 & 0 & 0 & 0 & 0 & 0 & 0 \\
\hline Pu-240 & 0 & 0 & 0 & 0 & 0 & 0 & 0 \\
\hline Pu-241 & 0 & 0 & 0 & 0 & 0 & 0 & 0 \\
\hline $\mathrm{Pu}-242$ & 0 & 0 & 0 & 0 & 0 & 0 & 0 \\
\hline Am-241 & 0 & 0 & 0 & 0 & 0 & 0 & 0 \\
\hline Am-243 & 0 & 0 & 0 & 0 & 0 & 0 & 0 \\
\hline $\mathrm{Cm}-242$ & 0 & 0 & 0 & 0 & 0 & 0 & 0 \\
\hline $\mathrm{Cm}-243$ & 0 & 0 & 0 & 0 & 0 & 0 & 0 \\
\hline $\mathrm{Cm}-244$ & 0 & 0 & 0 & 0 & 0 & 0 & 0 \\
\hline Totals & $\mathbf{M}$ & $\boldsymbol{n} \mathrm{n} / \mathrm{s}$ & Lo & $\begin{array}{c}\text { SCl } \\
(\mathrm{M} \text { ) } \\
(\mathrm{L})\end{array}$ & $\begin{array}{l}67 \mathrm{CI} \\
\mathrm{GHor} \\
/ \mathrm{L})\end{array}$ & $\begin{array}{l}+67 \mathrm{CP} \\
(\mathrm{Mor} \\
\mathrm{NC}\end{array}$ & $\begin{array}{c}+95 \mathrm{CI} \\
(\mathrm{Mor} \\
\mathrm{AL})\end{array}$ \\
\hline $\mathrm{Pu}$ & $0(\mathrm{~g} / \mathrm{L})$ & - & 0 & 0 & 0 & 0 & 0 \\
\hline $\mathrm{U}$ & 0 & 0 & 0 & 0 & 0 & 0 & 0 \\
\hline
\end{tabular}

- Density is calculated based on $\mathrm{Na}, \mathrm{OH}$-, and $\mathrm{AJO2}$.

†Water wt\% derived from the difference of density and total dissolved spocies. 
HDW Model Rov, 4

\begin{tabular}{|c|c|c|c|c|c|c|c|}
\hline \multicolumn{8}{|c|}{ Single-Shell Tank 24I-SX-109 } \\
\hline & \multicolumn{7}{|c|}{ Total Inventory Estimate* } \\
\hline $\begin{array}{l}\text { Phystcil } \\
\text { Propertles }\end{array}$ & & & & $-95 \mathrm{CI}$ & $-67 \mathrm{CI}$ & $+67 \mathrm{CI}$ & $+95 \mathrm{CI}$ \\
\hline Totnl Waste & $1.63 \mathrm{E}+06(\mathrm{~kg})$ & $(250 \mathrm{kgal})$ & $\infty$ & 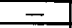 & - & $\ldots$ & - \\
\hline Hent Load & $8.61(\mathrm{~kW})$ & $(2,94 \mathrm{E}+04$ BTU/hr) & - & 8.32 & 8.49 & 8.71 & 8.81 \\
\hline Builk Density & $1.72(0 / \mathrm{cc})$ & - & - & 1.51 & 1.62 & 1.78 & 1.81 \\
\hline & & & & & & & \\
\hline Woter wt\%t & 33.1 & $\cdots$ & $\ldots$ & 25.2 & 28.8 & 41.2 & 49.4 \\
\hline TOC wt\% C (w) & $3,97 E-03$ & $\ldots$ & $\ldots$ & 3.7.EE-03 & $3.83 E-03$ & 4.23E-03 & $4.69 \mathrm{E}-03$ \\
\hline $\begin{array}{l}\text { Rodplosed } \\
\text { Conntlituents }\end{array}$ & $\mathrm{Cl}$ & $\mu C V_{s}$ & $\mathrm{Cl}$ & $\begin{array}{l}90 \mathrm{CL} \\
(\mathrm{CH} / \mathrm{L})\end{array}$ & $\begin{array}{l}6701 \\
(c / 1)\end{array}$ & $\begin{array}{l}+67 \mathrm{C} \\
(\mathrm{Cl} / \mathrm{l})\end{array}$ & $\begin{array}{l}+95 \mathrm{CI} \\
\text { (CV) }\end{array}$ \\
\hline $\mathrm{H}-3$ & $1.84 \mathrm{E}-04$ & 0.107 & 174 & $1.46 \mathrm{E}-05$ & $1.46 \mathrm{E}-05$ & $1.92 \mathrm{E}-04$ & 2.08E-04 \\
\hline C-14 & $9.50 \mathrm{E}-06$ & S.SIE- 03 & 8.99 & S.03E-07 & $0.03 \mathrm{E}-07$ & 9.85E-06 & $1.06 \mathrm{E}-05$ \\
\hline Ni-59 & $1.30 \mathrm{E}-05$ & 7.54E-03 & 12.3 & $7.80 \mathrm{E}-06$ & $7.80 \mathrm{E}-\infty 6$ & $1.33 \mathrm{E}-05$ & 1.35E-OS \\
\hline $\mathrm{Ni}-63$ & I 26E-03 & 0.712 & $1.19 \mathrm{E}+03$ & $7.57 \mathrm{E}-04$ & $7.57 \mathrm{E}-04$ & $1.29 \mathrm{E}-03$ & $1.31 E-03$ \\
\hline $\operatorname{co-60}$ & $8.43 \mathrm{E}-06$ & $4.90 \mathrm{E}-03$ & 7.98 & $6.10 E-07$ & $6.10 \mathrm{E}-07$ & $8.77 E-06$ & 9.48E-06 \\
\hline Se-79 & 7.60E-O6 & $4.41 \mathrm{E}-03$ & 7.19 & $2.31 \mathrm{E}-\infty$ & $2.31 E-06$ & $9.99 \mathrm{E}-06$ & $1.23 \mathrm{E}-05$ \\
\hline Sr-90. & 1.16 & 675 & $1.10 \mathrm{E}+06$ & 1.11 & 1.14 & $1,1 \mathrm{~B}$ & 1.19 \\
\hline$Y-90$ & 1.16 & 675 & $1.10 \mathrm{E}+06$ & 0.962 & 0.962 & 1.18 & 1.19 \\
\hline Zr-93 & $3.4 \pi-05$ & $2.02 \mathrm{E}-02$ & 32.9 & $9.73 \mathrm{E}-06$ & $9.73 \mathrm{E}-06$ & 4.67E-05 & $5.81 \mathrm{E}-05$ \\
\hline $\mathrm{Nb}-93 \mathrm{~m}$ & $2.95 \mathrm{E}-05$ & $1.71 \mathrm{E}-02$ & 27.9 & $9.17 \mathrm{E}-06$ & $9.17 \mathrm{E}-06$ & $3.78 \mathrm{E}-05$ & 4.59E-05 \\
\hline Te-99 & $7.37 \mathrm{E}-0 \mathrm{~S}$ & $4.28 \mathrm{E}-02$ & 69.7 & $7,12 \mathrm{E}-05$ & $7.16 \mathrm{E}-05$ & $7.87 \mathrm{E}-05$ & 845E-05 \\
\hline Ru-106 & $1.93 \mathrm{E}-09$ & 1.12E-06 & $1.63 \mathrm{E}-03$ & $1.23 \mathrm{E}-10$ & $1.23 \mathrm{E}-10$ & $2.210-09$ & $7.95 \mathrm{E}-09$ \\
\hline Cd-113m & $3.83 \mathrm{E}-0 \mathrm{~s}$ & $2.22 \mathrm{E}-02$ & 36.2 & $3.09 \mathrm{E}-06$ & $3.09 \mathrm{E}-06$ & B. 13E-05 & 1.44E-04 \\
\hline Sb-125 & 2.99E-05 & $1.74 \mathrm{E}-02$ & 28.3 & 1.98E-05 & $1.98 \mathrm{E}-06$ & $3,12 \mathrm{E}-0 \mathrm{~S}$ & 3.37E-0s \\
\hline $\mathrm{Sn}-126$ & $1.10 \mathrm{E}-05$ & 6.41E-03 & 10.5 & 2.94E-06 & 2.94E-06 & $1.46 \mathrm{E}-05$ & 1.1E-05 \\
\hline I-129 & $1.40 \mathrm{E}-07$ & C.12E-05 & 0.132 & $1,35 \mathrm{E}-0)$ & 1.36E-07 & $1.49 \mathrm{E}-07$ & $1.61 \mathrm{E}-07$ \\
\hline $\mathrm{C} 2-134$ & $1.91 \mathrm{E}-00$ & 1.11E-03 & I.8I & $1.60 \mathrm{E}-07$ & 1.60E-07 & $1.98 \mathrm{E}-06$ & $2.12 \mathrm{E}-06$ \\
\hline $\mathrm{Ce}-137$ & 0.273 & 151 & $2.50 E+05$ & 0.265 & 0.266 & 0.291 & 0.311 \\
\hline $\mathrm{Ba}-137 \mathrm{~m}$ & 0.258 & 150 & $244 E+05$ & $2.52 \mathrm{E}-02$ & $2.52 \mathrm{E}-02$ & 0.267 & 0.285 \\
\hline Sm-15I & 2.71E-02 & 15.8 & $2.57 \mathrm{E}+04$ & $8.30 \mathrm{E}-03$ & $8.305-03$ & $3.55 \mathrm{E}-02$ & 4.35E-02 \\
\hline Eu-152 & $1.51 \mathrm{E}-05$ & 879E-03 & 14.3 & $8.40 \mathrm{E}-06$ & $8.40 E-06$ & 1.52E-05 & $1.53 \mathrm{E}-05$ \\
\hline Eu-154 & $202 \mathrm{E}-04$ & 0.117 & 191 & $1.48 \mathrm{E}-05$ & 1.48E-0S & $2.10 \mathrm{E}-04$ & $6.92 \mathrm{E}-04$ \\
\hline Eu-15s & $7.67 \mathrm{E}-04$ & 0.445 & 725 & 4.25E-04 & $4.25 E-04$ & $7,69 \mathrm{E}-04$ & $7.73 \mathrm{E}-04$ \\
\hline Ra-226 & $1.02 \mathrm{E}-09$ & 5.93E-07 & 9.67E-04 & $6.65 \mathrm{E}-10$ & 6.65E-50 & $1.15 E-09$ & $1.27 \mathrm{E}-09$ \\
\hline $\mathrm{Ra-228}$ & 1.20E-0s & $6.95 \mathrm{E}-06$ & $1.13 E-02$ & $1.42 \mathrm{E}-14$ & $1,42 \mathrm{E}-14$ & $1.21 \mathrm{E}-\mathrm{OB}$ & 1.22E-08 \\
\hline Ac-227 & S.04E-09 & $293 E-06$ & 4.77E-03 & $2.96 \mathrm{E}-09$ & $2.96 \mathrm{e}-09$ & $5.79 \mathrm{E}-09$ & $6.26 E-09$ \\
\hline$P_{4-231}$ & $1.03 \mathrm{E}-08$ & $5.99 \mathrm{E}-06$ & S.T7E-03 & $2.46 \mathrm{E}-09$ & 2. $46 \mathrm{E}-09$ & $1.27 \mathrm{E}-0 \mathrm{~s}$ & $1,50 \mathrm{E}-08$ \\
\hline \begin{tabular}{|l|} 
Th-229 \\
\end{tabular} & 2.80E-10 & $1.6 \mathrm{E}-07$ & $2.73 \mathrm{E}-\mathrm{A4}$ & $1.09 \mathrm{E}-12$ & 1.09E-12 & $2.91 \mathrm{E}-10$ & 2.94E-10 \\
\hline Th-232 & $1.60 \mathrm{E}-10$ & 9.32E-04 & $1.52 \mathrm{E}-04$ & $5.01 \mathrm{E}-16$ & $5.0 \mathrm{tE}-16$ & $2.02 E-10$ & $2.43 \mathrm{E}-10$ \\
\hline U-232 & $5.35 E-08$ & $3.11 \mathrm{E}-05$ & $5.06 \mathrm{E}-02$ & 1.09E-08 & $3.49 \mathrm{E}-0 \mathrm{~s}$ & 7.62E-08 & $1.01 \mathrm{E}-07$ \\
\hline $\mathrm{U}-233$ & 2.05E-97 & 1.19E-04 & 0.194 & 7.24E-08 & $1.34 \mathrm{E}-07$ & $2.92 \mathrm{E}-07$ & 3. 18 E- 07 \\
\hline $\mathrm{U}-234$ & $9.97 E-07$ & $5.790-04$ & 0.943 & 4.53E-07 & $7.18 \mathrm{E}-07$ & 1.30E-06 & $1.60 \mathrm{E}-06$ \\
\hline L-235 & 4.03E- $0 \mathrm{~s}$ & 2.34E-0S & $3.81 \mathrm{E}-02$ & $1.83 \mathrm{E}-08$ & $2.90 \mathrm{E}-08$ & $5.26 \mathrm{E}-08$ & $6.49 \mathrm{E}-08$ \\
\hline U-236 & $4.10 \mathrm{E}-0 \mathrm{~B}$ & $2.38 \mathrm{E}-0 \mathrm{~s}$ & 3. $38 \mathrm{E}-02$ & $1.92 E-08$ & $3.00 \mathrm{E}-08$ & $5.25 \mathrm{E}-0 \mathrm{~s}$ & $6.38 \mathrm{E}-08$ \\
\hline $\mathrm{U}-238$ & 8.75E-07 & $5,02 E-04$ & 0.828 & $3.96 \mathrm{E}-07$ & $6.28 \mathrm{E}-07$ & $1.14 \mathrm{E}-06$ & 1.41E-06 \\
\hline$N p-237$ & $3.25 \mathrm{E}-07$ & 1.98E-04 & 0.307 & 3.09E-07 & 3.15E-07 & $3.49 \mathrm{E}-07$ & $3.76 \mathrm{E}-07$ \\
\hline Pu-238 & 7.77E-06 & $4.51 \mathrm{E}-03$ & 7.35 & $6.51 E-06$ & $7.13 \mathrm{E}-06$ & 8.41E-06 & $9,03 E-06$ \\
\hline Pu-239 & $2.34 \mathrm{E}-04$ & 0.130 & 222 & 1.94E-04 & $2.14 \mathrm{E}-04$ & $2.55 \mathrm{E}-04$ & $2.75 \mathrm{E}-04$ \\
\hline $\mathrm{Pu}-240$ & 3.95E-05 & $2.30 E-02$ & 37.4 & $3.21 \mathrm{E}-0 \mathrm{~S}$ & $3.61 \mathrm{E}-05$ & $4.30 \mathrm{E}-05$ & 4.63E-05 \\
\hline$P(v-24 !$ & $3.93 \mathrm{E}-04$ & 0.228 & 372 & $3.29 \mathrm{E}-04$ & $3.60 \mathrm{E}-04$ & $4.26 \mathrm{E}-04$ & $4.57 \mathrm{E}-04$ \\
\hline Pu-242 & $2.13 E-09$ & $1.24 E-06$ & $2.02 \mathrm{E}-03$ & $1.79 \mathrm{E}-09$ & $1.96 \mathrm{E}-09$ & $2.31 E-09$ & $2.495-09$ \\
\hline Am-241 & $2.31 E-04$ & 0.134 & 219 & $1.61 \mathrm{E}-04$ & 1.95E-04 & $2.67 \mathrm{E}-04$ & $3.01 \mathrm{E}-04$ \\
\hline Am-243 & 9.19E-09 & $5.34 \mathrm{E}-06$ & 8.70E-03 & $6.02 \mathrm{E}-09$ & $7.58 \mathrm{E}-09$ & 1.08E-08 & $1.24 \mathrm{E}-08$ \\
\hline $\mathrm{Cm}-242$ & $3.69 \mathrm{E}-07$ & $2.14 \mathrm{E}-04$ & 0.349 & $3.65 \mathrm{E}-07$ & $3.67 \mathrm{E}-07$ & $3.69 \mathrm{E}-07$ & $3.69 \mathrm{E}-07$ \\
\hline $\mathrm{Cm}-243$ & $3.51 \mathrm{E}-08$ & 2.04E-05 & 3.32E 02 & $3.50 \mathrm{E}-08$ & 3,48E-08 & $3.51 \mathrm{E}-08$ & 3.51E-0E \\
\hline $\mathrm{Cm}-244$ & $1.04 E-\infty$ & 6.05E-04 & 0.985 & $8.07 \mathrm{E}-07$ & $9,22 \mathrm{E}-07$ & $1.16 \mathrm{E}-06$ & $1.28 \mathrm{E}-06$ \\
\hline Totals: & $\mathbf{M}$ & 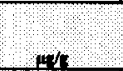 & $\mathrm{l}_{\mathrm{n}}$ & $\begin{array}{l}3 \mathrm{~d} \\
(\mathrm{Mqr} \\
\mathrm{N} / \mathrm{d})\end{array}$ & $\begin{array}{l}6 \mathrm{CO} \\
(\mathrm{M} \mathrm{d}) \\
(1 \mathrm{~d})\end{array}$ & 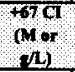 & $\begin{array}{l}+5 \mathrm{Cl} \\
(\mathrm{M} \text { or } \\
\text { (1) }\end{array}$ \\
\hline Pu & $3.93 \mathrm{E}-03(r / \mathrm{L})$ & - & 3.72 & $3.25 \mathrm{E}-03$ & $3.59 \mathrm{E}-03$ & 4.28E-03 & $4.62 \mathrm{E}-03$ \\
\hline$\overline{\mathbf{U}}$ & $1.08 \mathrm{E}-02$ & $1.49 \mathrm{E}+03$ & $2.44 \mathrm{E}+03$ & $4.67 \mathrm{E}-03$ & $7.75 \mathrm{E}-03$ & 1.42E-02 & $1.75 \mathrm{E}-02$ \\
\hline
\end{tabular}

- Unknowns in tank solida imventory are ansigned by Tank Layering Model (TLM).

$\uparrow$ Volume average for density, mass average Water wt\% and TOC w $\% \mathrm{C}$. 
G. K. Patel10

P7-28

PAGE 3 OF 5

L. R. Pederson

$\mathrm{K} 2-44$

K. M. Remund

$\mathrm{K} 5-12$

P. A. Scott

K9-46

$x$

G. L. Smith

$\mathrm{K} 7-74$

$\mathrm{K} 7-94$

$\mathrm{H} 2-53$

T. L. Traub

P8-37

K6-51

$\mathrm{K} 5-12$

$\mathrm{K} 4-16$

K. D. Wiemers

P. D. Whitney

J. Y. Young

T6-30 $\quad X$

T6-16 X

Rust Federal Services Hanford, Inc.

T. H. Bushaw

C. T. Narquis

$\hat{X}$

SGN Eurisys Services Corp.

D. B. Engelman

B4-51

H6-12

H5-27

D. E. Place

L5-55

S3-90

B4-51

E. F. Riedel

K. V. Scott

\section{OFFSITE}

LOS Alamos National Laboratory

S. F Agnew

Los Alamos National Laboratory

CST-14, MS-J586

Bikini Atoll Rd, SM30

Los ATamos, NM 87545

Los Alamos Technical Associates

T. T. Tran

$X$

903 Bradley Boulevard

Richland, Washington 99352

Ogden Evironmental

C. R. Ungerich

$x$

1404 Potter

Richland, Washington 99352

Tank Advisory Panel

D. 0. Campbe11

$x$

102 Windham Road

0ak Ridge, TN 37830 
D. Powers

Sandia National Laboratory

P. 0. Box 5800

MS-0744, Dept. 6404

Albuquerque, NM 87815

J. L. Kovach

$x$

Nuclear Consulting Services Inc.

P. 0. Box 29151

Columbus, $\mathrm{OH}$ 43229-0151

B. C. Hudson

Chemical Reaction Sub-TAP

$x$

202 Northridge Court

Lindsborg, KS 67456

Department of Energy - Headquarters

K. T. Lang

$x$

J. A. Poppitti

U. S. Department of Energy

Office of Environmental Restoration

and Waste Management (EM-563)

12800 Middlebrook Road

Germantown, MD 20874

Science Applications International Corporation

H. Sutter

$x$

555 Quince Orchard Rd.

Suite 500

Germantown, MD 20874

Columbia Bas in College

D. E. Campbe 11

$x$

2600 North 20th Avenue

Pasco, Washington 99301

Wastren Inc.

E. E. Oscarson

B2-69 X

Wastren, Inc.

$1050 \mathrm{Gilmore}$ Ave, Suite C

Richland, WA 99352

MACTEC - ERS

J. R. Brodeur

B1-45 X

MACTEC - ERS

303 Bradley Boulevard, Suite 104

Richland, WA 99352 
HNF-SD-WM-ER-352, Rev. 1 DISTRIBUTION SHEET

PAGE 5 OF 5

Washington State Department of Ecology

A. B. Stone

B5-18

$x$ Washington State Department of Ecology 1315 W. 4th

Kennewick, WA 99336 


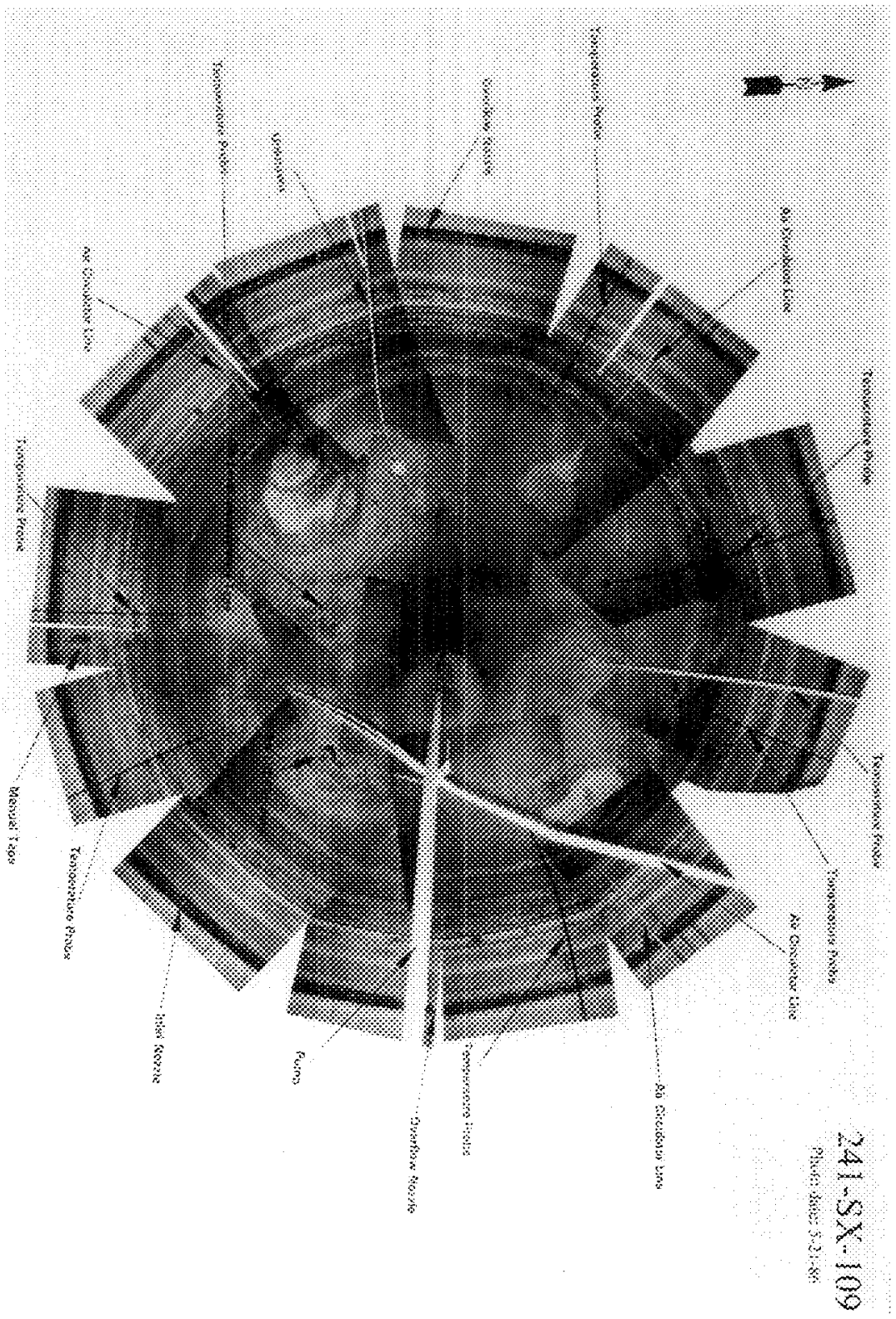


TANK 241-SX-110 SUMMARY

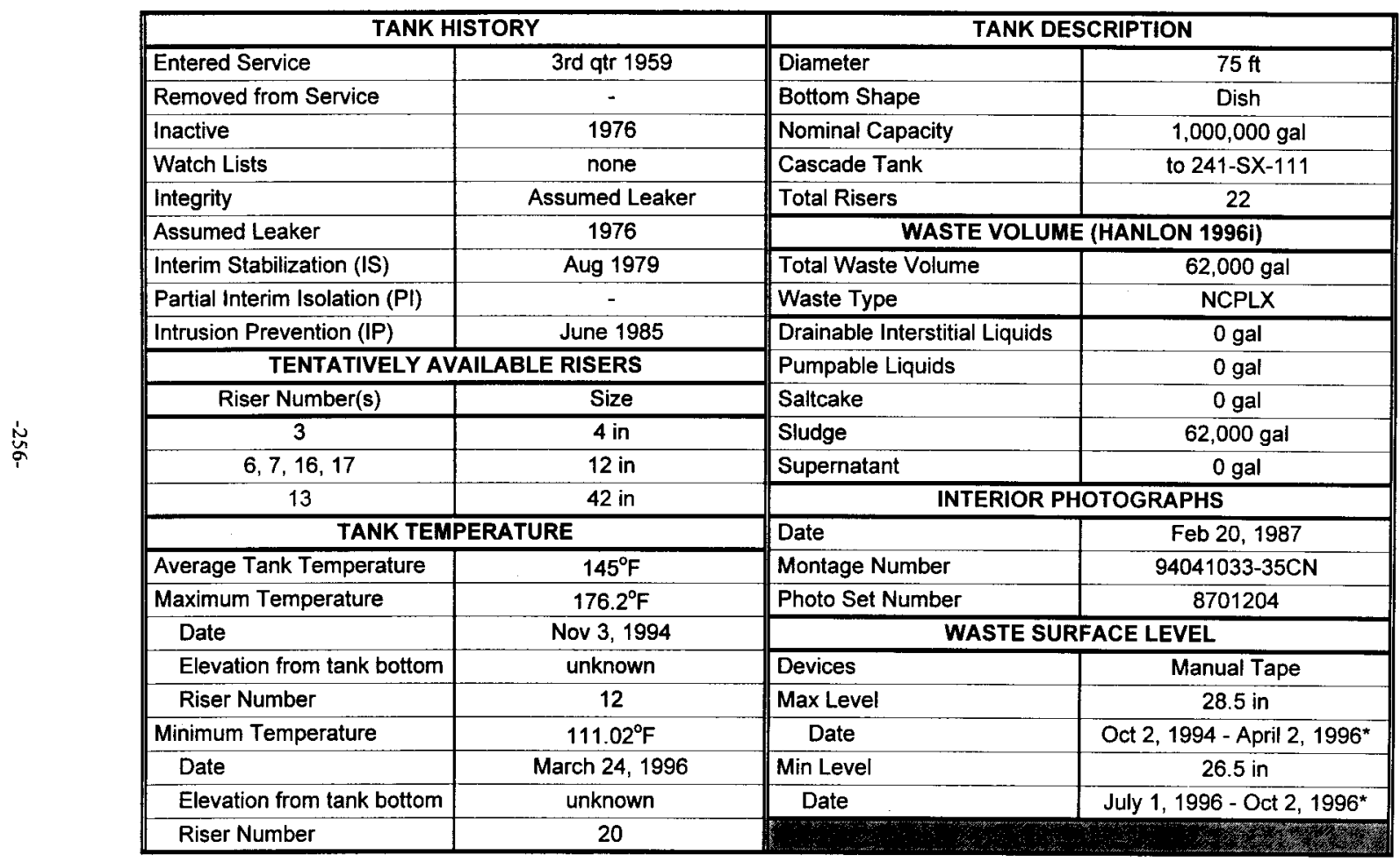

\footnotetext{
- Numerous dates in this time span
} 


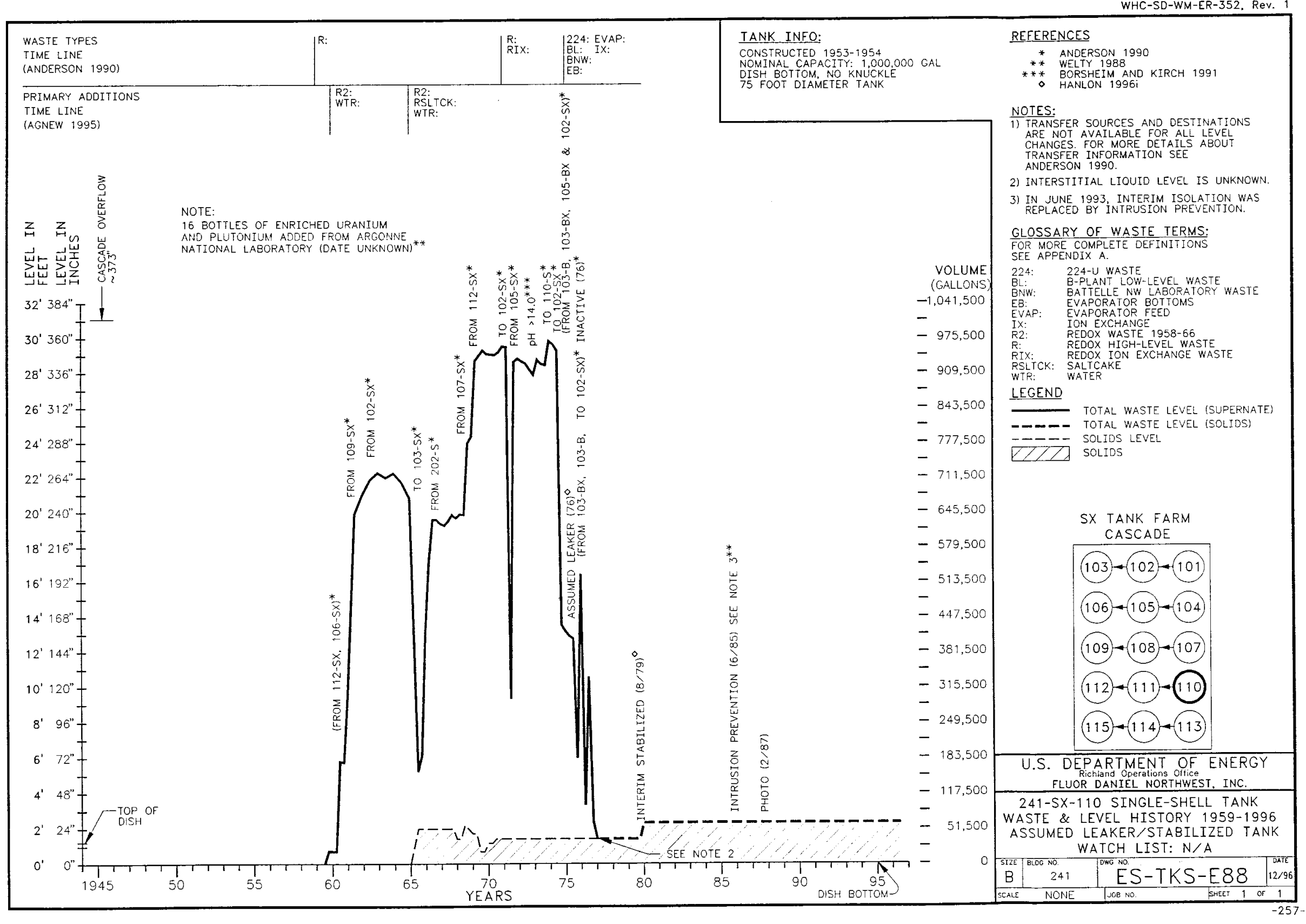


HOW Model Rẹv. 4

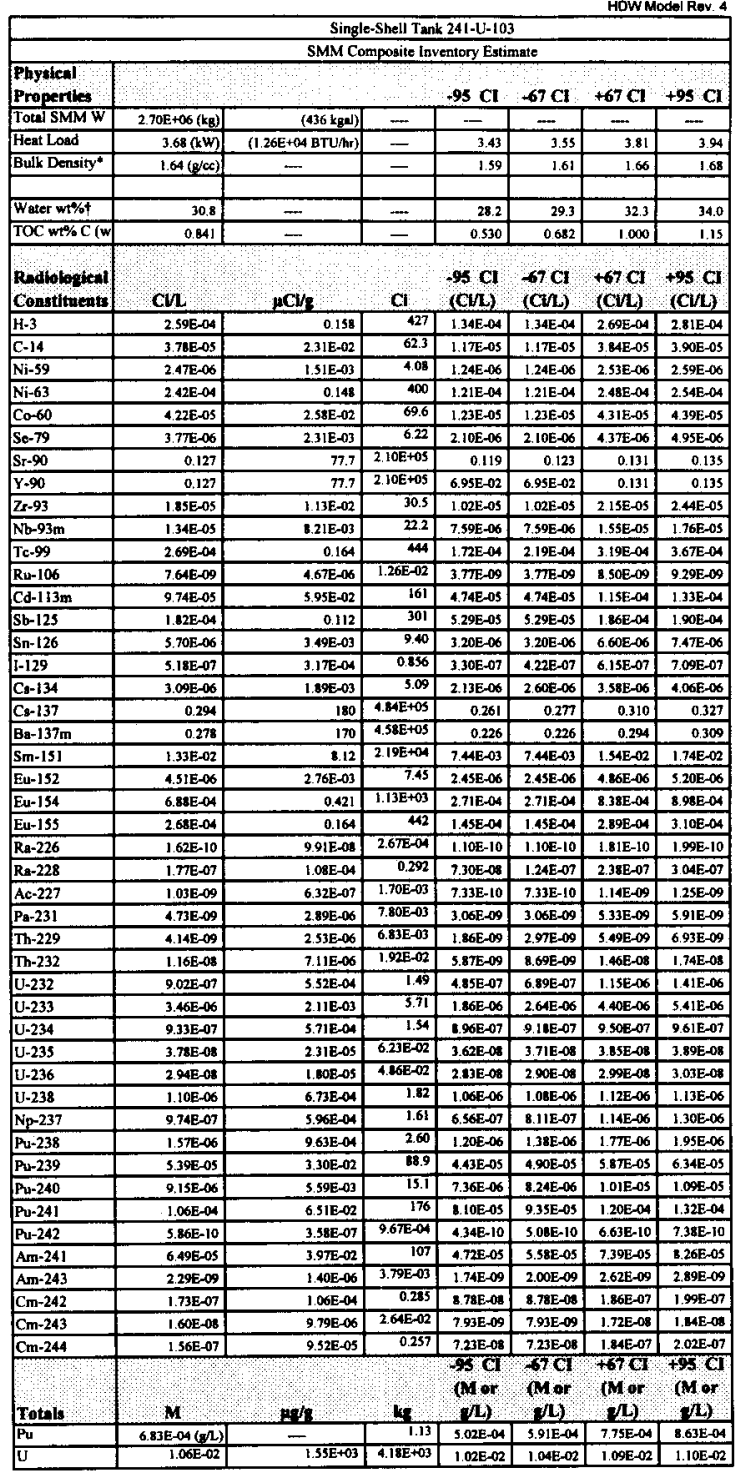

-Density is calculated boued on $\mathrm{Ne}, \mathrm{OH}-$, and AlO2-

Water wr\% derived from the difference of density and total diswolved apecies. 